Supporting Information for

\title{
Diverse Asymmetric Hydrofunctionalization of Aliphatic Internal Alkenes Through Catalytic Regioselective Hydroboration
}

\author{
Yumeng Xi and John F. Hartwig* \\ Division of Chemical Sciences, Lawrence Berkeley National Laboratory, \\ and Department of Chemistry, University of California, Berkeley, CA 94720, United States \\ Email: jhartwig@berkeley.edu
}




\section{Table of Contents}

$\begin{array}{ll}\text { I. General Methods and Materials } & \text { S3 }\end{array}$

II. General Procedure for Catalytic Hydroboration $\quad$ S4

$\begin{array}{lr}\text { III. Compounds Characterization } & \text { S5-S12 }\end{array}$

IV. Oxidation of Secondary Boronates $\quad$ S13-S17

V. Derivatization of Secondary Boronates $\quad$ S18-S23

$\begin{array}{ll}\text { VI. Synthesis of Substrates } & \text { S24-S26 }\end{array}$

$\begin{array}{ll}\text { VII. Summary of the Effect of Reaction Parameters } & \text { S27 }\end{array}$

VIII. Hydroboration of Homoallyl Benzoate Derivatives and Homoallyl Acetate (5) and Pivalate

S28

IX. Effect of Olefin Position and Geometry on the Hydroboration $\quad$ S29

$\begin{array}{ll}X . \text { Hydroboration of 1a on a Gram Scale } & \text { S30 }\end{array}$

XI. Assignment of Absolute Stereochemistry $\quad$ S31

XII. Crystallographic Information of Compound 2c S32-S45

$\begin{array}{lr}\text { XIII. DFT Calculation } & \text { S46-S128 }\end{array}$

$\begin{array}{lr}\text { XIV. Reference } & \text { S129 }\end{array}$

XV. SFC and HPLC Traces $\quad$ S130-S149

XVI. NMR Spectra $\quad$ S149-S256 


\section{General Methods and Materials}

All reagents were purchased from commercial suppliers, stored in the glove box and used as received. Glassware was dried at $130{ }^{\circ} \mathrm{C}$ for at least 4 hours before use. Anhydrous cyclohexane was purchase from Aldrich, stored in a dry box, and used as received without further purification. $\mathrm{CuCl}$ was purchased from Aldrich. ( $S$ )-DTBM-SEGPHOS was used as received from Takasago. $(R)$-DTBM-SEGPHOS was purchased from Strem. Pinacolborane (HBPin) was purchased from Aldrich and stored in a dry box. All catalytic reactions were set up in an argon-filled dry box with oven-dried glassware and were stirred with Teflon-coated magnetic stirring bars. All products for hydroboration, oxidation and derivatization were purified by flash column chromatography with a Teledyne Isco CombiFlash ${ }^{\circledR} \mathrm{R}_{f}$ system using RediSep $\mathrm{R}_{f}$ Gold $^{\mathrm{TM}}$ columns.

${ }^{1} \mathrm{H}$ NMR spectra were recorded on Bruker AVB-400, AVQ-400, AV-500 and AV-600 instruments with $400,400,500$, and $600 \mathrm{MHz}$ frequencies, and ${ }^{13} \mathrm{C}$ were recorded on a Bruker AV-600 instrument with a ${ }^{13} \mathrm{C}$ operating frequency of $150 \mathrm{MHz} .{ }^{19} \mathrm{~F}$ NMR spectra were recorded on a Bruker AVQ-400 spectrometer with a

${ }^{19} \mathrm{~F}$ operating frequency of $376 \mathrm{MHz}$. Chemical shifts $(\delta)$ are reported in ppm relative to the residual solvent signal $\left(\mathrm{CDCl}_{3} \delta=7.26\right.$ for ${ }^{1} \mathrm{H}$ NMR and $\delta=77.0$ for ${ }^{13} \mathrm{C}$ NMR $=7.26 \mathrm{ppm}$; acetone- $d_{6}=2.05 \mathrm{ppm}$ for ${ }^{1} \mathrm{H}$ and $29.9 \mathrm{ppm}$ for ${ }^{13} \mathrm{C}$ ). Crude reaction mixtures were analyzed by ${ }^{1} \mathrm{H}$ NMR spectroscopy recorded on the Bruker AV-500. Quantitative analysis by GC was performed with dodecane as an internal standard. High-resolution mass spectral data were obtained with a Thermo Finnigan LTQ FT Instrument in the QB3/Chemistry Mass Spectrometry Facility, University of California, Berkeley. GC analysis was performed on an Agilent 7890 GC equipped with an HP-5 column ( $25 \mathrm{~m} \times 0.20 \mathrm{~mm} \times 0.33 \mu \mathrm{m}$ film) and an FID detector. Chiral SFC analysis was conducted on a JASCO SFC system. Chiral HPLC analysis was conducted on Waters chromatography system. Racemic samples were obtained using racemic DTBM-SEGPHOS as the ligand, which was prepared by mixing equal amounts of $(S)$-DTBM-SEGPHOS and $(R)$-DTBM-SEGPHOS. 


\section{General Procedure for Catalytic Hydroboration}

Representative procedure for the catalytic hydroboration on a $0.4 \mathrm{mmol}$ scale using Condition A (General Procedure)

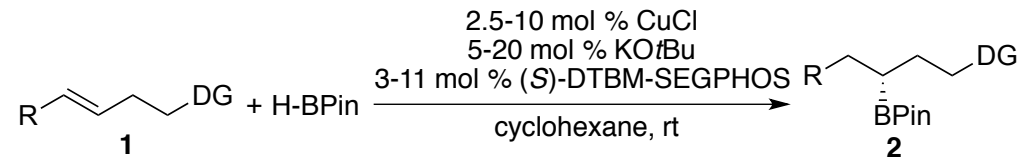

In an argon-filled dry box, a 1-dram vial was charged with $\mathrm{CuCl}(1.0 \mathrm{mg}, 2.5 \mathrm{~mol} \%), \mathrm{KO} t \mathrm{Bu}(2.2 \mathrm{mg}, 5 \mathrm{~mol}$ $\%),(S)$-DTBM-SEGPHOS (14.2 mg, $3 \mathrm{~mol} \%)$ and cyclohexane (400 $\mu \mathrm{L})$. The mixture was allowed to stir at ambient temperature for 3 minutes before the addition of pinacolborane ( $71 \mu \mathrm{L}, 0.48 \mathrm{mmol}, 1.2$ equiv). After brief stirring ( $30-60$ seconds), the solution was added the alkene ( $0.4 \mathrm{mmol}, 1$ equiv), which was pre-weighed in another vial. The second vial was then washed four times with $100 \mu \mathrm{L}$ cyclohexane each time. If the alkene is a solid, it was added in one portion, followed by cyclohexane $(400 \mu \mathrm{L})$. The vial was then capped, sealed with electrical tape, and removed from the box. After $36 \mathrm{~h}$ of stirring at $\mathrm{rt}$, an aliquot of $20 \mu \mathrm{L}$ was removed and analyzed by GC. The reaction vial was diluted with $2 \mathrm{~mL}$ of ethyl acetate and the resulting solution was filtered through Celite. The crude material was concentrated in vacuo and purified by flash column chromatography (CombiFlash) to afford the pure product.

Summary of Conditions A-D in Table 2:

Condition A: Alkene (1 equiv), pinacolborane (1.2 equiv), $\mathrm{CuCl}(2.5 \mathrm{~mol} \%), \mathrm{KO} t \mathrm{Bu}(5 \mathrm{~mol} \%),(S)-\mathrm{DTBM}-$ SEGPHOS (3 mol \%), $36 \mathrm{~h}$, rt.

Condition B: Alkene (1 equiv), pinacolborane (1.2 equiv), $\mathrm{CuCl}$ (5 mol \%), $\mathrm{KO} t \mathrm{Bu}(10 \mathrm{~mol} \%),(S)$-DTBMSEGPHOS (5.5 mol \%), $36 \mathrm{~h}$, rt.

Condition C: Alkene (1 equiv), pinacolborane (2 equiv), $\mathrm{CuCl}(10 \mathrm{~mol} \%), \mathrm{KO} t \mathrm{Bu}(20 \mathrm{~mol} \%),(S)-\mathrm{DTBM}-$ SEGPHOS (11 mol \%), $84 \mathrm{~h}$, rt.

Condition D: Alkene (3 equiv), pinacolborane (1 equiv), $\mathrm{CuCl}$ (10 mol \%), $\mathrm{KO} t \mathrm{Bu}(20 \mathrm{~mol} \%),(S)-\mathrm{DTBM}-$ SEGPHOS (11 mol \%), $84 \mathrm{~h}$, rt. 


\section{Compound Characterization}<smiles>CCC(CCOC(=O)c1c(Cl)cc(Cl)cc1Cl)[Pb]c1ccccc1</smiles>

Compound 2a was synthesized according to the General Procedure Condition A with 1a (123 mg, 0.400 mmol), HBPin (71 $\mu \mathrm{L}, 0.48 \mathrm{mmol}, 1.2$ equiv), $\mathrm{CuCl}(1.0 \mathrm{mg}, 2.5 \mathrm{~mol} \%), \mathrm{KO} t \mathrm{Bu}(2.2 \mathrm{mg}, 5 \mathrm{~mol} \%)$, and $(S)$ DTBM-SEGPHOS (14.2 mg, $3 \mathrm{~mol} \%$ ). Compound 2a was purified by flash column chromatography (5\% ethyl acetate in hexane) and obtained as a colorless oil in $80 \%$ yield.

${ }^{1} \mathrm{H}$ NMR $\left(500 \mathrm{MHz}, \mathrm{CDCl}_{3}\right) \delta 7.32(\mathrm{~s}, 2 \mathrm{H}), 4.46-4.31(\mathrm{~m}, 2 \mathrm{H}), 1.91-1.74(\mathrm{~m}, 2 \mathrm{H}), 1.50-1.21(\mathrm{~m}, 5 \mathrm{H})$, $1.21(\mathrm{~s}, 12 \mathrm{H}), 0.88(\mathrm{t}, J=7.0 \mathrm{~Hz}, 3 \mathrm{H})$.

${ }^{13} \mathrm{C}$ NMR $\left(151 \mathrm{MHz}, \mathrm{CDCl}_{3}\right) \delta 163.96,135.81,132.55,132.43,127.82,82.98,66.21,33.12,29.57,24.71$, 24.60, 21.99, 19.68, 14.29.

${ }^{11} \mathrm{~B}$ NMR $\left(192 \mathrm{MHz}, \mathrm{CDCl}_{3}\right) \delta 33.2$.

HRMS (EI+) Calculated for $\mathrm{C}_{19} \mathrm{H}_{26} \mathrm{~B}^{35} \mathrm{Cl}_{3} \mathrm{O}_{4}[\mathrm{M}]^{+}:$434.0990, Found: 434.0989 .

$[\alpha]_{\mathrm{D}}^{23}=-8.2(\mathrm{c}=1.0, \mathrm{DCM})$.

SFC analysis indicated 97\% ee (compound 3a) after oxidation (see section IV for details).<smiles>CC(C)CC(Br)CCOC(=O)c1c(Cl)cc(Cl)cc1Cl</smiles>

Compound 2b was synthesized according to the General Procedure Condition A with 1b (129 mg, 0.401 mmol), HBPin (71 $\mu \mathrm{L}, 0.48 \mathrm{mmol}, 1.2$ equiv), $\mathrm{CuCl}$ (1.0 mg, $2.5 \mathrm{~mol} \%), \mathrm{KO} t \mathrm{Bu}(2.2 \mathrm{mg}, 5 \mathrm{~mol} \%)$, and $(S)$ DTBM-SEGPHOS (14.2 mg, $3 \mathrm{~mol} \%$ ). Compound $\mathbf{2 b}$ was purified by flash column chromatography (5\% ethyl acetate in hexane) and obtained as colorless oil in $80 \%$ yield.

${ }^{1} \mathrm{H}$ NMR (500 MHz, $\left.\mathrm{CDCl}_{3}\right) \delta 7.33$ (s, 2H), $4.44-4.35$ (m, 2H), $1.88-1.76$ (m, 2H), 1.59 (septet, $J=6.8 \mathrm{~Hz}$, $1 \mathrm{H}), 1.44-1.31(\mathrm{~m}, 1 \mathrm{H}), 1.27-1.18(\mathrm{~m}, 2 \mathrm{H}), 1.22(\mathrm{~s}, 12 \mathrm{H}), 0.87(\mathrm{~d}, J=6.6 \mathrm{~Hz}, 6 \mathrm{H})$.

${ }^{13} \mathrm{C}$ NMR $\left(151 \mathrm{MHz}, \mathrm{CDCl}_{3}\right) \delta 164.01,135.84,132.58,132.44,127.85,83.01,66.20,40.18,29.94,27.04$, 24.72, 24.63, 22.97, 22.41, 17.69.

${ }^{11} \mathrm{~B}$ NMR $\left(192 \mathrm{MHz}, \mathrm{CDCl}_{3}\right) \delta 33.7$.

HRMS (EI+) Calculated for $\mathrm{C}_{20} \mathrm{H}_{28} \mathrm{~B}^{35} \mathrm{Cl}_{3} \mathrm{O}_{4}[\mathrm{M}]^{+}: 448.1146$, Found: 448.1141 .

$[\alpha]_{\mathrm{D}}^{23}=-7.5(\mathrm{c}=1.0, \mathrm{DCM})$.

SFC analysis indicated 99\% ee (compound $\mathbf{3 b}$ ) after oxidation (see section IV for details). 
<smiles>CC(C)(C)C[C@@H](Br)c1ccccc1</smiles>

Compound 2c was synthesized according to the General Procedure Condition B with 1c (134 mg, 0.399 mmol), HBPin (71 $\mu \mathrm{L}, 0.48 \mathrm{mmol}, 1.2$ equiv), $\mathrm{CuCl}$ (2.0 mg, $5 \mathrm{~mol} \%), \mathrm{KO} t \mathrm{Bu}(4.4 \mathrm{mg}, 5 \mathrm{~mol} \%$ ), and $(S)$ DTBM-SEGPHOS (25.9 mg, $5.5 \mathrm{~mol} \%$ ). Compound $2 \mathrm{c}$ was purified by flash column chromatography (5\% ethyl acetate in hexane) and obtained as white solid in $79 \%$ yield.

${ }^{1} \mathrm{H}$ NMR $\left(500 \mathrm{MHz}, \mathrm{CDCl}_{3}\right) \delta 7.30(\mathrm{~s}, 2 \mathrm{H}), 4.37(\mathrm{t}, J=7.1 \mathrm{~Hz}, 2 \mathrm{H}), 1.84(\mathrm{dq}, J=14.5,7.3 \mathrm{~Hz}, 1 \mathrm{H}), 1.73(\mathrm{dt}, J$ $=13.7,7.0 \mathrm{~Hz}, 1 \mathrm{H}), 1.57(\mathrm{dd}, J=13.4,10.2 \mathrm{~Hz}, 1 \mathrm{H}), 1.21(\mathrm{~s}, 6 \mathrm{H}), 1.20(\mathrm{~s}, 6 \mathrm{H}), 1.17-1.05(\mathrm{~m}, 2 \mathrm{H}), 0.85(\mathrm{~s}$, 9H).

${ }^{13} \mathrm{C}$ NMR $\left(151 \mathrm{MHz}, \mathrm{CDCl}_{3}\right) \delta 163.93,135.83,132.56,132.38,127.84,83.03,65.81,45.16,31.68,30.96$, 29.57, 24.75, 24.72, 15.52 .

${ }^{11} \mathrm{~B}$ NMR $\left(192 \mathrm{MHz}, \mathrm{CDCl}_{3}\right) \delta 33.8$.

HRMS (ESI+) Calculated for $\mathrm{C}_{21} \mathrm{H}_{30} \mathrm{~B}^{35} \mathrm{Cl}_{3} \mathrm{O}_{4} \mathrm{Na}[\mathrm{M}+\mathrm{Na}]^{+}$: 485.1195 , Found: 485.1191.

$[\alpha]_{\mathrm{D}}^{23}=-10.9(\mathrm{c}=1.0, \mathrm{DCM})$.

SFC analysis indicated 99\% ee (compound 3c) after oxidation (see section IV for details).<smiles>O=C(OCCC(Br)CC1CCCCC1)c1c(Cl)cc(Cl)cc1Cl</smiles>

Compound 2d was synthesized according to the General Procedure Condition A with 1d (145 mg, 0.401 mmol), HBPin (71 $\mu \mathrm{L}, 0.48 \mathrm{mmol}, 1.2$ equiv), $\mathrm{CuCl}(1.0 \mathrm{mg}, 2.5 \mathrm{~mol} \%), \mathrm{KO} t \mathrm{Bu}(2.2 \mathrm{mg}, 5 \mathrm{~mol} \%)$, and $(S)$ DTBM-SEGPHOS (14.2 mg, $3 \mathrm{~mol} \%$ ). Compound 2d was purified by flash column chromatography (5\% ethyl acetate in hexane) and obtained as colorless liquid in $91 \%$ yield.

${ }^{1} \mathrm{H}$ NMR (500 MHz, CDCl$) \delta 7.31(\mathrm{~s}, 2 \mathrm{H}), 4.44-4.30(\mathrm{~m}, 2 \mathrm{H}), 1.88-1.57(\mathrm{~m}, 7 \mathrm{H}), 1.41-1.05(\mathrm{~m}, 7 \mathrm{H})$, $1.21(\mathrm{~s}, 12 \mathrm{H}), 0.91-0.74(\mathrm{~m}, 2 \mathrm{H})$.

${ }^{13} \mathrm{C}$ NMR $\left(151 \mathrm{MHz}, \mathrm{CDCl}_{3}\right) \delta 164.03,135.86,132.60,132.45,127.87,83.01,66.24,38.60,36.76,33.66$, $33.15,30.04,26.60,26.37,26.33,24.77,24.60,17.07$.

${ }^{11} \mathrm{~B}$ NMR (192 MHz, $\left.\mathrm{CDCl}_{3}\right) \delta 34.1$.

HRMS (ESI+) Calculated for $\mathrm{C}_{23} \mathrm{H}_{32} \mathrm{~B}^{35} \mathrm{Cl}_{3} \mathrm{O}_{4} \mathrm{Na}[\mathrm{M}+\mathrm{Na}]^{+}:$511.1347, Found: 511.1351 .

$[\alpha]_{\mathrm{D}}^{23}=-3.3(\mathrm{c}=1.0, \mathrm{DCM})$.

SFC analysis indicated 99\% ee (compound 3d) after oxidation (see section IV for details).<smiles>C/C=C/CCCCC([B]c1ccccc1)CCOC(=O)c1c(Cl)cc(Cl)cc1Cl</smiles> 
Compound 2e was synthesized according to the General Procedure Condition A with 1e (72.3 mg, 0.200 mmol), HBPin (59 $\mu \mathrm{L}, 0.4 \mathrm{mmol}, 2$ equiv), $\mathrm{CuCl}$ (2.0 mg, $10 \mathrm{~mol} \%$ ), KOtBu (4.4 mg, $20 \mathrm{~mol} \%$ ), and $(S)$ DTBM-SEGPHOS (25.9 mg, $11 \mathrm{~mol} \%$ ). Compound $2 \mathrm{e}$ was purified by flash column chromatography (5\% ethyl acetate in hexane) and obtained as colorless liquid in $66 \%$ yield (contains $<5 \%$ constitutional isomers).

${ }^{1} \mathrm{H}$ NMR (500 MHz, $\left.\mathrm{CDCl}_{3}\right) \delta 7.32(\mathrm{~s}, 2 \mathrm{H}), 5.39-5.36(\mathrm{~m}, 2 \mathrm{H}), 4.45-4.30(\mathrm{~m}, 2 \mathrm{H}), 1.98-1.73(\mathrm{~m}, 4 \mathrm{H})$, $1.66-1.55(\mathrm{~m}, 3 \mathrm{H}), 1.49-1.24(\mathrm{~m}, 6 \mathrm{H}), 1.21(\mathrm{~s}, 12 \mathrm{H}), 1.17-1.11(\mathrm{~m}, 1 \mathrm{H})$.

${ }^{13} \mathrm{C}$ NMR $\left(151 \mathrm{MHz}, \mathrm{CDCl}_{3}\right) \delta 164.04,135.87,132.61,132.49,131.46,127.89,124.58,83.06,66.27,32.38$, $30.78,29.72,29.65,28.38,24.76,24.66,19.93,17.86$.

${ }^{11} \mathrm{~B}$ NMR $\left(192 \mathrm{MHz}, \mathrm{CDCl}_{3}\right) \delta 33.8$.

HRMS (EI+) Calculated for $\mathrm{C}_{22} \mathrm{H}_{29} \mathrm{~B}^{35} \mathrm{Cl}_{3} \mathrm{O}_{4}\left[\mathrm{M}-\mathrm{CH}_{3}\right]^{+}$: 473.1224, Found: 473.1222.

$[\alpha]_{\mathrm{D}}^{23}=-2.5(\mathrm{c}=1.0, \mathrm{DCM})$.

SFC analysis indicated 97\% ee (compound 3e) after oxidation (see section IV for details).<smiles>CCC([B]c1ccccc1)CCOc1c(F)c(F)c(F)c(F)c1F</smiles>

Compound 2f was synthesized according to the General Procedure Condition A with 1f (106 mg, 0.398 mmol), HBPin (71 $\mu \mathrm{L}, 0.48 \mathrm{mmol}, 1.2$ equiv), $\mathrm{CuCl}$ (1.0 mg, $2.5 \mathrm{~mol} \%), \mathrm{KO} t \mathrm{Bu}(2.2 \mathrm{mg}, 5 \mathrm{~mol} \%$ ), and $(S)$ DTBM-SEGPHOS (14.2 mg, $3 \mathrm{~mol} \%$ ). 2 f was purified by flash column chromatography $(2.5 \%$ ethyl acetate in hexane) and obtained as colorless liquid in $77 \%$ yield (contains $<5 \%$ constitutional isomer $\mathbf{2 f}$ ').

${ }^{1} \mathrm{H}$ NMR (500 MHz, $\left.\mathrm{CDCl}_{3}\right) \delta 4.21-4.06(\mathrm{~m}, 2 \mathrm{H}), 1.91-1.73(\mathrm{~m}, 2 \mathrm{H}), 1.49-1.39$ (m, 1H), $1.38-1.27$ (m, $3 \mathrm{H}), 1.20(\mathrm{~s}, 12 \mathrm{H}), 1.20-1.11(\mathrm{~m}, 1 \mathrm{H}), 0.87(\mathrm{t}, J=7.1 \mathrm{~Hz}, 3 \mathrm{H})$.

${ }^{13} \mathrm{C}$ NMR $\left(151 \mathrm{MHz}, \mathrm{CDCl}_{3}\right) \delta 141.91\left(J_{\mathrm{C}-\mathrm{F}}=246 \mathrm{~Hz}\right), 137.95\left(J_{\mathrm{C}-\mathrm{F}}=246 \mathrm{~Hz}\right), 137.14\left(J_{\mathrm{C}-\mathrm{F}}=246 \mathrm{~Hz}\right), 133.80$, 83.06, 75.49, 33.35, 31.12, 24.70, 24.62, 22.07, 19.53, 14.22.

${ }^{11} \mathrm{~B}$ NMR $\left(192 \mathrm{MHz}, \mathrm{CDCl}_{3}\right) \delta 33.5$.

${ }^{19} \mathrm{~F} \mathrm{NMR}\left(376 \mathrm{MHz}, \mathrm{CDCl}_{3}\right) \delta-156.0(\mathrm{~d}, J=20.5 \mathrm{~Hz}, 2 \mathrm{~F}),-163.19(\mathrm{t}, J=22.8 \mathrm{~Hz}, 2 \mathrm{~F}),-163.52(\mathrm{t}, J=21.8 \mathrm{~Hz}$, $1 \mathrm{~F})$.

HRMS (EI+) Calculated for $\mathrm{C}_{18} \mathrm{H}_{25} \mathrm{BF}_{5} \mathrm{O}_{3}[\mathrm{M}+\mathrm{H}]^{+}: 395.1817$, Found: 395.1821.

$[\alpha]_{\mathrm{D}}^{23}=-6.8(\mathrm{c}=1.0, \mathrm{DCM})$.

HPLC analysis indicated $98 \%$ ee (compound 3f) after oxidation (see section IV for details).<smiles>CCC(Br)CCOc1ccc(Br)cc1</smiles>

Compound 2g was synthesized according to the General Procedure Condition A with 1g (102 mg, 0.400 mmol), HBPin (71 $\mu \mathrm{L}, 0.48 \mathrm{mmol}, 1.2$ equiv), $\mathrm{CuCl}$ (1.0 mg, $2.5 \mathrm{~mol} \%$ ), $\mathrm{KO} t \mathrm{Bu}(2.2 \mathrm{mg}, 5 \mathrm{~mol} \%$ ), and $(S)$ DTBM-SEGPHOS (14.2 mg, $3 \mathrm{~mol} \%)$. Compound $\mathbf{2 g}$ was purified by flash column chromatography $(2.5 \%$ 
ethyl acetate in hexane) and obtained as white solid in $62 \%$ yield along with its constitutional isomer $\mathbf{2 g}$ ' in $16 \%$ yield ( $78 \%$ combined yield).

${ }^{1} \mathrm{H}$ NMR $\left(500 \mathrm{MHz}, \mathrm{CDCl}_{3}\right) \delta 7.33(\mathrm{~d}, J=8.9 \mathrm{~Hz}, 2 \mathrm{H}), 6.77(\mathrm{~d}, J=8.9 \mathrm{~Hz}, 2 \mathrm{H}), 3.96-3.88(\mathrm{~m}, 2 \mathrm{H}), 1.97-$ $1.71(\mathrm{~m}, 2 \mathrm{H}), 1.51-1.43(\mathrm{~m}, 1 \mathrm{H}), 1.42-1.28(\mathrm{~m}, 3 \mathrm{H}), 1.23(\mathrm{~s}, 12 \mathrm{H}), 1.19-1.13(\mathrm{~m}, 1 \mathrm{H}), 0.90$ (t, $J=6.9 \mathrm{~Hz}$, $3 \mathrm{H})$.

${ }^{13} \mathrm{C}$ NMR $\left(151 \mathrm{MHz}, \mathrm{CDCl}_{3}\right) \delta 158.20,132.02,116.37,112.39,82.94,67.93,33.40,30.35,24.77,24.71,22.07$, 19.91, 14.30 .

${ }^{11} \mathrm{~B}$ NMR $\left(192 \mathrm{MHz}, \mathrm{CDCl}_{3}\right) \delta 33.8$.

HRMS (EI+) Calculated for $\mathrm{C}_{18} \mathrm{H}_{28} \mathrm{BBrO}_{3}[\mathrm{M}]^{+}: 382.1315$, Found: 382.1320 .

$[\alpha]_{\mathrm{D}}^{23}=-9.2(\mathrm{c}=1.0, \mathrm{DCM})$.

SFC analysis indicated 98\% ee (compound 3g) after oxidation (see section IV for details).

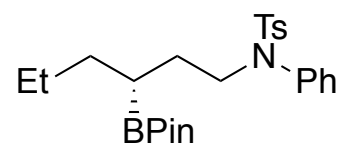

Compound 2h was synthesized according to the General Procedure Condition B with $\mathbf{1 h}$ (132 $\mathbf{m g}, 0.401$ mmol), HBPin (71 $\mu \mathrm{L}, 0.48 \mathrm{mmol}, 1.2$ equiv), $\mathrm{CuCl}$ (2.0 mg, $5 \mathrm{~mol} \%), \mathrm{KO} t \mathrm{Bu}(4.4 \mathrm{mg}, 5 \mathrm{~mol} \%$ ), and $(S)$ DTBM-SEGPHOS (25.9 mg, $5.5 \mathrm{~mol} \%$ ). Compound $\mathbf{2 h}$ was purified by flash column chromatography $(10 \%$ ethyl acetate in hexane) and obtained as colorless sticky oil in $84 \%$ yield.

${ }^{1} \mathrm{H}$ NMR (500 MHz, $\left.\mathrm{CDCl}_{3}\right) \delta 7.45(\mathrm{~d}, J=8.4 \mathrm{~Hz}, 2 \mathrm{H}), 7.32-7.24(\mathrm{~m}, 3 \mathrm{H}), 7.21(\mathrm{~d}, J=8.0 \mathrm{~Hz}, 2 \mathrm{H}), 7.03(\mathrm{dd}$, $J=7.9,1.8 \mathrm{~Hz}, 2 \mathrm{H}), 3.58-3.47(\mathrm{~m}, 2 \mathrm{H}), 2.39(\mathrm{~s}, 3 \mathrm{H}), 1.60-1.41(\mathrm{~m}, 2 \mathrm{H}), 1.39-1.28(\mathrm{~m}, 1 \mathrm{H}), 1.28-1.12$ $(\mathrm{m}, 3 \mathrm{H}), 1.19(\mathrm{~s}, 6 \mathrm{H}), 1.19(\mathrm{~s}, 6 \mathrm{H}), 1.03-0.99(\mathrm{~m}, 1 \mathrm{H}), 0.81(\mathrm{t}, J=7.0 \mathrm{~Hz}, 3 \mathrm{H})$.

${ }^{13} \mathrm{C}$ NMR $\left(151 \mathrm{MHz}, \mathrm{CDCl}_{3}\right) \delta 142.98,139.17,135.31,129.11,128.65,128.62,127.52,127.48,82.85,50.02$, $33.08,29.60,24.66,24.54,21.93,21.35,20.27,14.15$.

${ }^{11} \mathrm{~B}$ NMR $\left(192 \mathrm{MHz}, \mathrm{CDCl}_{3}\right) \delta 33.5$.

HRMS (ESI+) Calculated for $\mathrm{C}_{25} \mathrm{H}_{37} \mathrm{BNO}_{4} \mathrm{~S}[\mathrm{M}+\mathrm{H}]^{+}:$458.2531, Found: 458.2531.

$[\alpha]_{\mathrm{D}}^{23}=-10.1(\mathrm{c}=1.0, \mathrm{DCM})$.

SFC analysis indicated $96 \%$ ee (compound $3 \mathrm{~h}$ ) after oxidation (see section IV for details).<smiles>CCC([B]c1ccccc1)CCN(C)Cc1ccccc1</smiles>

Compound 2i was synthesized according to the General Procedure Condition B with 1i (137 mg, 0.399 mmol), HBPin ( $71 \mu \mathrm{L}, 0.48 \mathrm{mmol}, 1.2$ equiv), $\mathrm{CuCl}$ (2.0 mg, $5 \mathrm{~mol} \%), \mathrm{KO} t \mathrm{Bu}(4.4 \mathrm{mg}, 5 \mathrm{~mol} \%$ ), and $(S)$ DTBM-SEGPHOS (25.9 mg, $5.5 \mathrm{~mol} \%$ ). Compound $\mathbf{2 i}$ was purified by flash column chromatography (10\% ethyl acetate in hexane) and obtained as colorless sticky oil in $81 \%$ yield. 
${ }^{1} \mathrm{H}$ NMR (500 MHz, $\left.\mathrm{CDCl}_{3}\right) 7.74(\mathrm{~d}, J=8.3 \mathrm{~Hz}, 2 \mathrm{H}), 7.32-7.21(\mathrm{~m}, 7 \mathrm{H}), 4.37(\mathrm{~d}, J=15.0 \mathrm{~Hz}, 1 \mathrm{H}), 4.32(\mathrm{~d}, J$ $=15.0 \mathrm{~Hz}, 1 \mathrm{H}), 3.11(\mathrm{t}, J=8.3 \mathrm{~Hz}, 2 \mathrm{H}), 2.42(\mathrm{~s}, 3 \mathrm{H}), 1.51-1.41(\mathrm{~m}, 1 \mathrm{H}), 1.41-1.32(\mathrm{~m}, 1 \mathrm{H}), 1.30-1.23(\mathrm{~m}$, 1H), $1.20-1.07$ (m, 3H), 1.15 (s, 6H), 1.14 (s, 6H), 0.80 (t, J=7.0 Hz, 3H), $0.80-0.74(\mathrm{~m}, 1 \mathrm{H})$.

${ }^{13} \mathrm{C}$ NMR $\left(151 \mathrm{MHz}, \mathrm{CDCl}_{3}\right) \delta 142.82,137.39,136.47,129.44,128.28,128.04,127.40,127.00,82.80,51.14$, $47.04,32.89,28.65,24.57,24.51,21.83,21.32,20.59,14.11$.

${ }^{11} \mathrm{~B}$ NMR $\left(192 \mathrm{MHz}, \mathrm{CDCl}_{3}\right) \delta 33.5$.

HRMS (ESI+) Calculated for $\mathrm{C}_{26} \mathrm{H}_{38} \mathrm{BNO}_{4} \mathrm{~S}[\mathrm{M}+\mathrm{H}]^{+}: 472.2690$, Found: 472.2690 .

$[\alpha]_{\mathrm{D}}^{23}=-1.7(\mathrm{c}=1.0, \mathrm{DCM})$.

SFC analysis indicated 99\% ee (compound 3i) after oxidation (see section IV for details).<smiles>CCC(Br)CCCN([Tl])C([14CH2])c1ccccc1</smiles>

Compound $2 \mathbf{j}$ was synthesized according to the General Procedure Condition $\mathbf{C}$ with $\mathbf{1 j}(67.1 \mathrm{mg}, 0.200$ mmol), HBPin (59 $\mu \mathrm{L}, 0.4 \mathrm{mmol}, 2$ equiv), $\mathrm{CuCl}$ (2.0 mg, $10 \mathrm{~mol} \%$ ), $\mathrm{KO} t \mathrm{Bu}$ (4.4 mg, $20 \mathrm{~mol} \%$ ), and $(S)$ DTBM-SEGPHOS (25.9 mg, $11 \mathrm{~mol} \%$ ). Compound $\mathbf{2} \mathbf{j}$ was purified by flash column chromatography (5\% ethyl acetate in hexane) and obtained as colorless liquid in $86 \%$ yield.

${ }^{1} \mathrm{H}$ NMR (500 MHz, $\left.\mathrm{CDCl}_{3}\right) \delta 7.44(\mathrm{~d}, J=7.6 \mathrm{~Hz}, 2 \mathrm{H}), 7.37(\mathrm{t}, J=7.5 \mathrm{~Hz}, 2 \mathrm{H}), 7.32(\mathrm{t}, J=7.2 \mathrm{~Hz}, 1 \mathrm{H}), 5.23$ $(\mathrm{q}, J=7.2 \mathrm{~Hz}, 1 \mathrm{H}), 3.19-3.08(\mathrm{~m}, 2 \mathrm{H}), 1.71(\mathrm{~d}, J=7.1 \mathrm{~Hz}, 3 \mathrm{H}), 1.63-1.50(\mathrm{~m}, 1 \mathrm{H}), 1.34-1.23(\mathrm{~m}, 2 \mathrm{H})$, $1.21-1.12(\mathrm{~m}, 3 \mathrm{H}), 1.17(\mathrm{~s}, 12 \mathrm{H}), 0.81(\mathrm{t}, J=6.9 \mathrm{~Hz}, 3 \mathrm{H}), 0.73-0.65(\mathrm{~m}, 1 \mathrm{H})$.

${ }^{13} \mathrm{C}$ NMR $\left(151 \mathrm{MHz}, \mathrm{CDCl}_{3}\right) \delta 138.67,128.64,128.29,127.58,119.99\left(\mathrm{q}, J_{\mathrm{C}-\mathrm{F}}=323.5 \mathrm{~Hz}\right), 83.07,57.62$, 45.26, 33.11, 31.85, 24.70, 24.68, 21.85, 21.07, 17.03, 14.15 .

${ }^{11} \mathrm{~B}$ NMR $\left(192 \mathrm{MHz}, \mathrm{CDCl}_{3}\right) \delta 33.5$.

${ }^{19} \mathrm{~F} \mathrm{NMR}\left(376 \mathrm{MHz}, \mathrm{CDCl}_{3}\right) \delta-75.2(\mathrm{~s})$.

HRMS (EI+) Calculated for $\mathrm{C}_{20} \mathrm{H}_{30} \mathrm{BF}_{3} \mathrm{NO}_{4} \mathrm{~S}\left[\mathrm{M}-\mathrm{CH}_{3}\right]^{+}: 448.1931$, Found: 448.1935 .

$[\alpha]_{\mathrm{D}}^{23}=14.4(\mathrm{c}=1.0, \mathrm{DCM})$.

${ }^{1} \mathrm{H}$ NMR analysis indicated $>20: 1$ d.r..<smiles>CCCCCCC(CCCCC)CC(OC(=O)c1c(Cl)cc(Cl)cc1Cl)c1ccccc1</smiles>

Compound 2k was synthesized according to the General Procedure Condition $\mathbf{C}$ with $\mathbf{1 k}(82.3 \mathrm{mg}, 0.200$ mmol), HBPin (59 $\mu \mathrm{L}, 0.4 \mathrm{mmol}, 2$ equiv), $\mathrm{CuCl}$ (2.0 mg, $10 \mathrm{~mol} \%), \mathrm{KO} t \mathrm{Bu}(4.4 \mathrm{mg}, 20 \mathrm{~mol} \%$ ), and $(S)$ - 
DTBM-SEGPHOS (25.9 mg, $11 \mathrm{~mol} \%$ ). Compound $\mathbf{2 k}$ was purified by flash column chromatography (3\% ethyl acetate in hexane) and obtained as colorless sticky oil in $77 \%$ yield.

${ }^{1} \mathrm{H}$ NMR (500 MHz, $\left.\mathrm{CDCl}_{3}\right) \delta 7.46-7.40(\mathrm{~m}, 2 \mathrm{H}), 7.38-7.33(\mathrm{~m}, 2 \mathrm{H}), 7.31-7.27(\mathrm{~m}, 1 \mathrm{H}), 7.31(\mathrm{~s}, 2 \mathrm{H})$, $6.04(\mathrm{dd}, J=8.2,6.5 \mathrm{~Hz}, 1 \mathrm{H}), 2.20(\mathrm{dt}, J=13.7,7.8 \mathrm{~Hz}, 1 \mathrm{H}), 1.86(\mathrm{ddd}, J=14.2,8.0,6.5 \mathrm{~Hz}, 1 \mathrm{H}), 1.51-1.43$ $(\mathrm{m}, 2 \mathrm{H}), 1.33-1.21(\mathrm{~m}, 6 \mathrm{H}), 1.24(\mathrm{~s}, 12 \mathrm{H}), 1.11$ (quintet, $J=7.6 \mathrm{~Hz}, 1 \mathrm{H}), 0.87(\mathrm{t}, J=6.9 \mathrm{~Hz}, 3 \mathrm{H})$.

${ }^{13} \mathrm{C}$ NMR $\left(151 \mathrm{MHz}, \mathrm{CDCl}_{3}\right) \delta 163.30,139.77,135.82,132.63,132.44,128.26,128.06,127.88,127.05,83.09$, $77.67,37.39,32.08,30.33,28.46,24.83,24.71,22.52,19.35,13.98$.

${ }^{11} \mathrm{~B}$ NMR $\left(192 \mathrm{MHz}, \mathrm{CDCl}_{3}\right) \delta 33.8$.

HRMS (ESI+) Calculated for $\mathrm{C}_{27} \mathrm{H}_{34} \mathrm{~B}^{35} \mathrm{Cl}_{3} \mathrm{O}_{4} \mathrm{Na}[\mathrm{M}+\mathrm{Na}]^{+}$: 561.1508, Found: 561.1509 .

$[\alpha]_{\mathrm{D}}^{23}=30.4(\mathrm{c}=1.0, \mathrm{DCM})$.

${ }^{1} \mathrm{H}$ NMR analysis indicated $>20: 1$ d.r..

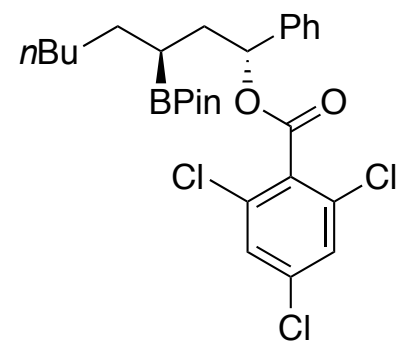

Compound 2l was synthesized according to the General Procedure Condition $\mathbf{C}$ with $\mathbf{1 k}(82.3 \mathrm{mg}, 0.200$ mmol), HBPin (59 $\mu \mathrm{L}, 0.4 \mathrm{mmol}, 2$ equiv), $\mathrm{CuCl}(2.0 \mathrm{mg}, 10 \mathrm{~mol} \%), \mathrm{KO} t \mathrm{Bu}(4.4 \mathrm{mg}, 20 \mathrm{~mol} \%$ ), and $(R)-$ DTBM-SEGPHOS (25.9 mg, $11 \mathrm{~mol} \%$ ). Compound $2 \mathbf{l}$ was purified by flash column chromatography (3\% ethyl acetate in hexane) and obtained as white solid in $76 \%$ yield.

${ }^{1} \mathrm{H}$ NMR (500 MHz, $\left.\mathrm{CDCl}_{3}\right) \delta 7.44(\mathrm{~d}, J=7.5 \mathrm{~Hz}, 2 \mathrm{H}), 7.35(\mathrm{t}, J=7.5 \mathrm{~Hz}, 2 \mathrm{H}), 7.32(\mathrm{~s}, 2 \mathrm{H}), 7.29(\mathrm{t}, J=7.3$ $\mathrm{Hz}, 1 \mathrm{H}), 6.11$ (dd, $J=9.6,4.3 \mathrm{~Hz}, 1 \mathrm{H}), 2.05$ (ddd, $J=14.6,9.6,5.3 \mathrm{~Hz}, 1 \mathrm{H}), 1.97$ (ddd, $J=14.3,10.3,4.4 \mathrm{~Hz}$, $1 \mathrm{H}), 1.52-1.38(\mathrm{~m}, 2 \mathrm{H}), 1.36-1.23(\mathrm{~m}, 7 \mathrm{H}), 1.28(\mathrm{~s}, 6 \mathrm{H}), 1.26(\mathrm{~s}, 6 \mathrm{H}), 0.87(\mathrm{t}, J=6.8 \mathrm{~Hz}, 3 \mathrm{H})$.

${ }^{13} \mathrm{C}$ NMR $\left(151 \mathrm{MHz}, \mathrm{CDCl}_{3}\right) \delta 163.46,140.25,135.78,132.64,132.50,128.23,127.94,127.85,126.70,83.06$, $79.04,38.17,32.06,31.28,28.37,24.94,24.69,22.49,20.23,14.00$.

${ }^{11} \mathrm{~B}$ NMR $\left(192 \mathrm{MHz}, \mathrm{CDCl}_{3}\right) \delta 33.8$.

HRMS (ESI+) Calculated for $\mathrm{C}_{27} \mathrm{H}_{34} \mathrm{~B}^{35} \mathrm{Cl}_{3} \mathrm{O}_{4} \mathrm{Na}[\mathrm{M}+\mathrm{Na}]^{+}:$561.1508, Found: 561.1505.

$[\alpha]_{\mathrm{D}}^{23}=46.5(\mathrm{c}=1.0, \mathrm{DCM})$.

${ }^{1} \mathrm{H}$ NMR analysis indicated $>20: 1$ d.r..

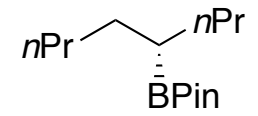

Compound 2m was synthesized according to the General Procedure Condition D with trans-4-octene (471 $\mu \mathrm{L}, 3.00 \mathrm{mmol}, 3$ equiv), HBPin (145 $\mu \mathrm{L}, 1.00 \mathrm{mmol}, 1$ equiv), $\mathrm{CuCl}(10.0 \mathrm{mg}, 10 \mathrm{~mol} \%), \mathrm{KO} t \mathrm{Bu}(22.0 \mathrm{mg}$, 
$5 \mathrm{~mol} \%$ ), and (S)-DTBM-SEGPHOS (130 $\mathrm{mg}, 11 \mathrm{~mol} \%$ ). Compound $\mathbf{2 m}$ was purified by flash column chromatography (2.5\% ethyl acetate in hexane) and obtained as colorless liquid in $34 \%$ yield.

${ }^{1} \mathrm{H}$ NMR (500 MHz, $\left.\mathrm{CDCl}_{3}\right) \delta 1.43-1.20(\mathrm{~m}, 10 \mathrm{H}), 1.24(\mathrm{~s}, 12 \mathrm{H}), 0.99-0.93(\mathrm{~m}, 1 \mathrm{H}), 0.89-0.86(\mathrm{~m}, 6 \mathrm{H})$,

${ }^{13} \mathrm{C}$ NMR $\left(151 \mathrm{MHz}, \mathrm{CDCl}_{3}\right) \delta 82.66,33.74,31.53,31.06,24.73,24.73,23.73,22.94,22.37,14.39,14.04$.

${ }^{11} \mathrm{~B}$ NMR $\left(192 \mathrm{MHz}, \mathrm{CDCl}_{3}\right) \delta 33.9$.

$[\alpha]_{\mathrm{D}}^{23}=2.2(\mathrm{c}=1.0, \mathrm{DCM})$.

${ }^{19}$ F NMR analysis indicated $98 \%$ ee (compound $\mathbf{3 m}$ ) after oxidation (see section IV for details).

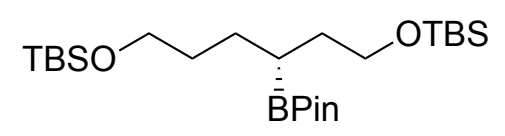

Compound 2n was synthesized according to the General Procedure Condition $\mathbf{C}$ with 1n (138 $\mathrm{mg}, 0.400$ mmol), HBPin (118 $\mu \mathrm{L}, 0.8 \mathrm{mmol}, 2$ equiv), $\mathrm{CuCl}$ (4.0 mg, $10 \mathrm{~mol} \%), \mathrm{KO} t \mathrm{Bu}(8.8 \mathrm{mg}, 10 \mathrm{~mol} \%$ ), and $(S)$ DTBM-SEGPHOS (11 mol \%, $51.9 \mathrm{mg}$ ). Compound $\mathbf{2 n}$ was purified by flash column chromatography (2.5\% ethyl acetate in hexane) and obtained as colorless liquid in $74 \%$ yield.

${ }^{1} \mathrm{H}$ NMR (500 MHz, $\left.\mathrm{CDCl}_{3}\right) \delta 3.62-3.51(\mathrm{~m}, 4 \mathrm{H}), 1.63$ (ddd, $\left.J=14.2,9.1,5.5 \mathrm{~Hz}, 1 \mathrm{H}\right), 1.59-1.32(\mathrm{~m}, 5 \mathrm{H})$, $1.19(\mathrm{~s}, 12 \mathrm{H}), 1.03-0.97(\mathrm{~m}, 1 \mathrm{H}), 0.85(\mathrm{~s}, 18 \mathrm{H}), 0.00(\mathrm{~s}, 6 \mathrm{H}), 0.00(\mathrm{~s}, 6 \mathrm{H})$.

${ }^{13} \mathrm{C}$ NMR $\left(151 \mathrm{MHz}, \mathrm{CDCl}_{3}\right) \delta 82.78,63.40,62.86,34.29,32.37,27.25,25.98,25.95,24.78,24.74,19.40$, $18.33,18.30,-5.25,-5.28,-5.29,-5.29$.

${ }^{11} \mathrm{~B}$ NMR $\left(192 \mathrm{MHz}, \mathrm{CDCl}_{3}\right) \delta 33.8$.

HRMS (EI+) Calculated for $\mathrm{C}_{24} \mathrm{H}_{54} \mathrm{BO}_{4} \mathrm{Si}_{2}[\mathrm{M}+\mathrm{H}]^{+}: 473.3654$, Found: 473.3650 .

$[\alpha]_{\mathrm{D}}^{23}=-0.6(\mathrm{c}=1.0, \mathrm{DCM})$.

${ }^{19}$ F NMR analysis indicated $>98 \%$ ee (compound 3n) after oxidation (see section IV for details).<smiles>O=C(OCCCC([B]c1ccccc1)CCOC(=O)c1c(Cl)cc(Cl)cc1Cl)c1c(Cl)cc(Cl)cc1Cl</smiles>

Compound 2o was synthesized according to the General Procedure Condition C with 1o (106 mg, 0.200 mmol), HBPin (59 $\mu \mathrm{L}, 0.4 \mathrm{mmol}, 2$ equiv), $\mathrm{CuCl}$ (2.0 mg, $10 \mathrm{~mol} \%), \mathrm{KO} t \mathrm{Bu}(4.4 \mathrm{mg}, 20 \mathrm{~mol} \%$ ), and $(S)$ DTBM-SEGPHOS $(25.9 \mathrm{mg}, 11 \mathrm{~mol} \%)$. Compound 20 was purified by flash column chromatography $(10 \%$ ethyl acetate in hexane) and obtained as white solid in $82 \%$ yield.

${ }^{1} \mathrm{H}$ NMR (500 MHz, $\left.\mathrm{CDCl}_{3}\right) \delta 7.33(\mathrm{~s}, 2 \mathrm{H}), 7.32(\mathrm{~s}, 2 \mathrm{H}), 4.46-4.31(\mathrm{~m}, 4 \mathrm{H}), 1.96-1.71(\mathrm{~m}, 4 \mathrm{H}), 1.63-1.46$ $(\mathrm{m}, 2 \mathrm{H}), 1.23-1.16(\mathrm{~m}, 1 \mathrm{H}), 1.21(\mathrm{~s}, 12 \mathrm{H})$.

${ }^{13} \mathrm{C}$ NMR $\left(151 \mathrm{MHz}, \mathrm{CDCl}_{3}\right) \delta 164.03,164.02,135.94,135.93,132.56,132.55,132.35,132.32,127.93$, 127.90, 83.26, 66.62, 65.99, 29.60, 27.78, 27.14, 24.74, 24.66, 19.55 .

${ }^{11} \mathrm{~B}$ NMR $\left(192 \mathrm{MHz}, \mathrm{CDCl}_{3}\right) \delta 33.8$.

HRMS (ESI+) Calculated for $\mathrm{C}_{26} \mathrm{H}_{27} \mathrm{BCl}_{6} \mathrm{O}_{6} \mathrm{Na}[\mathrm{M}+\mathrm{Na}]^{+}$: 678.9924, Found: 678.9930 .

$[\alpha]_{\mathrm{D}}^{23}=-0.7(\mathrm{c}=1.0, \mathrm{DCM})$. 
SFC analysis indicated 97\% ee (compound 3o) after oxidation (see section IV for details). 


\section{Oxidation of Secondary Boronates}

\section{Representative procedure for oxidation of secondary boronates}
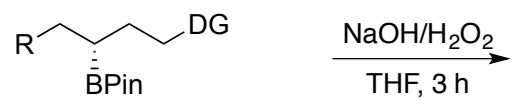

$\overbrace{\text { Ōं }}^{\sim D G}$

In a 20 -mL scintillation vial, the secondary boronate $(20-100 \mathrm{mg}$ ) was dissolved in $2 \mathrm{~mL}$ THF. The vial was cooled in an ice bath and added a premixed solution of $\mathrm{NaOH}(2 \mathrm{M}$, aq. $) / 30 \% \mathrm{H}_{2} \mathrm{O}_{2}(2: 1,3 \mathrm{~mL})$. The reaction was warmed to $\mathrm{rt}$ and allowed to stir for 3 hours before the addition of water $(5 \mathrm{~mL})$ and ethyl acetate $(5 \mathrm{~mL})$. The phases were separated and the aqueous layer was extracted twice with $5 \mathrm{~mL}$ ethyl acetate. The organic phases were combined, dried over $\mathrm{Na}_{2} \mathrm{SO}_{4}$, and concentrated in vacuo. The pure product was obtained by flash column chromatography (CombiFlash).<smiles>CCC[C@H](O)CCOC(=O)c1c(Cl)cc(Cl)cc1Cl</smiles>

Compound 3a was purified by flash column chromatography (16\% ethyl acetate in hexane) and obtained as colorless liquid in $90 \%$ yield.

${ }^{1} \mathrm{H}$ NMR $\left(500 \mathrm{MHz}, \mathrm{CDCl}_{3}\right) \delta 7.34(\mathrm{~s}, 2 \mathrm{H}), 4.61(\mathrm{ddd}, J=11.0,8.7,5.2 \mathrm{~Hz}, 1 \mathrm{H}), 4.49(\mathrm{dt}, J=11.1,5.6 \mathrm{~Hz}$, $1 \mathrm{H}), 3.83-3.79$ (m, 1H), 1.96 (dddd, $J=14.6,9.1,6.1,3.3 \mathrm{~Hz}, 1 \mathrm{H}), 1.88$ (brs, $1 \mathrm{H}), 1.78$ (ddt, $J=14.5,9.2$, $5.2 \mathrm{~Hz}, 1 \mathrm{H}), 1.53-1.31(\mathrm{~m}, 4 \mathrm{H}), 0.92(\mathrm{t}, J=6.8 \mathrm{~Hz}, 3 \mathrm{H})$.

${ }^{13} \mathrm{C} \mathrm{NMR}\left(151 \mathrm{MHz}, \mathrm{CDCl}_{3}\right) \delta 164.23,136.14,132.54,132.14,128.02,68.14,63.84,39.70,36.07,18.71$, 13.95 .

HRMS (EI+) Calculated for $\mathrm{C}_{13} \mathrm{H}_{15}{ }^{35} \mathrm{Cl}_{3} \mathrm{O}_{3}[\mathrm{M}]^{+}: 324.0087$, Found: 324.0086 .

$[\alpha]_{\mathrm{D}}^{23}=-9.4(\mathrm{c}=1.0, \mathrm{DCM})$.

$\mathrm{SFC}$ analysis (OZ-H, 10\% IPA/CO $2,2.5 \mathrm{~mL} / \mathrm{min}, 220 \mathrm{~nm})$ indicated $97 \%$ ee: $\mathrm{t}_{\mathrm{R}}$ (major) $=3.4 \mathrm{~min}, \mathrm{t}_{\mathrm{R}}$ (minor) $=3.0 \mathrm{~min}$.<smiles>CC(C)C[C@H](O)CCOC(=O)c1c(Cl)cc(Cl)cc1Cl</smiles>

Compound 3b was purified by flash column chromatography (16\% ethyl acetate in hexane) and obtained as colorless liquid in $99 \%$ yield.

${ }^{1} \mathrm{H}$ NMR $\left(500 \mathrm{MHz}, \mathrm{CDCl}_{3}\right) \delta 7.34(\mathrm{~s}, 2 \mathrm{H}), 4.64(\mathrm{ddd}, J=11.0,8.9,5.1 \mathrm{~Hz}, 1 \mathrm{H}), 4.47(\mathrm{ddd}, J=11.0,6.0,5.0$ $\mathrm{Hz}, 1 \mathrm{H}), 3.88$ (tt, $J=9.1,3.7 \mathrm{~Hz}, 1 \mathrm{H}), 1.97-1.91(\mathrm{~m}, 1 \mathrm{H}), 1.91(\mathrm{brs}, 1 \mathrm{H}), 1.83-1.70$ (m, 2H), 1.45 (ddd, $J=$ 14.1, 9.1, 5.3 Hz, 1H), $1.23(\mathrm{ddd}, J=13.5,8.9,4.1 \mathrm{~Hz}, 1 \mathrm{H}), 0.91(\mathrm{~d}, J=6.6 \mathrm{~Hz}, 3 \mathrm{H}), 0.89$ (d, $J=6.6 \mathrm{~Hz}, 3 \mathrm{H})$.

${ }^{13} \mathrm{C}$ NMR $\left(151 \mathrm{MHz}, \mathrm{CDCl}_{3}\right) \delta 164.27,136.14,132.54,132.13,128.02,66.39,63.76,46.75,36.66,24.50$, 23.37, 21.92.

HRMS (EI+) Calculated for $\mathrm{C}_{14} \mathrm{H}_{17}{ }^{35} \mathrm{Cl}_{3} \mathrm{O}_{3}[\mathrm{M}]^{+}: 338.0243$, Found: 338.0244 . 
$[\alpha]_{\mathrm{D}}^{23}=-6.3(\mathrm{c}=1.0, \mathrm{DCM})$.

$\mathrm{SFC}$ analysis (OZ-H, 20\% IPA/CO $2,1.5 \mathrm{~mL} / \mathrm{min}, 220 \mathrm{~nm}$ ) indicated $99 \%$ ee: $\mathrm{t}_{\mathrm{R}}$ (major) $=2.2 \mathrm{~min}, \mathrm{t}_{\mathrm{R}}$ (minor) $=2.0 \mathrm{~min}$.<smiles>CC(C)(C)C[C@H](O)CCOC(=O)c1c(Cl)cc(Cl)cc1Cl</smiles>

Compound 3c was purified by flash column chromatography (16\% ethyl acetate in hexane) and obtained as colorless liquid in $99 \%$ yield.

${ }^{1} \mathrm{H}$ NMR $\left(500 \mathrm{MHz}, \mathrm{CDCl}_{3}\right) \delta 7.35(\mathrm{~s}, 2 \mathrm{H}), 4.65(\mathrm{ddd}, J=11.1,9.0,5.0 \mathrm{~Hz}, 1 \mathrm{H}), 4.45(\mathrm{dt}, J=10.9,5.6 \mathrm{~Hz}$, $1 \mathrm{H}), 3.99-3.93(\mathrm{~m}, 1 \mathrm{H}), 1.94-1.87(\mathrm{~m}, 1 \mathrm{H}), 1.83-1.76(\mathrm{~m}, 2 \mathrm{H}), 1.45(\mathrm{dd}, J=14.6,8.4 \mathrm{~Hz}, 1 \mathrm{H}), 1.36(\mathrm{dd}, J$ $=14.6,2.5 \mathrm{~Hz}, 1 \mathrm{H}), 0.96(\mathrm{~s}, 9 \mathrm{H})$.

${ }^{13} \mathrm{C}$ NMR $\left(151 \mathrm{MHz}, \mathrm{CDCl}_{3}\right) \delta 164.35,136.17,132.56,132.13,128.05,66.04,63.70,51.25,38.13,30.30$, 30.10 .

HRMS (EI+) Calculated for $\mathrm{C}_{15} \mathrm{H}_{19}{ }^{35} \mathrm{Cl}_{3} \mathrm{O}_{3}[\mathrm{M}]^{+}: 352.0400$, Found: 352.0392 .

$[\alpha]_{\mathrm{D}}^{23}=-6.5(\mathrm{c}=1.0, \mathrm{DCM})$.

$\mathrm{SFC}$ analysis (OD-H, 20\% IPA/CO $2,1.5 \mathrm{~mL} / \mathrm{min}, 220 \mathrm{~nm})$ indicated $99 \%$ ee: $\mathrm{t}_{\mathrm{R}}$ (major) $=2.1 \mathrm{~min}, \mathrm{t}_{\mathrm{R}}($ minor) $=1.7 \mathrm{~min}$.<smiles>O=C(OCC[C@H](O)CC1CCCCC1)c1c(Cl)cc(Cl)cc1Cl</smiles>

Compound 3d was purified by flash column chromatography (16\% ethyl acetate in hexane) and obtained as colorless liquid in $92 \%$ yield.

${ }^{1} \mathrm{H}$ NMR $\left(500 \mathrm{MHz}, \mathrm{CDCl}_{3}\right) \delta 7.35(\mathrm{~s}, 2 \mathrm{H}), 4.63(\mathrm{ddd}, J=11.0,8.9,5.1 \mathrm{~Hz}, 1 \mathrm{H}), 4.54-4.42(\mathrm{~m}, 1 \mathrm{H}), 3.92(\mathrm{tt}$, $J=8.5,3.5 \mathrm{~Hz}, 1 \mathrm{H}), 1.94$ (dddd, $J=14.7,9.1,6.0,3.3 \mathrm{~Hz}, 1 \mathrm{H}), 1.84$ (brs, $1 \mathrm{H}), 1.79-1.74$ (m, 2H), $1.72-$ $1.60(\mathrm{~m}, 4 \mathrm{H}), 1.45-1.39(\mathrm{~m}, 2 \mathrm{H}), 1.33-1.08(\mathrm{~m}, 4 \mathrm{H}), 1.01-0.90(\mathrm{~m}, 1 \mathrm{H}), 0.90-0.78(\mathrm{~m}, 1 \mathrm{H})$.

${ }^{13} \mathrm{C}$ NMR $\left(151 \mathrm{MHz}, \mathrm{CDCl}_{3}\right) \delta 164.28,136.15,132.56,132.14,128.04,65.80,63.79,45.41,36.73,34.14$, $33.99,32.77,26.51,26.30,26.13$.

HRMS (EI+) Calculated for $\mathrm{C}_{17} \mathrm{H}_{21}{ }^{35} \mathrm{Cl}_{2}{ }^{37} \mathrm{ClO}_{3}[\mathrm{M}]^{+}: 380.0527$, Found: 380.0514 .

$[\alpha]_{\mathrm{D}}^{23}=-4.3(\mathrm{c}=1.0, \mathrm{DCM})$.

SFC analysis (OZ-H, 20\% IPA/CO $/ 1.5 \mathrm{~mL} / \mathrm{min}, 220 \mathrm{~nm}$ ) indicated $99 \%$ ee: $\mathrm{t}_{\mathrm{R}}$ (major) $=3.6 \mathrm{~min}, \mathrm{t}_{\mathrm{R}}$ (minor) $=3.0 \mathrm{~min}$. 
<smiles>C/C=C/CCCC[C@H](O)CCOC(=O)c1c(Cl)cc(Cl)cc1Cl</smiles>

Compound 3e was purified by flash column chromatography (12\% ethyl acetate in hexane) and obtained as colorless liquid in $95 \%$ yield.

${ }^{1} \mathrm{H}$ NMR (500 MHz, $\left.\mathrm{CDCl}_{3}\right) \delta 7.37(\mathrm{~s}, 2 \mathrm{H}), 5.46-5.37(\mathrm{~m}, 2 \mathrm{H}), 4.66-4.61(\mathrm{~m}, 1 \mathrm{H}), 4.53-4.49(\mathrm{~m}, 1 \mathrm{H})$, $3.86-3.78(\mathrm{~m}, 1 \mathrm{H}), 2.02-1.95(\mathrm{~m}, 3 \mathrm{H}), 1.85-1.77(\mathrm{~m}, 2 \mathrm{H}), 1.71-1.59(\mathrm{~d}, J=3.5 \mathrm{~Hz}, 3 \mathrm{H}), 1.56-1.30(\mathrm{~m}$, $6 \mathrm{H})$.

${ }^{13} \mathrm{C}$ NMR $\left(151 \mathrm{MHz}, \mathrm{CDCl}_{3}\right) \delta 164.23,136.16,132.56,132.14,131.17,128.04,124.91,68.44,63.83,37.43$, 36.06, 32.43, 29.43, 25.02, 17.88 .

HRMS (ESI+) Calculated for $\mathrm{C}_{17} \mathrm{H}_{21}{ }^{35} \mathrm{Cl}_{3} \mathrm{O}_{3} \mathrm{Na}[\mathrm{M}+\mathrm{Na}]^{+}: 401.0448$, Found: 401.0452 .

$[\alpha]_{\mathrm{D}}^{23}=-6.0(\mathrm{c}=1.0, \mathrm{DCM})$.

SFC analysis $\left(\mathrm{AD}-\mathrm{H}, 10 \% \mathrm{MeOH} / \mathrm{CO}_{2}, 2.5 \mathrm{~mL} / \mathrm{min}, 220 \mathrm{~nm}\right.$ ) indicated $97 \%$ ee: $\mathrm{t}_{\mathrm{R}}$ (major) $=3.7 \mathrm{~min}, \mathrm{t}_{\mathrm{R}}$ $($ minor $)=5.0 \mathrm{~min}$.<smiles>CC[C@H](O)CCOc1c(F)c(F)c(F)c(F)c1F</smiles>

Compound 3f was purified by flash column chromatography (12\% ethyl acetate in hexane) and obtained as colorless liquid in $64 \%$ yield.

${ }^{1} \mathrm{H}$ NMR $\left(500 \mathrm{MHz}, \mathrm{CDCl}_{3}\right) \delta 4.38-4.34(\mathrm{~m}, 1 \mathrm{H}), 4.31-4.27(\mathrm{~m}, 1 \mathrm{H}), 3.95-3.91(\mathrm{~m}, 1 \mathrm{H}), 1.98$ (dddd, $J=$ 14.0, 8.4, 5.7, 3.2 Hz, 1H), $1.88-1.77$ (m, 1H), 1.76 (brs, 1H), $1.54-1.45$ (m, 3H), $1.43-1.37$ (m, $1 \mathrm{H}), 0.95$ $(\mathrm{t}, J=6.7 \mathrm{~Hz}, 3 \mathrm{H})$.

${ }^{13} \mathrm{C}$ NMR $\left(151 \mathrm{MHz}, \mathrm{CDCl}_{3}\right) \delta 141.80\left(J_{\mathrm{C}-\mathrm{F}}=246 \mathrm{~Hz}\right), 138.01\left(J_{\mathrm{C}-\mathrm{F}}=251 \mathrm{~Hz}\right), 137.41\left(J_{\mathrm{C}-\mathrm{F}}=239 \mathrm{~Hz}\right), 133.66$, 73.40, 68.63, 39.91, 37.15, 18.73, 13.96 .

${ }^{19} \mathrm{~F}$ NMR $\left(376 \mathrm{MHz}, \mathrm{CDCl}_{3}\right) \delta-156.10(\mathrm{~d}, J=18.8 \mathrm{~Hz}, 2 \mathrm{~F}),-162.10--163.03(\mathrm{~m}, 3 \mathrm{~F})$.

HRMS (EI+) Calculated for $\mathrm{C}_{12} \mathrm{H}_{13} \mathrm{~F}_{5} \mathrm{O}_{2}[\mathrm{M}]^{+}: 284.0836$, Found: 284.0835 .

$[\alpha]_{\mathrm{D}}^{23}=-10.2(\mathrm{c}=1.0, \mathrm{DCM})$.

HPLC analysis (AD-H, 3\% IPA/Hex, $1.0 \mathrm{~mL} / \mathrm{min}, 254 \mathrm{~nm}$ ) indicated $98 \%$ ee: $\mathrm{t}_{\mathrm{R}}$ (major) $=11.8 \mathrm{~min}, \mathrm{t}_{\mathrm{R}}$ (minor) $=10.6 \mathrm{~min}$.<smiles>CCC(O)CCOc1ccc(Br)cc1</smiles>

Compound 3g was purified by flash column chromatography (16\% ethyl acetate in hexane) and obtained as colorless liquid in $90 \%$ yield. 
${ }^{1} \mathrm{H}$ NMR $\left(500 \mathrm{MHz}, \mathrm{CDCl}_{3}\right) \delta 7.36(\mathrm{~d}, J=9.0 \mathrm{~Hz}, 2 \mathrm{H}), 6.78(\mathrm{~d}, J=9.0 \mathrm{~Hz}, 2 \mathrm{H}), 4.13(\mathrm{ddd}, J=9.4,7.3,5.2$ $\mathrm{Hz}, 1 \mathrm{H}), 4.08-4.04$ (m, 1H), $3.92-3.83$ (m, 1H), 2.00 (brs, 1H), $1.98-1.90$ (m, 1H), $1.90-1.77$ (m, 1H), $1.54-1.44(\mathrm{~m}, 3 \mathrm{H}), 1.44-1.32(\mathrm{~m}, 1 \mathrm{H}), 0.94(\mathrm{t}, J=6.7 \mathrm{~Hz}, 3 \mathrm{H})$.

${ }^{13} \mathrm{C} \mathrm{NMR}\left(151 \mathrm{MHz}, \mathrm{CDCl}_{3}\right) \delta 157.85,132.22,116.27,112.94,69.39,65.95,39.83,36.35,18.74,14.02$.

HRMS (EI+) Calculated for $\mathrm{C}_{12} \mathrm{H}_{17} \mathrm{BrO}_{2}[\mathrm{M}]^{+}:$272.0412, Found: 272.0410 .

$[\alpha]_{\mathrm{D}}^{23}=-5.3(\mathrm{c}=1.0, \mathrm{DCM})$.

$\mathrm{SFC}$ analysis (AS-H, 15\% IPA/CO $2,2.5 \mathrm{~mL} / \mathrm{min}, 220 \mathrm{~nm}$ ) indicated $98 \%$ ee: $\mathrm{t}_{\mathrm{R}}($ major $)=4.7 \mathrm{~min}, \mathrm{t}_{\mathrm{R}}($ minor $)=$ $2.4 \mathrm{~min}$.<smiles>CCC[C@H](O)CCN([AsH3])c1ccccc1</smiles>

Compound 3h was purified by flash column chromatography (25\% ethyl acetate in hexane) and obtained as white solid in $97 \%$ yield.

${ }^{1} \mathrm{H}$ NMR $\left(500 \mathrm{MHz}, \mathrm{CDCl}_{3}\right) \delta 7.48(\mathrm{~d}, J=8.3 \mathrm{~Hz}, 2 \mathrm{H}), 7.36-7.27(\mathrm{~m}, 3 \mathrm{H}), 7.24(\mathrm{~d}, J=8.0 \mathrm{~Hz}, 2 \mathrm{H}), 7.09-$ $7.00(\mathrm{~m}, 2 \mathrm{H}), 3.97-3.80(\mathrm{~m}, 2 \mathrm{H}), 3.45(\mathrm{ddd}, J=13.6,6.4,4.1 \mathrm{~Hz}, 1 \mathrm{H}), 2.45$ (brs, 1H), 2.41 (s, 3H), 1.57 $1.50(\mathrm{~m}, 1 \mathrm{H}), 1.49-1.26(\mathrm{~m}, 5 \mathrm{H}), 0.89(\mathrm{t}, J=7.0 \mathrm{~Hz}, 3 \mathrm{H})$.

${ }^{13} \mathrm{C}$ NMR $\left(151 \mathrm{MHz}, \mathrm{CDCl}_{3}\right) \delta 143.41,139.01,135.20,129.40,129.03,128.75,127.92,127.59,67.83,47.57$, 39.35, 35.45, 21.47, 18.83, 13.97 .

HRMS (ESI+) Calculated for $\mathrm{C}_{19} \mathrm{H}_{25} \mathrm{NO}_{3} \mathrm{SNa}[\mathrm{M}+\mathrm{Na}]^{+}: 370.1447$, Found: 370.1443 .

$[\alpha]_{\mathrm{D}}^{23}=-48.2(\mathrm{c}=1.0, \mathrm{DCM})$.

$\mathrm{SFC}$ analysis (AS-H, 10\% IPA/CO $2,2.5 \mathrm{~mL} / \mathrm{min}, 220 \mathrm{~nm}$ ) indicated $96 \%$ ee: $\mathrm{t}_{\mathrm{R}}($ major $)=3.6 \mathrm{~min}, \mathrm{t}_{\mathrm{R}}($ minor $)=$ $3.0 \mathrm{~min}$.<smiles>CCC[C@H](O)CCN([13CH3])Br</smiles>

Compound 3i was purified by flash column chromatography (25\% ethyl acetate in hexane) and obtained as white solid in $97 \%$ yield.

${ }^{1} \mathrm{H}$ NMR (500 MHz, $\left.\mathrm{CDCl}_{3}\right) \delta 7.72(\mathrm{~d}, J=8.3 \mathrm{~Hz}, 2 \mathrm{H}), 7.32(\mathrm{~d}, J=8.0 \mathrm{~Hz}, 2 \mathrm{H}), 7.30-7.25(\mathrm{~m}, 5 \mathrm{H}), 4.51(\mathrm{~d}$, $J=14.6 \mathrm{~Hz}, 1 \mathrm{H}), 4.06(\mathrm{~d}, J=14.5 \mathrm{~Hz}, 1 \mathrm{H}), 3.63-3.40$ (m, 2H), 2.97 (ddd, $J=14.5,6.9,3.7 \mathrm{~Hz}, 1 \mathrm{H}), 2.44$ (s, $3 \mathrm{H}), 2.33$ (brs, $1 \mathrm{H}), 1.39-1.06(\mathrm{~m}, 6 \mathrm{H}), 0.83(\mathrm{t}, J=7.1 \mathrm{~Hz}, 3 \mathrm{H})$.

${ }^{13} \mathrm{C}$ NMR $\left(151 \mathrm{MHz}, \mathrm{CDCl}_{3}\right) \delta 143.38,136.51,136.37,129.73,128.53,128.46,127.84,127.12,67.68,53.15$, 45.71, 39.16, 35.78, 21.45, 18.67, 13.96 .

HRMS (ESI+) Calculated for $\mathrm{C}_{20} \mathrm{H}_{27} \mathrm{NO}_{3} \mathrm{SNa}[\mathrm{M}+\mathrm{Na}]^{+}$: 384.1604 , Found: 384.1599.

$[\alpha]_{\mathrm{D}}^{23}=-11.9(\mathrm{c}=1.0, \mathrm{DCM})$.

$\mathrm{SFC}$ analysis (OZ-H, 20\% IPA/CO $\mathrm{CO}_{2}, 2.5 \mathrm{~mL} / \mathrm{min}, 220 \mathrm{~nm}$ ) indicated $99 \%$ ee: $\mathrm{t}_{\mathrm{R}}$ (major) $=3.8 \mathrm{~min}, \mathrm{t}_{\mathrm{R}}$ (minor) $=2.9 \mathrm{~min}$. 


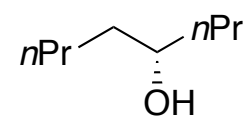

Compound 3m was purified by flash column chromatography (12\% ethyl acetate in hexane) and obtained as colorless liquid in $80 \%$ yield.

$[\alpha]_{\mathrm{D}}^{23}=-0.3(\mathrm{c}=1.0, \mathrm{DCM})$.

${ }^{19} \mathrm{~F}$ NMR analysis with Mosher ester indicated $98 \%$ ee.<smiles>OC(CCCO[SbH3])CCCO[SbH3]</smiles>

Compound 3n was purified by flash column chromatography (12\% ethyl acetate in hexane) and obtained as colorless liquid in $91 \%$ yield.

${ }^{1} \mathrm{H}$ NMR $\left(500 \mathrm{MHz}, \mathrm{CDCl}_{3}\right) \delta 3.90-3.86(\mathrm{~m}, 1 \mathrm{H}), 3.84-3.77(\mathrm{~m}, 2 \mathrm{H}), 3.67-3.60(\mathrm{~m}, 3 \mathrm{H}), 1.70-1.45(\mathrm{~m}$, $6 \mathrm{H}), 0.89$ (s, 9H), 0.88 (s, 9H), 0.07 (s, 6H), 0.04 (s, 6H)

${ }^{13} \mathrm{C}$ NMR $\left(151 \mathrm{MHz}, \mathrm{CDCl}_{3}\right) \delta$ 71.37, 63.35, 62.47, 38.60, 34.23, 29.00, 25.94, 25.86, 18.31, 18.14, -5.34, $5.34,-5.51,-5.54$.

HRMS (ESI+) Calculated for $\mathrm{C}_{18} \mathrm{H}_{43} \mathrm{O}_{3} \mathrm{Si}_{2}[\mathrm{M}+\mathrm{H}]^{+}: 363.2745$, Found: 363.2745 .

$[\alpha]_{\mathrm{D}}^{23}=1.0(\mathrm{c}=1.0, \mathrm{DCM})$.

${ }^{19} \mathrm{~F}$ NMR analysis with Mosher ester indicated $>98 \%$ ee.<smiles>O=C(OCCC[C@H](O)CCOC(=O)c1c(Cl)cc(Cl)cc1Cl)c1c(Cl)cc(Cl)cc1Cl</smiles>

Compound 30 was purified by flash column chromatography (25\% ethyl acetate in hexane) and obtained as white solid in $92 \%$ yield.

${ }^{1} \mathrm{H}$ NMR (500 MHz, $\left.\mathrm{CDCl}_{3}\right) \delta 7.35(\mathrm{~s}, 2 \mathrm{H}), 7.34(\mathrm{~s}, 2 \mathrm{H}), 4.64(\mathrm{ddd}, J=11.1,8.9,4.9 \mathrm{~Hz}, 1 \mathrm{H}), 4.49-4.45(\mathrm{~m}$, 1H), $4.43-4.39$ (m, 2H), $3.89-3.82$ (m, 1H), 2.07 (brs, 1H), $2.02-1.90$ (m, 2H), $1.89-1.78$ (m, 2H), $1.69-$ $1.56(\mathrm{~m}, 2 \mathrm{H})$.

${ }^{13} \mathrm{C}$ NMR $\left(151 \mathrm{MHz}, \mathrm{CDCl}_{3}\right) \delta 164.26,164.05,136.24,136.10,132.53,132.53,132.19,131.98,128.06$, $128.00,67.86,66.30,63.60,36.24,33.67,24.72$.

HRMS (ESI+) Calculated for $\mathrm{C}_{20} \mathrm{H}_{16}{ }^{35} \mathrm{Cl}_{6} \mathrm{O}_{5} \mathrm{Na}[\mathrm{M}+\mathrm{Na}]^{+}: 568.9021$, Found: 568.9021 .

$[\alpha]_{\mathrm{D}}^{23}=-3.4(\mathrm{c}=0.5, \mathrm{DCM})$.

SFC analysis (OD-H, 20\% IPA/CO $2,2.5 \mathrm{~mL} / \mathrm{min}, 220 \mathrm{~nm}$ ) indicated $97 \%$ ee: $\mathrm{t}_{\mathrm{R}}$ (major) $=5.3 \mathrm{~min}, \mathrm{t}_{\mathrm{R}}$ (minor) $=6.2 \mathrm{~min}$. 


\section{Derivatization of Secondary Boronates}<smiles>CCC(CCOc1ccc(Br)cc1)N[R6](=O)OCc1ccccc1</smiles>

Compound 4a was synthesized according to the following procedure.

In an argon-filled drybox, an oven-dried flask was charged with a freshly prepared solution of $O$ methylhydroxylamine ( $880 \mu \mathrm{L}, 0.68 \mathrm{M}$ in THF, 3 equiv). The solution was diluted with $1 \mathrm{~mL}$ of THF. The flask was capped, removed from the box, and cooled to $-78{ }^{\circ} \mathrm{C}$. A solution of $n \mathrm{BuLi}(240 \mu \mathrm{L}, 2.5 \mathrm{M}$ in hexanes, 3 equiv) was added dropwise. The resulting mixture was allowed to stir for $30 \mathrm{~min}$. A separate flask was charged with $\mathbf{2 g}$ (77 $\mathrm{mg}, 0.20 \mathrm{mmol})$ and THF $(1 \mathrm{~mL})$ in the drybox. The solution of $\mathbf{2 g}$ was then added dropwise to the solution of lithiated $O$-methylhydroxylamine. The reaction mixture was warmed to rt and then heated at $60{ }^{\circ} \mathrm{C}$. After 12 hours, the flask was cooled to rt and Boc anhydride ( $155 \mu \mathrm{L}, 3.3$ equiv) was added. After $1 \mathrm{~h}$ at $\mathrm{rt}$, the reaction was quenched with water $(10 \mathrm{~mL})$. The mixture was extracted with ethyl acetate $(15 \mathrm{~mL})$ twice. The combined organic layers were combined, dried over $\mathrm{Na}_{2} \mathrm{SO}_{4}$, and concentrated in vacuo. The crude material was purified by flash column chromatography (12\% ethyl acetate in hexane). Compound 4a was obtained as white solid in $72 \%$ yield.

${ }^{1} \mathrm{H}$ NMR $\left(500 \mathrm{MHz}, \mathrm{CDCl}_{3}\right) \delta 7.34(\mathrm{~d}, J=8.8 \mathrm{~Hz}, 2 \mathrm{H}), 6.76(\mathrm{~d}, J=8.9 \mathrm{~Hz}, 2 \mathrm{H}), 4.48(\mathrm{~d}, J=9.2 \mathrm{~Hz}, 1 \mathrm{H}), 4.02$ - $3.94(\mathrm{~m}, 2 \mathrm{H}), 3.79-3.72(\mathrm{~m}, 1 \mathrm{H}), 2.07-1.89(\mathrm{~m}, 1 \mathrm{H}), 1.87-1.75(\mathrm{~m}, 1 \mathrm{H}), 1.51-1.32(\mathrm{~m}, 4 \mathrm{H}), 1.40(\mathrm{~s}$, 9H), 0.91 (t, $J=7.1 \mathrm{~Hz}, 3 \mathrm{H})$.

${ }^{13} \mathrm{C}$ NMR $\left(151 \mathrm{MHz}, \mathrm{DCl}_{3}\right) \delta 157.93,155.58,132.14,116.32,112.76,79.00,65.63,48.17,37.68,34.77,28.33$, 19.07, 13.89 .

HRMS (ESI-) Calculated for $\mathrm{C}_{17} \mathrm{H}_{25} \mathrm{BrNO}_{3}[\mathrm{M}-\mathrm{H}]^{-}: 370.1023$, Found: 370.1021.

$[\alpha]_{\mathrm{D}}^{23}=-7.5(\mathrm{c}=1.0, \mathrm{DCM})$.

$\mathrm{SFC}$ analysis $\left(\mathrm{OJ}-\mathrm{H}, 5 \% \mathrm{IPA} / \mathrm{CO}_{2}, 2.5 \mathrm{~mL} / \mathrm{min}, 220 \mathrm{~nm}\right)$ indicated $97 \%$ ee: $\mathrm{t}_{\mathrm{R}}($ major $)=3.4 \mathrm{~min}, \mathrm{t}_{\mathrm{R}}(\operatorname{minor})=$ 2.1 min.<smiles></smiles>

Compound $\mathbf{4 b}$ was synthesized according to the following procedure.

In an argon-filled drybox, an oven-dried flask was charged with 3,5-bis(trifluoromethyl)-1-bromobenzene (69 $\mu \mathrm{L}, 2$ equiv) and $1 \mathrm{~mL}$ THF. The flask was capped, removed from the box, and cooled to $-78{ }^{\circ} \mathrm{C}$. A solution of $n \mathrm{BuLi}(160 \mu \mathrm{L}, 2.5 \mathrm{M}$ in hexanes, 2 equiv) was added dropwise. The resulting mixture was allowed to stir for $1 \mathrm{~h}$. A separate flask was charged with $\mathbf{2 h}(91 \mathrm{mg}, 0.20 \mathrm{mmol})$ and THF $(1 \mathrm{~mL})$ in the drybox. The solution of $\mathbf{2 h}$ was then dropwise added to the solution of aryllithium. The reaction mixture was allowed to stir at $-78{ }^{\circ} \mathrm{C}$ for $30 \mathrm{~min}$ and at $\mathrm{rt}$ for $30 \mathrm{~min}$. The resulting solution was added a solution of NBS (71 mg, 2 equiv) in THF (1 $\mathrm{mL}$ ) dropwise. After stirring at $\mathrm{rt}$ for $1 \mathrm{~h}, 20 \% \mathrm{Na}_{2} \mathrm{~S}_{2} \mathrm{O}_{3}$ solution was added. The mixture was extracted with ethyl acetate $(15 \mathrm{~mL})$ twice. The combined organic layers were combined, dried over $\mathrm{Na}_{2} \mathrm{SO}_{4}$, and 
concentrated in vacuo. The crude material was purified by flash column chromatography $(10 \%$ ethyl acetate in hexane). Compound $\mathbf{4 b}$ was obtained as light brown oil in $85 \%$ yield.

${ }^{1} \mathrm{H}$ NMR $\left(500 \mathrm{MHz}, \mathrm{CDCl}_{3}\right) \delta 7.48(\mathrm{~d}, J=7.9 \mathrm{~Hz}, 2 \mathrm{H}), 7.35-7.29(\mathrm{~m}, 3 \mathrm{H}), 7.26(\mathrm{~d}, J=7.9 \mathrm{~Hz}, 2 \mathrm{H}), 7.06$ (dd, $J=7.4 \mathrm{~Hz}, 2 \mathrm{H}), 4.08$ (tt, $J=8.8,4.3 \mathrm{~Hz}, 1 \mathrm{H}), 3.81-3.68(\mathrm{~m}, 2 \mathrm{H}), 2.43$ (s, 3H), $2.05-1.92(\mathrm{~m}, 2 \mathrm{H}), 1.82-$ $1.68(\mathrm{~m}, 2 \mathrm{H}), 1.58-1.45(\mathrm{~m}, 1 \mathrm{H}), 1.44-1.35(\mathrm{~m}, 1 \mathrm{H}), 0.90(\mathrm{t}, J=7.4 \mathrm{~Hz}, 3 \mathrm{H})$.

${ }^{13} \mathrm{C}$ NMR $\left(151 \mathrm{MHz}, \mathrm{CDCl}_{3}\right) \delta 143.44,139.20,134.85,129.35,129.01,128.53,127.91,127.67,53.91,49.09$, 40.99, 37.92, 21.46, 20.53, 13.25.

HRMS (EI+) Calculated for $\mathrm{C}_{19} \mathrm{H}_{24} \mathrm{BrNO}_{2} \mathrm{~S}[\mathrm{M}]^{+}$: 407.0711, Found: 407.0709.

$[\alpha]_{\mathrm{D}}^{23}=14.6(\mathrm{c}=1.0, \mathrm{DCM})$.

$\mathrm{SFC}$ analysis $\left(\mathrm{AD}-\mathrm{H}, 10 \% \mathrm{MeOH} / \mathrm{CO}_{2}, 2.5 \mathrm{~mL} / \mathrm{min}, 220 \mathrm{~nm}\right.$ ) indicated $97 \%$ ee: $\mathrm{t}_{\mathrm{R}}$ (major) $=2.6 \mathrm{~min}, \mathrm{t}_{\mathrm{R}}$ $($ minor $)=2.8 \mathrm{~min}$.

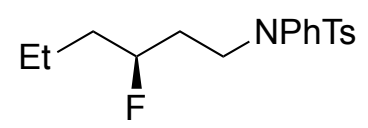

Compound $4 \mathbf{c}$ was synthesized according to the following procedure.

To a solution of $\mathbf{2 h}(77 \mathrm{mg}, 0.20 \mathrm{mmol})$ in THF $(2 \mathrm{~mL})$ at $0{ }^{\circ} \mathrm{C}$ was added $\mathrm{PhLi}(106 \mu \mathrm{L}, 1.8 \mathrm{M}$ in hexanes, 0.95 equiv) dropwise. The solution was allowed to stir for $30 \mathrm{~min}$. Anhydrous $\mathrm{MeCN}(0.5 \mathrm{~mL})$ was added, and the volatile materials were evaporated by high vacuum. $\mathrm{MeCN}(2.5 \mathrm{~mL})$ was then added to the flask. A separate flask was charged with Selecfluor I (92 mg, 1.3 equiv) and $4 \AA$ molecular sieves $(150 \mathrm{mg})$. To the latter flask was added $\mathrm{MeCN}(2.5 \mathrm{~mL})$, and the flask was cooled to $-20{ }^{\circ} \mathrm{C}$. Styrene (11 $\mu \mathrm{L}, 0.5$ equiv) was added. The resulting mixture was stirred briefly, and the solution of ate complex was added dropwise at -20 ${ }^{\circ} \mathrm{C}$. The flask was allowed to stir at $-20{ }^{\circ} \mathrm{C}$ for $20 \mathrm{~h}$. A premixed solution of $\mathrm{NaOH}(2 \mathrm{M}$, aq. $) / 30 \% \mathrm{H}_{2} \mathrm{O}_{2}(2: 1,3$ $\mathrm{mL}$ ) was added dropwise at $0{ }^{\circ} \mathrm{C}$. The resulting solution was warmed to $\mathrm{rt}$ and allowed to stir for $3 \mathrm{~h}$ before being quenched with water $(10 \mathrm{~mL})$. The mixture was extracted with ethyl acetate $(15 \mathrm{~mL})$ twice. The organic layers were combined, dried over $\mathrm{Na}_{2} \mathrm{SO}_{4}$, and concentrated in vacuo. The crude material was purified by flash column chromatography ( $8 \%$ ethyl acetate in hexane). Compound $\mathbf{4 c}$ was obtained as white solid in $67 \%$ yield. ${ }^{1} \mathrm{H}$ NMR $\left(500 \mathrm{MHz}, \mathrm{CDCl}_{3}\right) \delta 7.46(\mathrm{~d}, J=8.0 \mathrm{~Hz}, 2 \mathrm{H}), 7.34-7.29(\mathrm{~m}, 3 \mathrm{H}), 7.24(\mathrm{~d}, J=8.0 \mathrm{~Hz}, 2 \mathrm{H}), 7.04$ (dd, $J=7.6,2.2 \mathrm{~Hz}, 2 \mathrm{H}), 4.57$ (dtt, $\left.J=49.5\left({ }^{1} J_{\mathrm{H}-\mathrm{F}}\right), 8.2,4.0 \mathrm{~Hz}, 1 \mathrm{H}\right), 3.75-3.69(\mathrm{~m}, 1 \mathrm{H}), 3.62(\mathrm{ddd}, J=13.5,8.3$, $5.4 \mathrm{~Hz}, 1 \mathrm{H}), 2.42(\mathrm{~s}, 3 \mathrm{H}), 1.84-1.64(\mathrm{~m}, 2 \mathrm{H}), 1.58-1.28(\mathrm{~m}, 4 \mathrm{H}), 0.89$ (t, $J=7.1 \mathrm{~Hz}, 3 \mathrm{H})$.

${ }^{13} \mathrm{C}$ NMR $\left(151 \mathrm{MHz}, \mathrm{CDCl}_{3}\right) \delta 143.41,139.22,135.01,129.37,129.04,128.65,127.91,127.73,91.47\left(\mathrm{~d}, J_{\mathrm{C}-\mathrm{F}}\right.$ $=167.6 \mathrm{~Hz}), 47.16-47.11\left(\mathrm{~d}, J_{\mathrm{C}-\mathrm{F}}=45.3 \mathrm{~Hz}\right), 37.08\left(\mathrm{~d}, J_{\mathrm{C}-\mathrm{F}}=20.4 \mathrm{~Hz}\right), 34.30\left(\mathrm{~d}, J_{\mathrm{C}-\mathrm{F}}=21.0 \mathrm{~Hz}\right), 21.51,18.20$ $\left(\mathrm{d}, J_{\mathrm{C}-\mathrm{F}}=4.8 \mathrm{~Hz}\right), 13.79$.

${ }^{19} \mathrm{~F}$ NMR $\left(376 \mathrm{MHz}, \mathrm{CDCl}_{3}\right) \delta-181.9--182.2(\mathrm{~m})$.

HRMS (EI+) Calculated for $\mathrm{C}_{27} \mathrm{H}_{29} \mathrm{O}_{2} \mathrm{~N}^{35} \mathrm{Cl}_{3}[\mathrm{M}]^{+}: 349.1512$, Found: 349.1512 .

$[\alpha]_{\mathrm{D}}^{23}=8.0(\mathrm{c}=1.0, \mathrm{DCM})$.

HPLC analysis (AS-H, 5\% IPA/Hex, $0.6 \mathrm{~mL} / \mathrm{min}, 254 \mathrm{~nm}$ ) indicated $70 \%$ ee: $\mathrm{t}_{\mathrm{R}}$ (major) $=24.9 \mathrm{~min}, \mathrm{t}_{\mathrm{R}}($ minor) $=23.0 \mathrm{~min}$. 
<smiles>CC(C)C[C@H](C)CCOC(=O)c1c(Cl)cc(Cl)cc1Cl</smiles>

Compound 4d was synthesized according to the following procedure.

In an argon-filled drybox, an oven-dried flask was charged with $\mathbf{1 b}(90 \mathrm{mg}, 0.20 \mathrm{mmol})$, dibromomethane (35 $\mu \mathrm{L}, 2.5$ equiv) and THF $(2 \mathrm{~mL})$. The flask was capped, removed from the drybox, and cooled to $-78{ }^{\circ} \mathrm{C}$. A solution of $n \mathrm{BuLi}(176 \mu \mathrm{L}, 2.5 \mathrm{M}$ in hexanes, 2.2 equiv) was added dropwise. The resulting mixture was allowed to stir for $10 \mathrm{~min}$, warmed to $\mathrm{rt}$ and stirred for an additional $2 \mathrm{~h}$. The reaction mixture was cooled to 0 ${ }^{\circ} \mathrm{C}$, and a premixed solution of $\mathrm{NaOH}(2 \mathrm{M}$, aq. $) / 30 \% \mathrm{H}_{2} \mathrm{O}_{2}(2: 1,3 \mathrm{~mL})$ was added dropwise. The resulting solution was warmed to $\mathrm{rt}$ and allowed to stir for $3 \mathrm{~h}$ before being quenched with water $(10 \mathrm{~mL})$. The mixture was extracted with ethyl acetate $(15 \mathrm{~mL})$ twice. The organic layers were combined, dried over $\mathrm{Na}_{2} \mathrm{SO}_{4}$, and concentrated in vacuo. The crude material was purified by flash column chromatography $(20 \%$ ethyl acetate in hexane). Compound $\mathbf{4 d}$ was obtained as a colorless oil in $70 \%$ yield.

${ }^{1} \mathrm{H}$ NMR $\left(500 \mathrm{MHz}, \mathrm{CDCl}_{3}\right) \delta 7.35(\mathrm{~s}, 2 \mathrm{H}), 4.52-4.45(\mathrm{~m}, 2 \mathrm{H}), 3.65(\mathrm{dd}, J=10.8,4.0 \mathrm{~Hz}, 1 \mathrm{H}), 3.55(\mathrm{dd}, J=$ 10.8, $5.4 \mathrm{~Hz}, 1 \mathrm{H}), 1.92-1.83(\mathrm{~m}, 1 \mathrm{H}), 1.82-1.72(\mathrm{~m}, 3 \mathrm{H}), 1.71-1.62(\mathrm{~m}, 1 \mathrm{H}), 1.30-1.24(\mathrm{~m}, 1 \mathrm{H}), 1.17-$ $1.12(\mathrm{~m}, 1 \mathrm{H}), 0.89(\mathrm{~d}, J=6.6 \mathrm{~Hz}, 6 \mathrm{H})$.

${ }^{13} \mathrm{C}$ NMR $\left(151 \mathrm{MHz}, \mathrm{CDCl}_{3}\right) \delta 164.13,136.07,132.54,132.26,128.01,65.27,64.96,40.48,35.09,30.42$, $25.30,22.95,22.65$.

HRMS (EI+) Calculated for $\mathrm{C}_{15} \mathrm{H}_{19} \mathrm{Cl}_{3} \mathrm{O}_{3}[\mathrm{M}]^{+}: 352.0400$, Found: 352.0399 .

$[\alpha]_{\mathrm{D}}^{23}=2.1(\mathrm{c}=1.0, \mathrm{DCM})$.

SFC analysis (OD-H, 10\% IPA/CO $\mathrm{CO}_{2}, 10 \mathrm{MPa}, 2.5 \mathrm{~mL} / \mathrm{min}, 220 \mathrm{~nm}$ ) indicated $99 \%$ ee: $\mathrm{t}_{\mathrm{R}}$ (major) $=4.1 \mathrm{~min}, \mathrm{t}_{\mathrm{R}}$ $($ minor $)=3.3 \mathrm{~min}$.

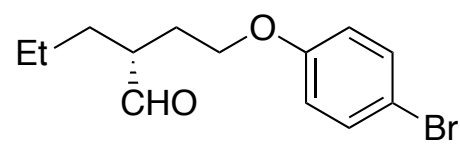

Compound 4e was synthesized according to the following procedure.

In an argon-filled drybox, an oven-dried flask was charged with DCM $(78 \mu \mathrm{L}, 6$ equiv $)$ and THF $(2 \mathrm{~mL})$. The flask was capped, removed from the box, and cooled to $-100{ }^{\circ} \mathrm{C}$. A solution of $n \mathrm{BuLi}(320 \mu \mathrm{L}, 2.5 \mathrm{M}$ in hexanes, 4 equiv) was added dropwise. The resulting mixture was allowed to stir for $10 \mathrm{~min}$. A separate flask was charged with $2 \mathrm{~g}(77 \mathrm{mg}, 0.20 \mathrm{mmol})$ and THF $(1.5 \mathrm{~mL})$ in the drybox. The solution of $2 \mathrm{~g}$ then was added dropwise to the solution of $\mathrm{LiCHCl}_{2}$. The reaction mixture was allowed to warm to rt over $2 \mathrm{~h}$. The reaction mixture was cooled to $0{ }^{\circ} \mathrm{C}$, and a premixed solution of $\mathrm{NaOH}(2 \mathrm{M}$, aq. $) / 30 \% \mathrm{H}_{2} \mathrm{O}_{2}(2: 1,3 \mathrm{~mL})$ was added dropwise. The resulting solution was warmed to $\mathrm{rt}$ and allowed to stir for $3 \mathrm{~h}$ before being quenched with water $(10 \mathrm{~mL})$. The mixture was extracted with ethyl acetate $(15 \mathrm{~mL})$ twice. The organic layers were combined, 
dried over $\mathrm{Na}_{2} \mathrm{SO}_{4}$, and concentrated in vacuo. The crude material was purified by flash column chromatography (5\% ethyl acetate in hexane). Compound $4 \mathrm{e}$ was obtained as colorless oil in $65 \%$ yield.

${ }^{1} \mathrm{H}$ NMR $\left(500 \mathrm{MHz}, \mathrm{CDCl}_{3}\right) \delta 9.66(\mathrm{~d}, J=2.4 \mathrm{~Hz}, 1 \mathrm{H}), 7.41-7.30(\mathrm{~m}, 2 \mathrm{H}), 6.78-6.71(\mathrm{~m}, 2 \mathrm{H}), 3.95(\mathrm{t}, J=$ $6.1 \mathrm{~Hz}, 2 \mathrm{H}), 2.59-2.47(\mathrm{~m}, 1 \mathrm{H}), 2.15$ (ddt, $J=14.6,8.5,6.1 \mathrm{~Hz}, 1 \mathrm{H}), 1.91$ (dtd, $J=14.5,6.3,4.6 \mathrm{~Hz}, 1 \mathrm{H})$, $1.77-1.65(\mathrm{~m}, 1 \mathrm{H}), 1.54-1.45(\mathrm{~m}, 1 \mathrm{H}), 1.41-1.33(\mathrm{~m}, 2 \mathrm{H}), 0.94(\mathrm{t}, J=7.3 \mathrm{~Hz}, 3 \mathrm{H})$.

${ }^{13} \mathrm{C}$ NMR $\left(151 \mathrm{MHz}, \mathrm{CDCl}_{3}\right) \delta 204.25,157.70,132.24,116.26,113.01,65.76,48.78,30.94,28.32,20.11$, 14.04 .

HRMS (EI+) Calculated for $\mathrm{C}_{13} \mathrm{H}_{17} \mathrm{BrO}_{2}[\mathrm{M}]^{+}: 284.0412$, Found: 284.0410s.

$[\alpha]_{\mathrm{D}}^{23}=8.2(\mathrm{c}=1.0, \mathrm{DCM})$.

$\mathrm{SFC}$ analysis $\left(\mathrm{AS}-\mathrm{H}, 5 \% \mathrm{IPA} / \mathrm{CO}_{2}, 2.5 \mathrm{~mL} / \mathrm{min}, 220 \mathrm{~nm}\right)$ indicated $93 \%$ ee: $\mathrm{t}_{\mathrm{R}}($ major $)=4.7 \mathrm{~min}, \mathrm{t}_{\mathrm{R}}($ minor $)=$ $2.8 \mathrm{~min}$.

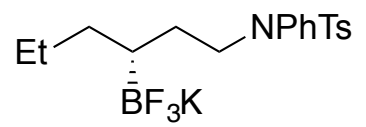

Compound $2 \mathbf{h}-\mathbf{B F}_{3} \mathbf{K}$ was synthesized according to a literature procedure ${ }^{1}$ and obtained as white solid in $80 \%$ yield.

${ }^{1} \mathrm{H}$ NMR (600 MHz, acetone- $\left.d_{6}\right) \delta 7.48(\mathrm{~d}, J=8.3 \mathrm{~Hz}, 2 \mathrm{H}), 7.36-7.24(\mathrm{~m}, 5 \mathrm{H}), 7.10-7.04(\mathrm{~m}, 2 \mathrm{H}), 3.70$ (ddd, $J=13.0,11.1,5.1 \mathrm{~Hz}, 1 \mathrm{H}$ ), 3.59 (ddd, $J=13.0,10.9,5.5 \mathrm{~Hz}, 1 \mathrm{H}$ ), 2.40 (s, 3H), 1.46 (dddd, $J=12.7$, 10.9, 7.7, $5.1 \mathrm{~Hz}, 1 \mathrm{H}), 1.36-1.26(\mathrm{~m}, 2 \mathrm{H}), 1.26-1.19(\mathrm{~m}, 1 \mathrm{H}), 1.19-1.09$ (m, 1H), 0.99 (dddd, $J=12.7$, $10.1,7.9,5.0 \mathrm{~Hz}, 1 \mathrm{H}), 0.75(\mathrm{t}, J=7.3 \mathrm{~Hz}, 3 \mathrm{H}), 0.27-0.18(\mathrm{~m}, 1 \mathrm{H})$.

${ }^{13} \mathrm{C}$ NMR $\left(151 \mathrm{MHz}\right.$, acetone- $\left.d_{6}\right) \delta 143.93,141.01,137.28,130.19,129.57,129.43,128.45,128.02,51.83$, $34.69,31.22,26.31,22.72,21.47,15.27$.

${ }^{11} \mathrm{~B}$ NMR $\left(192 \mathrm{MHz}\right.$, acetone- $\left.d_{6}\right) \delta 4.9$.

${ }^{19} \mathrm{~F}$ NMR $\left(376 \mathrm{MHz}\right.$, acetone- $\left.d_{6}\right) \delta-142.8$ (s).

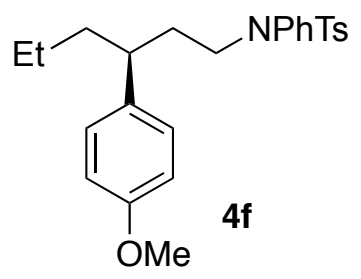

Compound $\mathbf{4 f}$ was synthesized according to the following procedure.

In an argon-filled drybox, a one dram-vial was charged with $\mathbf{2} \mathbf{h}-\mathbf{B F}_{3} \mathbf{K}(53 \mathrm{mg}, 0.12 \mathrm{mmol}), \mathrm{K}_{2} \mathrm{CO}_{3}(50 \mathrm{mg}, 3$ equiv), $\mathrm{Pd}-t \mathrm{Bu}_{3} \mathrm{P} \mathrm{G} 3$ catalyst (13.7 mg, $\left.20 \mathrm{~mol} \%\right)$, 4-chloroanisole (18 $\mu \mathrm{L}, 1.2$ equiv) and toluene $(0.5 \mathrm{~mL})$. The vial was capped with a teflon-coated cap with septum and removed from the drybox. Degassed water $(0.25$ $\mathrm{mL}$ ) was added to the vial. The cap was quickly replaced by a teflon-coated cap without septum. The vial was sealed with electric tape, and heated at $100{ }^{\circ} \mathrm{C}$ for $48 \mathrm{~h}$. The reaction mixture was added water $(10 \mathrm{~mL})$. The mixture was extracted with ethyl acetate $(15 \mathrm{~mL})$ twice. The combined organic layers were combined, dried 
over $\mathrm{Na}_{2} \mathrm{SO}_{4}$, and concentrated in vacuo. The crude material was purified by flash column chromatography ( $10 \%$ ethyl acetate in hexane). Compound $\mathbf{4 f}$ was obtained as white solid in $38 \%$ yield.

${ }^{1} \mathrm{H}$ NMR $\left(600 \mathrm{MHz}, \mathrm{CDCl}_{3}\right) \delta 7.37(\mathrm{~d}, J=8.2 \mathrm{~Hz}, 2 \mathrm{H}), 7.34-7.27(\mathrm{~m}, 3 \mathrm{H}), 7.18(\mathrm{~d}, J=7.9 \mathrm{~Hz}, 2 \mathrm{H}), 7.02-$ $6.97(\mathrm{~m}, 2 \mathrm{H}), 6.97-6.92(\mathrm{~m}, 2 \mathrm{H}), 6.84-6.74(\mathrm{~m}, 2 \mathrm{H}), 3.78(\mathrm{~s}, 3 \mathrm{H}), 3.45-3.41(\mathrm{~m}, 1 \mathrm{H}), 3.29-3.25(\mathrm{~m}, 1 \mathrm{H})$, 2.53 (tt, $J=9.7,4.8 \mathrm{~Hz}, 1 \mathrm{H}), 2.39(\mathrm{~s}, 3 \mathrm{H}), 1.80-1.72(\mathrm{~m}, 1 \mathrm{H}), 1.65-1.59(\mathrm{~m}, 1 \mathrm{H}), 1.49-1.35(\mathrm{~m}, 2 \mathrm{H}), 1.11$ $-1.03(\mathrm{~m}, 2 \mathrm{H}), 0.78(\mathrm{t}, J=7.3 \mathrm{~Hz}, 3 \mathrm{H})$.

${ }^{13} \mathrm{C}$ NMR $\left(151 \mathrm{MHz}, \mathrm{CDCl}_{3}\right) \delta 157.85,143.13,139.36,136.69,135.25,129.24,128.85,128.73,128.40$, 127.66, 127.66, 113.68, 55.17, 48.92, 41.80, 39.14, 35.53, 21.47, 20.42, 13.96.

HRMS (EI+) Calculated for $\mathrm{C}_{26} \mathrm{H}_{31} \mathrm{NO}_{3} \mathrm{~S}[\mathrm{M}]^{+}:$437.2025, Found: 437.2016.

$[\alpha]_{\mathrm{D}}^{23}=-6.3(\mathrm{c}=1.0, \mathrm{DCM})$.

HPLC analysis (OD-H, 3\% IPA/Hex, $1.0 \mathrm{~mL} / \mathrm{min}, 254 \mathrm{~nm}$ ) indicated $87 \%$ ee: $\mathrm{t}_{\mathrm{R}}$ (major) $=7.1 \mathrm{~min}, \mathrm{t}_{\mathrm{R}}$ (minor) $=6.3 \mathrm{~min}$.<smiles>CC(C)C[C@H](CCOC(=O)c1c(Cl)cc(Cl)cc1Cl)c1cc2ccccc2o1</smiles>

Compound $4 \mathrm{~g}$ was synthesized according to the following procedure.

In an argon-filled drybox, an oven-dried flask was charged with benzofuran ( $28 \mu \mathrm{L}, 1.25$ equiv). The solution was diluted with $1 \mathrm{~mL}$ THF. The flask was capped, removed from the box, and cooled to $-78^{\circ} \mathrm{C}$. A solution of $n \mathrm{BuLi}(100 \mu \mathrm{L}, 1.25$ equiv) was added dropwise. The resulting mixture was warmed to rt and stirred for $1 \mathrm{~h}$. A separate flask was charged with $\mathbf{1 b}(90 \mathrm{mg}, 0.20 \mathrm{mmol})$ and THF $(1 \mathrm{~mL})$ in the drybox. Then the solution of 1b was added dropwise to the solution of lithiated benzofuran at $-78{ }^{\circ} \mathrm{C}$. After $1 \mathrm{~h}$ at $-78{ }^{\circ} \mathrm{C}$, the resulting solution was added dropwise to a solution of NBS (71 mg, 2 equiv) in THF $(1 \mathrm{~mL})$. After stirring at $-78{ }^{\circ} \mathrm{C}$ for $1 \mathrm{~h}$, a $20 \%$ aqueous solution of $\mathrm{Na}_{2} \mathrm{~S}_{2} \mathrm{O}_{3}$ was added. The mixture was warmed to rt and extracted with ethyl acetate $(15 \mathrm{~mL})$ twice. The combined organic layers were combined, dried over $\mathrm{Na}_{2} \mathrm{SO}_{4}$, and concentrated in vacuo. The crude material was purified by flash column chromatography ( $2.5 \%$ ethyl acetate in hexane). Compound $\mathbf{4 g}$ was obtained as white solid in $71 \%$ yield.

${ }^{1} \mathrm{H}$ NMR $\left(500 \mathrm{MHz}, \mathrm{CDCl}_{3}\right) \delta 7.54-7.47(\mathrm{~m}, 1 \mathrm{H}), 7.46-7.38(\mathrm{~m}, 1 \mathrm{H}), 7.35(\mathrm{~s}, 2 \mathrm{H}), 7.25-7.16(\mathrm{~m}, 2 \mathrm{H})$, $6.46(\mathrm{~s}, 1 \mathrm{H}), 4.41-4.37(\mathrm{~m}, 1 \mathrm{H}), 4.31-4.27(\mathrm{~m}, 1 \mathrm{H}), 3.17-3.11(\mathrm{~m}, 1 \mathrm{H}), 2.20-2.09(\mathrm{~m}, 2 \mathrm{H}), 1.86-1.79$ $(\mathrm{m}, 1 \mathrm{H}), 1.52-1.41(\mathrm{~m}, 2 \mathrm{H}), 0.91(\mathrm{~d}, J=6.2 \mathrm{~Hz}, 3 \mathrm{H}), 0.87(\mathrm{~d}, J=6.3 \mathrm{~Hz}, 3 \mathrm{H})$.

${ }^{13} \mathrm{C}$ NMR $\left(151 \mathrm{MHz}, \mathrm{CDCl}_{3}\right) \delta 163.95,160.02,154.65,136.05,132.51,132.20,128.51,127.99,123.27$, $122.46,120.34,110.86,103.19,64.52,43.03,34.23,33.19,25.61,23.25,21.78$.

HRMS (EI+) Calculated for $\mathrm{C}_{22} \mathrm{H}_{21}{ }^{35} \mathrm{Cl}_{3} \mathrm{O}_{3}[\mathrm{M}]^{+}:$438.0556, Found: 438.0558 .

$[\alpha]_{\mathrm{D}}^{23}=-40.6(\mathrm{c}=1.0, \mathrm{DCM})$. 
SFC analysis $\left(\mathrm{OJ}-\mathrm{H}, 5 \% \mathrm{IPA} / \mathrm{CO}_{2}, 2.5 \mathrm{~mL} / \mathrm{min}, 220 \mathrm{~nm}\right)$ indicated $99 \%$ ee: $\mathrm{t}_{\mathrm{R}}($ major $)=3.8 \mathrm{~min}, \mathrm{t}_{\mathrm{R}}($ minor $)=$ $3.4 \mathrm{~min}$.<smiles>CCC(CCOc1ccc(Br)cc1)c1ccncc1F</smiles>

Compound $4 \mathrm{~h}$ was synthesized according to the following procedure.

In an argon-filled drybox, an oven-dried flask was charged with diisopropylamine ( $61 \mu \mathrm{L}, 2.2$ equiv) and THF $(0.5 \mathrm{~mL})$. The flask was capped, removed from the drybox, and cooled to $-78^{\circ} \mathrm{C}$. A solution of $n \mathrm{BuLi}(160 \mu \mathrm{L}$, $2.5 \mathrm{M}$ in hexanes, 2 equiv) was added dropwise. The resulting mixture was warmed to $\mathrm{rt}$ and allowed to stir for $15 \mathrm{~min}$. A solution of 3-fluoropyridine ( $34 \mu \mathrm{L}, 2$ equiv) in THF ( $1 \mathrm{~mL}$ ) was added dropwise to the solution of LDA at $-60{ }^{\circ} \mathrm{C}$. A solution of $\mathbf{2 g}(77 \mathrm{mg}, 0.20 \mathrm{mmol})$ in THF $(1 \mathrm{~mL})$ was added after $30 \mathrm{~min}$. The reaction mixture was allowed to stir for $2 \mathrm{~h}$ at the same temperature. TrocCl (61 $\mu \mathrm{L}, 2.2$ equiv) was added, and the mixture was allowed to warm to rt overnight. The mixture was quenched with water and extracted with ethyl acetate $(15 \mathrm{~mL})$ twice. The organic layers were combined, dried over $\mathrm{Na}_{2} \mathrm{SO}_{4}$, and concentrated in vacuo. The obtained material was dissolved in THF $(2 \mathrm{~mL})$, and the reaction mixture was cooled to $0{ }^{\circ} \mathrm{C}$. A premixed solution of $\mathrm{NaOH}(2 \mathrm{M}$, aq. $) / 30 \% \mathrm{H}_{2} \mathrm{O}_{2}(2: 1,3 \mathrm{~mL})$ was added dropwise. The resulting solution was warmed to $\mathrm{rt}$ and allowed to stir for $3 \mathrm{~h}$ before being quenched with water $(10 \mathrm{~mL})$. The mixture was extracted with ethyl acetate $(15 \mathrm{~mL})$ twice. The organic layers were combined, dried over $\mathrm{Na}_{2} \mathrm{SO}_{4}$, and concentrated in vacuo. The crude material was purified by flash column chromatography ( $20 \%$ ethyl acetate in hexane). Compound 4h was obtained as colorless oil in $83 \%$ yield.

${ }^{1} \mathrm{H}$ NMR $\left(500 \mathrm{MHz}, \mathrm{CDCl}_{3}\right) \delta 8.36(\mathrm{~s}, 1 \mathrm{H}), 8.32(\mathrm{~d}, J=4.9 \mathrm{~Hz}, 1 \mathrm{H}), 7.31(\mathrm{~d}, J=9.0 \mathrm{~Hz}, 2 \mathrm{H}), 7.13(\mathrm{t}, J=5.6$ $\mathrm{Hz}, 1 \mathrm{H}), 6.64(\mathrm{~d}, J=8.9 \mathrm{~Hz}, 2 \mathrm{H}), 3.82(\mathrm{dt}, J=9.4,5.9 \mathrm{~Hz}, 1 \mathrm{H}), 3.73-3.69(\mathrm{~m}, 1 \mathrm{H}), 3.21(\mathrm{tt}, J=9.9,5.5 \mathrm{~Hz}$, 1H), $2.21-2.14(\mathrm{~m}, 1 \mathrm{H}), 2.08-2.00(\mathrm{~m}, 1 \mathrm{H}), 1.73-1.62(\mathrm{~m}, 2 \mathrm{H}), 1.27-1.12(\mathrm{~m}, 2 \mathrm{H}), 0.87$ (t, $J=7.3 \mathrm{~Hz}$, $3 \mathrm{H})$.

${ }^{13} \mathrm{C}$ NMR $\left(151 \mathrm{MHz}, \mathrm{CDCl}_{3}\right) \delta 158.43\left(J_{\mathrm{C}-\mathrm{F}}=254 \mathrm{~Hz}\right), 157.70,145.71\left(J_{\mathrm{C}-\mathrm{F}}=4.8 \mathrm{~Hz}\right), 140.27\left(\mathrm{~d}, J_{\mathrm{C}-\mathrm{F}}=12.2\right.$ $\mathrm{Hz}), 138.09\left(\mathrm{~d}, J_{\mathrm{C}-\mathrm{F}}=25.8 \mathrm{~Hz}\right), 132.14,123.32,116.13,112.84,65.84,37.11,35.29,34.25,20.43,13.82$.

${ }^{19} \mathrm{~F} \mathrm{NMR}\left(376 \mathrm{MHz}, \mathrm{CDCl}_{3}\right) \delta-131.4(\mathrm{~s})$.

HRMS (EI+) Calculated for $\mathrm{C}_{17} \mathrm{H}_{19}$ BrFNOs [M] $]^{+}: 351.0634$, Found: 351.0636 .

$[\alpha]_{\mathrm{D}}^{23}=-49.5(\mathrm{c}=1.0, \mathrm{DCM})$.

$\mathrm{SFC}$ analysis (OJ-H, 10\% IPA/CO $2,2.5 \mathrm{~mL} / \mathrm{min}, 220 \mathrm{~nm})$ indicated $97 \%$ ee: $\mathrm{t}_{\mathrm{R}}$ (major) $=5.3 \mathrm{~min}, \mathrm{t}_{\mathrm{R}}(\operatorname{minor})=$ $4.2 \mathrm{~min}$. 


\section{Synthesis of Substrates}

Procedures for synthesizing compounds $\mathbf{1 a}, \mathbf{1 b}, \mathbf{1 c}, \mathbf{1 d}, \mathbf{1 e}, \mathbf{1 f}, \mathbf{1 g}, \mathbf{1 k}$, and $1 \mathrm{o}$ have been reported previously. ${ }^{2}$

General procedure for the synthesis of the sulfonamides through Mitsunobu reactions
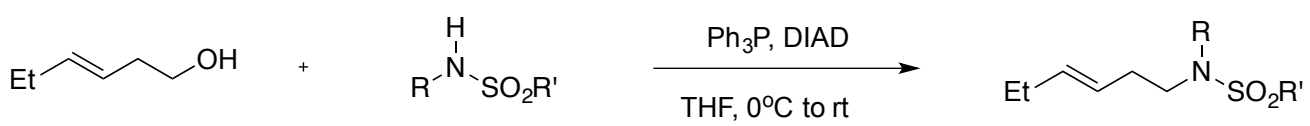

A $100 \mathrm{~mL}$ oven-dried round-bottom flask was charged with trans-3-hexenol (1 equiv, $5 \mathrm{mmol}$ ), sulfonamide (1.2 equiv), $\mathrm{Ph}_{3} \mathrm{P}$ (1.0 equiv) and THF $\left(20 \mathrm{~mL}\right.$ ). The flask was cooled to $0{ }^{\circ} \mathrm{C}$, and DIAD (1.2 equiv) was added dropwise. The mixture was allowed to warm to room temperature and was stirred for $24-48 \mathrm{~h}$. After cooling to $\mathrm{rt}$, saturated $\mathrm{NH}_{4} \mathrm{Cl}(\mathrm{aq})$ was added to quench the reaction. The mixture was extracted with ethyl acetate (twice) and dried over anhydrous $\mathrm{Na}_{2} \mathrm{SO}_{4}$. The crude product obtained after filtration and concentration in vасио was purified by flash column chromatography with hexanes and ethyl acetate as eluents to afford the pure product.

$\mathrm{Et} \sim \mathrm{NTsPh}$

1h was obtained in $84 \%$ isolated yield as white solid.

${ }^{1} \mathrm{H}$ NMR $\left(600 \mathrm{MHz}, \mathrm{CDCl}_{3}\right) \delta 7.47(\mathrm{~d}, J=8.3 \mathrm{~Hz}, 2 \mathrm{H}), 7.33-7.27(\mathrm{~m}, 3 \mathrm{H}), 7.23(\mathrm{~d}, J=8.0 \mathrm{~Hz}, 2 \mathrm{H}), 7.07-$ $7.00(\mathrm{~m}, 2 \mathrm{H}), 5.44-5.39(\mathrm{~m}, 1 \mathrm{H}), 5.31-5.25(\mathrm{~m}, 1 \mathrm{H}), 3.54(\mathrm{t}, J=7.5 \mathrm{~Hz}, 2 \mathrm{H}), 2.41(\mathrm{~s}, 3 \mathrm{H}), 2.11(\mathrm{q}, J=7.1$ $\mathrm{Hz}, 2 \mathrm{H}), 1.98-1.93(\mathrm{~m}, 2 \mathrm{H}), 0.93(\mathrm{t}, J=7.5 \mathrm{~Hz}, 3 \mathrm{H})$.

${ }^{13} \mathrm{C}$ NMR $\left(151 \mathrm{MHz}, \mathrm{CDCl}_{3}\right) \delta 143.19,139.10,135.42,134.77,129.28,128.91,128.86,127.76,127.64$, 124.61, 50.49, 31.65, 25.52, 21.48, 13.62.

HRMS (EI+) Calculated for $\mathrm{C}_{19} \mathrm{H}_{23} \mathrm{NO}_{2} \mathrm{~S}[\mathrm{M}]^{+}: 329.1450$, Found: 329.1446.<smiles>CC/C=C/CCN[SnH2]c1ccccc1</smiles>

$\mathbf{1 i}$ was obtained in $59 \%$ isolated yield as sticky oil.

${ }^{1} \mathrm{H}$ NMR $\left(500 \mathrm{MHz}, \mathrm{CDCl}_{3}\right) \delta 7.74(\mathrm{~d}, J=8.2 \mathrm{~Hz}, 2 \mathrm{H}), 7.37-7.25(\mathrm{~m}, 7 \mathrm{H}), 5.34-5.29(\mathrm{~m}, 1 \mathrm{H}), 5.13-5.07$ (m, 1H), 4.34 (s, 2H), $3.16-3.09$ (m, 2H), $2.43(\mathrm{~s}, 3 \mathrm{H}), 2.05-1.99$ (q, J= $7.9 \mathrm{~Hz}, 2 \mathrm{H}), 1.92-1.87(\mathrm{~m}, 2 \mathrm{H})$, 0.89 (t, $J=7.4 \mathrm{~Hz}, 3 \mathrm{H})$.

${ }^{13} \mathrm{C}$ NMR $\left(151 \mathrm{MHz}, \mathrm{CDCl}_{3}\right) \delta 143.04,137.16,136.38,134.49,129.56,128.39,128.15,127.57,127.03$, $124.70,51.75,47.76,31.37,25.38,21.37,13.48$.

HRMS (EI+) Calculated for $\mathrm{C}_{20} \mathrm{H}_{25} \mathrm{NO}_{2} \mathrm{~S}[\mathrm{M}]^{+}: 344.1606$, Found: 344.1601 .

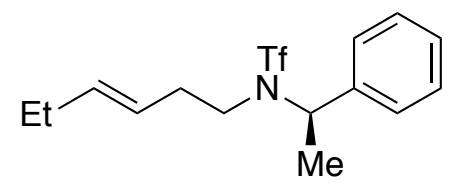

$\mathbf{1 j}$ was obtained in $64 \%$ isolated yield as colorless liquid. 
${ }^{1} \mathrm{H}$ NMR $\left(600 \mathrm{MHz}, \mathrm{CDCl}_{3}\right) \delta 7.62-7.29(\mathrm{~m}, 5 \mathrm{H}), 5.35-5.26(\mathrm{~m}, 2 \mathrm{H}), 5.11-5.06(\mathrm{~m}, 1 \mathrm{H}), 3.18(\mathrm{t}, J=8.4$ $\mathrm{Hz}, 2 \mathrm{H}), 2.18-2.09(\mathrm{~m}, 1 \mathrm{H}), 1.96-1.91(\mathrm{~m}, 2 \mathrm{H}), 1.82-1.70(\mathrm{~m}, 1 \mathrm{H}), 1.72(\mathrm{~d}, J=7.2 \mathrm{~Hz}, 3 \mathrm{H}), 0.93(\mathrm{t}, J=$ $7.5 \mathrm{~Hz}, 3 \mathrm{H})$.

${ }^{13} \mathrm{C}$ NMR $\left(151 \mathrm{MHz}, \mathrm{CDCl}_{3}\right) \delta 138.49,135.26,128.73,128.48,127.63,123.85,120.07\left(\mathrm{q}, J_{\mathrm{C}-\mathrm{F}}=323.6 \mathrm{~Hz}\right)$, 57.41, 45.27, 33.63, 25.39, 16.39, 13.42 .

${ }^{19} \mathrm{~F}$ NMR $\left(376 \mathrm{MHz}, \mathrm{CDCl}_{3}\right) \delta-131.4(\mathrm{~m})$.

HRMS (EI+) Calculated for $\mathrm{C}_{15} \mathrm{H}_{20} \mathrm{~F}_{3} \mathrm{NO}_{2} \mathrm{~S}[\mathrm{M}]^{+}: 335.1167$, Found: 335.1164 .

$[\alpha]_{\mathrm{D}}^{23}=18.2(\mathrm{c}=1.0, \mathrm{DCM})$.

Compound S1a was reported previously. ${ }^{2}$ Compounds S1b, S1c, and S1d were synthesized following the literature procedure. ${ }^{2}$ Compounds $\mathbf{5}$ and $\mathbf{S 2}$ are known compounds and were synthesized following the literature procedure. ${ }^{3}$<smiles>CC/C=C/CCOC(=O)c1ccc(OC)cc1</smiles>

S1b was obtained in $66 \%$ isolated yield as colorless liquid.

${ }^{1} \mathrm{H}$ NMR $\left(500 \mathrm{MHz}, \mathrm{CDCl}_{3}\right) \delta 8.33(\mathrm{~d}, J=8.8 \mathrm{~Hz}, 2 \mathrm{H}), 7.25(\mathrm{~d}, J=8.7,2 \mathrm{H}), 5.98-5.91(\mathrm{~m}, 1 \mathrm{H}), 5.82-5.75$ $(\mathrm{m}, 1 \mathrm{H}), 4.63(\mathrm{t}, J=6.8 \mathrm{~Hz}, 2 \mathrm{H}), 4.19(\mathrm{~s}, 3 \mathrm{H}), 2.78(\mathrm{q}, J=6.9 \mathrm{~Hz}, 2 \mathrm{H}), 2.41-2.31(\mathrm{~m}, 2 \mathrm{H}), 1.31(\mathrm{t}, J=7.5 \mathrm{~Hz}$, $3 \mathrm{H})$.

${ }^{13} \mathrm{C}$ NMR $\left(151 \mathrm{MHz}, \mathrm{CDCl}_{3}\right) \delta 165.93,163.02,134.79,131.25,123.97,122.63,113.26,55.01,31.89,25.42$, 13.50 .

EI-MS Calculated for $\mathrm{C}_{14} \mathrm{H}_{18} \mathrm{O}_{3}[\mathrm{M}]^{+}:$234.1, Found: 234.0.<smiles>CC/C=C/CCOC(=O)c1ccc(C(F)(F)F)cc1</smiles>

S1c was obtained in $41 \%$ isolated yield as colorless liquid.

${ }^{1} \mathrm{H}$ NMR $\left(500 \mathrm{MHz}, \mathrm{CDCl}_{3}\right) \delta 8.23-8.10(\mathrm{~d}, J=8.2 \mathrm{~Hz}, 2 \mathrm{H}), 7.71(\mathrm{~d}, J=8.4 \mathrm{~Hz}, 2 \mathrm{H}), 5.65-5.58(\mathrm{~m}, 1 \mathrm{H})$, $5.47-5.40(\mathrm{~m}, 1 \mathrm{H}), 4.37(\mathrm{t}, J=6.8 \mathrm{~Hz}, 2 \mathrm{H}), 2.48(\mathrm{q}, J=6.7 \mathrm{~Hz}, 2 \mathrm{H}), 2.09-1.96(\mathrm{~m}, 2 \mathrm{H}), 0.98(\mathrm{t}, J=7.5 \mathrm{~Hz}$, $3 \mathrm{H})$.

${ }^{13} \mathrm{C}$ NMR (151 MHz, $\left.\mathrm{CDCl}_{3}\right) \delta 165.12,135.32,134.20$ (q, $\left.J_{\mathrm{C}-\mathrm{F}}=32.6 \mathrm{~Hz}\right), 133.61,129.82,125.19,123.74$, $123.59\left(\mathrm{q}, J_{\mathrm{C}-\mathrm{F}}=272.7 \mathrm{~Hz}\right), 64.96,31.89,25.53,13.51$.

EI-MS Calculated for $\mathrm{C}_{14} \mathrm{H}_{15} \mathrm{O}_{2} \mathrm{~F}_{2}[\mathrm{M}-\mathrm{F}]^{+}:$: 253.1, Found: 253.0. 


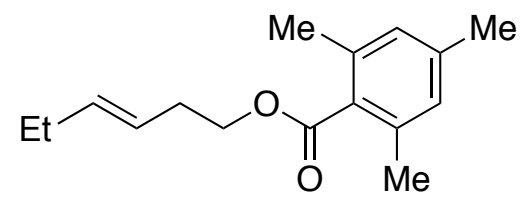

S1d was obtained in $99 \%$ isolated yield as colorless liquid (10\% of an unidentified impurity is present).

${ }^{1} \mathrm{H}$ NMR $\left(600 \mathrm{MHz}, \mathrm{CDCl}_{3}\right) \delta 6.87(\mathrm{~s}, 2 \mathrm{H}), 5.66-5.61(\mathrm{~m}, 1 \mathrm{H}), 5.49-5.44(\mathrm{~m}, 1 \mathrm{H}), 4.36(\mathrm{t}, J=6.8 \mathrm{~Hz}, 2 \mathrm{H})$,

$2.47(\mathrm{q}, J=6.7 \mathrm{~Hz}, 2 \mathrm{H}), 2.33(\mathrm{~s}, 6 \mathrm{H}), 2.30(\mathrm{~s}, 3 \mathrm{H}), 2.11-2.00(\mathrm{~m}, 2 \mathrm{H}), 1.01(\mathrm{t}, J=7.5 \mathrm{~Hz}, 3 \mathrm{H})$.

${ }^{13} \mathrm{C}$ NMR $\left(151 \mathrm{MHz}, \mathrm{CDCl}_{3}\right) \delta 170.07,139.10,136.29,135.06,131.16,128.35,124.23,64.55,32.00,25.60$,

21.06, 19.77, 13.62.

HRMS (EI+) Calculated for $\mathrm{C}_{16} \mathrm{H}_{22} \mathrm{O}_{2}[\mathrm{M}]^{+}: 246.1620$, Found: 246.1618 . 


\section{Summary of the Effect of Reaction Parameters}

Table S1 Evaluation of effect of reaction parameters for regioselective hydroboration ${ }^{a}$

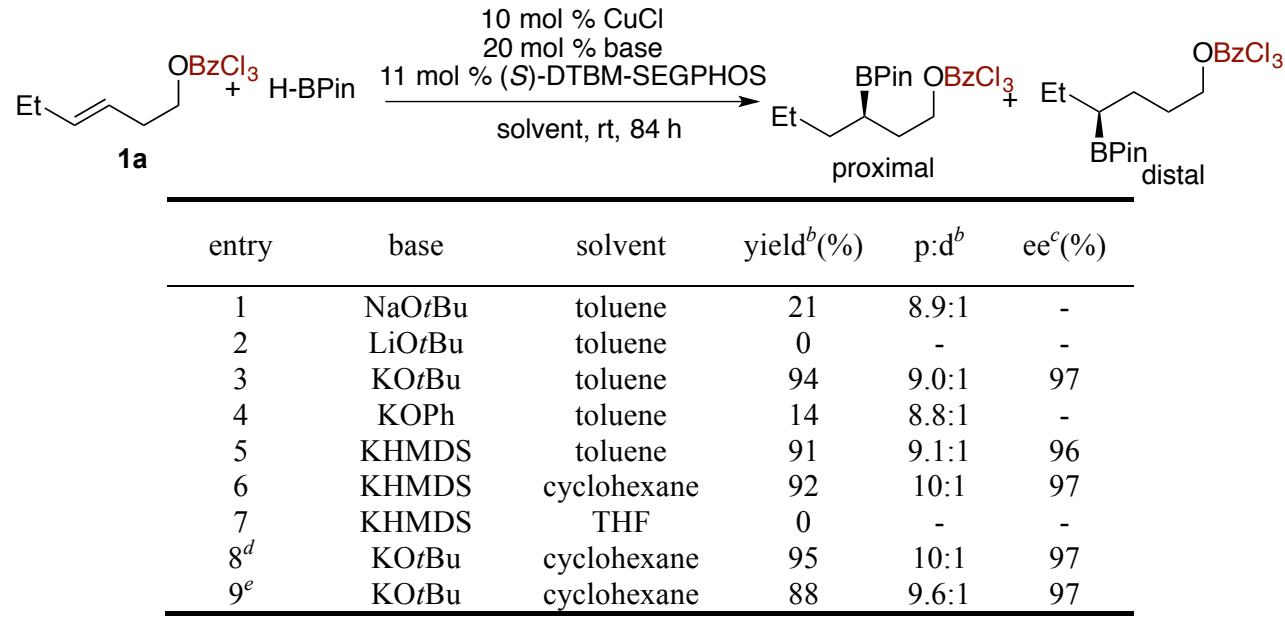

${ }^{a}$ Reaction conditions: 1a $(0.1 \mathrm{mmol})$, HBPin (2 equiv), $\mathrm{CuCl}(10 \mathrm{~mol} \%)$ and $(S)$-DTBM-SEGPHOS $(11 \mathrm{~mol} \%)$, base (20 mol $\%)$ in solvent $(0.2 \mathrm{~mL}), \mathrm{rt}, 84 \mathrm{~h} ;{ }^{b}$ Determined by GC analysis; ${ }^{c}$ Determined by SFC for the major isomer; ${ }^{d} 12 \mathrm{~h} ;{ }^{e}$ Reaction conditions: $1 \mathrm{a}(0.1 \mathrm{mmol}), \mathrm{HBPin}(1.2$ equiv), $\mathrm{CuCl}(2.5 \mathrm{~mol} \%),(S)$-DTBM-SEGPHOS (3 mol \%), base (5 mol \%), rt, $36 \mathrm{~h}$. 


\section{Hydroboration of Homoallyl Benzoate Derivatives and Homoallyl Acetate (5) and Pivalate}

Table S2 Evaluation of directing groups for regioselective hydroboration ${ }^{a}$

$$
\begin{aligned}
& 5 \mathrm{~mol} \% \mathrm{CuCl} \\
& 10 \mathrm{~mol} \% \mathrm{KOtBu}
\end{aligned}
$$

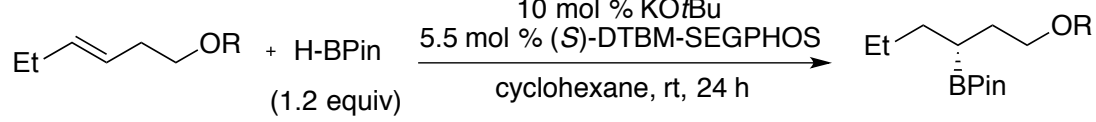
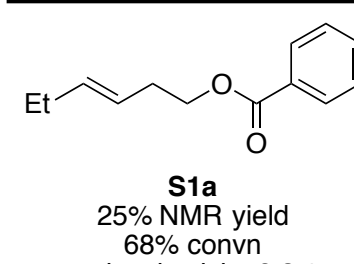

regioselectivity $6.2: 1$

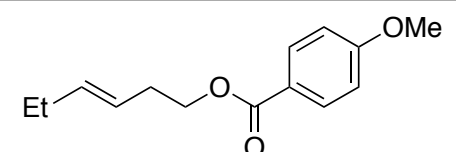

s1b $35 \%$ NMR yield $50 \%$ convn regioselectivity $5.8: 1$

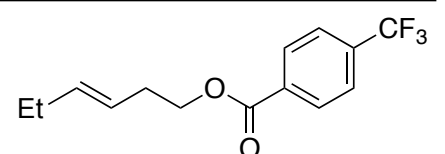

O

$<5 \%$ NMR yield

$100 \%$ convn

regioselectivity $7.3: 1$

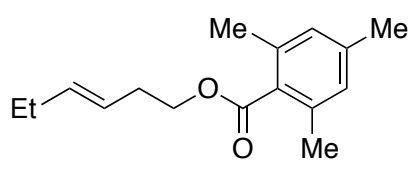

S1d $66 \%$ NMR yield $69 \%$ convn regioselectivity 8.0:1<smiles>CC/C=C/CCOC(=O)c1c(Cl)cc(Cl)cc1Cl</smiles>

$1 \mathrm{a}$ 95\% NMR yield $100 \%$ convn regioselectivity $9.7: 1$

${ }^{a}$ Regioselectivity was determined by GC analysis; yields and conversions were determined by ${ }^{1} \mathrm{H}$ NMR spectroscopy.

Table S3 Hydroboration of homoallyl acetate and pivalate ${ }^{a}$

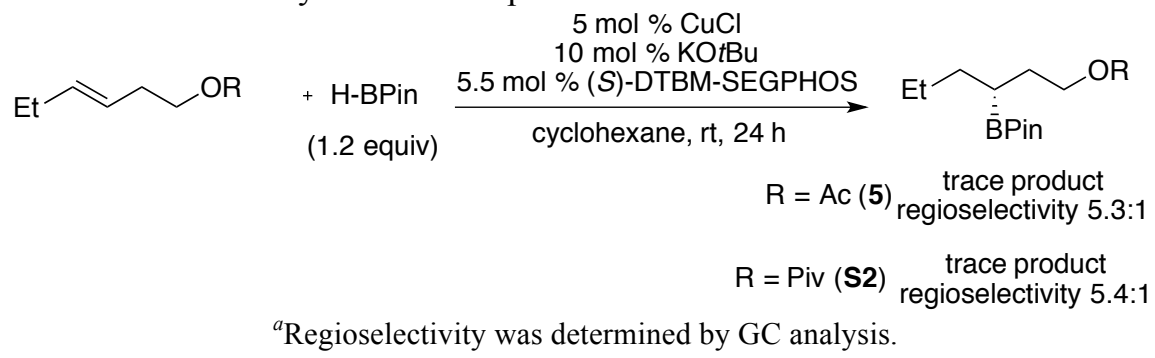

The hydroboration reactions of S1a-S1d, 1a, 5, and S2 were conducted following the General Procedure Condition B with a slight alteration:

In an argon-filled dry box, a 1-dram vial was charged with $\mathrm{CuCl}(1.0 \mathrm{mg}, 5 \mathrm{~mol} \%), \mathrm{KO} t \mathrm{Bu}(2.2 \mathrm{mg}, 10 \mathrm{~mol}$ $\%),(S)$-DTBM-SEGPHOS (13.0 mg, $5.5 \mathrm{~mol} \%)$ and cyclohexane $(200 \mu \mathrm{L})$. The mixture was allowed to stir at ambient temperature for 3 minutes before the addition of pinacolborane ( $35 \mu \mathrm{L}, 0.24 \mathrm{mmol}, 1.2$ equiv). After brief stirring ( $30-60$ seconds), the solution was added the alkene ( $0.2 \mathrm{mmol}, 1$ equiv), which was preweighed in another vial. The second vial was then washed twice with $100 \mu \mathrm{L}$ cyclohexane each time. The vial was then capped, sealed with electrical tape, and removed from the box. After $24 \mathrm{~h}$ of stirring at $\mathrm{rt}$, The reaction vial was added 1,3,5-trimethoxybenzene $(16.8 \mathrm{mg}, 0.1 \mathrm{mmol})$ as an internal standard and diluted with $2 \mathrm{~mL}$ of ethyl acetate and the resulting solution was filtered through silica gel. An aliquot of $0.3 \mathrm{~mL}$ was taken for GC analysis. The rest of the crude material was concentrated in vacuo and analyzed by ${ }^{1} \mathrm{H}$ NMR spectroscopy. 


\section{Effect of Olefin Position and Geometry on the Hydroboration}
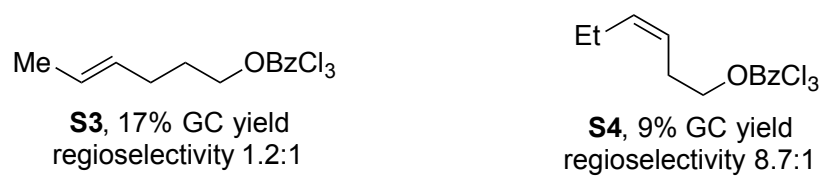

Both the reactivity and regioselectivity of the hydroboration are sensitive to the structure of the substrate. cisAlkene $\mathbf{S 4},{ }^{2}$ which is analogous to the trans-alkene 1a, underwent hydroboration in low yield (9\% by GC) and with regioselectivity that is slightly lower than that of its trans-counterpart. The homoallylic trichlorobenzoate $\mathbf{S 3}^{2}$ underwent hydroboration with poor regioselectivity (1.2:1) and low yield (17\% by GC).

\section{Experimental procedure:}

In an argon-filled dry box, a 1-dram vial was charged with $\mathrm{CuCl}(1.0 \mathrm{mg}, 10 \mathrm{~mol} \%), \mathrm{KO} t \mathrm{Bu}(2.2 \mathrm{mg}, 20 \mathrm{~mol}$ $\%),(S)$-DTBM-SEGPHOS (13.0 mg, $11 \mathrm{~mol} \%)$ and cyclohexane $(100 \mu \mathrm{L})$. The mixture was allowed to stir at ambient temperature for 3 minutes before the addition of pinacolborane ( $29 \mu \mathrm{L}, 0.2 \mathrm{mmol}, 2.0$ equiv). After brief stirring ( $30-60$ seconds), the solution was added the alkene ( $0.1 \mathrm{mmol}, 1$ equiv), which was pre-weighed in another vial. The second vial was then washed twice with $50 \mu \mathrm{L}$ cyclohexane each time. The vial was added $10 \mu \mathrm{L}$ dodecane, then capped, sealed with electrical tape, and removed from the box. After $84 \mathrm{~h}$ of stirring at $\mathrm{rt}$, the reaction mixture was filtered through celite. An aliquot was taken for GC analysis. 


\section{Hydroboration of 1a on a Gram Scale}

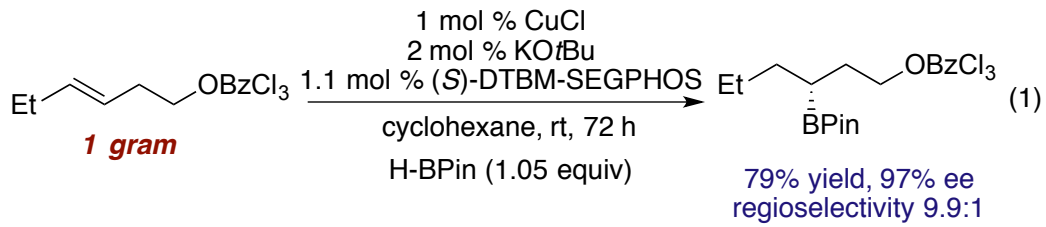

In an argon-filled dry box, a $20 \mathrm{~mL}$ vial was charged with $\mathrm{CuCl}(3.3 \mathrm{mg}, 1 \mathrm{~mol} \%), \mathrm{KO} t \mathrm{Bu}(7.3 \mathrm{mg}, 2 \mathrm{~mol} \%)$, $(S)$-DTBM-SEGPHOS (42.2 $\mathrm{mg}, 1.1 \mathrm{~mol} \%)$ and cyclohexane $(2 \mathrm{~mL})$. The mixture was allowed to stir at ambient temperature for 3 minutes before the addition of pinacolborane (500 $\mu \mathrm{L}, 3.45 \mathrm{mmol}$, 1.06 equiv). After brief stirring ( 30 - 60 seconds), the solution was added $1 \mathrm{a}(1.00 \mathrm{~g}, 3.25 \mathrm{mmol}, 1$ equiv), which was preweighed in another vial. The second vial was then washed five times with $500 \mu \mathrm{L}$ cyclohexane each time. The vial was then capped, sealed with electrical tape, and removed from the box. After $72 \mathrm{~h}$ of stirring at rt, an aliquot of $20 \mu \mathrm{L}$ was removed and analyzed by GC. The reaction vial was diluted with $20 \mathrm{~mL}$ of ethyl acetate and the resulting solution was filtered through Celite. The crude material was concentrated in vacuo and purified by flash column chromatography (CombiFlash, 2.4\% ethyl acetate in hexanes) to afford the pure product in colorless oil (1.124 g, 79\% yield). 


\section{Assignment of Absolute Stereochemistry}

The absolute configuration of $\mathbf{2 c}$ was unambiguously determined to be $R$ at the boron-bound stereocenters, as established by single-crystal x-ray crystallography. The absolute configurations of all other hydroboration products were assigned by analogy. The absolute configurations of all secondary alcohols were assigned by the established retention of configuration for oxidation of boronates. ${ }^{4}$ The absolute configurations of all products from derivatization were assigned by the established retention of configuration for the transformations, ${ }^{5-9}$ except for compounds $\mathbf{4 b}, \mathbf{4 c}$ and $\mathbf{4 f}$, which were assigned as by the established inversion of configuration. ${ }^{10-12}$

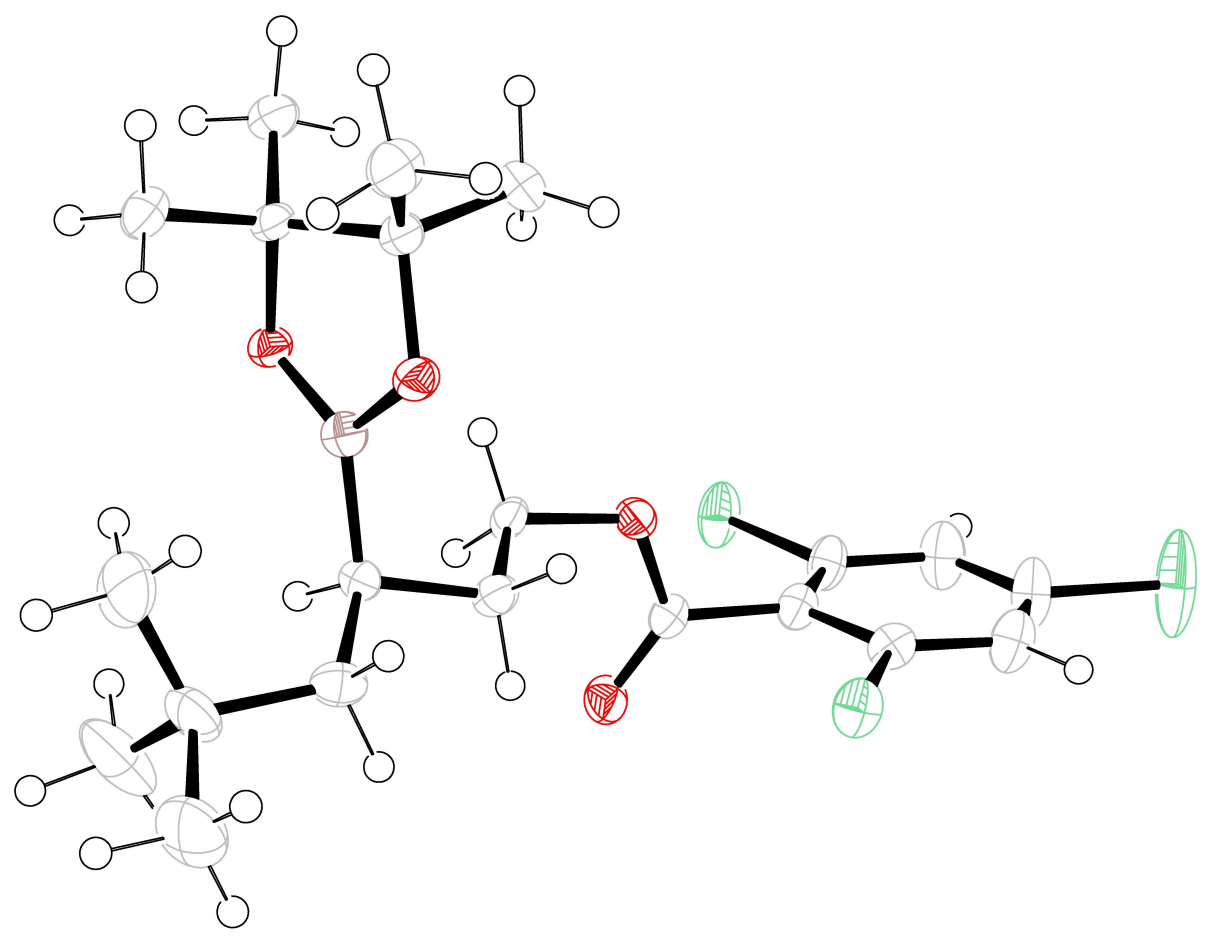

Figure S1 ORTEP drawing of compound 2c 


\section{Crystallographic Information of Compound 2c}

A colorless block $0.150 \times 0.120 \times 0.100 \mathrm{~mm}$ in size was mounted on a cryoloop with Paratone oil. Data were collected in a nitrogen gas stream at $100(2) \mathrm{K}$. The crystal-to-detector distance was $50 \mathrm{~mm}$, and the exposure time was 10 seconds per frame using a scan width of $2.0^{\circ}$. Data collection was $100.0 \%$ complete to $25.000^{\circ}$ in $\theta$. A total of 29929 reflections were collected covering the indices, $-11<=h<=11,-13<=k<=13$, $14<=k<=14.8930$ reflections were found to be symmetry independent, with an $\mathrm{R}_{\text {int }}$ of 0.0282 . Indexing and unit cell refinement indicated a primitive, triclinic lattice. The space group was found to be P 1 (No. 1). The data were integrated using the Bruker SAINT software program and scaled using the SADABS software program. Solution by iterative methods (SHELXT-2014) produced a complete heavy-atom phasing model consistent with the proposed structure. All non-hydrogen atoms were refined anisotropically by full-matrix least squares (SHELXL-2014). All hydrogen atoms were placed using a riding model. Their positions were constrained, relative to their parent atom, using the appropriate HFIX command in SHELXL-2014. The absolute configuration was unambiguously determined to be $R$ at all chiral centers. 
Table S4 Crystal data and structure refinement.

Empirical formula

Formula weight

Temperature

Wavelength

Crystal system

Space group

Unit cell dimensions

Volume

Z

Density (calculated)

Absorption coefficient

$\mathrm{F}(000)$

Crystal size

Theta range for data collection

Index ranges

Reflections collected

Independent reflections

Completeness to theta $=25.000^{\circ}$

Absorption correction

Max. and min. transmission

Refinement method

Data / restraints / parameters

Goodness-of-fit on $\mathrm{F}^{2}$

Final $\mathrm{R}$ indices [I>2sigma(I)]

$\mathrm{R}$ indices (all data)

Absolute structure parameter

Extinction coefficient

Largest diff. peak and hole
$\mathrm{C}_{21} \mathrm{H}_{30} \mathrm{BCl}_{3} \mathrm{O}_{4}$

463.61

100(2) K

$0.71073 \AA$

Triclinic

P 1

$\mathrm{a}=9.8418(5) \AA \quad \alpha=101.9250(10)^{\circ}$.

$\mathrm{b}=11.2380(6) \AA \quad \beta=109.9040(10)^{\circ}$.

$\mathrm{c}=12.2846(7) \AA \quad \gamma=94.5190(10)^{\circ}$.

1233.43(12) $\AA^{3}$

2

$1.248 \mathrm{Mg} / \mathrm{m}^{3}$

$0.394 \mathrm{~mm}^{-1}$

488

$0.150 \times 0.120 \times 0.100 \mathrm{~mm}^{3}$

1.820 to $25.354^{\circ}$.

$-11<=\mathrm{h}<=11,-13<=\mathrm{k}<=13,-14<=1<=14$

29929

$8930[\mathrm{R}(\mathrm{int})=0.0282]$

$100.0 \%$

Semi-empirical from equivalents

0.928 and 0.855

Full-matrix least-squares on $\mathrm{F}^{2}$

8930 / 3 / 537

1.047

$\mathrm{R} 1=0.0371, \mathrm{wR} 2=0.0920$

$\mathrm{R} 1=0.0406, \mathrm{wR} 2=0.0952$

$-0.026(15)$

$\mathrm{n} / \mathrm{a}$

0.380 and -0.330 e. $\AA^{-3}$ 
Table S5. Atomic coordinates $\left(\times 10^{4}\right)$ and equivalent isotropic displacement parameters $\left(\AA^{2} \times 10^{3}\right)$. $U(e q)$ is defined as one third of the trace of the orthogonalized $U^{i j}$ tensor.

\begin{tabular}{|c|c|c|c|c|}
\hline & $\mathrm{x}$ & $\mathrm{y}$ & $\mathrm{z}$ & $\mathrm{U}(\mathrm{eq})$ \\
\hline $\mathrm{C}(1)$ & $4451(4)$ & 2421(3) & $2340(3)$ & $24(1)$ \\
\hline$C(2)$ & $5152(4)$ & $2552(3)$ & $3563(3)$ & $25(1)$ \\
\hline$C(3)$ & $6489(5)$ & $2186(4)$ & $4047(4)$ & $33(1)$ \\
\hline$C(4)$ & $7135(5)$ & $1677(4)$ & $3252(4)$ & $40(1)$ \\
\hline$C(5)$ & $6494(5)$ & $1515(4)$ & 2033(4) & $39(1)$ \\
\hline$C(6)$ & $5138(5)$ & $1897(4)$ & $1596(3)$ & $29(1)$ \\
\hline$C(7)$ & 2990(4) & 2813(3) & $1862(3)$ & $21(1)$ \\
\hline $\mathrm{C}(8)$ & $1813(4)$ & $4530(3)$ & $1509(3)$ & $22(1)$ \\
\hline $\mathrm{C}(9)$ & $1224(4)$ & $4816(3)$ & $2501(3)$ & $24(1)$ \\
\hline$C(10)$ & $-55(4)$ & $5550(3)$ & 2183(3) & $21(1)$ \\
\hline $\mathrm{C}(11)$ & $-791(4)$ & $5608(4)$ & $3113(3)$ & $29(1)$ \\
\hline $\mathrm{C}(12)$ & $-2330(4)$ & $5956(4)$ & 2774(4) & $38(1)$ \\
\hline $\mathrm{C}(13)$ & $-2289(6)$ & $7269(5)$ & $2616(6)$ & $59(2)$ \\
\hline$C(14)$ & $-3387(5)$ & $5052(6)$ & $1644(5)$ & $66(2)$ \\
\hline$C(15)$ & $-2861(6)$ & $5891(6)$ & $3795(5)$ & $57(1)$ \\
\hline$C(16)$ & $986(4)$ & $8499(3)$ & $1360(3)$ & $22(1)$ \\
\hline $\mathrm{C}(17)$ & 2167(4) & $8650(3)$ & $2628(3)$ & $25(1)$ \\
\hline $\mathrm{C}(18)$ & $-206(4)$ & $9284(3)$ & $1358(4)$ & $27(1)$ \\
\hline $\mathrm{C}(19)$ & $1570(5)$ & $8662(4)$ & $396(4)$ & $29(1)$ \\
\hline$C(20)$ & $2386(5)$ & 9899(4) & $3488(4)$ & $36(1)$ \\
\hline $\mathrm{C}(21)$ & $3636(4)$ & $8330(4)$ & $2602(4)$ & $35(1)$ \\
\hline $\mathrm{C}(22)$ & $5003(4)$ & $7722(3)$ & $7615(4)$ & $22(1)$ \\
\hline$C(23)$ & $4229(4)$ & $7524(3)$ & $6394(3)$ & $22(1)$ \\
\hline$C(24)$ & 2917(4) & 7933(4) & $5939(3)$ & $26(1)$ \\
\hline$C(25)$ & $2347(5)$ & $8538(4)$ & $6737(4)$ & $34(1)$ \\
\hline$C(26)$ & $3060(5)$ & $8761(4)$ & $7976(4)$ & $34(1)$ \\
\hline$C(27)$ & $4380(4)$ & $8350(4)$ & $8386(3)$ & $26(1)$ \\
\hline $\mathrm{C}(28)$ & $6473(4)$ & $7324(3)$ & $8072(3)$ & $23(1)$ \\
\hline $\mathrm{C}(29)$ & $7660(5)$ & $5592(4)$ & $8345(4)$ & $27(1)$ \\
\hline$C(30)$ & 7972(4) & $5196(4)$ & $7189(4)$ & $29(1)$ \\
\hline $\mathrm{C}(31)$ & $9163(4)$ & $4361(3)$ & $7289(3)$ & $27(1)$ \\
\hline
\end{tabular}




\begin{tabular}{|c|c|c|c|c|}
\hline$C(32)$ & $10686(4)$ & $5038(4)$ & $8140(4)$ & $34(1)$ \\
\hline$C(33)$ & 11992(4) & $4552(4)$ & $7873(4)$ & $40(1)$ \\
\hline$C(34)$ & $13382(5)$ & $5191(6)$ & $8924(5)$ & $57(1)$ \\
\hline$C(35)$ & $12069(5)$ & $4867(5)$ & $6740(4)$ & $53(1)$ \\
\hline$C(36)$ & $11881(5)$ & $3148(5)$ & $7710(5)$ & $48(1)$ \\
\hline$C(37)$ & $8415(4)$ & $1664(3)$ & $8572(3)$ & $24(1)$ \\
\hline $\mathrm{C}(38)$ & $7120(4)$ & $1388(4)$ & $7330(4)$ & $29(1)$ \\
\hline$C(39)$ & $7970(5)$ & $1611(4)$ & $9621(4)$ & $32(1)$ \\
\hline $\mathrm{C}(40)$ & $9606(4)$ & $886(3)$ & $8554(4)$ & $30(1)$ \\
\hline $\mathrm{C}(41)$ & $5693(5)$ & $1724(5)$ & $7420(4)$ & $43(1)$ \\
\hline $\mathrm{C}(42)$ & $6883(5)$ & $83(4)$ & $6553(4)$ & $41(1)$ \\
\hline $\mathrm{B}(1)$ & $568(5)$ & $6849(4)$ & 2097(4) & $22(1)$ \\
\hline $\mathrm{B}(2)$ & $8640(5)$ & $3162(4)$ & $7605(4)$ & $25(1)$ \\
\hline $\mathrm{O}(1)$ & 1841(3) & $2134(2)$ & $1517(2)$ & $29(1)$ \\
\hline $\mathrm{O}(2)$ & $3160(3)$ & $4003(2)$ & 1883(2) & $23(1)$ \\
\hline $\mathrm{O}(3)$ & $296(3)$ & $7207(2)$ & $1057(2)$ & $22(1)$ \\
\hline $\mathrm{O}(4)$ & $1554(3)$ & $7704(2)$ & $3066(2)$ & $24(1)$ \\
\hline $\mathrm{O}(5)$ & $7605(3)$ & $8001(2)$ & $8360(3)$ & $30(1)$ \\
\hline $\mathrm{O}(6)$ & $6320(3)$ & $6143(2)$ & $8076(2)$ & $26(1)$ \\
\hline $\mathrm{O}(7)$ & $9047(3)$ & $2940(2)$ & $8714(2)$ & $25(1)$ \\
\hline $\mathrm{O}(8)$ & $7609(3)$ & $2240(2)$ & $6742(2)$ & $28(1)$ \\
\hline $\mathrm{Cl}(1)$ & $4312(1)$ & $3208(1)$ & $4523(1)$ & $31(1)$ \\
\hline $\mathrm{Cl}(2)$ & $8835(2)$ & $1235(2)$ & $3839(1)$ & $74(1)$ \\
\hline $\mathrm{Cl}(3)$ & $4309(1)$ & $1744(1)$ & $71(1)$ & $38(1)$ \\
\hline $\mathrm{Cl}(4)$ & $4962(1)$ & $6755(1)$ & $5407(1)$ & $30(1)$ \\
\hline $\mathrm{Cl}(5)$ & $691(1)$ & $9045(2)$ & $6183(1)$ & $66(1)$ \\
\hline $\mathrm{Cl}(6)$ & $5308(1)$ & $8595(1)$ & $9917(1)$ & $36(1)$ \\
\hline
\end{tabular}


Table S6. Bond lengths $[\AA]$ and angles $\left[{ }^{\circ}\right]$.

\begin{tabular}{|c|c|c|c|}
\hline$C(1)-C(6)$ & $1.379(6)$ & $\mathrm{C}(14)-\mathrm{H}(14 \mathrm{~B})$ & 0.9800 \\
\hline $\mathrm{C}(1)-\mathrm{C}(2)$ & $1.393(5)$ & $\mathrm{C}(14)-\mathrm{H}(14 \mathrm{C})$ & 0.9800 \\
\hline $\mathrm{C}(1)-\mathrm{C}(7)$ & $1.496(5)$ & $\mathrm{C}(15)-\mathrm{H}(15 \mathrm{~A})$ & 0.9800 \\
\hline$C(2)-C(3)$ & $1.381(6)$ & $\mathrm{C}(15)-\mathrm{H}(15 \mathrm{~B})$ & 0.9800 \\
\hline $\mathrm{C}(2)-\mathrm{Cl}(1)$ & $1.737(4)$ & $\mathrm{C}(15)-\mathrm{H}(15 \mathrm{C})$ & 0.9800 \\
\hline$C(3)-C(4)$ & $1.391(6)$ & $\mathrm{C}(16)-\mathrm{O}(3)$ & $1.469(4)$ \\
\hline $\mathrm{C}(3)-\mathrm{H}(3)$ & 0.9500 & $\mathrm{C}(16)-\mathrm{C}(19)$ & $1.517(5)$ \\
\hline$C(4)-C(5)$ & $1.379(6)$ & $C(16)-C(18)$ & $1.521(5)$ \\
\hline $\mathrm{C}(4)-\mathrm{Cl}(2)$ & $1.739(5)$ & $\mathrm{C}(16)-\mathrm{C}(17)$ & $1.560(5)$ \\
\hline$C(5)-C(6)$ & $1.397(6)$ & $\mathrm{C}(17)-\mathrm{O}(4)$ & $1.463(4)$ \\
\hline $\mathrm{C}(5)-\mathrm{H}(5)$ & 0.9500 & $\mathrm{C}(17)-\mathrm{C}(20)$ & $1.521(5)$ \\
\hline $\mathrm{C}(6)-\mathrm{Cl}(3)$ & $1.735(4)$ & $\mathrm{C}(17)-\mathrm{C}(21)$ & $1.527(6)$ \\
\hline $\mathrm{C}(7)-\mathrm{O}(1)$ & $1.202(4)$ & $\mathrm{C}(18)-\mathrm{H}(18 \mathrm{~A})$ & 0.9800 \\
\hline $\mathrm{C}(7)-\mathrm{O}(2)$ & $1.328(4)$ & $\mathrm{C}(18)-\mathrm{H}(18 \mathrm{~B})$ & 0.9800 \\
\hline $\mathrm{C}(8)-\mathrm{O}(2)$ & $1.463(4)$ & $\mathrm{C}(18)-\mathrm{H}(18 \mathrm{C})$ & 0.9800 \\
\hline $\mathrm{C}(8)-\mathrm{C}(9)$ & $1.507(5)$ & $\mathrm{C}(19)-\mathrm{H}(19 \mathrm{~A})$ & 0.9800 \\
\hline $\mathrm{C}(8)-\mathrm{H}(8 \mathrm{~A})$ & 0.9900 & $\mathrm{C}(19)-\mathrm{H}(19 \mathrm{~B})$ & 0.9800 \\
\hline $\mathrm{C}(8)-\mathrm{H}(8 \mathrm{~B})$ & 0.9900 & $\mathrm{C}(19)-\mathrm{H}(19 \mathrm{C})$ & 0.9800 \\
\hline $\mathrm{C}(9)-\mathrm{C}(10)$ & $1.542(5)$ & $\mathrm{C}(20)-\mathrm{H}(20 \mathrm{~A})$ & 0.9800 \\
\hline $\mathrm{C}(9)-\mathrm{H}(9 \mathrm{~A})$ & 0.9900 & $\mathrm{C}(20)-\mathrm{H}(20 \mathrm{~B})$ & 0.9800 \\
\hline $\mathrm{C}(9)-\mathrm{H}(9 \mathrm{~B})$ & 0.9900 & $\mathrm{C}(20)-\mathrm{H}(20 \mathrm{C})$ & 0.9800 \\
\hline $\mathrm{C}(10)-\mathrm{C}(11)$ & $1.541(5)$ & $\mathrm{C}(21)-\mathrm{H}(21 \mathrm{~A})$ & 0.9800 \\
\hline $\mathrm{C}(10)-\mathrm{B}(1)$ & $1.576(5)$ & $\mathrm{C}(21)-\mathrm{H}(21 \mathrm{~B})$ & 0.9800 \\
\hline $\mathrm{C}(10)-\mathrm{H}(10)$ & 1.0000 & $\mathrm{C}(21)-\mathrm{H}(21 \mathrm{C})$ & 0.9800 \\
\hline $\mathrm{C}(11)-\mathrm{C}(12)$ & $1.533(5)$ & $\mathrm{C}(22)-\mathrm{C}(23)$ & $1.392(5)$ \\
\hline $\mathrm{C}(11)-\mathrm{H}(11 \mathrm{~A})$ & 0.9900 & $\mathrm{C}(22)-\mathrm{C}(27)$ & $1.399(5)$ \\
\hline $\mathrm{C}(11)-\mathrm{H}(11 \mathrm{~B})$ & 0.9900 & $\mathrm{C}(22)-\mathrm{C}(28)$ & $1.503(6)$ \\
\hline$C(12)-C(14)$ & $1.519(7)$ & $\mathrm{C}(23)-\mathrm{C}(24)$ & $1.378(6)$ \\
\hline $\mathrm{C}(12)-\mathrm{C}(15)$ & $1.526(6)$ & $\mathrm{C}(23)-\mathrm{Cl}(4)$ & $1.732(4)$ \\
\hline$C(12)-C(13)$ & $1.527(7)$ & $C(24)-C(25)$ & $1.377(6)$ \\
\hline $\mathrm{C}(13)-\mathrm{H}(13 \mathrm{~A})$ & 0.9800 & $\mathrm{C}(24)-\mathrm{H}(24)$ & 0.9500 \\
\hline $\mathrm{C}(13)-\mathrm{H}(13 \mathrm{~B})$ & 0.9800 & $C(25)-C(26)$ & $1.401(6)$ \\
\hline $\mathrm{C}(13)-\mathrm{H}(13 \mathrm{C})$ & 0.9800 & $\mathrm{C}(25)-\mathrm{Cl}(5)$ & $1.731(4)$ \\
\hline $\mathrm{C}(14)-\mathrm{H}(14 \mathrm{~A})$ & 0.9800 & $\mathrm{C}(26)-\mathrm{C}(27)$ & $1.379(6)$ \\
\hline
\end{tabular}




\begin{tabular}{|c|c|c|c|}
\hline $\mathrm{C}(26)-\mathrm{H}(26)$ & 0.9500 & $\mathrm{C}(36)-\mathrm{H}(36 \mathrm{~A})$ & 0.9800 \\
\hline $\mathrm{C}(27)-\mathrm{Cl}(6)$ & $1.738(4)$ & $\mathrm{C}(36)-\mathrm{H}(36 \mathrm{~B})$ & 0.9800 \\
\hline $\mathrm{C}(28)-\mathrm{O}(5)$ & $1.197(4)$ & $\mathrm{C}(36)-\mathrm{H}(36 \mathrm{C})$ & 0.9800 \\
\hline $\mathrm{C}(28)-\mathrm{O}(6)$ & $1.325(4)$ & $\mathrm{C}(37)-\mathrm{O}(7)$ & $1.468(4)$ \\
\hline $\mathrm{C}(29)-\mathrm{O}(6)$ & $1.466(5)$ & $C(37)-C(39)$ & $1.506(5)$ \\
\hline$C(29)-C(30)$ & $1.538(6)$ & $C(37)-C(40)$ & $1.520(6)$ \\
\hline $\mathrm{C}(29)-\mathrm{H}(29 \mathrm{~A})$ & 0.9900 & $\mathrm{C}(37)-\mathrm{C}(38)$ & $1.570(5)$ \\
\hline $\mathrm{C}(29)-\mathrm{H}(29 \mathrm{~B})$ & 0.9900 & $\mathrm{C}(38)-\mathrm{O}(8)$ & $1.457(5)$ \\
\hline$C(30)-C(31)$ & $1.546(6)$ & $\mathrm{C}(38)-\mathrm{C}(41)$ & $1.517(6)$ \\
\hline $\mathrm{C}(30)-\mathrm{H}(30 \mathrm{~A})$ & 0.9900 & $\mathrm{C}(38)-\mathrm{C}(42)$ & $1.527(6)$ \\
\hline $\mathrm{C}(30)-\mathrm{H}(30 \mathrm{~B})$ & 0.9900 & $\mathrm{C}(39)-\mathrm{H}(39 \mathrm{~A})$ & 0.9800 \\
\hline$C(31)-C(32)$ & $1.531(5)$ & $\mathrm{C}(39)-\mathrm{H}(39 \mathrm{~B})$ & 0.9800 \\
\hline $\mathrm{C}(31)-\mathrm{B}(2)$ & $1.573(5)$ & $\mathrm{C}(39)-\mathrm{H}(39 \mathrm{C})$ & 0.9800 \\
\hline $\mathrm{C}(31)-\mathrm{H}(31)$ & 1.0000 & $\mathrm{C}(40)-\mathrm{H}(40 \mathrm{~A})$ & 0.9800 \\
\hline$C(32)-C(33)$ & $1.544(6)$ & $\mathrm{C}(40)-\mathrm{H}(40 \mathrm{~B})$ & 0.9800 \\
\hline $\mathrm{C}(32)-\mathrm{H}(32 \mathrm{~A})$ & 0.9900 & $\mathrm{C}(40)-\mathrm{H}(40 \mathrm{C})$ & 0.9800 \\
\hline $\mathrm{C}(32)-\mathrm{H}(32 \mathrm{~B})$ & 0.9900 & $\mathrm{C}(41)-\mathrm{H}(41 \mathrm{~A})$ & 0.9800 \\
\hline$C(33)-C(34)$ & $1.521(6)$ & $\mathrm{C}(41)-\mathrm{H}(41 \mathrm{~B})$ & 0.9800 \\
\hline$C(33)-C(35)$ & $1.529(6)$ & $\mathrm{C}(41)-\mathrm{H}(41 \mathrm{C})$ & 0.9800 \\
\hline$C(33)-C(36)$ & $1.539(7)$ & $\mathrm{C}(42)-\mathrm{H}(42 \mathrm{~A})$ & 0.9800 \\
\hline $\mathrm{C}(34)-\mathrm{H}(34 \mathrm{~A})$ & 0.9800 & $\mathrm{C}(42)-\mathrm{H}(42 \mathrm{~B})$ & 0.9800 \\
\hline $\mathrm{C}(34)-\mathrm{H}(34 \mathrm{~B})$ & 0.9800 & $\mathrm{C}(42)-\mathrm{H}(42 \mathrm{C})$ & 0.9800 \\
\hline $\mathrm{C}(34)-\mathrm{H}(34 \mathrm{C})$ & 0.9800 & $\mathrm{~B}(1)-\mathrm{O}(3)$ & $1.365(5)$ \\
\hline $\mathrm{C}(35)-\mathrm{H}(35 \mathrm{~A})$ & 0.9800 & $\mathrm{~B}(1)-\mathrm{O}(4)$ & $1.369(5)$ \\
\hline $\mathrm{C}(35)-\mathrm{H}(35 \mathrm{~B})$ & 0.9800 & $\mathrm{~B}(2)-\mathrm{O}(7)$ & $1.365(5)$ \\
\hline $\mathrm{C}(35)-\mathrm{H}(35 \mathrm{C})$ & 0.9800 & $\mathrm{~B}(2)-\mathrm{O}(8)$ & $1.369(5)$ \\
\hline$C(6)-C(1)-C(2)$ & $117.6(4)$ & $C(5)-C(4)-C(3)$ & $123.1(4)$ \\
\hline$C(6)-C(1)-C(7)$ & $121.8(3)$ & $\mathrm{C}(5)-\mathrm{C}(4)-\mathrm{Cl}(2)$ & $119.1(4)$ \\
\hline$C(2)-C(1)-C(7)$ & $120.6(4)$ & $\mathrm{C}(3)-\mathrm{C}(4)-\mathrm{Cl}(2)$ & $117.8(3)$ \\
\hline$C(3)-C(2)-C(1)$ & $122.9(4)$ & $C(4)-C(5)-C(6)$ & $117.4(4)$ \\
\hline $\mathrm{C}(3)-\mathrm{C}(2)-\mathrm{Cl}(1)$ & $118.5(3)$ & $\mathrm{C}(4)-\mathrm{C}(5)-\mathrm{H}(5)$ & 121.3 \\
\hline $\mathrm{C}(1)-\mathrm{C}(2)-\mathrm{Cl}(1)$ & $118.6(3)$ & $\mathrm{C}(6)-\mathrm{C}(5)-\mathrm{H}(5)$ & 121.3 \\
\hline $\mathrm{C}(2)-\mathrm{C}(3)-\mathrm{C}(4)$ & $116.8(4)$ & $C(1)-C(6)-C(5)$ & $122.2(4)$ \\
\hline $\mathrm{C}(2)-\mathrm{C}(3)-\mathrm{H}(3)$ & 121.6 & $\mathrm{C}(1)-\mathrm{C}(6)-\mathrm{Cl}(3)$ & $118.7(3)$ \\
\hline $\mathrm{C}(4)-\mathrm{C}(3)-\mathrm{H}(3)$ & 121.6 & $\mathrm{C}(5)-\mathrm{C}(6)-\mathrm{Cl}(3)$ & 119.1(3) \\
\hline
\end{tabular}




\begin{tabular}{|c|c|c|c|}
\hline $\mathrm{O}(1)-\mathrm{C}(7)-\mathrm{O}(2)$ & $125.9(4)$ & $\mathrm{C}(12)-\mathrm{C}(13)-\mathrm{H}(13 \mathrm{C})$ & 109.5 \\
\hline $\mathrm{O}(1)-\mathrm{C}(7)-\mathrm{C}(1)$ & $123.9(3)$ & $\mathrm{H}(13 \mathrm{~A})-\mathrm{C}(13)-\mathrm{H}(13 \mathrm{C})$ & 109.5 \\
\hline $\mathrm{O}(2)-\mathrm{C}(7)-\mathrm{C}(1)$ & $110.2(3)$ & $\mathrm{H}(13 \mathrm{~B})-\mathrm{C}(13)-\mathrm{H}(13 \mathrm{C})$ & 109.5 \\
\hline $\mathrm{O}(2)-\mathrm{C}(8)-\mathrm{C}(9)$ & $111.3(3)$ & $\mathrm{C}(12)-\mathrm{C}(14)-\mathrm{H}(14 \mathrm{~A})$ & 109.5 \\
\hline $\mathrm{O}(2)-\mathrm{C}(8)-\mathrm{H}(8 \mathrm{~A})$ & 109.4 & $\mathrm{C}(12)-\mathrm{C}(14)-\mathrm{H}(14 \mathrm{~B})$ & 109.5 \\
\hline $\mathrm{C}(9)-\mathrm{C}(8)-\mathrm{H}(8 \mathrm{~A})$ & 109.4 & $\mathrm{H}(14 \mathrm{~A})-\mathrm{C}(14)-\mathrm{H}(14 \mathrm{~B})$ & 109.5 \\
\hline $\mathrm{O}(2)-\mathrm{C}(8)-\mathrm{H}(8 \mathrm{~B})$ & 109.4 & $\mathrm{C}(12)-\mathrm{C}(14)-\mathrm{H}(14 \mathrm{C})$ & 109.5 \\
\hline $\mathrm{C}(9)-\mathrm{C}(8)-\mathrm{H}(8 \mathrm{~B})$ & 109.4 & $\mathrm{H}(14 \mathrm{~A})-\mathrm{C}(14)-\mathrm{H}(14 \mathrm{C})$ & 109.5 \\
\hline $\mathrm{H}(8 \mathrm{~A})-\mathrm{C}(8)-\mathrm{H}(8 \mathrm{~B})$ & 108.0 & $\mathrm{H}(14 \mathrm{~B})-\mathrm{C}(14)-\mathrm{H}(14 \mathrm{C})$ & 109.5 \\
\hline $\mathrm{C}(8)-\mathrm{C}(9)-\mathrm{C}(10)$ & $111.5(3)$ & $\mathrm{C}(12)-\mathrm{C}(15)-\mathrm{H}(15 \mathrm{~A})$ & 109.5 \\
\hline $\mathrm{C}(8)-\mathrm{C}(9)-\mathrm{H}(9 \mathrm{~A})$ & 109.3 & $\mathrm{C}(12)-\mathrm{C}(15)-\mathrm{H}(15 \mathrm{~B})$ & 109.5 \\
\hline $\mathrm{C}(10)-\mathrm{C}(9)-\mathrm{H}(9 \mathrm{~A})$ & 109.3 & $\mathrm{H}(15 \mathrm{~A})-\mathrm{C}(15)-\mathrm{H}(15 \mathrm{~B})$ & 109.5 \\
\hline $\mathrm{C}(8)-\mathrm{C}(9)-\mathrm{H}(9 \mathrm{~B})$ & 109.3 & $\mathrm{C}(12)-\mathrm{C}(15)-\mathrm{H}(15 \mathrm{C})$ & 109.5 \\
\hline $\mathrm{C}(10)-\mathrm{C}(9)-\mathrm{H}(9 \mathrm{~B})$ & 109.3 & $\mathrm{H}(15 \mathrm{~A})-\mathrm{C}(15)-\mathrm{H}(15 \mathrm{C})$ & 109.5 \\
\hline $\mathrm{H}(9 \mathrm{~A})-\mathrm{C}(9)-\mathrm{H}(9 \mathrm{~B})$ & 108.0 & $\mathrm{H}(15 \mathrm{~B})-\mathrm{C}(15)-\mathrm{H}(15 \mathrm{C})$ & 109.5 \\
\hline $\mathrm{C}(11)-\mathrm{C}(10)-\mathrm{C}(9)$ & 109.1(3) & $\mathrm{O}(3)-\mathrm{C}(16)-\mathrm{C}(19)$ & $107.8(3)$ \\
\hline $\mathrm{C}(11)-\mathrm{C}(10)-\mathrm{B}(1)$ & $114.2(3)$ & $\mathrm{O}(3)-\mathrm{C}(16)-\mathrm{C}(18)$ & $107.0(3)$ \\
\hline $\mathrm{C}(9)-\mathrm{C}(10)-\mathrm{B}(1)$ & $108.0(3)$ & $\mathrm{C}(19)-\mathrm{C}(16)-\mathrm{C}(18)$ & $110.0(3)$ \\
\hline $\mathrm{C}(11)-\mathrm{C}(10)-\mathrm{H}(10)$ & 108.5 & $\mathrm{O}(3)-\mathrm{C}(16)-\mathrm{C}(17)$ & $102.3(3)$ \\
\hline $\mathrm{C}(9)-\mathrm{C}(10)-\mathrm{H}(10)$ & 108.5 & $C(19)-C(16)-C(17)$ & $115.6(3)$ \\
\hline $\mathrm{B}(1)-\mathrm{C}(10)-\mathrm{H}(10)$ & 108.5 & $C(18)-C(16)-C(17)$ & $113.4(3)$ \\
\hline$C(12)-C(11)-C(10)$ & $117.1(3)$ & $\mathrm{O}(4)-\mathrm{C}(17)-\mathrm{C}(20)$ & $108.8(3)$ \\
\hline $\mathrm{C}(12)-\mathrm{C}(11)-\mathrm{H}(11 \mathrm{~A})$ & 108.0 & $\mathrm{O}(4)-\mathrm{C}(17)-\mathrm{C}(21)$ & $106.7(3)$ \\
\hline $\mathrm{C}(10)-\mathrm{C}(11)-\mathrm{H}(11 \mathrm{~A})$ & 108.0 & $C(20)-C(17)-C(21)$ & $110.4(3)$ \\
\hline $\mathrm{C}(12)-\mathrm{C}(11)-\mathrm{H}(11 \mathrm{~B})$ & 108.0 & $\mathrm{O}(4)-\mathrm{C}(17)-\mathrm{C}(16)$ & $102.4(3)$ \\
\hline $\mathrm{C}(10)-\mathrm{C}(11)-\mathrm{H}(11 \mathrm{~B})$ & 108.0 & $C(20)-C(17)-C(16)$ & $114.4(3)$ \\
\hline $\mathrm{H}(11 \mathrm{~A})-\mathrm{C}(11)-\mathrm{H}(11 \mathrm{~B})$ & 107.3 & $C(21)-C(17)-C(16)$ & $113.4(3)$ \\
\hline$C(14)-C(12)-C(15)$ & $108.4(4)$ & $\mathrm{C}(16)-\mathrm{C}(18)-\mathrm{H}(18 \mathrm{~A})$ & 109.5 \\
\hline$C(14)-C(12)-C(13)$ & $110.5(5)$ & $\mathrm{C}(16)-\mathrm{C}(18)-\mathrm{H}(18 \mathrm{~B})$ & 109.5 \\
\hline$C(15)-C(12)-C(13)$ & $108.7(4)$ & $\mathrm{H}(18 \mathrm{~A})-\mathrm{C}(18)-\mathrm{H}(18 \mathrm{~B})$ & 109.5 \\
\hline$C(14)-C(12)-C(11)$ & $110.9(4)$ & $\mathrm{C}(16)-\mathrm{C}(18)-\mathrm{H}(18 \mathrm{C})$ & 109.5 \\
\hline $\mathrm{C}(15)-\mathrm{C}(12)-\mathrm{C}(11)$ & $107.8(4)$ & $\mathrm{H}(18 \mathrm{~A})-\mathrm{C}(18)-\mathrm{H}(18 \mathrm{C})$ & 109.5 \\
\hline$C(13)-C(12)-C(11)$ & $110.5(3)$ & $\mathrm{H}(18 \mathrm{~B})-\mathrm{C}(18)-\mathrm{H}(18 \mathrm{C})$ & 109.5 \\
\hline $\mathrm{C}(12)-\mathrm{C}(13)-\mathrm{H}(13 \mathrm{~A})$ & 109.5 & $\mathrm{C}(16)-\mathrm{C}(19)-\mathrm{H}(19 \mathrm{~A})$ & 109.5 \\
\hline $\mathrm{C}(12)-\mathrm{C}(13)-\mathrm{H}(13 \mathrm{~B})$ & 109.5 & $\mathrm{C}(16)-\mathrm{C}(19)-\mathrm{H}(19 \mathrm{~B})$ & 109.5 \\
\hline $\mathrm{H}(13 \mathrm{~A})-\mathrm{C}(13)-\mathrm{H}(13 \mathrm{~B})$ & 109.5 & $\mathrm{H}(19 \mathrm{~A})-\mathrm{C}(19)-\mathrm{H}(19 \mathrm{~B})$ & 109.5 \\
\hline
\end{tabular}




\begin{tabular}{|c|c|c|c|}
\hline $\mathrm{C}(16)-\mathrm{C}(19)-\mathrm{H}(19 \mathrm{C})$ & 109.5 & $\mathrm{O}(6)-\mathrm{C}(29)-\mathrm{C}(30)$ & $108.8(3)$ \\
\hline $\mathrm{H}(19 \mathrm{~A})-\mathrm{C}(19)-\mathrm{H}(19 \mathrm{C})$ & 109.5 & $\mathrm{O}(6)-\mathrm{C}(29)-\mathrm{H}(29 \mathrm{~A})$ & 109.9 \\
\hline H(19B)-C(19)-H(19C) & 109.5 & $\mathrm{C}(30)-\mathrm{C}(29)-\mathrm{H}(29 \mathrm{~A})$ & 109.9 \\
\hline $\mathrm{C}(17)-\mathrm{C}(20)-\mathrm{H}(20 \mathrm{~A})$ & 109.5 & $\mathrm{O}(6)-\mathrm{C}(29)-\mathrm{H}(29 \mathrm{~B})$ & 109.9 \\
\hline $\mathrm{C}(17)-\mathrm{C}(20)-\mathrm{H}(20 \mathrm{~B})$ & 109.5 & $\mathrm{C}(30)-\mathrm{C}(29)-\mathrm{H}(29 \mathrm{~B})$ & 109.9 \\
\hline $\mathrm{H}(20 \mathrm{~A})-\mathrm{C}(20)-\mathrm{H}(20 \mathrm{~B})$ & 109.5 & H(29A)-C(29)-H(29B) & 108.3 \\
\hline $\mathrm{C}(17)-\mathrm{C}(20)-\mathrm{H}(20 \mathrm{C})$ & 109.5 & $\mathrm{C}(29)-\mathrm{C}(30)-\mathrm{C}(31)$ & $113.7(3)$ \\
\hline $\mathrm{H}(20 \mathrm{~A})-\mathrm{C}(20)-\mathrm{H}(20 \mathrm{C})$ & 109.5 & $\mathrm{C}(29)-\mathrm{C}(30)-\mathrm{H}(30 \mathrm{~A})$ & 108.8 \\
\hline $\mathrm{H}(20 \mathrm{~B})-\mathrm{C}(20)-\mathrm{H}(20 \mathrm{C})$ & 109.5 & $\mathrm{C}(31)-\mathrm{C}(30)-\mathrm{H}(30 \mathrm{~A})$ & 108.8 \\
\hline $\mathrm{C}(17)-\mathrm{C}(21)-\mathrm{H}(21 \mathrm{~A})$ & 109.5 & $\mathrm{C}(29)-\mathrm{C}(30)-\mathrm{H}(30 \mathrm{~B})$ & 108.8 \\
\hline $\mathrm{C}(17)-\mathrm{C}(21)-\mathrm{H}(21 \mathrm{~B})$ & 109.5 & $\mathrm{C}(31)-\mathrm{C}(30)-\mathrm{H}(30 \mathrm{~B})$ & 108.8 \\
\hline $\mathrm{H}(21 \mathrm{~A})-\mathrm{C}(21)-\mathrm{H}(21 \mathrm{~B})$ & 109.5 & $\mathrm{H}(30 \mathrm{~A})-\mathrm{C}(30)-\mathrm{H}(30 \mathrm{~B})$ & 107.7 \\
\hline $\mathrm{C}(17)-\mathrm{C}(21)-\mathrm{H}(21 \mathrm{C})$ & 109.5 & $C(32)-C(31)-C(30)$ & $112.7(3)$ \\
\hline $\mathrm{H}(21 \mathrm{~A})-\mathrm{C}(21)-\mathrm{H}(21 \mathrm{C})$ & 109.5 & $\mathrm{C}(32)-\mathrm{C}(31)-\mathrm{B}(2)$ & 114.1(3) \\
\hline $\mathrm{H}(21 \mathrm{~B})-\mathrm{C}(21)-\mathrm{H}(21 \mathrm{C})$ & 109.5 & $\mathrm{C}(30)-\mathrm{C}(31)-\mathrm{B}(2)$ & $108.5(3)$ \\
\hline $\mathrm{C}(23)-\mathrm{C}(22)-\mathrm{C}(27)$ & $117.0(4)$ & $\mathrm{C}(32)-\mathrm{C}(31)-\mathrm{H}(31)$ & 107.0 \\
\hline $\mathrm{C}(23)-\mathrm{C}(22)-\mathrm{C}(28)$ & $120.9(3)$ & $\mathrm{C}(30)-\mathrm{C}(31)-\mathrm{H}(31)$ & 107.0 \\
\hline $\mathrm{C}(27)-\mathrm{C}(22)-\mathrm{C}(28)$ & $122.1(4)$ & $\mathrm{B}(2)-\mathrm{C}(31)-\mathrm{H}(31)$ & 107.0 \\
\hline$C(24)-C(23)-C(22)$ & $122.7(3)$ & $\mathrm{C}(31)-\mathrm{C}(32)-\mathrm{C}(33)$ & $116.0(3)$ \\
\hline $\mathrm{C}(24)-\mathrm{C}(23)-\mathrm{Cl}(4)$ & $118.7(3)$ & $\mathrm{C}(31)-\mathrm{C}(32)-\mathrm{H}(32 \mathrm{~A})$ & 108.3 \\
\hline $\mathrm{C}(22)-\mathrm{C}(23)-\mathrm{Cl}(4)$ & $118.6(3)$ & $\mathrm{C}(33)-\mathrm{C}(32)-\mathrm{H}(32 \mathrm{~A})$ & 108.3 \\
\hline $\mathrm{C}(25)-\mathrm{C}(24)-\mathrm{C}(23)$ & $117.9(4)$ & $\mathrm{C}(31)-\mathrm{C}(32)-\mathrm{H}(32 \mathrm{~B})$ & 108.3 \\
\hline $\mathrm{C}(25)-\mathrm{C}(24)-\mathrm{H}(24)$ & 121.1 & $\mathrm{C}(33)-\mathrm{C}(32)-\mathrm{H}(32 \mathrm{~B})$ & 108.3 \\
\hline $\mathrm{C}(23)-\mathrm{C}(24)-\mathrm{H}(24)$ & 121.1 & $\mathrm{H}(32 \mathrm{~A})-\mathrm{C}(32)-\mathrm{H}(32 \mathrm{~B})$ & 107.4 \\
\hline$C(24)-C(25)-C(26)$ & $122.4(4)$ & $C(34)-C(33)-C(35)$ & $108.9(4)$ \\
\hline $\mathrm{C}(24)-\mathrm{C}(25)-\mathrm{Cl}(5)$ & $118.7(3)$ & $\mathrm{C}(34)-\mathrm{C}(33)-\mathrm{C}(36)$ & 109.3(4) \\
\hline $\mathrm{C}(26)-\mathrm{C}(25)-\mathrm{Cl}(5)$ & $118.9(3)$ & $\mathrm{C}(35)-\mathrm{C}(33)-\mathrm{C}(36)$ & $108.9(4)$ \\
\hline$C(27)-C(26)-C(25)$ & $117.4(4)$ & $\mathrm{C}(34)-\mathrm{C}(33)-\mathrm{C}(32)$ & $107.6(4)$ \\
\hline $\mathrm{C}(27)-\mathrm{C}(26)-\mathrm{H}(26)$ & 121.3 & $\mathrm{C}(35)-\mathrm{C}(33)-\mathrm{C}(32)$ & $110.1(4)$ \\
\hline $\mathrm{C}(25)-\mathrm{C}(26)-\mathrm{H}(26)$ & 121.3 & $\mathrm{C}(36)-\mathrm{C}(33)-\mathrm{C}(32)$ & $111.9(3)$ \\
\hline $\mathrm{C}(26)-\mathrm{C}(27)-\mathrm{C}(22)$ & $122.5(4)$ & $\mathrm{C}(33)-\mathrm{C}(34)-\mathrm{H}(34 \mathrm{~A})$ & 109.5 \\
\hline $\mathrm{C}(26)-\mathrm{C}(27)-\mathrm{Cl}(6)$ & 119.3(3) & $\mathrm{C}(33)-\mathrm{C}(34)-\mathrm{H}(34 \mathrm{~B})$ & 109.5 \\
\hline $\mathrm{C}(22)-\mathrm{C}(27)-\mathrm{Cl}(6)$ & 118.2(3) & $\mathrm{H}(34 \mathrm{~A})-\mathrm{C}(34)-\mathrm{H}(34 \mathrm{~B})$ & 109.5 \\
\hline $\mathrm{O}(5)-\mathrm{C}(28)-\mathrm{O}(6)$ & $126.2(4)$ & $\mathrm{C}(33)-\mathrm{C}(34)-\mathrm{H}(34 \mathrm{C})$ & 109.5 \\
\hline $\mathrm{O}(5)-\mathrm{C}(28)-\mathrm{C}(22)$ & $123.0(3)$ & $\mathrm{H}(34 \mathrm{~A})-\mathrm{C}(34)-\mathrm{H}(34 \mathrm{C})$ & 109.5 \\
\hline $\mathrm{O}(6)-\mathrm{C}(28)-\mathrm{C}(22)$ & $110.7(3)$ & $\mathrm{H}(34 \mathrm{~B})-\mathrm{C}(34)-\mathrm{H}(34 \mathrm{C})$ & 109.5 \\
\hline
\end{tabular}




\begin{tabular}{|c|c|c|c|}
\hline $\mathrm{C}(33)-\mathrm{C}(35)-\mathrm{H}(35 \mathrm{~A})$ & 109.5 & $\mathrm{C}(38)-\mathrm{C}(41)-\mathrm{H}(41 \mathrm{~A})$ & 109.5 \\
\hline $\mathrm{C}(33)-\mathrm{C}(35)-\mathrm{H}(35 \mathrm{~B})$ & 109.5 & $\mathrm{C}(38)-\mathrm{C}(41)-\mathrm{H}(41 \mathrm{~B})$ & 109.5 \\
\hline $\mathrm{H}(35 \mathrm{~A})-\mathrm{C}(35)-\mathrm{H}(35 \mathrm{~B})$ & 109.5 & $\mathrm{H}(41 \mathrm{~A})-\mathrm{C}(41)-\mathrm{H}(41 \mathrm{~B})$ & 109.5 \\
\hline C(33)-C(35)-H(35C) & 109.5 & $\mathrm{C}(38)-\mathrm{C}(41)-\mathrm{H}(41 \mathrm{C})$ & 109.5 \\
\hline $\mathrm{H}(35 \mathrm{~A})-\mathrm{C}(35)-\mathrm{H}(35 \mathrm{C})$ & 109.5 & $\mathrm{H}(41 \mathrm{~A})-\mathrm{C}(41)-\mathrm{H}(41 \mathrm{C})$ & 109.5 \\
\hline $\mathrm{H}(35 \mathrm{~B})-\mathrm{C}(35)-\mathrm{H}(35 \mathrm{C})$ & 109.5 & $\mathrm{H}(41 \mathrm{~B})-\mathrm{C}(41)-\mathrm{H}(41 \mathrm{C})$ & 109.5 \\
\hline $\mathrm{C}(33)-\mathrm{C}(36)-\mathrm{H}(36 \mathrm{~A})$ & 109.5 & $\mathrm{C}(38)-\mathrm{C}(42)-\mathrm{H}(42 \mathrm{~A})$ & 109.5 \\
\hline $\mathrm{C}(33)-\mathrm{C}(36)-\mathrm{H}(36 \mathrm{~B})$ & 109.5 & $\mathrm{C}(38)-\mathrm{C}(42)-\mathrm{H}(42 \mathrm{~B})$ & 109.5 \\
\hline $\mathrm{H}(36 \mathrm{~A})-\mathrm{C}(36)-\mathrm{H}(36 \mathrm{~B})$ & 109.5 & $\mathrm{H}(42 \mathrm{~A})-\mathrm{C}(42)-\mathrm{H}(42 \mathrm{~B})$ & 109.5 \\
\hline $\mathrm{C}(33)-\mathrm{C}(36)-\mathrm{H}(36 \mathrm{C})$ & 109.5 & $\mathrm{C}(38)-\mathrm{C}(42)-\mathrm{H}(42 \mathrm{C})$ & 109.5 \\
\hline $\mathrm{H}(36 \mathrm{~A})-\mathrm{C}(36)-\mathrm{H}(36 \mathrm{C})$ & 109.5 & $\mathrm{H}(42 \mathrm{~A})-\mathrm{C}(42)-\mathrm{H}(42 \mathrm{C})$ & 109.5 \\
\hline $\mathrm{H}(36 \mathrm{~B})-\mathrm{C}(36)-\mathrm{H}(36 \mathrm{C})$ & 109.5 & $\mathrm{H}(42 \mathrm{~B})-\mathrm{C}(42)-\mathrm{H}(42 \mathrm{C})$ & 109.5 \\
\hline $\mathrm{O}(7)-\mathrm{C}(37)-\mathrm{C}(39)$ & 108.7(3) & $\mathrm{O}(3)-\mathrm{B}(1)-\mathrm{O}(4)$ & $112.7(3)$ \\
\hline $\mathrm{O}(7)-\mathrm{C}(37)-\mathrm{C}(40)$ & $106.8(3)$ & $\mathrm{O}(3)-\mathrm{B}(1)-\mathrm{C}(10)$ & $124.5(3)$ \\
\hline C(39)-C(37)-C(40) & $110.5(3)$ & $\mathrm{O}(4)-\mathrm{B}(1)-\mathrm{C}(10)$ & $122.6(4)$ \\
\hline $\mathrm{O}(7)-\mathrm{C}(37)-\mathrm{C}(38)$ & 102.1(3) & $\mathrm{O}(7)-\mathrm{B}(2)-\mathrm{O}(8)$ & $112.9(3)$ \\
\hline C(39)-C(37)-C(38) & $115.3(3)$ & $\mathrm{O}(7)-\mathrm{B}(2)-\mathrm{C}(31)$ & $126.5(4)$ \\
\hline $\mathrm{C}(40)-\mathrm{C}(37)-\mathrm{C}(38)$ & $112.8(3)$ & $\mathrm{O}(8)-\mathrm{B}(2)-\mathrm{C}(31)$ & $120.5(3)$ \\
\hline $\mathrm{O}(8)-\mathrm{C}(38)-\mathrm{C}(41)$ & $106.6(3)$ & $\mathrm{C}(7)-\mathrm{O}(2)-\mathrm{C}(8)$ & $116.0(3)$ \\
\hline $\mathrm{O}(8)-\mathrm{C}(38)-\mathrm{C}(42)$ & $107.9(3)$ & $\mathrm{B}(1)-\mathrm{O}(3)-\mathrm{C}(16)$ & $107.9(3)$ \\
\hline $\mathrm{C}(41)-\mathrm{C}(38)-\mathrm{C}(42)$ & $110.9(4)$ & $\mathrm{B}(1)-\mathrm{O}(4)-\mathrm{C}(17)$ & $108.0(3)$ \\
\hline $\mathrm{O}(8)-\mathrm{C}(38)-\mathrm{C}(37)$ & $102.4(3)$ & $\mathrm{C}(28)-\mathrm{O}(6)-\mathrm{C}(29)$ & $116.3(3)$ \\
\hline $\mathrm{C}(41)-\mathrm{C}(38)-\mathrm{C}(37)$ & $113.6(3)$ & $\mathrm{B}(2)-\mathrm{O}(7)-\mathrm{C}(37)$ & $107.8(3)$ \\
\hline $\mathrm{C}(42)-\mathrm{C}(38)-\mathrm{C}(37)$ & $114.6(3)$ & $\mathrm{B}(2)-\mathrm{O}(8)-\mathrm{C}(38)$ & $108.1(3)$ \\
\hline $\mathrm{C}(37)-\mathrm{C}(39)-\mathrm{H}(39 \mathrm{~A})$ & 109.5 & & \\
\hline $\mathrm{C}(37)-\mathrm{C}(39)-\mathrm{H}(39 \mathrm{~B})$ & 109.5 & & \\
\hline H(39A)-C(39)-H(39B) & 109.5 & & \\
\hline $\mathrm{C}(37)-\mathrm{C}(39)-\mathrm{H}(39 \mathrm{C})$ & 109.5 & & \\
\hline H(39A)-C(39)-H(39C) & 109.5 & & \\
\hline H(39B)-C(39)-H(39C) & 109.5 & & \\
\hline $\mathrm{C}(37)-\mathrm{C}(40)-\mathrm{H}(40 \mathrm{~A})$ & 109.5 & & \\
\hline $\mathrm{C}(37)-\mathrm{C}(40)-\mathrm{H}(40 \mathrm{~B})$ & 109.5 & & \\
\hline $\mathrm{H}(40 \mathrm{~A})-\mathrm{C}(40)-\mathrm{H}(40 \mathrm{~B})$ & 109.5 & & \\
\hline $\mathrm{C}(37)-\mathrm{C}(40)-\mathrm{H}(40 \mathrm{C})$ & 109.5 & & \\
\hline $\mathrm{H}(40 \mathrm{~A})-\mathrm{C}(40)-\mathrm{H}(40 \mathrm{C})$ & 109.5 & & \\
\hline $\mathrm{H}(40 \mathrm{~B})-\mathrm{C}(40)-\mathrm{H}(40 \mathrm{C})$ & 109.5 & & \\
\hline
\end{tabular}


Symmetry transformations used to generate equivalent atoms: 
Table S7. Anisotropic displacement parameters $\left(\AA^{2} \times 10^{3}\right)$. The anisotropic displacement factor exponent takes the form: $-2 \pi^{2}\left[h^{2} a * 2 U^{11}+\ldots+2 h k a^{*} b^{*} U^{12}\right]$

\begin{tabular}{|c|c|c|c|c|c|c|}
\hline & $\mathrm{U}^{11}$ & $\mathrm{U}^{22}$ & $\mathrm{U}^{33}$ & $\mathrm{U}^{23}$ & $\mathrm{U}^{13}$ & $\mathrm{U}^{12}$ \\
\hline $\mathrm{C}(1)$ & $27(2)$ & $19(2)$ & $20(2)$ & $2(1)$ & $4(2)$ & $4(1)$ \\
\hline$C(2)$ & $32(2)$ & $20(2)$ & $23(2)$ & $2(2)$ & $11(2)$ & $5(2)$ \\
\hline $\mathrm{C}(3)$ & $40(2)$ & $33(2)$ & $22(2)$ & $1(2)$ & $5(2)$ & $16(2)$ \\
\hline $\mathrm{C}(4)$ & $35(2)$ & $50(3)$ & $27(2)$ & $2(2)$ & $2(2)$ & $21(2)$ \\
\hline$C(5)$ & $37(2)$ & $49(2)$ & $29(2)$ & $1(2)$ & $11(2)$ & $22(2)$ \\
\hline$C(6)$ & $32(2)$ & $30(2)$ & $18(2)$ & $2(2)$ & $2(2)$ & $9(2)$ \\
\hline$C(7)$ & $26(2)$ & $19(2)$ & $15(2)$ & $2(1)$ & $5(2)$ & $5(2)$ \\
\hline $\mathrm{C}(8)$ & $23(2)$ & $23(2)$ & $23(2)$ & 11(1) & $9(2)$ & $10(2)$ \\
\hline $\mathrm{C}(9)$ & $27(2)$ & $24(2)$ & $24(2)$ & $10(1)$ & $12(2)$ & $6(2)$ \\
\hline$C(10)$ & $20(2)$ & $22(2)$ & $22(2)$ & $6(1)$ & $8(1)$ & $2(1)$ \\
\hline $\mathrm{C}(11)$ & $33(2)$ & $32(2)$ & $28(2)$ & $10(2)$ & $17(2)$ & $3(2)$ \\
\hline$C(12)$ & $24(2)$ & $53(2)$ & $44(2)$ & $15(2)$ & $19(2)$ & $5(2)$ \\
\hline$C(13)$ & $46(3)$ & $78(4)$ & $84(4)$ & $44(3)$ & $41(3)$ & $40(3)$ \\
\hline$C(14)$ & $31(3)$ & $106(5)$ & $54(3)$ & $5(3)$ & $20(2)$ & $-10(3)$ \\
\hline$C(15)$ & $44(3)$ & $86(4)$ & $55(3)$ & $24(3)$ & $32(2)$ & $15(3)$ \\
\hline$C(16)$ & $24(2)$ & $17(2)$ & $24(2)$ & $9(1)$ & $5(2)$ & $1(1)$ \\
\hline$C(17)$ & $24(2)$ & $23(2)$ & $26(2)$ & $10(2)$ & $4(2)$ & $1(2)$ \\
\hline $\mathrm{C}(18)$ & $31(2)$ & $22(2)$ & $26(2)$ & $7(2)$ & $7(2)$ & $7(2)$ \\
\hline$C(19)$ & $32(2)$ & $28(2)$ & $30(2)$ & $13(2)$ & $12(2)$ & $4(2)$ \\
\hline $\mathrm{C}(20)$ & $37(2)$ & $30(2)$ & $26(2)$ & $5(2)$ & $-2(2)$ & $-3(2)$ \\
\hline $\mathrm{C}(21)$ & $20(2)$ & $40(2)$ & $42(2)$ & $16(2)$ & $6(2)$ & $0(2)$ \\
\hline $\mathrm{C}(22)$ & $22(2)$ & $20(2)$ & $23(2)$ & $6(1)$ & $8(2)$ & $0(1)$ \\
\hline$C(23)$ & $23(2)$ & $21(2)$ & $22(2)$ & $2(2)$ & $10(2)$ & $3(2)$ \\
\hline$C(24)$ & $26(2)$ & $32(2)$ & $18(2)$ & $4(2)$ & $5(2)$ & $6(2)$ \\
\hline$C(25)$ & $26(2)$ & $48(3)$ & $24(2)$ & $4(2)$ & $6(2)$ & $15(2)$ \\
\hline$C(26)$ & $30(2)$ & $47(2)$ & $22(2)$ & $-1(2)$ & $9(2)$ & $10(2)$ \\
\hline$C(27)$ & $27(2)$ & $32(2)$ & $16(2)$ & $2(2)$ & $6(2)$ & $2(2)$ \\
\hline $\mathrm{C}(28)$ & $30(2)$ & $24(2)$ & $15(2)$ & $5(1)$ & $7(2)$ & $2(2)$ \\
\hline$C(29)$ & $30(2)$ & $25(2)$ & $26(2)$ & $10(2)$ & $7(2)$ & $10(2)$ \\
\hline $\mathrm{C}(30)$ & $30(2)$ & $27(2)$ & $28(2)$ & $12(2)$ & $8(2)$ & $5(2)$ \\
\hline
\end{tabular}




\begin{tabular}{|c|c|c|c|c|c|c|}
\hline$C(31)$ & $27(2)$ & $23(2)$ & $29(2)$ & $9(2)$ & $8(2)$ & $2(2)$ \\
\hline$C(32)$ & $35(2)$ & $32(2)$ & $31(2)$ & $13(2)$ & $9(2)$ & $-1(2)$ \\
\hline$C(33)$ & $28(2)$ & $49(3)$ & $44(2)$ & $21(2)$ & $12(2)$ & $0(2)$ \\
\hline$C(34)$ & $33(2)$ & $85(4)$ & $52(3)$ & $28(3)$ & $12(2)$ & $-6(2)$ \\
\hline$C(35)$ & $43(3)$ & $73(3)$ & $51(3)$ & $26(3)$ & $23(2)$ & $1(2)$ \\
\hline$C(36)$ & $35(2)$ & $62(3)$ & $67(3)$ & $36(3)$ & $28(2)$ & $22(2)$ \\
\hline$C(37)$ & $23(2)$ & $23(2)$ & $25(2)$ & $9(2)$ & $3(2)$ & $-1(2)$ \\
\hline$C(38)$ & $28(2)$ & $31(2)$ & $25(2)$ & $11(2)$ & $5(2)$ & $-3(2)$ \\
\hline$C(39)$ & $31(2)$ & $36(2)$ & $30(2)$ & $15(2)$ & $8(2)$ & $3(2)$ \\
\hline$C(40)$ & $28(2)$ & $22(2)$ & $34(2)$ & $7(2)$ & $6(2)$ & $2(2)$ \\
\hline$C(41)$ & $23(2)$ & $58(3)$ & $45(3)$ & $25(2)$ & $2(2)$ & $3(2)$ \\
\hline$C(42)$ & $47(3)$ & $27(2)$ & $33(2)$ & $7(2)$ & $-1(2)$ & $-7(2)$ \\
\hline $\mathrm{B}(1)$ & $24(2)$ & $21(2)$ & $26(2)$ & $5(2)$ & $15(2)$ & $8(2)$ \\
\hline $\mathrm{B}(2)$ & $22(2)$ & $27(2)$ & $27(2)$ & $9(2)$ & $8(2)$ & $8(2)$ \\
\hline $\mathrm{O}(1)$ & $26(2)$ & $24(1)$ & $31(2)$ & $5(1)$ & $3(1)$ & $2(1)$ \\
\hline $\mathrm{O}(2)$ & $20(1)$ & $21(1)$ & $29(1)$ & $7(1)$ & $8(1)$ & $3(1)$ \\
\hline $\mathrm{O}(3)$ & $23(1)$ & $18(1)$ & $23(1)$ & $6(1)$ & $7(1)$ & $2(1)$ \\
\hline $\mathrm{O}(4)$ & $24(1)$ & $25(1)$ & $21(1)$ & $9(1)$ & $5(1)$ & $1(1)$ \\
\hline $\mathrm{O}(5)$ & $24(1)$ & $26(1)$ & $34(2)$ & $10(1)$ & $2(1)$ & $-1(1)$ \\
\hline $\mathrm{O}(6)$ & $26(1)$ & $21(1)$ & $30(2)$ & $9(1)$ & $8(1)$ & $3(1)$ \\
\hline $\mathrm{O}(7)$ & $25(1)$ & $22(1)$ & $26(1)$ & $8(1)$ & $8(1)$ & $3(1)$ \\
\hline $\mathrm{O}(8)$ & $30(1)$ & $26(1)$ & $24(1)$ & 11(1) & $4(1)$ & $0(1)$ \\
\hline $\mathrm{Cl}(1)$ & $36(1)$ & $35(1)$ & $22(1)$ & $3(1)$ & $10(1)$ & $11(1)$ \\
\hline $\mathrm{Cl}(2)$ & $57(1)$ & $119(1)$ & $34(1)$ & $2(1)$ & $2(1)$ & $63(1)$ \\
\hline $\mathrm{Cl}(3)$ & $38(1)$ & $57(1)$ & $18(1)$ & $6(1)$ & $7(1)$ & $20(1)$ \\
\hline $\mathrm{Cl}(4)$ & $32(1)$ & $36(1)$ & $22(1)$ & $4(1)$ & $11(1)$ & $13(1)$ \\
\hline $\mathrm{Cl}(5)$ & $45(1)$ & $106(1)$ & $36(1)$ & $-2(1)$ & $5(1)$ & $48(1)$ \\
\hline $\mathrm{Cl}(6)$ & $35(1)$ & $51(1)$ & $18(1)$ & $4(1)$ & $7(1)$ & $7(1)$ \\
\hline
\end{tabular}


Table S8. Hydrogen coordinates ( $\left.\times 10^{4}\right)$ and isotropic displacement parameters $\left(\AA^{2} \times 10^{3}\right)$.

\begin{tabular}{|c|c|c|c|c|}
\hline & $\mathrm{x}$ & $\mathrm{y}$ & $\mathrm{z}$ & $\mathrm{U}(\mathrm{eq})$ \\
\hline $\mathrm{H}(3)$ & 6946 & 2279 & 4883 & 40 \\
\hline $\mathrm{H}(5)$ & 6957 & 1158 & 1510 & 47 \\
\hline $\mathrm{H}(8 \mathrm{~A})$ & 1068 & 3941 & 805 & 26 \\
\hline $\mathrm{H}(8 \mathrm{~B})$ & 2013 & 5296 & 1272 & 26 \\
\hline $\mathrm{H}(9 \mathrm{~A})$ & 2018 & 5299 & 3242 & 29 \\
\hline $\mathrm{H}(9 \mathrm{~B})$ & 882 & 4036 & 2653 & 29 \\
\hline $\mathrm{H}(10)$ & -791 & 5103 & 1381 & 26 \\
\hline $\mathrm{H}(11 \mathrm{~A})$ & -844 & 4793 & 3296 & 35 \\
\hline $\mathrm{H}(11 \mathrm{~B})$ & -147 & 6212 & 3858 & 35 \\
\hline $\mathrm{H}(13 \mathrm{~A})$ & -2113 & 7291 & 1881 & 89 \\
\hline $\mathrm{H}(13 \mathrm{~B})$ & -1499 & 7821 & 3302 & 89 \\
\hline $\mathrm{H}(13 \mathrm{C})$ & -3227 & 7538 & 2564 & 89 \\
\hline $\mathrm{H}(14 \mathrm{~A})$ & -4389 & 5205 & 1520 & 99 \\
\hline $\mathrm{H}(14 \mathrm{~B})$ & -3314 & 4208 & 1725 & 99 \\
\hline $\mathrm{H}(14 \mathrm{C})$ & -3140 & 5161 & 959 & 99 \\
\hline $\mathrm{H}(15 \mathrm{~A})$ & -3834 & 6132 & 3611 & 85 \\
\hline $\mathrm{H}(15 \mathrm{~B})$ & -2176 & 6452 & 4537 & 85 \\
\hline $\mathrm{H}(15 \mathrm{C})$ & -2915 & 5047 & 3892 & 85 \\
\hline $\mathrm{H}(18 \mathrm{~A})$ & -991 & 9051 & 568 & 41 \\
\hline $\mathrm{H}(18 \mathrm{~B})$ & 209 & 10156 & 1542 & 41 \\
\hline $\mathrm{H}(18 \mathrm{C})$ & -603 & 9149 & 1962 & 41 \\
\hline $\mathrm{H}(19 \mathrm{~A})$ & 2233 & 8069 & 326 & 44 \\
\hline H(19B) & 2102 & 9502 & 609 & 44 \\
\hline H(19C) & 751 & 8521 & -369 & 44 \\
\hline $\mathrm{H}(20 \mathrm{~A})$ & 1469 & 10024 & 3609 & 53 \\
\hline $\mathrm{H}(20 \mathrm{~B})$ & 2677 & 10551 & 3152 & 53 \\
\hline $\mathrm{H}(20 \mathrm{C})$ & 3154 & 9926 & 4255 & 53 \\
\hline $\mathrm{H}(21 \mathrm{~A})$ & 4263 & 8283 & 3400 & 52 \\
\hline $\mathrm{H}(21 \mathrm{~B})$ & 4114 & 8968 & 2356 & 52 \\
\hline $\mathrm{H}(21 \mathrm{C})$ & 3476 & 7533 & 2032 & 52 \\
\hline
\end{tabular}




\begin{tabular}{|c|c|c|c|}
\hline $\mathrm{H}(24)$ & 2422 & 7802 & 5101 \\
\hline $\mathrm{H}(26)$ & 2650 & 9179 & 8514 \\
\hline $\mathrm{H}(29 \mathrm{~A})$ & 7537 & 4868 & 8664 \\
\hline $\mathrm{H}(29 \mathrm{~B})$ & 8492 & 6199 & 8956 \\
\hline $\mathrm{H}(30 \mathrm{~A})$ & 8285 & 5943 & 6970 \\
\hline $\mathrm{H}(30 \mathrm{~B})$ & 7054 & 4751 & 6536 \\
\hline $\mathrm{H}(31)$ & 9213 & 4100 & 6476 \\
\hline $\mathrm{H}(32 \mathrm{~A})$ & 10793 & 4995 & 8962 \\
\hline $\mathrm{H}(32 \mathrm{~B})$ & 10743 & 5917 & 8125 \\
\hline $\mathrm{H}(34 \mathrm{~A})$ & 14238 & 4965 & 8744 \\
\hline $\mathrm{H}(34 \mathrm{~B})$ & 13382 & 4935 & 9640 \\
\hline $\mathrm{H}(34 \mathrm{C})$ & 13420 & 6086 & 9065 \\
\hline $\mathrm{H}(35 \mathrm{~A})$ & 12133 & 5759 & 6833 \\
\hline $\mathrm{H}(35 \mathrm{~B})$ & 11187 & 4443 & 6055 \\
\hline $\mathrm{H}(35 \mathrm{C})$ & 12935 & 4600 & 6606 \\
\hline $\mathrm{H}(36 \mathrm{~A})$ & 12796 & 2894 & 7669 \\
\hline $\mathrm{H}(36 \mathrm{~B})$ & 11066 & 2729 & 6969 \\
\hline $\mathrm{H}(36 \mathrm{C})$ & 11712 & 2927 & 8390 \\
\hline $\mathrm{H}(39 \mathrm{~A})$ & 8842 & 1823 & 10356 \\
\hline $\mathrm{H}(39 \mathrm{~B})$ & 7480 & 776 & 9508 \\
\hline $\mathrm{H}(39 \mathrm{C})$ & 7298 & 2198 & 9681 \\
\hline $\mathrm{H}(40 \mathrm{~A})$ & 9896 & 942 & 7876 \\
\hline $\mathrm{H}(40 \mathrm{~B})$ & 9234 & 25 & 8474 \\
\hline $\mathrm{H}(40 \mathrm{C})$ & 10456 & 1189 & 9300 \\
\hline $\mathrm{H}(41 \mathrm{~A})$ & 5872 & 2561 & 7926 \\
\hline $\mathrm{H}(41 \mathrm{~B})$ & 5293 & 1143 & 7772 \\
\hline $\mathrm{H}(41 \mathrm{C})$ & 4990 & 1685 & 6620 \\
\hline $\mathrm{H}(42 \mathrm{~A})$ & 6004 & -38 & 5831 \\
\hline $\mathrm{H}(42 \mathrm{~B})$ & 6757 & -516 & 7002 \\
\hline $\mathrm{H}(42 \mathrm{C})$ & 7734 & -34 & 6325 \\
\hline
\end{tabular}




\section{DFT Calculations}

\section{General}

DFT calculations were conducted at the Molecular Graphics and Computation Facility at the University of California, Berkeley. The geometry optimizations of all structures were performed with the B3LYP functional with Gaussian 09 revision D01 package. SDD and 6-31G(d) basis sets were used for $\mathrm{Cu}$ and all other atoms, respectively. Single-point energies were calculated with the M06 functional. SDD and 6-311+G(d,p) basis sets were used for $\mathrm{Cu}$ and all other atoms, respectively. The SMD model for cyclohexane was used for the solvent corrections. Frequency calculations were also conducted with the optimized geometries to confirm that the stationary points were minima (zero imaginary frequencies) or transition states (one imaginary frequency).

\section{(R)-DTBM-SEGPHOSCuH}

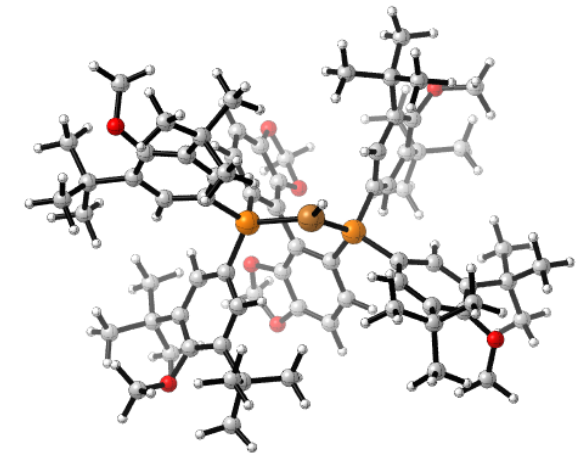

Thermal correction to Enthalpy=

1.699458

Thermal correction to Gibbs Free Energy=

1.472397

Sum of electronic and zero-point Energies=

$-4360.797109$

Sum of electronic and thermal Enthalpies=

$-4360.703004$

Sum of electronic and thermal Free Energies=

$-4360.930064$

SMD E $($ M06 $)=-4360.921482$

Cartesian coordinates:

$\mathrm{P}$

$\begin{array}{lll}-1.73666600 & -0.14203000 & -0.28711900\end{array}$

$\mathrm{O}$

$\begin{array}{lll}1.82551000 & 0.27561900 & -0.33213000\end{array}$

$$
\begin{array}{llll}
-0.86926800 & 1.78067500 & 4.03933600
\end{array}
$$

$\mathrm{O}$

$$
\begin{array}{llll}
0.07148300 & 3.88432500 & 4.11997800
\end{array}
$$

$\mathrm{O}$

$\begin{array}{llll}0.87405800 & -0.76045000 & 4.33128000\end{array}$

$\begin{array}{lllll}\mathrm{O} & -0.02432800 & -2.83150300 & 4.79223700\end{array}$

$\begin{array}{llll}\mathrm{O} & 4.42692300 & -4.81906000 & 1.46011600\end{array}$

$\begin{array}{lllll}\mathrm{O} & 6.41425300 & 3.28187300 & -2.78307600\end{array}$

$\begin{array}{llll}\mathrm{O} & -5.07648000 & 4.70959200 & 0.81682100\end{array}$ 


\begin{tabular}{|c|c|c|c|}
\hline $\mathrm{O}$ & -5.74854400 & -3.99899900 & -2.54689 \\
\hline $\mathrm{C}$ & 1.36448200 & 1.35625000 & 1.10306500 \\
\hline $\mathrm{C}$ & 0.35596900 & 0.96398900 & 2.04566600 \\
\hline $\mathrm{C}$ & 0.04100500 & 1.91070500 & 3.01065500 \\
\hline $\mathrm{C}$ & 0.60630100 & 3.18237700 & 3.06671700 \\
\hline $\mathrm{C}$ & 1.54591300 & 3.58884100 & 2.14656400 \\
\hline $\mathrm{C}$ & 1.91319900 & 2.64604800 & 1.17133600 \\
\hline $\mathrm{C}$ & -0.63462000 & 2.90677100 & 4.88766600 \\
\hline $\mathrm{C}$ & -1.26329400 & -0.97187100 & 1.30346500 \\
\hline $\mathrm{C}$ & -1.79139100 & -2.23628800 & 1.6054690 \\
\hline $\mathrm{C}$ & -1.43789700 & -2.96420600 & 2.7547110 \\
\hline $\mathrm{C}$ & -0.54005400 & -2.36170300 & 3.60680200 \\
\hline $\mathrm{C}$ & 0.00242100 & -1.11142800 & 3.32034300 \\
\hline $\mathrm{C}$ & -0.29244900 & -0.38167300 & 2.1772330 \\
\hline $\mathrm{C}$ & 0.61442400 & -1.69539700 & 5.3802990 \\
\hline $\mathrm{C}$ & 2.50190600 & -1.28303400 & 0.37755100 \\
\hline $\mathrm{C}$ & 2.28475800 & -2.45179400 & -0.34995800 \\
\hline $\mathrm{C}$ & 2.82224000 & -3.69300500 & 0.02967200 \\
\hline $\mathrm{C}$ & 3.69607400 & -3.68436700 & 1.14020000 \\
\hline $\mathrm{C}$ & 3.83611200 & -2.55072300 & 1.98868200 \\
\hline $\mathrm{C}$ & 3.22625300 & -1.36202900 & 1.57368100 \\
\hline $\mathrm{C}$ & 4.54966800 & -2.62946600 & 3.36418400 \\
\hline $\mathrm{C}$ & 6.05535200 & -2.96428900 & 3.24254400 \\
\hline $\mathrm{C}$ & 3.85338800 & -3.71297500 & 4.22571200 \\
\hline $\mathrm{C}$ & 4.45493800 & -1.29012500 & 4.12836100 \\
\hline $\mathrm{C}$ & 2.35245500 & -4.93779500 & -0.77592100 \\
\hline $\mathrm{C}$ & 2.66328900 & -6.30643600 & -0.12664300 \\
\hline $\mathrm{C}$ & 0.80319100 & -4.88032100 & -0.89401400 \\
\hline $\mathrm{C}$ & 2.95171800 & -4.90630800 & -2.20237900 \\
\hline $\mathrm{C}$ & 5.58322600 & -5.01105300 & 0.64396800 \\
\hline $\mathrm{C}$ & 3.30846400 & 1.09101600 & -1.07163600 \\
\hline $\mathrm{C}$ & 4.56730900 & 1.14651600 & -0.45634400 \\
\hline $\mathrm{C}$ & 3.16539400 & 1.62280500 & -2.35131500 \\
\hline $\mathrm{C}$ & 4.21424000 & 2.28144300 & -3.01829000 \\
\hline $\mathrm{C}$ & 5.40567000 & 2.47001800 & -2.28476700 \\
\hline $\mathrm{C}$ & 5.64379900 & 1.81858200 & -1.04017700 \\
\hline $\mathrm{C}$ & 7.05080000 & 1.76190600 & -0.390091 \\
\hline
\end{tabular}




\begin{tabular}{|c|c|c|c|}
\hline $\mathrm{C}$ & 7.05650200 & 0.86771600 & 0.86965000 \\
\hline $\mathrm{C}$ & 7.56480000 & 3.15439900 & 0.04781600 \\
\hline $\mathrm{C}$ & 6.17002200 & 4.67715300 & -2.60572900 \\
\hline $\mathrm{C}$ & 8.05024800 & 1.13969400 & -1.39722200 \\
\hline $\mathrm{C}$ & 3.95776800 & 2.67453100 & -4.50051800 \\
\hline $\mathrm{C}$ & 2.91983500 & 3.82043400 & -4.57116200 \\
\hline $\mathrm{C}$ & 5.21309100 & 3.07810700 & -5.30781000 \\
\hline $\mathrm{C}$ & 3.36796800 & 1.43590400 & -5.23192700 \\
\hline $\mathrm{C}$ & -2.64596800 & 1.38125600 & 0.20270500 \\
\hline $\mathrm{C}$ & -3.38231100 & 1.51528200 & 1.38589700 \\
\hline $\mathrm{C}$ & -4.15904900 & 2.64979400 & 1.64367600 \\
\hline $\mathrm{C}$ & -4.18144000 & 3.66346100 & 0.64572400 \\
\hline $\mathrm{C}$ & -3.30895300 & 3.64774100 & -0.46618600 \\
\hline $\mathrm{C}$ & -2.59171100 & 2.45882700 & -0.68041000 \\
\hline $\mathrm{C}$ & -3.02175900 & 4.82430400 & -1.44194800 \\
\hline $\mathrm{C}$ & -3.54301300 & 6.20719900 & -0.98742600 \\
\hline $\mathrm{C}$ & -3.59268900 & 4.50106800 & -2.84358800 \\
\hline $\mathrm{C}$ & -1.47997200 & 4.98347300 & -1.56558100 \\
\hline $\mathrm{C}$ & -4.38381600 & 4.10654500 & 3.69109800 \\
\hline $\mathrm{C}$ & -6.24073000 & 4.61300700 & -0.00446200 \\
\hline $\mathrm{C}$ & -4.88395600 & 2.81032900 & 3.00553600 \\
\hline $\mathrm{C}$ & -4.56918000 & 1.63292300 & 3.95521300 \\
\hline $\mathrm{C}$ & -4.88452500 & -3.00554700 & -2.10012500 \\
\hline $\mathrm{C}$ & -3.63159500 & -2.84695600 & -2.74362000 \\
\hline $\mathrm{C}$ & -4.37376300 & -2.93470100 & -5.13995500 \\
\hline $\mathrm{C}$ & -2.72396600 & -1.96527300 & -2.13888600 \\
\hline $\mathrm{C}$ & -3.04796200 & -1.23058300 & -0.99482700 \\
\hline $\mathrm{C}$ & -4.35789600 & -1.28625300 & -0.50817800 \\
\hline $\mathrm{C}$ & -5.32033900 & -2.14190800 & -1.06021500 \\
\hline $\mathrm{C}$ & -5.57857900 & -5.24749400 & -1.86399400 \\
\hline $\mathrm{C}$ & -3.29724900 & -4.99758200 & -4.17791200 \\
\hline $\mathrm{C}$ & -7.41361100 & -3.27462300 & -0.00318900 \\
\hline $\mathrm{C}$ & -6.81229000 & -2.00315500 & -0.64676400 \\
\hline $\mathrm{C}$ & -7.00837200 & -0.85839800 & 0.37265100 \\
\hline $\mathrm{C}$ & -1.92786500 & -2.98330300 & -4.64869600 \\
\hline $\mathrm{C}$ & -6.42309000 & 2.87050200 & 2.86083400 \\
\hline $\mathrm{C}$ & -3.30931100 & -3.45151200 & -4.13901400 \\
\hline
\end{tabular}




\begin{tabular}{|c|c|c|c|}
\hline $\mathrm{C}$ & -7.63588500 & -1.64114500 & -1.90864800 \\
\hline $\mathrm{H}$ & 1.98984100 & 4.57778900 & 2.17972600 \\
\hline & 2.66735300 & 2.93205600 & 0.44819100 \\
\hline & -1.58962400 & 3.32051600 & 5.21489000 \\
\hline & -0.01634100 & 2.59653000 & 5.74346500 \\
\hline $\mathrm{H}$ & -2.51872600 & -2.67421900 & 0.93280700 \\
\hline $\mathrm{H}$ & -1.86363700 & -3.93892800 & 2.96672600 \\
\hline & 1.55687900 & -2.00293700 & 5.83491100 \\
\hline & -0.06131100 & -1.23776200 & 6.11839800 \\
\hline $\mathrm{H}$ & 1.66924300 & -2.39089300 & -1.24135200 \\
\hline $\mathrm{H}$ & 3.30927800 & -0.47399000 & 2.18684500 \\
\hline & 6.55812400 & -2.28651600 & 2.54214000 \\
\hline$\mu$ & 6.53849600 & -2.84663100 & 4.22048100 \\
\hline $\mathrm{H}$ & 6.22112100 & -3.99158100 & 2.91858500 \\
\hline $\mathrm{H}$ & 3.94535800 & -4.70054200 & 3.76875100 \\
\hline & 4.30961600 & -3.75173900 & 5.22338100 \\
\hline & 2.78615700 & -3.49279300 & 4.34506600 \\
\hline & 3.42006600 & -0.96627600 & 4.27503400 \\
\hline U & 4.91401400 & -1.41061900 & 5.11638300 \\
\hline & 4.99578100 & -0.48530800 & 3.61611100 \\
\hline & 2.12817600 & -7.08225200 & -0.68771300 \\
\hline & 3.72107200 & -6.56929400 & -0.14631900 \\
\hline & 2.32494700 & -6.34846000 & 0.91265700 \\
\hline 11 & 0.44318900 & -5.77186800 & -1.42101100 \\
\hline & 0.33538700 & -4.85668300 & 0.09668400 \\
\hline & 0.44807100 & -4.00915100 & -1.45108800 \\
\hline & 2.68742900 & -3.98326600 & -2.72996900 \\
\hline 11 & 4.04516500 & -4.97646900 & -2.18488100 \\
\hline $\mathrm{H}$ & 2.57251000 & -5.75067100 & -2.79134700 \\
\hline U & 6.26877000 & -4.15869000 & 0.71649100 \\
\hline & 6.07922800 & -5.90974200 & 1.01982600 \\
\hline & 5.31890300 & -5.15813400 & -0.40861000 \\
\hline $\mathrm{H}$ & 4.70059400 & 0.65001800 & 0.49528200 \\
\hline & 2.20597200 & 1.49831400 & -2.84462800 \\
\hline & 8.07917700 & 0.80525300 & 1.25781800 \\
\hline & 6.42736100 & 1.27439200 & 1.67046200 \\
\hline & 6.72161900 & -0.15267100 & 0.65284800 \\
\hline
\end{tabular}




\begin{tabular}{|c|c|c|c|}
\hline t & 7.79825300 & 3.78845300 & -0.80755600 \\
\hline $\mathrm{H}$ & 6.82981600 & 3.66871000 & 0.67885400 \\
\hline & 8.48427500 & 3.04033300 & 0.63533600 \\
\hline & 5.28035100 & 5.00751700 & -3.15252500 \\
\hline H & 6.04308800 & 4.92782400 & -1.54565400 \\
\hline H & 7.04786300 & 5.19631200 & -2.99930100 \\
\hline $\mathrm{H}$ & 8.12018900 & 1.73641000 & -2.30887000 \\
\hline & 9.04924700 & 1.08030000 & -0.94668300 \\
\hline & 7.74337500 & 0.12311500 & -1.66990800 \\
\hline $\mathrm{H}$ & 1.97655200 & 3.53540200 & -4.09332900 \\
\hline $\mathrm{H}$ & 3.28438300 & 4.73020600 & -4.07964300 \\
\hline U & 2.70379400 & 4.06964600 & -5.61755200 \\
\hline 11 & 4.93293300 & 3.14884300 & -6.36582600 \\
\hline $\mathrm{H}$ & 5.62514700 & 4.04595100 & -5.01963800 \\
\hline $\mathrm{H}$ & 6.00939100 & 2.33327600 & -5.22031700 \\
\hline $\mathrm{H}$ & 4.06470800 & 0.59074400 & -5.18709600 \\
\hline$\Pi$ & 2.41021700 & 1.10901900 & -4.81888700 \\
\hline $\mathrm{H}$ & 3.20307400 & 1.68191600 & -6.28772500 \\
\hline $\mathrm{H}$ & -3.34497300 & 0.71401500 & 2.11224000 \\
\hline 11 & -1.96454600 & 2.36349700 & -1.56107500 \\
\hline $\mathrm{H}$ & -4.62772900 & 6.30449400 & -1.04123900 \\
\hline $\mathrm{H}$ & -3.23351200 & 6.44094700 & 0.03523400 \\
\hline $\mathrm{H}$ & -3.11813900 & 6.96972500 & -1.65139900 \\
\hline$\Pi$ & -3.34716200 & 5.30614000 & -3.54730900 \\
\hline $\mathrm{H}$ & -3.17730800 & 3.56906700 & -3.24197400 \\
\hline $\mathrm{H}$ & -4.68360300 & 4.39697800 & -2.82301700 \\
\hline$H$ & -1.25266500 & 5.82843700 & -2.22623900 \\
\hline $\mathrm{H}$ & -1.02936500 & 5.18560500 & -0.58713400 \\
\hline $\mathrm{H}$ & -0.99049000 & 4.10137400 & -1.98630900 \\
\hline $\mathrm{H}$ & -4.84750500 & 4.21420600 & 4.68010700 \\
\hline $\mathrm{H}$ & -3.29606500 & 4.07780400 & 3.82508200 \\
\hline $\mathrm{H}$ & -4.62847300 & 4.99019400 & 3.09836600 \\
\hline $\mathrm{H}$ & -5.99320600 & 4.66535000 & -1.07007900 \\
\hline $\mathrm{H}$ & -6.78434100 & 3.67950900 & 0.18331800 \\
\hline 11 & -6.87520600 & 5.46310400 & 0.25972300 \\
\hline $\mathrm{H}$ & -4.96726200 & 0.68231400 & 3.58045900 \\
\hline $\mathrm{H}$ & -3.49416100 & 1.51149900 & 4.12218200 \\
\hline
\end{tabular}




$\begin{array}{lrrr}\mathrm{H} & -5.04245800 & 1.82208200 & 4.92552900 \\ \mathrm{H} & -4.36000800 & -1.84003400 & -5.19114800 \\ \mathrm{H} & -4.16235800 & -3.32158200 & -6.14479000 \\ \mathrm{H} & -5.37776500 & -3.25708300 & -4.85276500 \\ \mathrm{H} & -1.74704700 & -1.80754000 & -2.57942100 \\ \mathrm{H} & -4.63113600 & -0.63094400 & 0.30815500 \\ \mathrm{H} & -6.31367700 & -5.93340400 & -2.29331200 \\ \mathrm{H} & -4.57355800 & -5.65240900 & -2.01151400 \\ \mathrm{H} & -5.75956500 & -5.14573700 & -0.78990700 \\ \mathrm{H} & -2.89208400 & -5.33253700 & -5.14042200 \\ \mathrm{H} & -2.65954200 & -5.41639000 & -3.38995200 \\ \mathrm{H} & -4.29938600 & -5.41770200 & -4.08487900 \\ \mathrm{H} & -7.55969800 & -4.07148900 & -0.73283100 \\ \mathrm{H} & -6.78410600 & -3.64955500 & 0.81282400 \\ \mathrm{H} & -8.39723900 & -3.03932600 & 0.42140400 \\ \mathrm{H} & -8.07849600 & -0.74599200 & 0.57897400 \\ \mathrm{H} & -6.51102300 & -1.06462500 & 1.32798100 \\ \mathrm{H} & -6.64171000 & 0.10160400 & -0.00570100 \\ \mathrm{H} & -1.84332500 & -1.89353200 & -4.70234200 \\ \mathrm{H} & -1.10830700 & -3.35702600 & -4.02324600 \\ \mathrm{H} & -1.77225600 & -3.37695000 & -5.65944300 \\ \mathrm{H} & -6.80006400 & 2.02218700 & 2.27698500 \\ \mathrm{H} & -6.88962000 & 2.82354800 & 3.85280000 \\ \mathrm{H} & -6.75256600 & 3.79588500 & 2.38819600 \\ \mathrm{H} & -8.69322300 & -1.51394200 & -1.64342200 \\ \mathrm{H} & -7.28241900 & -0.70090700 & -2.34715400 \\ \mathrm{H} & -7.56493400 & -2.42467100 & -2.66639000 \\ \mathrm{Cu} & 0.00478700 & 0.23221600 & -1.78631200 \\ \mathrm{H} & -0.07074900 & 0.52616500 & -3.31739300\end{array}$




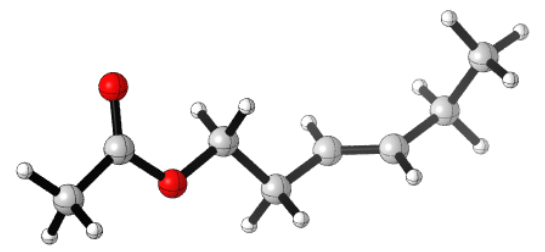

$\begin{array}{lc}\text { Thermal correction to Enthalpy }= & 0.222673 \\ \text { Thermal correction to Gibbs Free Energy= } & 0.168480 \\ \text { Sum of electronic and zero-point Energies }= & -463.521300 \\ \text { Sum of electronic and thermal Enthalpies }= & -463.507949 \\ \text { Sum of electronic and thermal Free Energies }= & -463.562142\end{array}$

SMD E $($ M06 $)=-463.543395$

Cartesian coordinates:

$\begin{array}{lrrr}\mathrm{C} & 1.60349900 & -0.65967900 & -0.54213200 \\ \mathrm{H} & 1.69253900 & -0.42578300 & -1.60541800 \\ \mathrm{C} & 2.68666000 & -0.55827400 & 0.23288100 \\ \mathrm{H} & 2.59481400 & -0.78898500 & 1.29721000 \\ \mathrm{C} & 4.05490200 & -0.13801100 & -0.23056100 \\ \mathrm{H} & 4.04172500 & 0.03031700 & -1.31492700 \\ \mathrm{H} & 4.76691900 & -0.95766600 & -0.05114700 \\ \mathrm{C} & 4.56579600 & 1.12335500 & 0.48685700 \\ \mathrm{H} & 4.60337600 & 0.97349300 & 1.57263600 \\ \mathrm{H} & 5.57557400 & 1.38473200 & 0.15082200 \\ \mathrm{H} & 3.90901400 & 1.97831700 & 0.29175800 \\ \mathrm{C} & 0.23547900 & -1.07312800 & -0.06800600 \\ \mathrm{H} & -0.11190500 & -1.95240600 & -0.62912400 \\ \mathrm{H} & 0.26794800 & -1.36095500 & 0.98949300 \\ \mathrm{C} & -0.78833000 & 0.04393100 & -0.25569200 \\ \mathrm{H} & -0.54545500 & 0.91977000 & 0.35439800 \\ \mathrm{H} & -0.84571000 & 0.37303500 & -1.29889900 \\ \mathrm{O} & -2.07563500 & -0.47446400 & 0.14636700 \\ \mathrm{C} & -3.12233000 & 0.37303100 & 0.01346000 \\ \mathrm{O} & -3.02199200 & 1.50157100 & -0.41521400\end{array}$




$$
\begin{array}{lrrr}
\mathrm{C} & -4.40531500 & -0.28338200 & 0.46976500 \\
\mathrm{H} & -4.59142600 & -1.19324200 & -0.11028400 \\
\mathrm{H} & -4.32420400 & -0.57821300 & 1.52127400 \\
\mathrm{H} & -5.23436000 & 0.41366200 & 0.34355000
\end{array}
$$

$5 \mathbf{a}$

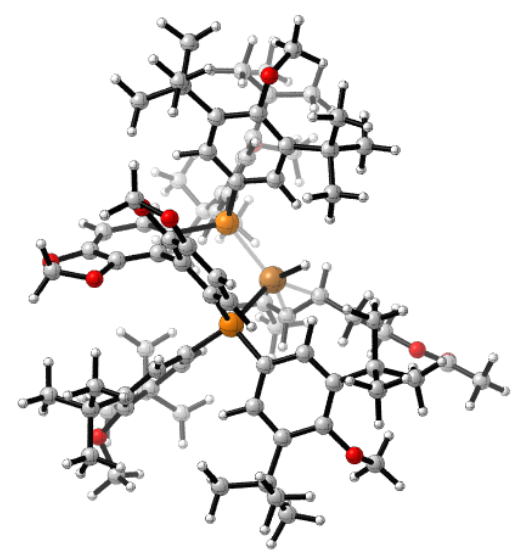

Thermal correction to Enthalpy= $\quad 1.924303$

Thermal correction to Gibbs Free Energy $=\quad 1.666342$

Sum of electronic and zero-point Energies $=\quad-4824.293878$

Sum of electronic and thermal Enthalpies $=\quad-4824.186121$

Sum of electronic and thermal Free Energies $=\quad-4824.444082$

SMD E $($ M06 $)=-4824.474162$

Cartesian coordinates:

$\begin{array}{llll}\mathrm{P} & -1.69304900 & -0.18562700 & -0.38257100 \\ \mathrm{P} & 1.90910300 & -0.24560800 & 0.28665100 \\ \mathrm{O} & -0.41420500 & -4.64997300 & -1.64663300 \\ \mathrm{O} & 0.52295100 & -6.08125100 & -0.10347500 \\ \mathrm{O} & 1.48081400 & -3.11724000 & -3.52358600 \\ \mathrm{O} & 0.62064000 & -2.29229500 & -5.49876200 \\ \mathrm{O} & 5.61843200 & 0.99256100 & -4.26878900 \\ \mathrm{O} & 5.81973200 & 0.95293000 & 4.71420300 \\ \mathrm{O} & -4.92067200 & -4.55699200 & 2.17920500 \\ \mathrm{O} & -6.21330900 & 3.66037800 & -1.33690400 \\ \mathrm{C} & 1.53071000 & -2.06786300 & 0.18277500 \\ \mathrm{C} & 0.62457700 & -2.55695000 & -0.81608600 \\ \mathrm{C} & 0.39039300 & -3.92651600 & -0.78921500 \\ \mathrm{C} & 0.95682400 & -4.79915300 & 0.13570600\end{array}$




\begin{tabular}{|c|c|c|c|}
\hline $\mathrm{C}$ & 1.82436100 & -4.34550300 & 1.10257600 \\
\hline $\mathrm{C}$ & 2.09948900 & -2.96750300 & 1.09627100 \\
\hline $\mathrm{C}$ & -0.08437000 & -6.01730500 & -1.39591400 \\
\hline $\mathrm{C}$ & -1.03152000 & -0.86238900 & -1.98089200 \\
\hline $\mathrm{C}$ & -1.53958700 & -0.38447400 & $-3.196433 c$ \\
\hline $\mathrm{C}$ & -1.05311400 & -0.79289700 & $-4.449220 c$ \\
\hline $\mathrm{C}$ & -0.03196300 & -1.71579000 & -4.43603600 \\
\hline $\mathrm{C}$ & 0.48455200 & -2.20531200 & -3.23988900 \\
\hline $\mathrm{C}$ & 0.03873000 & -1.81450100 & -1.9828740 \\
\hline $\mathrm{C}$ & 1.37331300 & -3.36407200 & -4.92719100 \\
\hline $\mathrm{C}$ & 2.91496800 & 0.03428200 & -1.24276500 \\
\hline $\mathrm{C}$ & 2.81872300 & 1.25645600 & -1.90110500 \\
\hline $\mathrm{C}$ & 3.63643000 & 1.58938400 & -2.99683500 \\
\hline $\mathrm{C}$ & 4.63844900 & 0.65745200 & -3.34379300 \\
\hline $\mathrm{C}$ & 4.67178700 & -0.65776300 & -2.7993260 \\
\hline $\mathrm{C}$ & 3.80520400 & -0.93001600 & -1.73670700 \\
\hline $\mathrm{C}$ & 5.55568500 & -1.78275400 & -3.39947500 \\
\hline $\mathrm{C}$ & 7.06861800 & -1.52604900 & -3.20421600 \\
\hline $\mathrm{C}$ & 5.25299600 & -1.91294100 & -4.9131390 \\
\hline $\mathrm{C}$ & 5.24107800 & -3.14968500 & -2.75174800 \\
\hline $\mathrm{C}$ & 3.30804500 & 2.92042600 & -3.72907200 \\
\hline $\mathrm{C}$ & 3.97827700 & 3.10707300 & -5.10953200 \\
\hline $\mathrm{C}$ & 1.77743500 & 2.95358600 & -3.99864200 \\
\hline $\mathrm{C}$ & 3.67053900 & 4.12301300 & -2.82482000 \\
\hline $\mathrm{C}$ & 6.66436900 & 1.80086000 & -3.73223700 \\
\hline $\mathrm{C}$ & 3.20927900 & -0.08638500 & 1.60952800 \\
\hline $\mathrm{C}$ & 2.94771500 & -0.46037900 & 2.93889500 \\
\hline $\mathrm{C}$ & 4.44186000 & 0.50501400 & 1.33535700 \\
\hline $\mathrm{C}$ & 5.39405900 & 0.79333200 & 2.32971100 \\
\hline $\mathrm{C}$ & 5.02112300 & 0.52485500 & 3.66323900 \\
\hline $\mathrm{C}$ & 3.84534000 & -0.20918800 & 3.98151600 \\
\hline $\mathrm{C}$ & 3.60020900 & -0.80275400 & 5.39418900 \\
\hline $\mathrm{C}$ & 2.34621000 & -1.70390100 & 5.42328800 \\
\hline $\mathrm{C}$ & 3.40086000 & 0.28203400 & 6.47917200 \\
\hline $\mathrm{C}$ & 5.64235700 & 2.33160500 & 5.04226400 \\
\hline $\mathrm{C}$ & 4.80845500 & -1.69383300 & 5.77923400 \\
\hline $\mathrm{C}$ & 6.77268500 & 1.32111700 & 1.838557 \\
\hline
\end{tabular}




\begin{tabular}{|c|c|c|c|}
\hline $\mathrm{C}$ & 6.61250600 & 2.74495200 & 1.25355500 \\
\hline $\mathrm{C}$ & 7.90235200 & 1.33704600 & 2.89408200 \\
\hline $\mathrm{C}$ & 7.27900200 & 0.37657300 & 0.71267800 \\
\hline $\mathrm{C}$ & -2.52826700 & -1.65731300 & 0.37268000 \\
\hline $\mathrm{C}$ & -3.16257500 & -2.64331900 & -0.39623200 \\
\hline $\mathrm{C}$ & -3.91433600 & -3.66864600 & 0.18556100 \\
\hline $\mathrm{C}$ & -4.03202900 & -3.66103000 & 1.60296200 \\
\hline $\mathrm{C}$ & -3.25619700 & -2.80743900 & 2.41847300 \\
\hline $\mathrm{C}$ & -2.55156100 & -1.78265300 & 1.75978700 \\
\hline $\mathrm{C}$ & -3.06054500 & -2.92714300 & 3.95662900 \\
\hline $\mathrm{C}$ & -3.59514800 & -4.22494500 & 4.60617500 \\
\hline $\mathrm{C}$ & -3.70355900 & -1.71348500 & 4.66910700 \\
\hline $\mathrm{C}$ & -1.53403400 & -2.92685100 & 4.24464700 \\
\hline $\mathrm{C}$ & -3.96244900 & -6.16240300 & -0.18102900 \\
\hline $\mathrm{C}$ & -6.15351300 & -3.96354400 & 2.58692300 \\
\hline $\mathrm{C}$ & -4.50674200 & -4.80397000 & -0.68963700 \\
\hline $\mathrm{C}$ & -4.07878100 & -4.65662700 & -2.16675300 \\
\hline $\mathrm{C}$ & -5.21233900 & 2.69471400 & -1.29498300 \\
\hline $\mathrm{C}$ & -5.53839800 & 1.38800800 & -0.85792300 \\
\hline $\mathrm{C}$ & -7.67391100 & 1.06864700 & -2.15344700 \\
\hline $\mathrm{C}$ & -4.46729600 & 0.50870900 & -0.63227200 \\
\hline $\mathrm{C}$ & -3.13884100 & 0.87708900 & -0.86361000 \\
\hline $\mathrm{C}$ & -2.88649500 & 2.12429900 & -1.45231100 \\
\hline $\mathrm{C}$ & -3.90899900 & 3.04008400 & -1.73509300 \\
\hline $\mathrm{C}$ & -6.29253800 & 4.43807500 & -0.13562500 \\
\hline $\mathrm{C}$ & -7.85692100 & 1.57434200 & 0.30713300 \\
\hline $\mathrm{C}$ & -3.81402900 & 5.64058100 & -1.90691800 \\
\hline $\mathrm{C}$ & -3.61382700 & 4.28290300 & -2.62148100 \\
\hline C & -2.15797400 & 4.26182500 & -3.13901800 \\
\hline $\mathrm{C}$ & -7.04508400 & -0.64163400 & -0.45509400 \\
\hline $\mathrm{C}$ & -6.05317400 & -4.83573700 & -0.67084100 \\
\hline $\mathrm{C}$ & -7.00093900 & 0.86988500 & -0.77174200 \\
\hline $\mathrm{C}$ & -4.54355800 & 4.22788000 & -3.86035900 \\
\hline $\mathrm{H}$ & 2.29161500 & -5.01654100 & 1.81518500 \\
\hline $\mathrm{H}$ & 2.81477000 & -2.60080900 & 1.82041400 \\
\hline $\mathrm{H}$ & -0.99460600 & -6.61821800 & -1.40175100 \\
\hline $\mathrm{H}$ & 0.63283100 & -6.36511200 & -2.15481800 \\
\hline
\end{tabular}




\begin{tabular}{|c|c|c|c|}
\hline 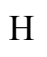 & -2.35684600 & 0.32552900 & -3.17983900 \\
\hline $\mathrm{H}$ & -1.47083400 & -0.41295400 & -5.37519300 \\
\hline $\mathrm{H}$ & 2.37021800 & -3.38690700 & -5.37046600 \\
\hline H & 0.84127300 & -4.31348300 & -5.09158800 \\
\hline & 2.06206000 & 1.95136700 & -1.55245700 \\
\hline & 3.81876600 & -1.90696800 & -1.27186200 \\
\hline $\mathrm{H}$ & 7.30673400 & -1.33444300 & -2.15120600 \\
\hline $\mathrm{H}$ & 7.63652100 & -2.41114500 & -3.51744000 \\
\hline & 7.41970300 & -0.68391200 & -3.80158500 \\
\hline 1 & 5.49782200 & -0.99545600 & -5.45110500 \\
\hline $\mathrm{H}$ & 5.83714100 & -2.73569100 & -5.34485700 \\
\hline $\mathrm{H}$ & 4.19060000 & -2.12958600 & -5.0735290 \\
\hline $\mathrm{U}$ & 4.18147500 & -3.41105500 & -2.84105300 \\
\hline 11 & 5.82497000 & -3.92663400 & -3.25855700 \\
\hline H & 5.51416200 & -3.17849900 & -1.69043300 \\
\hline $\mathrm{H}$ & 3.52632600 & 3.98087500 & -5.59475400 \\
\hline $\mathrm{H}$ & 5.05196000 & 3.29125500 & -5.05415800 \\
\hline 11 & 3.81732200 & 2.24157700 & -5.75862400 \\
\hline $\mathrm{H}$ & 1.52048200 & 3.87903900 & -4.52842600 \\
\hline$H$ & 1.47674400 & 2.10815800 & -4.62823500 \\
\hline 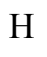 & 1.18097000 & 2.91836900 & -3.08339600 \\
\hline 11 & 3.14126800 & 4.07566300 & -1.86703800 \\
\hline $\mathrm{H}$ & 4.74568400 & 4.16176500 & -2.61251800 \\
\hline & 3.39350800 & 5.06413400 & -3.31631400 \\
\hline 11 & 7.16102000 & 1.30404800 & -2.88992300 \\
\hline 11 & 7.38447400 & 1.95093000 & -4.54108000 \\
\hline $\mathrm{H}$ & 6.29396700 & 2.77502200 & -3.39461200 \\
\hline & 2.01735500 & -0.96618100 & 3.15991500 \\
\hline 11 & 4.67578900 & 0.75515600 & 0.30957700 \\
\hline $\mathrm{H}$ & 2.25750100 & -2.15933200 & 6.41584200 \\
\hline H & 1.42385100 & -1.14182700 & 5.23917800 \\
\hline & 2.40391200 & -2.51799900 & 4.69252700 \\
\hline 11 & 4.32285200 & 0.82644300 & 6.68349400 \\
\hline $\mathrm{H}$ & 2.62427500 & 0.99933900 & 6.18812700 \\
\hline & 3.08226100 & -0.18942900 & 7.41708400 \\
\hline 11 & 5.90547000 & 2.98559000 & 4.20359400 \\
\hline & 4.60759300 & 2.54194100 & 5.337201 \\
\hline
\end{tabular}




\begin{tabular}{|c|c|c|c|}
\hline 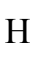 & 6.30952800 & 2.53701300 & 5.88343300 \\
\hline $\mathrm{H}$ & 5.73636100 & -1.11916400 & 5.80363900 \\
\hline & 4.64799400 & -2.13242000 & 6.77223000 \\
\hline & 4.92771100 & -2.51687500 & 5.06462600 \\
\hline & 5.88542400 & 2.76567400 & 0.43439400 \\
\hline $\mathrm{H}$ & 6.27412100 & 3.45728900 & 2.01482300 \\
\hline $\mathrm{H}_{\mathrm{H}}$ & 7.57236900 & 3.10482900 & 0.86229600 \\
\hline & 8.84891300 & 1.54437500 & 2.37995300 \\
\hline & 7.77762700 & 2.10806100 & 3.65524900 \\
\hline $\mathrm{H}$ & 7.99919800 & 0.37367600 & 3.40186700 \\
\hline $\mathrm{H}$ & 7.40772500 & -0.64385900 & 1.09158600 \\
\hline & 6.60489800 & 0.33144900 & -0.14655400 \\
\hline 11 & 8.25276200 & 0.72736700 & 0.35026600 \\
\hline $\mathrm{H}$ & -3.06870300 & -2.59185800 & -1.47301400 \\
\hline $\mathrm{H}$ & -1.99461600 & -1.05894100 & 2.34519000 \\
\hline$H$ & -4.68360300 & -4.27548100 & 4.64816600 \\
\hline$\Pi$ & -3.23453800 & -5.11758700 & 4.08822600 \\
\hline $\mathrm{H}$ & -3.23145000 & -4.26403700 & 5.64034900 \\
\hline $\mathrm{H}$ & -3.51956500 & -1.76460800 & 5.74935000 \\
\hline $\mathrm{H}$ & -3.29450100 & -0.76516000 & 4.30373300 \\
\hline $\mathrm{H}$ & -4.78858300 & -1.68616700 & 4.51590500 \\
\hline $\mathrm{H}$ & -1.36178600 & -3.01967700 & 5.32343600 \\
\hline $\mathrm{H}$ & -1.04389200 & -3.77110400 & 3.74705500 \\
\hline$\Pi$ & -1.03818900 & -2.01227900 & 3.91098200 \\
\hline $\mathrm{H}$ & -4.32386400 & -6.97714900 & -0.82125600 \\
\hline $\mathrm{H}$ & -2.86680400 & -6.17006500 & -0.20301200 \\
\hline$H$ & -4.28244400 & -6.36171600 & 0.84349900 \\
\hline $\mathrm{H}$ & -6.00210100 & -3.19689700 & 3.35510700 \\
\hline $\mathrm{H}$ & -6.67782900 & -3.50957500 & 1.73775600 \\
\hline $\mathrm{H}$ & -6.76255000 & -4.77169700 & 3.00033400 \\
\hline $\mathrm{H}$ & -4.49542500 & -3.75430800 & -2.62952100 \\
\hline $\mathrm{H}$ & -2.99053600 & -4.62889700 & -2.28018400 \\
\hline $\mathrm{H}$ & -4.45605100 & -5.51529900 & -2.73384000 \\
\hline $\mathrm{H}$ & -7.13213700 & 0.52076100 & -2.93319700 \\
\hline 11 & -8.70350400 & 0.68966700 & -2.12838500 \\
\hline $\mathrm{H}$ & -7.70384200 & 2.12489400 & -2.43004800 \\
\hline $\mathrm{H}$ & -4.67018700 & -0.48578600 & -0.2631020 \\
\hline
\end{tabular}




\begin{tabular}{|c|c|c|c|}
\hline $\mathrm{H}$ & -1.85657500 & 2.37579100 & -1.67392800 \\
\hline$H$ & -7.06022700 & 5.19655400 & -0.31159700 \\
\hline $\mathrm{H}$ & -6.57930300 & 3.82237200 & 0.72213200 \\
\hline $\mathrm{H}$ & -5.34011200 & 4.92582700 & 0.09027900 \\
\hline $\mathrm{H}$ & -8.80244100 & 1.03323600 & 0.43655200 \\
\hline $\mathrm{H}$ & -7.34853900 & 1.58893200 & 1.27865600 \\
\hline $\mathrm{H}$ & -8.10718200 & 2.59718700 & 0.02386200 \\
\hline $\mathrm{H}$ & -4.86989900 & 5.86333900 & -1.74383200 \\
\hline $\mathrm{H}$ & -3.28644600 & 5.67656100 & -0.94730000 \\
\hline $\mathrm{H}$ & -3.41062400 & 6.44294000 & -2.53721700 \\
\hline $\mathrm{H}$ & -2.01279200 & 5.10536500 & -3.82343200 \\
\hline $\mathrm{H}$ & -1.42522400 & 4.36135800 & -2.33121000 \\
\hline $\mathrm{H}$ & -1.92905900 & 3.34487200 & -3.69244700 \\
\hline $\mathrm{H}$ & -6.49015600 & -1.23377300 & -1.19035500 \\
\hline $\mathrm{H}$ & -6.64569600 & -0.86932600 & 0.54022000 \\
\hline $\mathrm{H}$ & -8.08731400 & -0.97929500 & -0.47725600 \\
\hline $\mathrm{H}$ & -6.47404500 & -3.85690000 & -0.92955400 \\
\hline $\mathrm{H}$ & -6.41489700 & -5.55859700 & -1.41261500 \\
\hline $\mathrm{H}$ & -6.44484200 & -5.13988300 & 0.30009600 \\
\hline $\mathrm{H}$ & -4.33537000 & 5.07877300 & -4.52123400 \\
\hline $\mathrm{H}$ & -4.37583500 & 3.30878500 & -4.43413300 \\
\hline $\mathrm{H}$ & -5.59592100 & 4.26791300 & -3.57003600 \\
\hline $\mathrm{Cu}$ & -0.00757800 & 1.24542000 & 0.44559200 \\
\hline $\mathrm{H}$ & 0.20043000 & 2.25082400 & -0.75705100 \\
\hline $\mathrm{C}$ & -0.25848400 & 2.90896100 & 1.87441600 \\
\hline $\mathrm{H}$ & 0.78129400 & 3.17978700 & 2.06820500 \\
\hline $\mathrm{C}$ & -0.77784700 & 1.83956800 & 2.55963600 \\
\hline $\mathrm{H}$ & -1.85185700 & 1.65915000 & 2.48027000 \\
\hline $\mathrm{C}$ & -0.07791300 & 1.13804400 & 3.69694500 \\
\hline $\mathrm{H}$ & -0.21259900 & 0.05162000 & 3.62645000 \\
\hline $\mathrm{H}$ & 1.00000700 & 1.32231300 & 3.63739800 \\
\hline $\mathrm{C}$ & -1.10324300 & 3.98489100 & 1.22722300 \\
\hline $\mathrm{H}$ & -0.57460800 & 4.41691600 & 0.37315100 \\
\hline $\mathrm{H}$ & -2.03789500 & 3.56181900 & 0.84589900 \\
\hline $\mathrm{C}$ & -1.42323000 & 5.08698800 & 2.23807000 \\
\hline $\mathrm{H}$ & -0.51592500 & 5.54201700 & 2.65012900 \\
\hline $\mathrm{H}$ & -2.01579600 & 4.71033900 & 3.07813800 \\
\hline
\end{tabular}




$\begin{array}{llll}\mathrm{O} & -2.18322400 & 6.10852300 & 1.54861000 \\ \mathrm{C} & -2.49987900 & 7.20400200 & 2.27607400 \\ \mathrm{C} & -3.27149200 & 8.20255100 & 1.44241000 \\ \mathrm{H} & -2.67333900 & 8.51590600 & 0.58032900 \\ \mathrm{H} & -4.18717900 & 7.74567800 & 1.05276100 \\ \mathrm{H} & -3.52125400 & 9.06964300 & 2.05498800 \\ \mathrm{O} & -2.19413100 & 7.34895300 & 3.43968400 \\ \mathrm{C} & -0.60822600 & 1.61379300 & 5.06352800 \\ \mathrm{H} & -0.10621900 & 1.08869100 & 5.88530600 \\ \mathrm{H} & -0.44322900 & 2.68929800 & 5.19449300 \\ \mathrm{H} & -1.68531200 & 1.42839100 & 5.15882200\end{array}$

$\mathbf{5 b}$

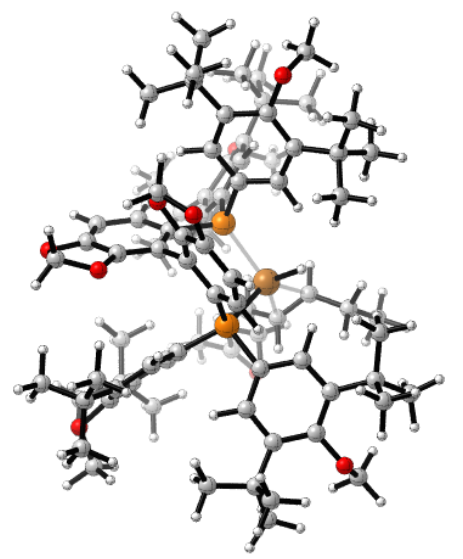

Thermal correction to Enthalpy= $\quad 1.924386$

Thermal correction to Gibbs Free Energy $=\quad 1.666060$

Sum of electronic and zero-point Energies $=\quad-4824.293753$

Sum of electronic and thermal Enthalpies $=\quad-4824.185849$

Sum of electronic and thermal Free Energies $=\quad-4824.444175$

SMD E $(\mathrm{M} 06)=\quad-4824.474911$

Cartesian coordinates:

$\begin{array}{lrrr}\mathrm{P} & -1.77768600 & -0.61852400 & -0.19553500 \\ \mathrm{P} & 1.80228400 & 0.08045800 & 0.13442900 \\ \mathrm{O} & -0.86476200 & -1.59395700 & 4.44063800 \\ \mathrm{O} & -0.08190400 & 0.05981300 & 5.84008300 \\ \mathrm{O} & 1.17896900 & -3.53168600 & 3.16503800 \\ \mathrm{O} & 0.39072400 & -5.56432100 & 2.40426100 \\ \mathrm{O} & 5.74885000 & -4.42943300 & -0.25534100\end{array}$




\begin{tabular}{|c|c|c|c|}
\hline $\mathrm{O}$ & 5.68199000 & 4.54142100 & -1.04940200 \\
\hline $\mathrm{O}$ & -5.28970500 & 2.25999400 & 3.73998900 \\
\hline $\mathrm{O}$ & -6.02047200 & -1.86225900 & -4.27297000 \\
\hline $\mathrm{C}$ & 1.25453200 & 0.11403700 & 1.91505900 \\
\hline $\mathrm{C}$ & 0.32807600 & -0.87205600 & 2.39049600 \\
\hline $\mathrm{C}$ & -0.01903300 & -0.76478700 & 3.73158400 \\
\hline $\mathrm{C}$ & 0.45495900 & 0.22745000 & 4.58548600 \\
\hline $\mathrm{C}$ & 1.33792900 & 1.18548000 & 4.14236700 \\
\hline $\mathrm{C}$ & 1.72920000 & 1.09712300 & 2.79537800 \\
\hline $\mathrm{C}$ & -0.65042800 & -1.25144600 & 5.81095300 \\
\hline $\mathrm{C}$ & -1.17696100 & -2.16010800 & 0.64666400 \\
\hline $\mathrm{C}$ & -1.64135200 & -3.40725900 & 0.20906100 \\
\hline $\mathrm{C}$ & -1.17877500 & -4.62683900 & 0.73014300 \\
\hline $\mathrm{C}$ & -0.22576300 & -4.54528800 & 1.72007400 \\
\hline $\mathrm{C}$ & 0.24629200 & -3.31609600 & 2.17145000 \\
\hline $\mathrm{C}$ & -0.18225400 & -2.09060300 & 1.67569400 \\
\hline $\mathrm{C}$ & 1.08383100 & -4.92331500 & 3.47703500 \\
\hline $\mathrm{C}$ & 2.87984900 & -1.42677700 & 0.10673900 \\
\hline $\mathrm{C}$ & 2.89792900 & -2.23693700 & -1.02462500 \\
\hline $\mathrm{C}$ & 3.77589100 & -3.32960500 & -1.15386000 \\
\hline $\mathrm{C}$ & 4.71466600 & -3.51357600 & -0.11507100 \\
\hline $\mathrm{C}$ & 4.62771400 & -2.80892800 & 1.11909200 \\
\hline $\mathrm{C}$ & 3.70618700 & -1.76071800 & 1.18914300 \\
\hline $\mathrm{C}$ & 5.44243900 & -3.22210100 & 2.37325800 \\
\hline $\mathrm{C}$ & 6.96122500 & -2.98189300 & 2.20461900 \\
\hline $\mathrm{C}$ & 5.19410900 & -4.72078600 & 2.67394800 \\
\hline $\mathrm{C}$ & 4.99373500 & -2.42769700 & 3.62002300 \\
\hline $\mathrm{C}$ & 3.57948300 & -4.23747900 & -2.40032200 \\
\hline $\mathrm{C}$ & 4.30545600 & -5.60162300 & -2.35212900 \\
\hline $\mathrm{C}$ & 2.06623100 & -4.57255900 & -2.51718600 \\
\hline $\mathrm{C}$ & 4.01159600 & -3.47929200 & -3.67835500 \\
\hline $\mathrm{C}$ & 6.83776000 & -3.95539100 & -1.04583800 \\
\hline $\mathrm{C}$ & 3.06700500 & 1.43642100 & -0.02102000 \\
\hline $\mathrm{C}$ & 2.73700100 & 2.78059100 & 0.22747500 \\
\hline $\mathrm{C}$ & 4.35237800 & 1.16063600 & -0.48583400 \\
\hline $\mathrm{C}$ & 5.29948500 & 2.16188800 & -0.76651900 \\
\hline $\mathrm{C}$ & 4.87234600 & 3.49803400 & -0.62305000 \\
\hline
\end{tabular}




\begin{tabular}{|c|c|c|c|}
\hline $\mathrm{C}$ & 3.62914900 & 3.83021700 & -0.01648900 \\
\hline $\mathrm{C}$ & 3.31397300 & 5.26798800 & 0.47632900 \\
\hline $\mathrm{C}$ & 1.97501900 & 5.32974900 & 1.24310300 \\
\hline $\mathrm{C}$ & 3.21741900 & 6.30039400 & -0.67169700 \\
\hline $\mathrm{C}$ & 5.61118100 & 4.78212700 & -2.45522400 \\
\hline $\mathrm{C}$ & 4.42923700 & 5.70367300 & 1.46079300 \\
\hline $\mathrm{C}$ & 6.72842200 & 1.68038600 & -1.14971000 \\
\hline $\mathrm{C}$ & 6.69923100 & 1.01102100 & -2.54478800 \\
\hline $\mathrm{C}$ & 7.83056900 & 2.76477700 & -1.13663400 \\
\hline $\mathrm{C}$ & 7.18287300 & 0.62509800 & -0.10308100 \\
\hline $\mathrm{C}$ & -2.71435200 & 0.24371900 & 1.15207400 \\
\hline $\mathrm{C}$ & -3.43518100 & -0.45314100 & 2.13286600 \\
\hline $\mathrm{C}$ & -4.25341700 & 0.20115600 & 3.05860500 \\
\hline $\mathrm{C}$ & -4.34368700 & 1.61642200 & 2.95565000 \\
\hline $\mathrm{C}$ & -3.48803600 & 2.36620700 & 2.11874400 \\
\hline $\mathrm{C}$ & -2.72097400 & 1.63575900 & 1.19237700 \\
\hline $\mathrm{C}$ & -3.26191900 & 3.90372000 & 2.16902400 \\
\hline $\mathrm{C}$ & -3.89719800 & 4.64216500 & 3.37002900 \\
\hline $\mathrm{C}$ & -3.76673000 & 4.55979500 & 0.86200200 \\
\hline $\mathrm{C}$ & -1.73359000 & 4.15092200 & 2.29758700 \\
\hline $\mathrm{C}$ & -4.49486500 & -0.00756200 & 5.55949800 \\
\hline $\mathrm{C}$ & -6.46104400 & 2.64727500 & 3.02123700 \\
\hline $\mathrm{C}$ & -4.94528300 & -0.59272100 & 4.19750100 \\
\hline $\mathrm{C}$ & -4.53347300 & -2.08152500 & 4.17460700 \\
\hline $\mathrm{C}$ & -5.08353700 & -1.74433800 & -3.25106300 \\
\hline $\mathrm{C}$ & -5.50438300 & -1.26330400 & -1.98728900 \\
\hline $\mathrm{C}$ & -7.62104400 & -2.61011300 & -1.75650700 \\
\hline $\mathrm{C}$ & -4.49755900 & -0.96486500 & -1.05552000 \\
\hline $\mathrm{C}$ & -3.14230400 & -1.17305900 & -1.32788400 \\
\hline $\mathrm{C}$ & -2.79463600 & -1.80878200 & -2.52825800 \\
\hline $\mathrm{C}$ & -3.74885100 & -2.15832000 & -3.49352300 \\
\hline $\mathrm{C}$ & -6.10609700 & -0.69525200 & -5.10010900 \\
\hline $\mathrm{C}$ & -7.83865600 & -0.17198400 & -2.35035600 \\
\hline $\mathrm{C}$ & -3.46433100 & -2.41217900 & -6.06933200 \\
\hline $\mathrm{C}$ & -3.34901300 & -3.08106000 & -4.67923500 \\
\hline $\mathrm{C}$ & -1.88879600 & -3.56660500 & -4.53690100 \\
\hline & -7.14333700 & -0.84474600 & -0.0667380 \\
\hline
\end{tabular}




\begin{tabular}{|c|c|c|c|}
\hline C & -6.48927400 & -0.54924400 & 4.11123800 \\
\hline $\mathrm{C}$ & -6.99821800 & -1.20449500 & -1.56237500 \\
\hline $\mathrm{C}$ & -4.25493000 & -4.33813300 & -4.65194300 \\
\hline $\mathrm{H}$ & 1.73249800 & 1.95134500 & 4.80131400 \\
\hline $\mathrm{H}$ & 2.46057800 & 1.81213400 & 2.44193100 \\
\hline $\mathrm{H}$ & -1.60468200 & -1.24736900 & 6.33888700 \\
\hline $\mathrm{H}$ & 0.05576300 & -1.96645700 & 6.25981100 \\
\hline $\mathrm{H}$ & -2.39971600 & -3.44194500 & -0.56326600 \\
\hline $\mathrm{H}$ & -1.56099900 & -5.57940100 & 0.37970800 \\
\hline $\mathrm{H}$ & 2.08608900 & -5.34656900 & 3.56684400 \\
\hline $\mathrm{H}$ & 0.51345400 & -5.05103800 & 4.40952700 \\
\hline $\mathrm{H}$ & 2.18337000 & -2.01324300 & -1.81025700 \\
\hline $\mathrm{H}$ & 3.62551800 & -1.17882200 & 2.09764100 \\
\hline H & 7.16845100 & -1.94808600 & 1.90381600 \\
\hline $\mathrm{H}$ & 7.47221700 & -3.15832100 & 3.15949300 \\
\hline $\mathrm{H}$ & 7.40165000 & -3.65565100 & 1.46877300 \\
\hline $\mathrm{H}$ & 5.52290200 & -5.35576200 & 1.84954100 \\
\hline $\mathrm{H}$ & 5.73696000 & -5.01806300 & 3.58021700 \\
\hline $\mathrm{H}$ & 4.12695200 & -4.90315300 & 2.84413000 \\
\hline $\mathrm{H}$ & 3.92127500 & -2.53918600 & 3.81208400 \\
\hline $\mathrm{H}$ & 5.53347000 & -2.80414000 & 4.49649600 \\
\hline $\mathrm{H}$ & 5.21939500 & -1.35847200 & 3.53436700 \\
\hline $\mathrm{H}$ & 3.94236800 & -6.21385900 & -3.18677100 \\
\hline $\mathrm{H}$ & 5.38831500 & -5.52550100 & -2.45791500 \\
\hline $\mathrm{H}$ & 4.09486100 & -6.14072000 & -1.42396700 \\
\hline $\mathrm{H}$ & 1.90370000 & -5.22631400 & -3.38280700 \\
\hline $\mathrm{H}$ & 1.71605600 & -5.10114400 & -1.62309800 \\
\hline $\mathrm{H}$ & 1.43929200 & -3.68681600 & -2.64859800 \\
\hline $\mathrm{H}$ & 3.44712800 & -2.54835300 & -3.79895100 \\
\hline $\mathrm{H}$ & 5.07796300 & -3.22458300 & -3.65875700 \\
\hline $\mathrm{H}$ & 3.83277600 & -4.09928500 & -4.56581300 \\
\hline $\mathrm{H}$ & 7.25964600 & -3.03142200 & -0.63192200 \\
\hline $\mathrm{H}$ & 7.59753200 & -4.74134500 & -1.02716400 \\
\hline $\mathrm{H}$ & 6.53864200 & -3.76924900 & -2.08330700 \\
\hline $\mathrm{H}$ & 1.76011000 & 3.00757700 & 0.63452500 \\
\hline $\mathrm{H}$ & 4.63373300 & 0.12730400 & -0.63704300 \\
\hline $\mathrm{H}$ & 1.84397800 & 6.34164600 & 1.64323900 \\
\hline
\end{tabular}




\begin{tabular}{|c|c|c|c|}
\hline t & 1.11656500 & 5.12331700 & 0.59537600 \\
\hline $\mathrm{H}$ & 1.94858000 & 4.63880700 & 2.09298700 \\
\hline & 4.18082700 & 6.46817300 & -1.15244300 \\
\hline & 2.48707000 & 5.98655900 & -1.42605700 \\
\hline H & 2.88289300 & 7.26385200 & -0.26573000 \\
\hline H & 5.95400100 & 3.91671000 & -3.03330800 \\
\hline $\mathrm{H}_{\mathrm{H}}$ & 4.58926700 & 5.02829700 & -2.76733700 \\
\hline & 6.26779500 & 5.63267000 & -2.65627500 \\
\hline & 5.40754600 & 5.71693700 & 0.97575300 \\
\hline H & 4.21979600 & 6.71151900 & 1.84118600 \\
\hline $\mathrm{H}$ & 4.47709700 & 5.02448100 & 2.32027000 \\
\hline & 5.99783000 & 0.16996800 & -2.57465200 \\
\hline 11 & 6.39979800 & 1.72029800 & -3.32497900 \\
\hline $\mathrm{H}$ & 7.69480700 & 0.62968300 & -2.80443000 \\
\hline $\mathrm{H}$ & 8.80275400 & 2.26627100 & -1.23440200 \\
\hline $\mathrm{H}$ & 7.75313700 & 3.47669800 & -1.95905500 \\
\hline$\Pi$ & 7.83507700 & 3.32962700 & -0.20050500 \\
\hline $\mathrm{H}$ & 7.21845600 & 1.06456700 & 0.90045400 \\
\hline $\mathrm{H}$ & 6.52945700 & -0.24997400 & -0.06128700 \\
\hline 11 & 8.19070900 & 0.27229600 & -0.35274500 \\
\hline $\mathrm{H}$ & -3.35666200 & -1.53208000 & 2.15666700 \\
\hline $\mathrm{H}$ & -2.09861000 & 2.16876300 & 0.48194400 \\
\hline $\mathrm{H}$ & -4.98406300 & 4.71250800 & 3.31209200 \\
\hline$\Pi$ & -3.63826300 & 4.17165900 & 4.32206100 \\
\hline $\mathrm{H}$ & -3.50938600 & 5.66825400 & 3.38330300 \\
\hline $\mathrm{H}$ & -3.56293900 & 5.63770600 & 0.87426500 \\
\hline $\mathrm{H}$ & -3.27585900 & 4.13728700 & -0.02136600 \\
\hline $\mathrm{H}$ & -4.84746800 & 4.42632300 & 0.73638600 \\
\hline $\mathrm{H}$ & -1.53500900 & 5.22806700 & 2.34716600 \\
\hline $\mathrm{H}$ & -1.34039600 & 3.68884200 & 3.20994600 \\
\hline 11 & -1.16578600 & 3.75295700 & 1.45331700 \\
\hline $\mathrm{H}$ & -4.92434000 & -0.59386500 & 6.38177600 \\
\hline $\mathrm{H}$ & -3.40323400 & -0.03958300 & 5.65017700 \\
\hline $\mathrm{H}$ & -4.81398400 & 1.03022900 & 5.67413500 \\
\hline 11 & -6.22796500 & 3.34836100 & 2.21200500 \\
\hline $\mathrm{H}$ & -6.97215000 & 1.77655400 & 2.59320600 \\
\hline $\mathrm{H}$ & -7.11920100 & 3.13642200 & 3.74401900 \\
\hline
\end{tabular}




$\begin{array}{llll}\mathrm{H} & -4.88870900 & -2.59487600 & 3.27334800 \\ \mathrm{H} & -3.44817500 & -2.20936600 & 4.23725500 \\ \mathrm{H} & -4.98431600 & -2.58896500 & 5.03515600 \\ \mathrm{H} & -7.09494200 & -3.35662900 & -1.15023400 \\ \mathrm{H} & -8.67260900 & -2.60129700 & -1.44297200 \\ \mathrm{H} & -7.57732900 & -2.92057300 & -2.80263900 \\ \mathrm{H} & -4.77491400 & -0.55839900 & -0.09418200 \\ \mathrm{H} & -1.74575200 & -2.02190000 & -2.69671900 \\ \mathrm{H} & -6.84766800 & -0.92057500 & -5.87126600 \\ \mathrm{H} & -6.42944200 & 0.17960400 & -4.52845000 \\ \mathrm{H} & -5.14697300 & -0.46615400 & -5.57291200 \\ \mathrm{H} & -8.81688400 & -0.05109700 & -1.86874700 \\ \mathrm{H} & -7.35494000 & 0.81199300 & -2.36821600 \\ \mathrm{H} & -8.02239700 & -0.49396500 & -3.37600100 \\ \mathrm{H} & -4.50281500 & -2.27296300 & -6.37326700 \\ \mathrm{H} & -2.95386200 & -1.44195000 & -6.09279300 \\ \mathrm{H} & -2.98973300 & -3.05244600 & -6.82299600 \\ \mathrm{H} & -1.67249900 & -4.27913800 & -5.34110100 \\ \mathrm{H} & -1.16434500 & -2.74909700 & -4.61511200 \\ \mathrm{H} & -1.71589400 & -4.07981000 & -3.58502700 \\ \mathrm{H} & -6.60310900 & -1.54434000 & 0.57986600 \\ \mathrm{H} & -6.67890600 & 0.16890500 & 0.15443800 \\ \mathrm{H} & -1.71061500 & 1.83019100 & -2.62030000 \\ \mathrm{H} & -8.20298400 & -0.89067500 & 0.20896900 \\ \mathrm{H} & -6.84174800 & -0.85879100 & 3.12032700 \\ \mathrm{H} & -6.91822500 & -1.24125200 & 4.84663800 \\ \mathrm{H} & -6.88274100 & 0.44428600 & 4.32871500 \\ \mathrm{H} & -3.97257100 & -5.01948800 & -5.46443900 \\ \mathrm{H} & -4.14051500 & -4.87964700 & -3.70558400 \\ \mathrm{H} & -5.30741100 & -4.07380500 & -4.77526100 \\ \mathrm{H} & 0.04728300 & 0.02887500 & -1.52520700 \\ \mathrm{H} & 0.19510100 & 1.29554200 & -3.35611900 \\ \mathrm{H} & 1.25534400 & 1.56041600 & -3.35380700 \\ \mathrm{H} & -0.63628400 & 1.99714300 & -2.52134500 \\ \mathrm{H} & -24668700 & -1.77536600 \\ \mathrm{H} & -27904500 & -0.77243300\end{array}$




$\begin{array}{lrrr}\mathrm{H} & 0.85287600 & 3.28773800 & -1.65291400 \\ \mathrm{C} & -0.27064700 & 0.50578400 & -4.55899700 \\ \mathrm{H} & 0.36265300 & -0.37674800 & -4.68668500 \\ \mathrm{H} & -1.28950400 & 0.13982400 & -4.38830100 \\ \mathrm{C} & -0.70934000 & 4.48355400 & -2.53940700 \\ \mathrm{H} & -1.78787600 & 4.45488000 & -2.72668600 \\ \mathrm{H} & -0.20717600 & 4.57600300 & -3.50792000 \\ \mathrm{O} & -0.40871000 & 5.64753600 & -1.73454600 \\ \mathrm{C} & -0.88435600 & 6.82813500 & -2.20003500 \\ \mathrm{C} & -0.53168700 & 7.96093000 & -1.26352600 \\ \mathrm{H} & -0.91809600 & 7.75637600 & -0.25971300 \\ \mathrm{H} & 0.55594400 & 8.05537500 & -1.18028200 \\ \mathrm{H} & -0.95614200 & 8.89062200 & -1.64417100 \\ \mathrm{O} & -1.50804700 & 6.93696000 & -3.23260800 \\ \mathrm{C} & -0.23397200 & 1.36505800 & -5.83816000 \\ \mathrm{H} & 0.77725400 & 1.74224000 & -6.03615500 \\ \mathrm{H} & -0.54465900 & 0.77642200 & -6.70976600 \\ \mathrm{H} & -0.90214400 & 2.23032500 & -5.75693300\end{array}$

$5 c$

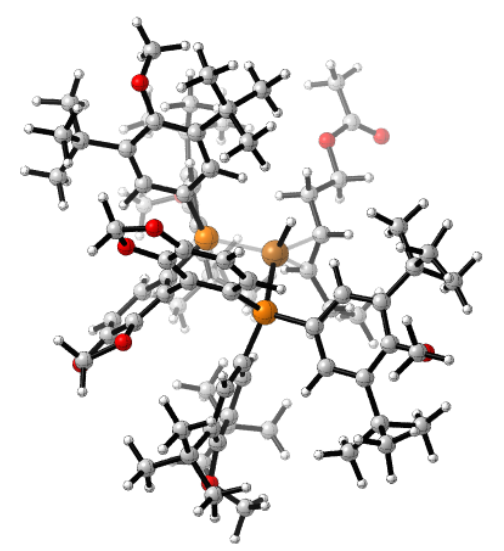

Thermal correction to Enthalpy=

1.924107

Thermal correction to Gibbs Free Energy=

1.663384

Sum of electronic and zero-point Energies=

$-4824.298627$

Sum of electronic and thermal Enthalpies=

$-4824.190362$

Sum of electronic and thermal Free Energies=

$-4824.451085$

$\operatorname{SMD~} \mathrm{E}(\mathrm{M} 06)=-4824.480391$ 
Cartesian coordinates:

\begin{tabular}{|c|c|c|c|}
\hline$P$ & 1.98396800 & 0.41480700 & 0.18 \\
\hline $\mathrm{P}$ & -1.53985700 & -0.47406700 & 0.06768300 \\
\hline $\mathrm{O}$ & 1.75503900 & -3.72968000 & 2.67610000 \\
\hline $\mathrm{O}$ & 1.12592700 & -5.63893000 & 1.55185400 \\
\hline $\mathrm{O}$ & -0.37211500 & -2.03534400 & 4.29068500 \\
\hline $\mathrm{O}$ & 0.42405900 & -0.55282900 & 5.88214800 \\
\hline $\mathrm{O}$ & -5.18523300 & 0.54168400 & 4.73692000 \\
\hline $\mathrm{O}$ & -5.76855200 & -1.66420000 & -4.07747500 \\
\hline $\mathrm{O}$ & 5.51542400 & -3.99479100 & -1.86876900 \\
\hline $\mathrm{O}$ & 6.12863300 & 4.77805100 & 0.55232500 \\
\hline $\mathrm{C}$ & -0.72287700 & -2.10078900 & 0.44571400 \\
\hline $\mathrm{C}$ & 0.28676400 & -2.14552700 & 1.45981700 \\
\hline $\mathrm{C}$ & 0.80132700 & -3.40575000 & 1.73272100 \\
\hline $\mathrm{C}$ & 0.42278000 & -4.56424700 & 1.05946800 \\
\hline $\mathrm{C}$ & -0.52252700 & -4.53161500 & 0.05852100 \\
\hline $\mathrm{C}$ & -1.09121200 & -3.27670400 & -0.22276200 \\
\hline $\mathrm{C}$ & 1.70786600 & -5.15442500 & 2.76538800 \\
\hline $\mathrm{C}$ & 1.63805100 & 0.04879800 & 1.97166800 \\
\hline $\mathrm{C}$ & 2.12448900 & 0.91581700 & 2.95767700 \\
\hline $\mathrm{C}$ & 1.78631000 & 0.79918400 & 4.31626600 \\
\hline $\mathrm{C}$ & 0.92884100 & -0.22532600 & 4.64803000 \\
\hline $\mathrm{C}$ & 0.44628000 & -1.10727500 & 3.68631300 \\
\hline $\mathrm{C}$ & 0.76244700 & -1.02504500 & 2.33657700 \\
\hline $\mathrm{C}$ & -0.29631300 & -1.77461200 & 5.69474300 \\
\hline $\mathrm{C}$ & -2.56016300 & -0.21863600 & 1.58968200 \\
\hline $\mathrm{C}$ & -2.57320300 & 1.03353700 & 2.19533900 \\
\hline $\mathrm{C}$ & -3.36382400 & 1.31425800 & 3.32579200 \\
\hline $\mathrm{C}$ & -4.23034500 & 0.28784500 & 3.76126200 \\
\hline $\mathrm{C}$ & -4.14697700 & -1.04271300 & 3.25847700 \\
\hline $\mathrm{C}$ & -3.30402200 & -1.25773300 & 2.16620500 \\
\hline $\mathrm{C}$ & -4.88253900 & -2.23485100 & 3.92482500 \\
\hline $\mathrm{C}$ & -6.42182500 & -2.12221000 & 3.81463500 \\
\hline $\mathrm{C}$ & -4.48213000 & -2.31553700 & 5.41872600 \\
\hline $\mathrm{C}$ & -4.48298600 & -3.57880000 & 3.27619200 \\
\hline $\mathrm{C}$ & -3.15585900 & 2.70392600 & 3.99135600 \\
\hline C & -3.75213100 & 2.86081700 & 5.40940100 \\
\hline
\end{tabular}




\begin{tabular}{|c|c|c|c|}
\hline $\mathrm{C}$ & -1.62773900 & 2.93697500 & 4.15528200 \\
\hline $\mathrm{C}$ & -3.72293600 & 3.81646300 & 3.07704300 \\
\hline $\mathrm{C}$ & -6.34544000 & 1.21615700 & 4.25087600 \\
\hline $\mathrm{C}$ & -2.83046300 & -0.86395300 & -1.20891700 \\
\hline $\mathrm{C}$ & -4.16503800 & -0.48934000 & -1.00714900 \\
\hline $\mathrm{C}$ & -2.47136300 & -1.33623600 & -2.47599800 \\
\hline $\mathrm{C}$ & -3.40737500 & -1.55961700 & -3.50185300 \\
\hline $\mathrm{C}$ & -4.76494700 & -1.31483800 & -3.18539200 \\
\hline $\mathrm{C}$ & -5.15149800 & -0.67027400 & -1.98171300 \\
\hline $\mathrm{C}$ & -6.57191800 & -0.08517600 & -1.76832100 \\
\hline $\mathrm{C}$ & -6.67608000 & 0.68160600 & -0.43135600 \\
\hline $\mathrm{C}$ & -7.67925000 & -1.16561400 & -1.75197600 \\
\hline $\mathrm{C}$ & -6.13838400 & -3.04193600 & -4.02427100 \\
\hline $\mathrm{C}$ & -6.85855100 & 0.93087100 & -2.90309800 \\
\hline $\mathrm{C}$ & -2.85771700 & -2.01296900 & -4.88639100 \\
\hline $\mathrm{C}$ & -2.38076000 & -3.48318200 & -4.80591500 \\
\hline $\mathrm{C}$ & -3.84144600 & -1.86740700 & -6.07194500 \\
\hline $\mathrm{C}$ & -1.64013900 & -1.12476100 & -5.26035500 \\
\hline $\mathrm{C}$ & 2.94823400 & -1.07077200 & -0.36393200 \\
\hline $\mathrm{C}$ & 3.86783000 & -1.71929600 & 0.47229200 \\
\hline $\mathrm{C}$ & 4.69066800 & -2.75150300 & 0.01181800 \\
\hline $\mathrm{C}$ & 4.58082500 & -3.10379700 & -1.36171800 \\
\hline $\mathrm{C}$ & 3.54350800 & -2.61100700 & -2.18441000 \\
\hline $\mathrm{C}$ & 2.77155300 & -1.55993800 & -1.65577300 \\
\hline $\mathrm{C}$ & 3.14874300 & -3.15109800 & -3.58818300 \\
\hline $\mathrm{C}$ & 3.74699300 & -4.52815100 & -3.96040400 \\
\hline $\mathrm{C}$ & 3.52535800 & -2.12211300 & -4.68097500 \\
\hline $\mathrm{C}$ & 1.60890300 & -3.35204100 & -3.61735800 \\
\hline $\mathrm{C}$ & 5.26037000 & -5.03159800 & 0.93241100 \\
\hline $\mathrm{C}$ & 6.54699500 & -3.37924900 & -2.64031600 \\
\hline $\mathrm{C}$ & 5.60906200 & -3.52318500 & 0.99534700 \\
\hline $\mathrm{C}$ & 5.38789600 & -3.05724400 & 2.45199700 \\
\hline $\mathrm{C}$ & 5.16797100 & 3.79738200 & 0.32401000 \\
\hline $\mathrm{C}$ & 3.79745300 & 4.12397600 & 0.48798600 \\
\hline $\mathrm{C}$ & 3.78226100 & 6.33888600 & -0.70817200 \\
\hline $\mathrm{C}$ & 2.88484900 & 3.06095800 & 0.44137500 \\
\hline $\mathrm{C}$ & 3.28760000 & 1.73822600 & 0.20651300 \\
\hline
\end{tabular}




\begin{tabular}{|c|c|c|c|}
\hline $\mathrm{C}$ & 4.62319600 & 1.50290000 & -0.13238700 \\
\hline $\mathrm{C}$ & 5.58796700 & 2.52180600 & -0.12557800 \\
\hline $\mathrm{C}$ & 6.61058100 & 4.79439900 & 1.90143200 \\
\hline $\mathrm{C}$ & 3.72794700 & 6.36534700 & 1.80944400 \\
\hline $\mathrm{C}$ & 8.15821300 & 2.39756700 & 0.27528400 \\
\hline $\mathrm{C}$ & 6.99449000 & 2.26330800 & -0.73488600 \\
\hline $\mathrm{C}$ & 7.09372100 & 0.84341900 & -1.33696600 \\
\hline $\mathrm{C}$ & 1.73443100 & 5.63503000 & 0.52766800 \\
\hline $\mathrm{C}$ & 7.11320700 & -3.33582400 & 0.68559700 \\
\hline $\mathrm{C}$ & 3.27844600 & 5.58738500 & 0.55001000 \\
\hline $\mathrm{C}$ & 7.21808200 & 3.26723200 & -1.89411400 \\
\hline $\mathrm{H}$ & -0.83294400 & -5.42888800 & -0.46601700 \\
\hline $\mathrm{H}$ & -1.87011000 & -3.23458500 & -0.97409800 \\
\hline $\mathrm{H}$ & 2.72014400 & -5.54677200 & 2.86633600 \\
\hline $\mathrm{H}$ & 1.07382400 & -5.44642500 & 3.61631000 \\
\hline $\mathrm{H}$ & 2.78585300 & 1.72259500 & 2.66696000 \\
\hline $\mathrm{H}$ & 2.17619700 & 1.48281300 & 5.06251800 \\
\hline $\mathrm{H}$ & -1.30612200 & -1.66630200 & 6.10088200 \\
\hline $\mathrm{H}$ & 0.24057700 & -2.59543300 & 6.19094600 \\
\hline $\mathrm{H}$ & -1.92574300 & 1.79815700 & 1.77673700 \\
\hline $\mathrm{H}$ & -3.22134300 & -2.24878400 & 1.73937500 \\
\hline $\mathrm{H}$ & -6.73410200 & -1.95625800 & 2.77637900 \\
\hline $\mathrm{H}$ & -6.88689700 & -3.05578200 & 4.15528700 \\
\hline $\mathrm{H}$ & -6.81720100 & -1.31560100 & 4.43248700 \\
\hline $\mathrm{H}$ & -4.74132300 & -1.39941700 & 5.95288600 \\
\hline $\mathrm{H}$ & -4.99667900 & -3.15594400 & 5.90190200 \\
\hline $\mathrm{H}$ & -3.40308900 & -2.48496300 & 5.51475800 \\
\hline $\mathrm{H}$ & -3.40191100 & -3.74984300 & 3.31664600 \\
\hline $\mathrm{H}$ & -4.96938100 & -4.39612600 & 3.82054200 \\
\hline $\mathrm{H}$ & -4.80319100 & -3.64645100 & 2.22993800 \\
\hline $\mathrm{H}$ & -3.37717900 & 3.79846300 & 5.83745500 \\
\hline $\mathrm{H}$ & -4.84127600 & 2.91550500 & 5.42409200 \\
\hline $\mathrm{H}$ & -3.44635900 & 2.04481900 & 6.07063000 \\
\hline $\mathrm{H}$ & -1.45835200 & 3.90993900 & 4.63273500 \\
\hline $\mathrm{H}$ & -1.18406900 & 2.16514600 & 4.79432300 \\
\hline $\mathrm{H}$ & -1.08813400 & 2.93678700 & 3.20479300 \\
\hline $\mathrm{H}$ & -3.25273300 & 3.79788900 & 2.08797600 \\
\hline
\end{tabular}




\begin{tabular}{|c|c|c|c|}
\hline 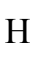 & -4.80528300 & 3.71407800 & 2.93528900 \\
\hline & -3.53578400 & 4.80280900 & 3.51983400 \\
\hline & -6.83198500 & 0.64698900 & 3.44968900 \\
\hline & -7.02856600 & 1.30486300 & 5.09985100 \\
\hline 1 & -6.10806600 & 2.21652700 & 3.87369200 \\
\hline H & -4.43224500 & -0.04063000 & -0.06206600 \\
\hline $\mathrm{H}$ & -1.42494700 & -1.54565900 & -2.67064300 \\
\hline & -7.67693900 & 1.12069100 & -0.35217700 \\
\hline & -6.53544100 & 0.02652900 & 0.43666200 \\
\hline $\mathrm{H}$ & -5.95137300 & 1.50004700 & -0.36558200 \\
\hline $\mathrm{H}$ & -7.83270500 & -1.60819300 & -2.73615800 \\
\hline & -7.44609800 & -1.96456300 & -1.03757300 \\
\hline 11 & -8.62982400 & -0.71311200 & -1.44369200 \\
\hline $\mathrm{H}$ & -5.32037000 & -3.69606800 & -4.34638500 \\
\hline $\mathrm{H}$ & -6.44474900 & -3.33497200 & -3.01347100 \\
\hline $\mathrm{H}$ & -6.98244900 & -3.16120500 & -4.70866900 \\
\hline$\Pi$ & -6.83326400 & 0.44953400 & -3.88324000 \\
\hline $\mathrm{H}$ & -7.85213000 & 1.37656800 & -2.76420400 \\
\hline $\mathrm{H}$ & -6.11726900 & 1.73874500 & -2.89087800 \\
\hline $\mathrm{H}$ & -1.61086000 & -3.61445600 & -4.03771100 \\
\hline $\mathrm{H}$ & -3.20607200 & -4.16394200 & -4.56925800 \\
\hline $\mathrm{H}$ & -1.95430400 & -3.79776500 & -5.76663000 \\
\hline $\mathrm{H}$ & -3.28429200 & -2.03031100 & -7.00252800 \\
\hline $\mathrm{H}$ & -4.65518400 & -2.59189400 & -6.05417600 \\
\hline $\mathrm{H}$ & -4.28108100 & -0.86696900 & -6.11369300 \\
\hline $\mathrm{H}$ & -1.92807000 & -0.06888200 & -5.31463100 \\
\hline $\mathrm{H}$ & -0.80943400 & -1.21388500 & -4.55589800 \\
\hline $\mathrm{H}$ & -1.25927800 & -1.42179900 & -6.24426600 \\
\hline $\mathrm{H}$ & 3.93909300 & -1.39538700 & 1.50237100 \\
\hline $\mathrm{H}$ & 1.99645800 & -1.11087700 & -2.26615500 \\
\hline $\mathrm{H}$ & 4.81715900 & -4.49781600 & -4.16502800 \\
\hline $\mathrm{H}$ & 3.57593200 & -5.26857100 & -3.17368600 \\
\hline $\mathrm{H}$ & 3.25274700 & -4.88368700 & -4.87273700 \\
\hline $\mathrm{H}$ & 3.20201200 & -2.47828400 & -5.66705700 \\
\hline $\mathrm{H}$ & 3.04716800 & -1.15274800 & -4.50117600 \\
\hline $\mathrm{H}$ & 4.60699300 & -1.95333300 & -4.72526900 \\
\hline 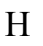 & 1.31162100 & -3.77367100 & -4.5845310 \\
\hline
\end{tabular}




\begin{tabular}{|c|c|c|c|}
\hline I & 1.28799500 & -4.04133200 & -2.82873000 \\
\hline $\mathrm{H}$ & 1.05722900 & -2.41698900 & -3.48922700 \\
\hline & 5.85010300 & -5.58505800 & 1.67421300 \\
\hline & 4.19848000 & -5.19309000 & 1.14898800 \\
\hline $\mathrm{H}$ & 5.47042100 & -5.44821300 & -0.05436600 \\
\hline $\mathrm{H}$ & 6.15687300 & -2.93367800 & -3.56205400 \\
\hline $\mathrm{H}$ & 7.06401500 & -2.60096400 & -2.06710300 \\
\hline & 7.25388400 & -4.17258500 & -2.89725900 \\
\hline$\pi$ & 5.69506000 & -2.01594900 & 2.60480100 \\
\hline $\mathrm{H}$ & 4.34257200 & -3.15538900 & 2.76184400 \\
\hline $\mathrm{H}$ & 5.99824900 & -3.67602600 & 3.11974500 \\
\hline$H$ & 3.41848400 & 5.85503900 & -1.62225600 \\
\hline $\mathrm{H}$ & 3.40914900 & 7.37093000 & -0.70309300 \\
\hline $\mathrm{H}$ & 4.87374500 & 6.36899800 & -0.74361500 \\
\hline $\mathrm{H}$ & 1.82568000 & 3.24049800 & 0.58457500 \\
\hline $\mathrm{H}$ & 4.91684400 & 0.50366700 & -0.41797900 \\
\hline $\mathrm{H}$ & 7.35743100 & 5.59143800 & 1.95003800 \\
\hline $\mathrm{H}$ & 5.80707900 & 5.00497000 & 2.61294100 \\
\hline $\mathrm{H}$ & 7.07555000 & 3.84248700 & 2.17432000 \\
\hline $\mathrm{H}$ & 3.17260900 & 7.30895500 & 1.87201200 \\
\hline $\mathrm{H}$ & 3.52190700 & 5.80074100 & 2.72669700 \\
\hline $\mathrm{H}$ & 4.78867800 & 6.61712400 & 1.77853500 \\
\hline $\mathrm{H}$ & 8.33721100 & 3.43591600 & 0.55625800 \\
\hline П & 7.97354500 & 1.81034700 & 1.18275000 \\
\hline $\mathrm{H}$ & 9.08306700 & 2.02105700 & -0.17906000 \\
\hline $\mathrm{H}$ & 8.06733100 & 0.73385900 & -1.82793200 \\
\hline $\mathrm{H}$ & 7.02185100 & 0.06191300 & -0.57132100 \\
\hline $\mathrm{H}$ & 6.32012400 & 0.65776200 & -2.08961300 \\
\hline $\mathrm{H}$ & 1.31696700 & 5.13521700 & -0.35208400 \\
\hline $\mathrm{H}$ & 1.28772000 & 5.17525500 & 1.41590800 \\
\hline $\mathrm{H}$ & 1.41273700 & 6.68234300 & 0.49865500 \\
\hline $\mathrm{H}$ & 7.37772500 & -2.27329000 & 0.62340600 \\
\hline $\mathrm{H}$ & 7.71323000 & -3.77914000 & 1.49007200 \\
\hline $\mathrm{H}$ & 7.40350000 & -3.82383300 & -0.24526600 \\
\hline $\mathrm{H}$ & 8.19833200 & 3.09520300 & -2.35692800 \\
\hline $\mathrm{H}$ & 6.45302000 & 3.14334300 & -2.66941000 \\
\hline $\mathrm{H}$ & 7.18389500 & 4.29861900 & -1.53657600 \\
\hline
\end{tabular}




$\begin{array}{llll}\mathrm{Cu} & -0.09273000 & 1.34567000 & -0.48961600 \\ \mathrm{H} & -0.25439600 & 2.47600000 & 0.60846800 \\ \mathrm{C} & -0.72691000 & 2.72488400 & -2.09055400 \\ \mathrm{C} & -0.13783600 & 1.69336600 & -2.77521100 \\ \mathrm{H} & -0.77254400 & 0.88761800 & -3.14272500 \\ \mathrm{H} & -0.12132800 & 3.60425400 & -1.87269200 \\ \mathrm{C} & 1.25723400 & 1.77840200 & -3.35478700 \\ \mathrm{H} & 1.85896400 & 2.47964800 & -2.76372300 \\ \mathrm{H} & 1.76377400 & 0.80841800 & -3.28917100 \\ \mathrm{C} & -2.22023200 & 2.91735500 & -1.94175900 \\ \mathrm{H} & -2.74518700 & 1.95956200 & -2.01712900 \\ \mathrm{H} & -2.44795900 & 3.34160800 & -0.95840500 \\ \mathrm{C} & -2.74751600 & 3.86273100 & -3.02187800 \\ \mathrm{H} & -2.62296300 & 3.44310900 & -4.02559600 \\ \mathrm{H} & -2.23777800 & 4.83176600 & -3.00042700 \\ \mathrm{O} & -4.15705900 & 4.07317500 & -2.77080800 \\ \mathrm{C} & -4.76977100 & 4.97936100 & -3.56734800 \\ \mathrm{O} & -4.20780300 & 5.58126200 & -4.45635300 \\ \mathrm{C} & 1.24002700 & 2.23027800 & -4.82787700 \\ \mathrm{H} & 0.79566000 & 3.22721800 & -4.92615100 \\ \mathrm{H} & 2.25631700 & 2.26767900 & -5.23868700 \\ \mathrm{H} & 0.65255900 & 1.54198800 & -5.44784500 \\ \mathrm{C} & -6.22820500 & 5.12775200 & -3.19566000 \\ \mathrm{H} & -6.75689700 & 4.18321900 & -3.36295900 \\ \mathrm{H} & -6.32692000 & 5.37314000 & -2.13335400 \\ \mathrm{H} & -6.67816000 & 5.91292200 & -3.80446800\end{array}$

5d 


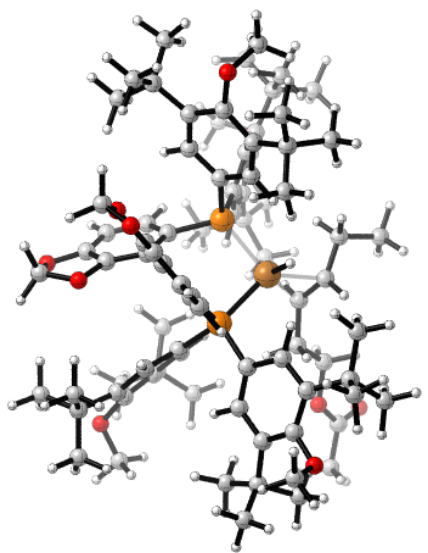

Thermal correction to Enthalpy=

1.924272

Thermal correction to Gibbs Free Energy=

1.664016

Sum of electronic and zero-point Energies= $-4824.297963$

Sum of electronic and thermal Enthalpies= $-4824.189853$

Sum of electronic and thermal Free Energies= $-4824.450109$

SMD E $(\mathrm{M} 06)=-4824.480572$

Cartesian coordinates:

$\begin{array}{llll}\mathrm{P} & -1.55065200 & -0.76793100 & -0.03881100 \\ \mathrm{P} & 1.91536900 & 0.27357000 & -0.04654100 \\ \mathrm{O} & -0.07764900 & -1.89131800 & 4.40201700 \\ \mathrm{O} & 0.60704300 & -0.19000200 & 5.79702300 \\ \mathrm{O} & 2.02601800 & -3.53397700 & 2.78087200 \\ \mathrm{O} & 1.36906600 & -5.59546300 & 1.96930700 \\ \mathrm{O} & 6.29032000 & -3.74594200 & -0.92288800 \\ \mathrm{O} & 5.29672200 & 5.26007600 & -0.49273600 \\ \mathrm{O} & -4.71281800 & 1.68071100 & 4.44429000 \\ \mathrm{O} & -6.22563500 & -2.73996900 & -3.29934200 \\ \mathrm{C} & 1.51921800 & 0.18640700 & 1.77137000 \\ \mathrm{C} & 0.79642000 & -0.93897500 & 2.28570000 \\ \mathrm{C} & 0.57535600 & -0.92988600 & 3.65704300 \\ \mathrm{C} & 0.98553900 & 0.09420400 & 4.50624300 \\ \mathrm{C} & 1.66176200 & 1.19251200 & 4.02417500 \\ \mathrm{C} & 1.92339400 & 1.20776000 & 2.64300500 \\ \mathrm{C} & 0.22204100 & -1.56691000 & 5.76029100 \\ \mathrm{C} & -0.73469700 & -2.29171100 & 0.63320600 \\ \mathrm{C} & -1.11658400 & -3.55413500 & 0.16289600 \\ \mathrm{C} & -0.47365500 & -4.74246000 & 0.54819500\end{array}$




$\begin{array}{llll}\mathrm{C} & 0.57447100 & -4.61273100 & 1.43127100 \\ \mathrm{C} & 0.96715300 & -3.36733800 & 1.91347500 \\ \mathrm{C} & 0.35711600 & -2.17255900 & 1.55285100 \\ \mathrm{C} & 2.12953400 & -4.94437200 & 2.98949900 \\ \mathrm{C} & 3.17445800 & -1.06980300 & -0.21835300 \\ \mathrm{C} & 3.14666700 & -1.88486300 & -1.34585100 \\ \mathrm{C} & 4.11699800 & -2.87655900 & -1.58104600 \\ \mathrm{C} & 5.18837500 & -2.94237700 & -0.66253900 \\ \mathrm{C} & 5.17617200 & -2.23153300 & 0.57150700 \\ \mathrm{C} & 4.15719900 & -1.29408200 & 0.75536600 \\ \mathrm{C} & 6.18544600 & -2.52311400 & 1.71262900 \\ \mathrm{C} & 7.63326200 & -2.12259500 & 1.34332100 \\ \mathrm{C} & 6.14542400 & -4.03128700 & 2.06191000 \\ \mathrm{C} & 5.81667100 & -1.75195800 & 2.99961600 \\ \mathrm{C} & 3.87375700 & -3.81681900 & -2.79533400 \\ \mathrm{C} & 4.72474100 & -5.10716600 & -2.81461500 \\ \mathrm{C} & 2.39360300 & -4.29099800 & -2.75222400 \\ \mathrm{C} & -2.97387100 & -0.83566000 & 2.43967000 \\ \mathrm{C} & -3.70607000 & -0.27992700 & 3.49276200 \\ \mathrm{C} & & & \\ \mathrm{C} & & & \end{array}$




\begin{tabular}{|c|c|c|c|}
\hline $\mathrm{C}$ & -3.83868900 & 1.13658400 & 3.51437100 \\
\hline $\mathrm{C}$ & -3.08755200 & 1.97615000 & 2.66103200 \\
\hline $\mathrm{C}$ & -2.38901100 & 1.34121800 & 1.61758100 \\
\hline $\mathrm{C}$ & -2.93373700 & 3.51807800 & 2.78995900 \\
\hline $\mathrm{C}$ & -3.34899100 & 4.10649700 & 4.15895900 \\
\hline $\mathrm{C}$ & -3.72712600 & 4.22991400 & 1.66760900 \\
\hline $\mathrm{C}$ & -1.43224600 & 3.87990300 & 2.62591500 \\
\hline $\mathrm{C}$ & -3.74172500 & -0.68226200 & 5.98654500 \\
\hline $\mathrm{C}$ & -5.96996500 & 2.08370100 & 3.90164200 \\
\hline $\mathrm{C}$ & -4.26932300 & -1.18478100 & 4.61926200 \\
\hline $\mathrm{C}$ & -3.79603100 & -2.64637900 & 4.45366900 \\
\hline $\mathrm{C}$ & -5.15478800 & -2.19173000 & -2.59973200 \\
\hline $\mathrm{C}$ & -3.83678200 & -2.40570900 & -3.08017800 \\
\hline $\mathrm{C}$ & -4.20406600 & -1.94728100 & -5.52543300 \\
\hline $\mathrm{C}$ & -2.78624800 & -1.98091200 & -2.25575500 \\
\hline $\mathrm{C}$ & -3.01042200 & -1.35891100 & -1.01858400 \\
\hline $\mathrm{C}$ & -4.32250900 & -1.02457800 & -0.67332800 \\
\hline $\mathrm{C}$ & -5.42098700 & -1.38158800 & -1.46942800 \\
\hline $\mathrm{C}$ & -6.60438900 & -4.03824200 & -2.82632000 \\
\hline $\mathrm{C}$ & -4.02834000 & -4.35137700 & -4.79989500 \\
\hline $\mathrm{C}$ & -7.92702400 & -1.77478700 & -0.86635200 \\
\hline $\mathrm{C}$ & -6.81392700 & -0.75134600 & -1.18988300 \\
\hline $\mathrm{C}$ & -6.75873100 & 0.23301100 & 0.00018000 \\
\hline $\mathrm{C}$ & -2.01446000 & -2.90591800 & -4.80738900 \\
\hline $\mathrm{C}$ & -5.81579600 & -1.21139800 & 4.64471400 \\
\hline $\mathrm{C}$ & -3.53189800 & -2.91371800 & -4.51762500 \\
\hline $\mathrm{C}$ & -7.22829400 & 0.06653600 & -2.43980400 \\
\hline $\mathrm{H}$ & 1.99757300 & 1.99087600 & 4.67719400 \\
\hline $\mathrm{H}$ & 2.48944900 & 2.04228800 & 2.24853200 \\
\hline $\mathrm{H}$ & -0.66737200 & -1.71324700 & 6.37427500 \\
\hline $\mathrm{H}$ & 1.05985600 & -2.18980500 & 6.10884000 \\
\hline $\mathrm{H}$ & -1.94704900 & -3.62824600 & -0.52827900 \\
\hline $\mathrm{H}$ & -0.79222800 & -5.71032900 & 0.17658500 \\
\hline $\mathrm{H}$ & 3.17542400 & -5.24935300 & 2.91280700 \\
\hline $\mathrm{H}$ & 1.71346700 & -5.19949000 & 3.97571500 \\
\hline $\mathrm{H}$ & 2.32296300 & -1.74855000 & -2.03956700 \\
\hline $\mathrm{H}$ & 4.11725100 & -0.71439700 & 1.6681640 \\
\hline
\end{tabular}




\begin{tabular}{|c|c|c|c|}
\hline I & 7.68163400 & -1.08274300 & 0.99788000 \\
\hline $\mathrm{H}$ & 8.27855000 & -2.20929800 & 2.22657500 \\
\hline & 8.05011200 & -2.76758200 & 0.56923400 \\
\hline & 6.41171500 & -4.64987800 & 1.20319800 \\
\hline $\mathrm{H}$ & 6.84650000 & -4.24802600 & 2.87791200 \\
\hline $\mathrm{H}$ & 5.14121400 & -4.31528300 & 2.39704200 \\
\hline $\mathrm{H}$ & 4.79884900 & -1.97902100 & 3.33463400 \\
\hline & 6.50600200 & -2.04372500 & 3.80015000 \\
\hline 11 & 5.90463800 & -0.66665800 & 2.87375300 \\
\hline $\mathrm{H}$ & 4.33564800 & -5.76465600 & -3.60170600 \\
\hline $\mathrm{H}$ & 5.77889500 & -4.93351700 & -3.03315000 \\
\hline $\mathrm{H}$ & 4.66166400 & -5.64677200 & -1.86507800 \\
\hline $\mathrm{H}$ & 2.20769600 & -4.97533300 & -3.58899900 \\
\hline $\mathrm{H}$ & 2.18412000 & -4.82982200 & -1.82109000 \\
\hline $\mathrm{H}$ & 1.67639900 & -3.46983900 & -2.83230300 \\
\hline $\mathrm{H}$ & 3.43554600 & -2.16820200 & -4.17929100 \\
\hline $\mathrm{H}$ & 5.12736400 & -2.68858400 & -4.21569000 \\
\hline $\mathrm{H}$ & 3.87822400 & -3.68812400 & -4.97608700 \\
\hline $\mathrm{H}$ & 7.57465500 & -2.19901000 & -1.50198800 \\
\hline $\mathrm{H}$ & 8.06031500 & -3.87155900 & -1.90409300 \\
\hline $\mathrm{H}$ & 6.78053400 & -3.05845700 & -2.84334100 \\
\hline $\mathrm{H}$ & 4.68964600 & 0.79008200 & -0.92756200 \\
\hline $\mathrm{H}$ & 1.33148400 & 3.11652400 & 0.25676100 \\
\hline $\mathrm{H}$ & 7.72645500 & 1.34202100 & -2.48651500 \\
\hline $\mathrm{H}$ & 6.81340400 & 0.65307200 & -1.13979700 \\
\hline $\mathrm{H}$ & 6.00756700 & 0.98230000 & -2.68732300 \\
\hline $\mathrm{H}$ & 7.58489200 & 4.27642900 & -0.34194000 \\
\hline $\mathrm{H}$ & 7.51771600 & 2.66165800 & 0.39887500 \\
\hline $\mathrm{H}$ & 8.55998100 & 2.96383000 & -0.99975300 \\
\hline $\mathrm{H}$ & 4.94758000 & 6.06719400 & 1.41209400 \\
\hline 11 & 6.20619400 & 4.80902700 & 1.33792200 \\
\hline $\mathrm{H}$ & 6.51210900 & 6.42442900 & 0.63825100 \\
\hline $\mathrm{H}$ & 6.31650100 & 4.74620500 & -2.52732600 \\
\hline $\mathrm{H}$ & 7.48486000 & 3.62505100 & -3.25468300 \\
\hline $\mathrm{H}$ & 5.74793800 & 3.38088000 & -3.50958200 \\
\hline $\mathrm{H}$ & 1.45674700 & 5.03426600 & 1.93776000 \\
\hline $\mathrm{H}$ & 2.94510100 & 5.97965700 & 2.0727500 \\
\hline
\end{tabular}




\begin{tabular}{|c|c|c|c|}
\hline I & 1.43249100 & 6.77666300 & 1.61367500 \\
\hline H & 2.28495100 & 7.67723900 & -0.60598500 \\
\hline $\mathrm{H}$ & 3.88207600 & 7.17054400 & -0.06681500 \\
\hline $\mathrm{H}$ & 3.29724300 & 6.67122200 & -1.65493200 \\
\hline H & 1.08971600 & 5.25181200 & -1.80123000 \\
\hline $\mathrm{H}$ & 0.28827000 & 4.73319800 & -0.30689800 \\
\hline $\mathrm{H}$ & 0.41906100 & 6.44691900 & -0.67689900 \\
\hline $\mathrm{H}$ & -2.87461800 & -1.91052000 & 2.36272300 \\
\hline$\Pi$ & -1.83545700 & 1.94147100 & 0.90485600 \\
\hline $\mathrm{H}$ & -4.42483600 & 4.09281700 & 4.33184800 \\
\hline $\mathrm{H}$ & -2.86955600 & 3.57603000 & 4.98708500 \\
\hline $\mathrm{H}$ & -3.02680500 & 5.15425300 & 4.19759600 \\
\hline $\mathrm{H}$ & -3.57066000 & 5.31457300 & 1.72084400 \\
\hline $\mathrm{H}$ & -3.41024200 & 3.89247800 & 0.67437300 \\
\hline $\mathrm{H}$ & -4.80361000 & 4.04424700 & 1.74922000 \\
\hline $\mathrm{H}$ & -1.29830400 & 4.95790600 & 2.77210600 \\
\hline $\mathrm{H}$ & -0.81673700 & 3.35364800 & 3.36362400 \\
\hline $\mathrm{H}$ & -1.04292200 & 3.63851300 & 1.63293600 \\
\hline $\mathrm{H}$ & -4.07879800 & -1.35091000 & 6.78870900 \\
\hline $\mathrm{H}$ & -2.64598500 & -0.66611800 & 5.99286600 \\
\hline $\mathrm{H}$ & -4.09555400 & 0.32645300 & 6.20581400 \\
\hline $\mathrm{H}$ & -5.86740500 & 2.94128600 & 3.22782700 \\
\hline $\mathrm{H}$ & -6.45082100 & 1.26388600 & 3.35544000 \\
\hline $\mathrm{H}$ & -6.59453900 & 2.36996200 & 4.75203700 \\
\hline $\mathrm{H}$ & -4.20390500 & -3.11456500 & 3.55033200 \\
\hline $\mathrm{H}$ & -2.70437900 & -2.72361100 & 4.41725500 \\
\hline $\mathrm{H}$ & -4.14806900 & -3.23330500 & 5.30967800 \\
\hline $\mathrm{H}$ & -3.81741800 & -0.92849200 & -5.40488200 \\
\hline $\mathrm{H}$ & -3.99122600 & -2.26805300 & -6.55315900 \\
\hline $\mathrm{H}$ & -5.28825900 & -1.92437300 & -5.39099300 \\
\hline $\mathrm{H}$ & -1.75819600 & -2.10130100 & -2.57435100 \\
\hline 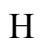 & -4.48939100 & -0.45229400 & 0.22707800 \\
\hline $\mathrm{H}$ & -7.43319000 & -4.36565200 & -3.45988900 \\
\hline $\mathrm{H}$ & -5.77954500 & -4.75164200 & -2.90900100 \\
\hline $\mathrm{H}$ & -6.93393200 & -4.00673400 & -1.78365600 \\
\hline $\mathrm{H}$ & -3.61611100 & -4.69763600 & -5.75552300 \\
\hline $\mathrm{H}$ & -3.69429000 & -5.05254500 & -4.0255750 \\
\hline
\end{tabular}




\begin{tabular}{|c|c|c|c|}
\hline $\mathrm{H}$ & -5.11475500 & -4.40243100 & -4.87994200 \\
\hline $\mathrm{H}$ & -8.22285100 & -2.34844900 & -1.74523800 \\
\hline $\mathrm{H}$ & -7.61725300 & -2.46907500 & -0.07599800 \\
\hline $\mathrm{H}$ & -8.81910900 & -1.24560100 & -0.50918200 \\
\hline $\mathrm{H}$ & -7.74113500 & 0.70505500 & 0.11515700 \\
\hline $\mathrm{H}$ & -6.52350200 & -0.27113200 & 0.94531300 \\
\hline $\mathrm{H}$ & -6.02426200 & 1.03025000 & -0.15541500 \\
\hline $\mathrm{H}$ & -1.56466200 & -1.91740800 & -4.67331100 \\
\hline $\mathrm{H}$ & -1.46758500 & -3.61029600 & -4.17019400 \\
\hline$\Pi$ & -1.85137100 & -3.20720600 & -5.84847100 \\
\hline $\mathrm{H}$ & -6.22385000 & -1.47573700 & 3.66159900 \\
\hline $\mathrm{H}$ & -6.16063600 & -1.96765100 & 5.36087500 \\
\hline $\mathrm{H}$ & -6.23759400 & -0.25397400 & 4.95202800 \\
\hline$\Pi$ & -8.19885400 & 0.55007100 & -2.26835000 \\
\hline $\mathrm{H}$ & -6.48997400 & 0.84943800 & -2.65035700 \\
\hline $\mathrm{H}$ & -7.31624500 & -0.57486100 & -3.31986700 \\
\hline $\mathrm{Cu}$ & 0.04978400 & 0.07803900 & -1.54060000 \\
\hline $\mathrm{H}$ & 0.30368000 & -1.18008500 & -2.46997100 \\
\hline $\mathrm{C}$ & -0.07171600 & 1.41249800 & -3.31179800 \\
\hline $\mathrm{C}$ & -0.72352700 & 2.10539800 & -2.32474400 \\
\hline $\mathrm{H}$ & -0.16355100 & 2.84282000 & -1.75108200 \\
\hline $\mathrm{H}$ & -0.67164300 & 0.80413000 & -3.98801000 \\
\hline $\mathrm{C}$ & 1.33921700 & 1.69096400 & -3.77564700 \\
\hline $\mathrm{H}$ & 1.89910000 & 2.20537200 & -2.98638900 \\
\hline$\Pi$ & 1.85500800 & 0.74364800 & -3.96943100 \\
\hline $\mathrm{C}$ & -2.23304500 & 2.16842700 & -2.22533600 \\
\hline $\mathrm{H}$ & -2.68064900 & 1.25956100 & -2.64245500 \\
\hline $\mathrm{H}$ & -2.56257900 & 2.23137300 & -1.18303400 \\
\hline $\mathrm{C}$ & -2.78132700 & 3.38544100 & -2.97015000 \\
\hline $\mathrm{H}$ & -2.34107400 & 4.32051900 & -2.60720900 \\
\hline$H$ & -2.58723200 & 3.32314700 & -4.04578200 \\
\hline $\mathrm{O}$ & -4.21078400 & 3.42397900 & -2.74675100 \\
\hline $\mathrm{C}$ & -4.86746200 & 4.47234300 & -3.29346300 \\
\hline$C$ & -6.34872300 & 4.39444400 & -2.99815000 \\
\hline $\mathrm{H}$ & -6.77263300 & 3.48488800 & -3.43699700 \\
\hline$U$ & -6.51824000 & 4.34358700 & -1.91767800 \\
\hline & -6.84771000 & 5.27103400 & -3.4130490 \\
\hline
\end{tabular}




$\begin{array}{llll}\mathrm{O} & -4.32412400 & 5.34686100 & -3.93265000 \\ \mathrm{C} & 1.35004900 & 2.54715800 & -5.05688200 \\ \mathrm{H} & 0.87905000 & 3.52207400 & -4.88588000 \\ \mathrm{H} & 2.37703400 & 2.72074100 & -5.39924800 \\ \mathrm{H} & 0.80608500 & 2.05157800 & -5.87038400\end{array}$

\section{5a-TS}

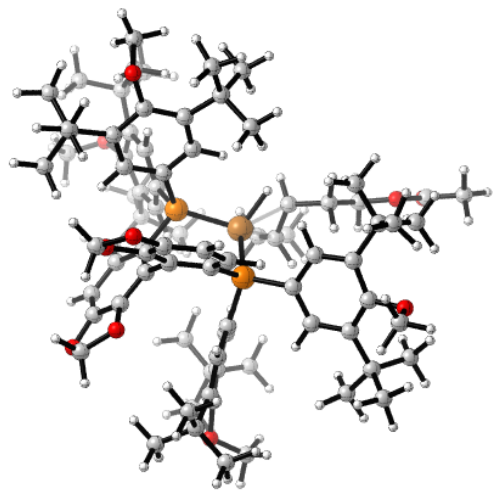

Thermal correction to Enthalpy= $\quad 1.922268$

Thermal correction to Gibbs Free Energy=

Sum of electronic and zero-point Energies $=$

1.665889

Sum of electronic and thermal Enthalpies=

$-4824.272558$

Sum of electronic and thermal Free Energies=

$-4824.165349$

SMD E $(\mathrm{M} 06)=-4824.455071$

Imaginary frequency: -805.35

Cartesian coordinates:

$P$

$$
\begin{array}{llll}
\mathrm{P} & -1.64524600 & -0.03453900 & -0.43898700 \\
\mathrm{P} & 1.90357200 & -0.14835100 & 0.25263300 \\
\mathrm{O} & -0.07975700 & -3.36240500 & -3.53948100 \\
\mathrm{O} & 0.98388100 & -5.26059800 & -2.77940300 \\
\mathrm{O} & 1.65642600 & -1.04221700 & -4.50911900 \\
\mathrm{O} & 0.69042900 & 0.48213800 & -5.94203600 \\
\mathrm{O} & 4.93588200 & 3.66874100 & -3.25202600 \\
\mathrm{O} & 6.64841000 & -1.42477700 & 3.76274700 \\
\mathrm{O} & -4.07765500 & -5.48119300 & 0.19985100 \\
\mathrm{O} & -6.74226400 & 3.17014000 & -0.72649700 \\
\mathrm{C} & 1.68952600 & -1.73430000 & -0.70779800 \\
\mathrm{C} & 0.81518900 & -1.79112700 & -1.84382000
\end{array}
$$




\begin{tabular}{|c|c|c|c|}
\hline $\mathrm{C}$ & 0.68446100 & -3.04235800 & -2.43496500 \\
\hline $\mathrm{C}$ & 1.32650800 & -4.19201300 & -1.98590400 \\
\hline $\mathrm{C}$ & 2.17079600 & -4.15445400 & $-0.899849 c$ \\
\hline $\mathrm{C}$ & 2.33633500 & -2.90359900 & -0.28171000 \\
\hline $\mathrm{C}$ & 0.34665500 & -4.67276800 & -3.9163540 \\
\hline $\mathrm{C}$ & -0.99310300 & 0.10258100 & -2.1741230 \\
\hline $\mathrm{C}$ & -1.56649600 & 1.01605100 & -3.07023 \\
\hline $\mathrm{C}$ & -1.07691400 & 1.23449200 & -4.36887500 \\
\hline $\mathrm{C}$ & 0.01600100 & 0.48617800 & -4.74382300 \\
\hline $\mathrm{C}$ & 0.59621800 & -0.42960900 & -3.8710330 \\
\hline $\mathrm{C}$ & 0.14995200 & -0.66158800 & -2.57581300 \\
\hline $\mathrm{C}$ & 1.51336500 & -0.68499700 & -5.8850790 \\
\hline $\mathrm{C}$ & 2.70938300 & 0.97336700 & -0.98037700 \\
\hline $\mathrm{C}$ & 2.38606500 & 2.32650400 & -0.9698090 \\
\hline $\mathrm{C}$ & 3.03630900 & 3.26557200 & -1.79128600 \\
\hline $\mathrm{C}$ & 4.11387800 & 2.78475800 & -2.5675550 \\
\hline $\mathrm{C}$ & 4.38140500 & 1.39416400 & -2.71587600 \\
\hline $\mathrm{C}$ & 3.66731000 & 0.51629200 & -1.89577600 \\
\hline $\mathrm{C}$ & 5.35538600 & 0.84595100 & -3.79121400 \\
\hline $\mathrm{C}$ & 6.82667600 & 1.24283800 & -3.52226100 \\
\hline $\mathrm{C}$ & 4.92770000 & 1.37821100 & -5.1818040 \\
\hline $\mathrm{C}$ & 5.31149600 & -0.69714000 & -3.85548700 \\
\hline $\mathrm{C}$ & 2.47037300 & 4.71358100 & -1.78898100 \\
\hline $\mathrm{C}$ & 2.93335400 & 5.60773900 & -2.96263800 \\
\hline $\mathrm{C}$ & 0.92390500 & 4.62919800 & -1.91702300 \\
\hline $\mathrm{C}$ & 2.80633500 & 5.41298300 & -0.44980200 \\
\hline $\mathrm{C}$ & 5.91444300 & 4.30912200 & -2.43361700 \\
\hline $\mathrm{C}$ & 3.31843400 & -0.48261000 & 1.41908400 \\
\hline $\mathrm{C}$ & 4.48547900 & 0.28953900 & 1.36801000 \\
\hline $\mathrm{C}$ & 3.21141200 & -1.43304200 & 2.44080000 \\
\hline $\mathrm{C}$ & 4.28066300 & -1.75361600 & 3.29834900 \\
\hline $\mathrm{C}$ & 5.49467100 & -1.05767100 & 3.08433500 \\
\hline $\mathrm{C}$ & 5.57750400 & 0.05630400 & 2.21033600 \\
\hline $\mathrm{C}$ & 6.78113200 & 1.03503700 & 2.21392800 \\
\hline $\mathrm{C}$ & 6.52490800 & 2.24687400 & 1.29125700 \\
\hline $\mathrm{C}$ & 8.09579100 & 0.37671300 & 1.73120200 \\
\hline $\mathrm{C}$ & 7.34213500 & -2.52713800 & 3.179142 \\
\hline
\end{tabular}




\begin{tabular}{|c|c|c|c|}
\hline $\mathrm{C}$ & 6.97901600 & 1.58548200 & 3.64845700 \\
\hline $\mathrm{C}$ & 4.03056100 & -2.83206400 & 4.39385200 \\
\hline $\mathrm{C}$ & 3.97093700 & -4.23456200 & 3.74014200 \\
\hline $\mathrm{C}$ & 5.06124900 & -2.85161100 & 5.54833700 \\
\hline $\mathrm{C}$ & 2.66326600 & -2.56530100 & 5.07457500 \\
\hline $\mathrm{C}$ & -2.24602300 & -1.77826700 & -0.34121100 \\
\hline $\mathrm{C}$ & -2.89591800 & -2.41514000 & -1.40693100 \\
\hline $\mathrm{C}$ & -3.45982600 & -3.68683800 & -1.27321700 \\
\hline $\mathrm{C}$ & -3.36624000 & -4.30591700 & 0.00495300 \\
\hline $\mathrm{C}$ & -2.55845300 & -3.78223300 & 1.03896600 \\
\hline $\mathrm{C}$ & -2.05557100 & -2.48262400 & 0.84428100 \\
\hline $\mathrm{C}$ & -2.12652500 & -4.51780400 & 2.33892200 \\
\hline $\mathrm{C}$ & -2.39766300 & -6.03944100 & 2.36614300 \\
\hline $\mathrm{C}$ & -2.79719900 & -3.86249900 & 3.56986100 \\
\hline $\mathrm{C}$ & -0.58563600 & -4.37114000 & 2.48035300 \\
\hline $\mathrm{C}$ & -3.38300000 & -5.77346600 & -2.69792200 \\
\hline $\mathrm{C}$ & -5.27715200 & -5.31269500 & 0.95562100 \\
\hline $\mathrm{C}$ & -4.07953800 & -4.40378300 & -2.50069600 \\
\hline $\mathrm{C}$ & -3.86270300 & -3.58803900 & -3.79491100 \\
\hline $\mathrm{C}$ & -5.56975200 & 2.45145300 & -0.52001900 \\
\hline $\mathrm{C}$ & -4.32530500 & 3.13266800 & -0.57336500 \\
\hline $\mathrm{C}$ & -4.88772900 & 5.17450100 & 0.78800700 \\
\hline $\mathrm{C}$ & -3.17029700 & 2.33872200 & -0.53442900 \\
\hline $\mathrm{C}$ & -3.22384700 & 0.93989200 & -0.44707800 \\
\hline $\mathrm{C}$ & -4.46584700 & 0.34063300 & -0.22337600 \\
\hline $\mathrm{C}$ & -5.66087100 & 1.07558900 & -0.19845400 \\
\hline $\mathrm{C}$ & -7.18540300 & 3.15683900 & -2.08942100 \\
\hline $\mathrm{C}$ & -4.86137200 & 5.40603500 & -1.71937700 \\
\hline $\mathrm{C}$ & -8.09598900 & 0.31640700 & -0.74245100 \\
\hline $\mathrm{C}$ & -6.96829900 & 0.40679600 & 0.31266000 \\
\hline $\mathrm{C}$ & -6.70408400 & -1.03457000 & 0.80449800 \\
\hline $\mathrm{C}$ & -2.73671500 & 5.13943000 & -0.47896700 \\
\hline $\mathrm{C}$ & -5.60377600 & -4.62271300 & -2.35341700 \\
\hline $\mathrm{C}$ & -4.21102600 & 4.68196400 & -0.51672000 \\
\hline $\mathrm{C}$ & -7.48502500 & 1.21654400 & 1.52899500 \\
\hline $\mathrm{H}$ & 2.69615500 & -5.03634500 & -0.54967700 \\
\hline $\mathrm{H}$ & 3.02316500 & -2.85090200 & 0.55201100 \\
\hline
\end{tabular}




\begin{tabular}{|c|c|c|c|}
\hline t & -0.52114800 & -5.27062900 & -4.19789400 \\
\hline $\mathrm{H}$ & 1.06991500 & -4.59873100 & -4.74268000 \\
\hline & -2.44017700 & 1.57585500 & -2.76214000 \\
\hline & -1.54761000 & 1.94053800 & -5.04442500 \\
\hline $\mathrm{H}$ & 2.49411600 & -0.46116700 & -6.30650200 \\
\hline $\mathrm{H}$ & 1.02021600 & -1.50683400 & -6.42643700 \\
\hline $\mathrm{H}$ & 1.59243600 & 2.64489700 & -0.30102100 \\
\hline & 3.85748400 & -0.54735400 & -1.95385100 \\
\hline$\Pi$ & 7.13306800 & 0.96626100 & -2.50604000 \\
\hline $\mathrm{H}$ & 7.48599500 & 0.71406500 & -4.22198200 \\
\hline $\mathrm{H}$ & 6.99459500 & 2.31134800 & -3.65945400 \\
\hline $\mathrm{H}$ & 5.00275900 & 2.46571200 & -5.23429800 \\
\hline $\mathrm{H}$ & 5.56764400 & 0.94838500 & -5.96295200 \\
\hline $\mathrm{H}$ & 3.88993100 & 1.09958200 & -5.39753100 \\
\hline $\mathrm{H}$ & 4.30029300 & -1.07245100 & -4.04290000 \\
\hline $\mathrm{H}$ & 5.95599000 & -1.03771100 & -4.67404000 \\
\hline $\mathrm{H}$ & 5.68477400 & -1.16072300 & -2.93479300 \\
\hline $\mathrm{H}$ & 2.32512300 & 6.52049200 & -2.96105200 \\
\hline $\mathrm{H}$ & 3.97635700 & 5.91744200 & -2.89323300 \\
\hline $\mathrm{H}$ & 2.79312400 & 5.11202300 & -3.92762700 \\
\hline $\mathrm{H}$ & 0.50553300 & 5.64183300 & -1.95982200 \\
\hline $\mathrm{H}$ & 0.63541500 & 4.10194800 & -2.83360100 \\
\hline $\mathrm{H}$ & 0.45481700 & 4.11748200 & -1.07299100 \\
\hline$\Pi$ & 2.41345200 & 4.84913400 & 0.40331700 \\
\hline $\mathrm{H}$ & 3.88729200 & 5.52277800 & -0.30657400 \\
\hline $\mathrm{H}$ & 2.36270500 & 6.41610800 & -0.42396000 \\
\hline $\mathrm{H}$ & 6.54504500 & 3.57599600 & -1.91687600 \\
\hline $\mathrm{H}$ & 6.53228300 & 4.91007100 & -3.10617900 \\
\hline $\mathrm{H}$ & 5.45558500 & 4.96381400 & -1.68528100 \\
\hline $\mathrm{H}$ & 4.54067400 & 1.08913400 & 0.64494400 \\
\hline 11 & 2.27096300 & -1.95902300 & 2.55900600 \\
\hline $\mathrm{H}$ & 7.36574100 & 2.94424000 & 1.37903500 \\
\hline $\mathrm{H}$ & 6.44667000 & 1.95713300 & 0.23683600 \\
\hline $\mathrm{H}$ & 5.61403700 & 2.79049300 & 1.56466300 \\
\hline $\mathrm{H}$ & 8.47827200 & -0.34784600 & 2.45030300 \\
\hline $\mathrm{H}$ & 7.95890900 & -0.12392100 & 0.76494000 \\
\hline $\mathrm{H}$ & 8.86695300 & 1.14592100 & 1.599800 \\
\hline
\end{tabular}




\begin{tabular}{|c|c|c|c|}
\hline t & 6.77280800 & -3.45903500 & 3.26639000 \\
\hline $\mathrm{H}$ & 7.56091300 & -2.34714500 & 2.12036700 \\
\hline & 8.27985200 & -2.62888700 & 3.73190300 \\
\hline & 7.19495400 & 0.78321900 & 4.35687700 \\
\hline & 7.81526700 & 2.29596900 & 3.66509700 \\
\hline $\mathrm{H}$ & 6.08131700 & 2.11568100 & 3.98755200 \\
\hline $\mathrm{H}_{\mathrm{H}}$ & 3.17895100 & -4.28698300 & 2.98428700 \\
\hline & 4.91516600 & -4.50027300 & 3.25216100 \\
\hline & 3.76051200 & -4.99877300 & 4.49874500 \\
\hline $\mathrm{H}$ & 4.67701600 & -3.50505600 & 6.34084600 \\
\hline $\mathrm{H}$ & 6.03841500 & -3.23727700 & 5.25913600 \\
\hline U & 5.20672700 & -1.85530300 & 5.97594300 \\
\hline 11 & 2.63350900 & -1.56663000 & 5.52284600 \\
\hline $\mathrm{H}$ & 1.81756600 & -2.65083900 & 4.38951000 \\
\hline $\mathrm{H}$ & 2.50615800 & -3.29979300 & 5.87285800 \\
\hline $\mathrm{H}$ & -2.96279900 & -1.89311300 & -2.35265200 \\
\hline$\Pi$ & -1.48685600 & -1.99625800 & 1.63048600 \\
\hline $\mathrm{H}$ & -3.45358700 & -6.29268800 & 2.46563200 \\
\hline $\mathrm{H}$ & -2.01453200 & -6.53628200 & 1.47035800 \\
\hline $\mathrm{H}$ & -1.88121400 & -6.46479200 & 3.23524700 \\
\hline $\mathrm{H}$ & -2.44862700 & -4.34242200 & 4.49272400 \\
\hline $\mathrm{H}$ & -2.56029000 & -2.79522500 & 3.63651900 \\
\hline $\mathrm{H}$ & -3.88848000 & -3.95961000 & 3.53567900 \\
\hline $\mathrm{H}$ & -0.24733400 & -4.89888300 & 3.37998000 \\
\hline $\mathrm{H}$ & -0.07105200 & -4.80409700 & 1.61508900 \\
\hline $\mathrm{H}$ & -0.26644300 & -3.32986000 & 2.57066400 \\
\hline $\mathrm{H}$ & -3.76702700 & -6.26338200 & -3.60165700 \\
\hline $\mathrm{H}$ & -2.30092300 & -5.64300100 & -2.81368400 \\
\hline $\mathrm{H}$ & -3.55423100 & -6.43571900 & -1.84744100 \\
\hline $\mathrm{H}$ & -5.07153100 & -4.97111800 & 1.97578300 \\
\hline $\mathrm{H}$ & -5.95341100 & -4.59474800 & 0.47623500 \\
\hline $\mathrm{H}$ & -5.75680100 & -6.29423200 & 0.99441700 \\
\hline $\mathrm{H}$ & -4.40364800 & -2.63459800 & -3.78049700 \\
\hline $\mathrm{H}$ & -2.80316700 & -3.37827800 & -3.97525700 \\
\hline $\mathrm{H}$ & -4.24334000 & -4.16317300 & -4.64673700 \\
\hline $\mathrm{H}$ & -4.39348800 & 4.75607700 & 1.67158900 \\
\hline $\mathrm{H}$ & -4.81987200 & 6.26841600 & 0.85128300 \\
\hline
\end{tabular}




\begin{tabular}{|c|c|c|c|}
\hline $\mathrm{H}$ & -5.94492300 & 4.89768400 & 0.81133300 \\
\hline$H$ & -2.19551400 & 2.80898400 & -0.56987200 \\
\hline $\mathrm{H}$ & -4.49974500 & -0.72477100 & -0.05120300 \\
\hline $\mathrm{H}$ & -8.12652500 & 3.71281400 & -2.11031100 \\
\hline $\mathrm{H}$ & -6.46252200 & 3.64151800 & -2.75180300 \\
\hline $\mathrm{H}$ & -7.35611400 & 2.13776700 & -2.44721100 \\
\hline $\mathrm{H}$ & -4.57430900 & 6.46444500 & -1.70332500 \\
\hline $\mathrm{H}$ & -4.52071100 & 4.98845100 & -2.67466600 \\
\hline $\mathrm{H}$ & -5.95018700 & 5.36628900 & -1.68144500 \\
\hline $\mathrm{H}$ & -8.54247800 & 1.28994700 & -0.94833500 \\
\hline $\mathrm{H}$ & -7.73585700 & -0.11544600 & -1.68394600 \\
\hline $\mathrm{H}$ & -8.89616200 & -0.33267000 & -0.36640500 \\
\hline $\mathrm{H}$ & -7.62747800 & -1.43639600 & 1.23654900 \\
\hline $\mathrm{H}$ & -6.40314900 & -1.70482100 & -0.00951900 \\
\hline $\mathrm{H}$ & -5.93266400 & -1.07002800 & 1.58088300 \\
\hline $\mathrm{H}$ & -2.19115300 & 4.72354200 & 0.37294100 \\
\hline $\mathrm{H}$ & -2.19789800 & 4.87370500 & -1.39587400 \\
\hline $\mathrm{H}$ & -2.70694900 & 6.23076000 & -0.38522400 \\
\hline $\mathrm{H}$ & -6.11821600 & -3.68324400 & -2.11747200 \\
\hline $\mathrm{H}$ & -6.01713100 & -4.99894000 & -3.29753200 \\
\hline $\mathrm{H}$ & -5.83799200 & -5.35371300 & -1.57900900 \\
\hline $\mathrm{H}$ & -8.39751600 & 0.75497300 & 1.92746500 \\
\hline $\mathrm{H}$ & -6.73848500 & 1.23351100 & 2.33136200 \\
\hline $\mathrm{H}$ & -7.71560000 & 2.24662500 & 1.24859600 \\
\hline $\mathrm{Cu}$ & -0.08903400 & 0.62044000 & 1.10337800 \\
\hline $\mathrm{H}$ & 0.00637600 & 2.24808800 & 1.28742500 \\
\hline $\mathrm{C}$ & -0.48904000 & 1.88761400 & 2.81463300 \\
\hline $\mathrm{H}$ & 0.44354300 & 2.26188300 & 3.24290900 \\
\hline $\mathrm{C}$ & -0.78244300 & 0.51960500 & 3.12352800 \\
\hline $\mathrm{H}$ & -1.84164800 & 0.25108100 & 3.08966000 \\
\hline $\mathrm{C}$ & 0.03456500 & -0.24066900 & 4.15067700 \\
\hline $\mathrm{H}$ & -0.03077000 & -1.32177700 & 3.96540500 \\
\hline $\mathrm{H}$ & 1.09621000 & 0.01713500 & 4.04114300 \\
\hline $\mathrm{C}$ & -1.60225500 & 2.92893000 & 2.80989600 \\
\hline $\mathrm{H}$ & -1.25258200 & 3.85955000 & 2.34738200 \\
\hline $\mathrm{H}$ & -2.44752200 & 2.56949600 & 2.21347600 \\
\hline $\mathrm{C}$ & -2.08612300 & 3.23103600 & 4.22547900 \\
\hline
\end{tabular}




$\begin{array}{lrrr}\mathrm{H} & -1.27680400 & 3.59710800 & 4.86709200 \\ \mathrm{H} & -2.52091100 & 2.35198500 & 4.70796200 \\ \mathrm{O} & -3.09921800 & 4.26155200 & 4.12154900 \\ \mathrm{C} & -3.64447100 & 4.67959300 & 5.28549100 \\ \mathrm{C} & -4.69378500 & 5.74098900 & 5.03872700 \\ \mathrm{H} & -4.26431900 & 6.57662700 & 4.47673100 \\ \mathrm{H} & -5.51006600 & 5.32904900 & 4.43567800 \\ \mathrm{H} & -5.08337900 & 6.09448300 & 5.99416200 \\ \mathrm{O} & -3.32479100 & 4.24889000 & 6.37225900 \\ \mathrm{C} & -0.39589800 & 0.01848900 & 5.60837400 \\ \mathrm{H} & 0.19622600 & -0.57611000 & 6.31734000 \\ \mathrm{H} & -0.27655500 & 1.07566800 & 5.87491200 \\ \mathrm{H} & -1.45180600 & -0.24087600 & 5.75785500\end{array}$

\section{5b-TS}

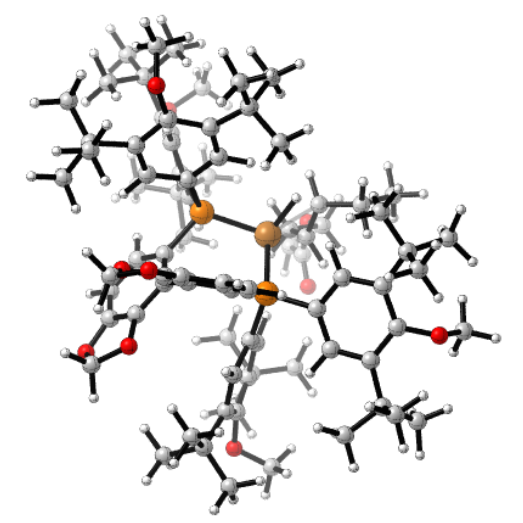

Thermal correction to Enthalpy=

1.923238

Thermal correction to Gibbs Free Energy= 1.672464

Sum of electronic and zero-point Energies $=\quad-4824.277137$

Sum of electronic and thermal Enthalpies $=\quad-4824.170803$

Sum of electronic and thermal Free Energies $=\quad-4824.421577$

SMD E $($ M06 $)=-4824.461300$

Imaginary frequency: -763.31

Cartesian coordinates:

$\begin{array}{llcc}\mathrm{P} & -1.75818400 & -0.55965500 & -0.13880100 \\ \mathrm{P} & 1.78165000 & 0.07722000 & 0.19119300 \\ \mathrm{O} & -0.94078600 & -1.22863200 & 4.60645200 \\ \mathrm{O} & -0.09849400 & 0.49566700 & 5.88153000\end{array}$




\begin{tabular}{|c|c|c|c|}
\hline $\mathrm{O}$ & 0.94578700 & -3.34123700 & 3.54617600 \\
\hline $\mathrm{O}$ & 0.10816800 & -5.38666100 & 2.90028000 \\
\hline $\mathrm{O}$ & 5.12589000 & -4.87004000 & -0.42862700 \\
\hline $\mathrm{O}$ & 6.16782700 & 4.04189700 & -0.96766700 \\
\hline $\mathrm{O}$ & -5.12343900 & 2.98093900 & 3.34128000 \\
\hline $\mathrm{O}$ & -6.09339700 & -2.11806300 & -4.00687800 \\
\hline $\mathrm{C}$ & 1.26144300 & 0.19125200 & 1.97581600 \\
\hline $\mathrm{C}$ & 0.29070200 & -0.71573300 & 2.51719600 \\
\hline $\mathrm{C}$ & -0.05425000 & -0.49403400 & 3.84505000 \\
\hline $\mathrm{C}$ & 0.45681100 & 0.53927600 & 4.62454600 \\
\hline $\mathrm{C}$ & 1.38356300 & 1.42134900 & 4.11793800 \\
\hline $\mathrm{C}$ & 1.77484500 & 1.21557700 & 2.78468700 \\
\hline $\mathrm{C}$ & -0.73439300 & -0.78244700 & 5.94831300 \\
\hline $\mathrm{C}$ & -1.24046700 & -2.05643100 & 0.83488700 \\
\hline $\mathrm{C}$ & -1.73786900 & -3.31537200 & 0.46632800 \\
\hline $\mathrm{C}$ & -1.34717300 & -4.51286000 & 1.08776100 \\
\hline $\mathrm{C}$ & -0.43811400 & -4.39831400 & 2.11510400 \\
\hline $\mathrm{C}$ & 0.06647700 & -3.15865200 & 2.49713400 \\
\hline $\mathrm{C}$ & -0.27698000 & -1.95608300 & 1.89086400 \\
\hline $\mathrm{C}$ & 0.71917400 & -4.68235500 & 3.98324500 \\
\hline $\mathrm{C}$ & 2.66326200 & -1.54693800 & 0.10578200 \\
\hline $\mathrm{C}$ & 2.62357700 & -2.26068900 & -1.08832700 \\
\hline $\mathrm{C}$ & 3.36024200 & -3.44195200 & -1.28893600 \\
\hline $\mathrm{C}$ & 4.22516500 & -3.83094400 & -0.24273400 \\
\hline $\mathrm{C}$ & 4.18929500 & -3.21607900 & 1.04066000 \\
\hline $\mathrm{C}$ & 3.40779500 & -2.06381100 & 1.17514500 \\
\hline $\mathrm{C}$ & 4.89410600 & -3.83638700 & 2.27587500 \\
\hline $\mathrm{C}$ & 6.43584700 & -3.86037400 & 2.14844000 \\
\hline $\mathrm{C}$ & 4.37690000 & -5.28443300 & 2.46704800 \\
\hline $\mathrm{C}$ & 4.56506400 & -3.05039500 & 3.56464700 \\
\hline $\mathrm{C}$ & 3.10337400 & -4.21184400 & -2.61490400 \\
\hline $\mathrm{C}$ & 3.61949100 & -5.66923200 & -2.64875600 \\
\hline $\mathrm{C}$ & 1.56700700 & -4.31133900 & -2.82665600 \\
\hline $\mathrm{C}$ & 3.70745500 & -3.42933700 & -3.80567700 \\
\hline $\mathrm{C}$ & 6.30341700 & -4.49514200 & -1.14266900 \\
\hline $\mathrm{C}$ & 3.19020900 & 1.27763000 & 0.01890700 \\
\hline $\mathrm{C}$ & 2.95526700 & 2.66000600 & 0.10881100 \\
\hline
\end{tabular}




$\begin{array}{llll}\mathrm{C} & 4.48826900 & 0.85209300 & -0.25637100 \\ \mathrm{C} & 5.54704200 & 1.74220200 & -0.51174100 \\ \mathrm{C} & 5.22831800 & 3.11528600 & -0.53877200 \\ \mathrm{C} & 3.96227200 & 3.60467700 & -0.11215200 \\ \mathrm{C} & 3.72489400 & 5.10376500 & 0.21009500 \\ \mathrm{C} & 2.33205000 & 5.33523300 & 0.83505600 \\ \mathrm{C} & 3.81979700 & 6.01686800 & -1.03449800 \\ \mathrm{C} & 6.25568900 & 4.14458400 & -2.38961400 \\ \mathrm{C} & 4.77597100 & 5.55657100 & 1.25563500 \\ \mathrm{C} & 6.95962000 & 1.11216800 & -0.67830500 \\ \mathrm{C} & 7.01595500 & 0.29074200 & -1.98870200 \\ \mathrm{C} & 8.14657500 & 2.10328200 & -0.66109200 \\ \mathrm{C} & 7.20686100 & 0.15537100 & 0.52079300 \\ \mathrm{C} & -2.64773800 & 0.49961300 & 1.08470200 \\ \mathrm{C} & -3.37269900 & -0.01299600 & 2.16974100 \\ \mathrm{C} & -4.15943900 & 0.80853700 & 2.98412900 \\ \mathrm{C} & -4.21317300 & 2.19165000 & 2.65312900 \\ \mathrm{C} & -3.35980400 & 2.77102000 & 1.68839400 \\ \mathrm{C} & -2.62218000 & 1.87857800 & 0.89003200 \\ \mathrm{C} & -3.11971700 & 4.28880900 & 1.44828300 \\ \mathrm{C} & -3.69481000 & 5.24177500 & 2.52164600 \\ \mathrm{C} & -3.67602100 & 4.69801500 & 0.06342500 \\ \mathrm{C} & -1.58659100 & 4.53959400 & 1.46254000 \\ \mathrm{C} & -4.31713300 & 0.98253200 & 5.49083200 \\ \mathrm{C} & -6.30208800 & 3.28132800 & 2.59326300 \\ \mathrm{C} & -4.85487100 & 0.23310100 & 4.24570100 \\ \mathrm{C} & -4.53986000 & -1.26786000 & 4.42946400 \\ \mathrm{C} & -5.12235500 & -1.90983600 & -3.03378500 \\ \mathrm{C} & -5.51461800 & -1.39172900 & -1.77539800 \\ \mathrm{C} & -7.55981300 & -2.81437100 & -1.39684400 \\ \mathrm{C} & -3.13522100 & -1.17022000 & -1.22177000 \\ \mathrm{C} & -2750500000 & -1.83792200 & -2.40852400 \\ \mathrm{C} & -2.27286300 & -3.31779700 \\ \mathrm{C} & -0.99228800 & -4.87616000 \\ \mathrm{C} & -0.41436400 & -2.08185000 \\ \mathrm{C} & -2.65609500 & -5.89203300\end{array}$




\begin{tabular}{|c|c|c|c|}
\hline $\mathrm{C}$ & -3.39552400 & -3.23385200 & -4.47760600 \\
\hline $\mathrm{C}$ & -1.90330800 & -3.62599000 & -4.40602500 \\
\hline $\mathrm{C}$ & -7.08843500 & -0.95821200 & 0.19505400 \\
\hline & -6.39657300 & 0.36189900 & 4.20511700 \\
\hline & -6.99085000 & -1.37703500 & -1.28934200 \\
\hline $\mathrm{C}$ & -4.21971200 & -4.53711500 & -4.32158600 \\
\hline & 1.80422700 & 2.22044300 & 4.71850600 \\
\hline & 2.52937400 & 1.87636300 & 2.37873600 \\
\hline & -1.69755900 & -0.68465400 & 6.45085000 \\
\hline $\mathrm{H}$ & -0.07581700 & -1.49216600 & 6.47152900 \\
\hline & -2.47220200 & -3.37632500 & -0.32709000 \\
\hline & -1.75510400 & -5.47157100 & 0.78648100 \\
\hline & 1.67263500 & -5.14998900 & 4.23123900 \\
\hline 11 & 0.03596100 & -4.67432700 & 4.84627400 \\
\hline T & 1.98433700 & -1.87955000 & -1.87842400 \\
\hline & 3.37692900 & -1.54509100 & 2.12431100 \\
\hline & 6.83153700 & -2.86547400 & 1.91150800 \\
\hline & 6.87835000 & -4.17211800 & 3.10279000 \\
\hline U & 6.77363400 & -4.56351800 & 1.38696900 \\
\hline & 4.64464100 & -5.91787400 & 1.61891500 \\
\hline & 4.81003100 & -5.72290300 & 3.37537800 \\
\hline & 3.28558600 & -5.29548400 & 2.56709000 \\
\hline & 3.48791500 & -2.99302100 & 3.74906000 \\
\hline 11 & 5.02796700 & -3.55802100 & 4.41871300 \\
\hline 11 & 4.96466000 & -2.02975700 & 3.53825900 \\
\hline $\mathrm{H}$ & 3.21373700 & -6.15726300 & -3.54330800 \\
\hline & 4.70560500 & -5.74876600 & -2.70492800 \\
\hline 11 & 3.28517200 & -6.23666700 & -1.77546400 \\
\hline $\mathrm{H}$ & 1.36031200 & -4.88094800 & -3.74055200 \\
\hline U & 1.09107700 & -4.83093800 & -1.98712800 \\
\hline & 1.08642800 & -3.33557700 & -2.93332000 \\
\hline & 3.29517600 & -2.41614900 & -3.86657000 \\
\hline $\mathrm{H}$ & 4.79703100 & -3.34147400 & -3.72429900 \\
\hline & 3.48361000 & -3.94153900 & -4.74984100 \\
\hline & 6.82638300 & -3.66973900 & -0.64510600 \\
\hline & 6.94889100 & -5.37737500 & -1.15773500 \\
\hline & 6.07820200 & -4.19710000 & -2.17245800 \\
\hline
\end{tabular}




\begin{tabular}{|c|c|c|c|}
\hline I & 1.95858300 & 2.99876300 & 0.36212100 \\
\hline $\mathrm{H}$ & 4.69089700 & -0.20989100 & -0.27996700 \\
\hline $\mathrm{H}$ & 2.23729000 & 6.39059800 & 1.11431600 \\
\hline $\mathrm{H}$ & 1.51921600 & 5.10649700 & 0.13802400 \\
\hline H & 2.18206700 & 4.74173500 & 1.74372100 \\
\hline $\mathrm{H}$ & 4.83330100 & 6.05780600 & -1.43349400 \\
\hline $\mathrm{H}$ & 3.13557300 & 5.68911000 & -1.82540300 \\
\hline $\mathrm{H}$ & 3.54031700 & 7.04063000 & -0.75381600 \\
\hline 11 & 6.57633500 & 3.20088400 & -2.84518100 \\
\hline $\mathrm{H}$ & 5.29426100 & 4.43533000 & -2.82873600 \\
\hline $\mathrm{H}$ & 7.00044200 & 4.91719900 & -2.59795200 \\
\hline $\mathrm{H}$ & 5.79249800 & 5.45533900 & 0.87007600 \\
\hline 11 & 4.61056500 & 6.60884900 & 1.51926300 \\
\hline $\mathrm{H}$ & 4.69158300 & 4.96410800 & 2.17445300 \\
\hline $\mathrm{H}$ & 6.24772100 & -0.49000400 & -2.00994300 \\
\hline $\mathrm{H}$ & 6.86505400 & 0.92750800 & -2.86808600 \\
\hline $\mathrm{H}$ & 7.99477900 & -0.19458100 & -2.09106200 \\
\hline $\mathrm{H}$ & 9.07624900 & 1.52405900 & -0.60064500 \\
\hline $\mathrm{H}$ & 8.21126700 & 2.71853800 & -1.55919600 \\
\hline $\mathrm{H}$ & 8.10828500 & 2.77082300 & 0.20381900 \\
\hline $\mathrm{H}$ & 7.17380100 & 0.70462600 & 1.46867200 \\
\hline $\mathrm{H}$ & 6.47709400 & -0.65652400 & 0.57914900 \\
\hline $\mathrm{H}$ & 8.20019300 & -0.30005000 & 0.42915100 \\
\hline $\mathrm{H}$ & -3.32231800 & -1.07580400 & 2.36842300 \\
\hline $\mathrm{H}$ & -1.99958400 & 2.26456200 & 0.09014400 \\
\hline $\mathrm{H}$ & -4.78253100 & 5.31838000 & 2.50044100 \\
\hline $\mathrm{H}$ & -3.39532400 & 4.94815600 & 3.53168600 \\
\hline $\mathrm{H}$ & -3.29937300 & 6.24664600 & 2.33033300 \\
\hline $\mathrm{H}$ & -3.44184500 & 5.74679100 & -0.14825000 \\
\hline $\mathrm{H}$ & -3.23455900 & 4.10237700 & -0.74167600 \\
\hline $\mathrm{H}$ & -4.76452500 & 4.57179700 & 0.01693300 \\
\hline $\mathrm{H}$ & -1.38699100 & 5.59679800 & 1.25558400 \\
\hline $\mathrm{H}$ & -1.16179700 & 4.29090300 & 2.44220700 \\
\hline $\mathrm{H}$ & -1.05439700 & 3.95588800 & 0.70878300 \\
\hline $\mathrm{H}$ & -4.75946000 & 0.56373300 & 6.40409500 \\
\hline $\mathrm{H}$ & -3.22749800 & 0.88735600 & 5.56275100 \\
\hline $\mathrm{H}$ & -4.56152700 & 2.04618700 & 5.44905100 \\
\hline
\end{tabular}




\begin{tabular}{|c|c|c|c|}
\hline $\mathrm{H}$ & -6.07304800 & 3.84164000 & 1.68047500 \\
\hline $\mathrm{H}$ & -6.84223000 & 2.36768900 & 2.31739100 \\
\hline $\mathrm{H}$ & -6.93258100 & 3.89345300 & 3.24354200 \\
\hline $\mathrm{H}$ & -4.95513900 & -1.87722200 & 3.61781500 \\
\hline $\mathrm{H}$ & -3.46468400 & -1.46207000 & 4.48754800 \\
\hline $\mathrm{H}$ & -4.99744500 & -1.61580600 & 5.36277000 \\
\hline $\mathrm{H}$ & -6.97619100 & -3.51156800 & -0.78449200 \\
\hline $\mathrm{H}$ & -8.59585500 & -2.83551500 & -1.03604000 \\
\hline $\mathrm{H}$ & -7.54919600 & -3.16850600 & -2.42993100 \\
\hline $\mathrm{H}$ & -4.73837200 & -0.57072900 & 0.05273500 \\
\hline $\mathrm{H}$ & -1.75767800 & -2.01768900 & -2.61537700 \\
\hline $\mathrm{H}$ & -7.05581300 & -1.27767600 & -5.58619400 \\
\hline $\mathrm{H}$ & -6.59238600 & -0.10463100 & -4.32187800 \\
\hline $\mathrm{H}$ & -5.35734700 & -0.75238600 & -5.42019700 \\
\hline $\mathrm{H}$ & -8.86722500 & -0.31204600 & -1.56210200 \\
\hline $\mathrm{H}$ & -7.46576200 & 0.58641900 & -2.16149500 \\
\hline $\mathrm{H}$ & -8.12169400 & -0.78711300 & -3.08403700 \\
\hline $\mathrm{H}$ & -4.69945100 & -2.60166300 & -6.13577000 \\
\hline $\mathrm{H}$ & -3.19571100 & -1.65870400 & -6.00155500 \\
\hline $\mathrm{H}$ & -3.16750100 & -3.30873900 & -6.63768800 \\
\hline $\mathrm{H}$ & -1.69336600 & -4.36779000 & -5.18482500 \\
\hline $\mathrm{H}$ & -1.23941700 & -2.77193000 & -4.57852200 \\
\hline $\mathrm{H}$ & -1.63862500 & -4.07551400 & -3.44323300 \\
\hline $\mathrm{H}$ & -6.49175900 & -1.60501400 & 0.84708600 \\
\hline $\mathrm{H}$ & -6.77082200 & 0.07835200 & 0.35722100 \\
\hline $\mathrm{H}$ & -8.13250000 & -1.03678100 & 0.51804000 \\
\hline $\mathrm{H}$ & -6.80645400 & -0.05216700 & 3.27618000 \\
\hline $\mathrm{H}$ & -6.83344100 & -0.20141500 & 5.03908800 \\
\hline $\mathrm{H}$ & -6.72238900 & 1.39721000 & 4.30333700 \\
\hline $\mathrm{H}$ & -3.95066900 & -5.24710000 & -5.11382900 \\
\hline $\mathrm{H}$ & -4.01325600 & -5.01657500 & -3.35743000 \\
\hline $\mathrm{H}$ & -5.29186700 & -4.33952700 & -4.38720000 \\
\hline $\mathrm{Cu}$ & 0.02187200 & 0.33607300 & -1.27853000 \\
\hline $\mathrm{H}$ & 0.42997700 & -0.44221900 & -2.65782500 \\
\hline $\mathrm{C}$ & 0.09329300 & 1.05449000 & -3.33019800 \\
\hline $\mathrm{H}$ & 1.13718600 & 1.20521300 & -3.61685200 \\
\hline $\mathrm{C}$ & -0.44214300 & 2.06362400 & -2.46772500 \\
\hline
\end{tabular}




$\begin{array}{lrrr}\mathrm{H} & -1.53191700 & 2.14575600 & -2.47132800 \\ \mathrm{C} & 0.27719800 & 3.37863700 & -2.23289300 \\ \mathrm{H} & -0.02441800 & 3.83019400 & -1.28041000 \\ \mathrm{H} & 1.36271300 & 3.22858500 & -2.18484800 \\ \mathrm{C} & -0.74838900 & 0.46087100 & -4.45494000 \\ \mathrm{H} & -0.28722300 & -0.47047300 & -4.80776200 \\ \mathrm{H} & -1.73678000 & 0.19303100 & -4.06469900 \\ \mathrm{C} & -0.04305100 & 4.38733800 & -3.34394700 \\ \mathrm{H} & -1.12375500 & 4.50583600 & -3.45332800 \\ \mathrm{H} & 0.38687800 & 4.07291600 & -4.29821800 \\ \mathrm{O} & 0.53726800 & 5.69953400 & -3.09813700 \\ \mathrm{C} & -0.21807800 & 6.58067400 & -2.40691700 \\ \mathrm{C} & 0.47636000 & 7.92068000 & -2.30267100 \\ \mathrm{H} & 1.47371200 & 7.80280600 & -1.86886400 \\ \mathrm{H} & 0.60417200 & 8.35124600 & -3.30194500 \\ \mathrm{H} & -0.12302900 & 8.59329600 & -1.68774800 \\ \mathrm{O} & -1.31501600 & 6.33180800 & -1.95022200 \\ \mathrm{C} & -0.90806300 & 1.43139700 & -5.63661400 \\ \mathrm{H} & -1.43210700 & 2.34473100 & -5.33621000 \\ \mathrm{H} & 0.06760800 & 1.72374600 & -6.04611700 \\ \mathrm{H} & -1.48012400 & 0.96459500 & -6.44802000\end{array}$

\section{5c-TS}

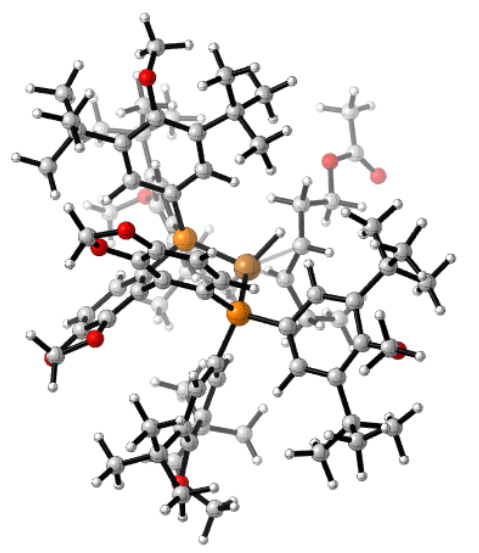

Thermal correction to Enthalpy= 1.922511

Thermal correction to Gibbs Free Energy $=\quad 1.665107$

Sum of electronic and zero-point Energies $=\quad-4824.278911$

Sum of electronic and thermal Enthalpies $=\quad-4824.171633$ 
Sum of electronic and thermal Free Energies $=\quad-4824.429037$

SMD $\mathrm{E}(\mathrm{M} 06)=\quad-4824.460361$

Imaginary frequency: -798.60

Cartesian coordinates:

\begin{tabular}{|c|c|c|c|}
\hline$P$ & -1.92835100 & -0.31659200 & 8300 \\
\hline $\mathrm{P}$ & 1.59007000 & 0.49153900 & 0.07137800 \\
\hline $\mathrm{O}$ & -1.64682700 & 3.74203600 & 2.81675800 \\
\hline $\mathrm{O}$ & -0.91042100 & 5.67199600 & 1.79728500 \\
\hline $\mathrm{O}$ & 0.27426300 & 2.01357000 & 4.45513900 \\
\hline $\mathrm{O}$ & -0.60971500 & 0.51774300 & 5.97076100 \\
\hline $\mathrm{O}$ & 4.83805900 & -1.59931300 & 4.66604300 \\
\hline $\mathrm{O}$ & 6.06932900 & 2.27886400 & -3.55401100 \\
\hline $\mathrm{O}$ & -5.35195600 & 4.21090600 & -1.77218700 \\
\hline $\mathrm{O}$ & -6.00069000 & -4.74870600 & 0.38984700 \\
\hline $\mathrm{C}$ & 0.84866500 & 2.11468800 & 0.60097600 \\
\hline $\mathrm{C}$ & -0.18951600 & 2.15550000 & 1.58855800 \\
\hline $\mathrm{C}$ & -0.66975600 & 3.42268600 & 1.89506200 \\
\hline $\mathrm{C}$ & -0.22740000 & 4.59362400 & 1.28615200 \\
\hline $\mathrm{C}$ & 0.75109500 & 4.56812700 & 0.31801600 \\
\hline $\mathrm{C}$ & 1.28039900 & 3.30578000 & -0.00016700 \\
\hline $\mathrm{C}$ & -1.56226700 & 5.16065300 & 2.96238500 \\
\hline $\mathrm{C}$ & -1.59293500 & -0.03596600 & 1.98524900 \\
\hline $\mathrm{C}$ & -2.11490400 & -0.93180600 & 2.92881900 \\
\hline $\mathrm{C}$ & -1.84440600 & -0.84516000 & 4.30496200 \\
\hline $\mathrm{C}$ & -1.02881900 & 0.18975800 & 4.70368700 \\
\hline $\mathrm{C}$ & -0.49855500 & 1.08882900 & 3.78212900 \\
\hline $\mathrm{C}$ & -0.73195300 & 1.02652000 & 2.41426700 \\
\hline $\mathrm{C}$ & -0.03762900 & 1.82063500 & 5.83619300 \\
\hline $\mathrm{C}$ & 2.45467500 & -0.09427300 & 1.59611800 \\
\hline $\mathrm{C}$ & 2.46384900 & -1.45972100 & 1.86548700 \\
\hline $\mathrm{C}$ & 3.16488400 & -2.01142600 & 2.95230900 \\
\hline $\mathrm{C}$ & 3.95310800 & -1.11784200 & 3.71158900 \\
\hline $\mathrm{C}$ & 3.85315900 & 0.29450600 & 3.56497200 \\
\hline $\mathrm{C}$ & 3.10190000 & 0.77242400 & 2.48711000 \\
\hline $\mathrm{C}$ & 4.47384400 & 1.28284300 & 4.58674200 \\
\hline $\mathrm{C}$ & 6.02051200 & 1.26602300 & 4.56561600 \\
\hline $\mathrm{C}$ & 3.97883500 & 0.92804100 & 6.01093400 \\
\hline
\end{tabular}




\begin{tabular}{|c|c|c|c|}
\hline $\mathrm{C}$ & 4.03311300 & 2.73611300 & 4.30053000 \\
\hline $\mathrm{C}$ & 2.95474800 & -3.52697700 & 3.22887200 \\
\hline $\mathrm{C}$ & 3.40861600 & -4.01228400 & 4.62509300 \\
\hline $\mathrm{C}$ & 1.43054400 & -3.82531100 & 3.16060700 \\
\hline $\mathrm{C}$ & 3.66285300 & -4.36903800 & 2.14070800 \\
\hline $\mathrm{C}$ & 6.05998500 & -2.10033800 & 4.12428300 \\
\hline $\mathrm{C}$ & 2.99522500 & 0.97250900 & -1.04248700 \\
\hline $\mathrm{C}$ & 4.33787300 & 0.83288400 & -0.67490400 \\
\hline $\mathrm{C}$ & 2.70924600 & 1.38622800 & -2.34727800 \\
\hline $\mathrm{C}$ & 3.70517300 & 1.77695900 & -3.25834100 \\
\hline $\mathrm{C}$ & 5.03865800 & 1.76399200 & -2.78095600 \\
\hline $\mathrm{C}$ & 5.38652300 & 1.18761600 & -1.53155300 \\
\hline $\mathrm{C}$ & 6.85379700 & 0.87318800 & -1.13697500 \\
\hline $\mathrm{C}$ & 6.92745700 & 0.09584600 & 0.19620200 \\
\hline $\mathrm{C}$ & 7.71454500 & 2.14678000 & -0.95925600 \\
\hline $\mathrm{C}$ & 6.20008300 & 3.69872700 & -3.49415000 \\
\hline $\mathrm{C}$ & 7.48860800 & -0.02939700 & -2.22465000 \\
\hline $\mathrm{C}$ & 3.25409000 & 2.15322700 & -4.70017200 \\
\hline $\mathrm{C}$ & 2.60891000 & 3.56060900 & -4.70004300 \\
\hline $\mathrm{C}$ & 4.37241900 & 2.10062900 & -5.76846500 \\
\hline $\mathrm{C}$ & 2.18254100 & 1.13866000 & -5.18300400 \\
\hline $\mathrm{C}$ & -2.87566800 & 1.18412200 & -0.32930300 \\
\hline $\mathrm{C}$ & -3.78959700 & 1.82580300 & 0.51677300 \\
\hline $\mathrm{C}$ & -4.58499200 & 2.88726500 & 0.07512300 \\
\hline $\mathrm{C}$ & -4.44881500 & 3.27671300 & -1.28642400 \\
\hline $\mathrm{C}$ & -3.41616600 & 2.78033800 & -2.11415900 \\
\hline $\mathrm{C}$ & -2.67592800 & 1.69588900 & -1.60937100 \\
\hline $\mathrm{C}$ & -2.99437700 & 3.34993600 & -3.49860800 \\
\hline $\mathrm{C}$ & -3.51409300 & 4.77390500 & -3.80667200 \\
\hline $\mathrm{C}$ & -3.43323000 & 2.38985500 & -4.63013400 \\
\hline $\mathrm{C}$ & -1.44463600 & 3.46113400 & -3.53238600 \\
\hline $\mathrm{C}$ & -5.11716500 & 5.14936500 & 1.06298700 \\
\hline $\mathrm{C}$ & -6.39191600 & 3.65666700 & -2.57819500 \\
\hline $\mathrm{C}$ & -5.50103200 & 3.64828200 & 1.06888800 \\
\hline $\mathrm{C}$ & -5.31466400 & 3.13073700 & 2.51283200 \\
\hline $\mathrm{C}$ & -5.05930100 & -3.74246400 & 0.20054800 \\
\hline $\mathrm{C}$ & -3.68077700 & -4.05901900 & 0.31719300 \\
\hline
\end{tabular}




\begin{tabular}{|c|c|c|c|}
\hline $\mathrm{C}$ & -3.63593100 & -6.19477900 & -1.00944400 \\
\hline $\mathrm{C}$ & -2.78366600 & -2.98265500 & 0.29811000 \\
\hline $\mathrm{C}$ & -3.20915900 & -1.65561300 & 0.14797200 \\
\hline $\mathrm{C}$ & -4.55624300 & -1.41847300 & -0.13605900 \\
\hline $\mathrm{C}$ & -5.50703600 & -2.44986900 & -0.16724900 \\
\hline $\mathrm{C}$ & -6.47243200 & -4.83809100 & 1.73999400 \\
\hline $\mathrm{C}$ & -3.59402200 & -6.37538500 & 1.50173100 \\
\hline $\mathrm{C}$ & -8.07415600 & -2.38220800 & 0.28216000 \\
\hline $\mathrm{C}$ & -6.92791300 & -2.17552400 & -0.73580800 \\
\hline $\mathrm{C}$ & -7.05359300 & -0.72471400 & -1.25404300 \\
\hline $\mathrm{C}$ & -1.60079000 & -5.55163700 & 0.28163000 \\
\hline $\mathrm{C}$ & -7.00358000 & 3.50461000 & 0.72947600 \\
\hline $\mathrm{C}$ & -3.14515000 & -5.51760900 & 0.29520000 \\
\hline $\mathrm{C}$ & -7.15817900 & -3.11462000 & -1.94707000 \\
\hline $\mathrm{H}$ & 1.11017500 & 5.47372700 & -0.15879000 \\
\hline $\mathrm{H}$ & 2.07288800 & 3.26440700 & -0.73666700 \\
\hline $\mathrm{H}$ & -2.56661900 & 5.57984000 & 3.03204000 \\
\hline $\mathrm{H}$ & -0.96132800 & 5.40131100 & 3.85257600 \\
\hline $\mathrm{H}$ & -2.76627100 & -1.72850700 & 2.59122900 \\
\hline $\mathrm{H}$ & -2.26943400 & -1.54590800 & 5.01538400 \\
\hline $\mathrm{H}$ & 0.87708500 & 1.88016400 & 6.42772900 \\
\hline $\mathrm{H}$ & -0.77122600 & 2.57701000 & 6.15431400 \\
\hline $\mathrm{H}$ & 1.88425000 & -2.10249500 & 1.21017500 \\
\hline $\mathrm{H}$ & 3.01645600 & 1.83879300 & 2.32308000 \\
\hline $\mathrm{H}$ & 6.40567800 & 1.42943600 & 3.55172600 \\
\hline $\mathrm{H}$ & 6.40817000 & 2.07208500 & 5.20113300 \\
\hline $\mathrm{H}$ & 6.42458400 & 0.32687500 & 4.94485500 \\
\hline $\mathrm{H}$ & 4.30401800 & -0.06897800 & 6.31266900 \\
\hline $\mathrm{H}$ & 4.36758500 & 1.65429800 & 6.73598600 \\
\hline $\mathrm{H}$ & 2.88420200 & 0.95707200 & 6.05416900 \\
\hline $\mathrm{H}$ & 2.94293400 & 2.83965800 & 4.29263700 \\
\hline $\mathrm{H}$ & 4.42832800 & 3.38976800 & 5.08654300 \\
\hline $\mathrm{H}$ & 4.42106200 & 3.10799400 & 3.34498400 \\
\hline $\mathrm{H}$ & 3.03846600 & -5.03426800 & 4.77205500 \\
\hline $\mathrm{H}$ & 4.49167400 & -4.04154100 & 4.74793100 \\
\hline $\mathrm{H}$ & 2.99654900 & -3.38772100 & 5.42319000 \\
\hline $\mathrm{H}$ & 1.25610400 & -4.88300300 & 3.39187400 \\
\hline
\end{tabular}




\begin{tabular}{|c|c|c|c|}
\hline I & 0.88094900 & -3.22168800 & 3.89169300 \\
\hline $\mathrm{H}$ & 1.00162400 & -3.62951400 & 2.17450600 \\
\hline $\mathrm{H}$ & 3.30275800 & -4.11046300 & 1.13884500 \\
\hline $\mathrm{H}$ & 4.74849500 & -4.21963300 & 2.15222200 \\
\hline $\mathrm{H}$ & 3.47136600 & -5.43722200 & 2.30251600 \\
\hline $\mathrm{H}$ & 6.56018700 & -1.34679900 & 3.50446000 \\
\hline $\mathrm{H}$ & 6.69574900 & -2.35010400 & 4.97788900 \\
\hline $\mathrm{H}$ & 5.90000200 & -2.99998800 & 3.52028900 \\
\hline 11 & 4.56515800 & 0.43665300 & 0.30357700 \\
\hline $\mathrm{H}$ & 1.67113300 & 1.39790100 & -2.65856900 \\
\hline $\mathrm{H}$ & 7.97320500 & -0.16072800 & 0.39938100 \\
\hline $\mathrm{H}$ & 6.56402500 & 0.68687500 & 1.04502200 \\
\hline $\mathrm{H}$ & 6.35796600 & -0.83936300 & 0.16201100 \\
\hline $\mathrm{H}$ & 7.90734200 & 2.64605400 & -1.90901300 \\
\hline $\mathrm{H}$ & 7.23502000 & 2.85954000 & -0.27742500 \\
\hline $\mathrm{H}$ & 8.68657200 & 1.87785600 & -0.52734000 \\
\hline $\mathrm{H}$ & 5.36984800 & 4.20678600 & -3.99654600 \\
\hline $\mathrm{H}$ & 6.25417200 & 4.05234400 & -2.45802000 \\
\hline $\mathrm{H}$ & 7.13191900 & 3.94437300 & -4.01051900 \\
\hline $\mathrm{H}$ & 7.49946200 & 0.46588300 & -3.19739800 \\
\hline $\mathrm{H}$ & 8.52237800 & -0.27544500 & -1.95045700 \\
\hline $\mathrm{H}$ & 6.93307000 & -0.96986700 & -2.31942300 \\
\hline $\mathrm{H}$ & 1.74799800 & 3.60351600 & -4.02345400 \\
\hline $\mathrm{H}$ & 3.31586100 & 4.33660700 & -4.38706200 \\
\hline $\mathrm{H}$ & 2.25753800 & 3.81683200 & -5.70743500 \\
\hline $\mathrm{H}$ & 3.90881000 & 2.18322600 & -6.75877000 \\
\hline $\mathrm{H}$ & 5.09954800 & 2.90782100 & -5.68480400 \\
\hline $\mathrm{H}$ & 4.91838100 & 1.15312000 & -5.73194900 \\
\hline $\mathrm{H}$ & 2.57175900 & 0.11478900 & -5.16413100 \\
\hline $\mathrm{H}$ & 1.26608500 & 1.16251800 & -4.58867200 \\
\hline $\mathrm{H}$ & 1.90021000 & 1.37306500 & -6.21572100 \\
\hline $\mathrm{H}$ & -3.87698600 & 1.47701400 & 1.53756400 \\
\hline $\mathrm{H}$ & -1.90671800 & 1.23668900 & -2.22012400 \\
\hline $\mathrm{H}$ & -4.58548500 & 4.81485000 & -4.00193700 \\
\hline $\mathrm{H}$ & -3.29501300 & 5.46809500 & -2.98973900 \\
\hline $\mathrm{H}$ & -3.00562000 & 5.14071200 & -4.70654000 \\
\hline $\mathrm{H}$ & -3.07828200 & 2.75958700 & -5.60026200 \\
\hline
\end{tabular}




\begin{tabular}{|c|c|c|c|}
\hline 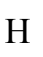 & -3.02323700 & 1.38494800 & -4.48235200 \\
\hline $\mathrm{H}$ & -4.52337200 & 2.29769800 & -4.68816100 \\
\hline & -1.13254100 & 3.91975300 & -4.47780500 \\
\hline & -1.07747500 & 4.08622000 & -2.71066900 \\
\hline & -0.94577600 & 2.49084700 & -3.46411400 \\
\hline $\mathrm{H}$ & -5.71359200 & 5.69308600 & 1.80670400 \\
\hline $\mathrm{H}$ & -4.05800000 & 5.27804200 & 1.31329800 \\
\hline & -5.29013400 & 5.60252500 & 0.08521900 \\
\hline & -6.00947800 & 3.26897100 & -3.52847800 \\
\hline $\mathrm{H}$ & -6.91529900 & 2.84830700 & -2.05422900 \\
\hline $\mathrm{H}$ & -7.09159300 & 4.47194200 & -2.78067100 \\
\hline & -5.64594700 & 2.09169500 & 2.62614500 \\
\hline 11 & -4.27287400 & 3.19674100 & 2.84272300 \\
\hline $\mathrm{H}$ & -5.92310500 & 3.74024300 & 3.19074900 \\
\hline $\mathrm{H}$ & -3.27456700 & -5.65185200 & -1.89046100 \\
\hline $\mathrm{H}$ & -3.25387000 & -7.22192600 & -1.06655500 \\
\hline $\mathrm{H}$ & -4.72711300 & -6.23240900 & -1.04962300 \\
\hline $\mathrm{H}$ & -1.71944800 & -3.16106700 & 0.39322500 \\
\hline $\mathrm{H}$ & -4.86759500 & -0.40691200 & -0.35216400 \\
\hline $\mathrm{H}$ & -7.23382000 & -5.62261000 & 1.74675400 \\
\hline $\mathrm{H}$ & -5.66779500 & -5.10703600 & 2.43037600 \\
\hline $\mathrm{H}$ & -6.91653300 & -3.89601400 & 2.07365800 \\
\hline $\mathrm{H}$ & -3.02914600 & -7.31539200 & 1.51027700 \\
\hline$\Pi$ & -3.39977400 & -5.86637100 & 2.45346500 \\
\hline $\mathrm{H}$ & -4.65158300 & -6.63503300 & 1.44728400 \\
\hline $\mathrm{H}$ & -8.23867400 & -3.43695400 & 0.50512200 \\
\hline $\mathrm{H}$ & -7.88378000 & -1.84591500 & 1.21955600 \\
\hline $\mathrm{H}$ & -9.00929000 & -1.99086600 & -0.13694000 \\
\hline $\mathrm{H}$ & -8.03637100 & -0.60007400 & -1.72237900 \\
\hline $\mathrm{H}$ & -6.97853100 & 0.01343900 & -0.44666000 \\
\hline $\mathrm{H}$ & -6.29503600 & -0.48717100 & -2.00732500 \\
\hline $\mathrm{H}$ & -1.17914100 & -5.00719900 & -0.56907200 \\
\hline $\mathrm{H}$ & -1.16726900 & -5.13755600 & 1.19922900 \\
\hline $\mathrm{H}$ & -1.27033700 & -6.59332200 & 0.20224100 \\
\hline $\mathrm{H}$ & -7.28978400 & 2.45058800 & 0.62820100 \\
\hline $\mathrm{H}$ & -7.60722100 & 3.93501300 & 1.53826000 \\
\hline $\mathrm{H}$ & -7.26771300 & 4.02797800 & -0.1897590 \\
\hline
\end{tabular}




$\begin{array}{lrrr}\mathrm{H} & -8.14751800 & -2.92769800 & -2.38380500 \\ \mathrm{H} & -6.40722300 & -2.93774100 & -2.72566000 \\ \mathrm{H} & -7.10773600 & -4.16425800 & -1.64946100 \\ \mathrm{Cu} & 0.01768100 & -0.89110300 & -0.85349200 \\ \mathrm{H} & 0.35308600 & -2.46258100 & -0.50270700 \\ \mathrm{C} & 0.44544600 & -2.57715000 & -2.13264500 \\ \mathrm{C} & 0.08864600 & -1.42586900 & -2.90983400 \\ \mathrm{H} & 0.92524300 & -0.88730900 & -3.35581100 \\ \mathrm{H} & -0.30466600 & -3.37080300 & -2.11267700 \\ \mathrm{C} & -1.20103500 & -1.40615300 & -3.71339500 \\ \mathrm{H} & -2.00420000 & -1.88161300 & -3.13265200 \\ \mathrm{H} & -1.53242900 & -0.37173700 & -3.88328200 \\ \mathrm{C} & 1.87536600 & -3.10504400 & -2.16843100 \\ \mathrm{H} & 2.58037800 & -2.27342200 & -2.05588300 \\ \mathrm{H} & 2.05341000 & -3.79336500 & -1.33352400 \\ \mathrm{C} & 2.16481400 & -3.83061000 & -3.47969900 \\ \mathrm{H} & 2.07790600 & -3.16710300 & -4.34424000 \\ \mathrm{H} & 1.48370400 & -4.67431700 & -3.63833000 \\ \mathrm{O} & 3.52014200 & -4.33310700 & -3.40246500 \\ \mathrm{C} & 3.95643900 & -5.03455900 & -4.47064800 \\ \mathrm{O} & 3.28310500 & -5.25889700 & -5.45330300 \\ \mathrm{C} & 5.38489300 & -5.49035700 & -4.26633500 \\ \mathrm{H} & 5.70557900 & -6.07734200 & -5.12780400 \\ \mathrm{H} & 6.04221600 & -4.62292500 & -4.14336600 \\ \mathrm{H} & 5.46440400 & -6.09113100 & -3.35433100 \\ \mathrm{C} & -1.09441100 & -2.10531200 & -5.08311900 \\ \mathrm{H} & -0.83584600 & -3.16502400 & -4.96749300 \\ \mathrm{H} & -2.03892800 & -2.04838200 & -5.64117500 \\ \mathrm{H} & -0.31369500 & -1.64227800 & -5.69978100\end{array}$

\section{5d-TS}




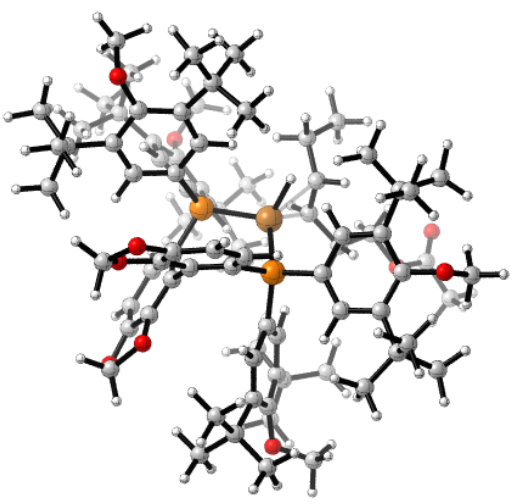

Thermal correction to Enthalpy=

1.922495

Thermal correction to Gibbs Free Energy=

1.665022

Sum of electronic and zero-point Energies $=$ $-4824.282307$

Sum of electronic and thermal Enthalpies= $-4824.175208$

Sum of electronic and thermal Free Energies= $-4824.432682$

SMD E $($ M06 $)=-4824.463088$

Imaginary frequency: -796.89

Cartesian coordinates:

$\mathrm{P}$

$\begin{array}{lll}1.58034000 & -0.62818600 & 0.18318900\end{array}$

$\begin{array}{llll}\mathrm{P} & -1.90201200 & 0.24371000 & -0.23231900\end{array}$

$\begin{array}{llll}\mathrm{O} & 0.31570400 & -2.96785500 & -3.88564100\end{array}$

$\begin{array}{llll}\mathrm{O} & -0.40195600 & -1.73601000 & -5.69448800\end{array}$

O $\quad-1.69459500 \quad-4.25458200 \quad-1.99370900$

$\begin{array}{llll}\mathrm{O} & -1.05427000 & -5.96322800 & -0.58704700\end{array}$

$\begin{array}{llll}\mathrm{O} & -5.78929500 & -3.59915300 & 2.24528000\end{array}$

$\begin{array}{llll}\mathrm{O} & -5.53776200 & 5.01635500 & -0.66498100\end{array}$

$\begin{array}{llll}\mathrm{O} & 5.10302700 & 0.51140900 & -4.54204300\end{array}$

$\begin{array}{lllll}\mathrm{O} & 5.94757400 & -0.97938500 & 4.30176100\end{array}$

C $\quad-1.47407700 \quad-0.36557800 \quad-1.93511200$

$\begin{array}{llll}\text { C } & -0.66912900 & -1.53828700 & -2.11464500\end{array}$

C $\quad-0.40515000 \quad-1.88048100 \quad-3.43478400$

$\begin{array}{lllll}\text { C } & -0.83780100 & -1.14241100 & -4.53339800\end{array}$

$\begin{array}{llll}\text { C } & -1.58519200 & 0.00276900 & -4.37537600\end{array}$

$\begin{array}{lllll}\text { C } & -1.89795100 & 0.36505300 & -3.05439200\end{array}$

$\begin{array}{llll}\text { C } & 0.04391800 & -3.03137400 & -5.28691000\end{array}$

$\begin{array}{lllll}\text { C } & 0.80981600 & -2.30058800 & -0.07184800\end{array}$

$\begin{array}{llll}\text { C } & 1.17703400 & -3.35694200 & 0.77390100\end{array}$ 


$\begin{array}{llll}\mathrm{C} & 0.60719400 & -4.63907200 & 0.69677600 \\ \mathrm{C} & -0.34501900 & -4.82623400 & -0.27991400 \\ \mathrm{C} & -0.72755300 & -3.78842800 & -1.12591200 \\ \mathrm{C} & -0.20422100 & -2.50310300 & -1.06421400 \\ \mathrm{C} & -1.66349100 & -5.67563700 & -1.84752200 \\ \mathrm{C} & -2.98895200 & -1.08207600 & 0.45600700 \\ \mathrm{C} & -2.93147800 & -1.32834200 & 1.82499700 \\ \mathrm{C} & -3.78537100 & -2.24372600 & 2.46518000 \\ \mathrm{C} & -4.78222900 & -2.84219400 & 1.66317100 \\ \mathrm{C} & -4.77940100 & -2.73664400 & 0.24403700 \\ \mathrm{C} & -3.87426600 & -1.83611000 & -0.32684200 \\ \mathrm{C} & -5.66283300 & -3.64024000 & -0.65530300 \\ \mathrm{C} & -7.17581300 & -3.36489200 & -0.48429100 \\ \mathrm{C} & -5.37258700 & -5.12320600 & -0.31390600 \\ \mathrm{C} & -5.33596100 & -3.43756700 & -2.15174900 \\ \mathrm{C} & -3.50603000 & -2.53320500 & 3.96709900 \\ \mathrm{C} & -4.18920000 & -3.79782600 & 4.53742000 \\ \mathrm{C} & -1.97977400 & -2.77238700 & 4.13867200 \\ \mathrm{C} & -3.90505600 & -1.30898100 & 4.82571200 \\ \mathrm{C} & -6.83761300 & -2.82093200 & 2.82193300 \\ \mathrm{C} & -3.08771000 & 1.63747300 & -0.52571600 \\ \mathrm{C} & -2.56567300 & 2.89477500 & -0.86780900 \\ \mathrm{C} & -4.46895900 & 1.50998200 & -0.39859500 \\ \mathrm{C} & -5.34825100 & 2.59975000 & -0.53179000 \\ \mathrm{C} & -4.76001700 & 3.86942100 & -0.72200400 \\ \mathrm{C} & -3.37457800 & 4.02647800 & -1.00410900 \\ \mathrm{C} & -2.77884400 & 5.36120900 & -1.52276700 \\ \mathrm{C} & -1.29162100 & 5.20497400 & -1.90914400 \\ \mathrm{C} & -2.85207800 & 6.49833400 & -0.47572400 \\ \mathrm{C} & -3.52464700 & 5.45351200 & 0.66430800 \\ \mathrm{C} & -6.87042200 & 2.27884000 & -0.50359500 \\ \mathrm{C} & -7.80079100 & 3.41171400 & -0.99638300 \\ \mathrm{C} & -13609100 & 1.08612400 & -1.46399500 \\ \mathrm{C} & -0.33605400 & -1.38277900 \\ \mathrm{C} & -1.36942600 & -2.13747900\end{array}$




\begin{tabular}{|c|c|c|c|}
\hline C & 3.91040200 & -1.11242500 & -3.23997700 \\
\hline $\mathrm{C}$ & 4.16031500 & 0.25105500 & -3.55812200 \\
\hline $\mathrm{C}$ & 3.45742000 & 1.31183900 & -2.94223800 \\
\hline $\mathrm{C}$ & 2.67750700 & 0.97681000 & -1.82112200 \\
\hline $\mathrm{C}$ & 3.42428700 & 2.79230600 & -3.41846700 \\
\hline $\mathrm{C}$ & 3.94083000 & 3.02968300 & -4.85716300 \\
\hline $\mathrm{C}$ & 4.21845100 & 3.69063300 & -2.43955400 \\
\hline $\mathrm{C}$ & 1.94536400 & 3.26868500 & -3.43000700 \\
\hline $\mathrm{C}$ & 3.94685200 & -2.06792200 & -5.57372300 \\
\hline $\mathrm{C}$ & 6.36721200 & 0.94237400 & -4.03885800 \\
\hline $\mathrm{C}$ & 4.43571100 & -2.27847300 & -4.11839500 \\
\hline $\mathrm{C}$ & 3.88609500 & -3.63934300 & -3.63618200 \\
\hline $\mathrm{C}$ & 4.93117800 & -1.07920000 & 3.35807500 \\
\hline $\mathrm{C}$ & 5.27399500 & -1.35284500 & 2.01023600 \\
\hline $\mathrm{C}$ & 6.95713500 & -3.18380300 & 2.41501600 \\
\hline $\mathrm{C}$ & 4.24856100 & -1.23246600 & 1.06089900 \\
\hline $\mathrm{C}$ & 2.93425200 & -0.91863600 & 1.41867700 \\
\hline $\mathrm{C}$ & 2.61529400 & -0.83299800 & 2.77926500 \\
\hline $\mathrm{C}$ & 3.58451000 & -0.95426100 & 3.78489800 \\
\hline $\mathrm{C}$ & 6.43119700 & 0.35909400 & 4.47578300 \\
\hline $\mathrm{C}$ & 7.82694900 & -0.90599300 & 1.77770900 \\
\hline $\mathrm{C}$ & 3.63435700 & 0.06819200 & 6.17849600 \\
\hline $\mathrm{C}$ & 3.14963800 & -1.08681200 & 5.27098700 \\
\hline $\mathrm{C}$ & 1.61094400 & -1.13889800 & 5.39845400 \\
\hline $\mathrm{C}$ & 6.67277000 & -2.31760400 & 0.09760500 \\
\hline $\mathrm{C}$ & 5.97870000 & -2.39182200 & -4.11128900 \\
\hline $\mathrm{C}$ & 6.66311300 & -1.90711500 & 1.58734200 \\
\hline $\mathrm{C}$ & 3.70076500 & -2.42496400 & 5.82550300 \\
\hline $\mathrm{H}$ & -1.93351500 & 0.58211700 & -5.22349800 \\
\hline $\mathrm{H}$ & -2.51312400 & 1.24387500 & -2.90463600 \\
\hline $\mathrm{H}$ & 0.95782700 & -3.28859000 & -5.82341700 \\
\hline $\mathrm{H}$ & -0.75342600 & -3.76749700 & -5.47076300 \\
\hline $\mathrm{H}$ & 1.94624500 & -3.18789300 & 1.51775200 \\
\hline $\mathrm{H}$ & 0.91498900 & -5.44124300 & 1.35866700 \\
\hline $\mathrm{H}$ & -2.68236400 & -6.06460700 & -1.85762200 \\
\hline $\mathrm{H}$ & -1.05685300 & -6.11246100 & -2.65542100 \\
\hline $\mathrm{H}$ & -2.17874500 & -0.79730200 & 2.39883200 \\
\hline
\end{tabular}




\begin{tabular}{|c|c|c|c|}
\hline [ & -3.85108600 & -1.70750300 & -1.40113900 \\
\hline $\mathrm{H}$ & -7.40563400 & -2.30186100 & -0.62533600 \\
\hline & -7.74018600 & -3.92764800 & -1.23833500 \\
\hline & -7.54012100 & -3.67426000 & 0.49556900 \\
\hline 1 & -5.64214700 & -5.35521600 & 0.71809600 \\
\hline H & -5.94553800 & -5.78256200 & -0.97834100 \\
\hline $\mathrm{H}$ & -4.30763700 & -5.34651400 & -0.44450200 \\
\hline & -4.27914900 & -3.62043200 & -2.37135100 \\
\hline & -5.93067600 & -4.14188000 & -2.74464500 \\
\hline $\mathrm{H}$ & -5.58952600 & -2.42852200 & -2.49784800 \\
\hline $\mathrm{H}$ & -3.77462500 & -3.99319900 & 5.53388300 \\
\hline U & -5.26892000 & -3.69823600 & 4.65198100 \\
\hline 11 & -3.99683200 & -4.67661700 & 3.91495100 \\
\hline $\mathrm{H}$ & -1.76489700 & -3.01977400 & 5.18506500 \\
\hline $\mathrm{H}$ & -1.64308300 & -3.60832200 & 3.51498100 \\
\hline $\mathrm{H}$ & -1.37783100 & -1.89775400 & 3.87937800 \\
\hline$\Pi$ & -3.37622900 & -0.40569000 & 4.50240300 \\
\hline $\mathrm{H}$ & -4.98012500 & -1.10380600 & 4.76749500 \\
\hline $\mathrm{H}$ & -3.65582100 & -1.48708400 & 5.87922600 \\
\hline $\mathrm{H}$ & -7.29095700 & -2.15114200 & 2.08118100 \\
\hline $\mathrm{H}$ & -7.58906600 & -3.53093700 & 3.17741200 \\
\hline $\mathrm{H}$ & -6.48229200 & -2.22040900 & 3.66629000 \\
\hline $\mathrm{H}$ & -1.49617400 & 2.97637200 & -1.01491200 \\
\hline $\mathrm{H}$ & -4.88012300 & 0.53195500 & -0.18494500 \\
\hline $\mathrm{H}$ & -0.93553400 & 6.14772800 & -2.33914100 \\
\hline $\mathrm{H}$ & -0.65731200 & 4.98083600 & -1.04390000 \\
\hline $\mathrm{H}$ & -1.13836100 & 4.42200800 & -2.65977100 \\
\hline $\mathrm{H}$ & -3.87591900 & 6.83331200 & -0.30773700 \\
\hline $\mathrm{H}$ & -2.42061300 & 6.18457800 & 0.48229400 \\
\hline $\mathrm{H}$ & -2.27778800 & 7.36295500 & -0.83052800 \\
\hline $\mathrm{H}$ & -6.43164500 & 4.72307400 & 1.21065500 \\
\hline $\mathrm{H}$ & -4.90487700 & 5.63805400 & 1.23104500 \\
\hline $\mathrm{H}$ & -6.38579100 & 6.38655200 & 0.56709100 \\
\hline $\mathrm{H}$ & -4.60352100 & 5.94095900 & -2.60205300 \\
\hline 11 & -3.12532400 & 6.72866300 & -3.18885600 \\
\hline $\mathrm{H}$ & -3.43955700 & 5.03044100 & -3.58667000 \\
\hline $\mathrm{H}$ & -6.72043500 & 0.99839300 & 1.27816900 \\
\hline
\end{tabular}




\begin{tabular}{|c|c|c|c|}
\hline 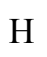 & -7.15112400 & 2.67370300 & 1.64419500 \\
\hline $\mathrm{H}$ & -8.35884500 & 1.58647500 & 0.93868200 \\
\hline & -8.81329300 & 3.00345300 & -1.10330000 \\
\hline & -7.86579500 & 4.25421000 & -0.30773400 \\
\hline $\mathrm{H}$ & -7.48866800 & 3.79722800 & -1.97107000 \\
\hline $\mathrm{H}$ & -6.83337700 & 1.33435800 & -2.48763700 \\
\hline $\mathrm{H}$ & -6.61055300 & 0.17396200 & -1.16970400 \\
\hline & -8.20801600 & 0.85596500 & -1.47543300 \\
\hline & 2.88724900 & -2.39189400 & -1.84565700 \\
\hline $\mathrm{H}$ & 2.16651100 & 1.75893300 & -1.27195700 \\
\hline $\mathrm{H}$ & 5.02105600 & 2.92304700 & -4.95707200 \\
\hline U & 3.46844000 & 2.35052300 & -5.57293400 \\
\hline $\mathrm{H}$ & 3.68884100 & 4.05632500 & -5.14979600 \\
\hline $\mathrm{H}$ & 4.15837500 & 4.73917400 & -2.75745400 \\
\hline $\mathrm{H}$ & 3.82623800 & 3.62798300 & -1.41896300 \\
\hline $\mathrm{H}$ & 5.27831200 & 3.41455900 & -2.40396300 \\
\hline$\Pi$ & 1.89645400 & 4.29866500 & -3.80202000 \\
\hline $\mathrm{H}$ & 1.33723300 & 2.63881400 & -4.08920200 \\
\hline $\mathrm{H}$ & 1.48366900 & 3.26004400 & -2.43930500 \\
\hline $\mathrm{H}$ & 4.25503300 & -2.91427000 & -6.20097700 \\
\hline $\mathrm{H}$ & 2.85376200 & -1.99603700 & -5.60818000 \\
\hline $\mathrm{H}$ & 4.35909400 & -1.15320200 & -6.00407900 \\
\hline $\mathrm{H}$ & 6.30100000 & 1.92228600 & -3.55410400 \\
\hline П & 6.77762800 & 0.22237200 & -3.32085600 \\
\hline $\mathrm{H}$ & 7.03386400 & 1.01423900 & -4.90232500 \\
\hline $\mathrm{H}$ & 4.25185300 & -3.90031000 & -2.63602100 \\
\hline $\mathrm{H}$ & 2.79218500 & -3.66024200 & -3.61823600 \\
\hline $\mathrm{H}$ & 4.22815800 & -4.42381200 & -4.32096400 \\
\hline $\mathrm{H}$ & 6.18871400 & -3.94604700 & 2.24138900 \\
\hline $\mathrm{H}$ & 7.92508100 & -3.60779100 & 2.11931700 \\
\hline 11 & 6.99096000 & -2.96353400 & 3.48410900 \\
\hline $\mathrm{H}$ & 4.47554700 & -1.38112900 & 0.01511500 \\
\hline $\mathrm{H}$ & 1.57770600 & -0.66356900 & 3.04151700 \\
\hline $\mathrm{H}$ & 7.21342500 & 0.30438600 & 5.23757300 \\
\hline 11 & 6.85218900 & 0.75742200 & 3.54827200 \\
\hline $\mathrm{H}$ & 5.63883100 & 1.03207200 & 4.81461800 \\
\hline $\mathrm{H}$ & 8.72713600 & -1.29255400 & 1.28412200 \\
\hline
\end{tabular}




\begin{tabular}{|c|c|c|c|}
\hline $\mathrm{H}$ & 7.59621100 & 0.06783900 & 1.32955600 \\
\hline$H$ & 8.07386300 & -0.76108600 & 2.83005600 \\
\hline $\mathrm{H}$ & 4.70718700 & 0.01605000 & 6.36857100 \\
\hline $\mathrm{H}$ & 3.39708500 & 1.04773200 & 5.74636200 \\
\hline $\mathrm{H}$ & 3.13018800 & 0.00544500 & 7.15052700 \\
\hline $\mathrm{H}$ & 1.34701300 & -1.31144200 & 6.44793200 \\
\hline $\mathrm{H}$ & 1.13272100 & -0.20270900 & 5.08921300 \\
\hline $\mathrm{H}$ & 1.17465700 & -1.95269400 & 4.80988700 \\
\hline $\mathrm{H}$ & 5.89513400 & -3.05352900 & -0.13376400 \\
\hline $\mathrm{H}$ & 6.54234200 & -1.45902700 & -0.57137400 \\
\hline $\mathrm{H}$ & 7.64027100 & -2.77444900 & -0.13868300 \\
\hline $\mathrm{H}$ & 6.36822900 & -2.44436200 & -3.08765600 \\
\hline $\mathrm{H}$ & 6.28166600 & -3.31120900 & -4.62771200 \\
\hline $\mathrm{H}$ & 6.45321600 & -1.55636200 & -4.62606600 \\
\hline $\mathrm{H}$ & 3.38536400 & -2.55648900 & 6.86824100 \\
\hline $\mathrm{H}$ & 3.31484600 & -3.27371100 & 5.24878100 \\
\hline $\mathrm{H}$ & 4.79216000 & -2.45003300 & 5.79119800 \\
\hline $\mathrm{Cu}$ & -0.02859500 & 0.80628600 & 0.95597500 \\
\hline $\mathrm{H}$ & -0.25369800 & 0.52061800 & 2.55407600 \\
\hline $\mathrm{C}$ & 0.05223300 & 2.15232600 & 2.65385300 \\
\hline $\mathrm{C}$ & 0.39281200 & 2.83810200 & 1.44326300 \\
\hline $\mathrm{H}$ & -0.40038900 & 3.45235400 & 1.01538500 \\
\hline $\mathrm{H}$ & 0.88972400 & 1.94804000 & 3.32502900 \\
\hline $\mathrm{C}$ & -1.23596200 & 2.50023000 & 3.39179000 \\
\hline $\mathrm{H}$ & -2.05620900 & 2.56887700 & 2.66639900 \\
\hline $\mathrm{H}$ & -1.49749100 & 1.69092100 & 4.08510300 \\
\hline $\mathrm{C}$ & 1.80467500 & 3.34022500 & 1.20099000 \\
\hline $\mathrm{H}$ & 2.54156600 & 2.60038700 & 1.54031900 \\
\hline $\mathrm{H}$ & 1.99176400 & 3.49137000 & 0.12985300 \\
\hline $\mathrm{C}$ & 2.08490100 & 4.66594400 & 1.90775200 \\
\hline $\mathrm{H}$ & 1.38494100 & 5.44818700 & 1.59431300 \\
\hline $\mathrm{H}$ & 2.01192000 & 4.57201900 & 2.99619900 \\
\hline $\mathrm{O}$ & 3.43230600 & 5.08250400 & 1.56179200 \\
\hline $\mathrm{C}$ & 3.84854500 & 6.25656900 & 2.07856300 \\
\hline $\mathrm{C}$ & 5.26192800 & 6.56951600 & 1.63514200 \\
\hline $\mathrm{H}$ & 5.94425700 & 5.77805000 & 1.96303900 \\
\hline $\mathrm{H}$ & 5.31440300 & 6.61163700 & 0.5421140 \\
\hline
\end{tabular}




$\begin{array}{lrrr}\mathrm{H} & 5.57298000 & 7.52523000 & 2.05880500 \\ \mathrm{O} & 3.17229000 & 6.95802200 & 2.80063800 \\ \mathrm{C} & -1.12170200 & 3.82320300 & 4.16650200 \\ \mathrm{H} & -2.05120900 & 4.03892700 & 4.70720600 \\ \mathrm{H} & -0.31088900 & 3.78196500 & 4.90491800 \\ \mathrm{H} & -0.91670700 & 4.66350300 & 3.49495100\end{array}$

6a

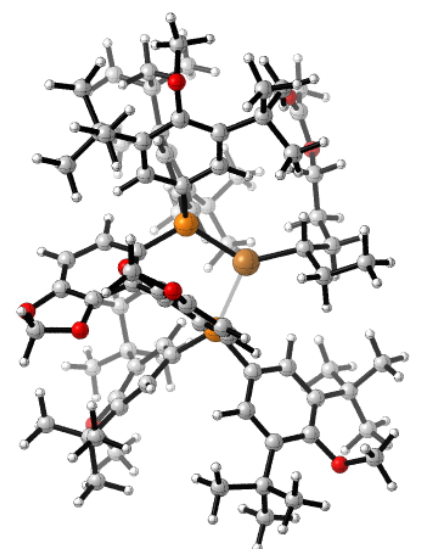

Thermal correction to Enthalpy=

1.928907

Thermal correction to Gibbs Free Energy=

1.675333

Sum of electronic and zero-point Energies=

$-4824.319573$

Sum of electronic and thermal Enthalpies=

$-4824.212383$

Sum of electronic and thermal Free Energies=

SMD E $($ M06 $)=-4824.505523$

Cartesian coordinates:

$\mathrm{P}$

$\begin{array}{lll}1.51739400 & -0.29567100 & 0.08483500\end{array}$

$\begin{array}{lllll}\mathrm{P} & -2.01801400 & 0.09991300 & -0.44181800\end{array}$

O $\quad 0.41525300 \quad-4.52366200 \quad-1.56130800$

$\begin{array}{llll}\mathrm{O} & -0.38095600 & -4.62772000 & -3.73187100\end{array}$

$\begin{array}{lllll}\mathrm{O} & -1.50679700 & -4.58842300 & 0.91004900\end{array}$

O $\quad-0.80268800 \quad-4.93189500 \quad 3.07665300$

$\begin{array}{llll}\mathrm{O} & -4.99092100 & -1.23151000 & 4.59509500\end{array}$

$\begin{array}{llll}\mathrm{O} & -6.81302000 & 2.65723100 & -3.05226300\end{array}$

$\begin{array}{lllll}\mathrm{O} & 6.09063100 & -2.84836800 & -2.85937300\end{array}$

$\begin{array}{lllll}\mathrm{O} & 4.56401700 & 2.71717800 & 4.30869700\end{array}$

$\begin{array}{llll}\text { C } & -1.63356700 & -1.42992600 & -1.41847300\end{array}$

C $\quad-0.73216600 \quad-2.42710200 \quad-0.91518500$ 


\begin{tabular}{|c|c|c|c|}
\hline $\mathrm{C}$ & -0.41208300 & -3.44873200 & -1.79920100 \\
\hline $\mathrm{C}$ & -0.88769400 & -3.51777600 & -3.10555800 \\
\hline $\mathrm{C}$ & -1.73075500 & -2.55499300 & -3.61418700 \\
\hline $\mathrm{C}$ & -2.08940600 & -1.51312800 & -2.74248400 \\
\hline $\mathrm{C}$ & 0.32329500 & -5.35553900 & -2.72185100 \\
\hline $\mathrm{C}$ & 0.76680300 & -1.66355800 & 1.08662500 \\
\hline $\mathrm{C}$ & 1.17447500 & -1.88581200 & 2.40935700 \\
\hline $\mathrm{C}$ & 0.69693500 & -2.95560800 & 3.18631700 \\
\hline $\mathrm{C}$ & -0.18891400 & -3.81122700 & 2.56849700 \\
\hline $\mathrm{C}$ & -0.61331600 & -3.59744500 & 1.25893400 \\
\hline $\mathrm{C}$ & -0.20025600 & -2.52566300 & 0.48111900 \\
\hline $\mathrm{C}$ & -1.37281000 & -5.57655100 & 1.93372200 \\
\hline $\mathrm{C}$ & -2.76166100 & -0.50981300 & 1.14657400 \\
\hline $\mathrm{C}$ & -2.53348700 & 0.23764200 & 2.29899800 \\
\hline $\mathrm{C}$ & -3.17575000 & -0.03478600 & 3.52099500 \\
\hline $\mathrm{C}$ & -4.15619300 & -1.04981600 & 3.50182800 \\
\hline $\mathrm{C}$ & -4.30694300 & -1.93745100 & 2.40038700 \\
\hline $\mathrm{C}$ & -3.59483300 & -1.63422000 & 1.23611900 \\
\hline $\mathrm{C}$ & -5.14733300 & -3.23789400 & 2.49888400 \\
\hline $\mathrm{C}$ & -6.65580100 & -2.96438200 & 2.71180100 \\
\hline $\mathrm{C}$ & -4.61353300 & -4.09008100 & 3.67803500 \\
\hline $\mathrm{C}$ & -5.02969500 & -4.08903500 & 1.21486500 \\
\hline $\mathrm{C}$ & -2.69600800 & 0.76461800 & 4.76521500 \\
\hline $\mathrm{C}$ & -3.20528100 & 0.25570800 & 6.13375900 \\
\hline $\mathrm{C}$ & -1.14882500 & 0.64434500 & 4.83556700 \\
\hline $\mathrm{C}$ & -3.07720700 & 2.25693000 & 4.61827600 \\
\hline $\mathrm{C}$ & -6.06744000 & -0.29489400 & 4.65475800 \\
\hline $\mathrm{C}$ & -3.52241300 & 0.83265700 & -1.24065500 \\
\hline $\mathrm{C}$ & -4.69782200 & 0.11383500 & -1.49801200 \\
\hline $\mathrm{C}$ & -3.52465100 & 2.20819100 & -1.46516700 \\
\hline $\mathrm{C}$ & -4.64254200 & 2.89480200 & -1.97735800 \\
\hline $\mathrm{C}$ & -5.74014100 & 2.09798900 & -2.37480300 \\
\hline $\mathrm{C}$ & -5.82659500 & 0.71177200 & -2.06164800 \\
\hline $\mathrm{C}$ & -7.13607800 & -0.10139000 & -2.24054800 \\
\hline $\mathrm{C}$ & -6.99239000 & -1.53458700 & -1.68313000 \\
\hline $\mathrm{C}$ & -7.55006900 & -0.24047700 & -3.72480800 \\
\hline$C$ & -6.59194100 & 2.88337300 & -4.44479200 \\
\hline
\end{tabular}




\begin{tabular}{|c|c|c|c|}
\hline $\mathrm{C}$ & -8.27760200 & 0.58229700 & -1.44699000 \\
\hline $\mathrm{C}$ & -4.58334200 & 4.44966800 & -2.01004800 \\
\hline $\mathrm{C}$ & -3.72363600 & 4.93915700 & -3.19985500 \\
\hline $\mathrm{C}$ & -5.96763400 & 5.13976200 & -2.05225500 \\
\hline $\mathrm{C}$ & -3.91461100 & 4.95451900 & -0.70103800 \\
\hline $\mathrm{C}$ & 2.80805700 & -1.18135200 & -0.89836300 \\
\hline $\mathrm{C}$ & 3.28234400 & -2.45223400 & -0.55322400 \\
\hline $\mathrm{C}$ & 4.33722500 & -3.06067200 & -1.24046400 \\
\hline $\mathrm{C}$ & 4.94088100 & -2.31943700 & -2.29191200 \\
\hline $\mathrm{C}$ & 4.38415900 & -1.11415500 & -2.78231900 \\
\hline $\mathrm{C}$ & 3.33762800 & -0.55496900 & -2.02767800 \\
\hline $\mathrm{C}$ & 4.79195800 & -0.38040400 & -4.09245800 \\
\hline $\mathrm{C}$ & 5.58562900 & -1.24462000 & -5.10149900 \\
\hline $\mathrm{C}$ & 5.60065300 & 0.89734600 & -3.76146400 \\
\hline $\mathrm{C}$ & 3.50122800 & 0.04229100 & -4.84725100 \\
\hline $\mathrm{C}$ & 4.66044100 & -5.39367900 & -2.16419500 \\
\hline $\mathrm{C}$ & 7.30056800 & -2.22171600 & -2.43180800 \\
\hline $\mathrm{C}$ & 4.75724100 & -4.51320400 & -0.89351000 \\
\hline $\mathrm{C}$ & 3.81619200 & -5.13477600 & 0.16301700 \\
\hline $\mathrm{C}$ & 3.91155000 & 2.09822800 & 3.24853200 \\
\hline $\mathrm{C}$ & 2.59300000 & 2.51232600 & 2.92113700 \\
\hline $\mathrm{C}$ & 2.89246700 & 5.00579300 & 3.00218500 \\
\hline $\mathrm{C}$ & 1.91627300 & 1.74970500 & 1.95860700 \\
\hline $\mathrm{C}$ & 2.51179600 & 0.65598900 & 1.31891500 \\
\hline $\mathrm{C}$ & 3.86258500 & 0.39512700 & 1.55916500 \\
\hline $\mathrm{C}$ & 4.61326200 & 1.12773500 & 2.48935800 \\
\hline $\mathrm{C}$ & 4.39079900 & 2.03604300 & 5.55814900 \\
\hline $\mathrm{C}$ & 1.79144700 & 3.89875100 & 4.97810100 \\
\hline $\mathrm{C}$ & 6.72928000 & 0.51559200 & 3.88744800 \\
\hline $\mathrm{C}$ & 6.16013000 & 0.97017500 & 2.52372000 \\
\hline $\mathrm{C}$ & 6.64491800 & -0.05810600 & 1.47609900 \\
\hline $\mathrm{C}$ & 0.58019600 & 4.09728200 & 2.82578700 \\
\hline $\mathrm{C}$ & 6.19283200 & -4.59556200 & -0.32237300 \\
\hline $\mathrm{C}$ & 1.97218900 & 3.83936900 & 3.44308700 \\
\hline $\mathrm{C}$ & 6.78822900 & 2.33572500 & 2.14550100 \\
\hline $\mathrm{H}$ & -2.09836900 & -2.59737900 & -4.63353100 \\
\hline $\mathrm{H}$ & -2.74892300 & -0.74060800 & -3.11889600 \\
\hline
\end{tabular}




\begin{tabular}{|c|c|c|c|}
\hline $\mathrm{H}$ & 1.32862800 & -5.59436800 & -3.07965600 \\
\hline $\mathrm{H}$ & -0.23389300 & -6.26973300 & -2.47329900 \\
\hline & 1.91072400 & -1.22312900 & 2.84926500 \\
\hline & 1.02741700 & -3.11392300 & 4.20715200 \\
\hline H & -2.35672600 & -5.96977600 & 2.19141900 \\
\hline $\mathrm{H}$ & -0.69544300 & -6.37126500 & 1.58717100 \\
\hline $\mathrm{H}_{\mathrm{H}}$ & -1.82756600 & 1.06043000 & 2.24490400 \\
\hline & -3.68414800 & -2.28010900 & 0.37245100 \\
\hline & -7.04402300 & -2.26704300 & 1.95963100 \\
\hline $\mathrm{H}$ & -7.21595300 & -3.90246000 & 2.61312800 \\
\hline $\mathrm{H}$ & -6.86330900 & -2.56257000 & 3.70360000 \\
\hline U & -4.73280900 & -3.56784000 & 4.62955600 \\
\hline 11 & -5.16290600 & -5.03862200 & 3.73538700 \\
\hline $\mathrm{H}$ & -3.54924300 & -4.31740000 & 3.54827600 \\
\hline $\mathrm{H}$ & -3.99220700 & -4.33849500 & 0.97366200 \\
\hline $\mathrm{H}$ & -5.57867200 & -5.02660000 & 1.35974800 \\
\hline$\Pi$ & -5.47139200 & -3.58766400 & 0.34564500 \\
\hline $\mathrm{H}$ & -2.64949000 & 0.77990400 & 6.92104400 \\
\hline $\mathrm{H}$ & -4.26398100 & 0.45288800 & 6.30560100 \\
\hline $\mathrm{H}$ & -3.03597700 & -0.81699800 & 6.25878300 \\
\hline $\mathrm{H}$ & -0.77470700 & 1.20345400 & 5.70189800 \\
\hline $\mathrm{H}$ & -0.84696200 & -0.40285000 & 4.94889700 \\
\hline $\mathrm{H}$ & -0.64585300 & 1.03603100 & 3.94814400 \\
\hline $\mathrm{H}$ & -2.66126200 & 2.69538900 & 3.70479000 \\
\hline $\mathrm{H}$ & -4.16439200 & 2.39182200 & 4.58359300 \\
\hline $\mathrm{H}$ & -2.69547500 & 2.83240900 & 5.47073100 \\
\hline$H$ & -6.70651800 & -0.36425000 & 3.76669700 \\
\hline $\mathrm{H}$ & -6.65007600 & -0.55107100 & 5.54349300 \\
\hline $\mathrm{H}$ & -5.70574100 & 0.73538300 & 4.74395200 \\
\hline $\mathrm{H}$ & -4.72146600 & -0.93923000 & -1.25617900 \\
\hline $\mathrm{H}$ & -2.62238500 & 2.75919600 & -1.22607000 \\
\hline $\mathrm{H}$ & -7.95337500 & -2.05229300 & -1.77732500 \\
\hline $\mathrm{H}$ & -6.24835400 & -2.12329100 & -2.23266400 \\
\hline $\mathrm{H}$ & -6.71942500 & -1.53690800 & -0.62217600 \\
\hline 11 & -7.87515600 & 0.70924400 & -4.14920300 \\
\hline $\mathrm{H}$ & -6.72532000 & -0.63345200 & -4.33144900 \\
\hline H & -8.38872800 & -0.94268000 & -3.8096850 \\
\hline
\end{tabular}




\begin{tabular}{|c|c|c|c|}
\hline I & -5.90935000 & 3.72117100 & -4.61840600 \\
\hline $\mathrm{H}$ & -6.18779400 & 1.99047100 & -4.93547300 \\
\hline & -7.56752800 & 3.12446500 & -4.87546200 \\
\hline & -8.46046800 & 1.59725800 & -1.80435100 \\
\hline H & -9.20511900 & 0.00556100 & -1.55379800 \\
\hline $\mathrm{H}$ & -8.03248400 & 0.62898400 & -0.37927000 \\
\hline $\mathrm{H}$ & -2.71128900 & 4.52540400 & -3.15598700 \\
\hline & -4.15395400 & 4.65453800 & -4.16549000 \\
\hline 1 & -3.64233400 & 6.03327400 & -3.18167300 \\
\hline $\mathrm{H}$ & -5.82724600 & 6.21209900 & -1.87044700 \\
\hline $\mathrm{H}$ & -6.48030400 & 5.03743800 & -3.00815900 \\
\hline $\mathrm{H}$ & -6.63244900 & 4.75155000 & -1.27393000 \\
\hline $\mathrm{H}$ & -4.45201700 & 4.59108600 & 0.18226900 \\
\hline $\mathrm{H}$ & -2.86775700 & 4.65362900 & -0.61005500 \\
\hline $\mathrm{H}$ & -3.93472400 & 6.05009900 & -0.68294600 \\
\hline$H$ & 2.81999200 & -2.96874100 & 0.27558300 \\
\hline$\Pi$ & 2.91571900 & 0.39618400 & -2.33079000 \\
\hline $\mathrm{H}$ & 6.60765400 & -1.45864100 & -4.78936700 \\
\hline $\mathrm{H}$ & 5.08686300 & -2.19948800 & -5.29509900 \\
\hline 11 & 5.64514800 & -0.69826700 & -6.05048700 \\
\hline $\mathrm{H}$ & 5.81978000 & 1.45096500 & -4.68327300 \\
\hline $\mathrm{H}$ & 5.06100500 & 1.56806700 & -3.08563200 \\
\hline $\mathrm{H}$ & 6.55837400 & 0.66219500 & -3.28416400 \\
\hline $\mathrm{H}$ & 3.77623500 & 0.51356600 & -5.79795200 \\
\hline $\mathrm{H}$ & 2.87260900 & -0.82766300 & -5.06975800 \\
\hline $\mathrm{H}$ & 2.89366900 & 0.76384600 & -4.29644000 \\
\hline$H$ & 4.94369500 & -6.42714700 & -1.92734900 \\
\hline $\mathrm{H}$ & 3.63237000 & -5.40618400 & -2.54613800 \\
\hline $\mathrm{H}$ & 5.31640500 & -5.02853200 & -2.95632100 \\
\hline $\mathrm{H}$ & 7.38672500 & -1.19865500 & -2.81155700 \\
\hline $\mathrm{H}$ & 7.37197700 & -2.19799100 & -1.33816900 \\
\hline $\mathrm{H}$ & 8.11803800 & -2.82411500 & -2.83706600 \\
\hline $\mathrm{H}$ & 3.89046600 & -4.63005600 & 1.13334500 \\
\hline $\mathrm{H}$ & 2.76874500 & -5.11332500 & -0.15579400 \\
\hline $\mathrm{H}$ & 4.09923200 & -6.18210700 & 0.31913200 \\
\hline $\mathrm{H}$ & 2.97336400 & 5.04436100 & 1.91038400 \\
\hline $\mathrm{H}$ & 2.47616900 & 5.96187100 & 3.34440900 \\
\hline
\end{tabular}




\begin{tabular}{|c|c|c|c|}
\hline $\mathrm{H}$ & 3.89350700 & 4.89812800 & 3.42840100 \\
\hline $\mathrm{H}$ & 0.90552700 & 2.01622700 & 1.67397100 \\
\hline $\mathrm{H}$ & 4.34006900 & -0.38864900 & 0.98866400 \\
\hline $\mathrm{H}$ & 4.97146400 & 2.59686000 & 6.29534100 \\
\hline $\mathrm{H}$ & 3.34040400 & 2.01553300 & 5.86213100 \\
\hline $\mathrm{H}$ & 4.76026500 & 1.00805600 & 5.50946500 \\
\hline $\mathrm{H}$ & 1.18696800 & 4.77597200 & 5.23850500 \\
\hline $\mathrm{H}$ & 1.26737800 & 3.01377000 & 5.35824300 \\
\hline $\mathrm{H}$ & 2.74469400 & 3.99824200 & 5.49749700 \\
\hline $\mathrm{H}$ & 6.66412300 & 1.30131500 & 4.64063300 \\
\hline $\mathrm{H}$ & 6.21886400 & -0.38059900 & 4.26065800 \\
\hline $\mathrm{H}$ & 7.79077700 & 0.26506300 & 3.77224600 \\
\hline $\mathrm{H}$ & 7.74030900 & -0.08336300 & 1.48845400 \\
\hline $\mathrm{H}$ & 6.29086200 & -1.07287800 & 1.69494700 \\
\hline $\mathrm{H}$ & 6.33363600 & 0.20646000 & 0.46050900 \\
\hline $\mathrm{H}$ & 0.61026400 & 4.14140800 & 1.73306300 \\
\hline $\mathrm{H}$ & -0.15210600 & 3.33788200 & 3.12352300 \\
\hline $\mathrm{H}$ & 0.20842300 & 5.06504400 & 3.18009200 \\
\hline $\mathrm{H}$ & 6.32485200 & -3.90267000 & 0.51747200 \\
\hline $\mathrm{H}$ & 6.38531500 & -5.60943300 & 0.05035000 \\
\hline $\mathrm{H}$ & 6.94747100 & -4.37566800 & -1.07795600 \\
\hline $\mathrm{H}$ & 7.88309900 & 2.25841000 & 2.15407000 \\
\hline $\mathrm{H}$ & 6.48027200 & 2.63942500 & 1.13894100 \\
\hline $\mathrm{H}$ & 6.49738100 & 3.11312800 & 2.85688500 \\
\hline $\mathrm{Cu}$ & 0.08902900 & 1.10243000 & -1.12499800 \\
\hline $\mathrm{H}$ & -0.68534000 & 4.08900700 & -2.20251000 \\
\hline $\mathrm{C}$ & 0.33336400 & 3.79149600 & -2.49337400 \\
\hline $\mathrm{H}$ & 0.54488000 & 4.35494700 & -3.42114400 \\
\hline $\mathrm{C}$ & 0.38240300 & 2.27099800 & -2.73367700 \\
\hline $\mathrm{H}$ & 1.41037700 & 2.03277400 & -3.06386800 \\
\hline $\mathrm{C}$ & -0.53946300 & 1.85582500 & -3.90498200 \\
\hline $\mathrm{H}$ & -0.51721500 & 0.76195400 & -4.00870200 \\
\hline $\mathrm{H}$ & -1.58382900 & 2.10642500 & -3.66352200 \\
\hline $\mathrm{C}$ & 1.29315000 & 4.33354700 & -1.42131300 \\
\hline $\mathrm{H}$ & 1.17803300 & 5.42299400 & -1.33918400 \\
\hline $\mathrm{H}$ & 1.04420400 & 3.90281900 & -0.44276100 \\
\hline $\mathrm{C}$ & 2.75115100 & 4.03014500 & -1.73186000 \\
\hline
\end{tabular}




$\begin{array}{lrrr}\mathrm{H} & 2.99803400 & 4.27122800 & -2.77312300 \\ \mathrm{H} & 3.00263100 & 2.98359900 & -1.55402200 \\ \mathrm{O} & 3.58144700 & 4.86492300 & -0.87894000 \\ \mathrm{C} & 4.88249800 & 4.53403000 & -0.77761700 \\ \mathrm{C} & 5.62733800 & 5.51723100 & 0.09871400 \\ \mathrm{H} & 5.30624800 & 6.54246800 & -0.10463800 \\ \mathrm{H} & 5.41234800 & 5.30301800 & 1.15138100 \\ \mathrm{H} & 6.70010900 & 5.41211900 & -0.07062700 \\ \mathrm{O} & 5.38672300 & 3.56395300 & -1.30883800 \\ \mathrm{C} & -0.20113900 & 2.46058200 & -5.28328300 \\ \mathrm{H} & -0.31061700 & 3.55122000 & -5.29740900 \\ \mathrm{H} & 0.83415300 & 2.23053900 & -5.56841600 \\ \mathrm{H} & -0.85709400 & 2.05720800 & -6.06679200\end{array}$

6b

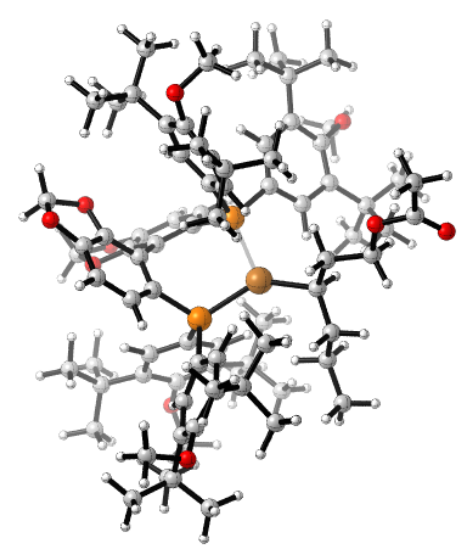

Thermal correction to Enthalpy=

1.928317

Thermal correction to Gibbs Free Energy $=\quad 1.669714$

Sum of electronic and zero-point Energies $=\quad-4824.322985$

Sum of electronic and thermal Enthalpies $=\quad-4824.215344$

Sum of electronic and thermal Free Energies $=\quad-4824.473947$

SMD E $($ M06 $)=-4824.506018$

Cartesian coordinates:

$\begin{array}{llll}\mathrm{P} & -1.84783800 & -0.21169700 & 0.15958600 \\ \mathrm{P} & 1.64283500 & 0.53207500 & -0.02226000 \\ \mathrm{O} & -1.38148400 & 3.62773200 & 3.13242800 \\ \mathrm{O} & -0.63803300 & 5.60991900 & 2.22631500 \\ \mathrm{O} & 0.46888200 & 1.73889200 & 4.57381300\end{array}$




\begin{tabular}{|c|c|c|c|}
\hline $\mathrm{O}$ & -0.33039800 & 0.08333500 & 5.96125600 \\
\hline $\mathrm{O}$ & 4.81358600 & -2.46238300 & 4.09856700 \\
\hline $\mathrm{O}$ & 5.98574800 & 2.92856300 & -3.41949300 \\
\hline $\mathrm{O}$ & -5.17299000 & 4.52308000 & -1.41148900 \\
\hline $\mathrm{O}$ & -6.01811500 & -4.53648100 & 0.41455800 \\
\hline $\mathrm{C}$ & 0.99223400 & 2.11141200 & 0.71225000 \\
\hline $\mathrm{C}$ & -0.01533800 & 2.10400700 & 1.73278400 \\
\hline $\mathrm{C}$ & -0.44758300 & 3.35494900 & 2.15322400 \\
\hline$C$ & 0.00014800 & 4.55745900 & 1.61267800 \\
\hline $\mathrm{C}$ & 0.93577800 & 4.58138400 & 0.60331000 \\
\hline $\mathrm{C}$ & 1.42392600 & 3.33491100 & 0.17606600 \\
\hline $\mathrm{C}$ & -1.25403300 & 5.02955300 & 3.37828700 \\
\hline $\mathrm{C}$ & -1.43018600 & -0.10328300 & 1.96934200 \\
\hline $\mathrm{C}$ & -1.90583300 & -1.09696000 & 2.838556 \\
\hline $\mathrm{C}$ & -1.59161300 & -1.14093200 & 4.20741200 \\
\hline $\mathrm{C}$ & -0.78877000 & -0.12901300 & 4.68241900 \\
\hline $\mathrm{C}$ & -0.30414400 & 0.86509300 & 3.83657300 \\
\hline $\mathrm{C}$ & -0.56281100 & 0.92132400 & 2.47331400 \\
\hline $\mathrm{C}$ & 0.19221400 & 1.41373600 & 5.93755600 \\
\hline $\mathrm{C}$ & 2.48032300 & -0.34338200 & 1.36797100 \\
\hline $\mathrm{C}$ & 2.51727100 & -1.73704600 & 1.32492300 \\
\hline $\mathrm{C}$ & 3.20418300 & -2.50750000 & 2.27928300 \\
\hline $\mathrm{C}$ & 3.95374600 & -1.79263700 & 3.24161300 \\
\hline $\mathrm{C}$ & 3.83240900 & -0.38515400 & 3.40768600 \\
\hline $\mathrm{C}$ & 3.08938900 & 0.31013400 & 2.44766200 \\
\hline $\mathrm{C}$ & 4.40753600 & 0.35498000 & 4.64418600 \\
\hline $\mathrm{C}$ & 5.95059100 & 0.27896700 & 4.73254600 \\
\hline $\mathrm{C}$ & 3.79196000 & -0.26659100 & 5.92332700 \\
\hline $\mathrm{C}$ & 4.03841600 & 1.85472700 & 4.62753400 \\
\hline $\mathrm{C}$ & 3.02308100 & -4.05113700 & 2.21091300 \\
\hline $\mathrm{C}$ & 3.43696100 & -4.81667200 & 3.48976000 \\
\hline $\mathrm{C}$ & 1.51100300 & -4.35685200 & 2.02003400 \\
\hline $\mathrm{C}$ & 3.79009400 & -4.63344700 & 0.99876400 \\
\hline $\mathrm{C}$ & 6.06346700 & -2.82043500 & 3.50756900 \\
\hline $\mathrm{C}$ & 3.05802100 & 1.11556900 & -1.06655800 \\
\hline $\mathrm{C}$ & 4.33225200 & 1.38356100 & -0.54897700 \\
\hline $\mathrm{C}$ & 2.85050300 & 1.28179600 & -2.436764 \\
\hline
\end{tabular}




\begin{tabular}{|c|c|c|c|}
\hline $\mathrm{C}$ & 3.83656400 & 1.80359500 & 2100 \\
\hline $\mathrm{C}$ & 5.04111600 & 2.22545500 & -2.68737800 \\
\hline $\mathrm{C}$ & 5.35568700 & 1.91973600 & -1.33516800 \\
\hline $\mathrm{C}$ & 6.78615900 & 2.08093100 & -0.75874600 \\
\hline $\mathrm{C}$ & 6.87886700 & 1.53972000 & 0.68496100 \\
\hline $\mathrm{C}$ & 7.25376100 & 3.55575800 & -0.71283200 \\
\hline $\mathrm{C}$ & 5.68352800 & 4.31552000 & -3.57016100 \\
\hline $\mathrm{C}$ & 7.77350400 & 1.25631100 & -1.62233000 \\
\hline $\mathrm{C}$ & 3.52100000 & 1.81921100 & -4.81915500 \\
\hline $\mathrm{C}$ & 2.42904900 & 2.87372500 & -5.12028300 \\
\hline $\mathrm{C}$ & 4.73065700 & 2.06826400 & -5.75075700 \\
\hline $\mathrm{C}$ & 2.97737300 & 0.42130100 & -5.22077500 \\
\hline $\mathrm{C}$ & -2.74931400 & 1.35088600 & -0.21408600 \\
\hline $\mathrm{C}$ & -3.57944000 & 2.00281400 & 0.70787400 \\
\hline $\mathrm{C}$ & -4.35717100 & 3.10819200 & 0.34815500 \\
\hline $\mathrm{C}$ & -4.28145900 & 3.54247400 & -1.00455200 \\
\hline $\mathrm{C}$ & -3.31807900 & 3.03697200 & -1.90671600 \\
\hline $\mathrm{C}$ & -2.60490400 & 1.90051100 & -1.48723000 \\
\hline $\mathrm{C}$ & -2.93497800 & 3.64327200 & -3.28696000 \\
\hline $\mathrm{C}$ & -3.45235400 & 5.07740600 & -3.54504100 \\
\hline $\mathrm{C}$ & -3.41404100 & 2.71671800 & -4.43007500 \\
\hline $\mathrm{C}$ & -1.38556500 & 3.74461200 & -3.35638600 \\
\hline $\mathrm{C}$ & -4.72203500 & 5.34363500 & 1.45652000 \\
\hline $\mathrm{C}$ & -6.26567800 & 4.02722300 & -2.18636200 \\
\hline $\mathrm{C}$ & -5.18871000 & 3.86640500 & 1.41563500 \\
\hline $\mathrm{C}$ & -4.97526000 & 3.27020700 & 2.82472100 \\
\hline $\mathrm{C}$ & -5.07793900 & -3.53638300 & 0.19469200 \\
\hline $\mathrm{C}$ & -3.71319700 & -3.89635400 & 0.04471800 \\
\hline $\mathrm{C}$ & -3.97777800 & -5.85074300 & -1.51690600 \\
\hline $\mathrm{C}$ & -2.78684600 & -2.84425400 & 0.01220900 \\
\hline $\mathrm{C}$ & -3.17566100 & -1.50147500 & 0.10423100 \\
\hline $\mathrm{C}$ & -4.53916800 & -1.20059000 & 0.07082100 \\
\hline $\mathrm{C}$ & -5.52486300 & -2.19856800 & 0.06177800 \\
\hline $\mathrm{C}$ & -6.27153500 & -4.78227000 & 1.80362600 \\
\hline $\mathrm{C}$ & -3.53974000 & -6.34218900 & 0.91473800 \\
\hline $\mathrm{C}$ & -7.97959300 & -2.11869400 & 0.92987400 \\
\hline & -7.00454000 & -1.82538300 & -0.2345480 \\
\hline
\end{tabular}




$\begin{array}{lrrr}\mathrm{C} & -7.14621600 & -0.32166200 & -0.56232500 \\ \mathrm{C} & -1.73495500 & -5.42385100 & -0.51319800 \\ \mathrm{C} & -6.71008700 & 3.82264200 & 1.13701300 \\ \mathrm{C} & -3.25335600 & -5.35353700 & -0.24017600 \\ \mathrm{C} & -7.46614600 & -2.61084700 & -1.48816100 \\ \mathrm{H} & 1.29270800 & 5.51125600 & 0.17404600 \\ \mathrm{H} & 2.18323400 & 3.32924700 & -0.59597200 \\ \mathrm{H} & -2.24359100 & 5.46505600 & 3.52076600 \\ \mathrm{H} & -0.61053700 & 5.18895500 & 4.25672400 \\ \mathrm{H} & -2.55990000 & -1.86656100 & 2.44797600 \\ \mathrm{H} & -1.97691200 & -1.91998900 & 4.85612900 \\ \mathrm{H} & 1.11650600 & 1.45248300 & 6.51502000 \\ \mathrm{H} & -0.56209900 & 2.11053800 & 6.33311500 \\ \mathrm{H} & 1.98857400 & -2.23923300 & 0.52263200 \\ \mathrm{H} & 2.98001000 & 1.38390600 & 2.52772900 \\ \mathrm{H} & 6.41930000 & 0.60326700 & 3.79572200 \\ \mathrm{H} & 6.30532700 & 0.94618400 & 5.52789200 \\ \mathrm{H} & 6.29810900 & -0.72631100 & 4.96963100 \\ \mathrm{H} & 4.07436800 & -1.31616200 & 6.02924500 \\ \mathrm{H} & 4.14142500 & 0.27503400 & 6.81191200 \\ \mathrm{H} & 2.69751900 & -0.21117200 & 5.89558000 \\ \mathrm{H} & 2.95740400 & 2.01565000 & 4.58231700 \\ \mathrm{H} & 4.41197600 & 2.32313200 & 5.54533500 \\ \mathrm{H} & 4.49942700 & 2.38218100 & 3.78365700 \\ \mathrm{H} & 3.09009400 & -5.85290200 & 3.39756100 \\ \mathrm{H} & 4.51479300 & -4.85210100 & 3.64815600 \\ \mathrm{H} & 2.97893900 & -4.38712100 & 4.38572300 \\ \mathrm{H} & 1.35528600 & -5.44193300 & 2.02755600 \\ \mathrm{H} & 0.91663200 & -3.92030900 & 2.83082800 \\ \mathrm{H} & 1.11738700 & -3.98254200 & 1.07166500 \\ \mathrm{H} & 3.44547100 & -4.20638300 & 0.05119200 \\ \mathrm{H} & & & \end{array}$




\begin{tabular}{|c|c|c|c|}
\hline t & 1.89131100 & 0.98422600 & -2.84733500 \\
\hline $\mathrm{H}$ & 7.91744300 & 1.61413200 & 1.02587300 \\
\hline & 6.26210500 & 2.11629400 & 1.38445100 \\
\hline & 6.58225600 & 0.48742800 & 0.75325600 \\
\hline 1 & 7.43677500 & 3.95590600 & -1.71010000 \\
\hline H & 6.51704500 & 4.19143300 & -0.20680400 \\
\hline $\mathrm{H}$ & 8.19379200 & 3.62704600 & -0.15180300 \\
\hline & 4.75113500 & 4.47065500 & -4.12370200 \\
\hline & 5.60141800 & 4.81355500 & -2.59674000 \\
\hline $\mathrm{H}$ & 6.51277600 & 4.75220900 & -4.13271300 \\
\hline $\mathrm{H}$ & 7.78164800 & 1.60210000 & -2.65802600 \\
\hline U & 8.79048100 & 1.34832200 & -1.22030700 \\
\hline 11 & 7.50306200 & 0.19392300 & -1.61314500 \\
\hline $\mathrm{H}$ & 1.51379300 & 2.67938100 & -4.55032500 \\
\hline $\mathrm{H}$ & 2.76426100 & 3.88790500 & -4.87386000 \\
\hline $\mathrm{H}$ & 2.17016000 & 2.85705700 & -6.18620300 \\
\hline 11 & 4.41483000 & 1.86569200 & -6.78122700 \\
\hline $\mathrm{H}$ & 5.10003300 & 3.09384100 & -5.72543700 \\
\hline $\mathrm{H}$ & 5.56603800 & 1.40195100 & -5.51886900 \\
\hline 11 & 3.71538000 & -0.36040000 & -5.00826600 \\
\hline $\mathrm{H}$ & 2.05139300 & 0.15435000 & -4.70690800 \\
\hline $\mathrm{H}$ & 2.76875100 & 0.40505900 & -6.29679700 \\
\hline $\mathrm{H}$ & -3.61811900 & 1.62491400 & 1.72122000 \\
\hline$\Pi$ & -1.90844800 & 1.42898800 & -2.17209700 \\
\hline $\mathrm{H}$ & -4.52847200 & 5.12903300 & -3.71082600 \\
\hline $\mathrm{H}$ & -3.20597800 & 5.75189800 & -2.71985100 \\
\hline$H$ & -2.96727900 & 5.46092000 & -4.45083700 \\
\hline $\mathrm{H}$ & -3.09078700 & 3.11478400 & -5.39995000 \\
\hline $\mathrm{H}$ & -3.00388100 & 1.70630000 & -4.32790400 \\
\hline $\mathrm{H}$ & -4.50629100 & 2.63111500 & -4.45319100 \\
\hline $\mathrm{H}$ & -1.09134900 & 4.21424600 & -4.30213600 \\
\hline $\mathrm{H}$ & -0.99497700 & 4.35578100 & -2.53500800 \\
\hline $\mathrm{H}$ & -0.89219400 & 2.76998300 & -3.31193700 \\
\hline $\mathrm{H}$ & -5.25665300 & 5.88545500 & 2.24726000 \\
\hline 11 & -3.64759600 & 5.40773600 & 1.66310200 \\
\hline $\mathrm{H}$ & -4.91309100 & 5.84744300 & 0.50690800 \\
\hline $\mathrm{H}$ & -5.92995700 & 3.60165000 & -3.13793300 \\
\hline
\end{tabular}




\begin{tabular}{|c|c|c|c|}
\hline $\mathrm{H}$ & -6.82728100 & 3.26157500 & -1.63839700 \\
\hline$H$ & -6.91547900 & 4.88351700 & -2.38474800 \\
\hline $\mathrm{H}$ & -5.35739600 & 2.24522200 & 2.90102500 \\
\hline $\mathrm{H}$ & -3.92072600 & 3.26532100 & 3.11723700 \\
\hline $\mathrm{H}$ & -5.52560900 & 3.87558300 & 3.55399800 \\
\hline $\mathrm{H}$ & -3.73813400 & -5.21235900 & -2.37481000 \\
\hline $\mathrm{H}$ & -3.65516500 & -6.87171200 & -1.75659700 \\
\hline $\mathrm{H}$ & -5.06188000 & -5.85756800 & -1.38084100 \\
\hline $\mathrm{H}$ & -1.73104300 & -3.06140400 & -0.09451900 \\
\hline $\mathrm{H}$ & -4.83751600 & -0.16264000 & 0.03602900 \\
\hline $\mathrm{H}$ & -7.04542600 & -5.55345400 & 1.84117900 \\
\hline $\mathrm{H}$ & -5.37563200 & -5.14126800 & 2.31822100 \\
\hline $\mathrm{H}$ & -6.62831800 & -3.88251200 & 2.31294500 \\
\hline $\mathrm{H}$ & -3.02551600 & -7.29028700 & 0.71688200 \\
\hline $\mathrm{H}$ & -3.16841800 & -5.96103200 & 1.87378700 \\
\hline $\mathrm{H}$ & -4.60265900 & -6.56586900 & 1.00827800 \\
\hline $\mathrm{H}$ & -8.15000700 & -3.18765300 & 1.06207200 \\
\hline $\mathrm{H}$ & -7.61721200 & -1.69690400 & 1.87510200 \\
\hline $\mathrm{H}$ & -8.95293700 & -1.66059800 & 0.71578200 \\
\hline $\mathrm{H}$ & -8.18321000 & -0.11830500 & -0.85187900 \\
\hline $\mathrm{H}$ & -6.91621700 & 0.31555400 & 0.29968800 \\
\hline $\mathrm{H}$ & -6.50326500 & -0.01752400 & -1.39508100 \\
\hline $\mathrm{H}$ & -1.43161700 & -4.78912100 & -1.35108600 \\
\hline $\mathrm{H}$ & -1.14200800 & -5.14333100 & 0.36536100 \\
\hline $\mathrm{H}$ & -1.46854500 & -6.45467400 & -0.77146500 \\
\hline $\mathrm{H}$ & -7.06054900 & 2.79302900 & 0.99588000 \\
\hline $\mathrm{H}$ & -7.25269600 & 4.24181000 & 1.99346400 \\
\hline $\mathrm{H}$ & -6.98198600 & 4.40817300 & 0.25880000 \\
\hline $\mathrm{H}$ & -8.50197000 & -2.34621600 & -1.73587600 \\
\hline $\mathrm{H}$ & -6.83938200 & -2.36609000 & -2.35334600 \\
\hline $\mathrm{H}$ & -7.41854000 & -3.68888500 & -1.32059900 \\
\hline $\mathrm{Cu}$ & -0.01628800 & -0.72185400 & -1.19921700 \\
\hline $\mathrm{H}$ & -1.44252200 & -3.08360800 & -2.90401200 \\
\hline $\mathrm{C}$ & -0.84061700 & -2.43437500 & -3.55976200 \\
\hline $\mathrm{H}$ & -0.53499800 & -3.07676700 & -4.40681300 \\
\hline $\mathrm{C}$ & 0.37604900 & -1.88604500 & -2.78747400 \\
\hline H & 0.93355200 & -1.24442400 & -3.4935790 \\
\hline
\end{tabular}




$\begin{array}{lrrr}\mathrm{C} & 1.35059100 & -3.00972200 & -2.36403200 \\ \mathrm{H} & 2.17516800 & -2.58827500 & -1.77251000 \\ \mathrm{H} & 0.84347600 & -3.73523400 & -1.70968800 \\ \mathrm{C} & -1.76107900 & -1.34919400 & -4.13217200 \\ \mathrm{H} & -2.13777700 & -0.73111100 & -3.30539500 \\ \mathrm{H} & -1.16750100 & -0.67841700 & -4.77243300 \\ \mathrm{C} & 1.99270500 & -3.78618500 & -3.51266000 \\ \mathrm{H} & 2.54093600 & -3.12153200 & -4.18977100 \\ \mathrm{H} & 1.26952900 & -4.34815500 & -4.10983200 \\ \mathrm{O} & 2.93583000 & -4.73319800 & -2.93415600 \\ \mathrm{C} & 3.59643400 & -5.52826500 & -3.79738700 \\ \mathrm{C} & 4.53767200 & -6.45933400 & -3.06114100 \\ \mathrm{H} & 5.26109300 & -5.88090000 & -2.47682400 \\ \mathrm{H} & 3.97709400 & -7.08455600 & -2.35801000 \\ \mathrm{H} & 5.06200000 & -7.08999000 & -3.78030700 \\ \mathrm{O} & 3.45159200 & -5.49682300 & -5.00150700 \\ \mathrm{C} & -2.94095300 & -1.90414900 & -4.93871300 \\ \mathrm{H} & -3.56917400 & -2.55755200 & -4.31941400 \\ \mathrm{H} & -3.58000900 & -1.10177300 & -5.32841200 \\ \mathrm{H} & -2.59345000 & -2.49702800 & -5.79426600\end{array}$

$6 c$

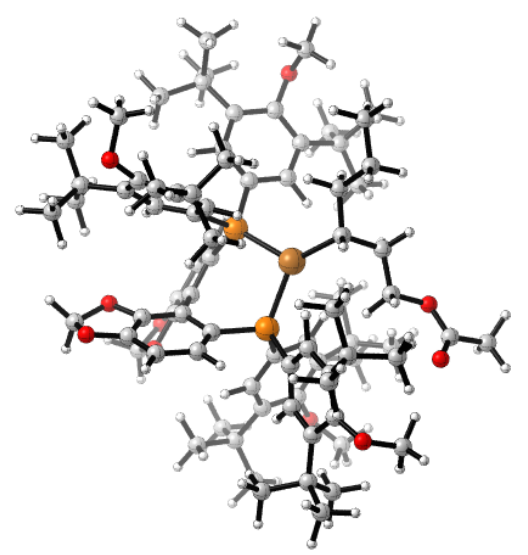

Thermal correction to Enthalpy=

1.928485

Thermal correction to Gibbs Free Energy $=\quad 1.671974$

Sum of electronic and zero-point Energies $=\quad-4824.326591$

Sum of electronic and thermal Enthalpies $=\quad-4824.219258$

Sum of electronic and thermal Free Energies $=\quad-4824.475768$ 
SMD E $($ M06 $)=-4824.505372$

Cartesian coordinates:

$\mathrm{P}$

$\mathrm{P}$

\section{O}

O

$\begin{array}{lllll}\mathrm{O} & 0.36714700 & -3.23343900 & -3.73639800\end{array}$

$\begin{array}{lllll}\mathrm{O} & -0.40457700 & -2.13944300 & -5.61129200\end{array}$

$\begin{array}{lllll}\mathrm{O} & 4.82759300 & 0.89347600 & -4.65285800\end{array}$

$\begin{array}{llll}\mathrm{O} & 6.24879500 & -0.44541300 & 3.90822000\end{array}$

$\begin{array}{lllll}\mathrm{O} & -5.31489000 & -3.60213700 & 2.91754400\end{array}$

$\begin{array}{lllll}\mathrm{O} & -5.99234400 & 4.49209500 & -1.00649300\end{array}$

$\begin{array}{lllll}\mathrm{C} & 0.88604800 & -2.25035100 & 0.02137000\end{array}$

$\begin{array}{llll}\text { C } & -0.12181000 & -2.58955500 & -0.94050100\end{array}$

$\begin{array}{lllll}\text { C } & -0.57035300 & -3.90293100 & -0.89432900\end{array}$

$\begin{array}{lllll}\text { C } & -0.13710000 & -4.84210400 & 0.03797000\end{array}$

$\begin{array}{lllll}\text { C } & 0.79978800 & -4.52107200 & 0.99422100\end{array}$

$\begin{array}{llll}\text { C } & 1.30461700 & -3.21043400 & 0.95590900\end{array}$

$\begin{array}{lllll}\text { C } & -1.40568000 & -5.89151400 & -1.44818900\end{array}$

$\begin{array}{llll}\text { C } & -1.50399600 & -0.58352100 & -1.93155200\end{array}$

$\begin{array}{lllll}\text { C } & -1.96155500 & 0.06198700 & -3.09067500\end{array}$

$\begin{array}{lllll}\text { C } & -1.64663800 & -0.37034700 & -4.38986800\end{array}$

$\begin{array}{lllll}\text { C } & -0.86171800 & -1.49713200 & -4.48458900\end{array}$

$\begin{array}{lllll}\text { C } & -0.39423900 & -2.15030000 & -3.34710000\end{array}$

$\begin{array}{lllll}\text { C } & -0.65449500 & -1.73251600 & -2.04878400\end{array}$

$\begin{array}{lllll}\text { C } & 0.10266700 & -3.38711700 & -5.13246500\end{array}$

$\begin{array}{lllll}\text { C } & 2.39145300 & -0.17687300 & -1.44263000\end{array}$

$\begin{array}{llll}\text { C } & 2.47006100 & 1.15405600 & -1.85007000\end{array}$

$\begin{array}{lllll}\text { C } & 3.19424600 & 1.55936000 & -2.98425700\end{array}$

$\begin{array}{lllll}\text { C } & 3.93194300 & 0.55407700 & -3.65030400\end{array}$

$\begin{array}{lllll}\text { C } & 3.76585600 & -0.82875100 & -3.35982800\end{array}$

$\begin{array}{llll}\text { C } & 2.98941200 & -1.15859100 & -2.24364300\end{array}$

$\begin{array}{lllll}\text { C } & 4.33628400 & -1.94232300 & -4.27694600\end{array}$

$\begin{array}{lllll}\text { C } & 5.88171800 & -1.92603900 & -4.36349500\end{array}$

$\begin{array}{lllll}\text { C } & 3.74601900 & -1.76200200 & -5.69822600\end{array}$

$\begin{array}{llll}\text { C } & 3.93925000 & -3.34874500 & -3.77606100\end{array}$

$\begin{array}{lllll}\text { C } & 3.06896500 & 3.05096900 & -3.40779700\end{array}$ 


\begin{tabular}{|c|c|c|c|}
\hline $\mathrm{C}$ & 3.55876900 & 3.37620400 & -4.83859300 \\
\hline $\mathrm{C}$ & 1.56406900 & 3.43681800 & -3.39159400 \\
\hline $\mathrm{C}$ & 3.81498500 & 3.95790700 & -2.39860800 \\
\hline $\mathrm{C}$ & 6.08022700 & 1.38111600 & -4.16919800 \\
\hline $\mathrm{C}$ & 2.99683000 & -0.69792900 & 1.31962600 \\
\hline $\mathrm{C}$ & 2.81873000 & -0.33294400 & 2.66114400 \\
\hline $\mathrm{C}$ & 4.24829800 & -1.16500700 & 0.91923100 \\
\hline $\mathrm{C}$ & 5.35288600 & -1.20482500 & 1.78445200 \\
\hline $\mathrm{C}$ & 5.16494100 & -0.65931200 & 3.07607200 \\
\hline $\mathrm{C}$ & 3.87761200 & -0.32533200 & 3.57543200 \\
\hline $\mathrm{C}$ & 3.62690500 & -0.02231200 & 5.07501300 \\
\hline $\mathrm{C}$ & 2.12210800 & 0.16072300 & 5.36929400 \\
\hline $\mathrm{C}$ & 4.34431800 & 1.26440300 & 5.55299300 \\
\hline $\mathrm{C}$ & 6.96828700 & 0.76168200 & 3.61503800 \\
\hline $\mathrm{C}$ & 4.11495900 & -1.22667700 & 5.91906200 \\
\hline $\mathrm{C}$ & 6.65149500 & -1.86763800 & 1.24497000 \\
\hline $\mathrm{C}$ & 7.30506700 & -0.95885400 & 0.17626800 \\
\hline $\mathrm{C}$ & 7.70401700 & -2.22463400 & 2.32008300 \\
\hline $\mathrm{C}$ & 6.27396400 & -3.21792600 & 0.57522300 \\
\hline $\mathrm{C}$ & -2.84454000 & -1.14074600 & 0.63235400 \\
\hline $\mathrm{C}$ & -3.66781100 & -2.09317500 & 0.01757000 \\
\hline $\mathrm{C}$ & -4.46596400 & -2.96829200 & 0.76233000 \\
\hline $\mathrm{C}$ & -4.41297000 & -2.85068300 & 2.17920100 \\
\hline $\mathrm{C}$ & -3.45869800 & -2.03994300 & 2.83413500 \\
\hline $\mathrm{C}$ & -2.72759800 & -1.15670900 & 2.02160500 \\
\hline $\mathrm{C}$ & -3.09818000 & -2.06541100 & 4.34688000 \\
\hline $\mathrm{C}$ & -3.63789000 & -3.28028000 & 5.13693400 \\
\hline $\mathrm{C}$ & -3.57689200 & -0.76240600 & 5.03069600 \\
\hline $\mathrm{C}$ & -1.55058800 & -2.14432300 & 4.47365300 \\
\hline $\mathrm{C}$ & -4.81355900 & -5.45632700 & 0.57059900 \\
\hline $\mathrm{C}$ & -6.41434800 & -2.84908300 & 3.43051100 \\
\hline $\mathrm{C}$ & -5.29084900 & -4.07514800 & 0.05482200 \\
\hline $\mathrm{C}$ & -5.08024500 & -4.04736400 & -1.47530800 \\
\hline $\mathrm{C}$ & -5.05580900 & 3.46534700 & -1.01537900 \\
\hline $\mathrm{C}$ & -5.51320300 & 2.12273700 & -1.02343900 \\
\hline $\mathrm{C}$ & -7.30601100 & 2.34895000 & -2.77731000 \\
\hline & -4.54901000 & 1.12826100 & -0.81028000 \\
\hline
\end{tabular}




\begin{tabular}{|c|c|c|c|}
\hline C & -3.19183500 & 1.42668200 & -0.65826000 \\
\hline $\mathrm{C}$ & -2.77408000 & 2.74905300 & -0.83790500 \\
\hline $\mathrm{C}$ & -3.67824900 & 3.79665300 & -1.06553100 \\
\hline $\mathrm{C}$ & -6.34464000 & 4.92584300 & 0.31507100 \\
\hline $\mathrm{C}$ & -8.03084200 & 2.18284400 & -0.36989300 \\
\hline $\mathrm{C}$ & -3.49686800 & 6.32919700 & -0.48571800 \\
\hline $\mathrm{C}$ & -3.15985900 & 5.19957700 & -1.48725400 \\
\hline $\mathrm{C}$ & -1.62432300 & 5.20033800 & -1.65731100 \\
\hline $\mathrm{C}$ & -7.11620300 & 0.19629400 & -1.53892900 \\
\hline $\mathrm{C}$ & -6.81377700 & -3.94055200 & 0.29435700 \\
\hline $\mathrm{C}$ & -6.96868700 & 1.72826000 & -1.39822900 \\
\hline $\mathrm{C}$ & -3.77494500 & 5.55142200 & -2.86553200 \\
\hline $\mathrm{H}$ & 1.14465700 & -5.24411500 & 1.72527300 \\
\hline $\mathrm{H}$ & 2.06638400 & -2.93971900 & 1.67701800 \\
\hline $\mathrm{H}$ & -2.40334900 & -6.33143800 & -1.42398400 \\
\hline $\mathrm{H}$ & -0.77248800 & -6.36458800 & -2.21387800 \\
\hline $\mathrm{H}$ & -2.60257600 & 0.92918700 & -2.98872800 \\
\hline $\mathrm{H}$ & -2.01726200 & 0.14621700 & -5.26852100 \\
\hline $\mathrm{H}$ & 1.03016700 & -3.62756100 & -5.65362700 \\
\hline $\mathrm{H}$ & -0.65662400 & -4.17060400 & -5.27565400 \\
\hline $\mathrm{H}$ & 1.94816200 & 1.90144100 & -1.26190900 \\
\hline $\mathrm{H}$ & 2.84867700 & -2.19772200 & -1.97540600 \\
\hline $\mathrm{H}$ & 6.33774900 & -1.92947800 & -3.36607300 \\
\hline $\mathrm{H}$ & 6.22837800 & -2.82556000 & -4.88707900 \\
\hline $\mathrm{H}$ & 6.25179300 & -1.06290200 & -4.91603900 \\
\hline $\mathrm{H}$ & 4.04597600 & -0.80541400 & -6.13177200 \\
\hline $\mathrm{H}$ & 4.09991800 & -2.56448100 & -6.35823200 \\
\hline $\mathrm{H}$ & 2.65048000 & -1.79590300 & -5.67453700 \\
\hline $\mathrm{H}$ & 2.85548600 & -3.47346000 & -3.69486400 \\
\hline $\mathrm{H}$ & 4.31313400 & -4.09617400 & -4.48524900 \\
\hline $\mathrm{H}$ & 4.38407300 & -3.57785700 & -2.80000200 \\
\hline $\mathrm{H}$ & 3.24135900 & 4.39612700 & -5.08714300 \\
\hline $\mathrm{H}$ & 4.64296000 & 3.34323700 & -4.94686700 \\
\hline $\mathrm{H}$ & 3.12516600 & 2.69863800 & -5.57965100 \\
\hline $\mathrm{H}$ & 1.44852100 & 4.47722100 & -3.71674400 \\
\hline $\mathrm{H}$ & 0.99018300 & 2.80031100 & -4.07465100 \\
\hline $\mathrm{H}$ & 1.11361400 & 3.35834700 & -2.39895300 \\
\hline
\end{tabular}




\begin{tabular}{|c|c|c|c|}
\hline t & 3.42289600 & 3.84662400 & -1.38174600 \\
\hline $\mathrm{H}$ & 4.88720600 & 3.73270400 & -2.36863800 \\
\hline & 3.70267800 & 5.01096700 & -2.68574200 \\
\hline & 6.56748400 & 0.64832500 & -3.51557400 \\
\hline H & 6.70380800 & 1.55464400 & -5.05007200 \\
\hline H & 5.96567900 & 2.32024400 & -3.61780000 \\
\hline $\mathrm{H}$ & 1.83269200 & -0.01289900 & 2.97541400 \\
\hline & 4.37643900 & -1.49480100 & -0.10412500 \\
\hline & 1.98526300 & 0.29707400 & 6.44792100 \\
\hline $\mathrm{H}$ & 1.70416600 & 1.04308500 & 4.87222300 \\
\hline $\mathrm{H}$ & 1.53266100 & -0.71380800 & 5.07089400 \\
\hline$U$ & 5.42143900 & 1.11713100 & 5.63839200 \\
\hline 11 & 4.16454400 & 2.10352600 & 4.87245400 \\
\hline $\mathrm{H}$ & 3.97045900 & 1.54467600 & 6.54575000 \\
\hline $\mathrm{H}$ & 7.56510800 & 0.65675800 & 2.70150600 \\
\hline $\mathrm{H}$ & 6.29348100 & 1.61486500 & 3.49959700 \\
\hline$\Pi$ & 7.64067500 & 0.92785400 & 4.46086000 \\
\hline $\mathrm{H}$ & 5.18145000 & -1.40999400 & 5.77227000 \\
\hline $\mathrm{H}$ & 3.94266900 & -1.02954900 & 6.98478500 \\
\hline 11 & 3.56472700 & -2.13778200 & 5.65400200 \\
\hline $\mathrm{H}$ & 6.61412900 & -0.75364700 & -0.64952800 \\
\hline $\mathrm{H}$ & 7.61109300 & 0.00454800 & 0.59815200 \\
\hline $\mathrm{H}$ & 8.19751600 & -1.44270000 & -0.24070600 \\
\hline $\mathrm{H}$ & 8.48246800 & -2.83993000 & 1.85189100 \\
\hline $\mathrm{H}$ & 8.19510200 & -1.35418100 & 2.75411100 \\
\hline $\mathrm{H}$ & 7.26455000 & -2.80332500 & 3.13795800 \\
\hline $\mathrm{H}$ & 5.78295600 & -3.88594100 & 1.29199500 \\
\hline $\mathrm{H}$ & 5.60763100 & -3.09976400 & -0.28409500 \\
\hline $\mathrm{H}$ & 7.18209200 & -3.71634100 & 0.21616600 \\
\hline $\mathrm{H}$ & -3.68157100 & -2.14111100 & -1.06365100 \\
\hline 11 & -2.03503700 & -0.46098000 & 2.48382200 \\
\hline $\mathrm{H}$ & -4.71642200 & -3.25436300 & 5.29386600 \\
\hline $\mathrm{H}$ & -3.39126400 & -4.22599700 & 4.64577400 \\
\hline $\mathrm{H}$ & -3.16804400 & -3.28183200 & 6.12784400 \\
\hline $\mathrm{H}$ & -3.27137700 & -0.75366100 & 6.08430900 \\
\hline $\mathrm{H}$ & -3.14947400 & 0.12427400 & 4.54983100 \\
\hline $\mathrm{H}$ & -4.66794500 & -0.66400500 & 4.99892200 \\
\hline
\end{tabular}




\begin{tabular}{|c|c|c|c|}
\hline t & -1.27286100 & -2.21501300 & 5.53178600 \\
\hline $\mathrm{H}$ & -1.16048200 & -3.02912800 & 3.95818100 \\
\hline & -1.04322800 & -1.26635800 & 4.06508500 \\
\hline & -5.34574000 & -6.25985300 & 0.04526700 \\
\hline H & -3.73896500 & -5.58675800 & 0.39823800 \\
\hline H & -4.99914300 & -5.56592400 & 1.64112000 \\
\hline $\mathrm{H}_{\mathrm{H}}$ & -6.08652300 & -2.07767600 & 4.13541000 \\
\hline & -6.97978300 & -2.36897000 & 2.62316500 \\
\hline & -7.05883100 & -3.56182200 & 3.95161100 \\
\hline $\mathrm{H}$ & -5.46127000 & -3.12309000 & -1.92609600 \\
\hline $\mathrm{H}$ & -4.02732000 & -4.15435700 & -1.75307700 \\
\hline U & -5.63398900 & -4.87912400 & -1.92568600 \\
\hline 11 & -6.61638300 & 1.98500500 & -3.54793000 \\
\hline $\mathrm{H}$ & -8.32372400 & 2.06824300 & -3.07690300 \\
\hline $\mathrm{H}$ & -7.24616300 & 3.43900200 & -2.74594300 \\
\hline$H$ & -4.85883500 & 0.09409200 & -0.75501700 \\
\hline$\Pi$ & -1.71277300 & 2.95889900 & -0.78389300 \\
\hline $\mathrm{H}$ & -7.06702200 & 5.73628800 & 0.18720500 \\
\hline $\mathrm{H}$ & -6.80038600 & 4.11785000 & 0.89408100 \\
\hline $\mathrm{H}$ & -5.47310700 & 5.29549000 & 0.86174800 \\
\hline $\mathrm{H}$ & -8.99280000 & 1.71044900 & -0.60407400 \\
\hline $\mathrm{H}$ & -7.75590400 & 1.88667600 & 0.64939800 \\
\hline $\mathrm{H}$ & -8.18707400 & 3.26173400 & -0.39469000 \\
\hline $\mathrm{H}$ & -4.55593200 & 6.58952800 & -0.50456100 \\
\hline $\mathrm{H}$ & -3.21377900 & 6.05766900 & 0.53792400 \\
\hline $\mathrm{H}$ & -2.93659600 & 7.23302300 & -0.75407900 \\
\hline $\mathrm{H}$ & -1.30980600 & 6.17822800 & -2.03848200 \\
\hline $\mathrm{H}$ & -1.09959600 & 5.03475500 & -0.70938700 \\
\hline $\mathrm{H}$ & -1.28824700 & 4.44471000 & -2.37530900 \\
\hline $\mathrm{H}$ & -6.41844100 & -0.22078200 & -2.27328100 \\
\hline $\mathrm{H}$ & -6.96619500 & -0.32538800 & -0.58638700 \\
\hline $\mathrm{H}$ & -8.13123000 & -0.03258400 & -1.88209600 \\
\hline $\mathrm{H}$ & -7.16805000 & -2.93270000 & 0.04604100 \\
\hline $\mathrm{H}$ & -7.35022100 & -4.64699600 & -0.35107500 \\
\hline 11 & -7.08811100 & -4.16571400 & 1.32474800 \\
\hline $\mathrm{H}$ & -3.40953800 & 6.53059700 & -3.19996500 \\
\hline $\mathrm{H}$ & -3.48915800 & 4.81053700 & -3.62149700 \\
\hline
\end{tabular}




$\begin{array}{lrrr}\mathrm{H} & -4.86531100 & 5.59183200 & -2.81475800 \\ \mathrm{Cu} & -0.04980400 & 1.02526200 & 0.92348000 \\ \mathrm{H} & 0.63280500 & 4.11514000 & 0.93425600 \\ \mathrm{C} & 0.89605900 & 3.69586400 & 1.91728500 \\ \mathrm{C} & -0.03729600 & 2.51600300 & 2.27739000 \\ \mathrm{H} & 0.36762000 & 2.06841600 & 3.20532800 \\ \mathrm{H} & 0.81107100 & 4.53236100 & 2.63196200 \\ \mathrm{C} & -1.45524800 & 3.01973800 & 2.63292400 \\ \mathrm{H} & -1.87114700 & 3.59376700 & 1.78981000 \\ \mathrm{H} & -2.13269700 & 2.15978600 & 2.75205500 \\ \mathrm{C} & -1.57851900 & 3.87594100 & 3.91119600 \\ \mathrm{H} & -1.17235300 & 3.30707100 & 4.76105000 \\ \mathrm{H} & -0.95913400 & 4.77888200 & 3.82703800 \\ \mathrm{C} & -3.02197400 & 4.29040600 & 4.22129300 \\ \mathrm{H} & -3.66774300 & 3.41140000 & 4.34763900 \\ \mathrm{H} & -3.08819300 & 4.88696800 & 5.13965100 \\ \mathrm{H} & -3.44453600 & 4.89101400 & 3.40465800 \\ \mathrm{C} & 2.35719400 & 3.28228200 & 1.89898400 \\ \mathrm{H} & 2.55188300 & 2.49046900 & 1.16967900 \\ \mathrm{H} & 2.68611600 & 2.92543300 & 2.87715900 \\ \mathrm{O} & 3.17412500 & 4.43205800 & 1.52939900 \\ \mathrm{C} & 4.45041400 & 4.44876100 & 1.94893400 \\ \mathrm{O} & 4.97187100 & 3.57662200 & 2.61684600 \\ \mathrm{C} & 5.15570900 & 5.70218500 & 1.47503100 \\ \mathrm{H} & 5.20418300 & 5.71203500 & 0.38063200 \\ \mathrm{H} & 4.59788800 & 6.59189800 & 1.78399100 \\ \mathrm{H} & 6.16522500 & 5.73134100 & 1.88726100 \\ & & & \\ \mathrm{H} & & \end{array}$

6d 


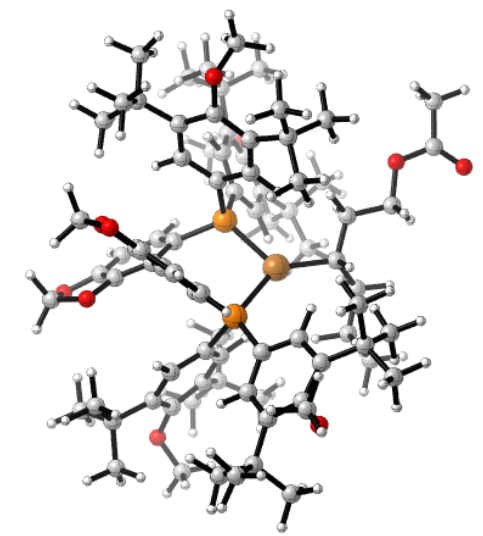

Thermal correction to Enthalpy=

1.928497

Thermal correction to Gibbs Free Energy=

1.671691

Sum of electronic and zero-point Energies= $-4824.321275$

Sum of electronic and thermal Enthalpies= $-4824.213884$

Sum of electronic and thermal Free Energies= $-4824.470690$

SMD E $(\mathrm{M} 06)=-4824.505544$

Cartesian coordinates:

$\begin{array}{lrrr}\mathrm{P} & 1.53678200 & -0.53601100 & -0.14459300 \\ \mathrm{P} & -1.92037800 & 0.30983500 & 0.01573800 \\ \mathrm{O} & 0.06420200 & -1.09724800 & -4.76445000 \\ \mathrm{O} & -0.74530600 & 0.76158700 & -5.85664100 \\ \mathrm{O} & -1.73235200 & -3.13751700 & -3.48902100 \\ \mathrm{O} & -0.98071000 & -5.23442500 & -2.90504900 \\ \mathrm{O} & -4.99241600 & -4.66781200 & 1.34505500 \\ \mathrm{O} & -6.13198600 & 4.58631200 & 0.33759300 \\ \mathrm{O} & 4.51604800 & 2.89875400 & -4.06141700 \\ \mathrm{O} & 5.97170500 & -3.41978300 & 2.70337100 \\ \mathrm{C} & -1.61369800 & 0.41523000 & -1.81732200 \\ \mathrm{C} & -0.81695000 & -0.55403900 & -2.51152600 \\ \mathrm{C} & -0.63323800 & -0.31359000 & -3.86732200 \\ \mathrm{C} & -1.12243800 & 0.80561600 & -4.53493900 \\ \mathrm{C} & -1.85165700 & 1.76799200 & -3.87408400 \\ \mathrm{C} & -2.09114700 & 1.53998900 & -2.50877600 \\ \mathrm{C} & -0.27818400 & -0.57548700 & -6.04997800 \\ \mathrm{C} & 0.80511100 & -1.99792300 & -1.03012600 \\ \mathrm{C} & 1.24257800 & -3.28793700 & -0.69101700 \\ \mathrm{C} & 0.70469000 & -4.45778400 & -1.25361300\end{array}$




\begin{tabular}{|c|c|c|c|}
\hline $\mathrm{C}$ & -0.28603900 & -4.28364700 & -2.19344300 \\
\hline $\mathrm{C}$ & -0.73912200 & -3.01326900 & -2.53871300 \\
\hline $\mathrm{C}$ & -0.25880300 & -1.83767500 & -1.97720100 \\
\hline $\mathrm{C}$ & -1.64927100 & -4.49433700 & -3.92932900 \\
\hline $\mathrm{C}$ & -2.76046600 & -1.31012300 & 0.28315500 \\
\hline $\mathrm{C}$ & -2.49280900 & -1.98339900 & 1.47419200 \\
\hline $\mathrm{C}$ & -3.13960500 & -3.17853000 & 1.83323800 \\
\hline $\mathrm{C}$ & -4.16674100 & -3.61973000 & 0.96882900 \\
\hline $\mathrm{C}$ & -4.37218900 & -3.05182200 & -0.31908800 \\
\hline $\mathrm{C}$ & -3.65225700 & -1.89267000 & -0.62819100 \\
\hline $\mathrm{C}$ & -5.27736200 & -3.72554700 & -1.38342800 \\
\hline $\mathrm{C}$ & -6.76535300 & -3.78422300 & -0.96217000 \\
\hline $\mathrm{C}$ & -4.76392400 & -5.16624100 & -1.63328000 \\
\hline $\mathrm{C}$ & -5.22673600 & -2.96821100 & -2.72884400 \\
\hline $\mathrm{C}$ & -2.62173400 & -3.90961100 & 3.10467200 \\
\hline $\mathrm{C}$ & -3.08857700 & -5.37533900 & 3.26467100 \\
\hline $\mathrm{C}$ & -1.07137000 & -3.97973600 & 3.02219000 \\
\hline $\mathrm{C}$ & -3.00878900 & -3.11335100 & 4.37386600 \\
\hline $\mathrm{C}$ & -6.02227700 & -4.29400200 & 2.26166900 \\
\hline $\mathrm{C}$ & -3.27235000 & 1.55178000 & 0.27629200 \\
\hline $\mathrm{C}$ & -4.63180400 & 1.26162100 & 0.10439500 \\
\hline $\mathrm{C}$ & -2.91524900 & 2.84134500 & 0.67483900 \\
\hline $\mathrm{C}$ & -3.85231400 & 3.88458900 & 0.79760800 \\
\hline $\mathrm{C}$ & -5.18448600 & 3.57875000 & 0.43727300 \\
\hline $\mathrm{C}$ & -5.61925900 & 2.24594700 & 0.20351300 \\
\hline $\mathrm{C}$ & -7.12068900 & 1.86137400 & 0.14486900 \\
\hline $\mathrm{C}$ & -7.30749600 & 0.33191000 & 0.04067900 \\
\hline $\mathrm{C}$ & -7.85574400 & 2.48451500 & -1.06596800 \\
\hline $\mathrm{C}$ & -6.07026300 & 5.31483100 & -0.88866400 \\
\hline $\mathrm{C}$ & -7.81013800 & 2.31936000 & 1.45454600 \\
\hline $\mathrm{C}$ & -3.33730300 & 5.24304700 & 1.35569500 \\
\hline $\mathrm{C}$ & -2.40602100 & 5.91630400 & 0.31893800 \\
\hline $\mathrm{C}$ & -4.43043000 & 6.25349700 & 1.77648300 \\
\hline $\mathrm{C}$ & -2.52020300 & 4.96395700 & 2.64577700 \\
\hline $\mathrm{C}$ & 2.31565200 & 0.48676000 & -1.46601000 \\
\hline $\mathrm{C}$ & 2.84988100 & -0.03759800 & -2.65027700 \\
\hline $\mathrm{C}$ & 3.54564200 & 0.76290700 & -3.56293600 \\
\hline
\end{tabular}




$\begin{array}{lrrr}\mathrm{C} & 3.70115600 & 2.13902400 & -3.23611700 \\ \mathrm{C} & 3.02419700 & 2.73861600 & -2.14927400 \\ \mathrm{C} & 2.37992900 & 1.86409200 & -1.25799500 \\ \mathrm{C} & 2.88559900 & 4.26337600 & -1.87389100 \\ \mathrm{C} & 3.19224900 & 5.17250300 & -3.08767200 \\ \mathrm{C} & 3.78108300 & 4.67975200 & -0.68151900 \\ \mathrm{C} & 1.40782400 & 4.56556000 & -1.49645600 \\ \mathrm{C} & 3.38314400 & 0.96221300 & -6.07234700 \\ \mathrm{C} & 5.80939200 & 3.17134700 & -3.52094600 \\ \mathrm{C} & 4.03671200 & 0.17137000 & -4.91091400 \\ \mathrm{C} & 3.62075500 & -1.30880000 & -5.06229500 \\ \mathrm{C} & 5.02020200 & -2.60643600 & 2.09862000 \\ \mathrm{C} & 3.82782900 & -2.30329300 & 2.80433300 \\ \mathrm{C} & 4.82788200 & -1.76221400 & 5.04875000 \\ \mathrm{C} & 2.81999300 & -1.64670600 & 2.08145100 \\ \mathrm{C} & 2.98444900 & -1.27359200 & 0.74230200 \\ \mathrm{C} & 4.24355800 & -1.43090200 & 0.15620700 \\ \mathrm{H} & -1.74929900 & -1.56241000 & 2.14242800 \\ \mathrm{H} & -3.78344300 & -1.42049400 & -1.59338900 \\ \mathrm{H} & & & \\ \mathrm{H} & & & \end{array}$




$\begin{array}{llll}\mathrm{H} & -7.13571300 & -2.79508700 & -0.66792800 \\ \mathrm{H} & -7.37243900 & -4.12635200 & -1.80946600 \\ \mathrm{H} & -6.93010400 & -4.48054400 & -0.14008300 \\ \mathrm{H} & -4.84088400 & -5.77513600 & -0.73001200 \\ \mathrm{H} & -5.35729700 & -5.64563100 & -2.42258000 \\ \mathrm{H} & -3.71447700 & -5.15981300 & -1.94863400 \\ \mathrm{H} & -4.20857700 & -2.88425800 & -3.12046800 \\ \mathrm{H} & -5.82882900 & -3.51196500 & -3.46578300 \\ \mathrm{H} & -5.64779600 & -1.95866300 & -2.64863200 \\ \mathrm{H} & -2.51323600 & -5.83147400 & 4.07940500 \\ \mathrm{H} & -4.14295900 & -5.47209800 & 3.52421700 \\ \mathrm{H} & -2.90974100 & -5.95949000 & 2.35745000 \\ \mathrm{H} & -0.68309900 & -4.52794900 & 3.88862900 \\ \mathrm{H} & -0.75015800 & -4.50265200 & 2.11432600 \\ \mathrm{H} & -0.59891900 & -2.99396300 & 3.02425200 \\ \mathrm{H} & -2.62672200 & -2.08747700 & 4.34024300 \\ \mathrm{H} & -4.09569200 & -3.05744800 & 4.50206100 \\ \mathrm{H} & -2.59270500 & -3.59741700 & 5.26605000 \\ \mathrm{H} & -6.67201500 & -3.51971100 & 1.83692600 \\ \mathrm{H} & -6.60849700 & -5.19702400 & 2.45056700 \\ \mathrm{H} & -5.61072400 & -3.92621400 & 3.20740200 \\ \mathrm{H} & -1.55923800 & 5.27170100 & 0.05853700 \\ \mathrm{H} & -7.91835900 & 0.24239400 & -0.11055100 \\ \mathrm{H} & -1.87193800 & 3.03351000 & 0.90182200 \\ \mathrm{H} & -8.37798500 & 0.10072500 & 0.07528500 \\ \mathrm{H} & -6.91651200 & -0.07024800 & -0.90136300 \\ \mathrm{H} & -6.82603800 & -0.20123000 & 0.86769400 \\ \mathrm{H} & -7.96798700 & 3.56386900 & -0.96244700 \\ \mathrm{H} & -7.32898500 & 2.27204600 & -2.00421200 \\ \mathrm{H} & -8.86217400 & 2.05539100 & -1.14687700 \\ \mathrm{H} & -6.22104300 & 4.65613300 & -1.75224000 \\ \mathrm{H} & -6.87572400 & 6.05272700 & -0.84961000 \\ \mathrm{H} & -74220700 & 3.40156200 & 1.58263500 \\ \mathrm{H} & -1560300 & -0.60842800\end{array}$




\begin{tabular}{|c|c|c|c|}
\hline I & -2.00203500 & 6.85230500 & 0.72400400 \\
\hline $\mathrm{H}$ & -3.94570900 & 7.07507400 & 2.31759800 \\
\hline & -4.96519000 & 6.69635200 & 0.93568200 \\
\hline & -5.16772300 & 5.80054800 & 2.44498400 \\
\hline $\mathrm{H}$ & -3.14485900 & 4.47975100 & 3.40486200 \\
\hline $\mathrm{H}$ & -1.64776800 & 4.32881200 & 2.47624500 \\
\hline $\mathrm{H}$ & -2.15774500 & 5.91140900 & 3.06145100 \\
\hline & 2.71690300 & -1.09244900 & -2.85222800 \\
\hline$\Pi$ & 1.90319100 & 2.26731800 & -0.37209500 \\
\hline $\mathrm{H}$ & 4.25031300 & 5.21624900 & -3.34558900 \\
\hline $\mathrm{H}$ & 2.64292600 & 4.85283500 & -3.97847800 \\
\hline $\mathrm{H}$ & 2.87540300 & 6.19372700 & -2.84396900 \\
\hline $\mathrm{H}$ & 3.63758800 & 5.74407500 & -0.45715600 \\
\hline $\mathrm{H}$ & 3.54325200 & 4.11095200 & 0.22396200 \\
\hline $\mathrm{H}$ & 4.84420400 & 4.52665300 & -0.89728400 \\
\hline$H$ & 1.28035800 & 5.64610300 & -1.36409700 \\
\hline$\Pi$ & 0.72468800 & 4.23561600 & -2.28742900 \\
\hline $\mathrm{H}$ & 1.09861400 & 4.08902400 & -0.56234200 \\
\hline $\mathrm{H}$ & 3.67003000 & 0.52169100 & -7.03588400 \\
\hline 11 & 2.29005700 & 0.93675900 & -5.99526500 \\
\hline $\mathrm{H}$ & 3.69669800 & 2.00797900 & -6.06648100 \\
\hline $\mathrm{H}$ & 5.75730900 & 3.84455400 & -2.65909500 \\
\hline $\mathrm{H}$ & 6.31654300 & 2.24822000 & -3.21712800 \\
\hline $\mathrm{H}$ & 6.38033000 & 3.65449300 & -4.31826200 \\
\hline $\mathrm{H}$ & 4.10837800 & -1.95223400 & -4.32004000 \\
\hline $\mathrm{H}$ & 2.53875000 & -1.44575700 & -4.97593300 \\
\hline $\mathrm{H}$ & 3.92923800 & -1.66549000 & -6.05179300 \\
\hline $\mathrm{H}$ & 4.78480400 & -0.69069600 & 4.82287900 \\
\hline $\mathrm{H}$ & 4.73715100 & -1.88124900 & 6.13573800 \\
\hline $\mathrm{H}$ & 5.80530900 & -2.14286000 & 4.74333000 \\
\hline $\mathrm{H}$ & 1.88245100 & -1.40119300 & 2.56576200 \\
\hline $\mathrm{H}$ & 4.39874200 & -1.04328700 & -0.84064500 \\
\hline $\mathrm{H}$ & 6.60504400 & -5.33702400 & 2.92407600 \\
\hline $\mathrm{H}$ & 4.83683500 & -5.18424300 & 2.73229800 \\
\hline $\mathrm{H}$ & 5.89797000 & -4.99615700 & 1.32050200 \\
\hline $\mathrm{H}$ & 3.41672100 & -4.08276900 & 5.82327000 \\
\hline $\mathrm{H}$ & 3.01367900 & -4.61371500 & 4.18563900 \\
\hline
\end{tabular}




\begin{tabular}{|c|c|c|c|}
\hline $\mathrm{H}$ & 4.71320900 & -4.43357700 & 4.68605000 \\
\hline$H$ & 7.62443100 & -3.91798100 & 0.81357200 \\
\hline $\mathrm{H}$ & 6.69312600 & -3.97621400 & -0.70261100 \\
\hline $\mathrm{H}$ & 8.29157200 & -3.22075000 & -0.65947300 \\
\hline $\mathrm{H}$ & 7.81364800 & -1.07809200 & -1.41044900 \\
\hline $\mathrm{H}$ & 6.20806600 & -1.64724900 & -1.87795100 \\
\hline $\mathrm{H}$ & 6.38417500 & -0.15218100 & -0.93559900 \\
\hline $\mathrm{H}$ & 2.24810800 & -0.87135100 & 4.63807700 \\
\hline $\mathrm{H}$ & 1.47706400 & -2.46039900 & 4.45169000 \\
\hline $\mathrm{H}$ & 2.31695000 & -2.04952700 & 5.94979800 \\
\hline $\mathrm{H}$ & 6.07556100 & -0.23836100 & -4.20185400 \\
\hline $\mathrm{H}$ & 5.87312000 & -0.34741100 & -5.95669100 \\
\hline $\mathrm{H}$ & 5.94599400 & 1.23611600 & -5.18550000 \\
\hline $\mathrm{H}$ & 8.66818400 & -1.16488500 & 0.85921100 \\
\hline $\mathrm{H}$ & 7.27530300 & -0.24688600 & 1.46258500 \\
\hline $\mathrm{H}$ & 7.70172800 & -1.80323300 & 2.20344000 \\
\hline $\mathrm{Cu}$ & 0.06086700 & 0.71200700 & 1.23312400 \\
\hline $\mathrm{H}$ & 0.58548900 & 0.64554300 & 4.49427900 \\
\hline $\mathrm{C}$ & 0.03131900 & 1.53290100 & 4.14790400 \\
\hline $\mathrm{C}$ & 0.54369000 & 1.94502300 & 2.75032500 \\
\hline $\mathrm{H}$ & 0.04440600 & 2.90053200 & 2.49985800 \\
\hline $\mathrm{H}$ & 0.24166200 & 2.31429300 & 4.90192300 \\
\hline $\mathrm{C}$ & -1.46847100 & 1.22187100 & 4.23462500 \\
\hline $\mathrm{H}$ & -1.70294500 & 0.38242000 & 3.56502900 \\
\hline $\mathrm{H}$ & -2.04126100 & 2.07632200 & 3.84885300 \\
\hline $\mathrm{C}$ & 2.05985900 & 2.24361900 & 2.78183000 \\
\hline $\mathrm{H}$ & 2.61881000 & 1.35473300 & 3.10938500 \\
\hline $\mathrm{H}$ & 2.42787000 & 2.46515500 & 1.77115300 \\
\hline $\mathrm{C}$ & 2.46825200 & 3.42363800 & 3.66242400 \\
\hline $\mathrm{H}$ & 1.91533600 & 4.33247600 & 3.39788800 \\
\hline $\mathrm{H}$ & 2.31258500 & 3.23943300 & 4.72887400 \\
\hline $\mathrm{O}$ & 3.88654600 & 3.67213300 & 3.44758500 \\
\hline $\mathrm{C}$ & 4.43162200 & 4.68874800 & 4.14226200 \\
\hline $\mathrm{C}$ & 5.90653300 & 4.82787100 & 3.82518800 \\
\hline $\mathrm{H}$ & 6.43579000 & 3.90341000 & 4.07962200 \\
\hline $\mathrm{H}$ & 6.04828400 & 4.99904800 & 2.75281200 \\
\hline $\mathrm{H}$ & 6.32378300 & 5.66116600 & 4.3921090 \\
\hline
\end{tabular}




$\begin{array}{llll}\mathrm{O} & 3.81804400 & 5.39396700 & 4.91587200 \\ \mathrm{C} & -1.94042900 & 0.89612700 & 5.65690300 \\ \mathrm{H} & -1.75867500 & 1.73790000 & 6.33716400 \\ \mathrm{H} & -3.01382600 & 0.67112100 & 5.68683000 \\ \mathrm{H} & -1.40616600 & 0.02737500 & 6.06366200\end{array}$




\section{Reference}

1. He, J.; Jiang, H.; Takise, R.; Zhu, R.-Y.; Chen, G.; Dai, H.-X.; Dhar, T. G. M.; Shi, J.; Zhang, H.; Cheng, P. T. W.;Yu, J.-Q. Angew. Chem. Int. Ed. 2016, 55, 785.

2. Xi, Y.; Butcher, T. W.; Zhang, J.; Hartwig, J. F. Angew. Chem. Int. Ed. 2016, 55, 776.

3. Bartoli, G.; Bosco, M.; Dalpozzo, R.; Marcantoni, E.; Massaccesi, M.; Sambri, L. Eur. J. Org. Chem. 2003, 4611.

4. Burns, M.; Essafi, S.; Bame, J. R.; Bull, S. P.; Webster, M. P.; Balieu, S.; Dale, J. W.; Butts, C. P.; Harvey, J. N.;Aggarwal, V. K. Nature, 2014, 513, 183-188.

5. Mlynarski, S. N.; Karns, A. S.; Morken, J. P. J. Am. Chem. Soc. 2012, 134, 16449.

6. Matteson, D. S.; Majumdar, D. J. Am. Chem. Soc. 1980, 102, 7588.

7. Sonawane, R. P.; Jheengut, V.; Rabalakos, C.; Larouche-Gauthier, R.; Scott, H. K.; Aggarwal, V. K. Angew. Chem. Int. Ed. 2011, 50, 3760.

8. Bonet, A.; Odachowski, M.; Leonori, D.; Essafi, S.; Aggarwal, V. K. Nat. Chem. 2014, 6, 584.

9. Llaveria, J.; Leonori, D.; Aggarwal, V. K. J. Am. Chem. Soc. 2015, 137, 10958.

10. Li, L.; Zhao, S.; Joshi-Pangu, A.; Diane, M.; Biscoe, M. R. J. Am. Chem. Soc. 2014, 136, 14027.

11. Larouche-Gauthier, R.; Elford, T. G.; Aggarwal, V. K. J. Am. Chem. Soc. 2011, 133, 16794.

12. Sandford, C.; Rasappan, R.; Aggarwal, V. K. J. Am. Chem. Soc. 2015, 137, 10100. 
XII. SFC and HPLC traces 


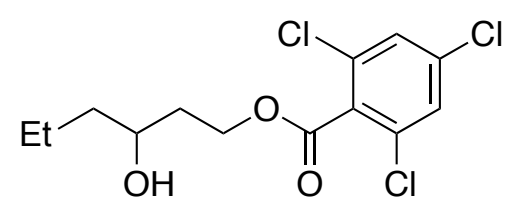

rac-3a

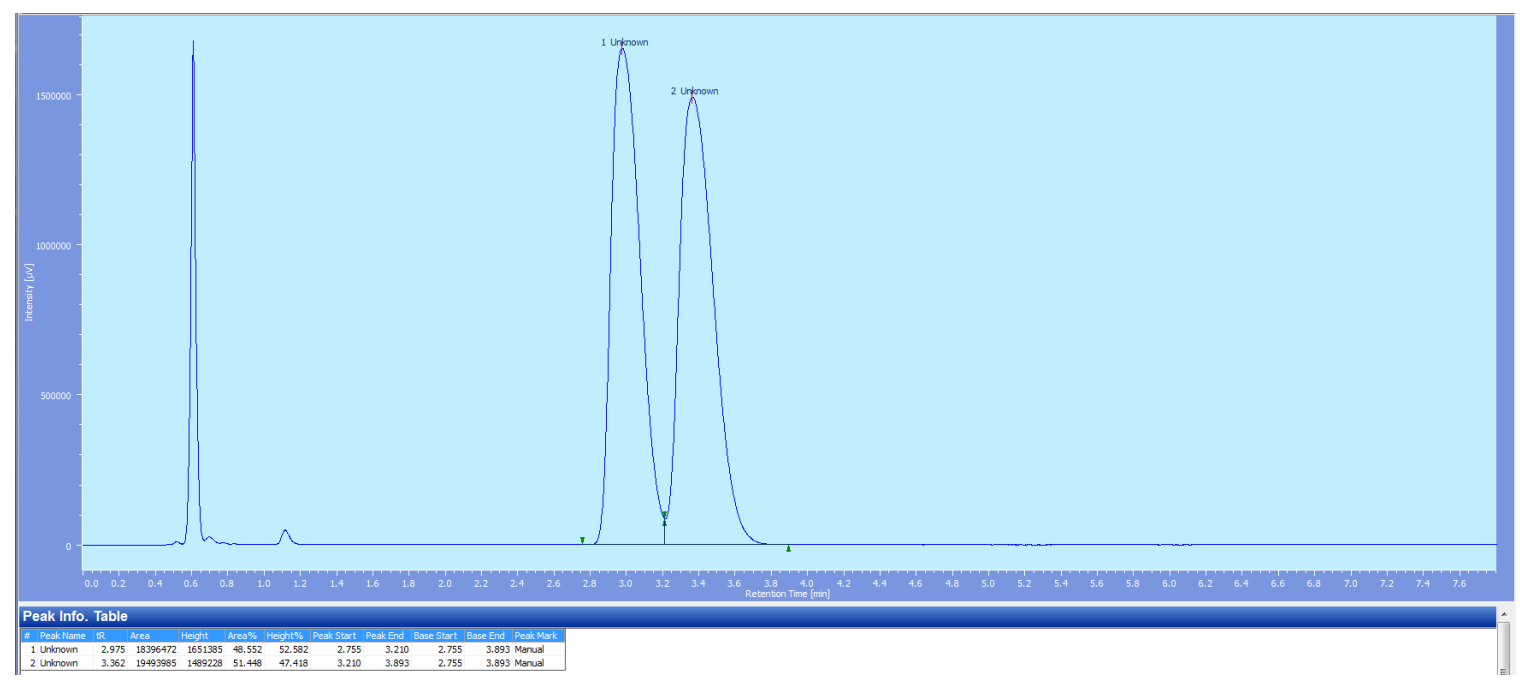<smiles>CCC(O)CCOC(=O)c1c(Cl)cc(Cl)cc1Cl</smiles>

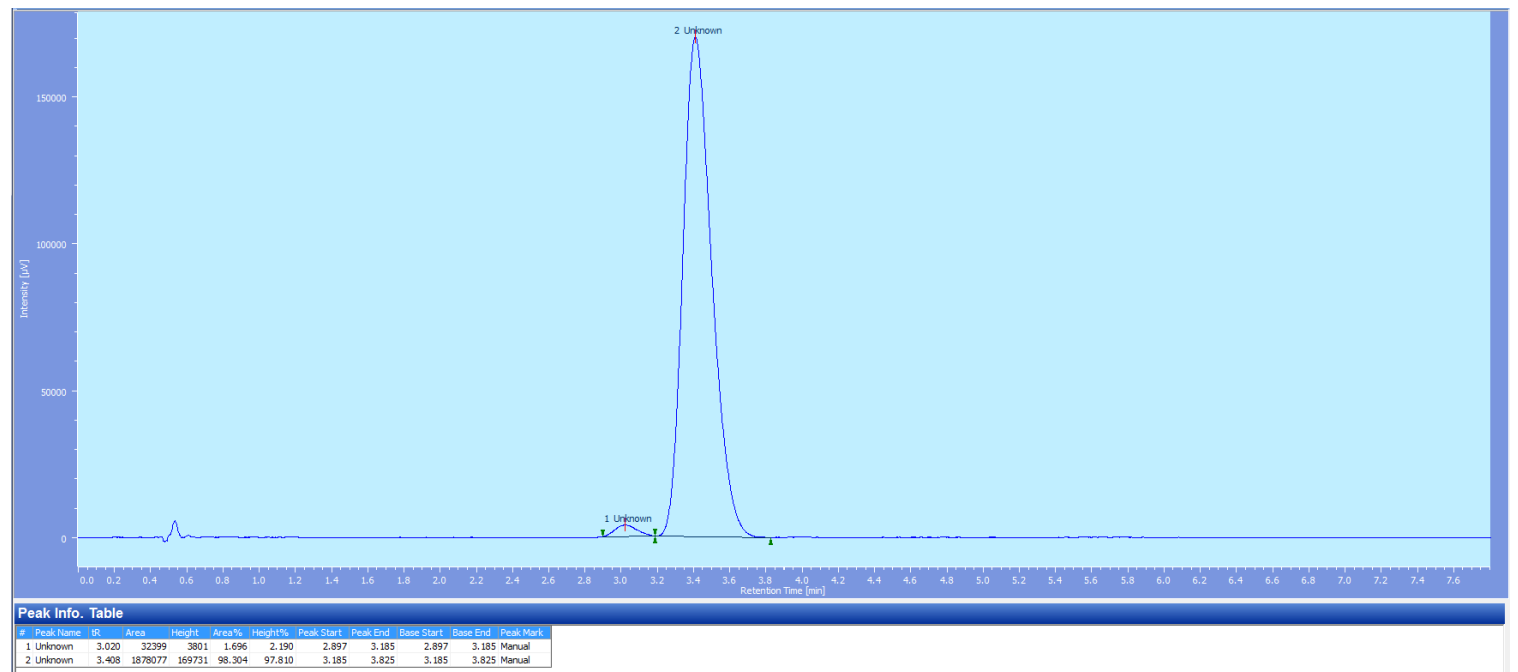


<smiles>CC(C)CC(O)CCOC(=O)c1c(Cl)cc(Cl)cc1Cl</smiles>

rac-3b

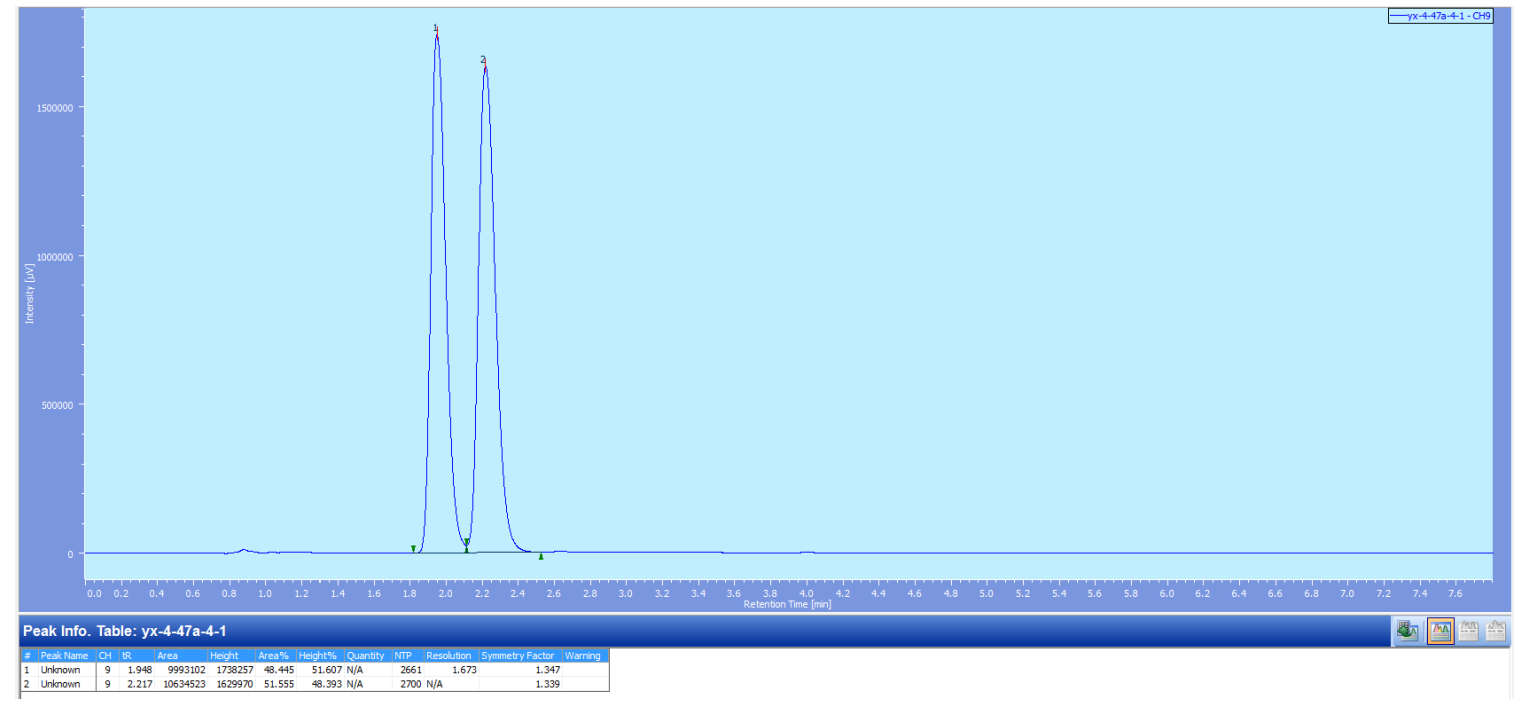<smiles>CC(C)C[C@H](O)CCOC(=O)c1c(Cl)cc(Cl)cc1Cl</smiles>

3b

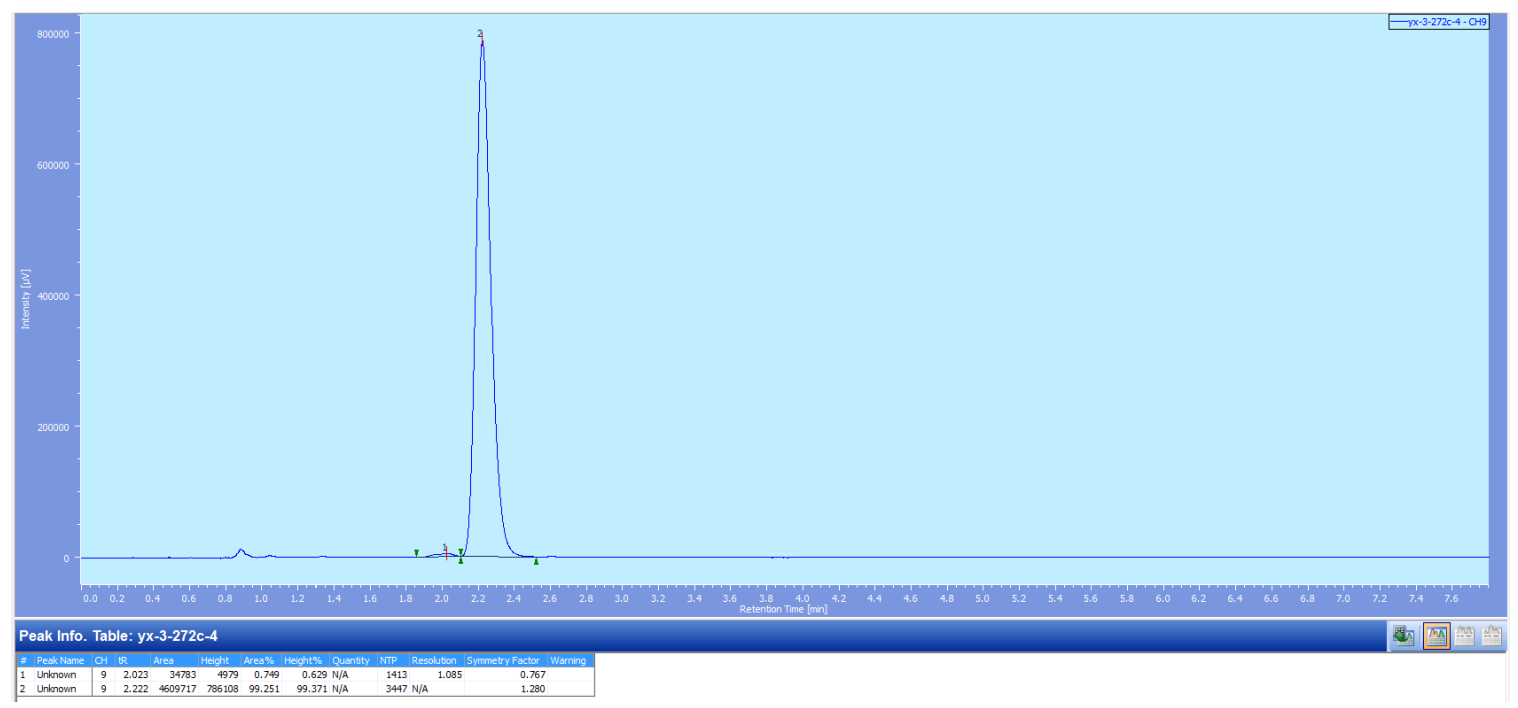


<smiles>CC(C)(C)CC(O)CCOC(=O)c1c(Cl)cc(Cl)cc1Cl</smiles>

rac-3c

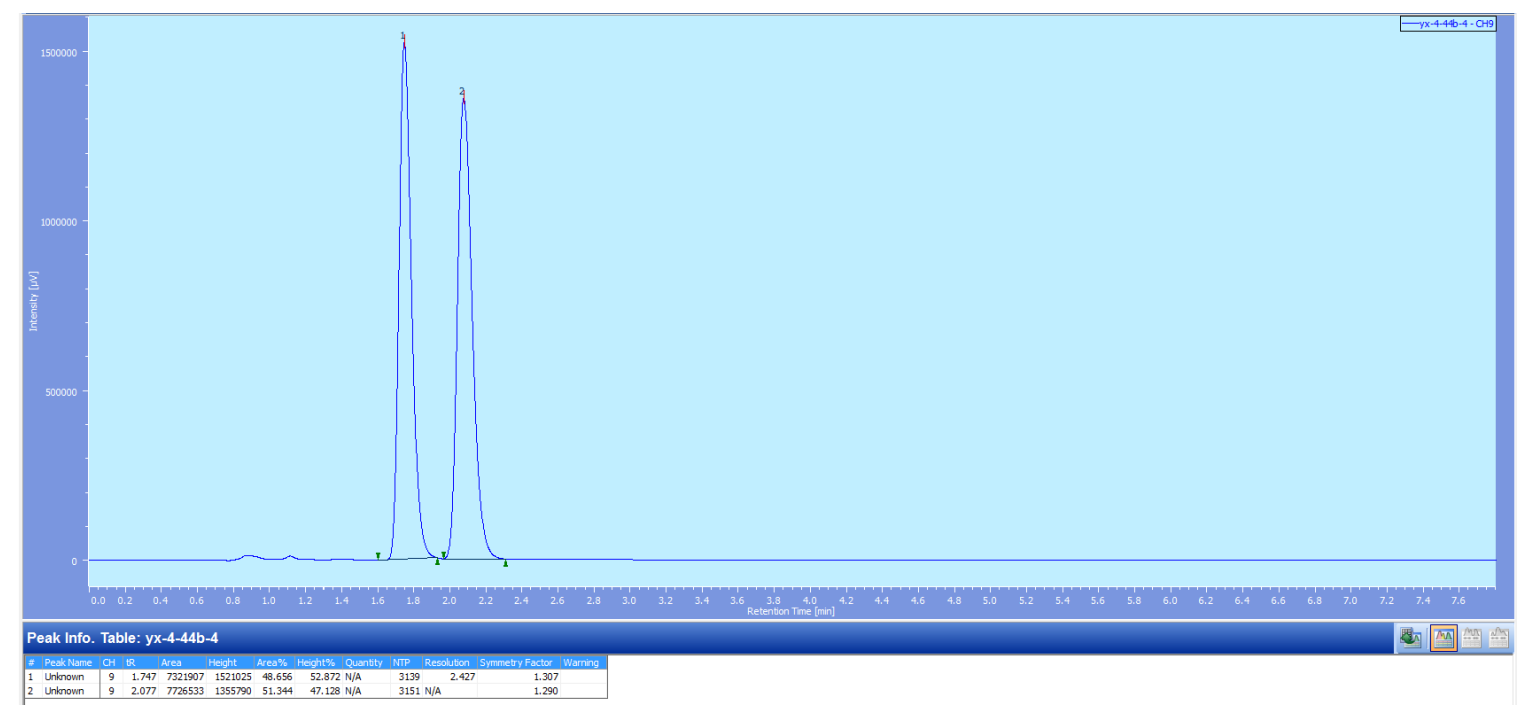<smiles>CC(C)(C)C[C@@H](O)CCOC(=O)c1c(Cl)cc(Cl)cc1Cl</smiles>

3c

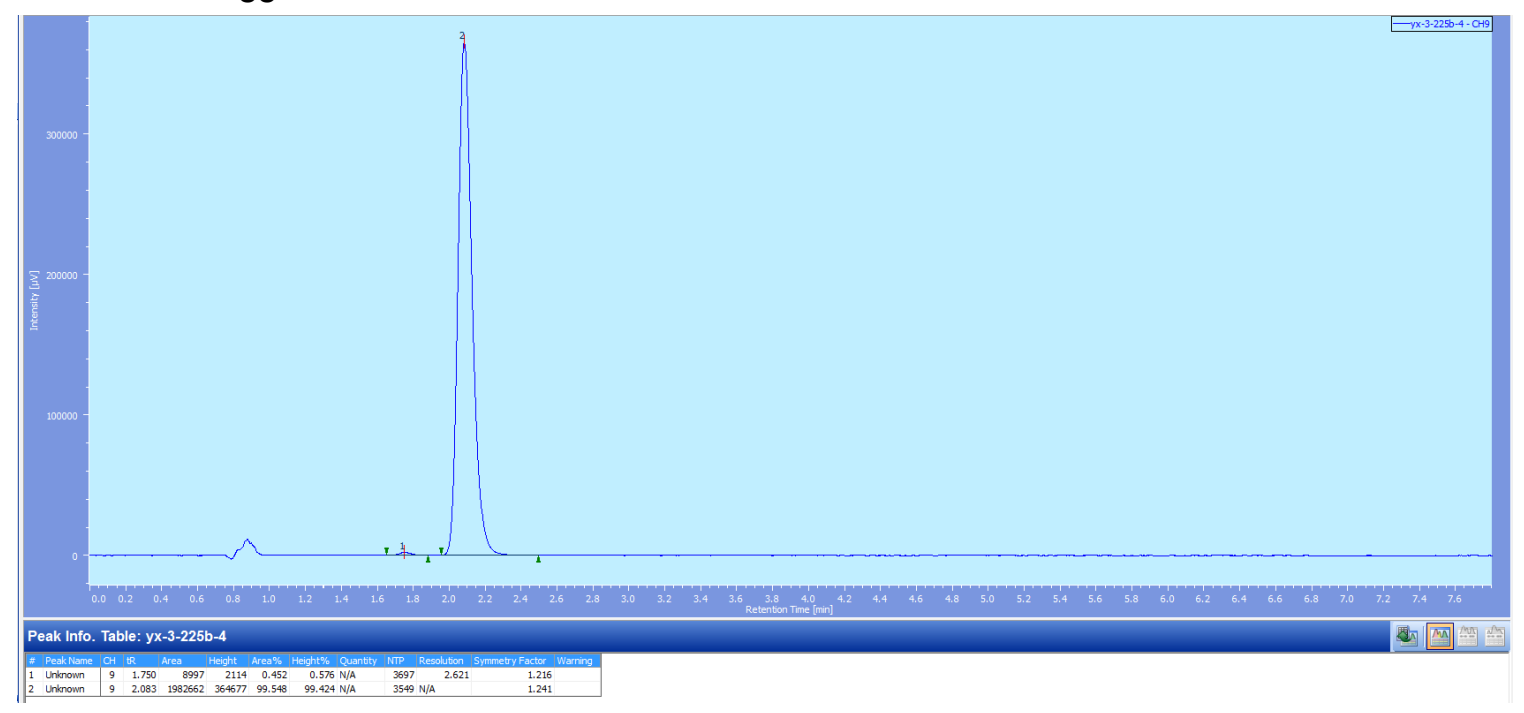



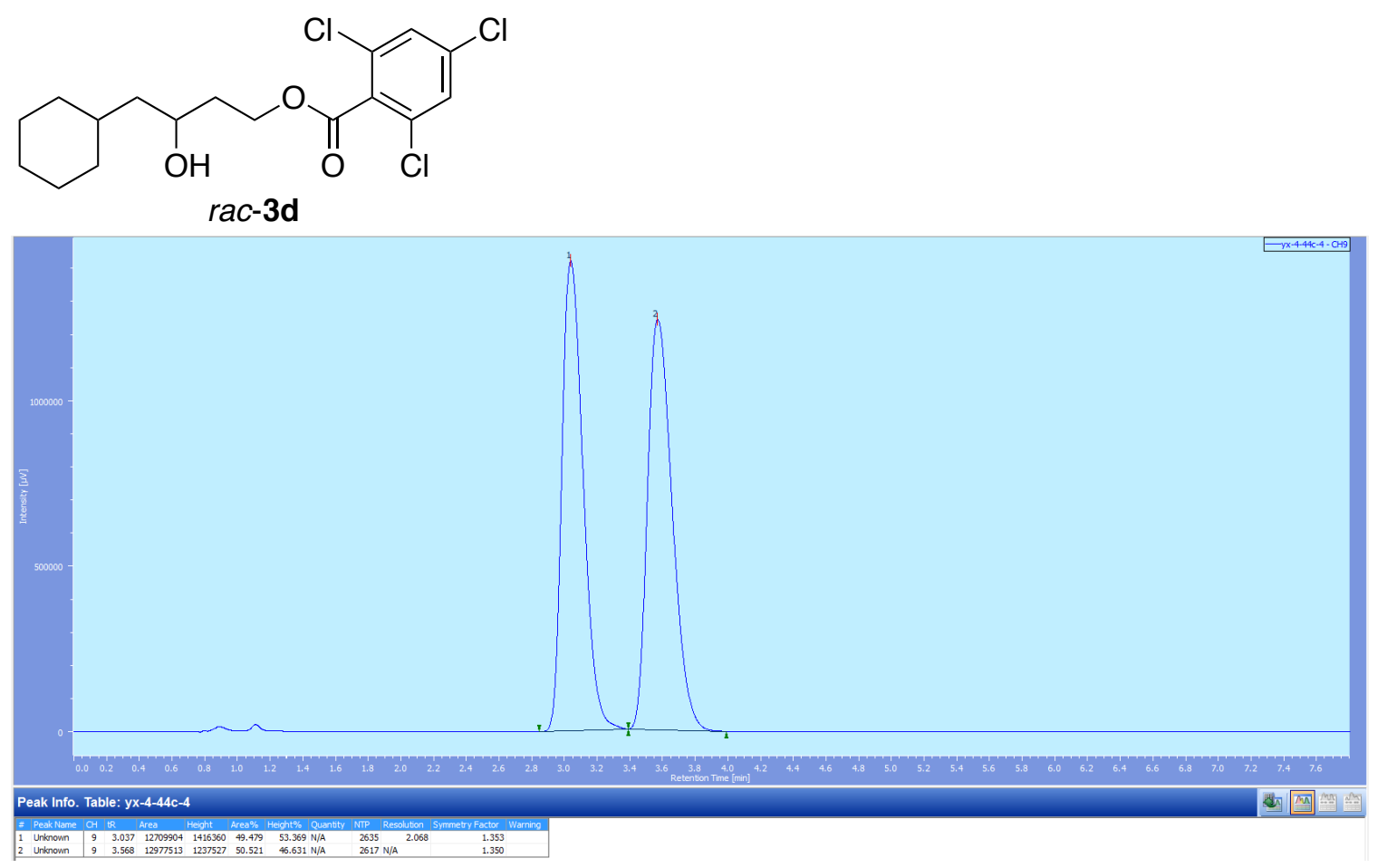<smiles>O=C(OCC[C@@H](O)CC1CCCCC1)c1c(Cl)cc(Cl)cc1Cl</smiles>

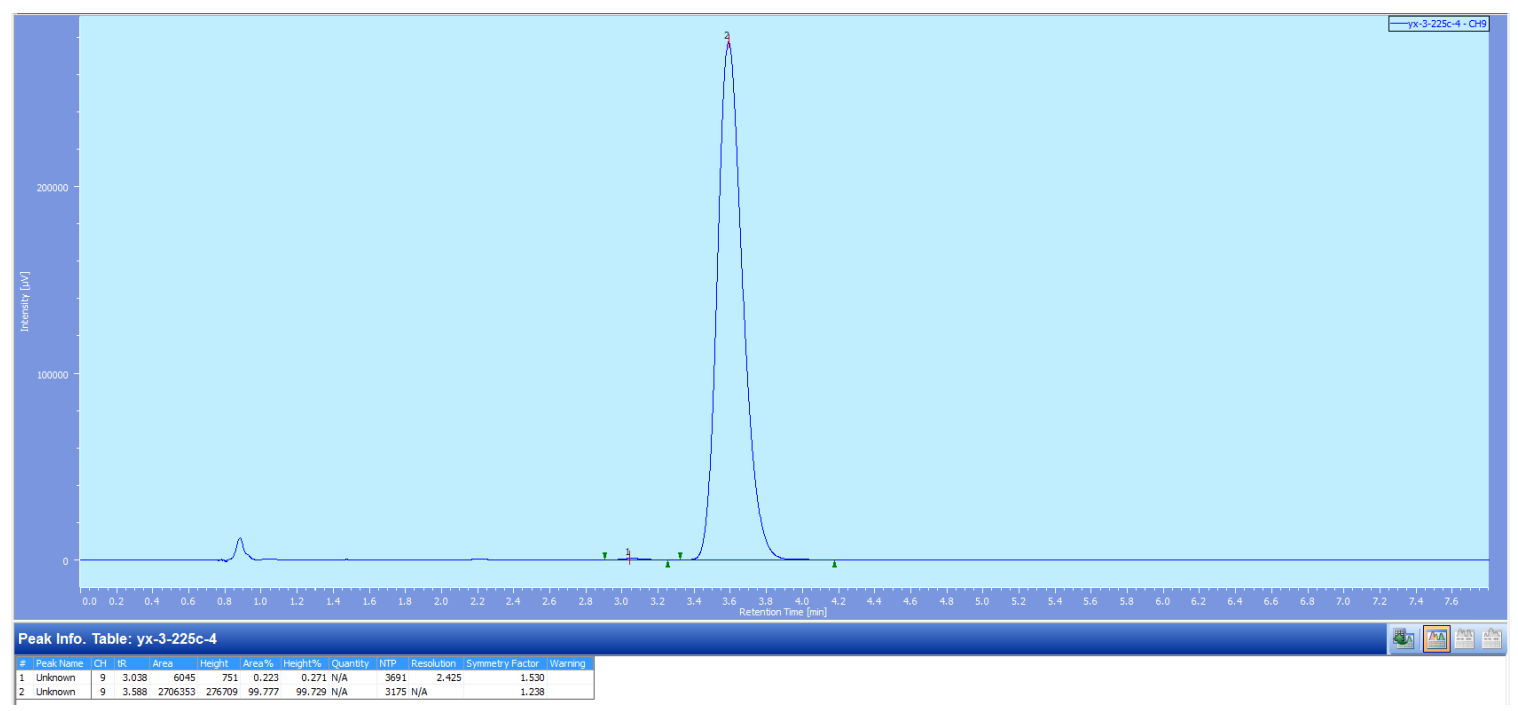




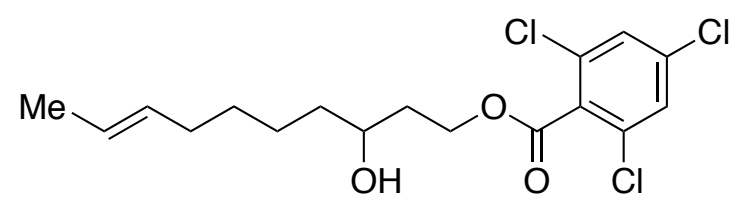

rac-3e

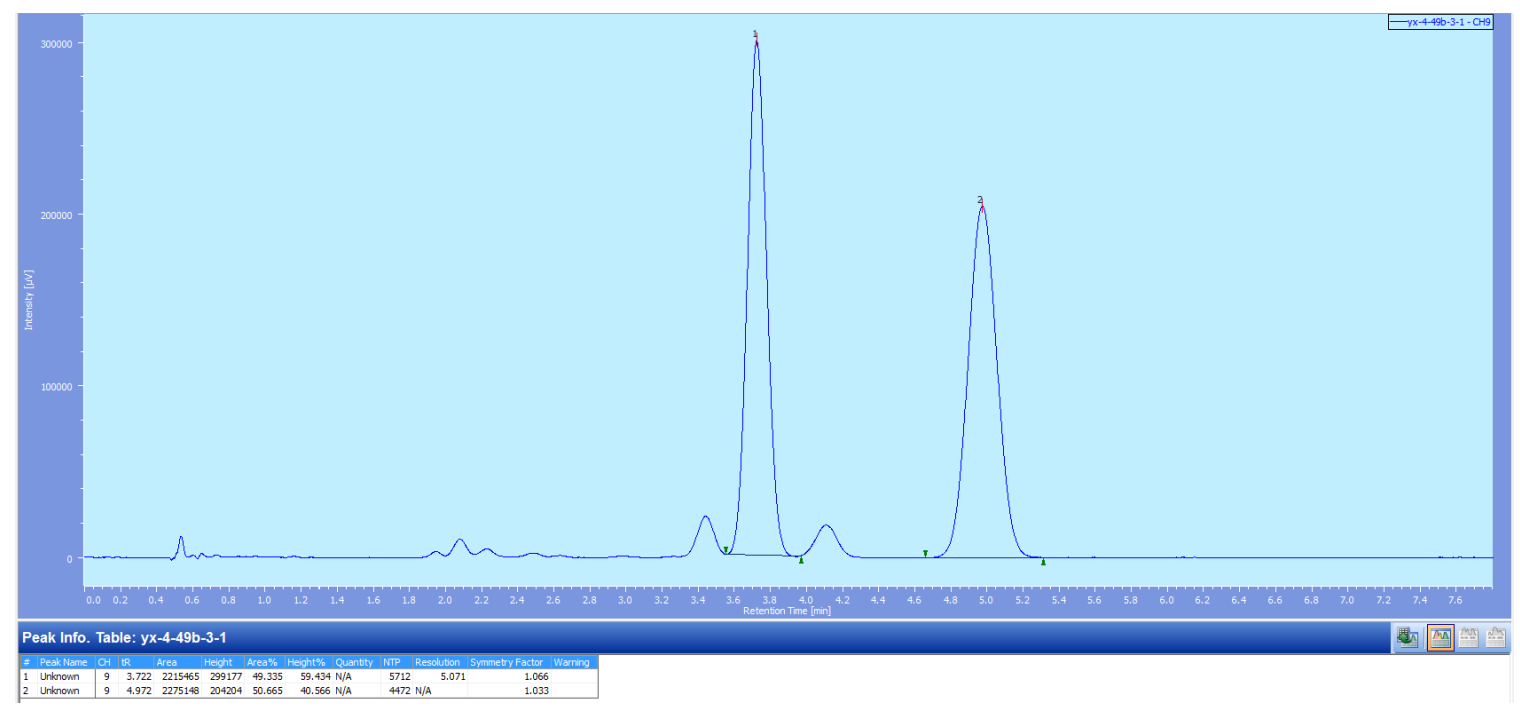<smiles>C/C=C/CCCC[C@H](O)CCOC(=O)c1c(Cl)cc(Cl)cc1Cl</smiles>

$3 e$

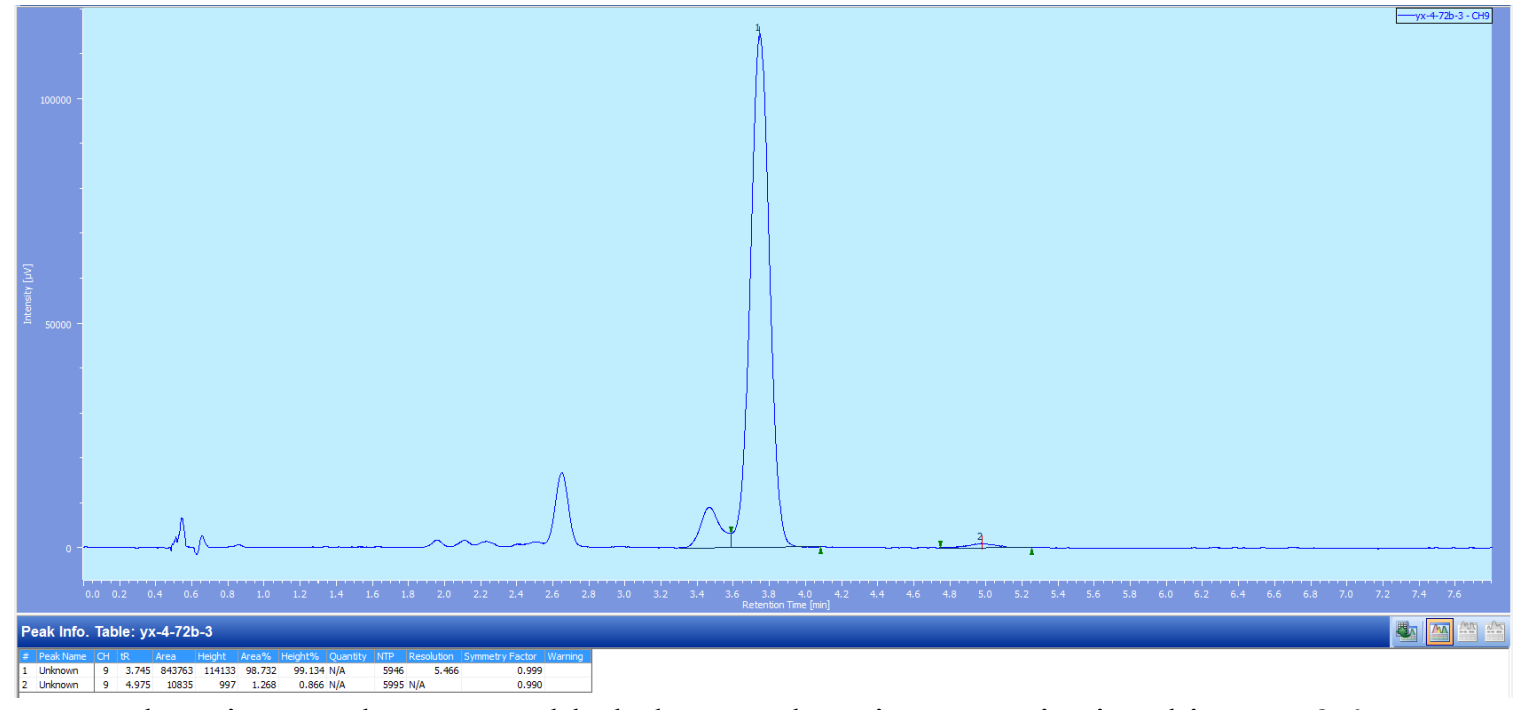

Note: The minor peaks presumably belong to the minor constitutional isomer $\mathbf{3 e}$ '. 
<smiles>CCCC(O)CCOc1c(F)c(F)c(F)c(F)c1F</smiles>

rac-3f

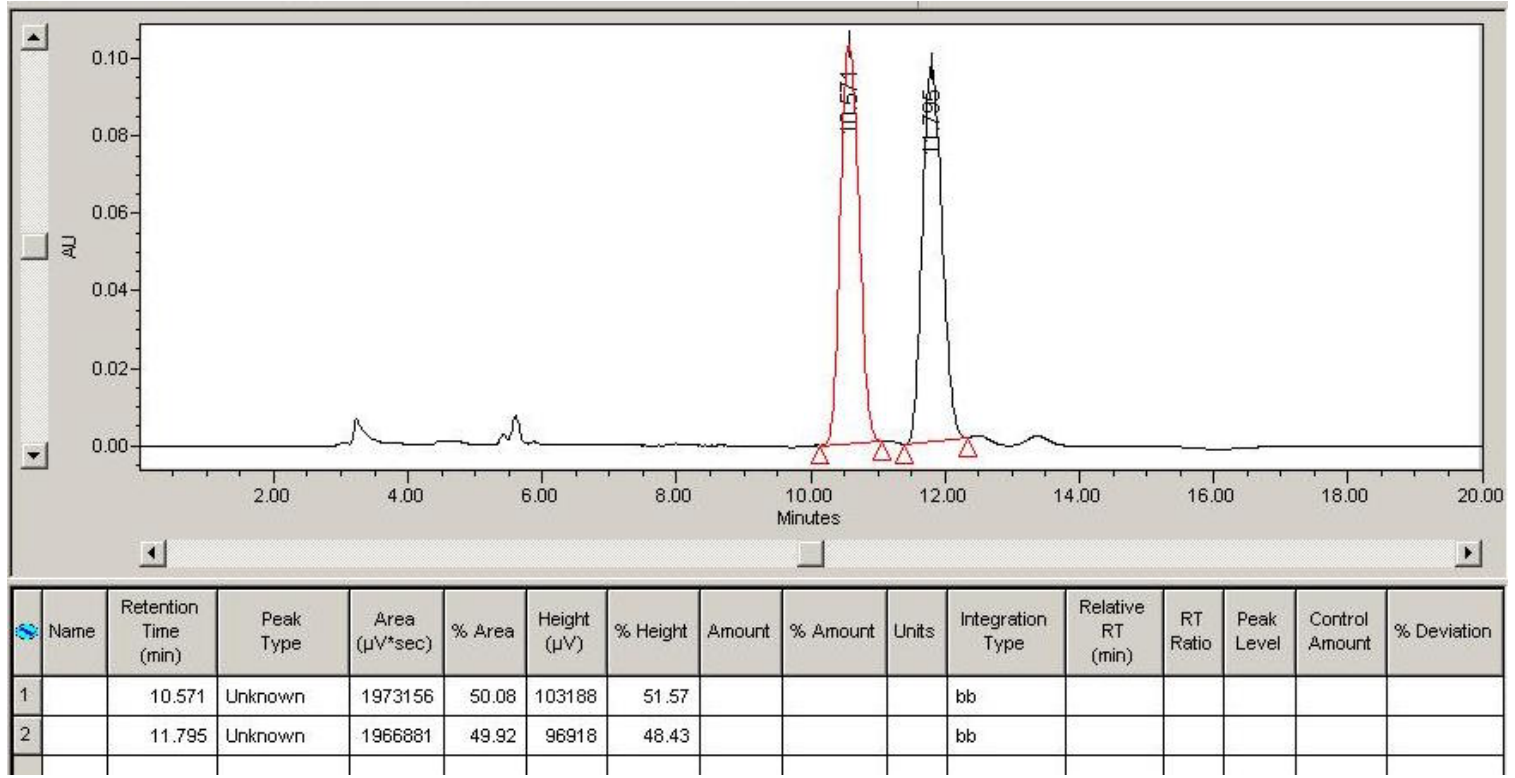<smiles>CCC[C@H](O)CCOc1c(F)c(F)c(F)c(F)c1F</smiles>

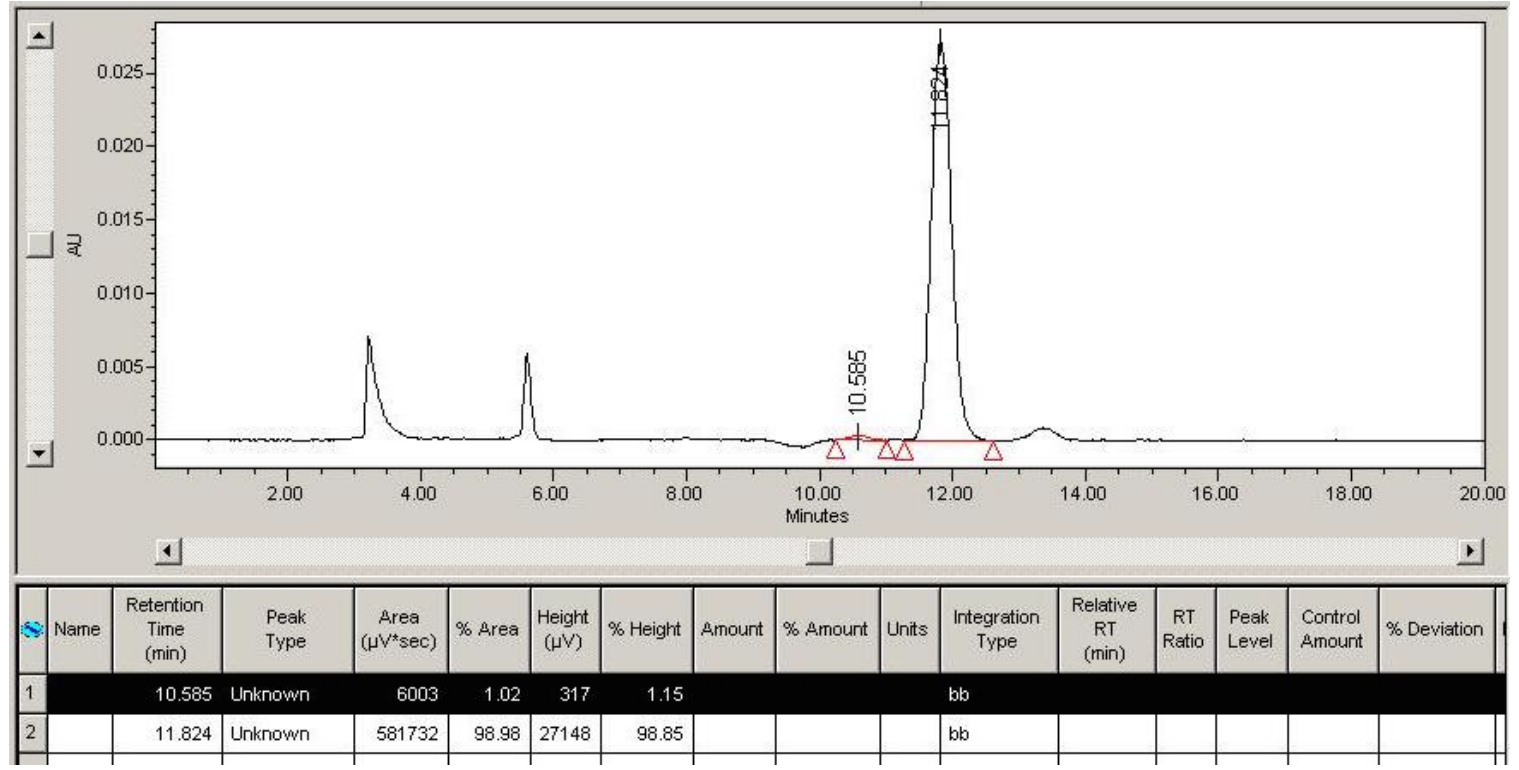



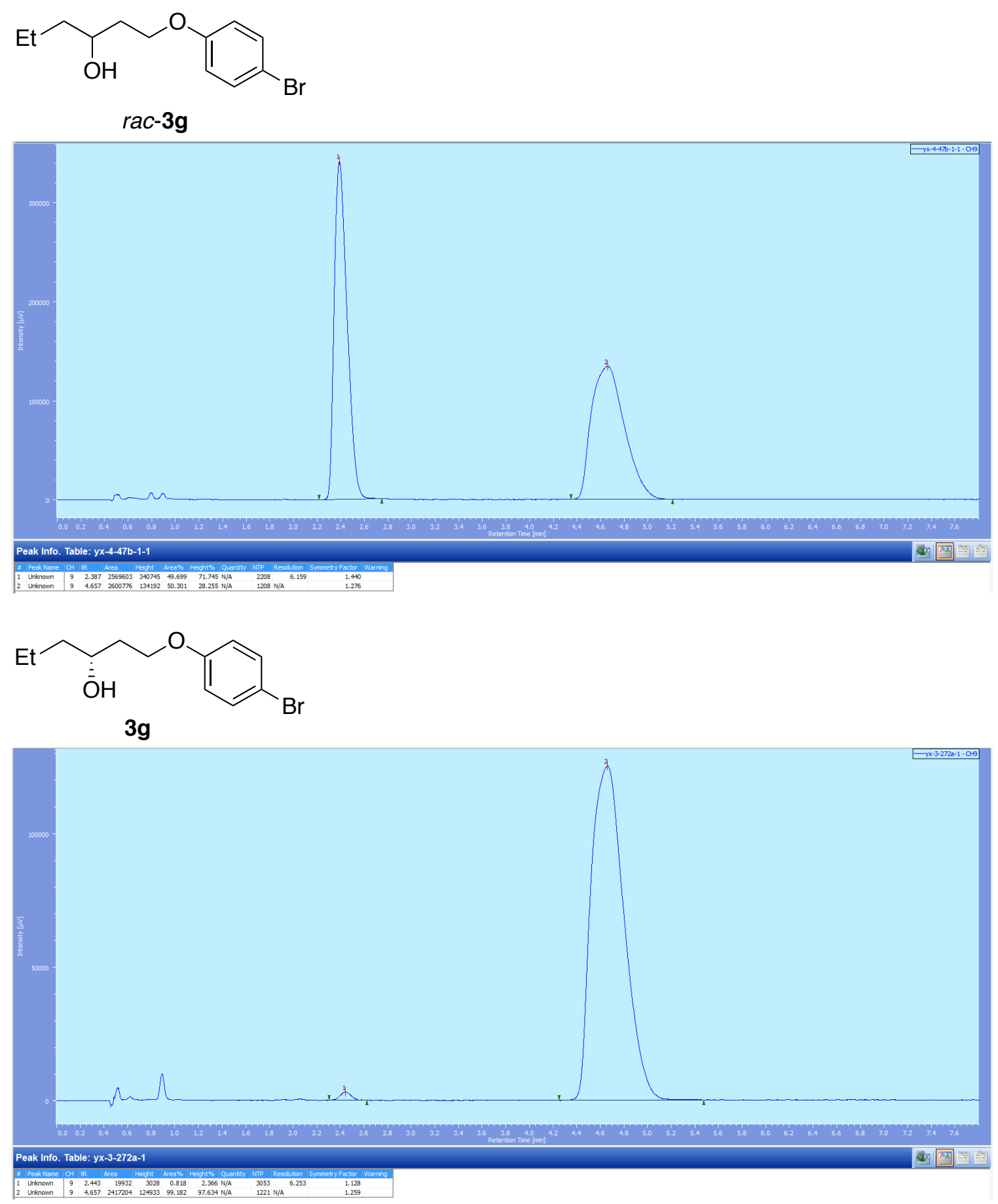

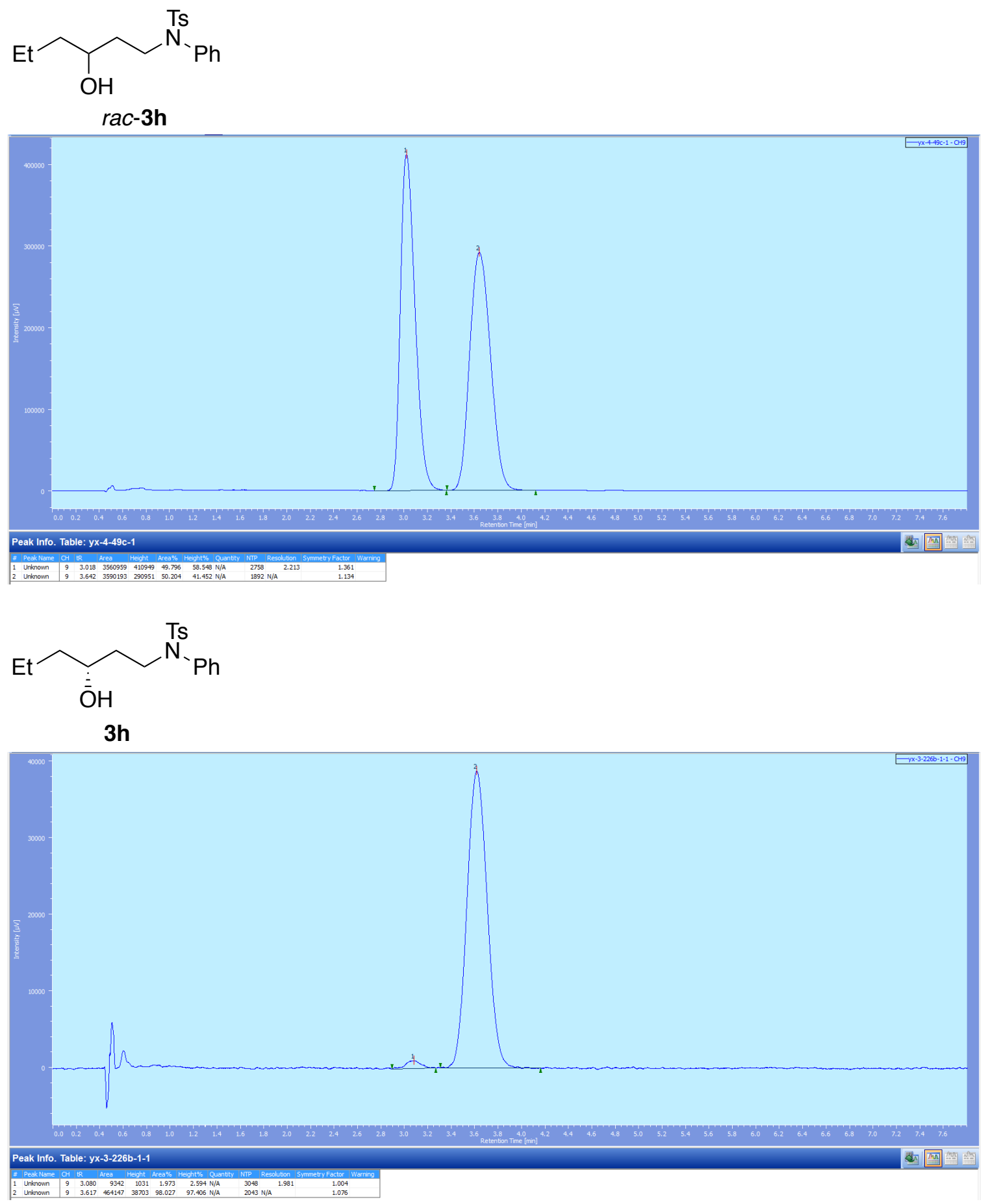
$\overbrace{\mathrm{OH}}^{\mathrm{TS}} \overbrace{\mathrm{Bn}}^{\mathrm{B}}$

rac-3i

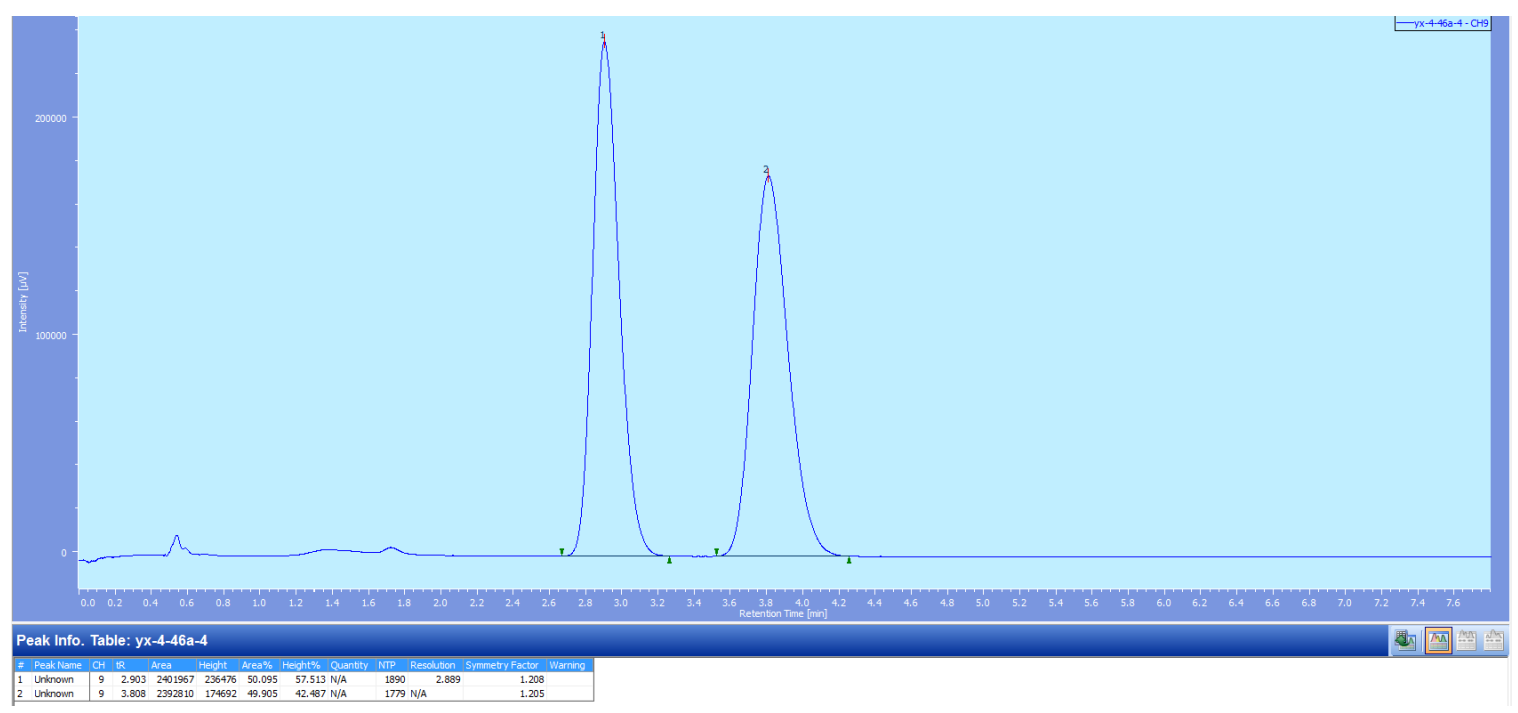

$\overbrace{\text { Ō }}^{\text {Et }} \overbrace{\text { Bn }}^{T s}$

3i

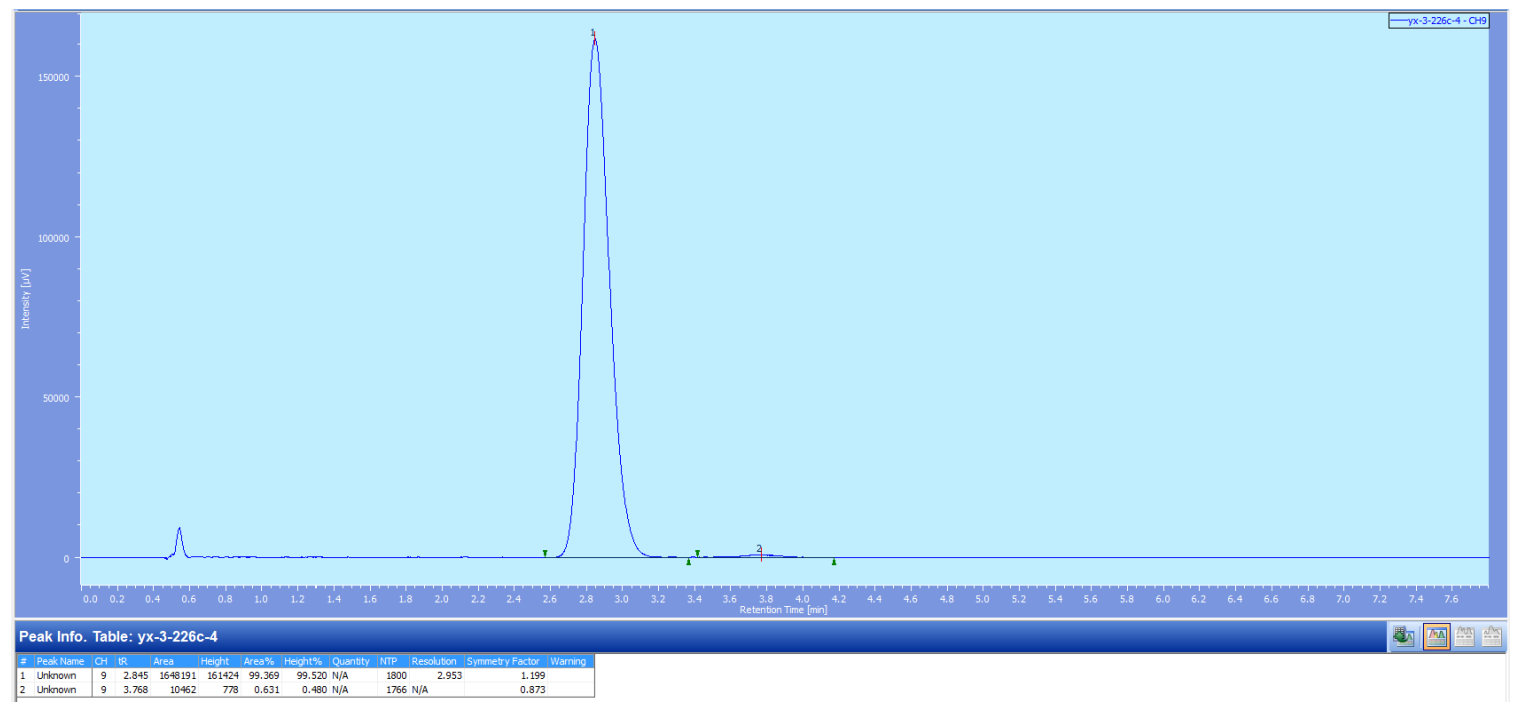


<smiles>O=C(OCCCC(O)CCOC(=O)c1c(Cl)cc(Cl)cc1Cl)c1c(Cl)cc(Cl)cc1Cl</smiles>

rac-3o

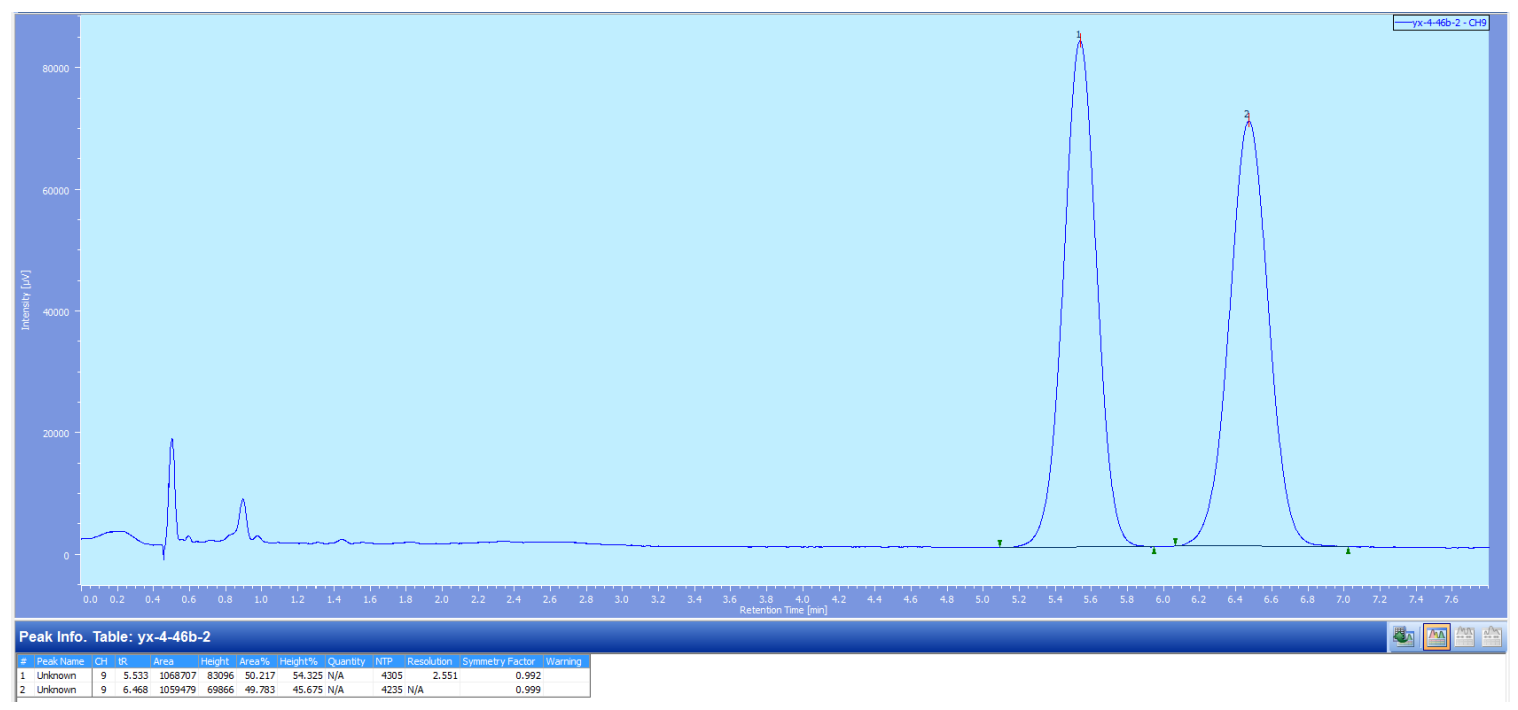<smiles>O=C(OCCC[C@@H](O)OCCC[C@@H](O)c1c(Cl)cc(Cl)cc1Cl)c1c(Cl)cc(Cl)cc1Cl</smiles>

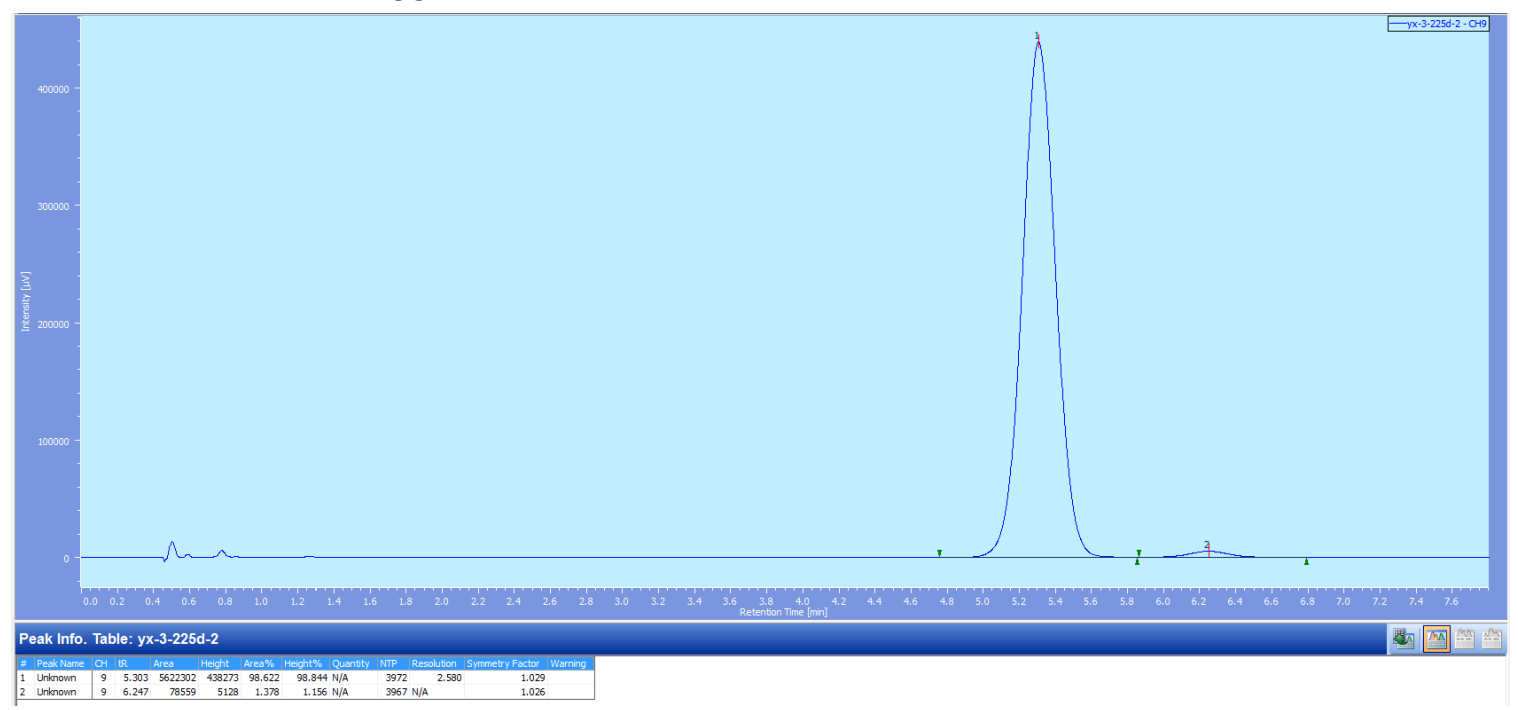



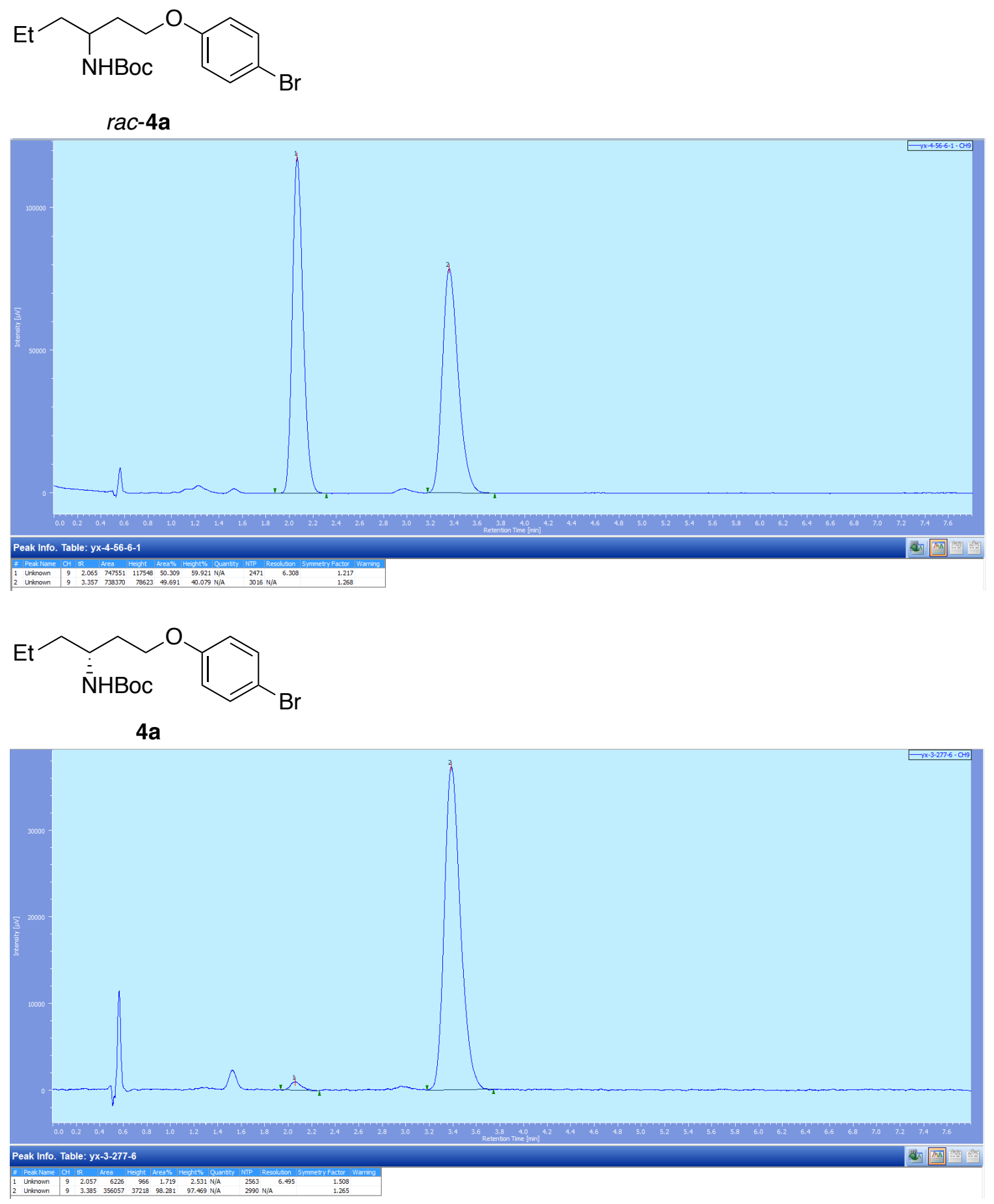

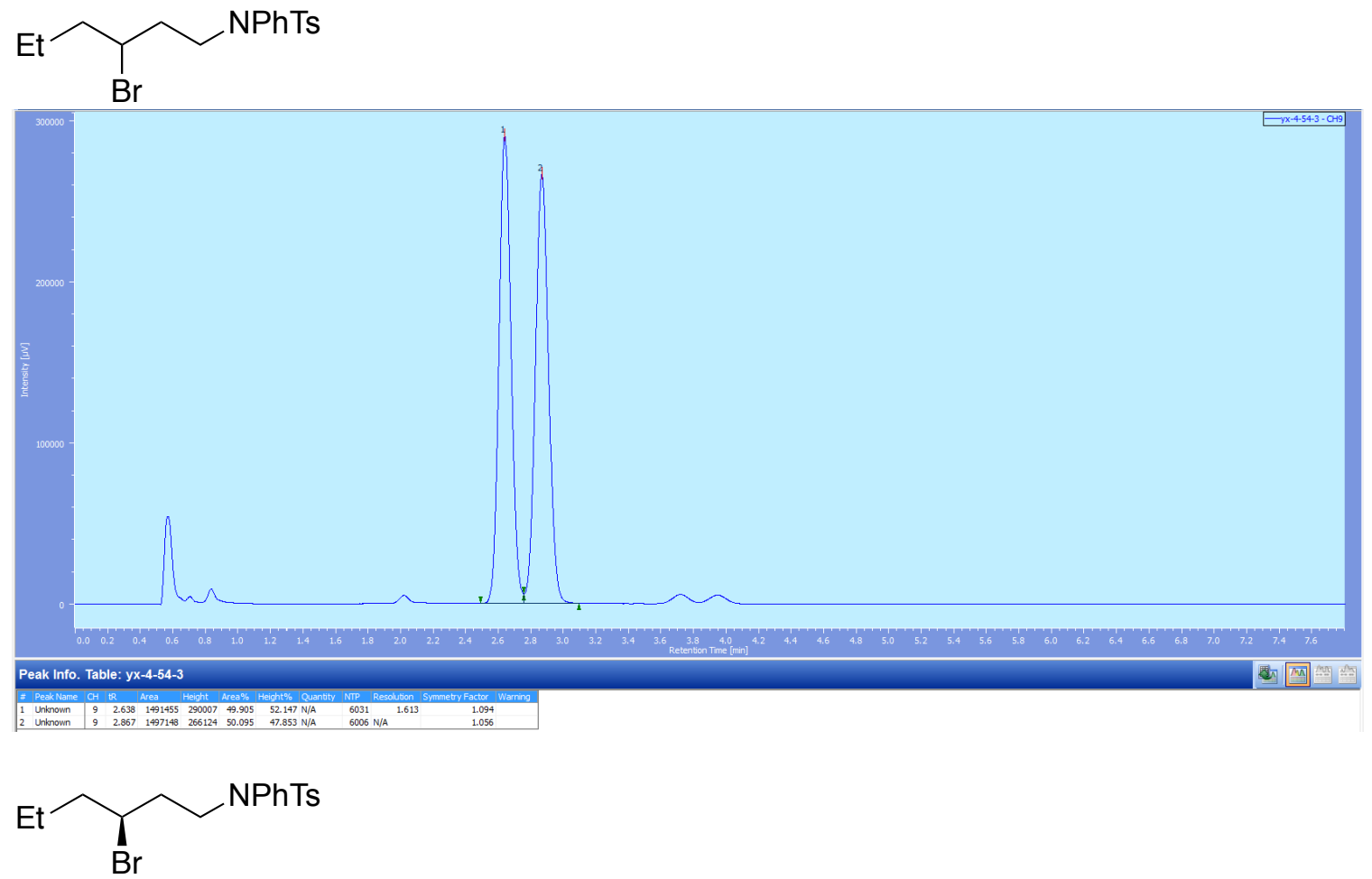

4b

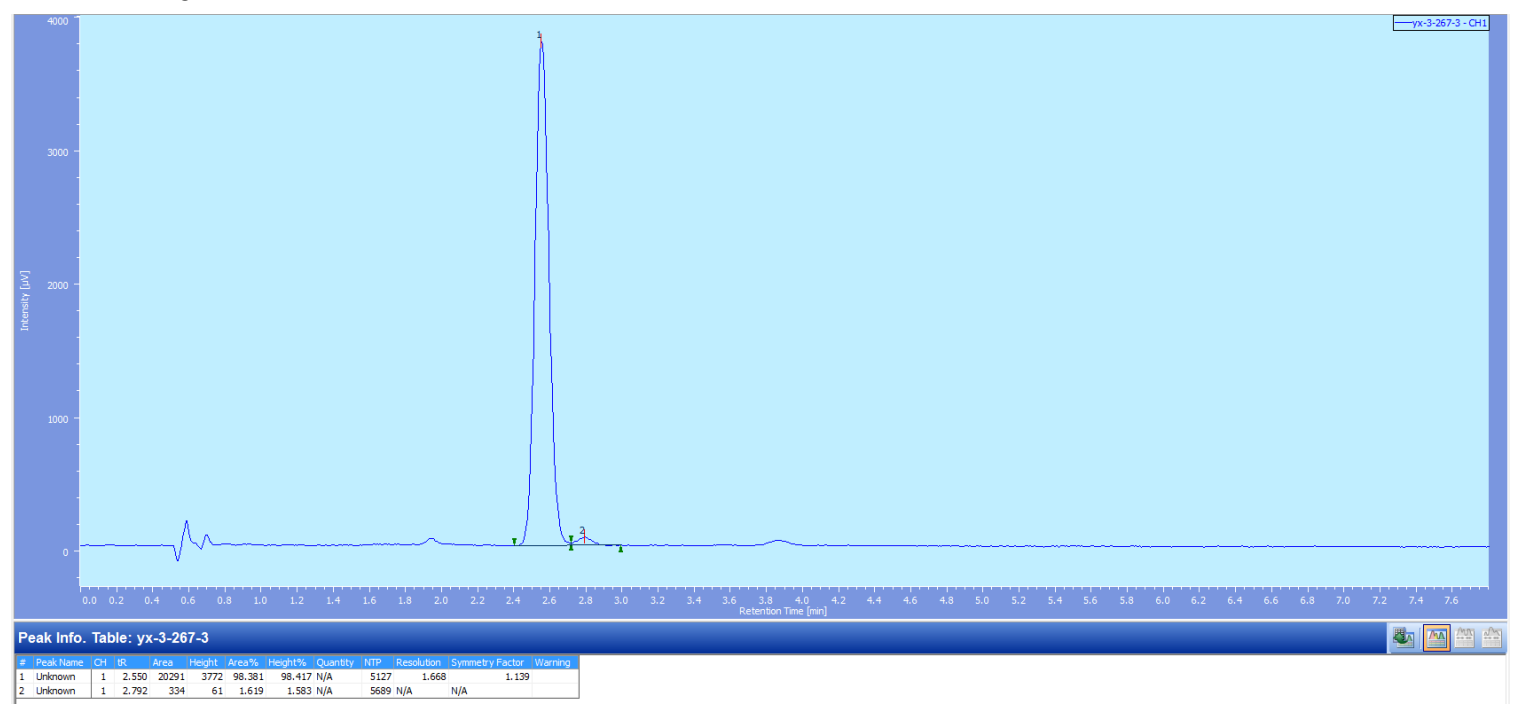




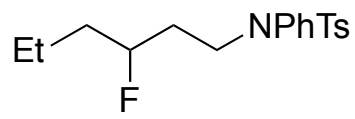

rac-4c

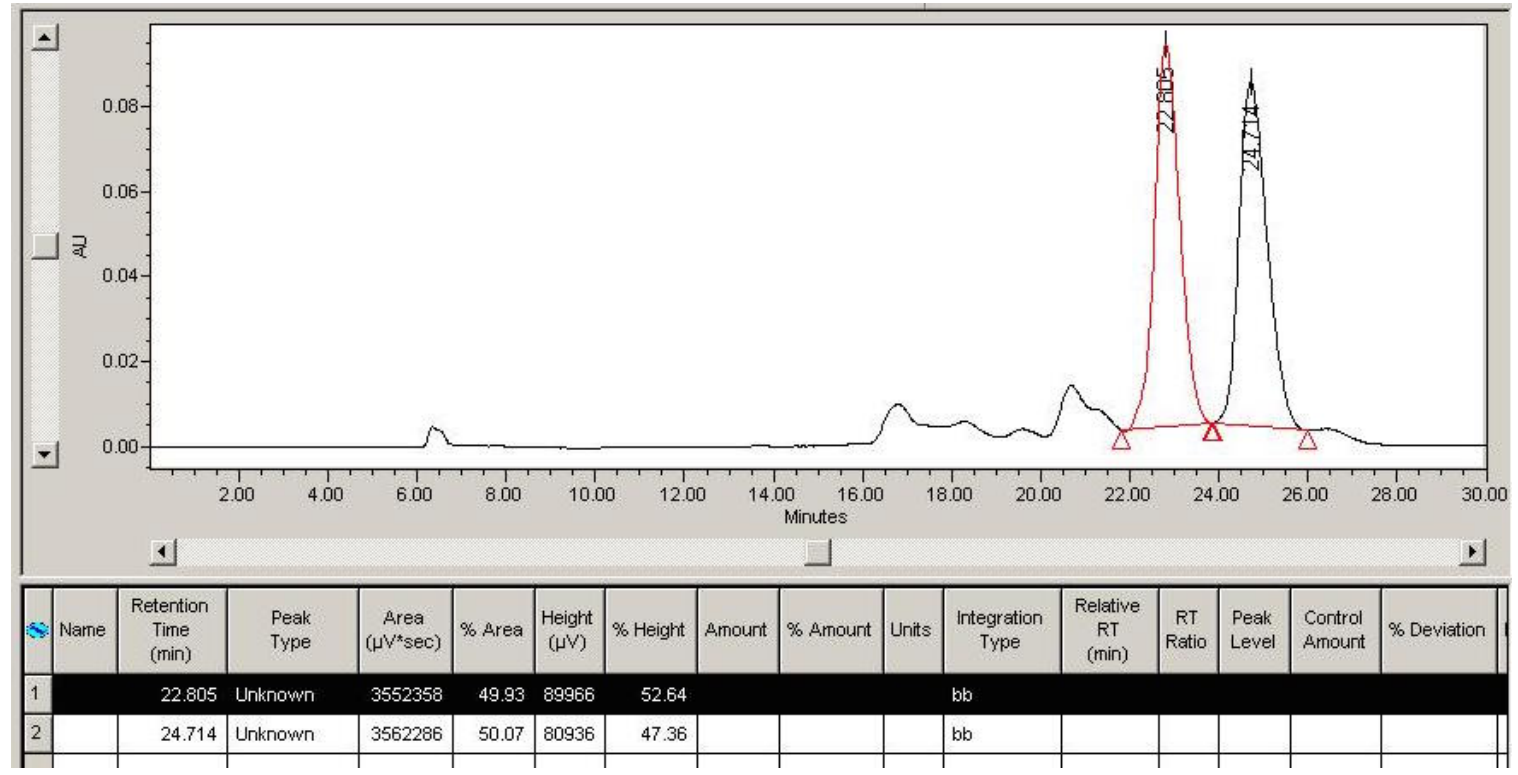<smiles></smiles>

4c

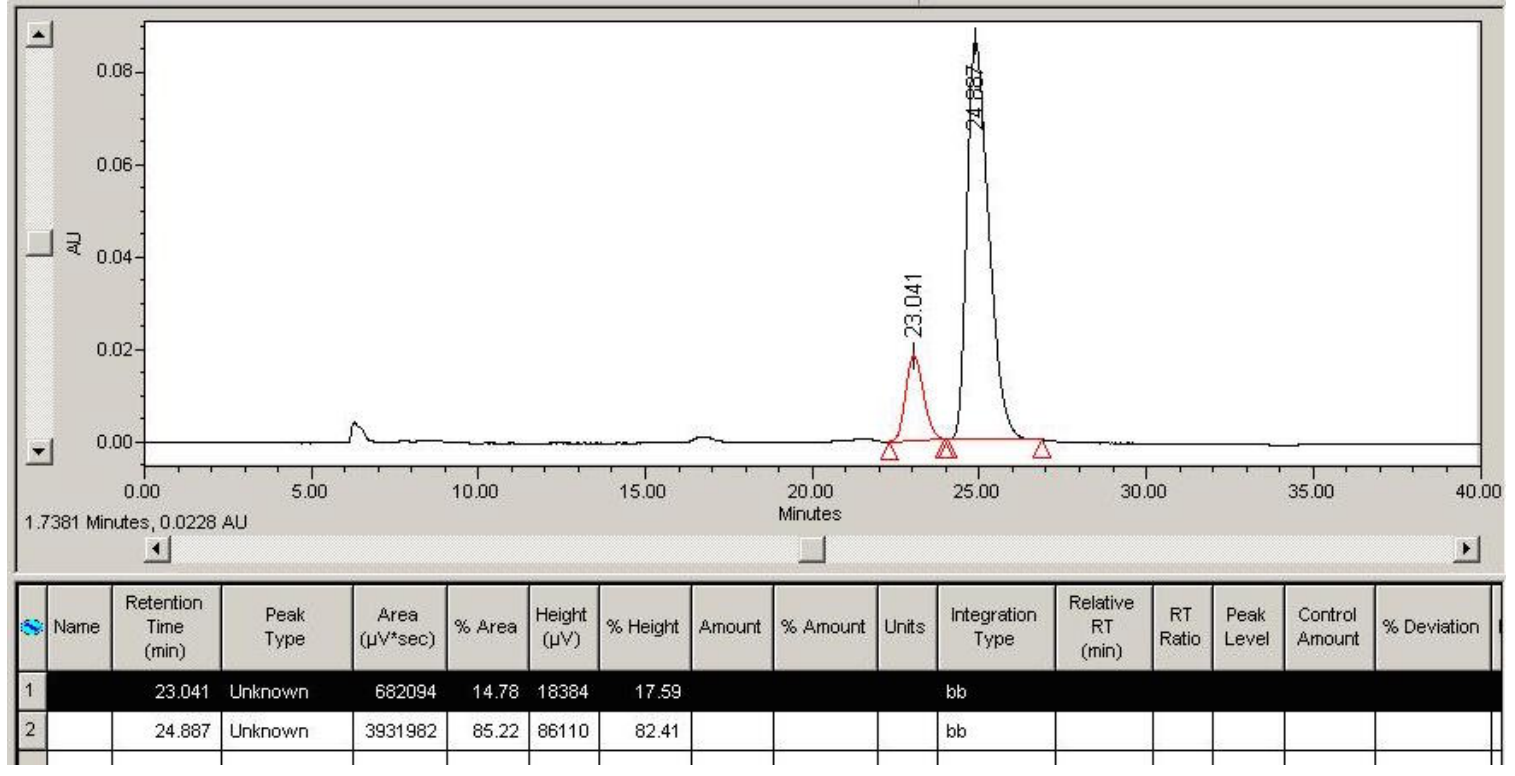




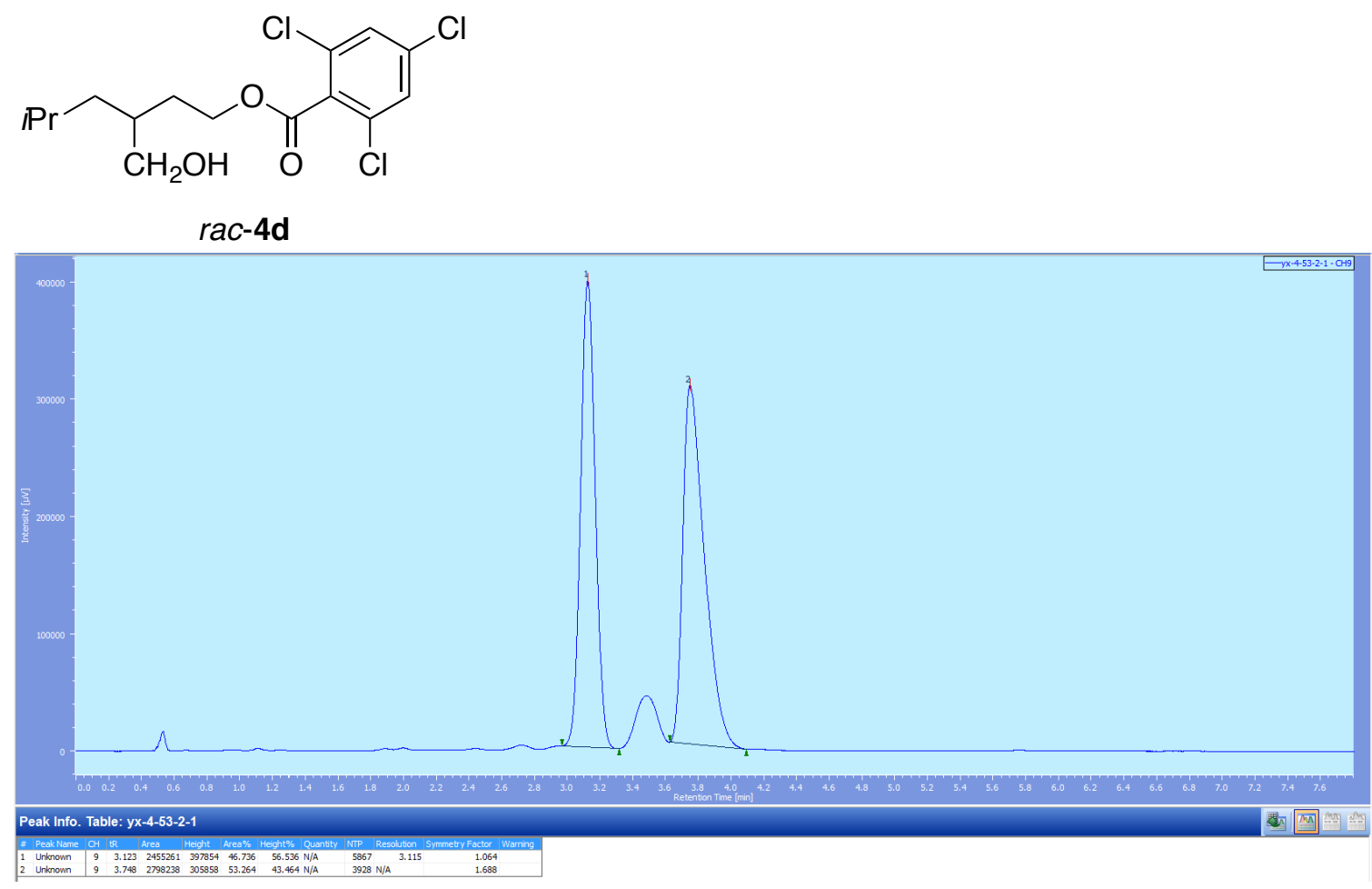<smiles>CC(C)C[C@H](C)CO</smiles>

4d

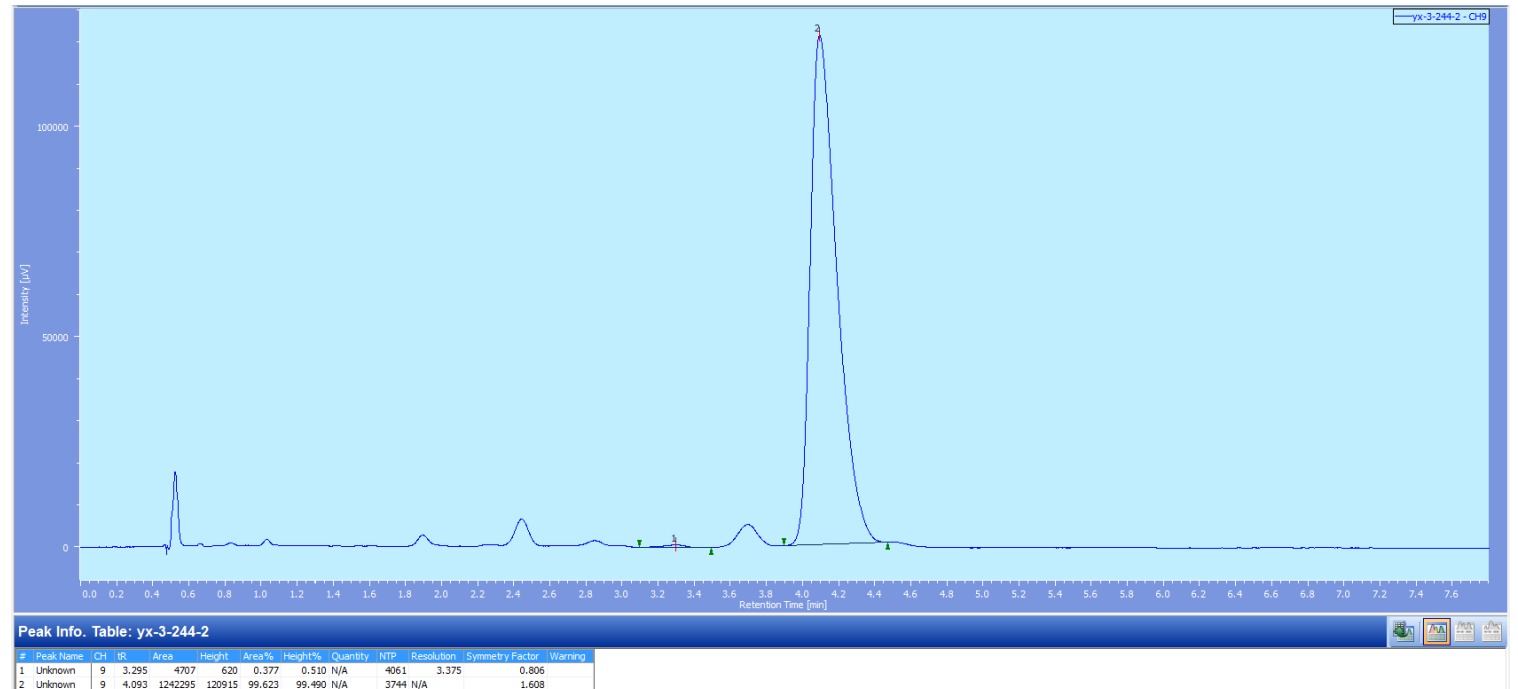

Note: The minor peaks belong to an unidentified impurity, which was not observed by ${ }^{1} \mathrm{H}$ NMR spectroscopy. 

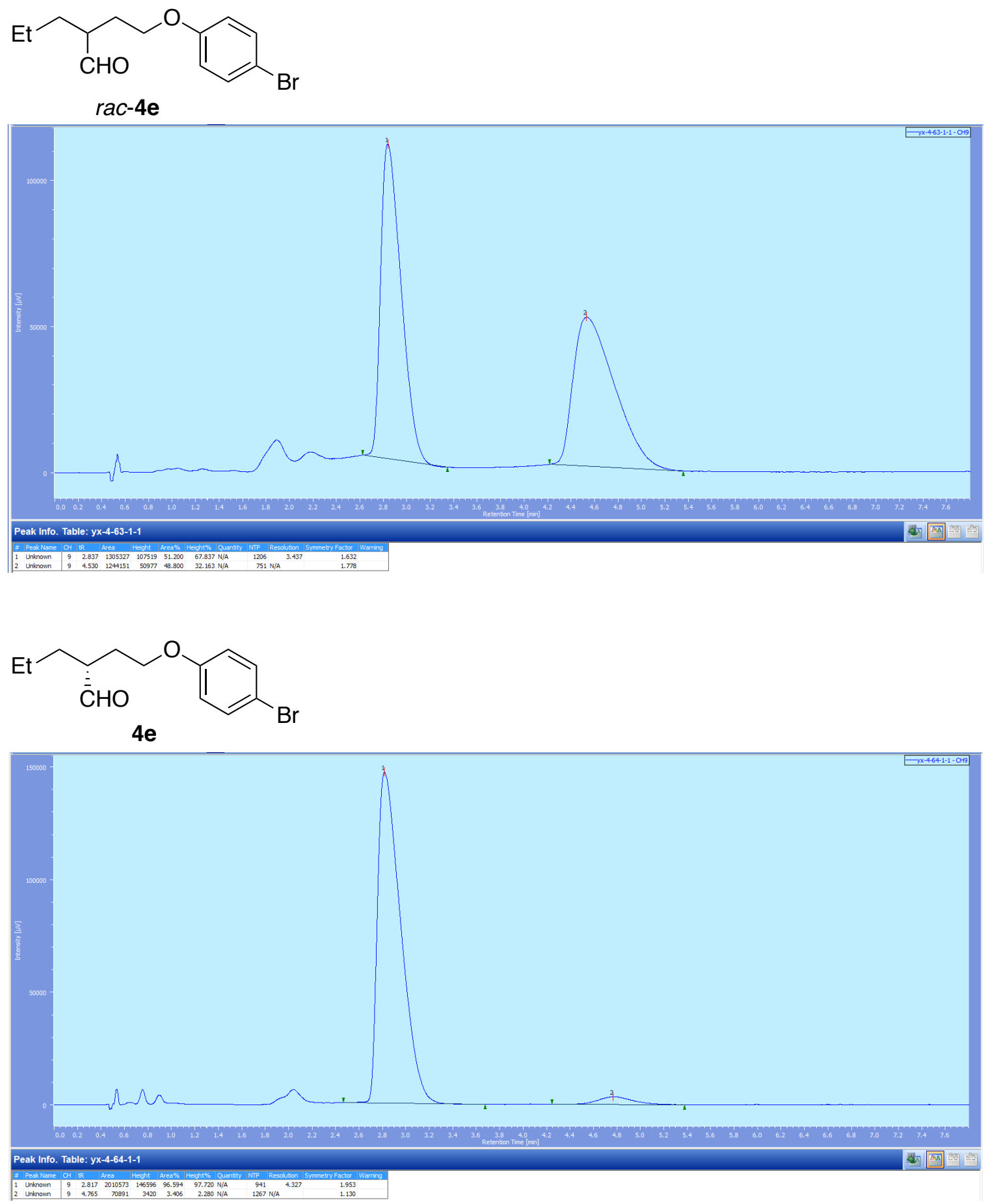

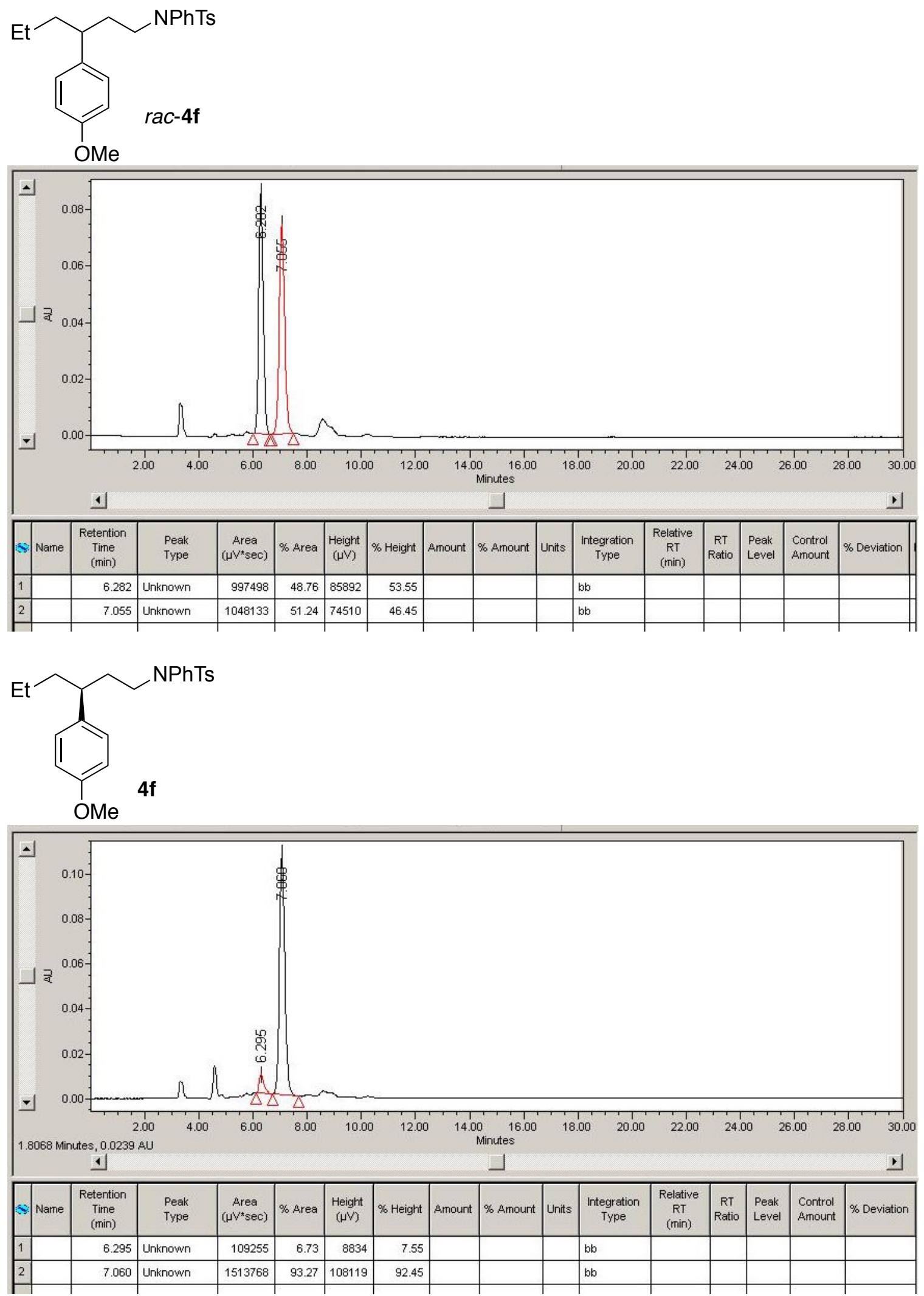

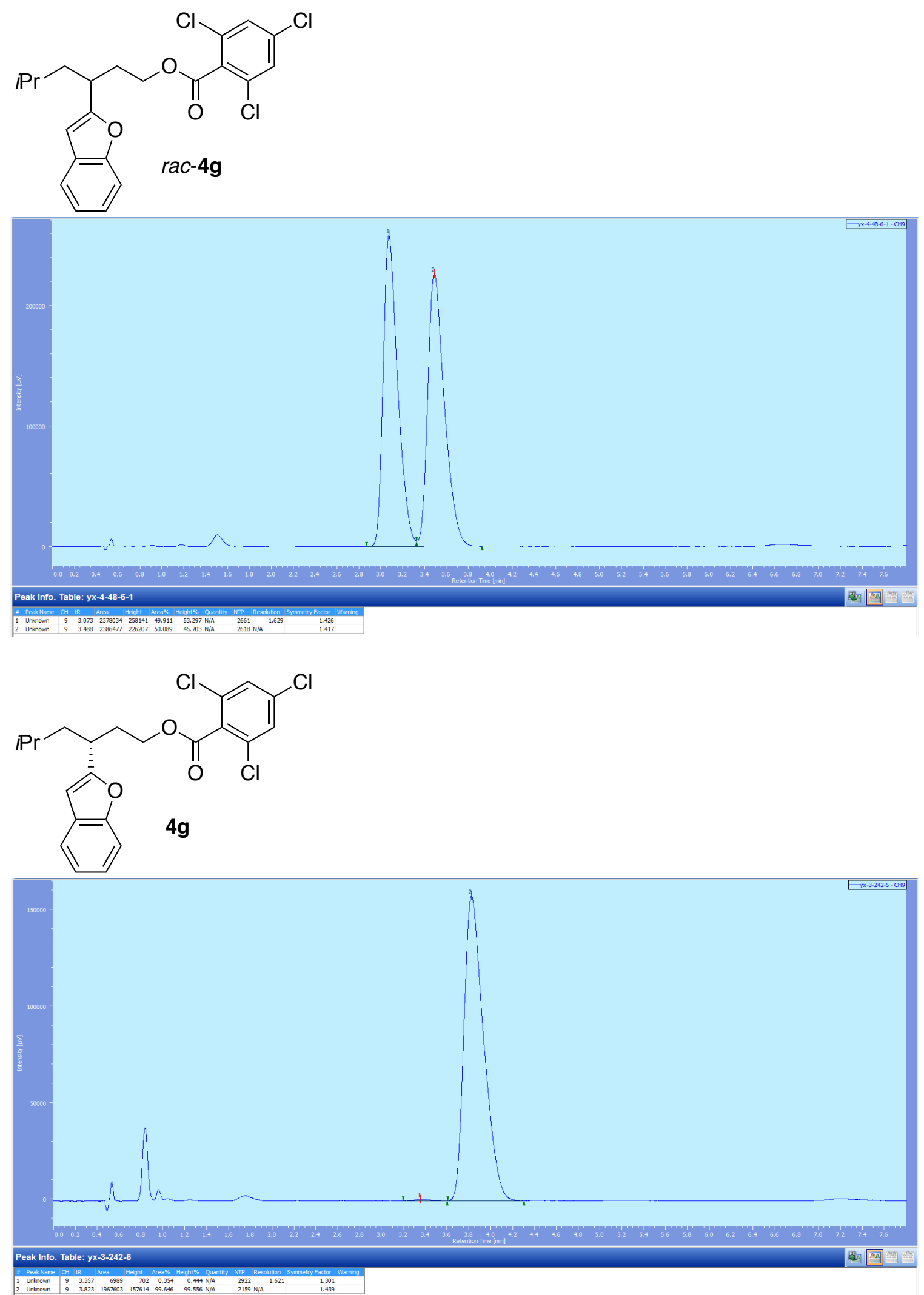

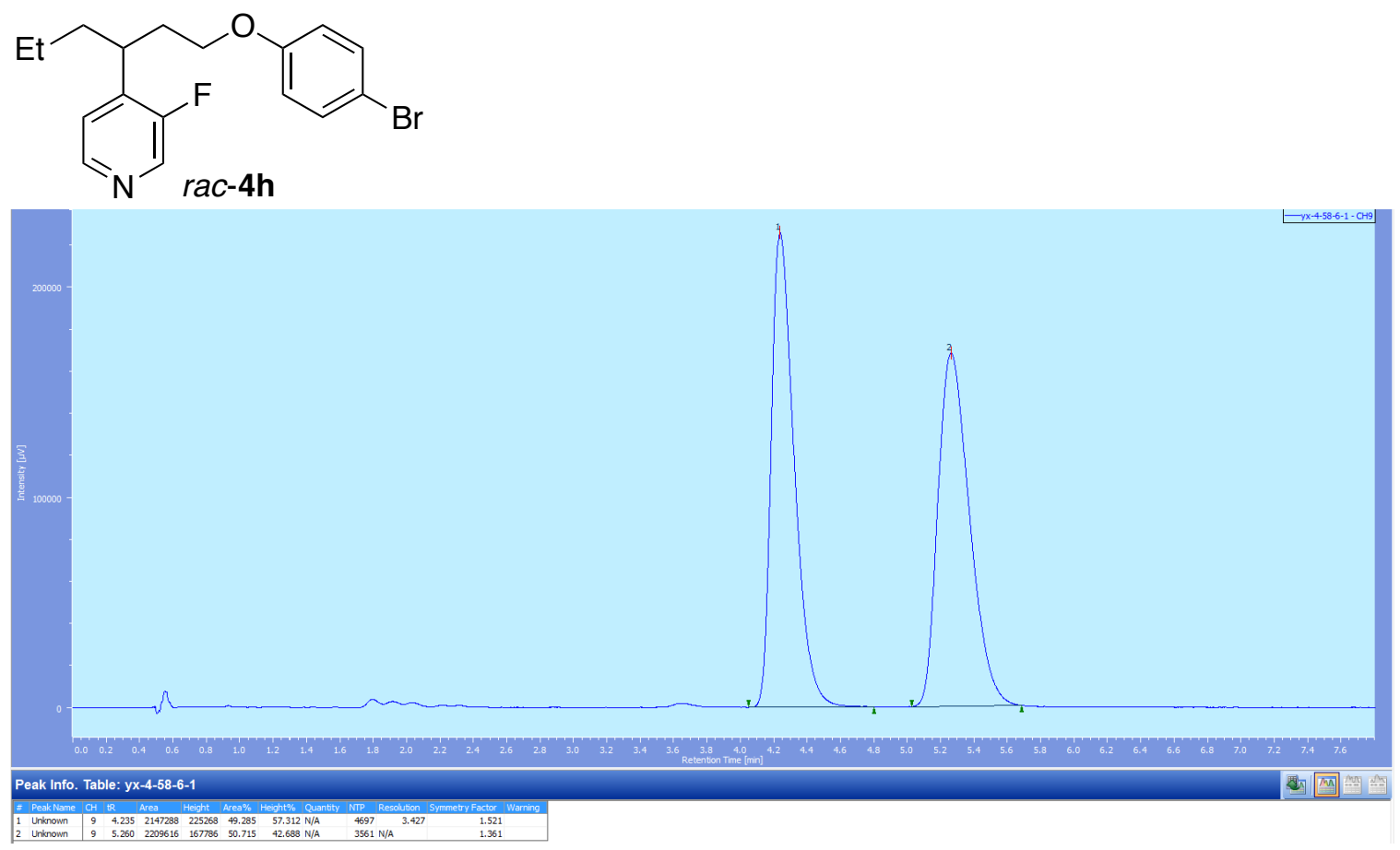<smiles>CCC(CCOc1ccc(Br)cc1)c1ccncc1F</smiles>

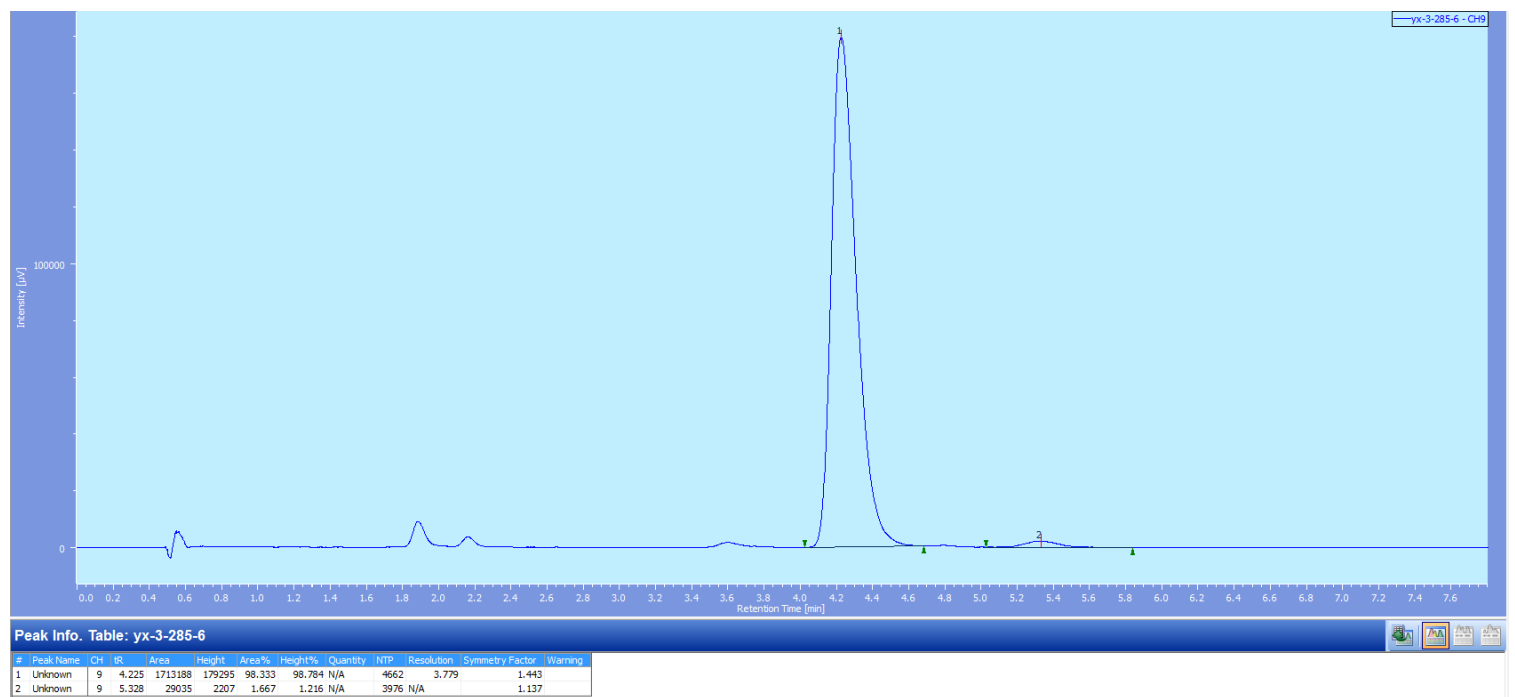


XIII. NMR Spectra 
YX-4-57-1H.1.fid

CC: 12182012 AV-500 TBIP probe

1H 1D NMR
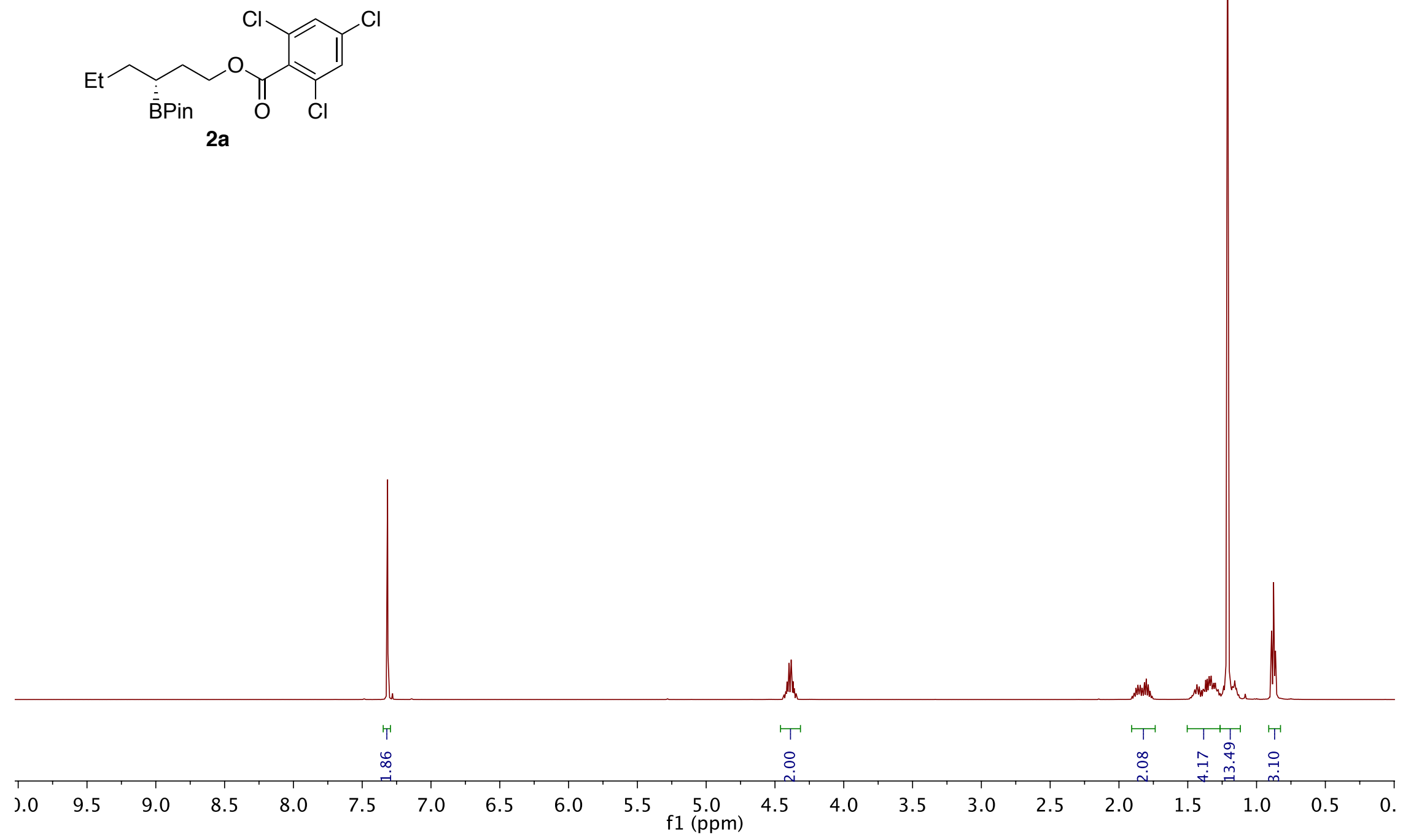


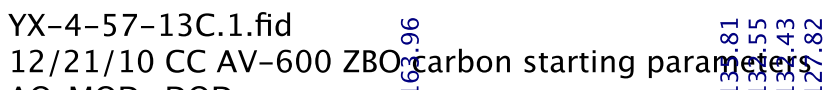
$A Q \angle M O D=D Q D$

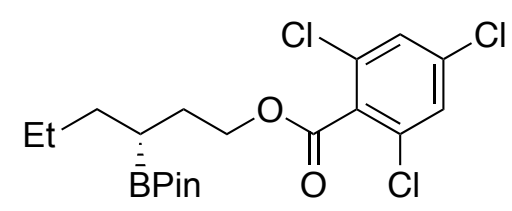

$2 a$

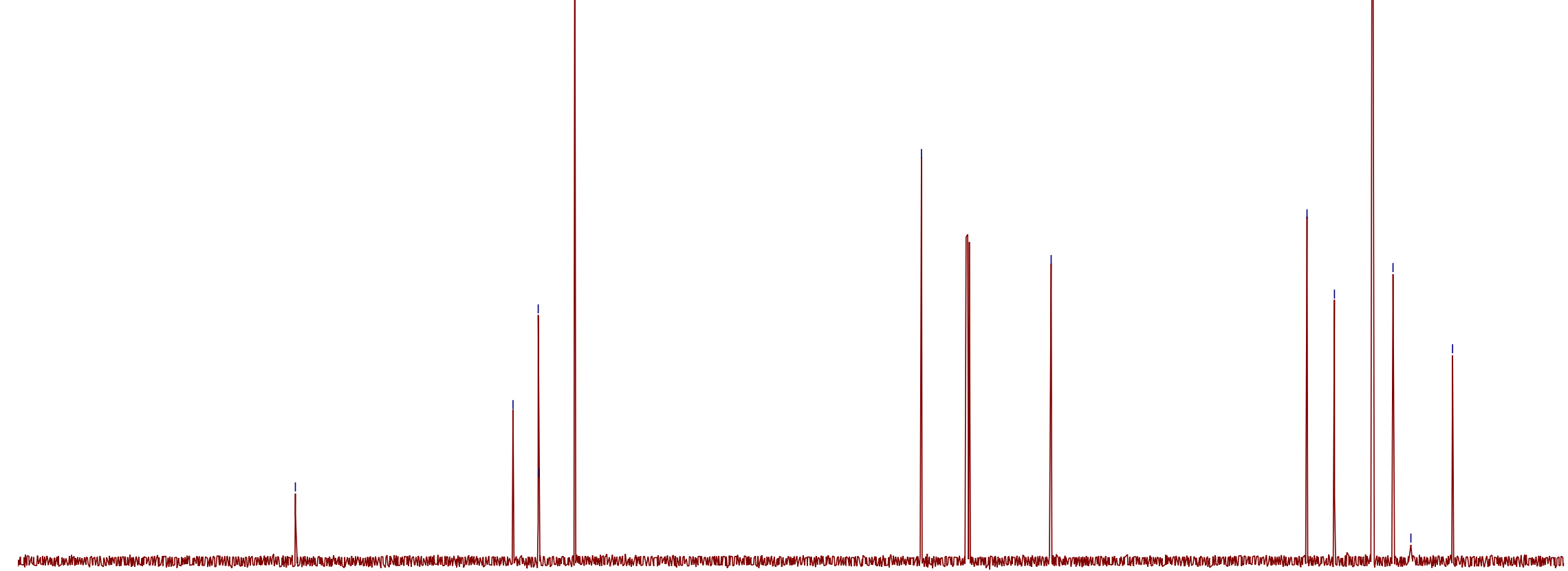

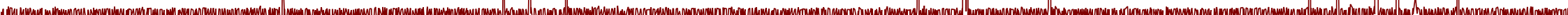

\begin{tabular}{|c|c|c|c|c|c|c|c|c|c|c|c|c|c|c|c|c|c|c|}
\hline 190 & 180 & 170 & 160 & 150 & 140 & 130 & 120 & 110 & $\begin{array}{l}100 \\
\mathrm{f} 1(\mathrm{ppm})\end{array}$ & 90 & 80 & 70 & 60 & 50 & 40 & 30 & 20 & 10 \\
\hline
\end{tabular}


YX-3-41A-P-11B.1.fid

AV-600 11B starting parameters్

with $1 \mathrm{H}$ decoupling

Using 90 pulses (for T1 $<0.7$ sec or less)

$\mathrm{SW}=265 \mathrm{ppm}$, O1P $=40 \mathrm{BF} 3$.Et2O $=0 \mathrm{ppm}$.

For $1 \mathrm{H}$ coupled experiments use ased to change pulse prog. to $\mathrm{zg}$

Use linear prediction (ME $\bmod =\mathrm{LPbc}$ ) to remove probe background<smiles>CCC[C@H]([B]c1ccccc1)CCOC(=O)c1c(Cl)cc(Cl)cc1Cl</smiles>

$2 \mathbf{a}$

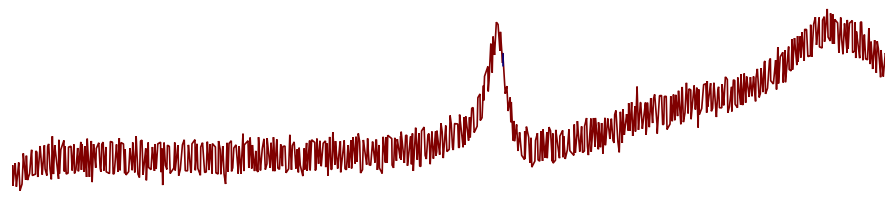

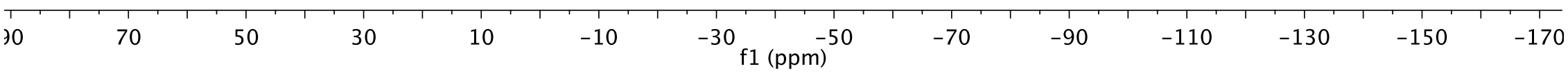


YX-3-239-1H.1.fid

CC: 12182012 AV-500 TBIP probe

1H 1D NMR

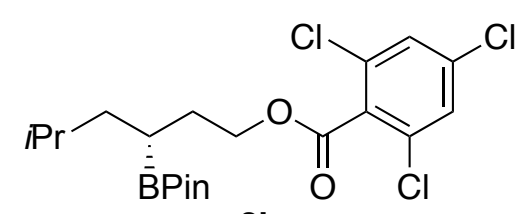

$2 b$

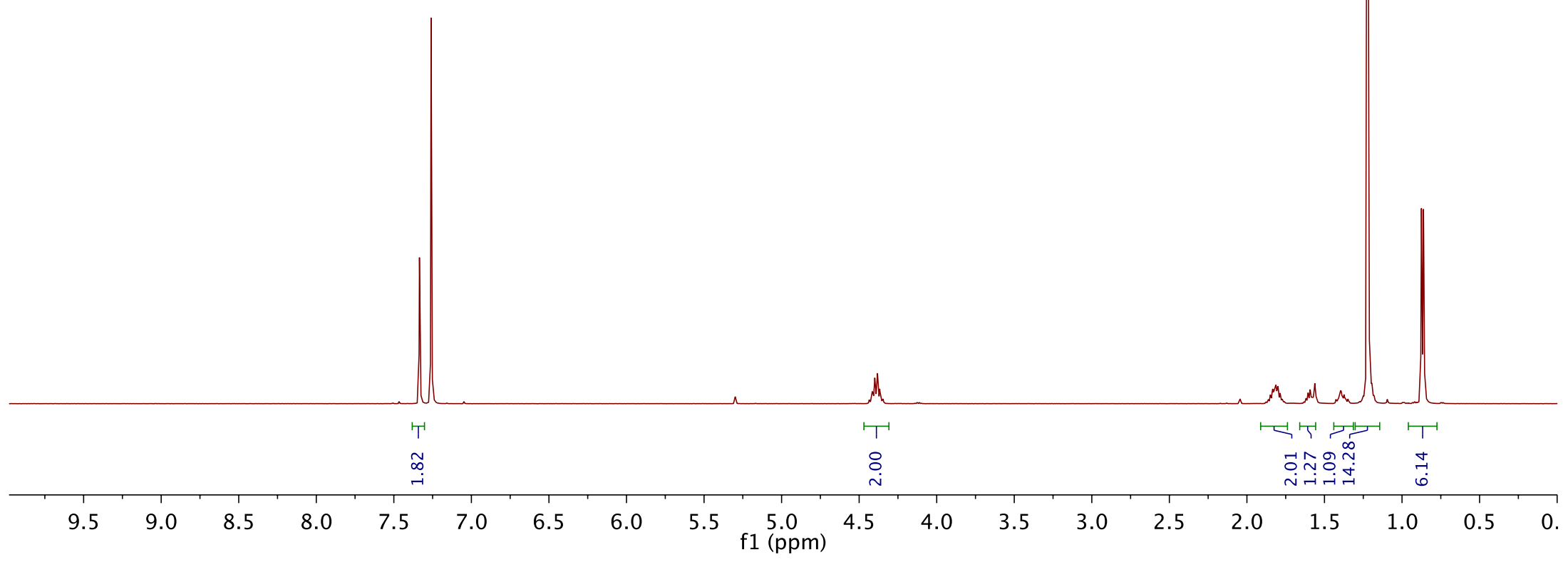


$\mathrm{YX}-3-215-13 \mathrm{C} .1$. fid

$12 / 21 / 10$ CC AV-600 ZBO

$A Q M O D=D Q D$

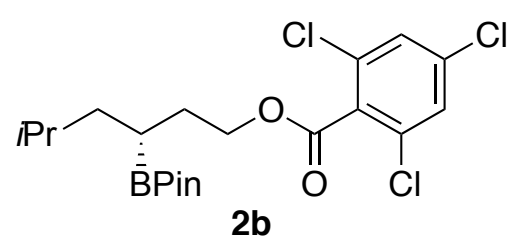

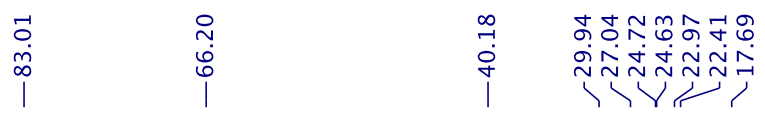

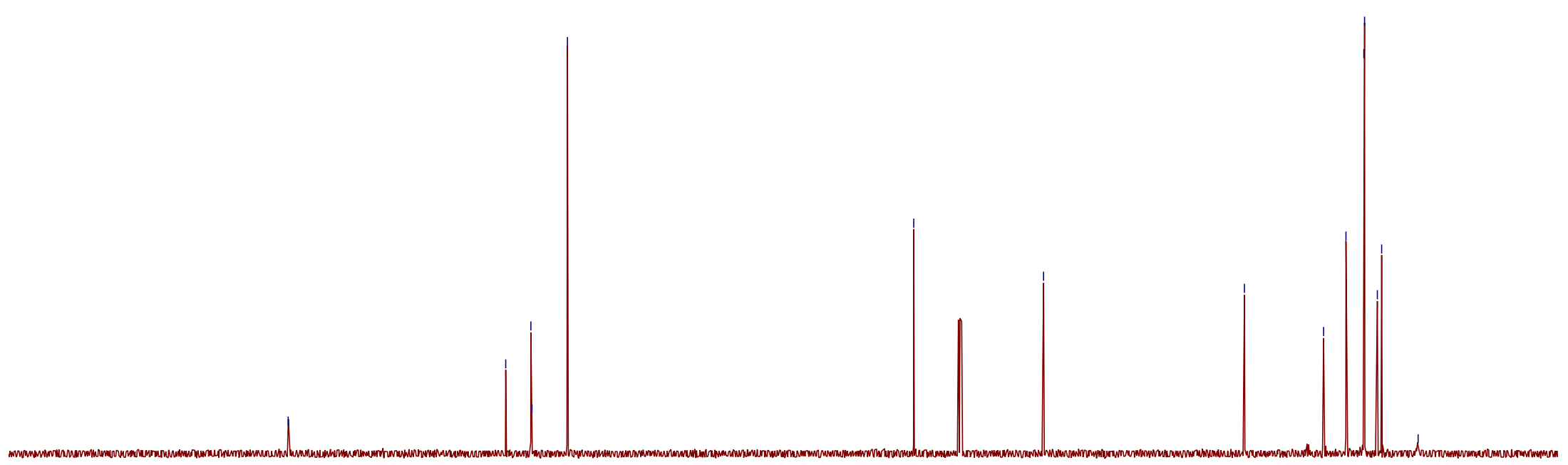

\begin{tabular}{|c|c|c|c|c|c|c|c|c|c|c|c|c|c|c|c|c|c|c|c|}
\hline 30 & 190 & 180 & 170 & 160 & 150 & 140 & 130 & 120 & 110 & $\begin{array}{c}100 \\
\mathrm{f} 1(\mathrm{ppm})\end{array}$ & 90 & 80 & 70 & 60 & 50 & 40 & 30 & 20 & 10 \\
\hline
\end{tabular}


YX-3-215-11B.1.fid

AV-600 11B starting parameters?

with $1 \mathrm{H}$ decoupling

Using 90 pulses (for T1 $<0.7$ sec or less).

$\mathrm{SW}=265 \mathrm{ppm}$, O1P $=40 \mathrm{BF} 3$.Et2O $=0 \mathrm{ppm}$.

For $1 \mathrm{H}$ coupled experiments use ased to change pulse prog. to $\mathrm{zg}$

Use linear prediction (ME $\bmod =\mathrm{LPbc}$ ) to remove probe background

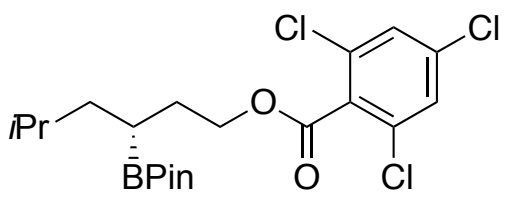

$2 \mathrm{~b}$

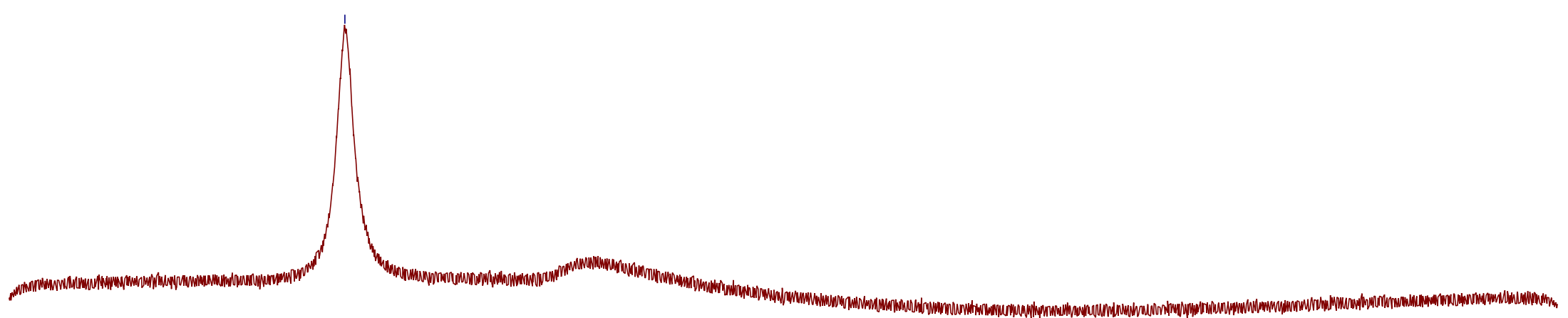

\begin{tabular}{llllllllllllllll}
\hline 0 & 70 & 50 & 30 & 10 & -10 & -30 & -50 & -70 & -90 & -110 & -130 & -150 & -170
\end{tabular}


YX-3-217B-P-1H.1.fid

CC: 12182012 AV-500 TBIP probe

1H 1D NMR

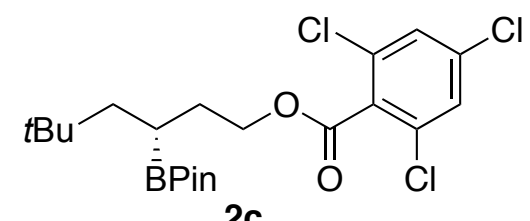

2c

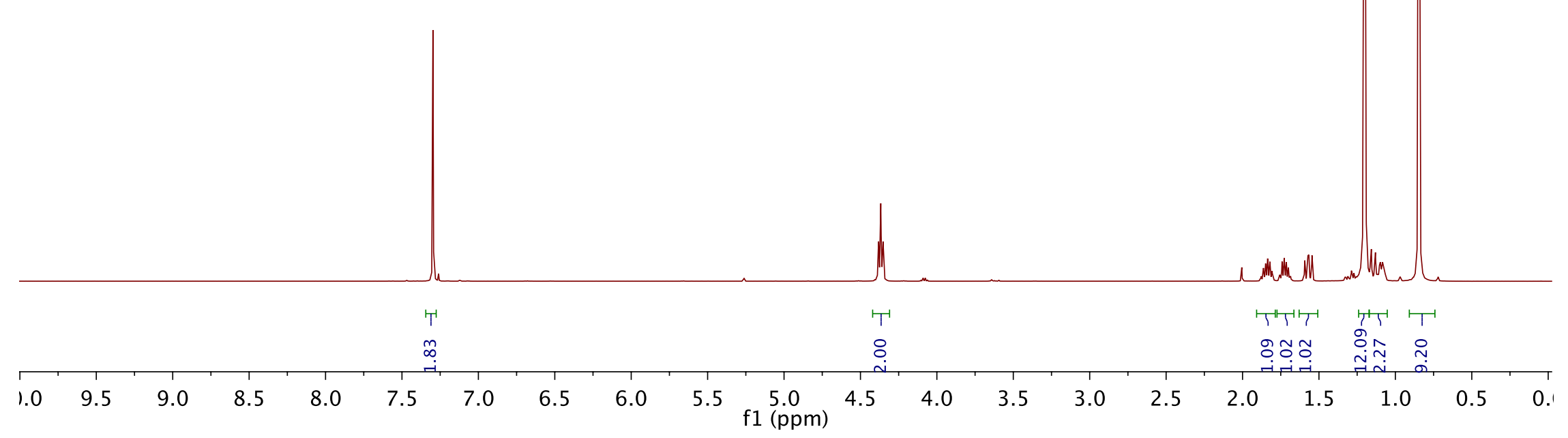


YX-3-217B-13C.1.fid

$12 / 21 / 10$ CC AV-600 $2 B$ ? $\quad$ m

$A Q \angle M O D=D Q D$

|

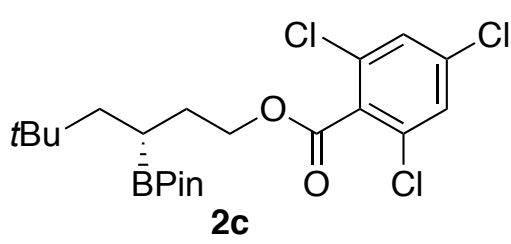

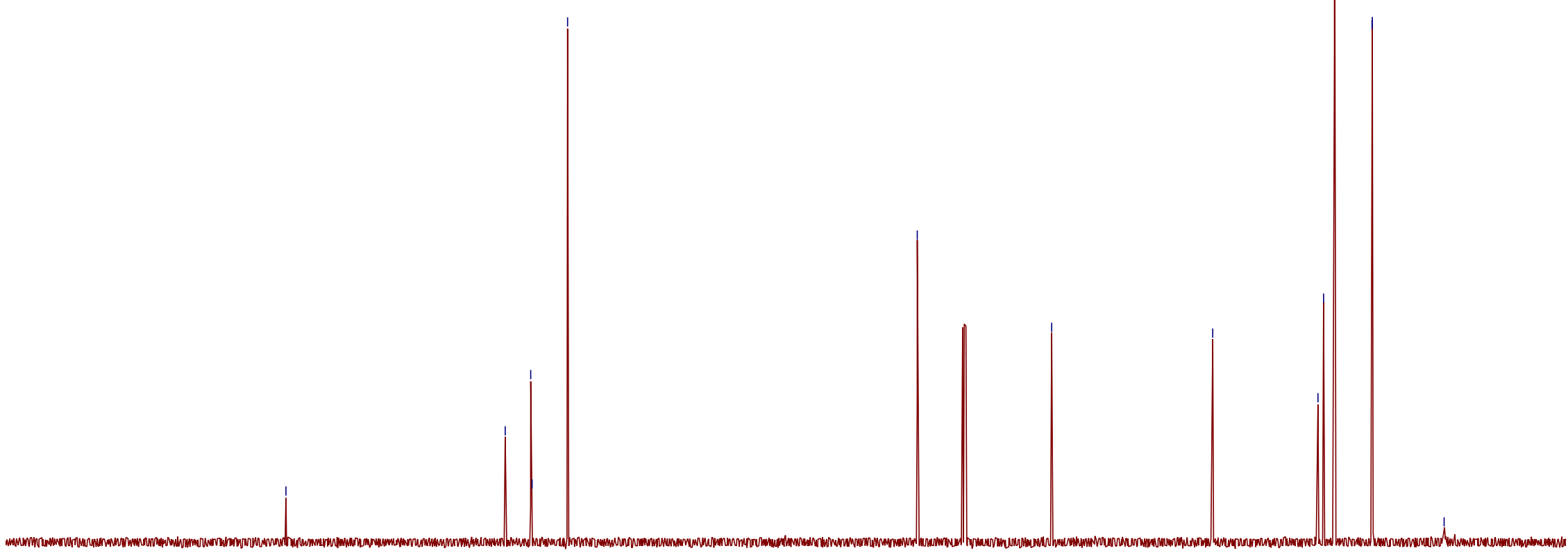

180

170

160

150

140

130

120

110 100

90

80

70

60

50

40

30

20 
YX-3-217B-11B.1.fid

AV-600 11B starting parameter

with $1 \mathrm{H}$ decoupling

Using 90 pulses (for T1 $<0.7$ sec or less).

$\mathrm{SW}=265 \mathrm{ppm}$, O1P $=40 \mathrm{BF} 3$.Et2O $=0 \mathrm{ppm}$.

For $1 \mathrm{H}$ coupled experiments use ased to change pulse prog. to $\mathrm{zg}$

Use linear prediction (ME $\bmod =\mathrm{LPbc}$ ) to remove probe background

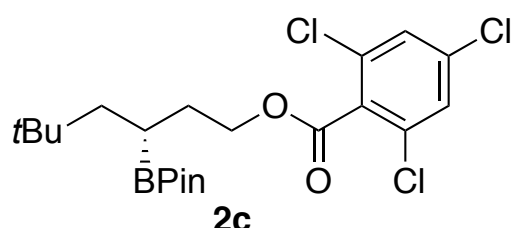

2c

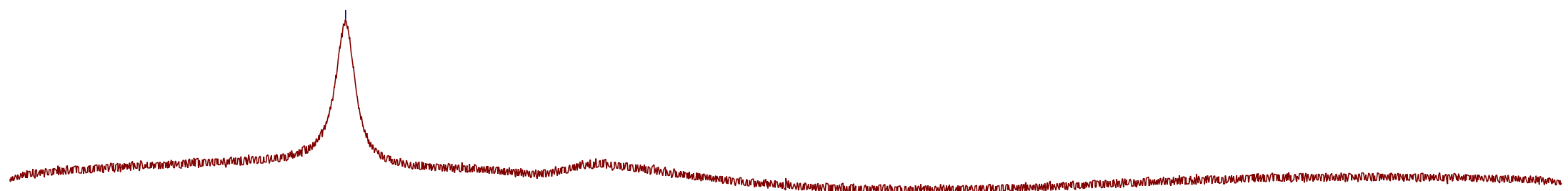

\begin{tabular}{llllllllllllllll}
\hline 0 & 70 & 50 & 30 & 10 & -10 & -30 & -50 & -70 & -90 & -110 & -130 & -150 & -170
\end{tabular}


YX-3-217C-P-1H.1.fid

CC: 12182012 AV-500 TBIP probe

1H 1D NMR
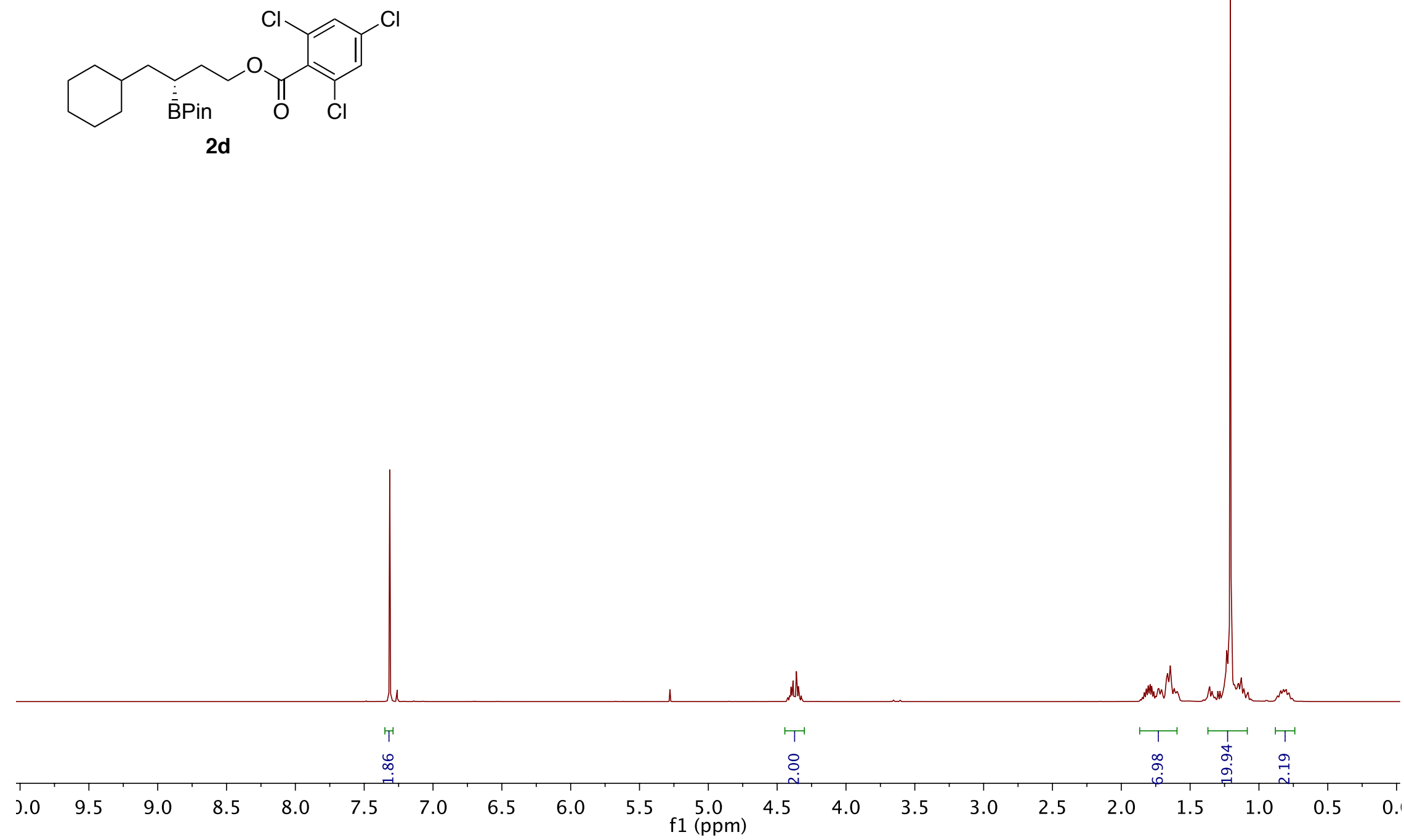


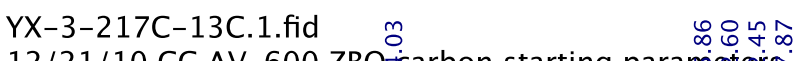

12/21/10 CC AV-600 ZBO Gicarbon starting parameiefers

$A Q M O D=D Q D$

।

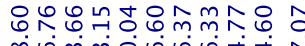

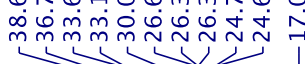<smiles>O=C(OCCC([B]c1ccccc1)CC1CCCCC1)c1c(Cl)cc(Cl)cc1Cl</smiles>

2d

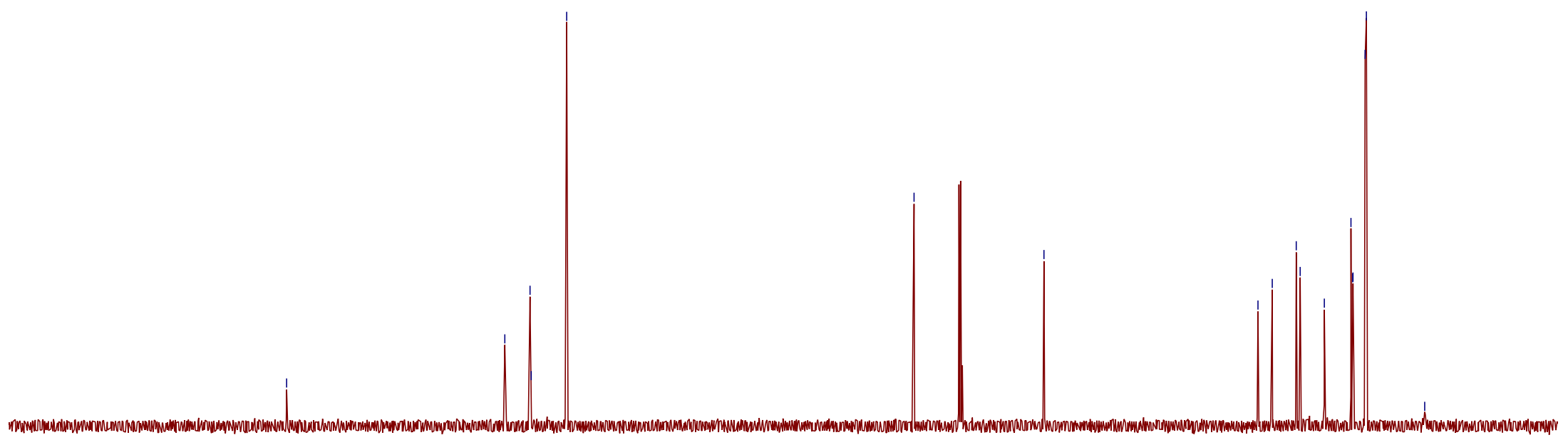


YX-3-217C-11B.1.fid

AV-600 11B starting parameters

with $1 \mathrm{H}$ decoupling

Using 90 pulses (for T1 $<0.7$ sec or less).

$\mathrm{SW}=265 \mathrm{ppm}$, O1P $=40 \mathrm{BF} 3$.Et2O $=0 \mathrm{ppm}$.

For $1 \mathrm{H}$ coupled experiments use ased to change pulse prog. to $\mathrm{zg}$

Use linear prediction (ME $\bmod =\mathrm{LPbc}$ ) to remove probe background

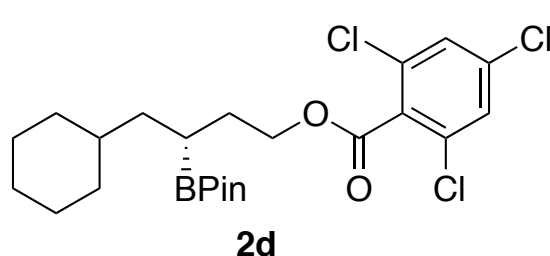

$\int_{\text {ming }}$

$\begin{array}{lllllllllllllll}70 & 70 & 50 & 30 & 10 & -10 & -30 & -50 & -70 & -90 & -110 & -130 & -150 & -170\end{array}$


YX-3-282B-1H.1.fid

CC: 12182012 AV-500 TBIP probe

$1 \mathrm{H}$ 1D NMR

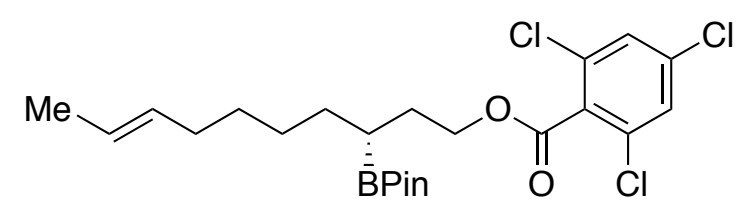

$2 e$

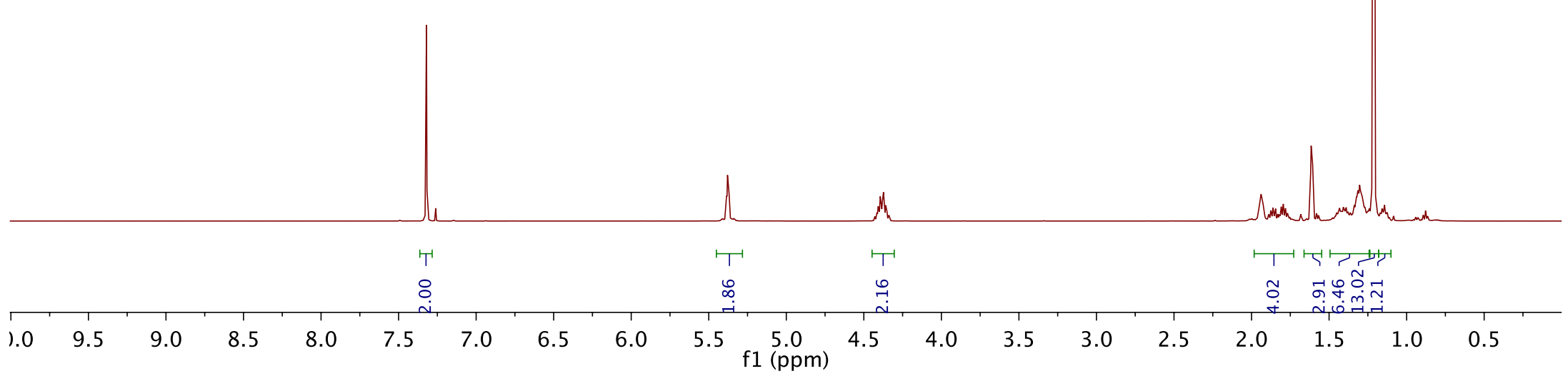




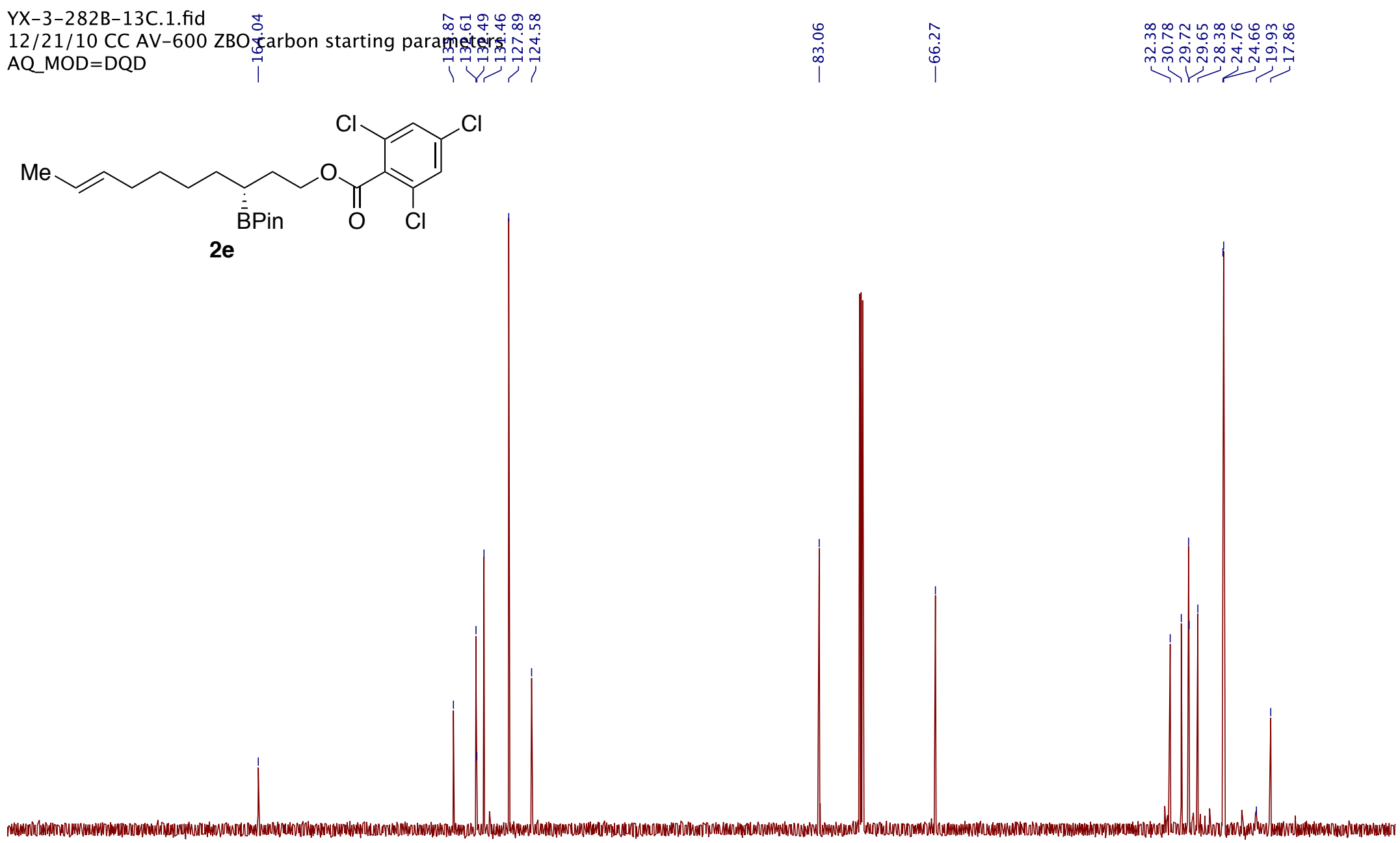

The small peaks correspond to the minor constitutional isomer $2 \mathrm{e}^{\prime}$

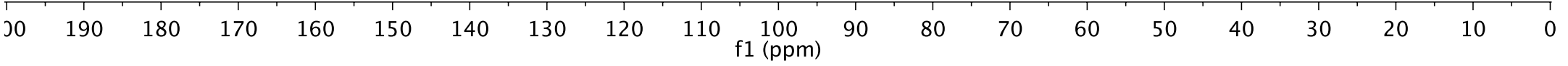


YX-3-282B-11B.1.fid

AV-600 11B starting parameters్

with $1 \mathrm{H}$ decoupling

Using 90 pulses (for T1 $<0.7$ sec or less).

$\mathrm{SW}=265 \mathrm{ppm}$, O1P $=40 \mathrm{BF} 3$.Et2O $=0 \mathrm{ppm}$.

For $1 \mathrm{H}$ coupled experiments use ased to change pulse prog. to $\mathrm{zg}$

Use linear prediction (ME $\mathrm{mod}=\mathrm{LPbc}$ ) to remove probe background

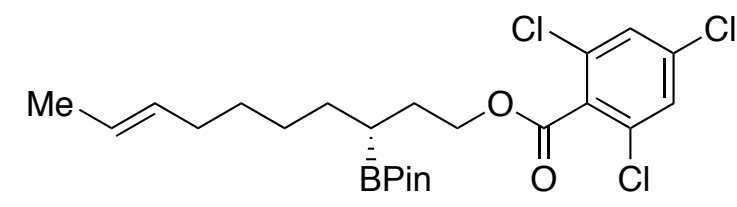

$2 e$

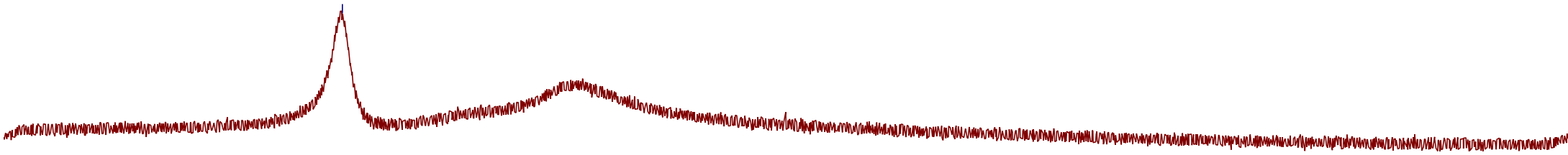

$\begin{array}{lllllllllllllllll} & 70 & 50 & 30 & 10 & -10 & -30 & -50 & -70 & -90 & -110 & -130 & -150 & -170\end{array}$


YX-3-217D-1H.1.fid

CC: 12182012 AV-500 TBIP probe

1H 1D NMR
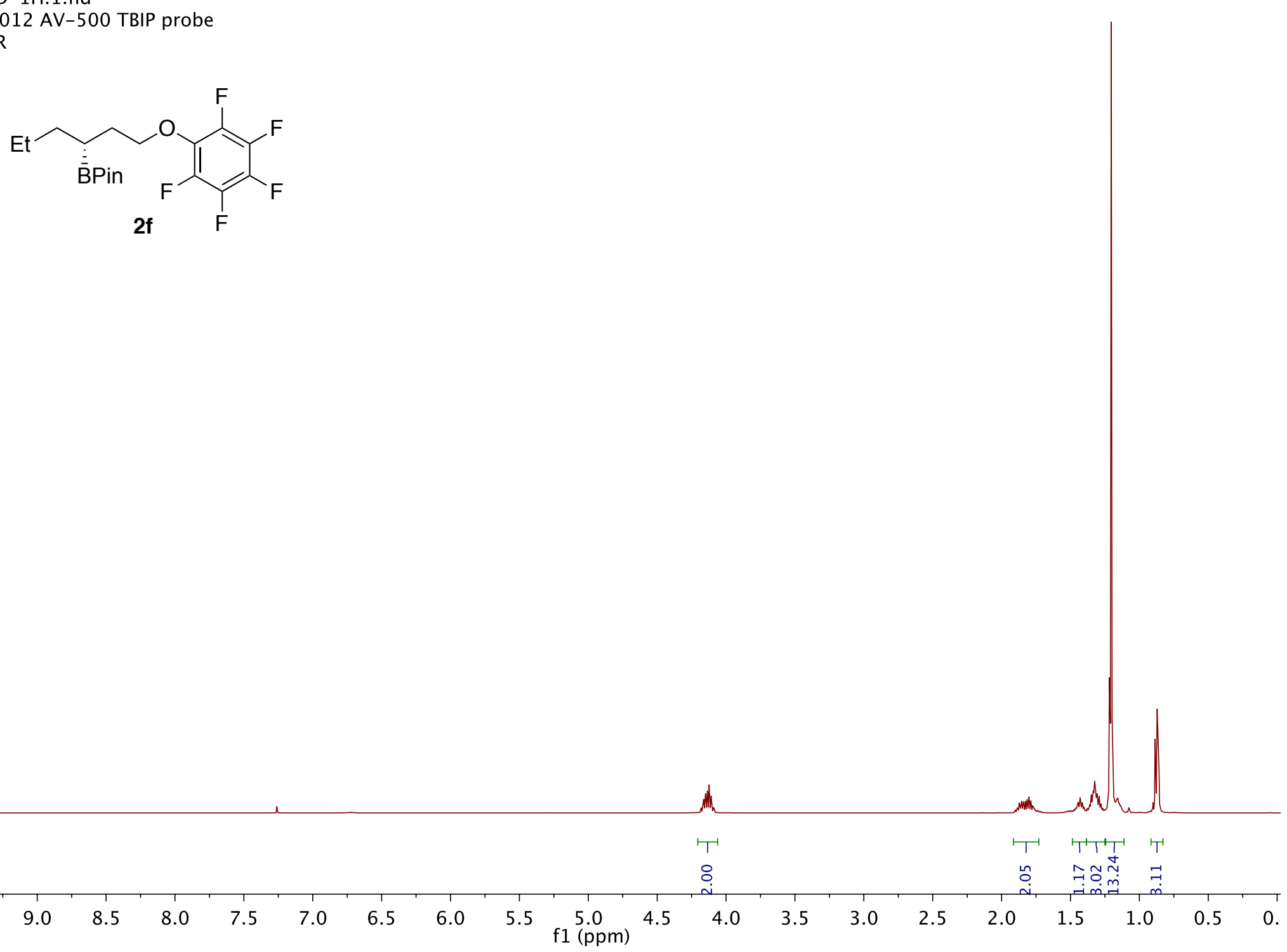
YX-3-217D-13C.1.fid

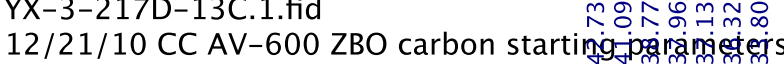
$A Q \_M O D=D Q D$

\रे।

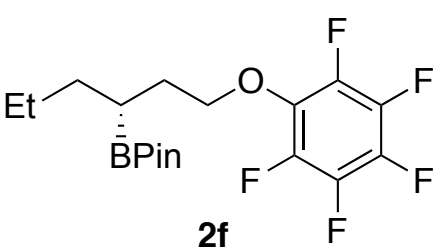

$2 f$
นกำ 종ำก

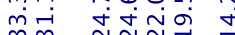

mi

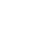
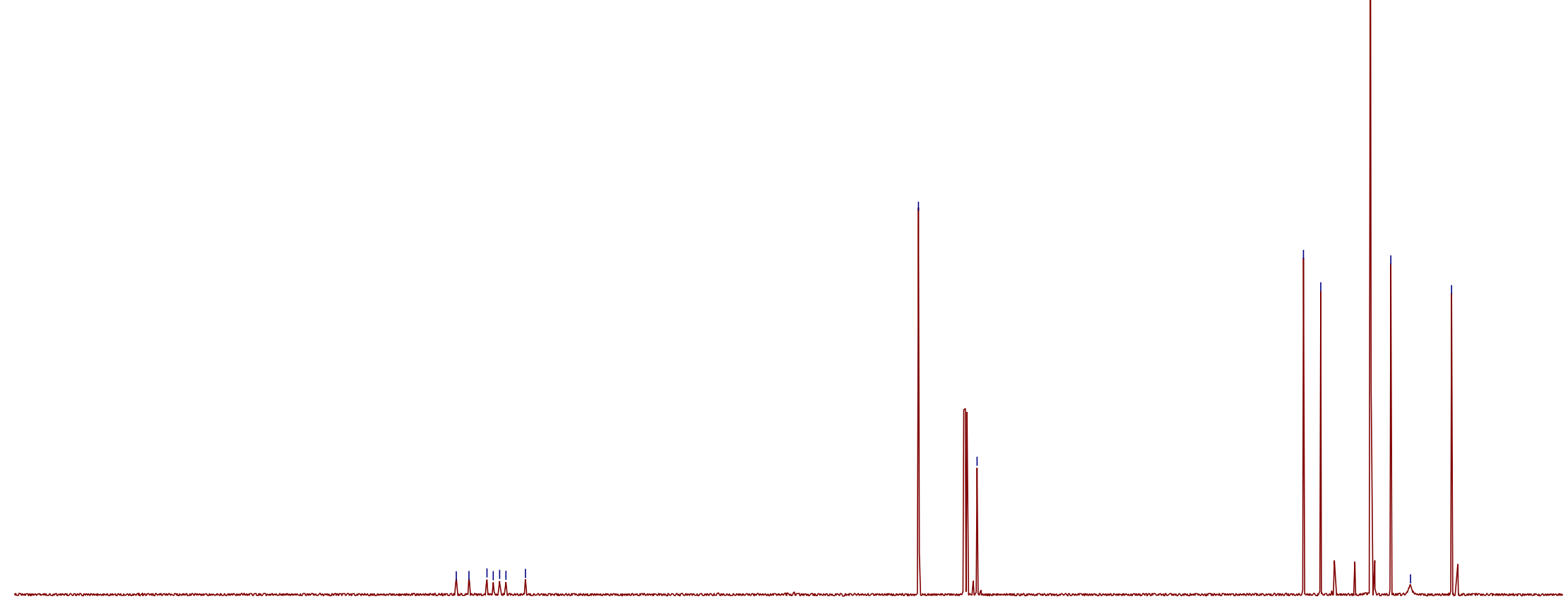

The small peaks correspond to the minor constitutional isomer $2 \mathrm{f}^{\prime}$

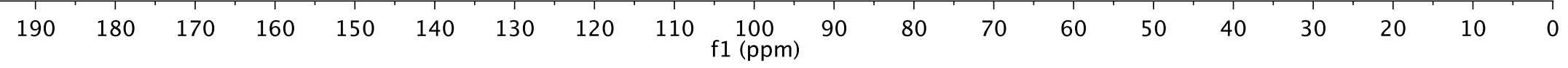


YX-3-217D-13Crifid

12/21/10 CC AV 600 ZBO carbon starifing parameters

AQ $M O D=D Q D$

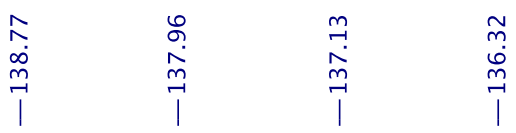

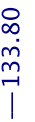

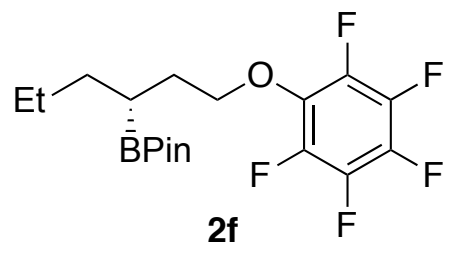

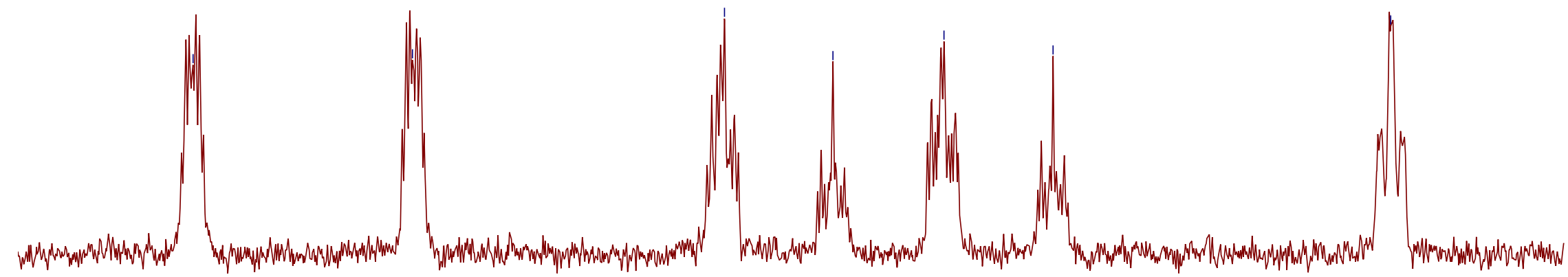

4.0143 .5143 .0142 .5142 .0141 .5141 .0140 .5140 .0139 .5139 .0138 .5138 .0137 .5137 .0136 .5136 .0135 .5135 .0134 .5134 .0133 .5133 .0 
YX-3-217D-11B.1.fid

AV-600 11B starting parameters़्ले

with $1 \mathrm{H}$ decoupling

Using 90 pulses (for T1 $<0.7$ sec or less).

$\mathrm{SW}=265 \mathrm{ppm}$, O1P $=40 \mathrm{BF} 3 . \mathrm{Et} 2 \mathrm{O}=0 \mathrm{ppm}$.

For $1 \mathrm{H}$ coupled experiments use ased to change pulse prog. to $\mathrm{zg}$

Use linear prediction (ME $\bmod =\mathrm{LPbC}$ ) to remove probe background
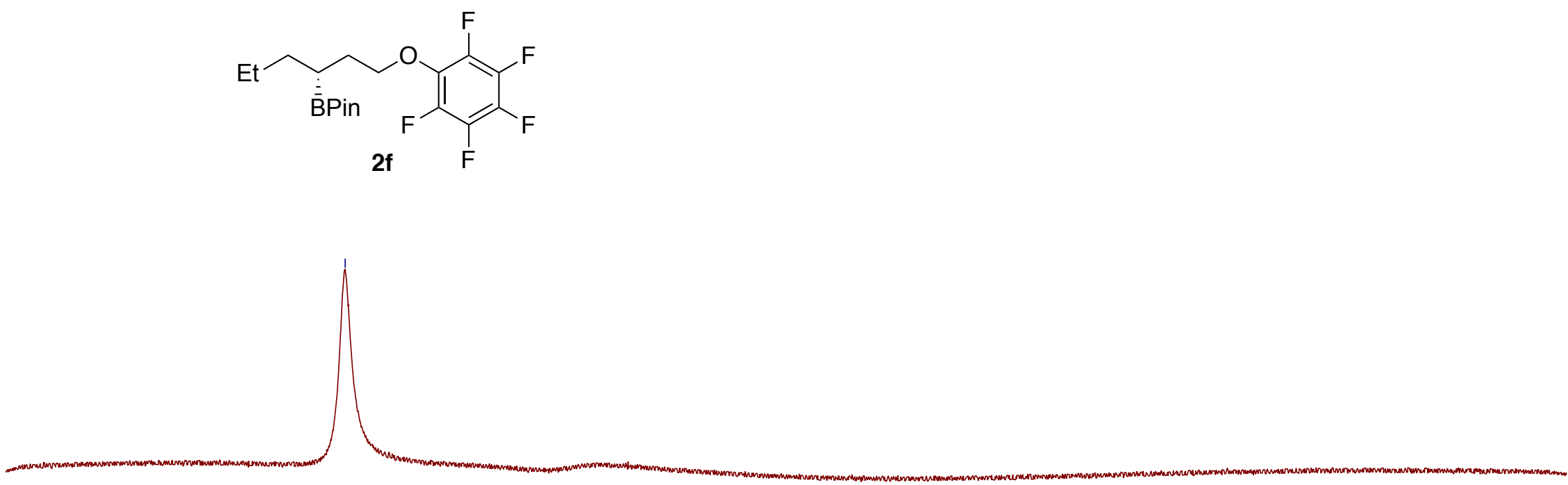

\begin{tabular}{|c|c|c|c|c|c|c|c|c|c|c|c|c|c|}
\hline 30 & 70 & 50 & 30 & 10 & -10 & -30 & m) & -70 & -90 & -110 & -130 & -150 & -170 \\
\hline
\end{tabular}


YX-3-217D-19F.1.fid

AVQ-400 QNP Probe 19F starting parameters. (revised P1, 2/12/04 RN)

chemical shifts relative to $\mathrm{CFCl} 3$ at $0 \mathrm{ppm}(082103 \mathrm{HvH})$

sw 239.28 ppm; o1p 0 ppm

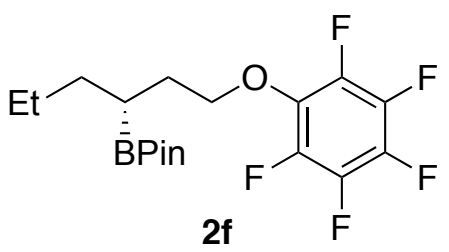

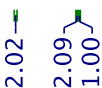

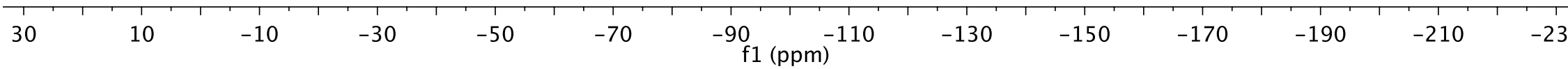


YX-3-253A1-1H.1.fid

CC: 12182012 AV-500 TBIP probe

$1 \mathrm{H}$ 1D NMR
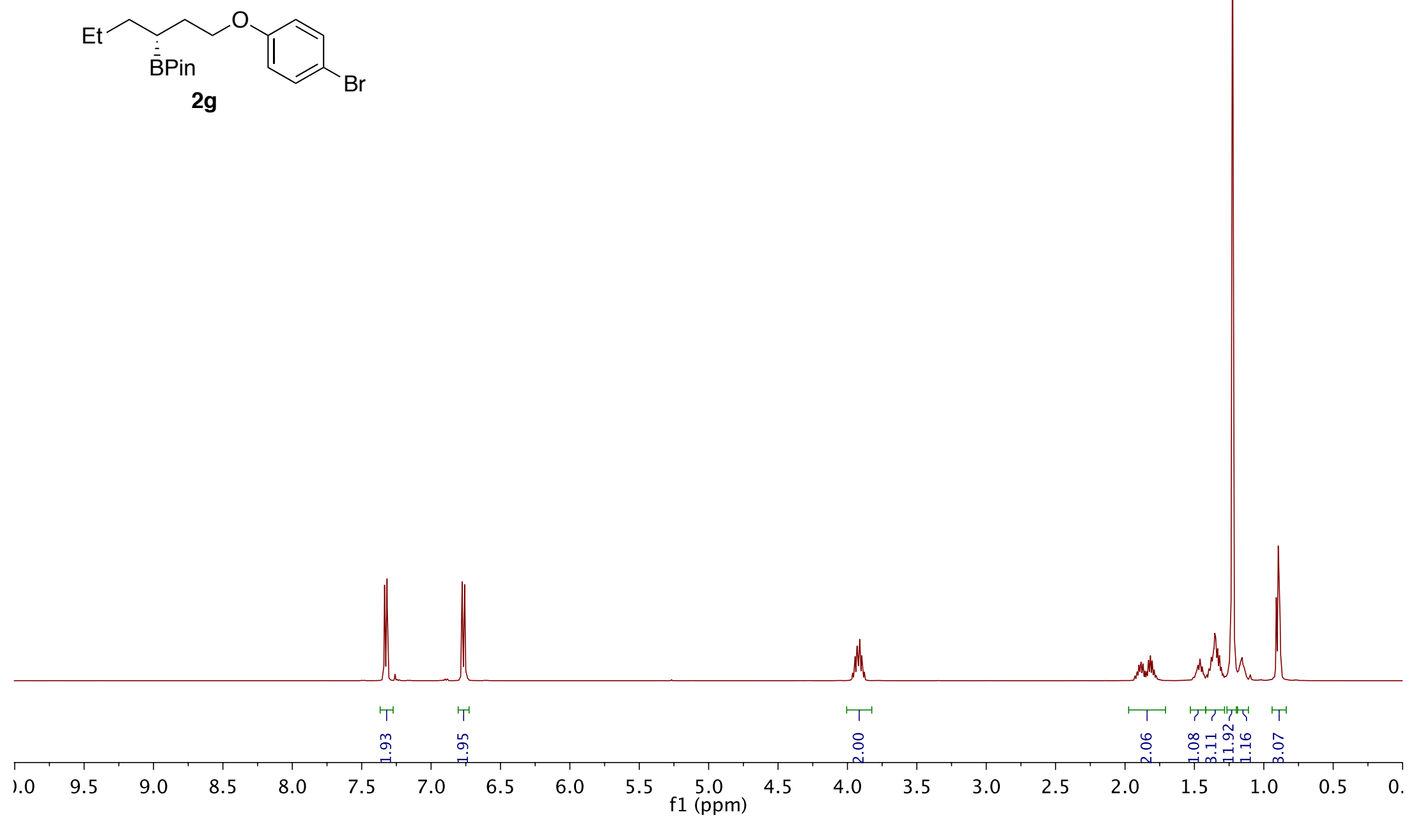
YX-3-253A1-13C.1.fid

$12 / 21 / 10$ CC AV-600 ZBO carbơn starting parameters $A Q \_M O D=D Q D$

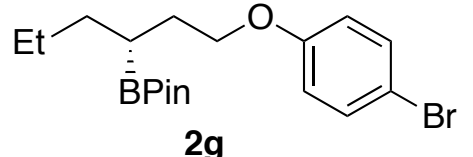

$2 \mathrm{~g}$
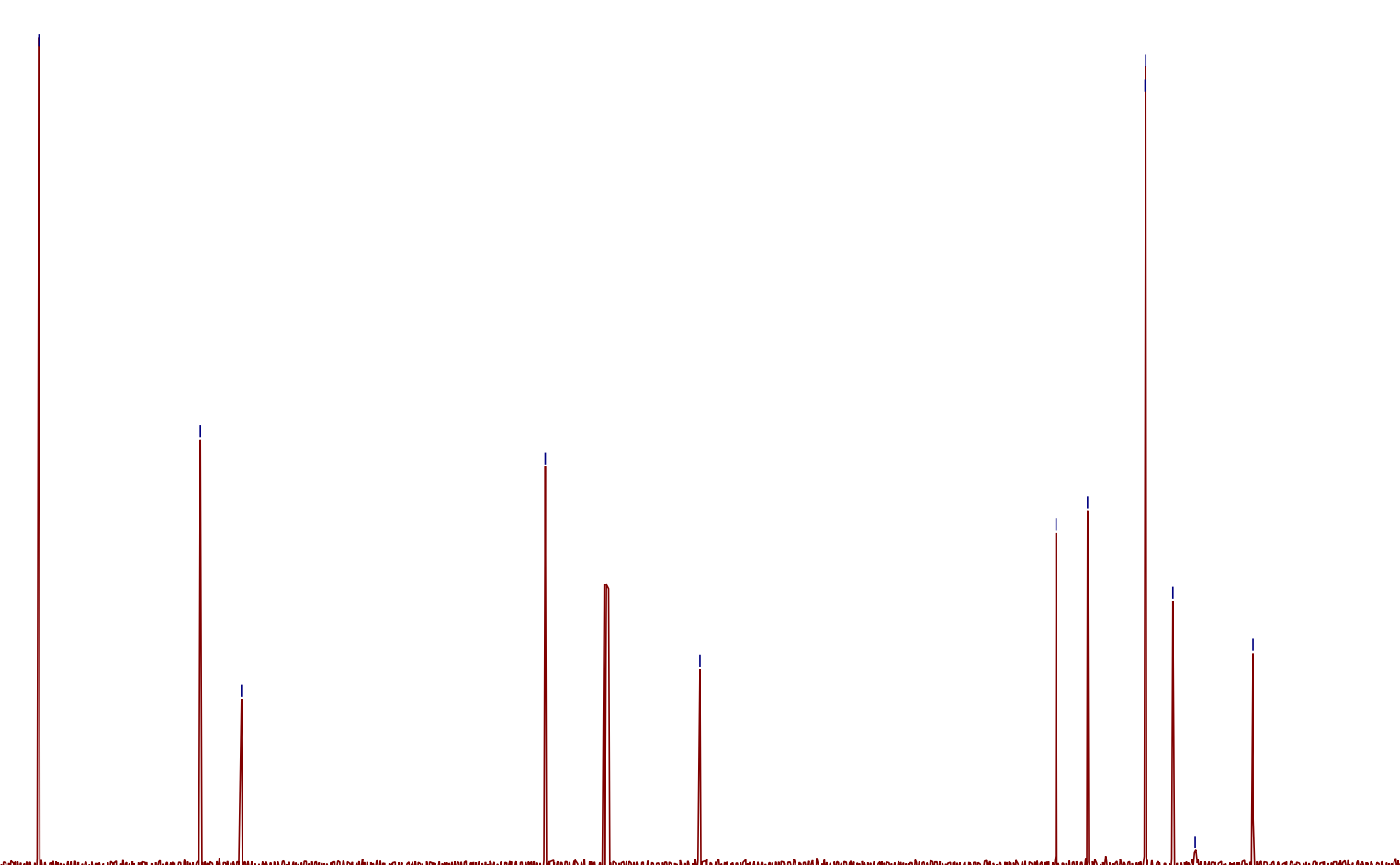

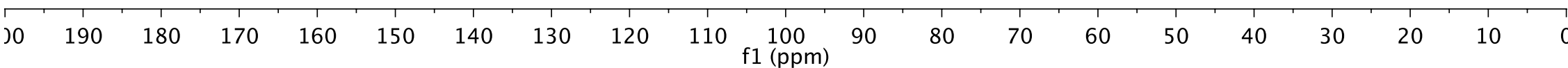


YX-3-253A1-11B.1.fid

AV-600 11B starting parameters్

with $1 \mathrm{H}$ decoupling

Using 90 pulses (for T1 $<0.7$ sec or less).

$\mathrm{SW}=265 \mathrm{ppm}$, O1P $=40 \mathrm{BF} 3$.Et2O $=0 \mathrm{ppm}$.

For $1 \mathrm{H}$ coupled experiments use ased to change pulse prog. to $\mathrm{zg}$

Use linear prediction (ME $\mathrm{mod}=\mathrm{LPbc}$ ) to remove probe background

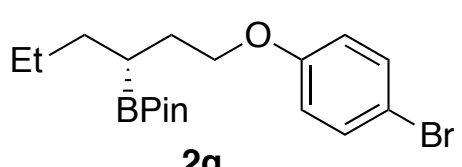

2g

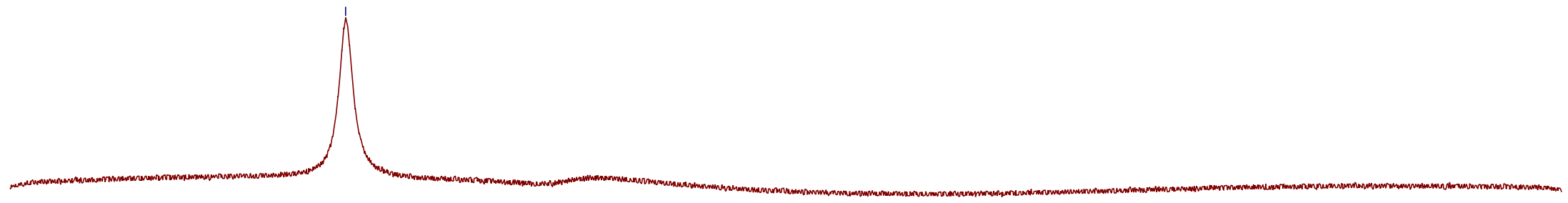


YX-3-217E-1H.1.fid

CC: 12182012 AV-500 TBIP probe

1H 1D NMR

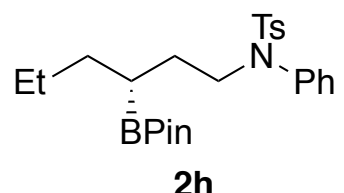

$2 \mathrm{~h}$

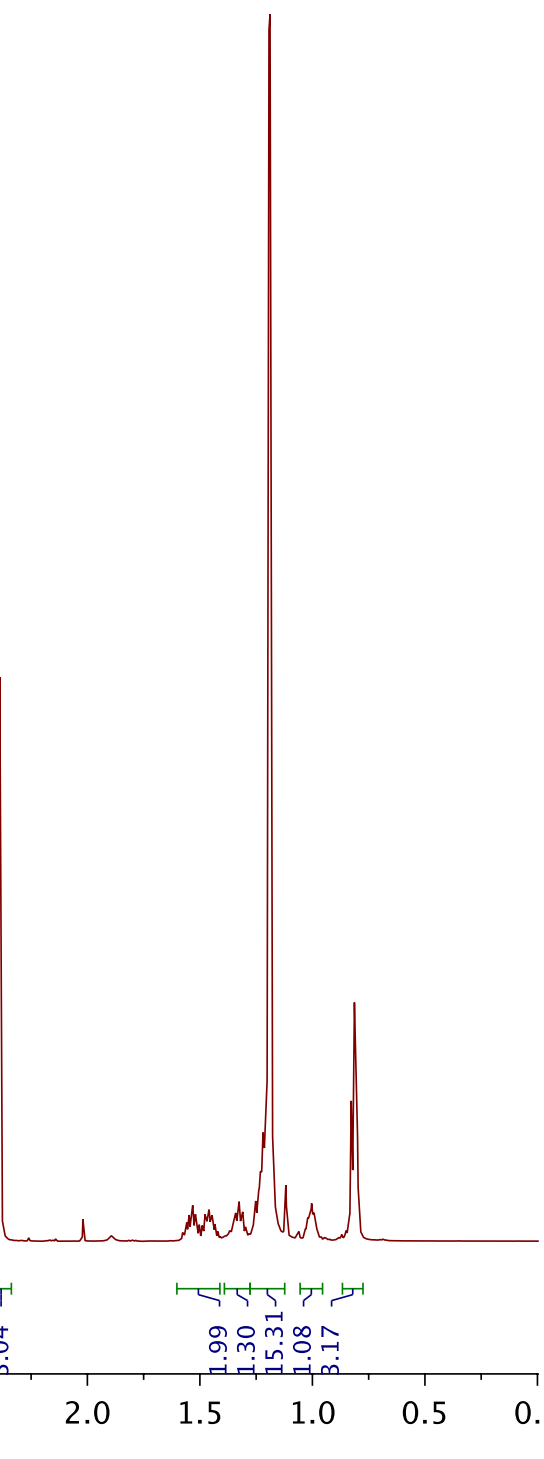


$\mathrm{YX}-3-217 \mathrm{E}-13 \mathrm{C} .1$. fid

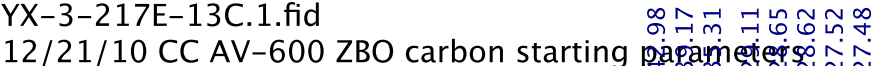
$A Q \quad M O D=D Q D$

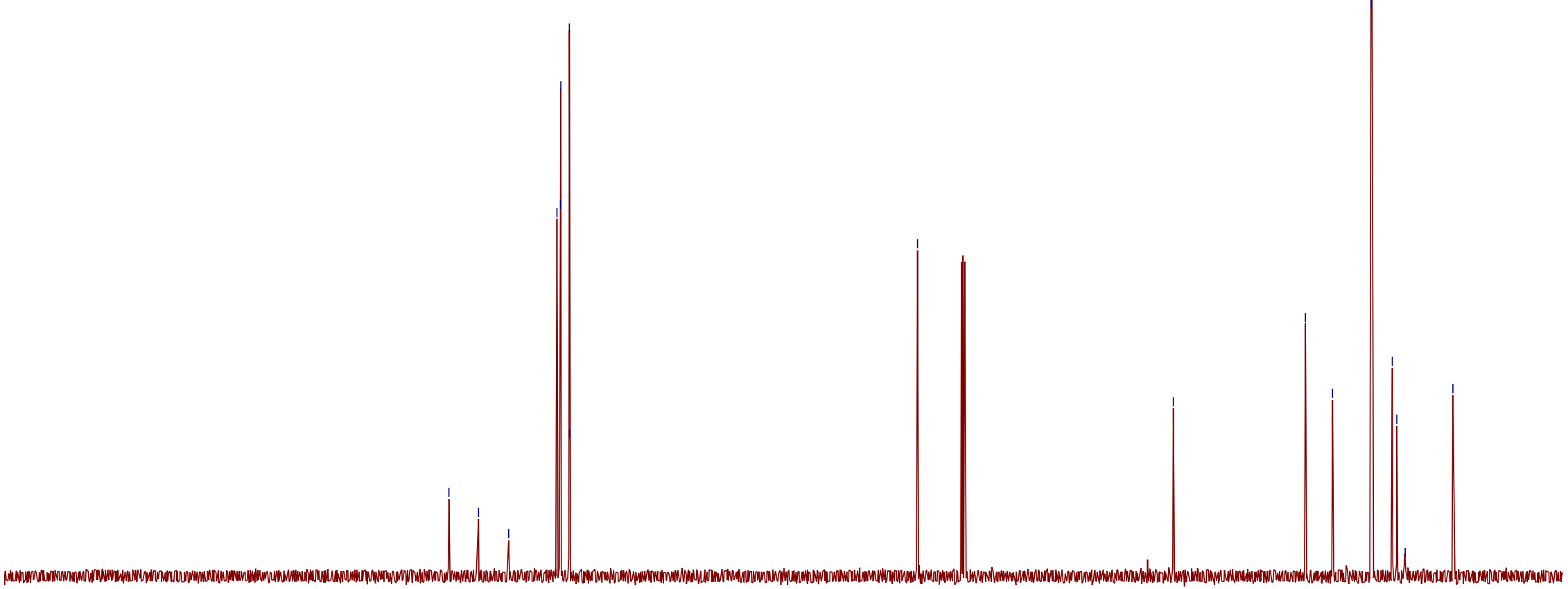

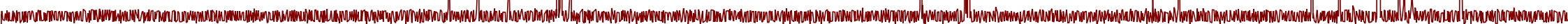

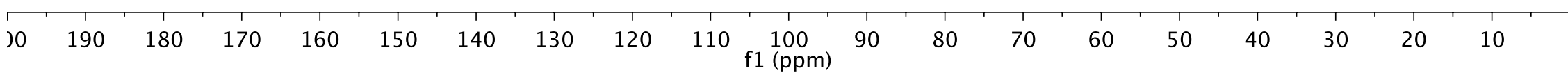


YX-3-217E-11B.1.fid

AV-600 11B starting parameter疋.

with $1 \mathrm{H}$ decoupling

Using 90 pulses (for $\mathrm{T} 1<0.7 \mathrm{sec}$ or less).

$\mathrm{SW}=265 \mathrm{ppm}$, O1P $=40 \mathrm{BF} 3$.Et2O $=0 \mathrm{ppm}$.

For $1 \mathrm{H}$ coupled experiments use ased to change pulse prog. to $\mathrm{zg}$

Use linear prediction (ME $\bmod =\mathrm{LPbc}$ ) to remove probe background

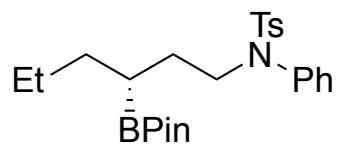

2h

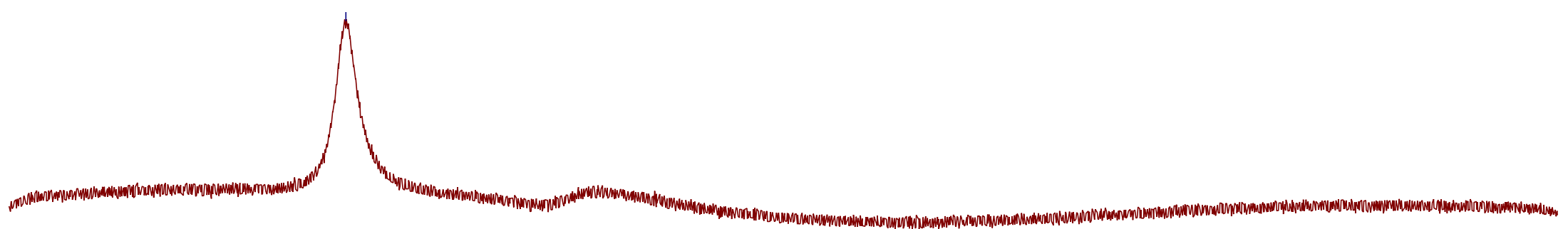

\begin{tabular}{lllllllllllllllll}
\hline 0 & 70 & 50 & 30 & 10 & -10 & -30 & -50 & -70 & -90 & -110 & -130 & -150 & -170
\end{tabular}


YX-3-217F-1H.1.fid

CC: 12182012 AV-500 TBIP probe

1H 1D NMR

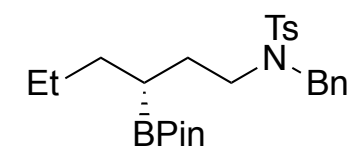

2i

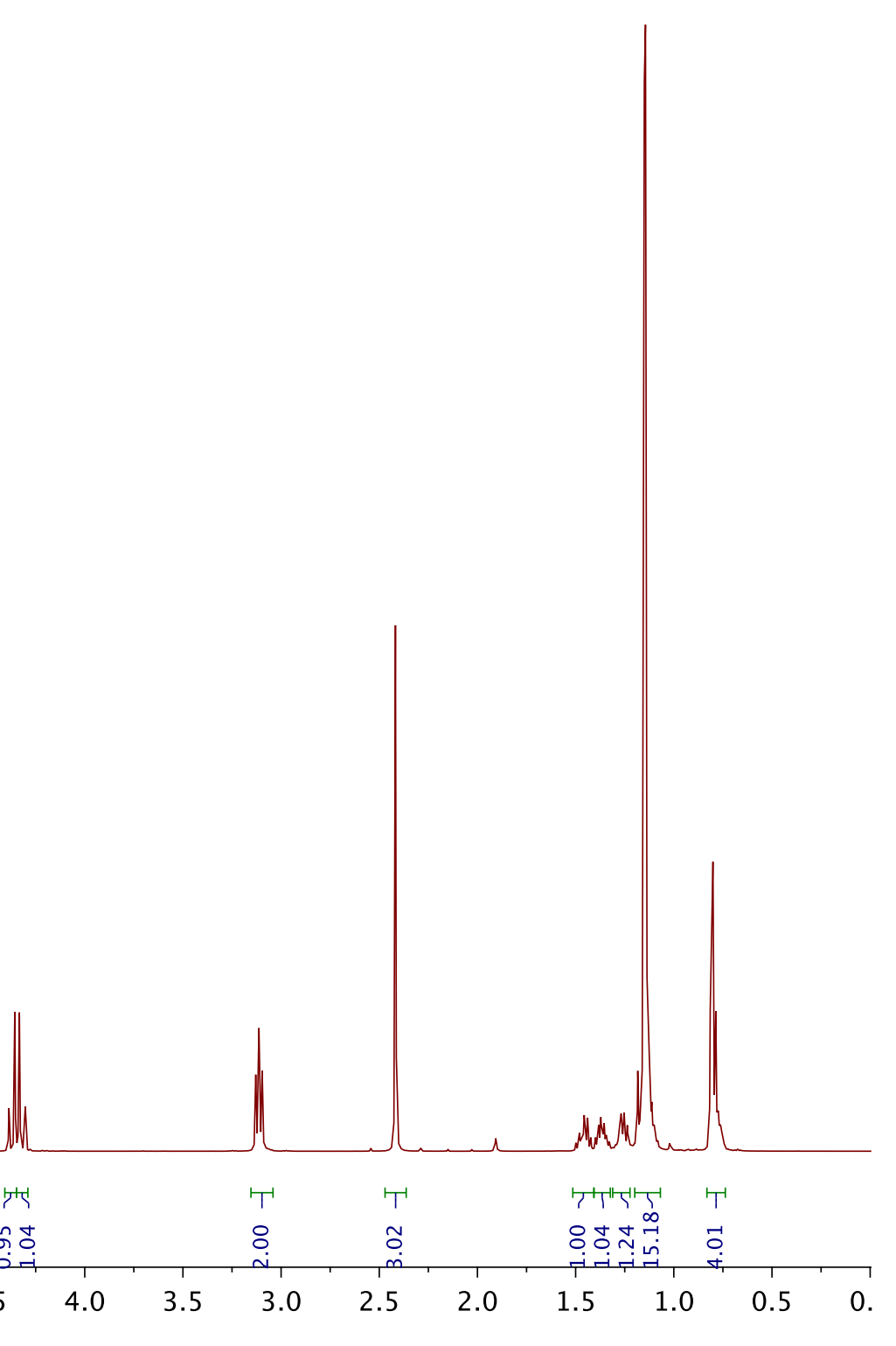


$\mathrm{YX}-3-217 \mathrm{~F}-13 \mathrm{C} .1$. fid

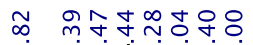

12/21/10 CC AV-600 ZBO carbon starting parangetexs

$A Q M O D=D Q D$

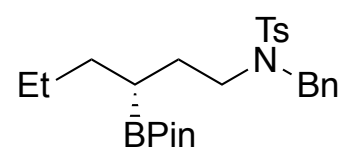

2i

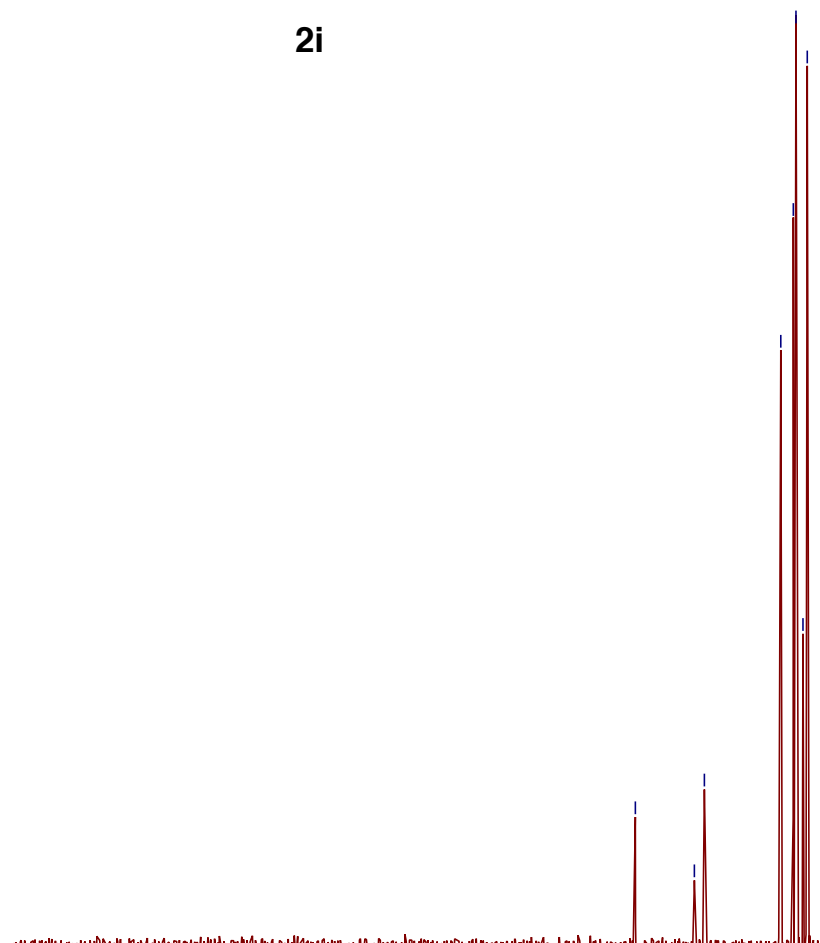

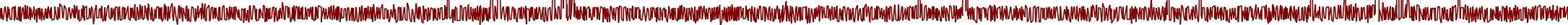
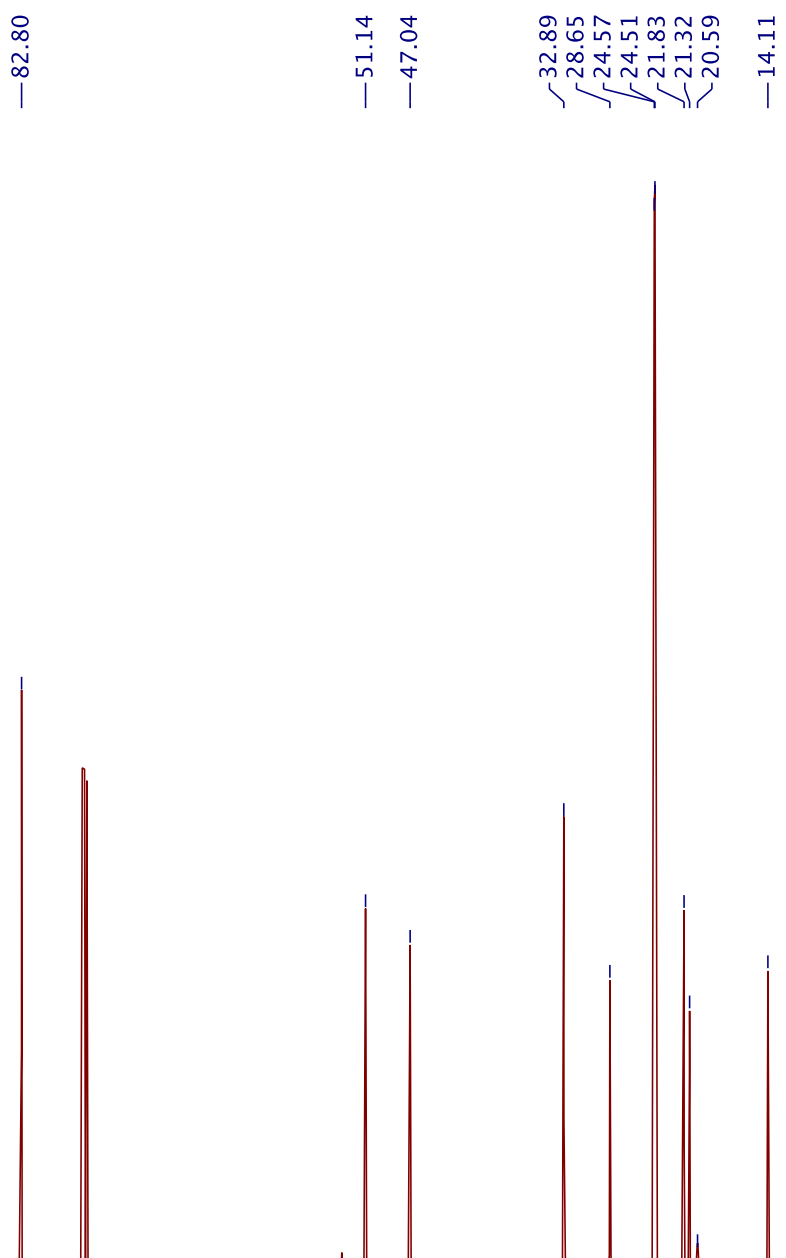
YX-3-217F-11B.1.fid

AV-600 11B starting parameter\$్ల

Using 90 pulses (for T1 $<0.7$ sec or less).

$\mathrm{SW}=265 \mathrm{ppm}$, O1P $=40 \mathrm{BF} 3$.Et2O $=0 \mathrm{ppm}$.

For $1 \mathrm{H}$ coupled experiments use ased to change pulse prog. to $\mathrm{zg}$

Use linear prediction (ME $\bmod =\mathrm{LPbc}$ ) to remove probe background

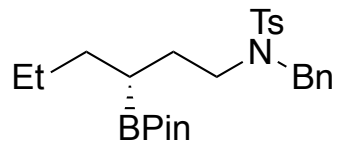

2i

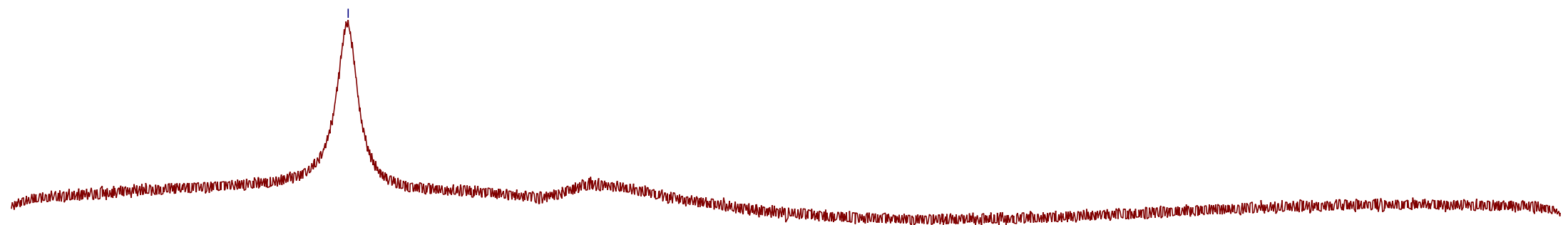

\begin{tabular}{|c|c|c|c|c|c|c|c|c|c|c|c|}
\hline 70 & 50 & 30 & 10 & -10 & \multicolumn{2}{|c|}{$-30 \mathrm{f} 1(\mathrm{ppm})$} & \multicolumn{3}{|c|}{$\begin{array}{ll}-70 & -90\end{array}$} & -110 & $-150 \quad-170$ \\
\hline
\end{tabular}


YX-3-282A-1H.1.fid

CC: 12182012 AV-500 TBIP probe

1H 1D NMR
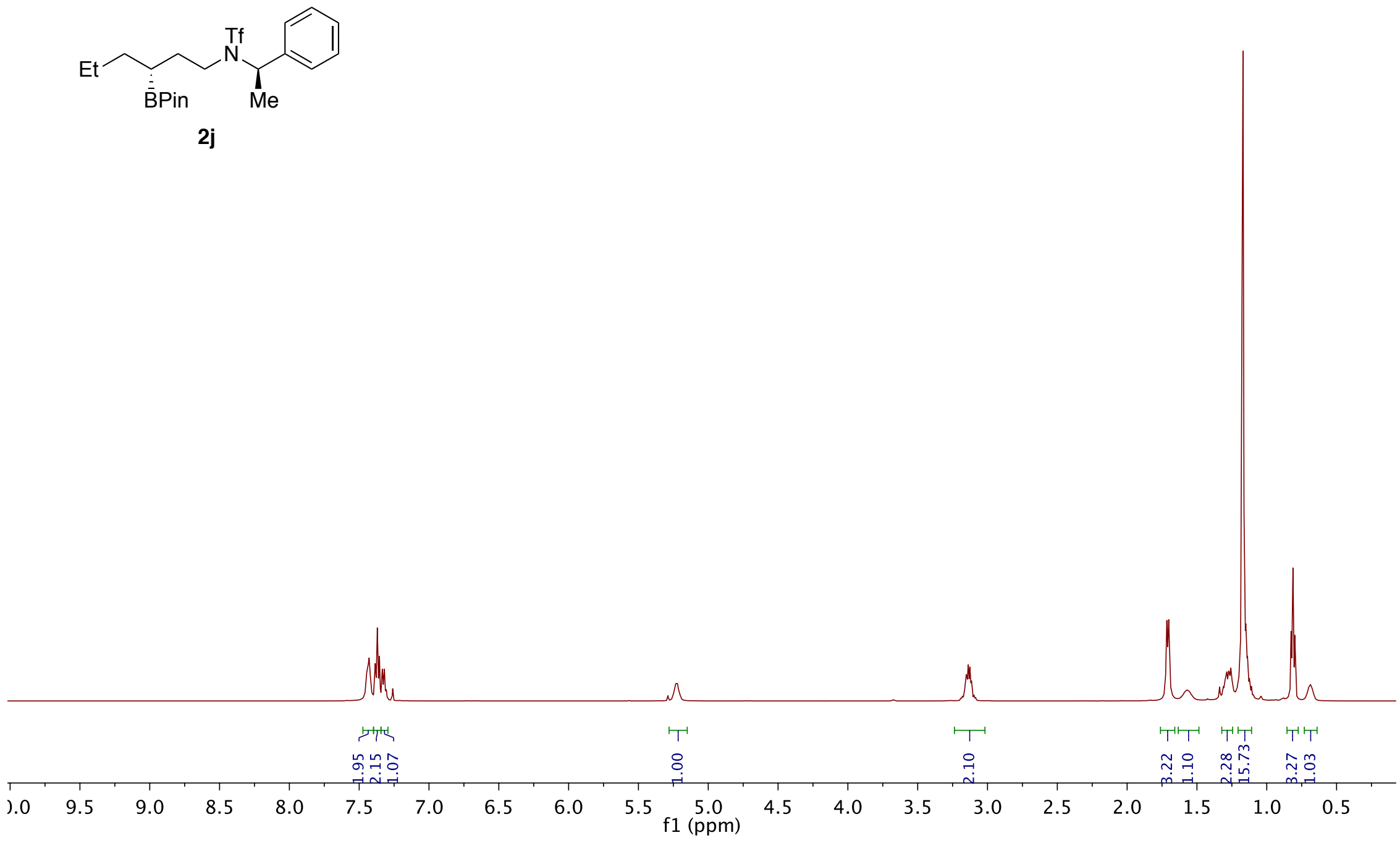
YX-3-282A-13C.1.fid

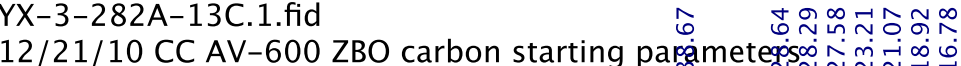
$A Q M O D=D Q D$

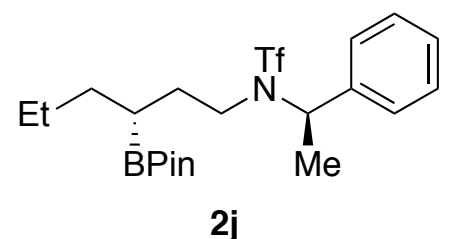

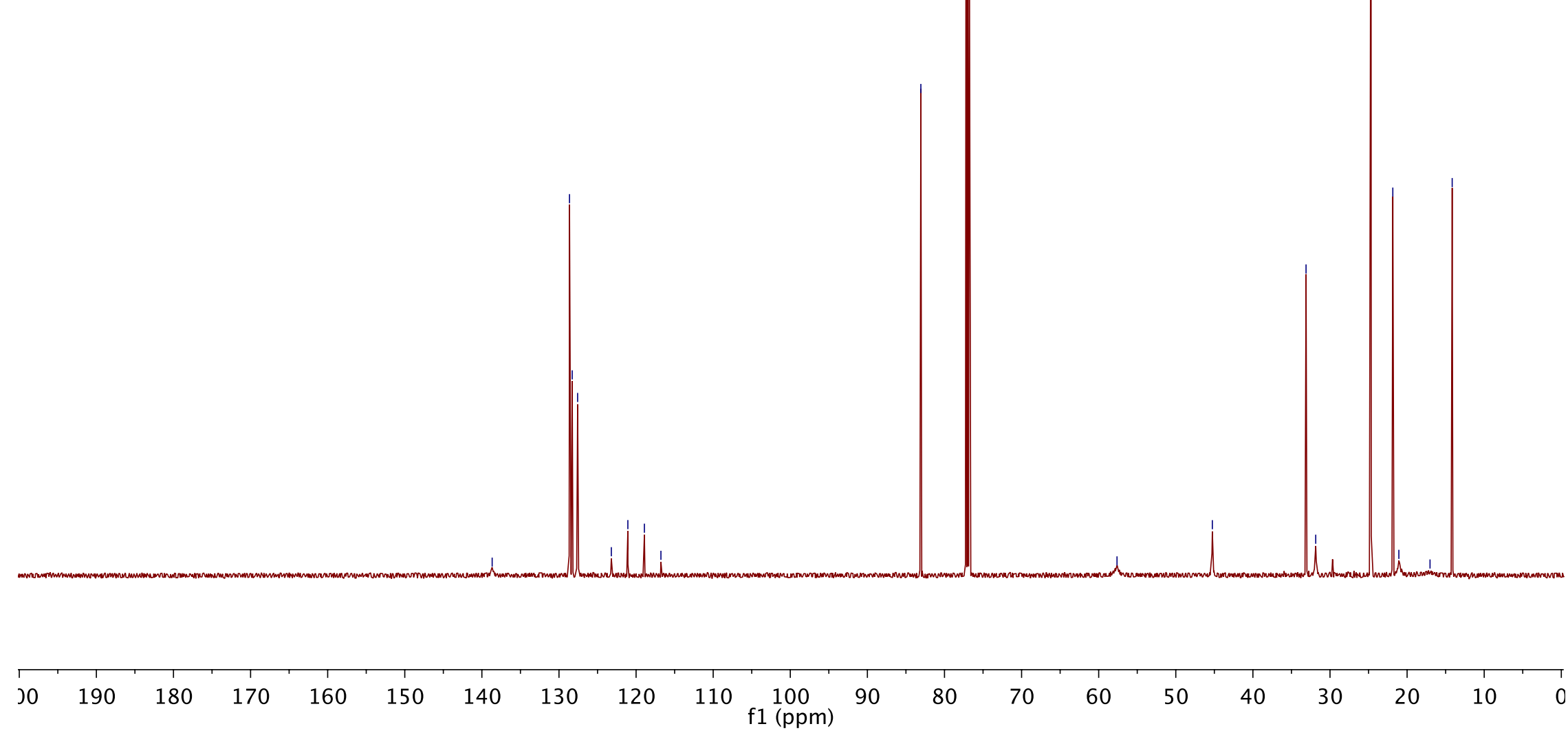


YX-3-282A-11B.1.fid

AV-600 11B starting parameter疋.

with $1 \mathrm{H}$ decoupling

Using 90 pulses (for $\mathrm{T} 1<0.7 \mathrm{sec}$ or less).

$\mathrm{SW}=265 \mathrm{ppm}$, O1P $=40 \mathrm{BF} 3$.Et2O $=0 \mathrm{ppm}$.

For $1 \mathrm{H}$ coupled experiments use ased to change pulse prog. to $\mathrm{zg}$

Use linear prediction (ME $\bmod =\mathrm{LPbc}$ ) to remove probe background

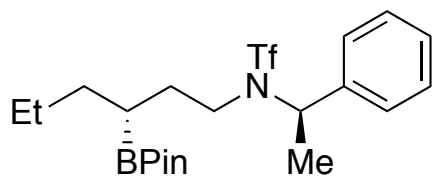

2j

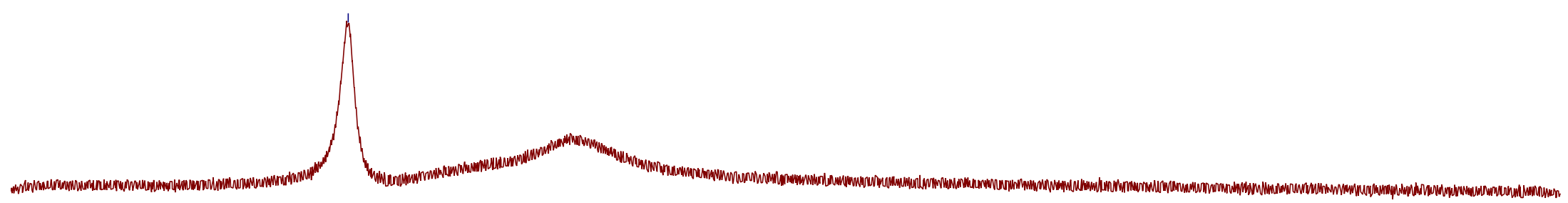

$\begin{array}{llllllllllllllll} & 70 & 50 & 30 & 10 & -10 & -30 & -50 & -70 & -90 & -110 & -130 & -150 & -170\end{array}$


YX-3-282A-19F.1.fid

AVQ-400 QNP Probe 19F starting parameters. (revised P1, 2ֶำ $12 / 04$ RN)

chemical shifts relative to $\mathrm{CFCl} 3$ at $0 \mathrm{ppm}(082103 \mathrm{HvH})$

sw 239.28 ppm; o1p 0 ppm

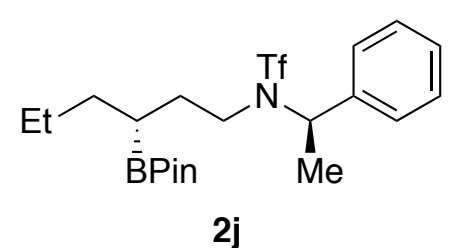

2j

0 
YX-3-220C-P-1H.1.fid

CC: 12182012 AV-500 TBIP probe

1H 1D NMR<smiles>CCCCCCCC(CCCC)CC(OC(=O)c1c(Cl)cc(Cl)cc1Cl)c1ccccc1</smiles>

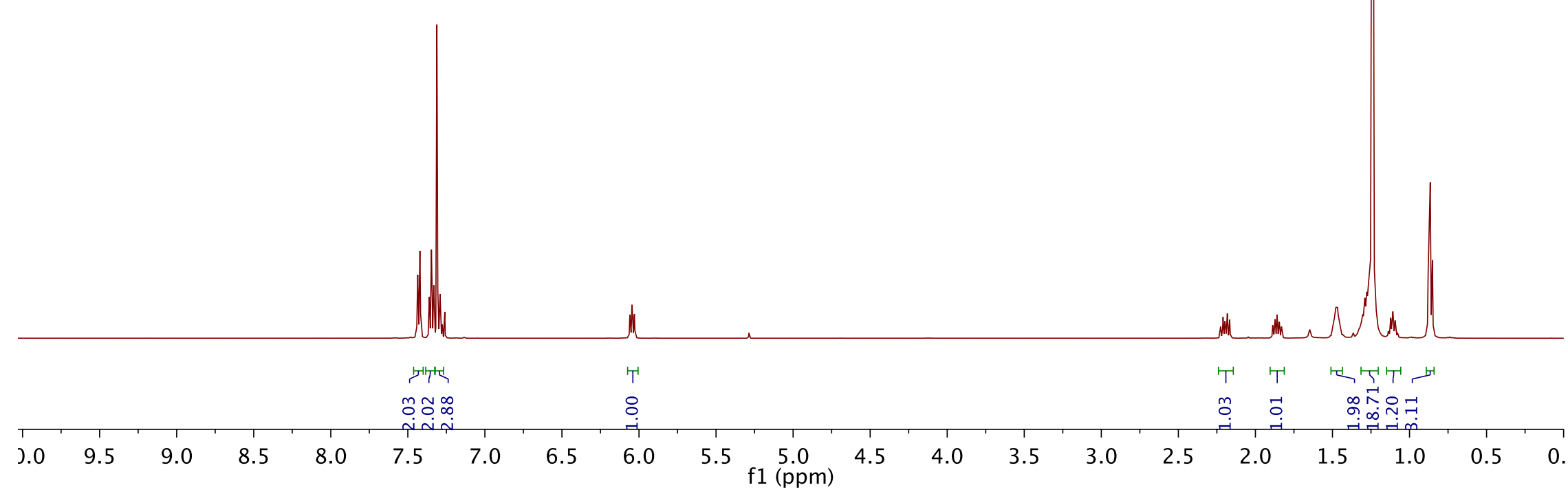


YX-3-220C-13C.1.fid

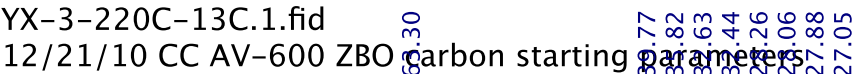

$A Q \_M O D=D Q D$

Dariametexis

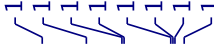

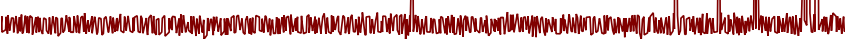

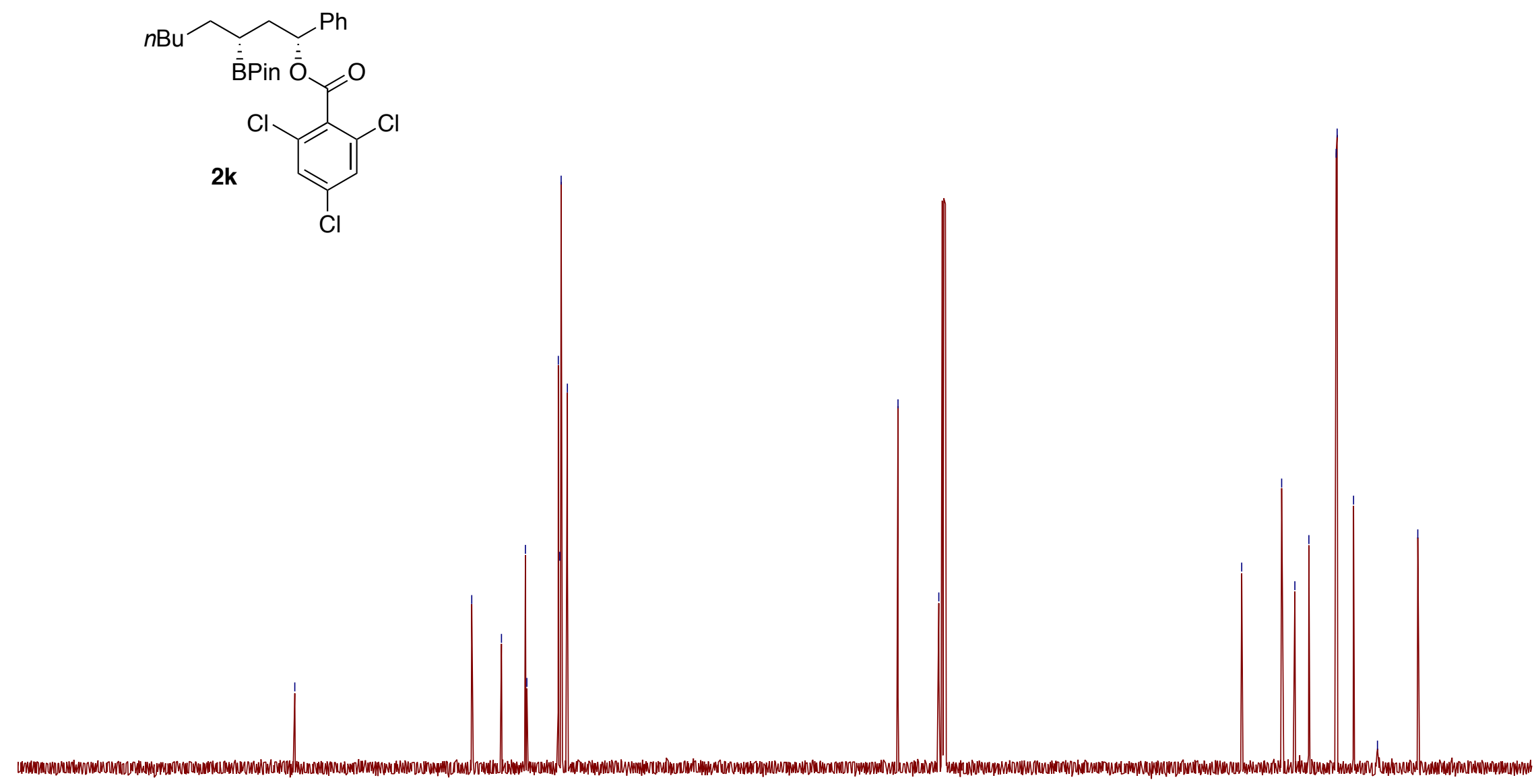

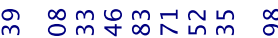
กิ

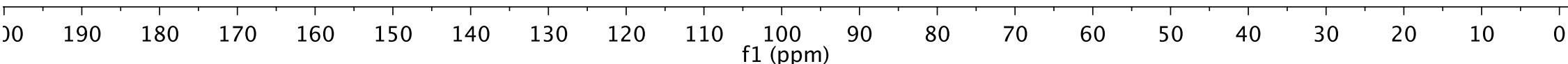


YX-3-220C-11B.1.fid
AV-600 11B starting parameters

with $1 \mathrm{H}$ decoupling

Using 90 pulses (for T1 $<0.7$ sec or less).

$\mathrm{SW}=265 \mathrm{ppm}$, O1P $=40 \mathrm{BF}$. Et2O $=0 \mathrm{ppm}$.

For $1 \mathrm{H}$ coupled experiments use ased to change pulse prog. to $\mathrm{zg}$

Use linear prediction (ME $\bmod =\mathrm{LPbC}$ ) to remove probe background<smiles>CCCCCC(C[C@H](Br)OC(=O)c1c(Cl)cc(Cl)cc1Cl)c1ccccc1</smiles>

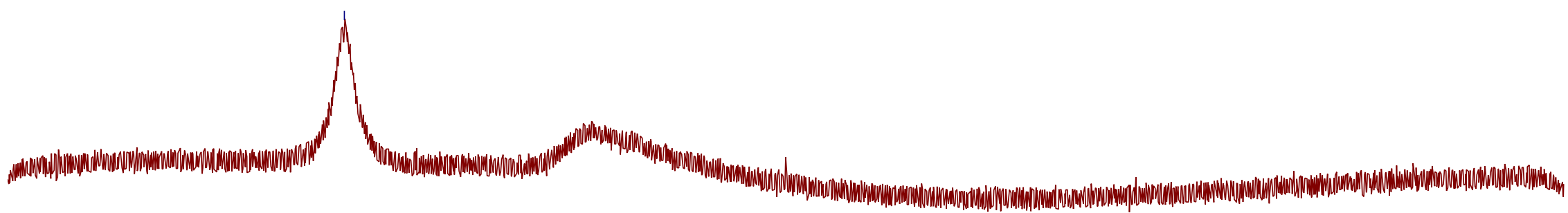

\begin{tabular}{|c|c|c|c|c|c|c|c|c|c|c|c|}
\hline 70 & 50 & 30 & 10 & -10 & -30 & $\frac{-50}{n)}$ & -70 & -90 & -110 & -130 & -150 \\
\hline
\end{tabular}


YX-3-253C-1H.1.fid

CC: 12182012 AV-500 TBIP probe

1H 1D NMR
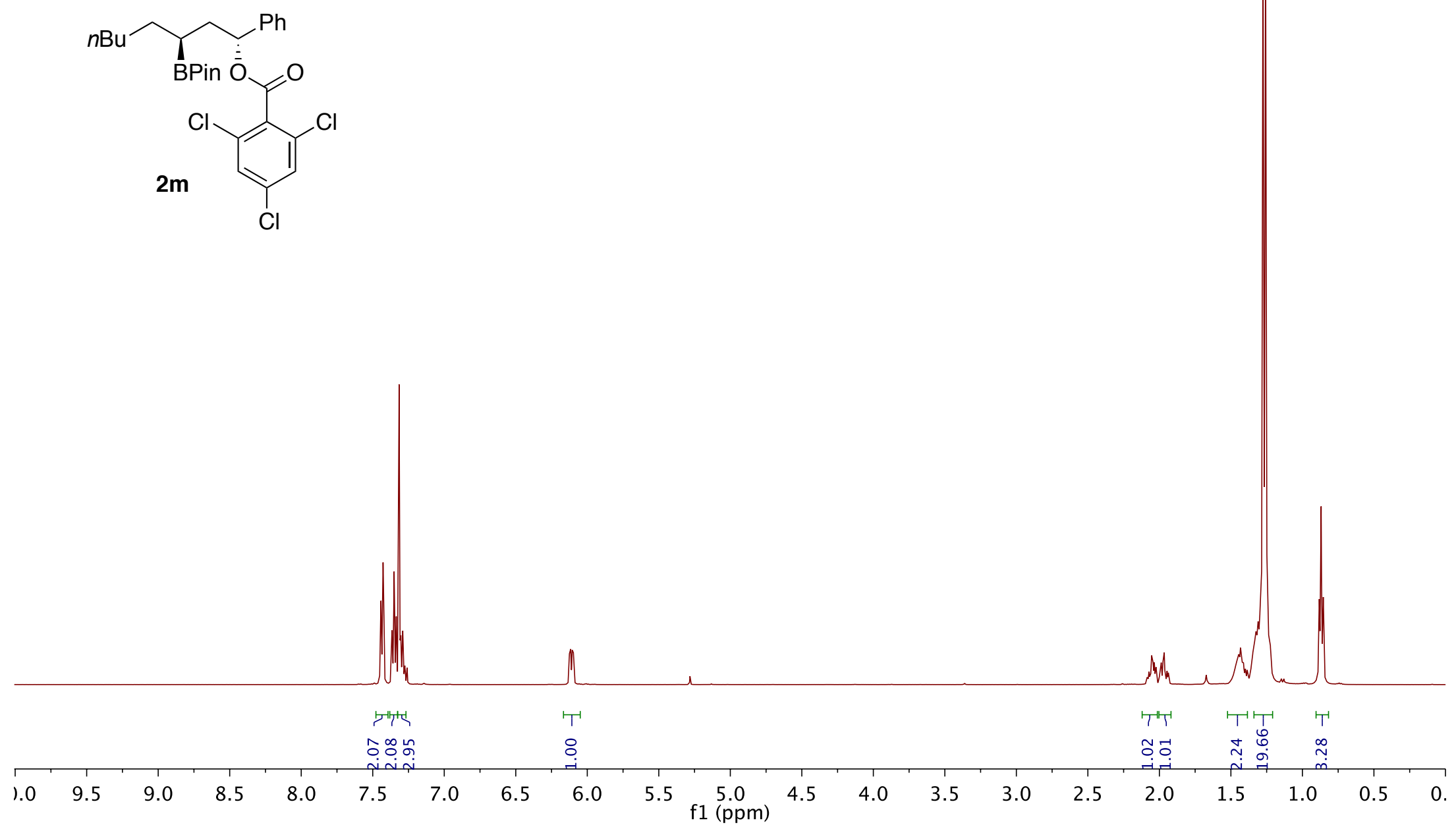
YX-3-253C-13C.1.fid

$12 / 21 / 10$ CC AV-600

$A Q \angle M O D=D Q D$

starting paragienterend

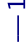

它匀

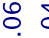

$\infty_{\infty}^{\infty}$ 구유ำ

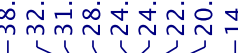

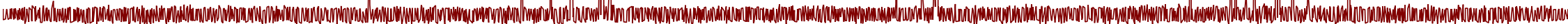<smiles>O=C(O[C@@H](Br)C[C@H](Br)Cc1ccccc1)c1c(Cl)cc(Cl)cc1Cl</smiles>
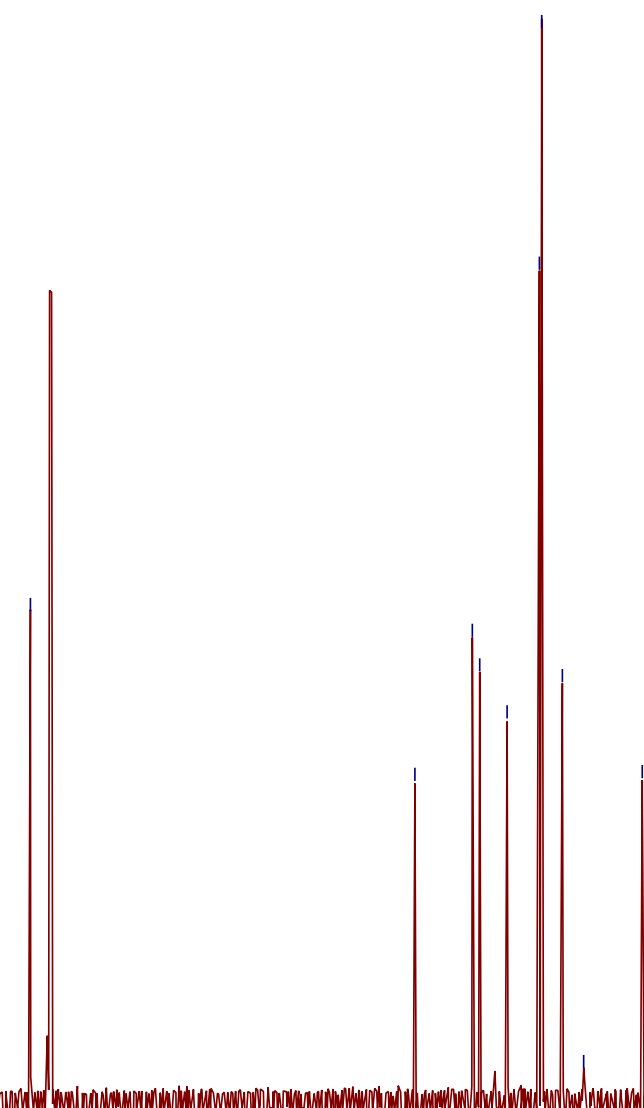

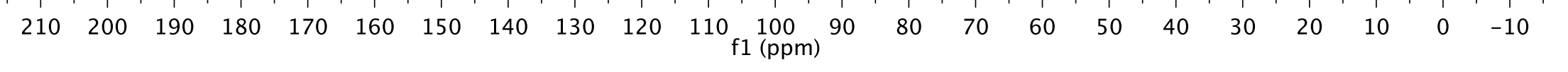


YX-3-253C-11B.1.fid

AV-600 11B starting parameters్

with $1 \mathrm{H}$ decoupling

Using 90 pulses (for T1 $<0.7$ sec or less).

$\mathrm{SW}=265 \mathrm{ppm}$, O1P $=40 \mathrm{BF}$. Et2O $=0 \mathrm{ppm}$.

For $1 \mathrm{H}$ coupled experiments use ased to change pulse prog. to $\mathrm{zg}$

Use linear prediction (ME $\bmod =\mathrm{LPbC}$ ) to remove probe background<smiles>O=C(O[C@@H](Br)C[C@H](Br)Cc1ccccc1)c1c(Cl)cc(Cl)cc1Cl</smiles>

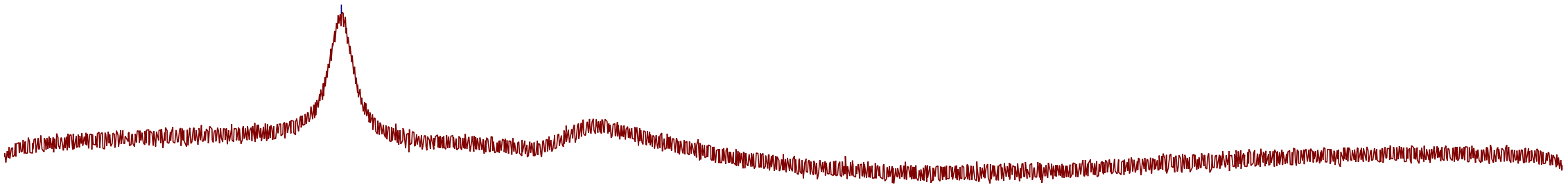

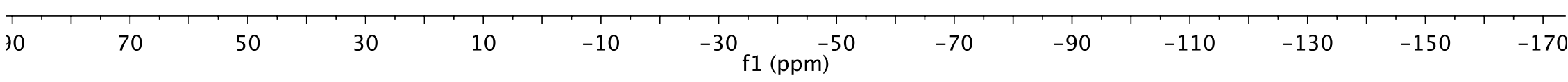


YX-3-212-1H.1.fid

$1 \mathrm{H}$ starting parameters (zg30)

DRX-500 TBIC

10/30/13 CGC

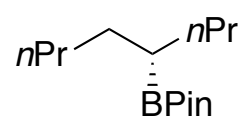

$2 \mathrm{~m}$

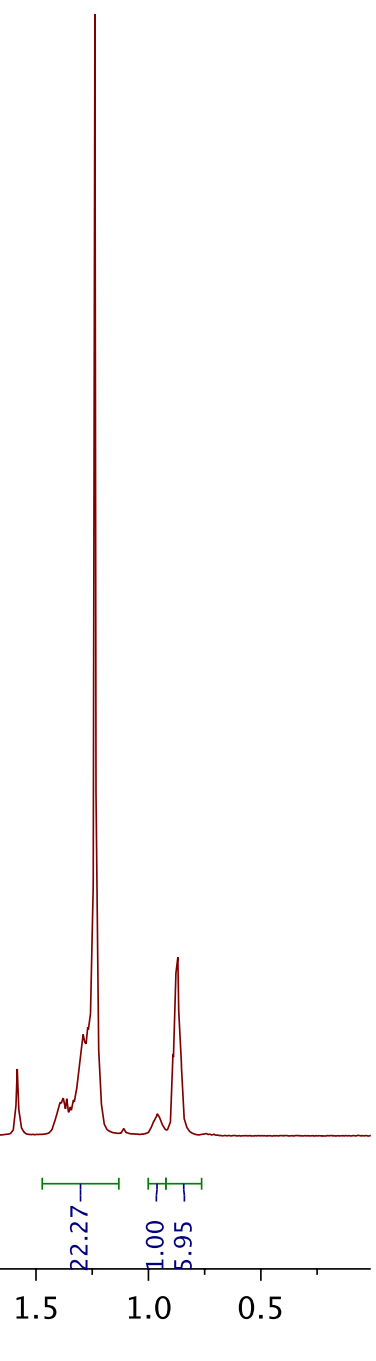


YX-3-231A-13C.1.fid

$12 / 21 / 10$ CC AV-600 ZBO carbon starting parameters

$A Q M O D=D Q D$

$2 m$

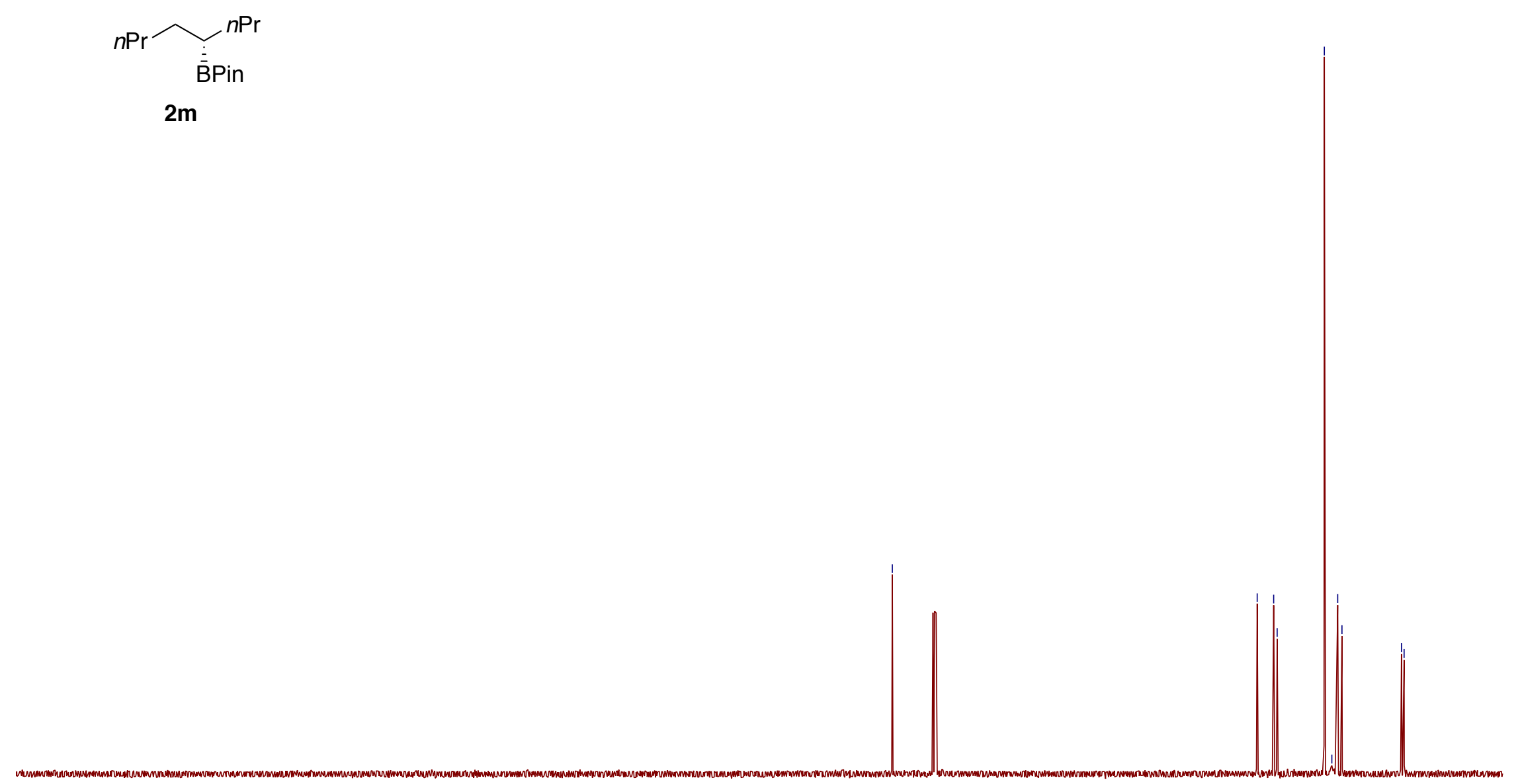

\begin{tabular}{|c|c|c|c|c|c|c|c|c|c|c|c|c|c|c|c|c|c|c|c|}
\hline 30 & 190 & 180 & 170 & 160 & 150 & 140 & 130 & 120 & 110 & $\begin{array}{l}100 \\
\mathrm{f} 1(\mathrm{ppm})\end{array}$ & 90 & 80 & 70 & 60 & 50 & 40 & 30 & 20 & 10 \\
\hline
\end{tabular}


YX-3-231A-11B.1.fid

AV-600 11B starting parameters్

with $1 \mathrm{H}$ decoupling

Using 90 pulses (for $\mathrm{T} 1<0.7$ sec or less)

$\mathrm{SW}=265 \mathrm{ppm}$, O1P $=40 \mathrm{BF} 3 . \mathrm{Et} 2 \mathrm{O}=0 \mathrm{ppm}$.

For $1 \mathrm{H}$ coupled experiments use ased to change pulse prog. to $\mathrm{zg}$

Use linear prediction (ME $\bmod =\mathrm{LPbC}$ ) to remove probe background

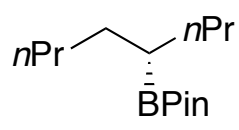

$2 \mathrm{~m}$

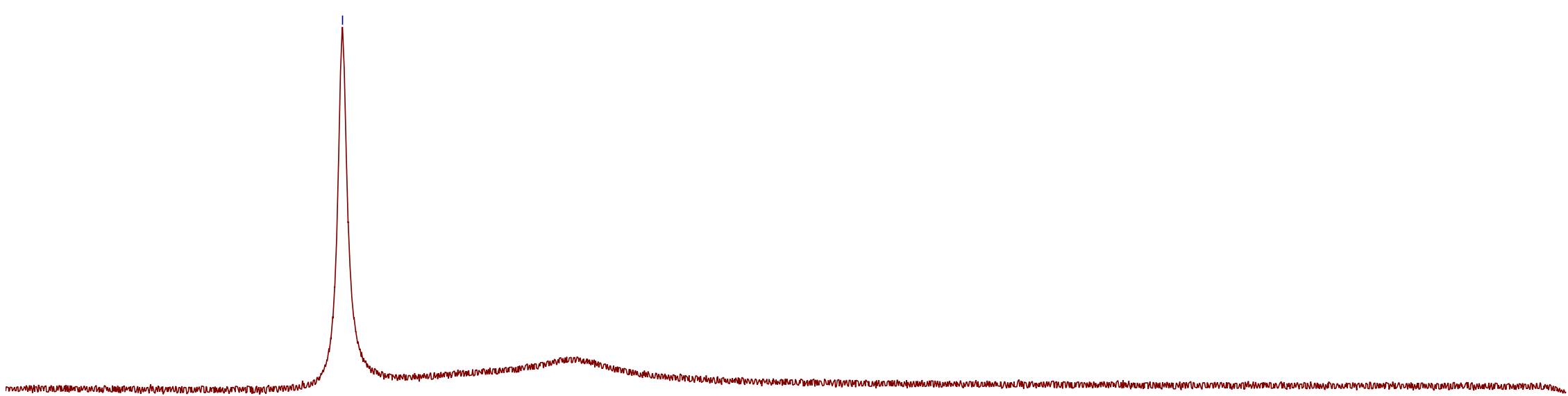

\begin{tabular}{|c|c|c|c|c|c|c|c|c|c|c|c|c|}
\hline 30 & 70 & 50 & 30 & 10 & -10 & -30 & $m)^{-50}$ & -70 & -90 & -110 & -130 & -150 \\
\hline
\end{tabular}


YX-3-262A-1H.1.fid

CC: 12182012 AV-500 TBIP probe

1H 1D NMR

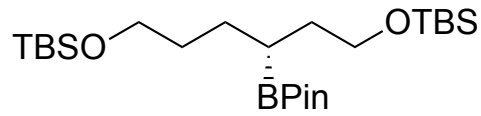

2n

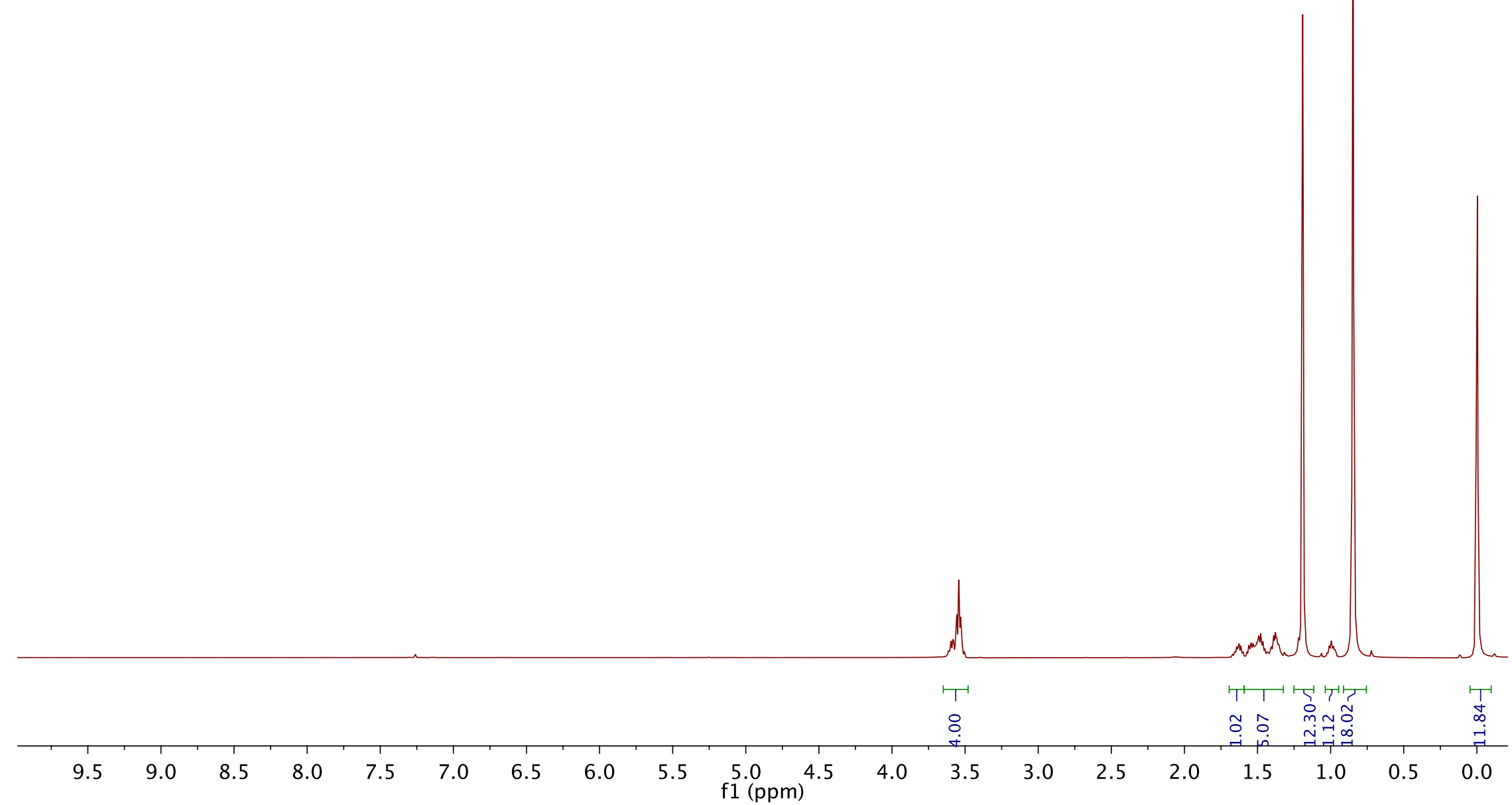


YX-3-262A-13C.1.fid

$12 / 21 / 10 \mathrm{CC}$ AV-600 ZBO carbon starting parameters

$A Q \_M O D=D Q D$

$2 n$

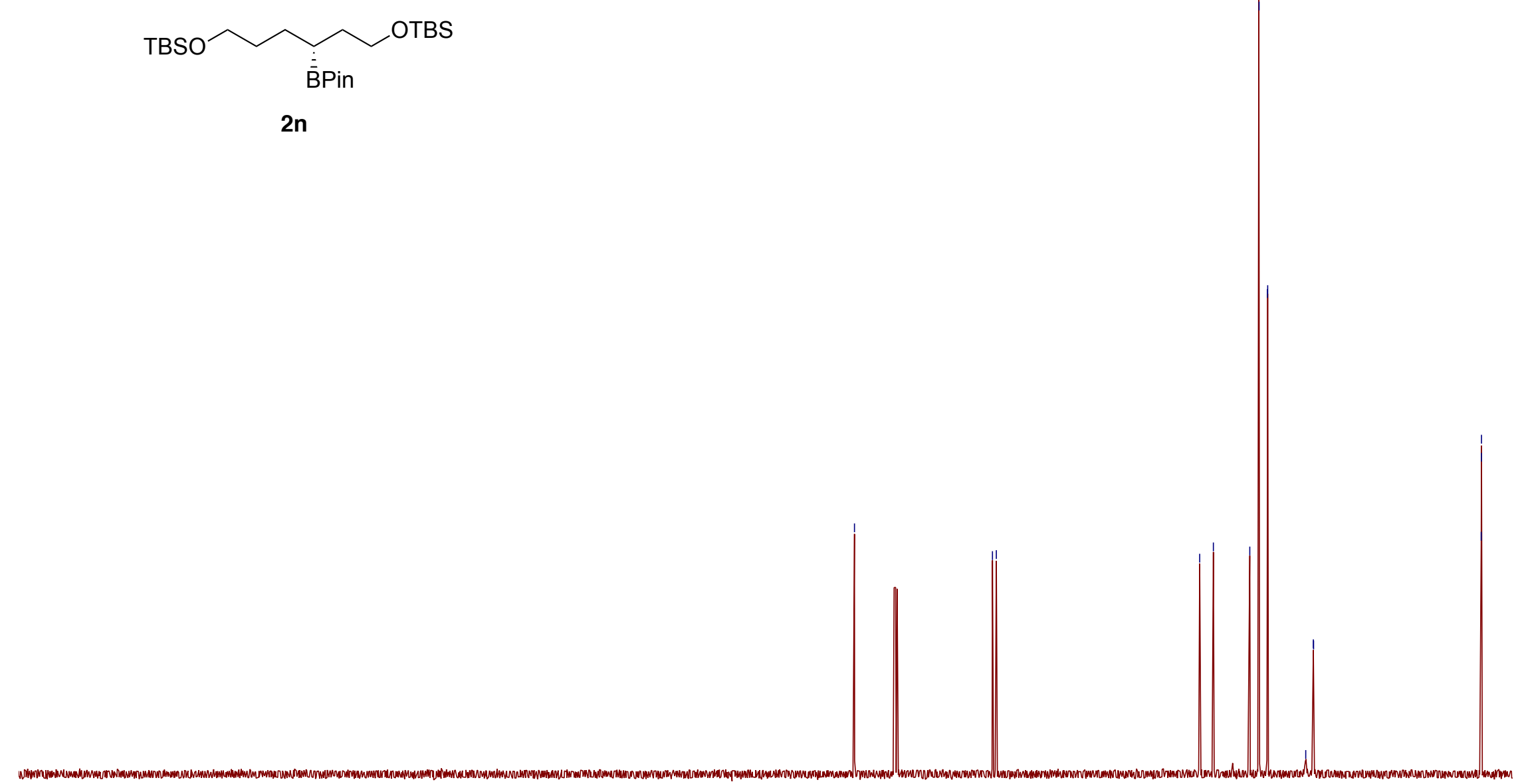

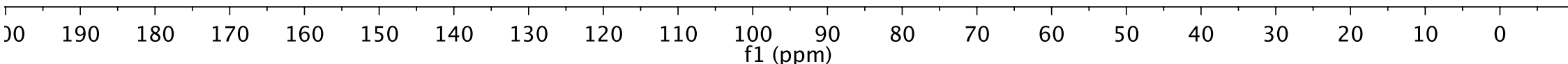


YX-3-262A-11B.1.fid

AV-600 11B starting parameters్

with $1 \mathrm{H}$ decoupling

Using 90 pulses (for T1 $<0.7$ sec or less).

$\mathrm{SW}=265 \mathrm{ppm}$, O1P $=40 \mathrm{BF} 3$.Et2O $=0 \mathrm{ppm}$.

For $1 \mathrm{H}$ coupled experiments use ased to change pulse prog. to $\mathrm{zg}$

Use linear prediction (ME_mod=LPbc) to remove probe background

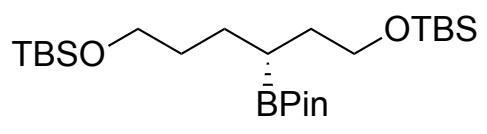

$2 n$

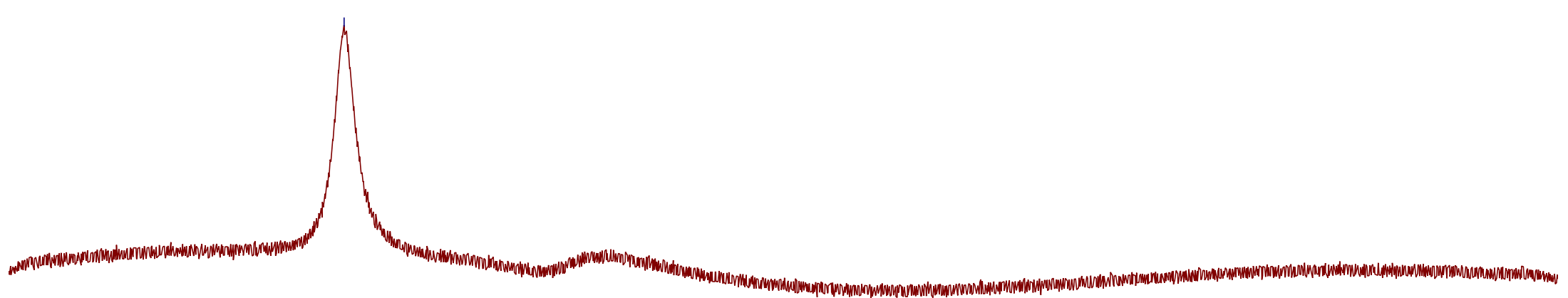

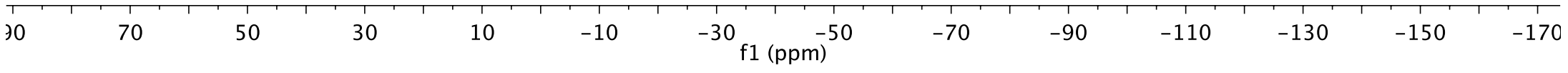


YX-3-220A-1H.1.fid

CC: 12182012 AV-500 TBIP probe

1H 1D NMR

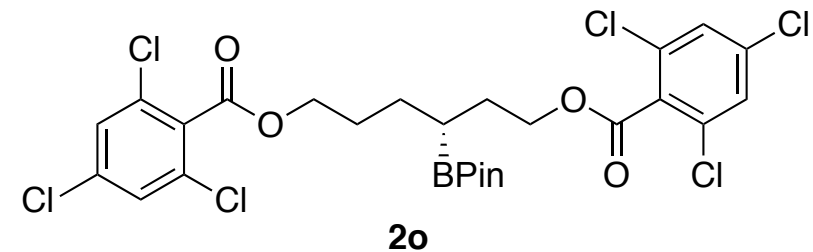

20

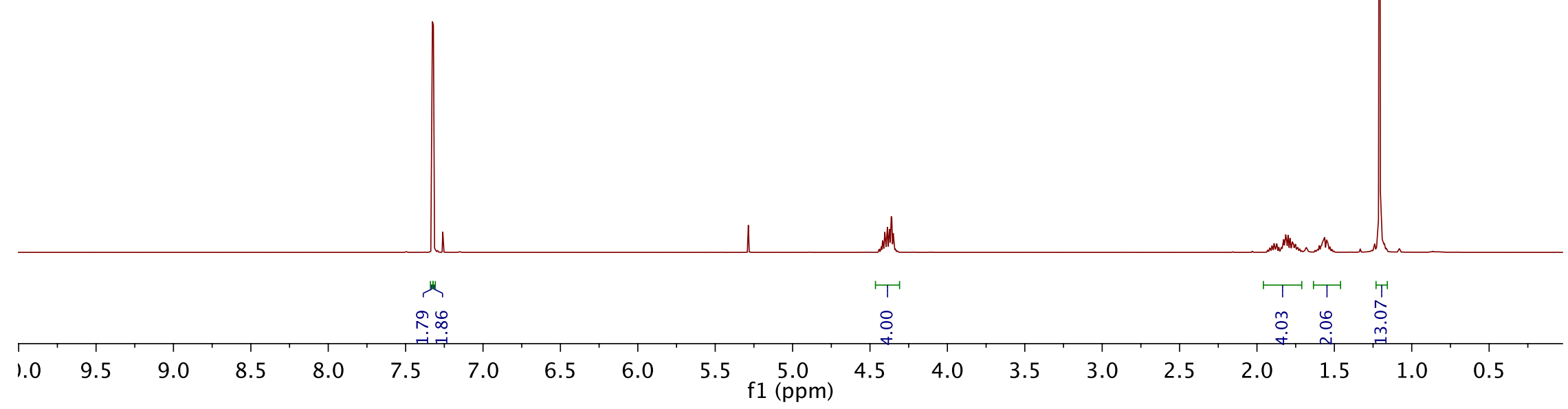




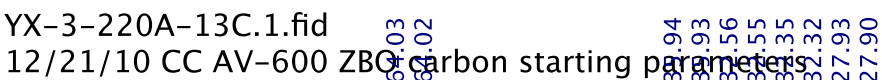

$A Q \_M O D=D Q D$

Y

giremietietsisn

皮为<smiles>O=C(OCCC[C@H](Br)c1ccccc1)c1c(Cl)cc(Cl)cc1Cl</smiles>

20

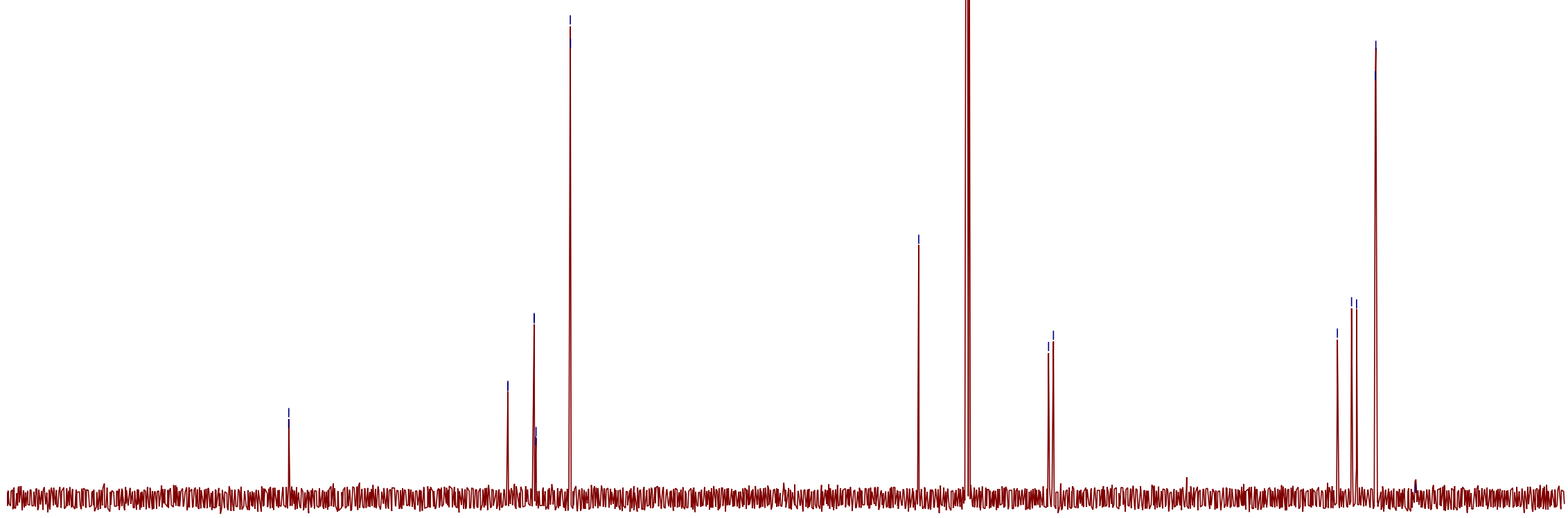


YX-3-220A-11B.1.fid

AV-600 11B starting parameters్

with $1 \mathrm{H}$ decoupling

Using 90 pulses (for T1 $<0.7$ sec or less).

$\mathrm{SW}=265 \mathrm{ppm}$, O1P $=40 \mathrm{BF} 3$.Et2O $=0 \mathrm{ppm}$.

For $1 \mathrm{H}$ coupled experiments use ased to change pulse prog. to $\mathrm{zg}$

Use linear prediction (ME $\bmod =\mathrm{LPbc}$ ) to remove probe background<smiles>O=C(OCCC[C@@H](Br)[Pb])c1c(Cl)cc(Cl)cc1Cl</smiles>

20

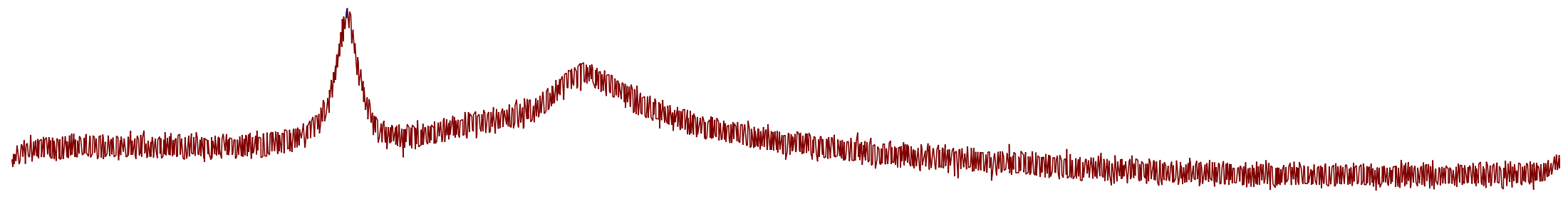

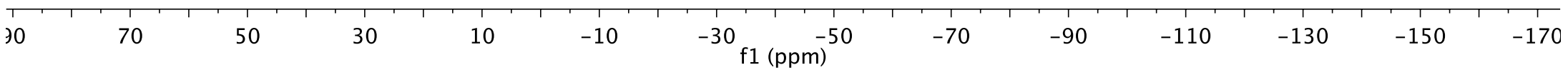


YX-4-80-1H.1.fid

CC: 12182012 AV-500 TBIP probe

1H 1D NMR

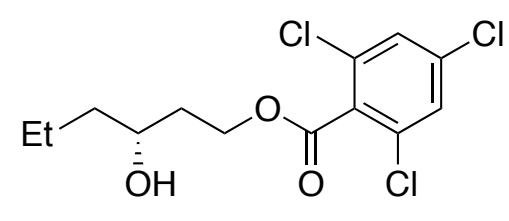

$3 a$

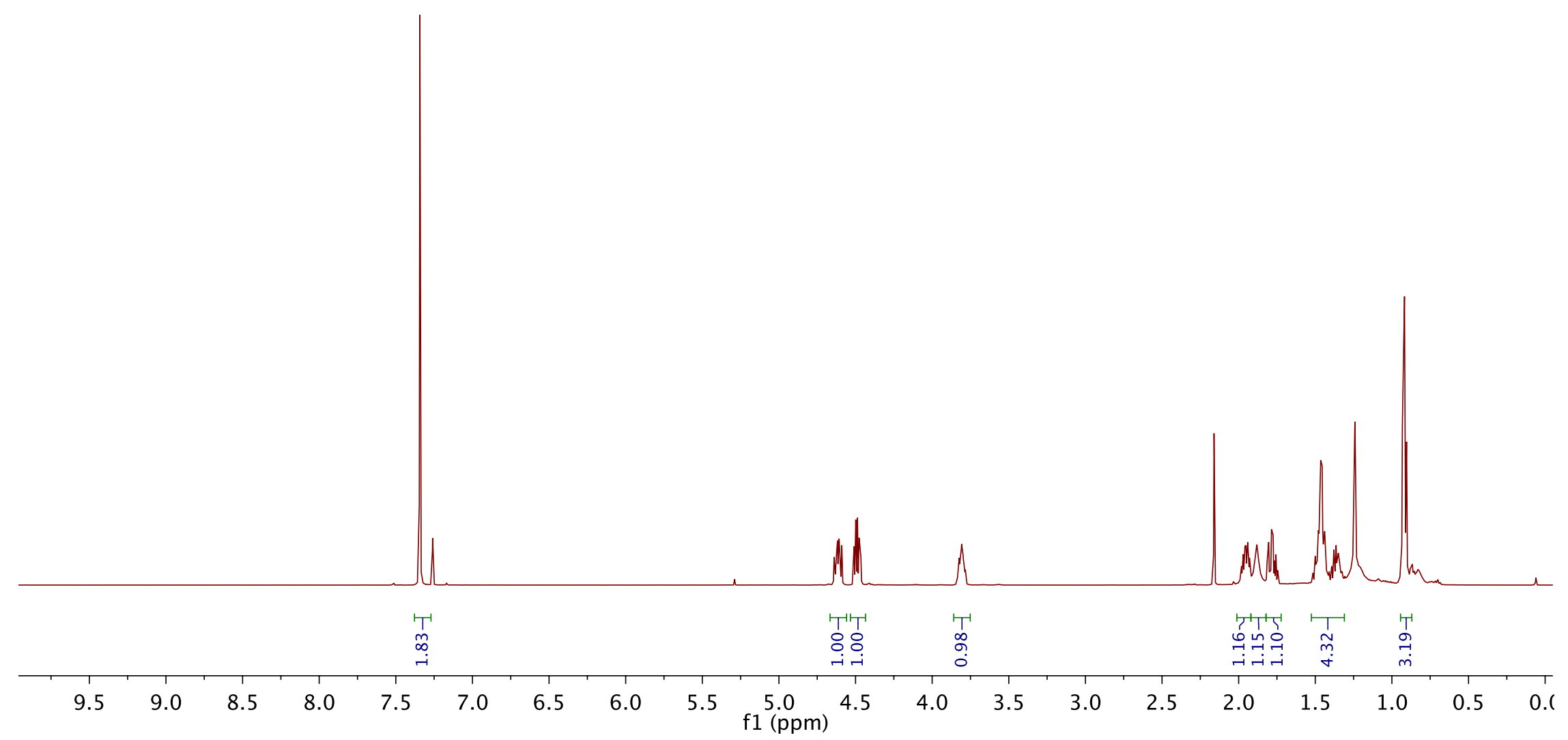


YX-4-80-13C.1.fid

$12 / 21 / 10$ CC AV-600 ZB $\stackrel{m}{\sim}$ ț

$A Q \angle M O D=D Q D$

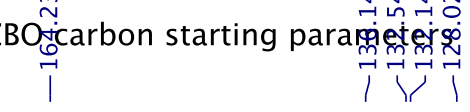

pientergo<smiles>CC[C@H](O)CCOC(=O)c1c(Cl)cc(Cl)cc1Cl</smiles>

$3 a$

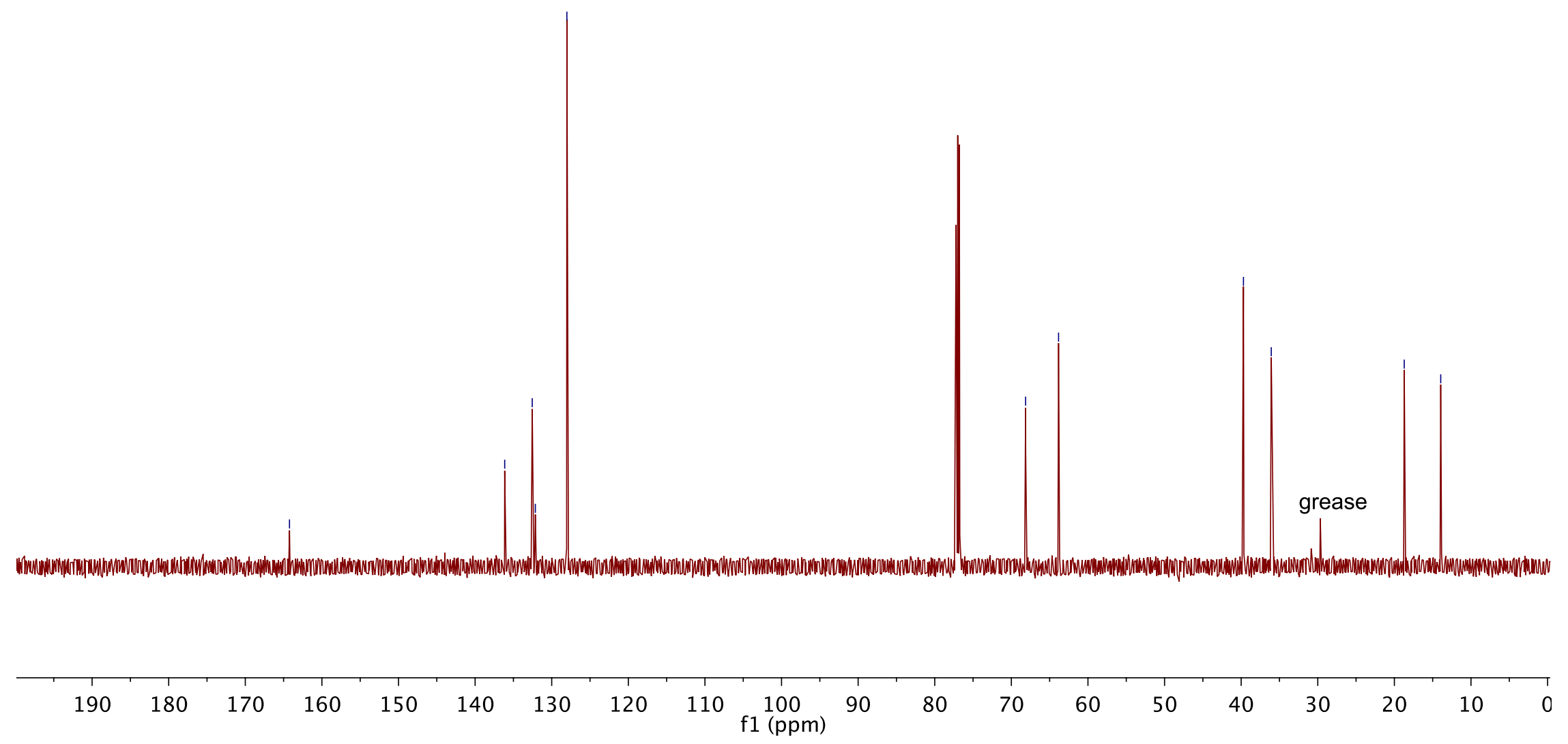

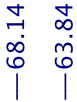

$\begin{array}{ll}\circ & \hat{0} \\ \text { مे } & 0 \\ \text { mे }\end{array}$

ㄴำ

$\stackrel{\infty}{\rightarrow} \stackrel{m}{\uparrow}$ 
YX-3-272C-1H.1.fid

CC: 12182012 AV-500 TBIP probe

1H 1D NMR

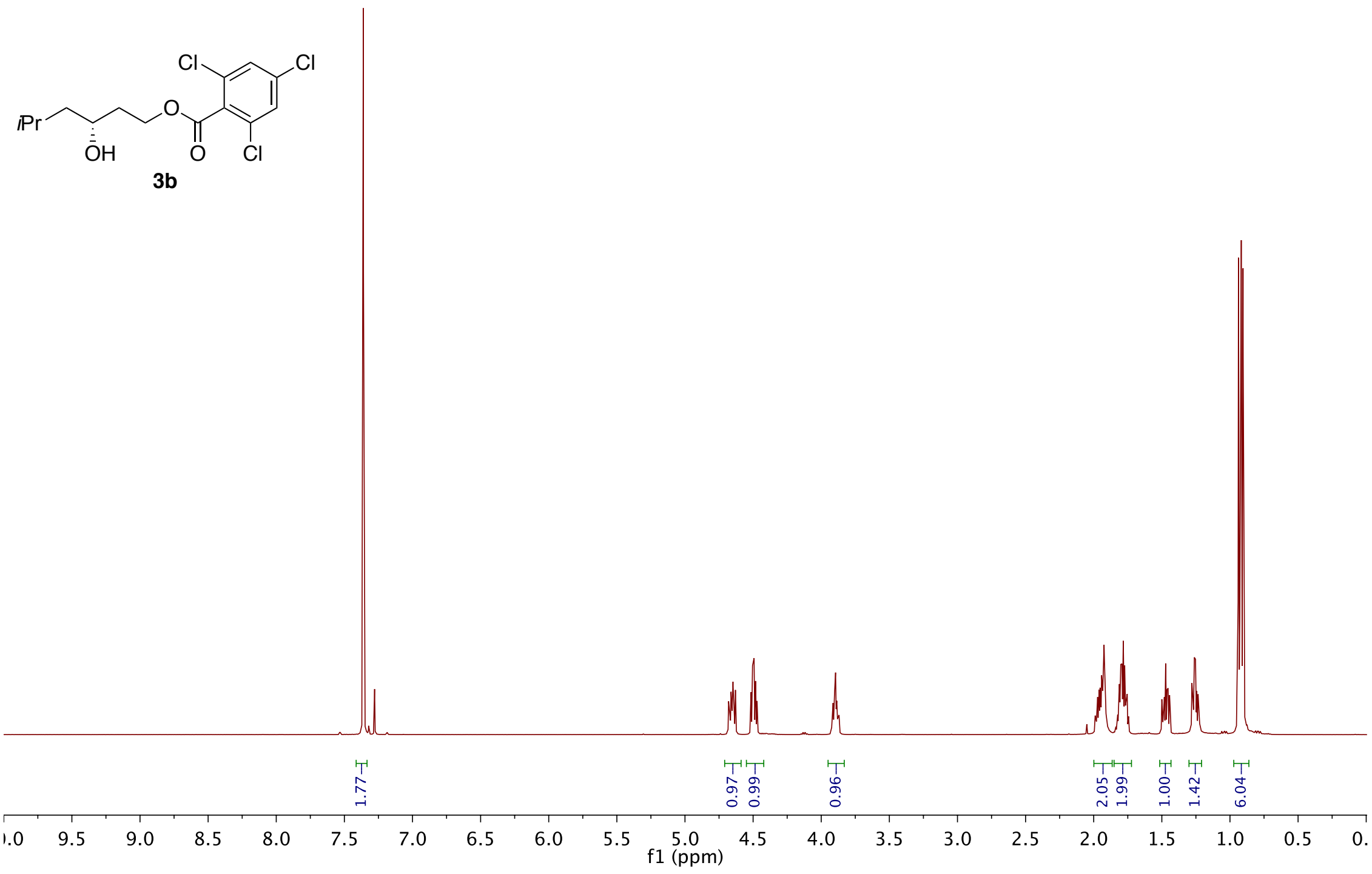




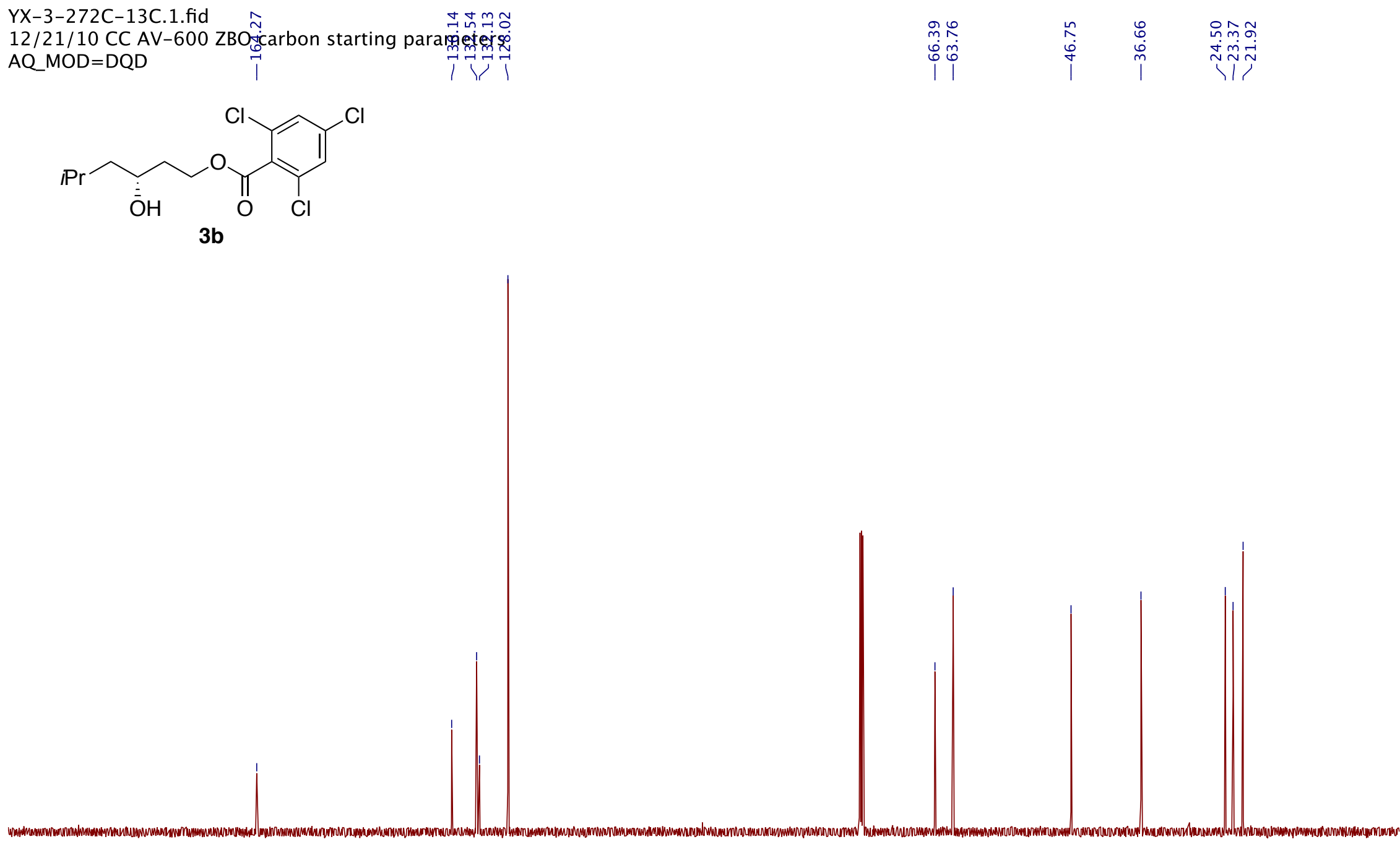

\begin{tabular}{|c|c|c|c|c|c|c|c|c|c|c|c|c|c|c|c|c|c|c|c|}
\hline 0 & 190 & 180 & 170 & 160 & 150 & 140 & 130 & 120 & 110 & $\begin{array}{c}100 \\
\mathrm{f} 1(\mathrm{ppm})\end{array}$ & 90 & 80 & 70 & 60 & 50 & 40 & 30 & 20 & 10 \\
\hline
\end{tabular}


YX-3-225B-1H.1.fid

CC: 12182012 AV-500 TBIP probe

1H 1D NMR
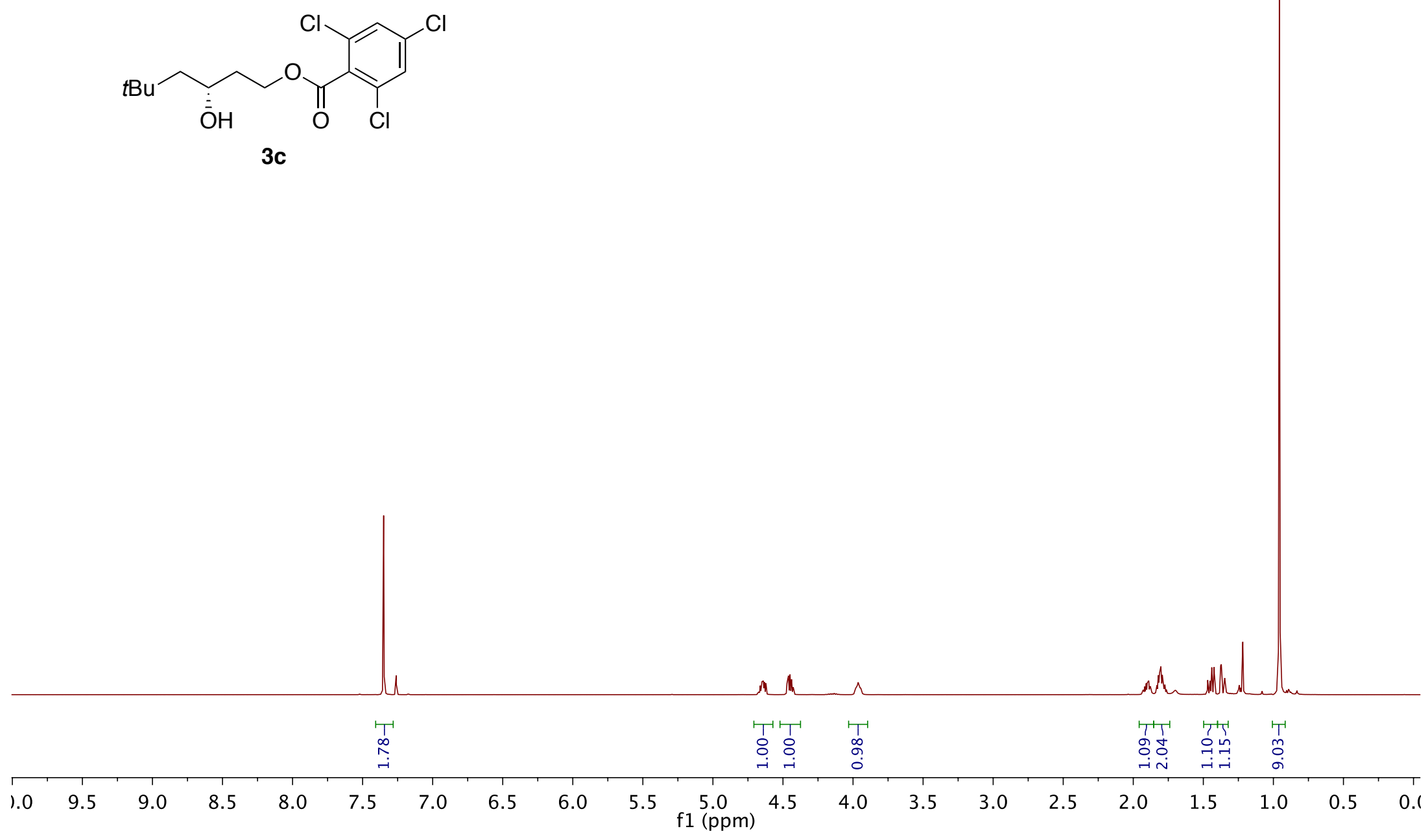
YX-3-225B-13C.1.fid

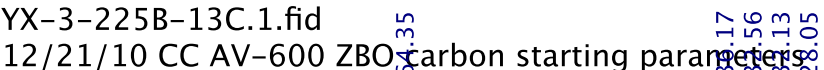

$A Q \_M O D=D Q D$

noteterso
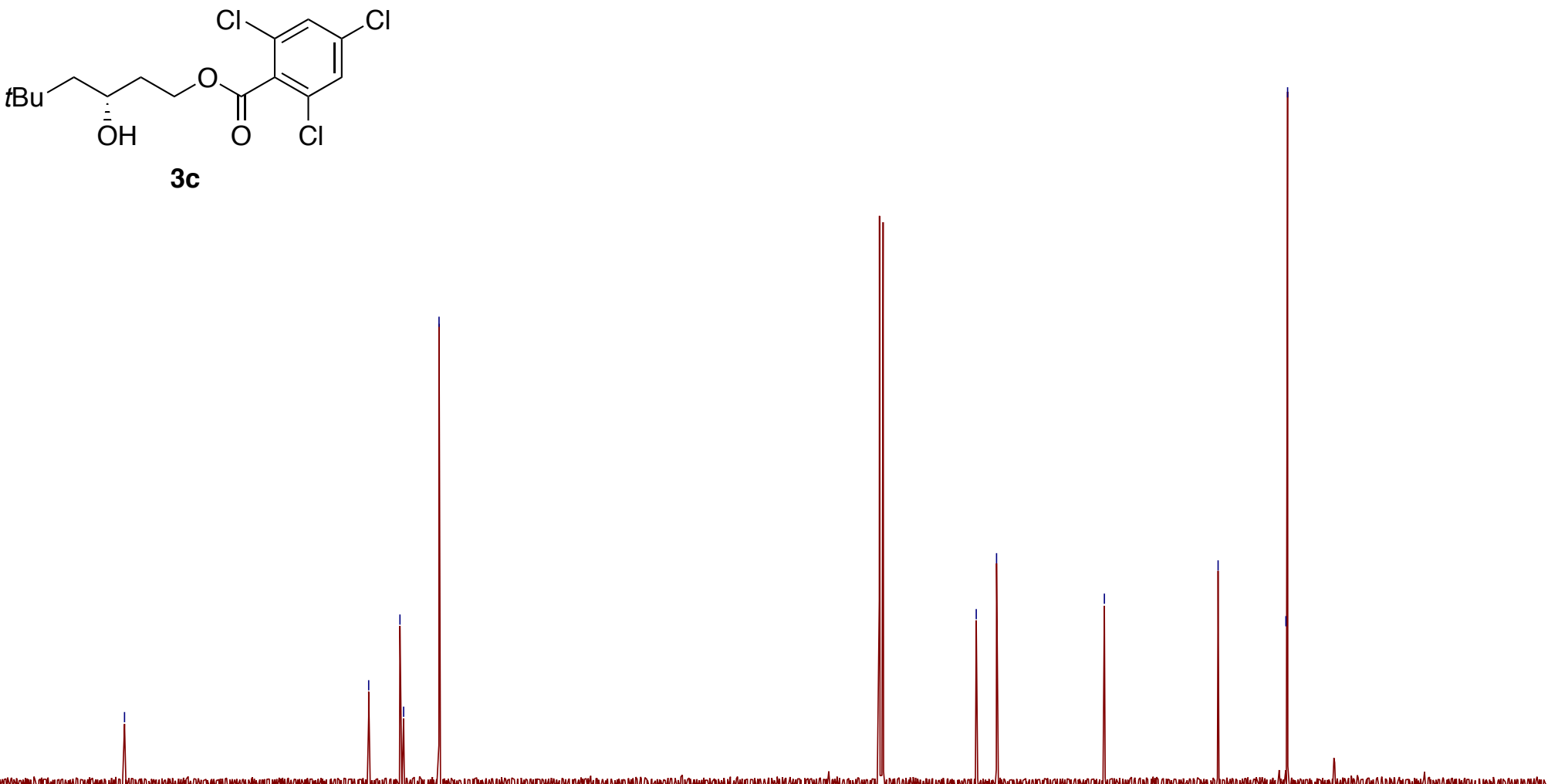

180

170

160

150

140

130

120

110100

90

80

70

60

50

$40 \quad 30$

20

10 
YX-3-225C-1H.1.fid

CC: 12182012 AV-500 TBIP probe

1H 1 D NMR
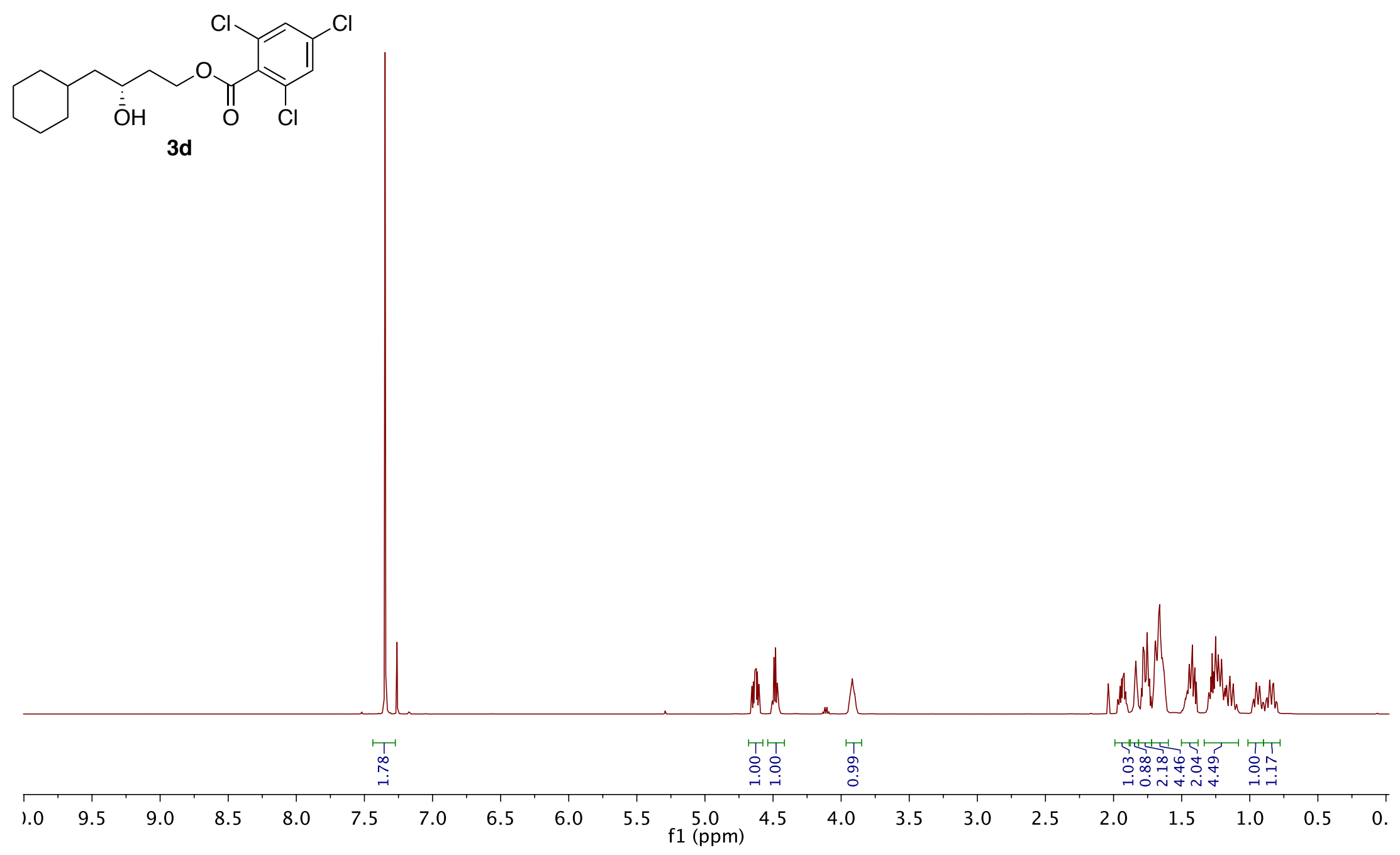
YX-3-225C-13C.1.fid

$12 / 21 / 10$ CC AV-600 ZB N

$A Q \_M O D=D Q D$

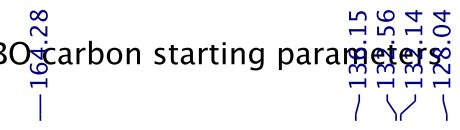

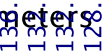

오

மิ

$\operatorname{ming}$

$\dot{m} \dot{m} m \tilde{m} \omega \dot{\omega}$<smiles>O=C(OCC[C@@H](O)CC1CCCCC1)c1c(Cl)cc(Cl)cc1Cl</smiles>

3d

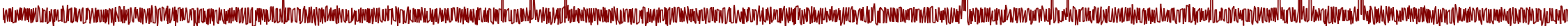

\begin{tabular}{|c|c|c|c|c|c|c|c|c|c|c|c|c|c|c|c|c|c|c|c|}
\hline 30 & 190 & 180 & 170 & 160 & 150 & 140 & 130 & 120 & 110 & $\begin{array}{c}100 \\
\mathrm{f} 1(\mathrm{ppm})\end{array}$ & 90 & 80 & 70 & 60 & 50 & 40 & 30 & 20 & 10 \\
\hline
\end{tabular}


YX-4-72B-1H.1.fid

CC: 12182012 AV-500 TBIP probe

1H 1D NMR

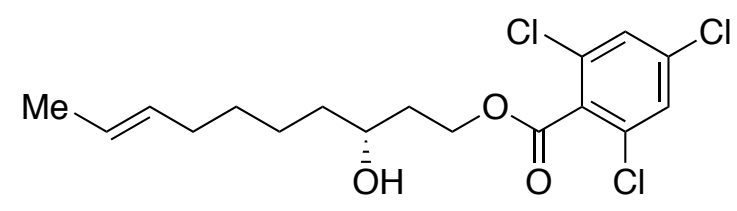

$3 e$

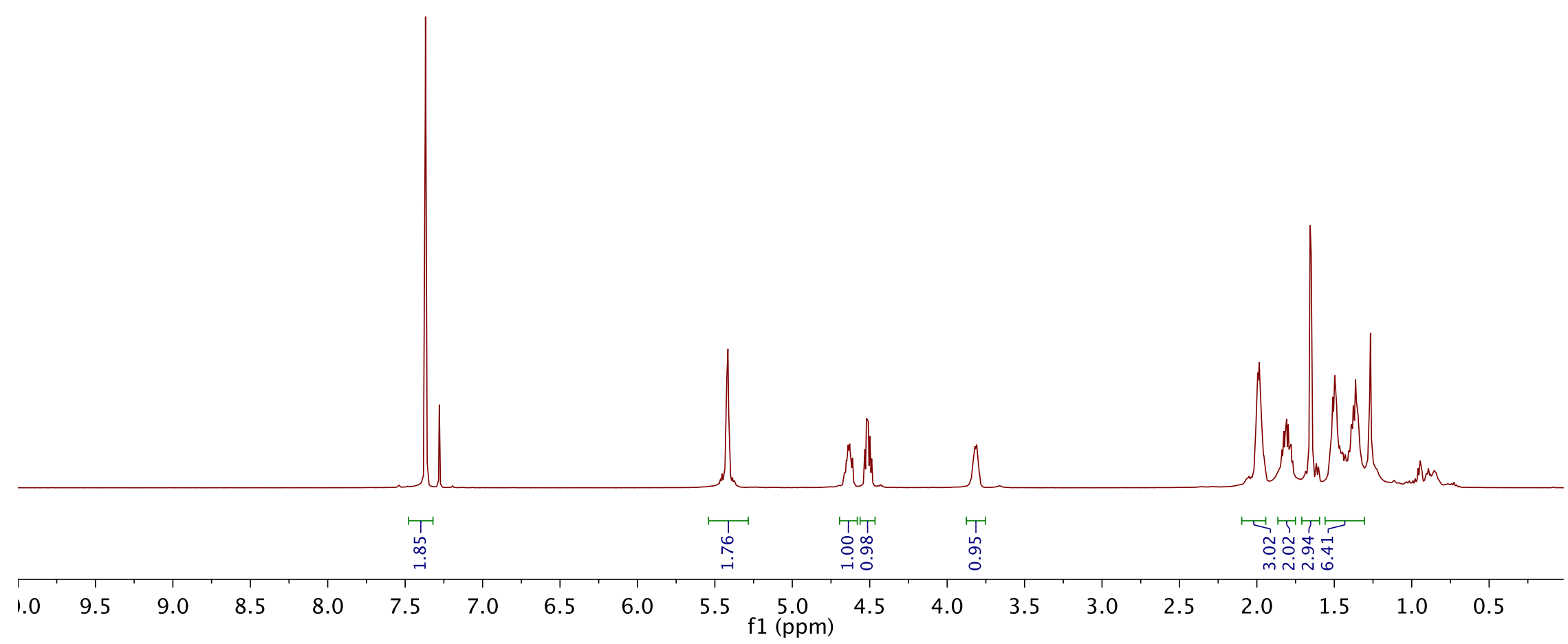




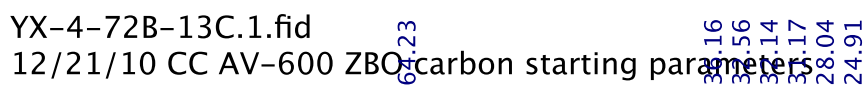

$12 / 21 / 10 \mathrm{CC}$ AV-600 ZBOficarbon starting parameters<smiles>C/C=C/CCCC[C@H](O)CCOC(=O)c1c(Cl)cc(Cl)cc1Cl</smiles>

$3 e$

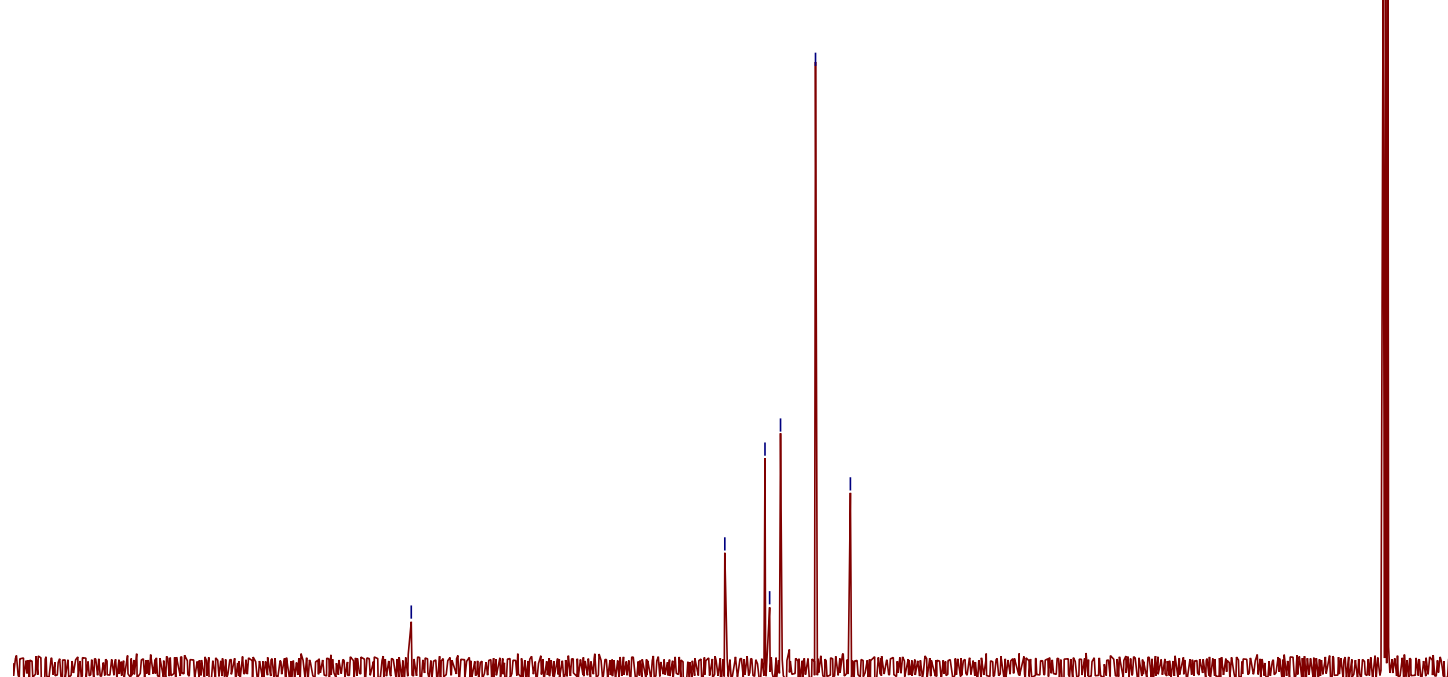

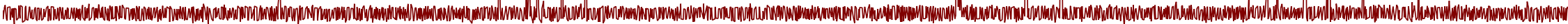

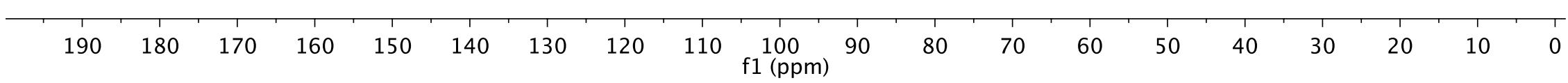


YX-3-226A-1H.1.fid

CC: 12182012 AV-500 TBIP probe

1H 1D NMR
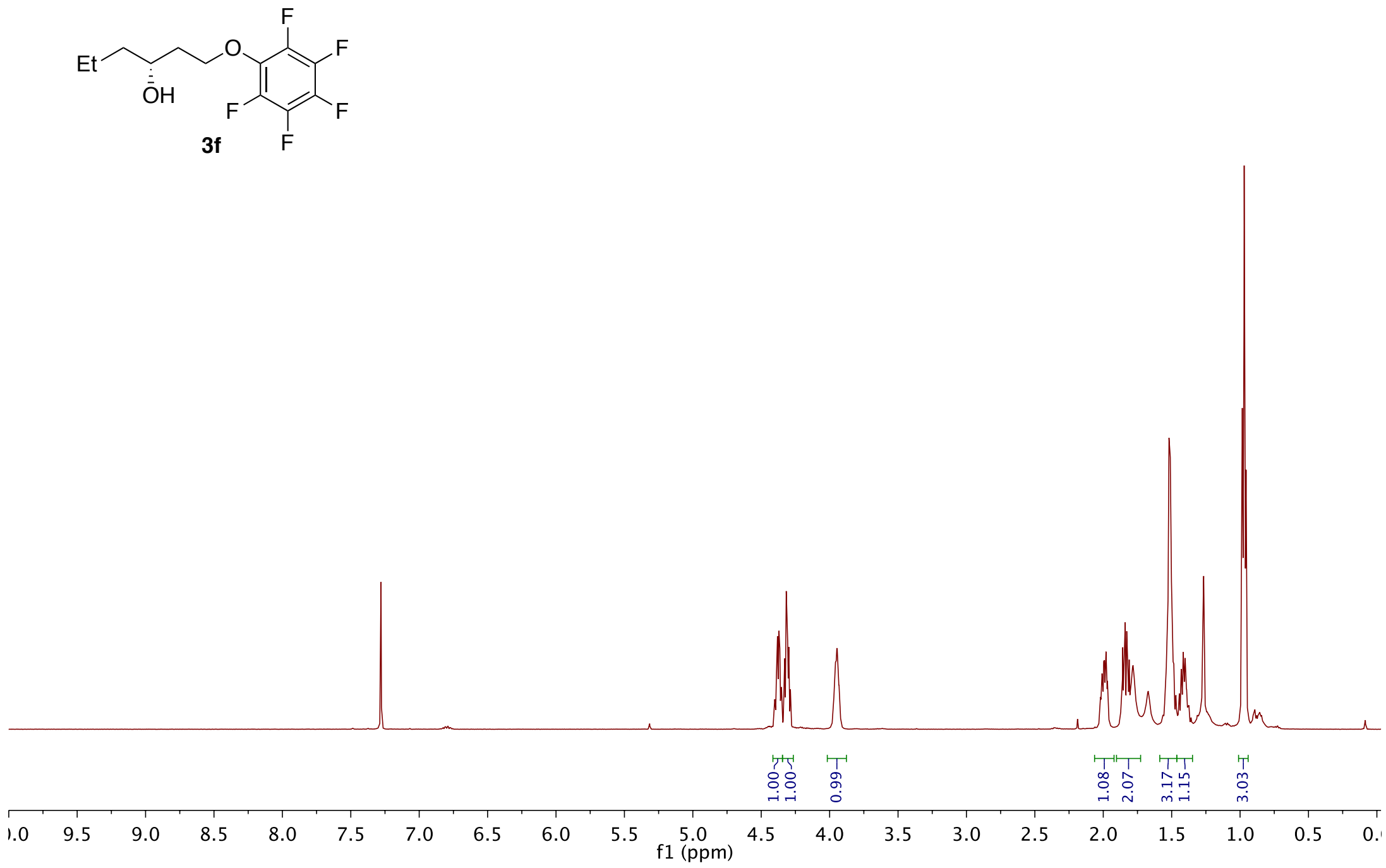
YX-3-226A-13C-1.1.fid

12/21/10 CC AV-600

$\mathrm{AQ} M O D=D Q D$

g9oprom

牙

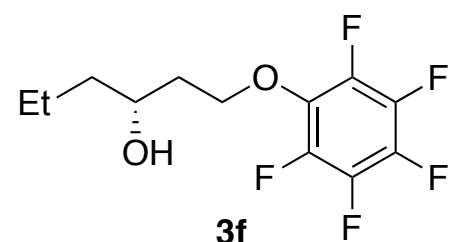

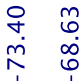

ตุ

min

$\Re$ o

$\stackrel{\infty}{\rightarrow} \stackrel{m}{1}$

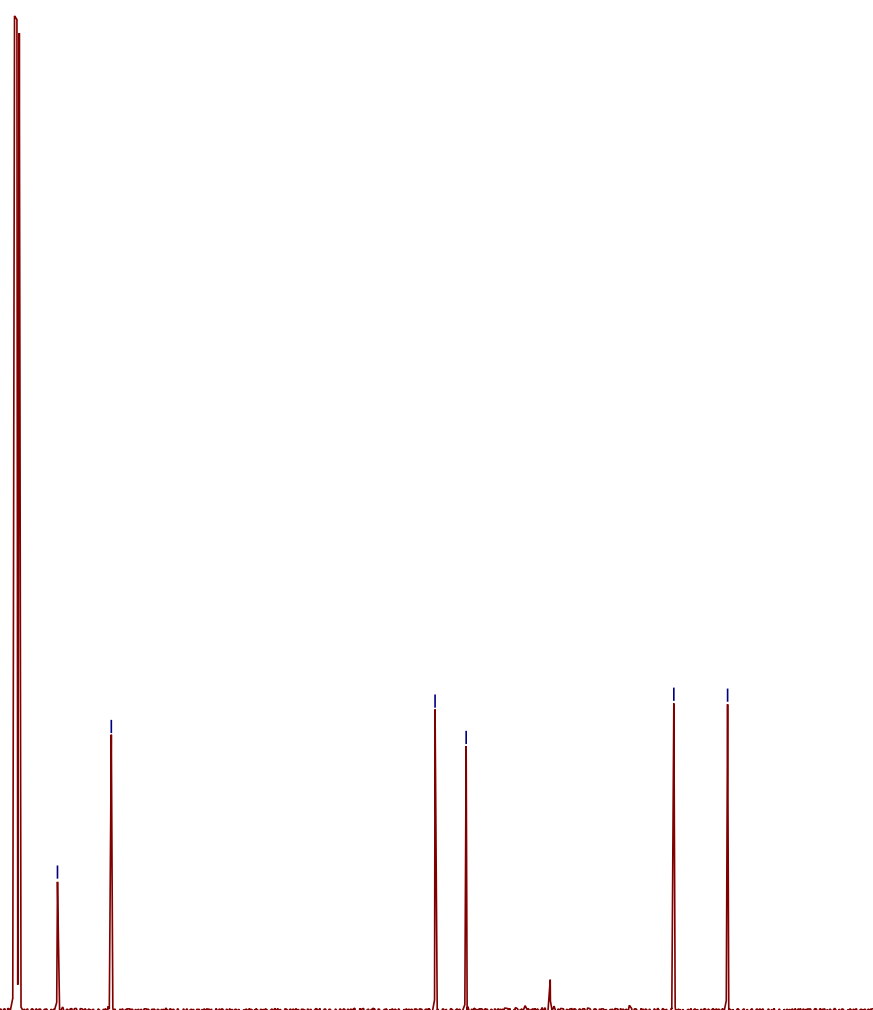


YX-3-226A-13C-1.1.fid

12/21/10 CC AV-600 ZBO ca্rbon starting pågameters

$A Q \_M O D=D Q D$

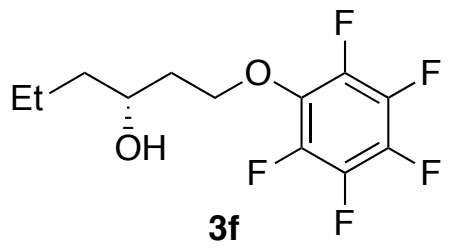

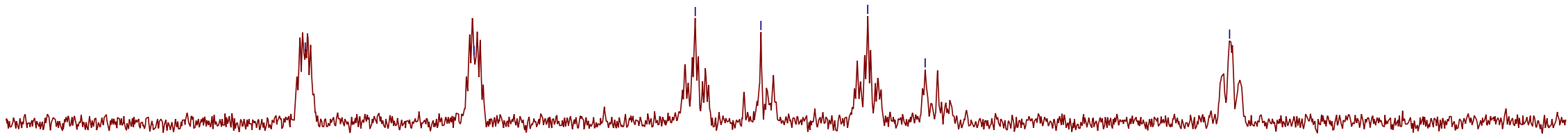

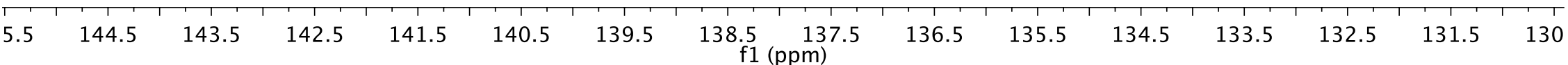


YX-3-226A-19F.1.fid

AVQ-400 QNP Probe 19F starting parameters. (revised P1, 2/12/04 RN)

chemical shifts relative to $\mathrm{CFCl} 3$ at $0 \mathrm{ppm}(082103 \mathrm{HvH})$

sw 239.28 ppm; o1p 0 ppm
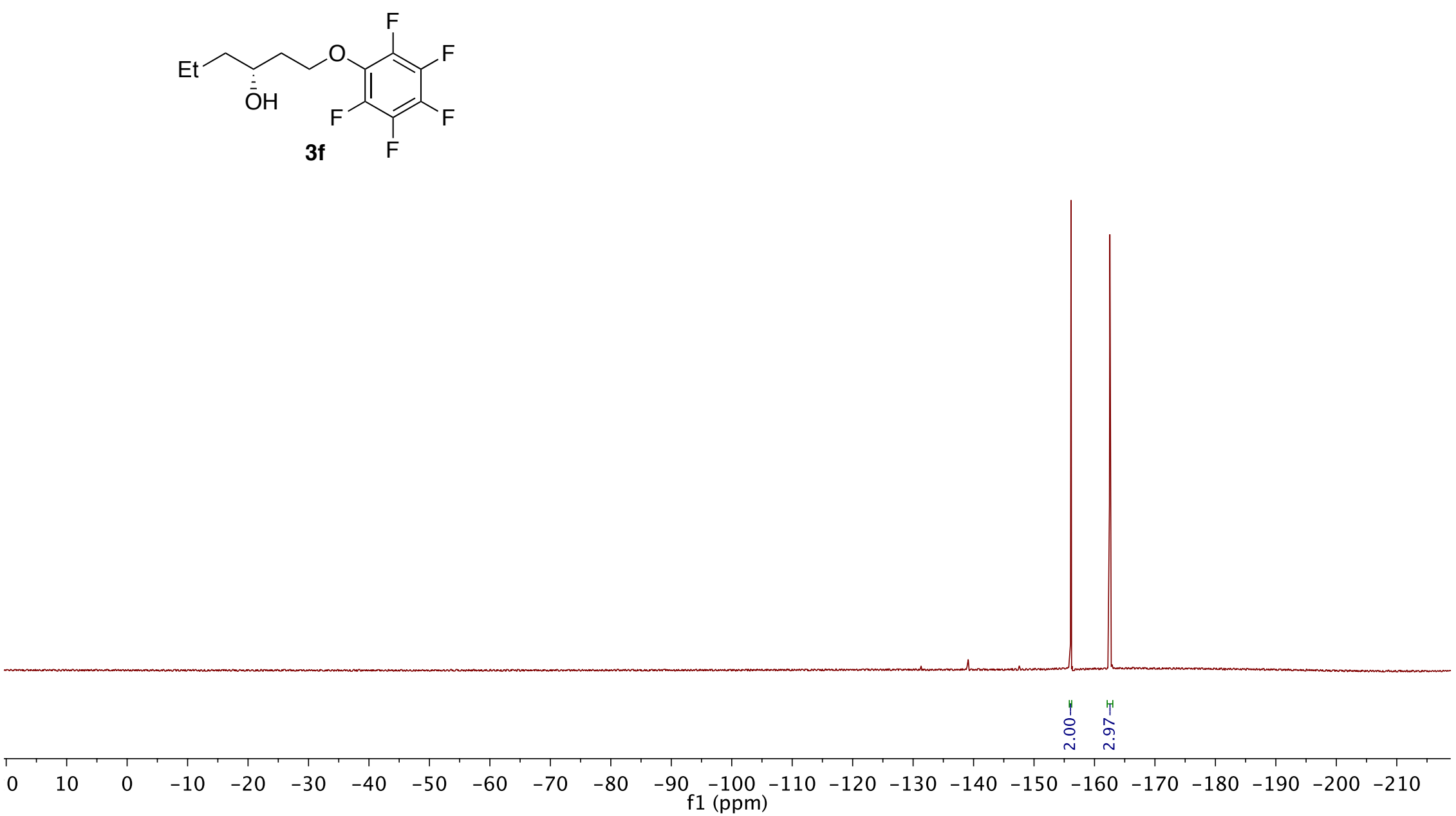
YX-3-272A-1H.1.fid

CC: 12182012 AV-500 TBIP probe

1H 1D NMR
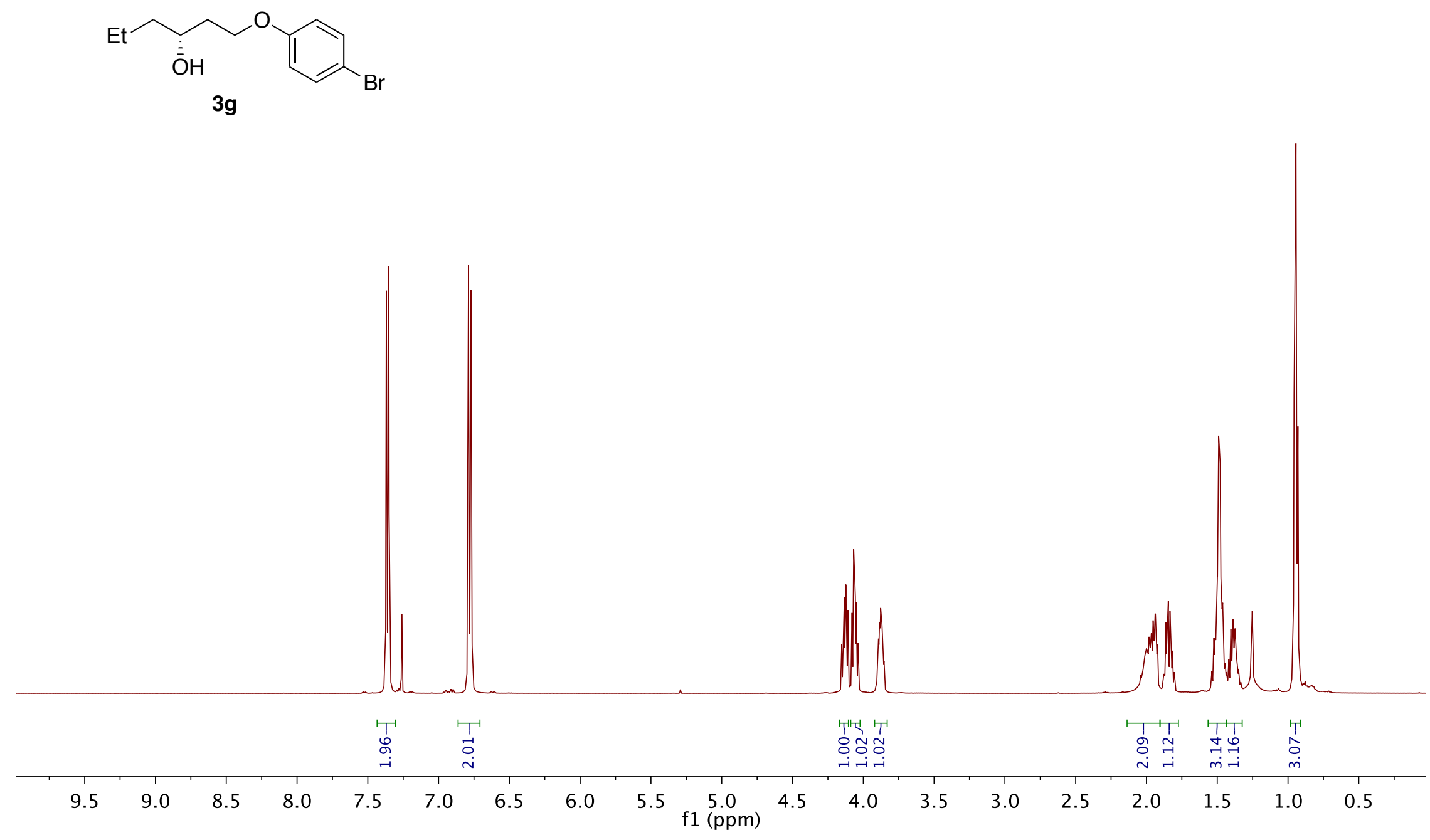


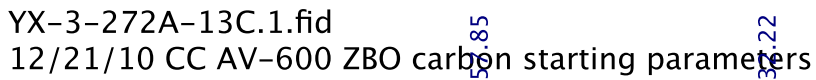
$A Q M O D=D Q D$

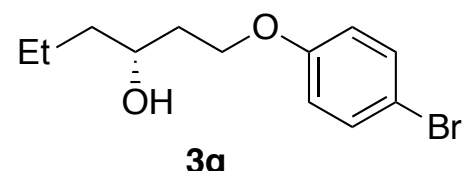

$3 g$

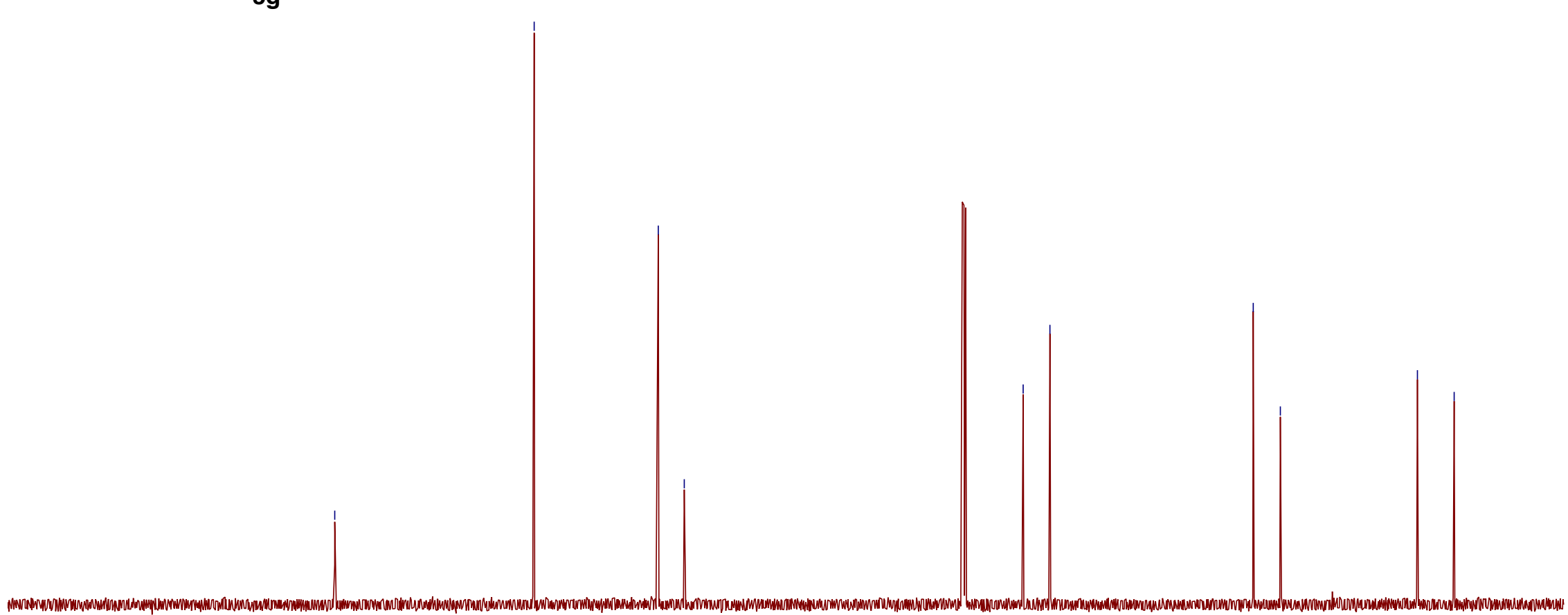

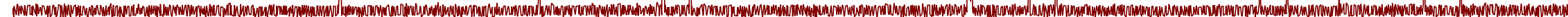


YX-3-226B-1H.1.fid

CC: 12182012 AV-500 TBIP probe

1H 1D NMR

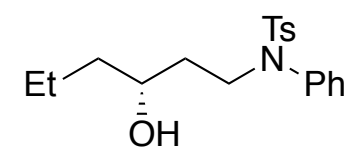

3h

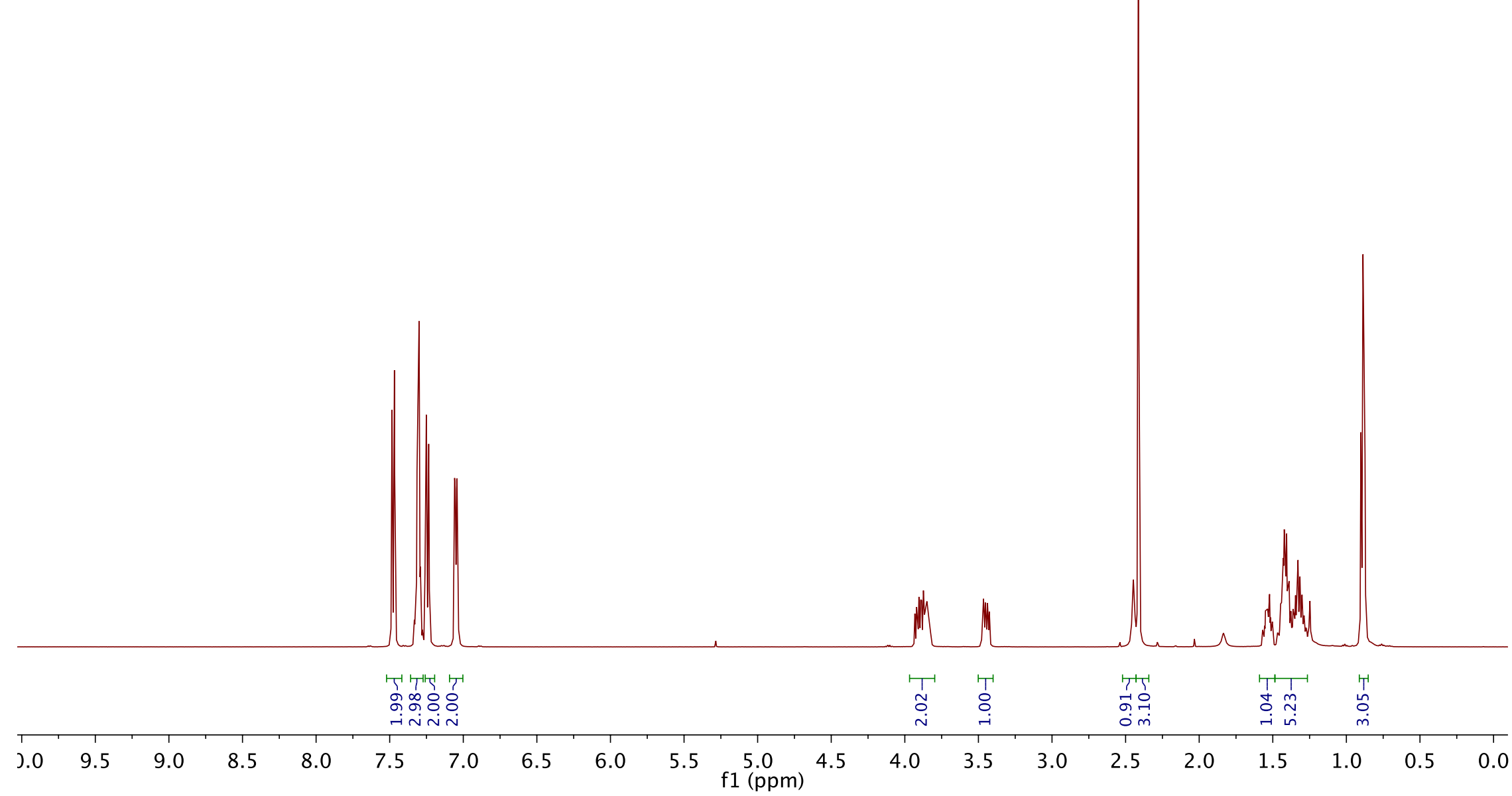


$\mathrm{YX}-3-226 \mathrm{~B}-13 \mathrm{C} .1$. fid

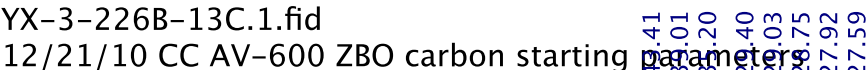

$A Q M O D=D Q D$

parametersis

1<smiles>CCC[C@@H](O)CCN([AsH3])c1ccccc1</smiles>

3h

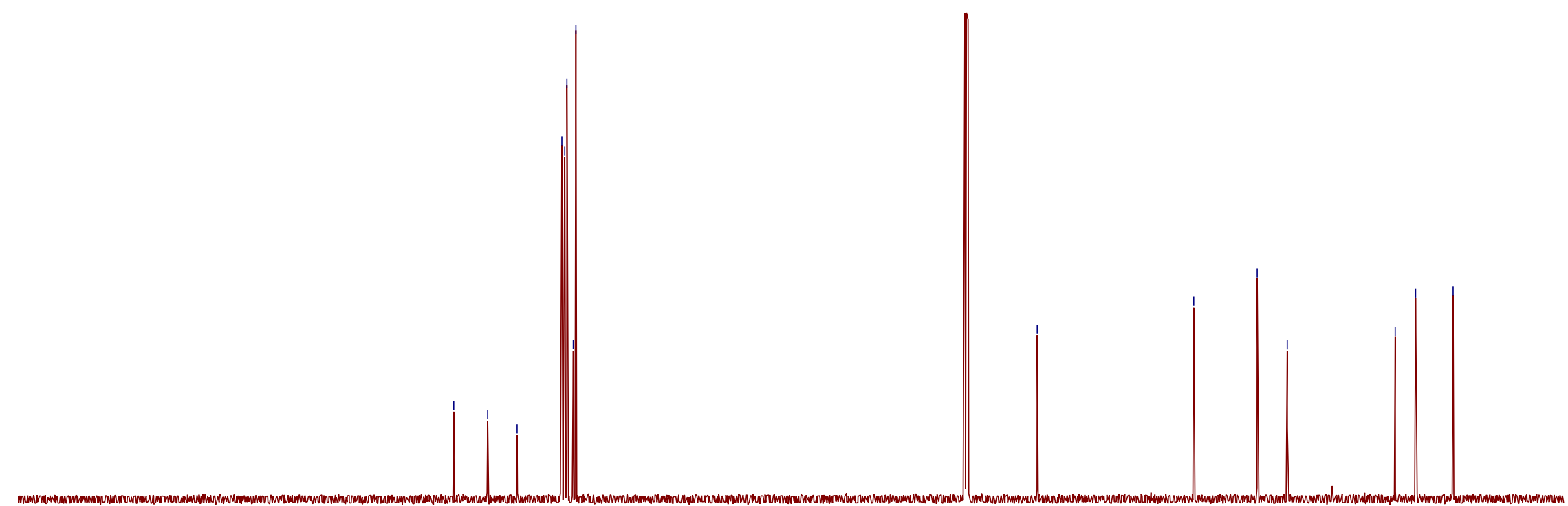


YX-3-226C-1H.1.fid

CC: 12182012 AV-500 TBIP probe

1H 1D NMR

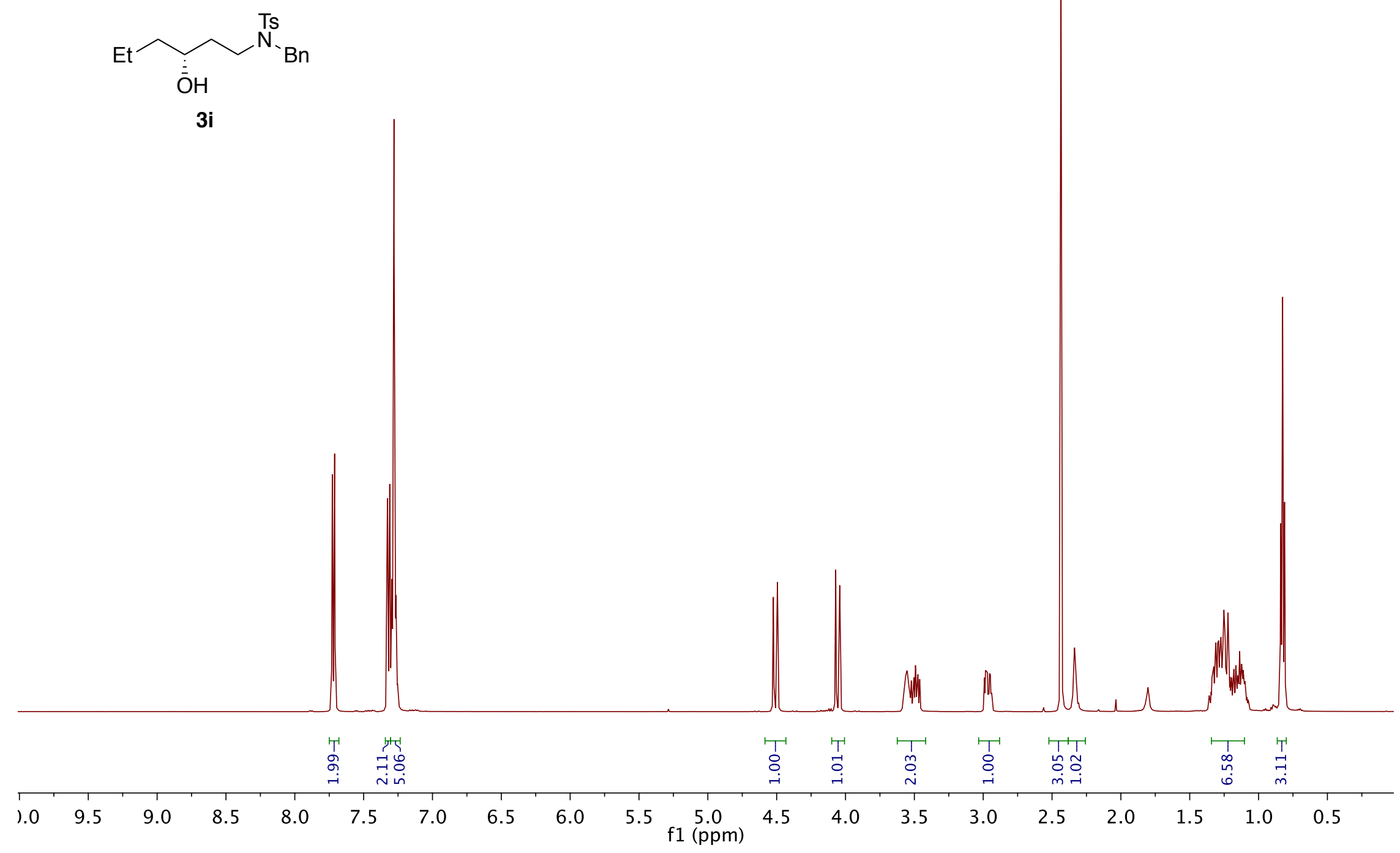




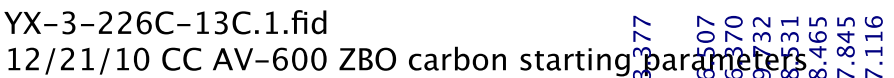

$A Q \_M O D=D Q D$<smiles>CCC[C@@H](O)CCN([AsH3])Cc1ccccc1</smiles>

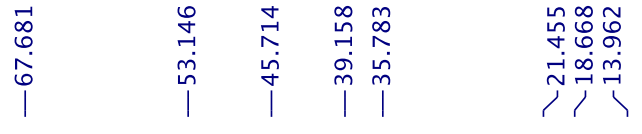

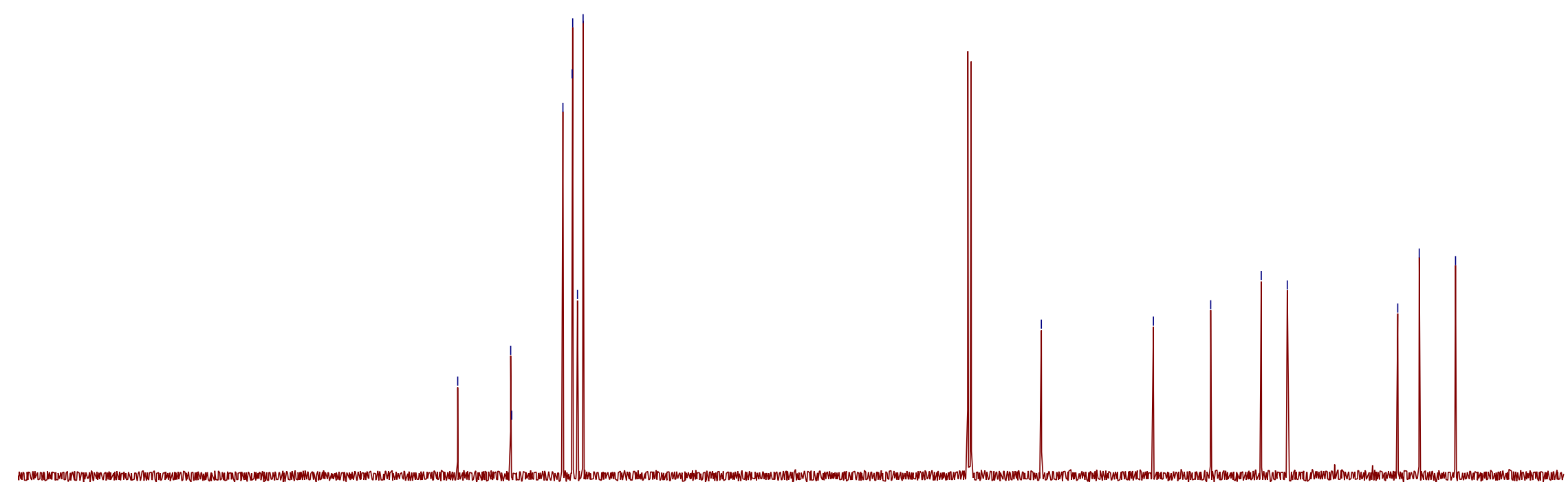

180

170

160

140

130

120

110

100

90

80

70

60

50

40

30

20

10 
YX-3-272B-1H.1.fid

CC: 12182012 AV-500 TBIP probe

1H 1D NMR

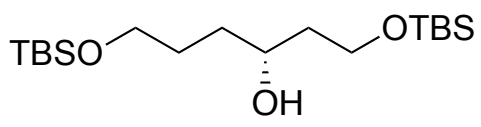

3n

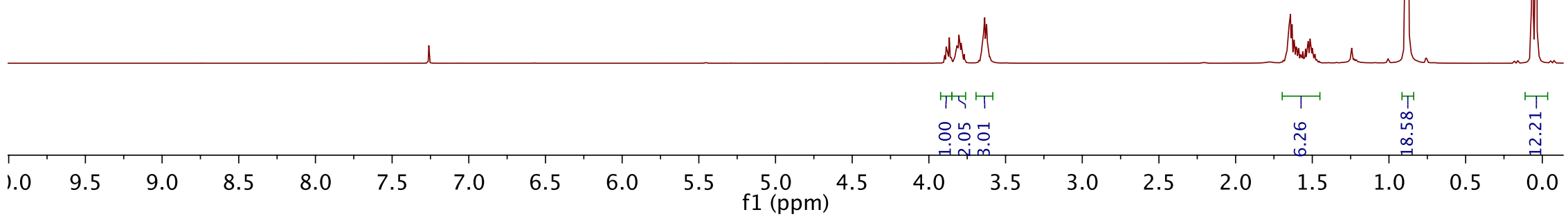


$\mathrm{YX}-3-272 \mathrm{~B}-13 \mathrm{C} .1$.fid

$12 / 21 / 10$ CC AV-600 ZBO carbon starting parameters

$A Q M O D=D Q D$

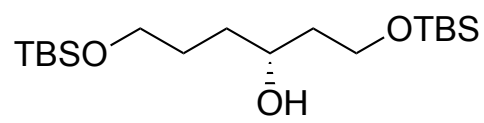

$3 n$

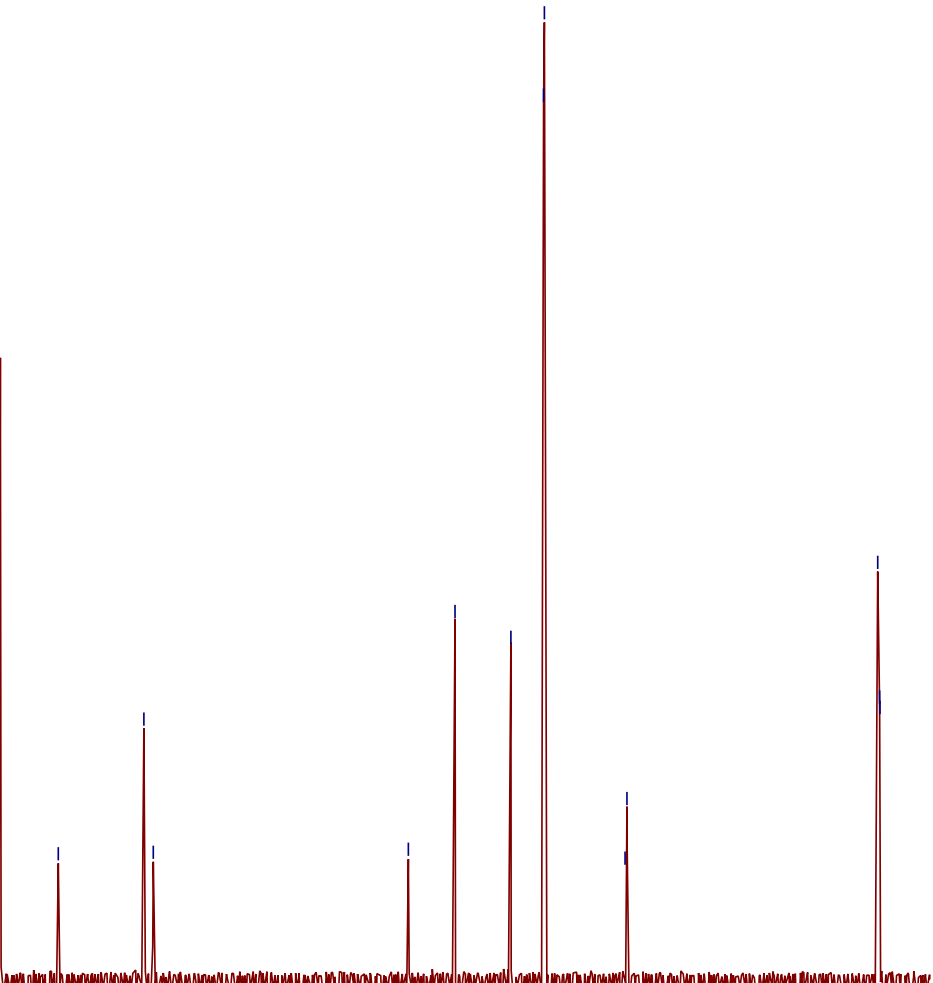

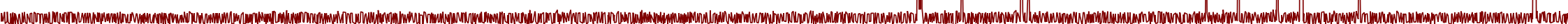

\begin{tabular}{|c|c|c|c|c|c|c|c|c|c|c|c|c|c|c|c|c|c|c|c|c|}
\hline 0 & & 180 & 170 & 160 & 150 & 140 & 130 & 120 & 110 & \multicolumn{2}{|c|}{$\begin{array}{l}100 \\
\mathrm{f} 1(\mathrm{ppm})\end{array}$} & 80 & 70 & & 50 & 40 & 30 & 20 & 10 & 0 \\
\hline
\end{tabular}


YX-3-225D-1H.1.fid

CC: 12182012 AV-500 TBIP probe

1H 1D NMR<smiles>O=C(OCCCC(O)O)c1c(Cl)cc(Cl)cc1Cl</smiles>

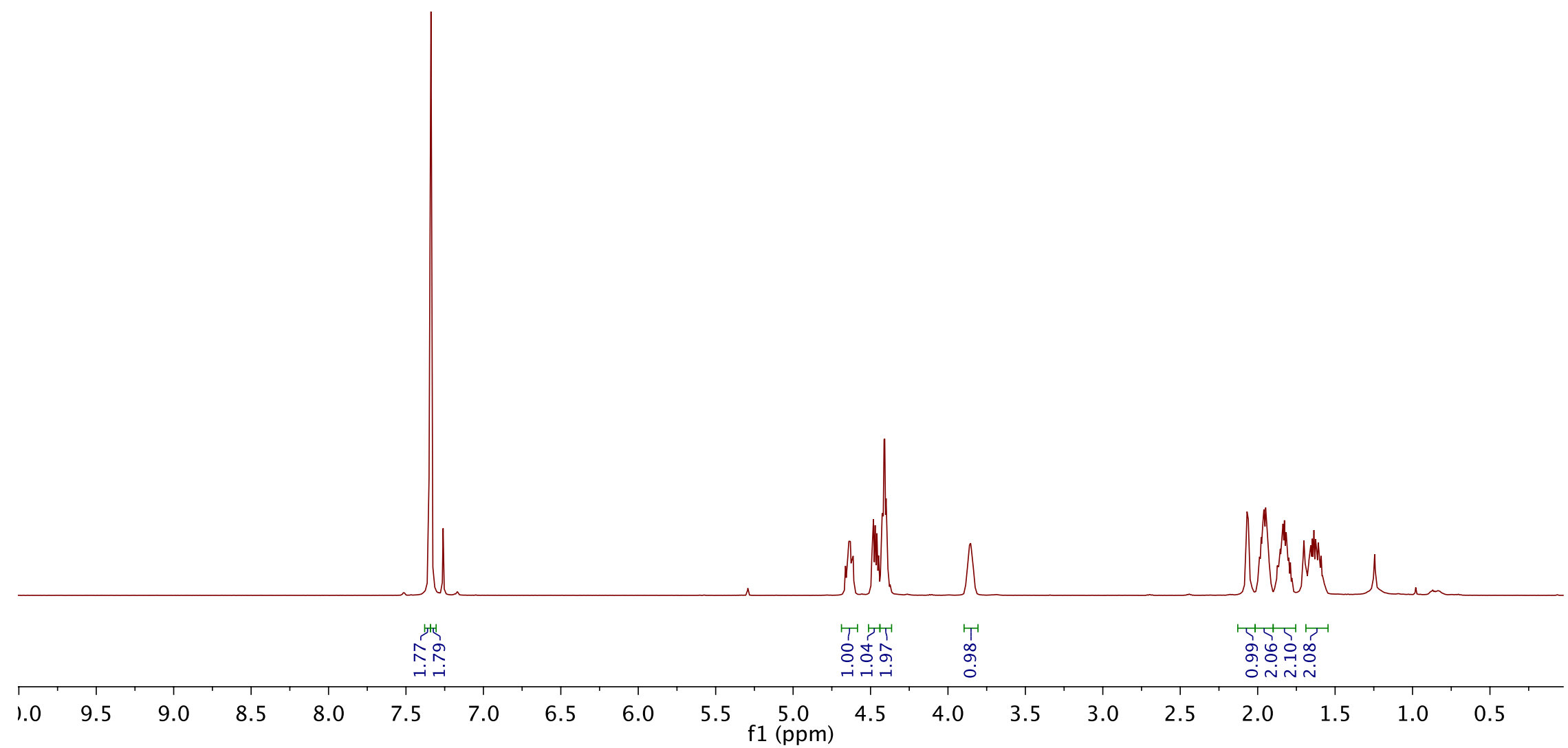




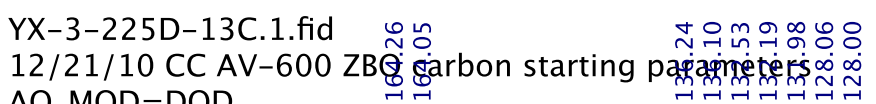

$A Q \_M O D=D Q D$

r

प

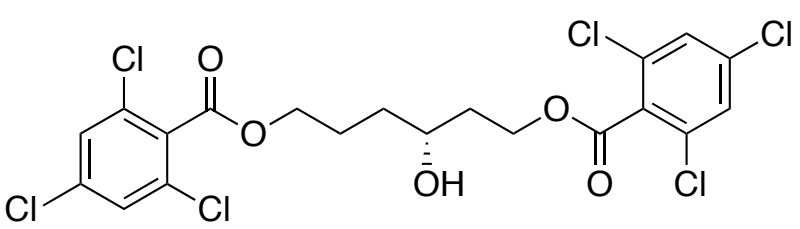

30

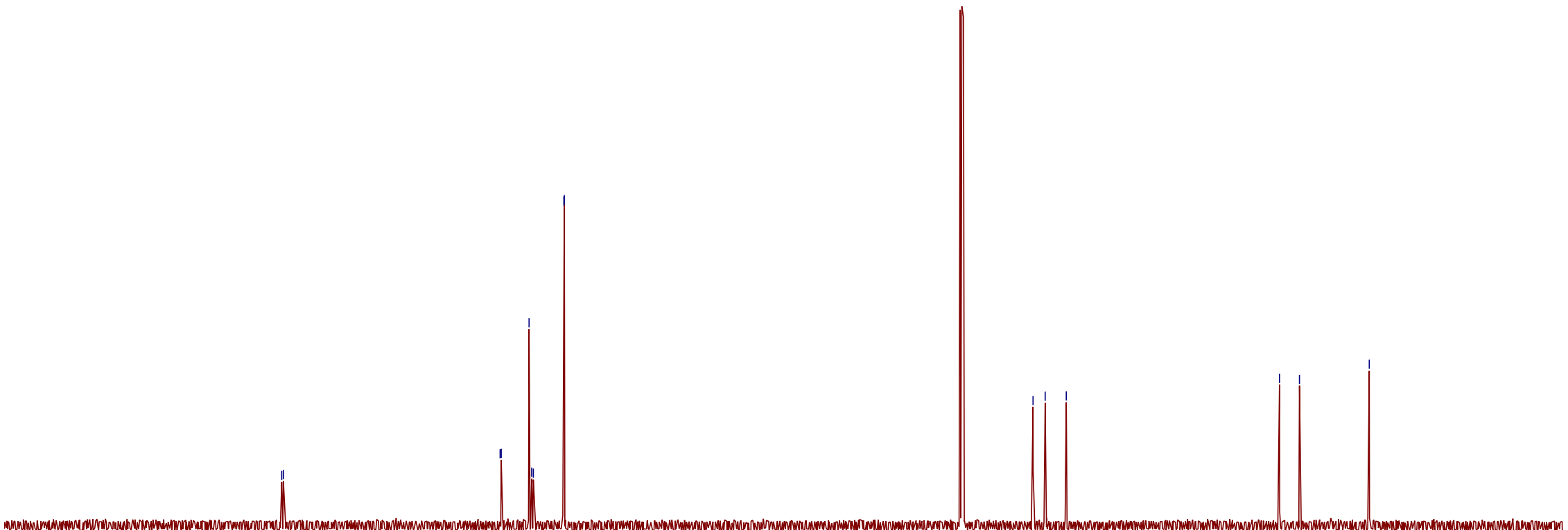


YX-3-277-1H.1.fid

CC: 12182012 AV-500 TBIP probe

1H 1D NMR

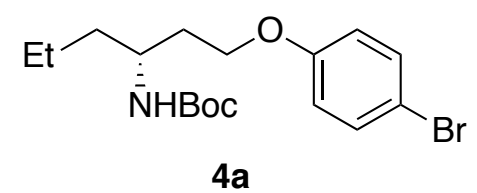

$4 a$

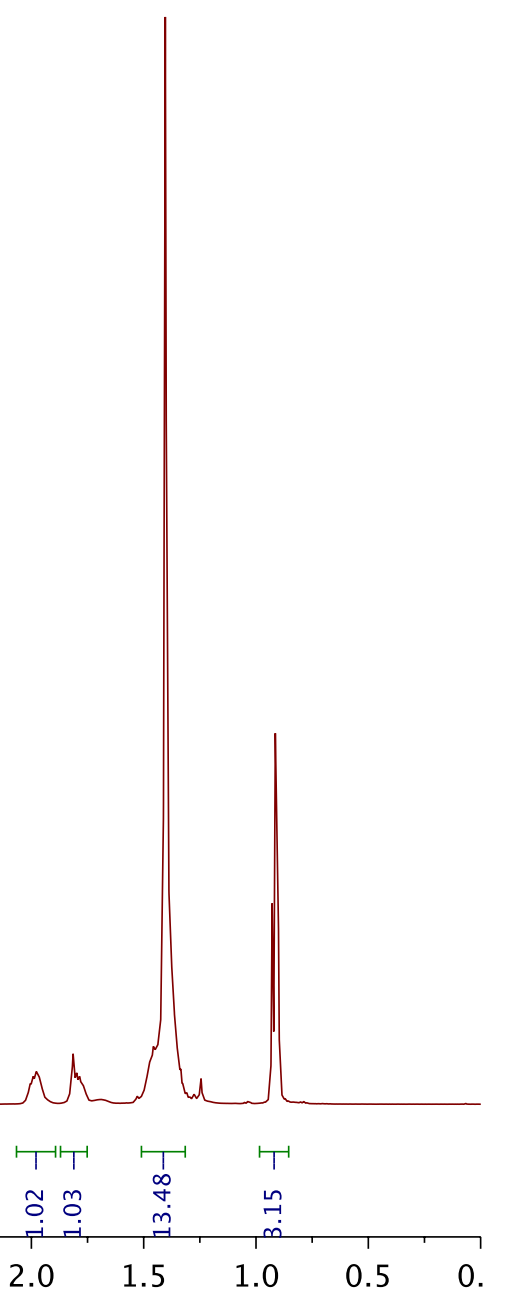


YX-3-277-13C.1.fid
12/21/10 CC AV-600 ZBO carbogn starting parameterers

$A Q M O D=D Q D$

年

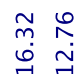

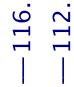

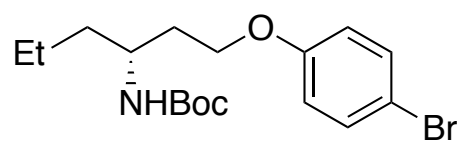

$4 a$

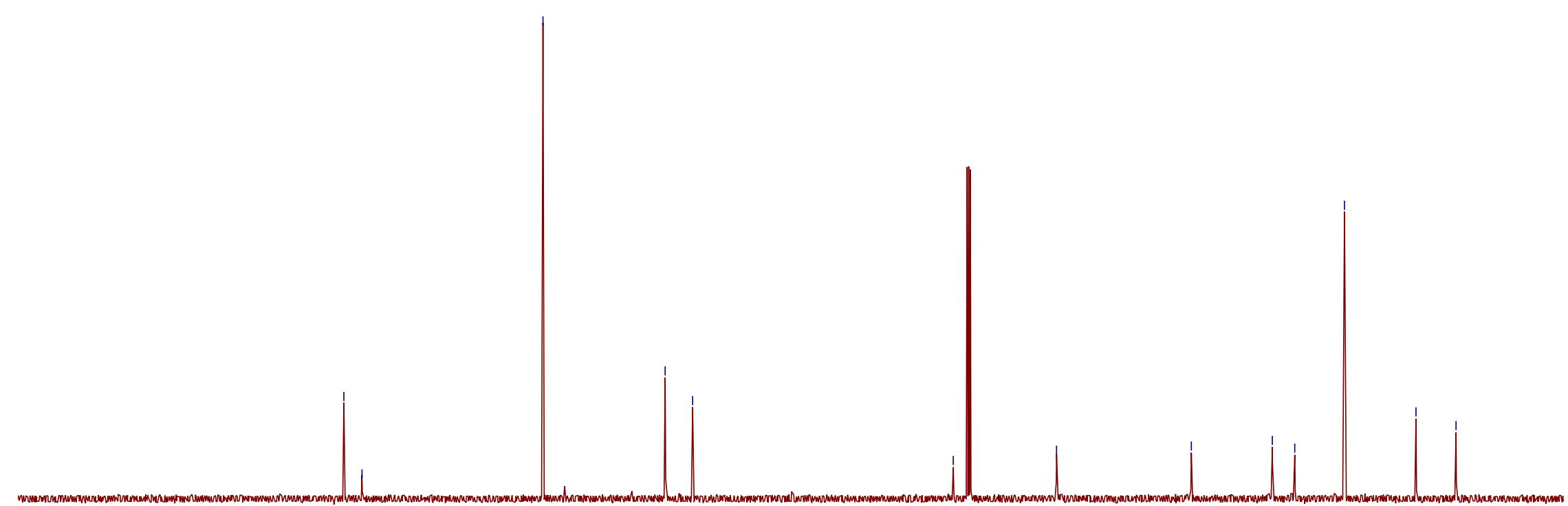

\begin{tabular}{|c|c|c|c|c|c|c|c|c|c|c|c|c|c|c|c|c|c|c|c|}
\hline )0 & 190 & 180 & 170 & 160 & 150 & 140 & 130 & 120 & 110 & $\begin{array}{c}100 \\
\mathrm{f} 1(\mathrm{ppm})\end{array}$ & 90 & 80 & 70 & 60 & 50 & 40 & 30 & 20 & 10 \\
\hline
\end{tabular}


YX-3-267-1H.1.fid

CC: 12182012 AV-500 TBIP probe

1H 1D NMR<smiles>CCC(Br)CC[Nb]</smiles>

$4 b$

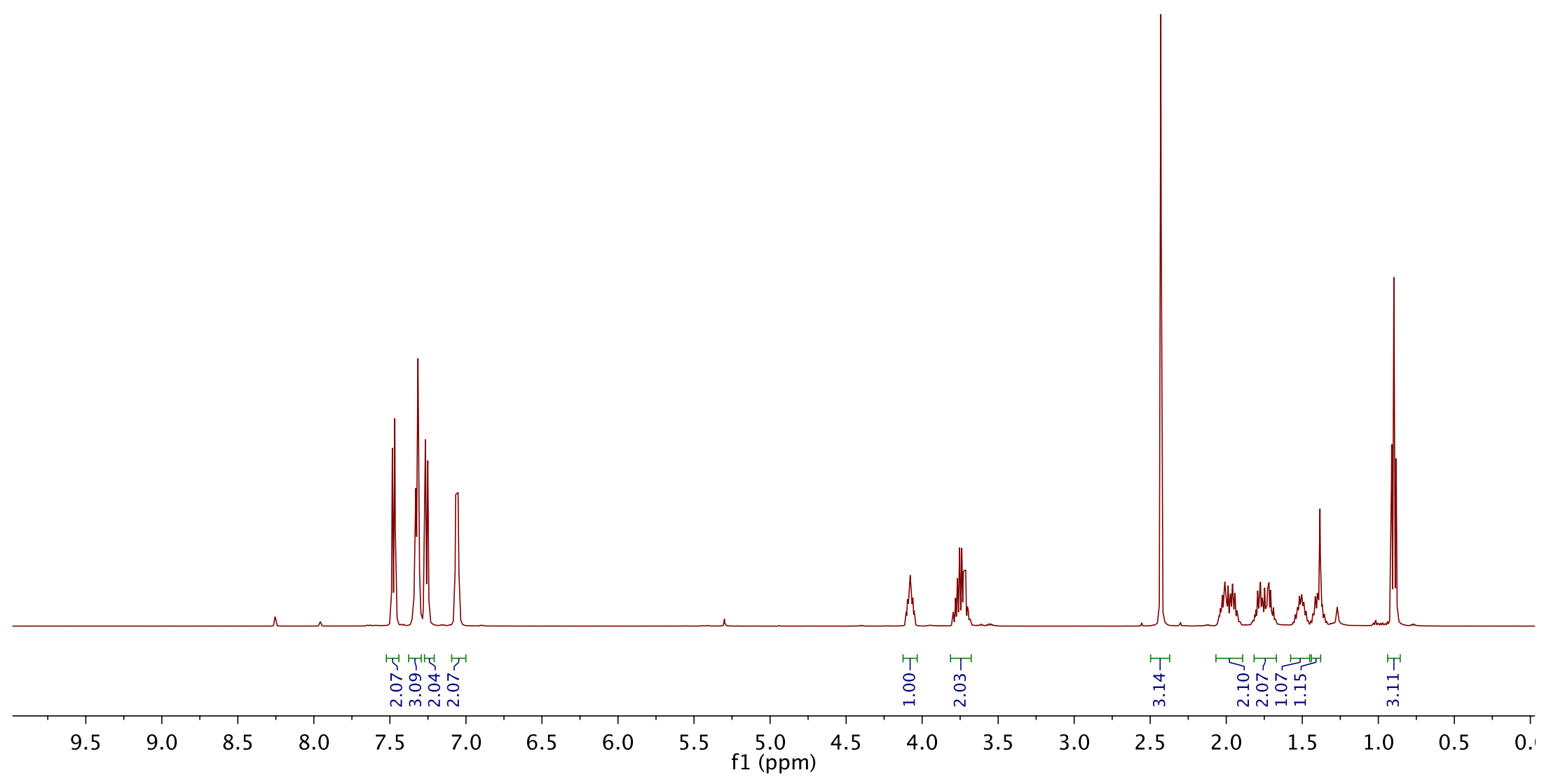


YX-3-267-13C.1.fid

寸ㅇํㅇํำ $A Q \_M O D=D Q D$

$$
\overbrace{\mathrm{Br}}^{\mathrm{NPhTS}}
$$

$4 b$

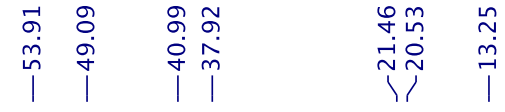

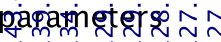

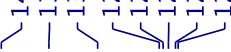

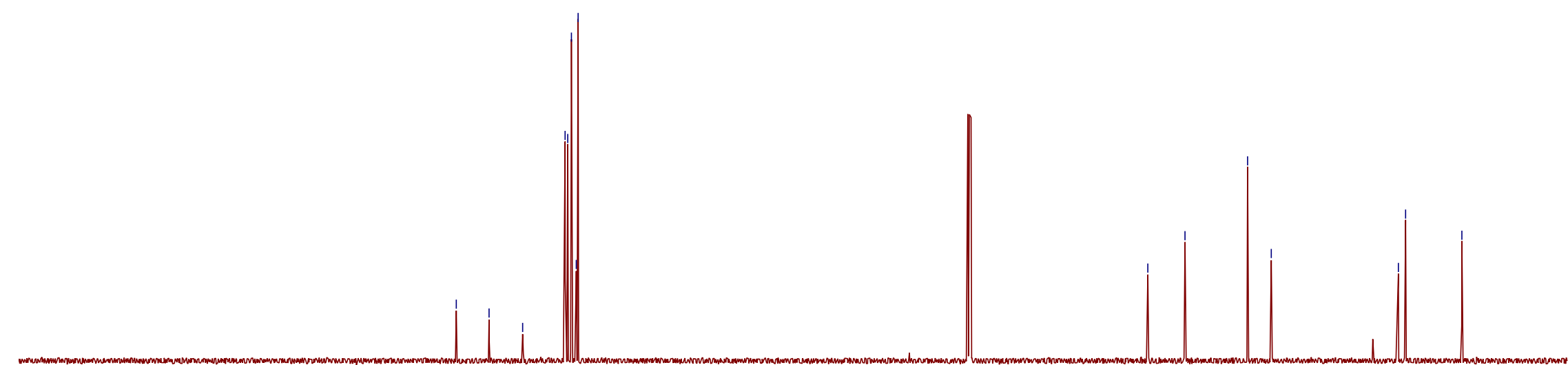

\begin{tabular}{|c|c|c|c|c|c|c|c|c|c|c|c|c|c|c|c|c|c|c|c|}
\hline 30 & 190 & 180 & 170 & 160 & 150 & 140 & 130 & 120 & 110 & $\mathrm{f} 1(\mathrm{ppm})$ & 90 & 80 & 70 & 60 & 50 & 40 & 30 & 20 & 10 \\
\hline
\end{tabular}


YX-4-73-1H-P.1.fid

CC: 12182012 AV-500 TBIP probe

1H 1D NMR
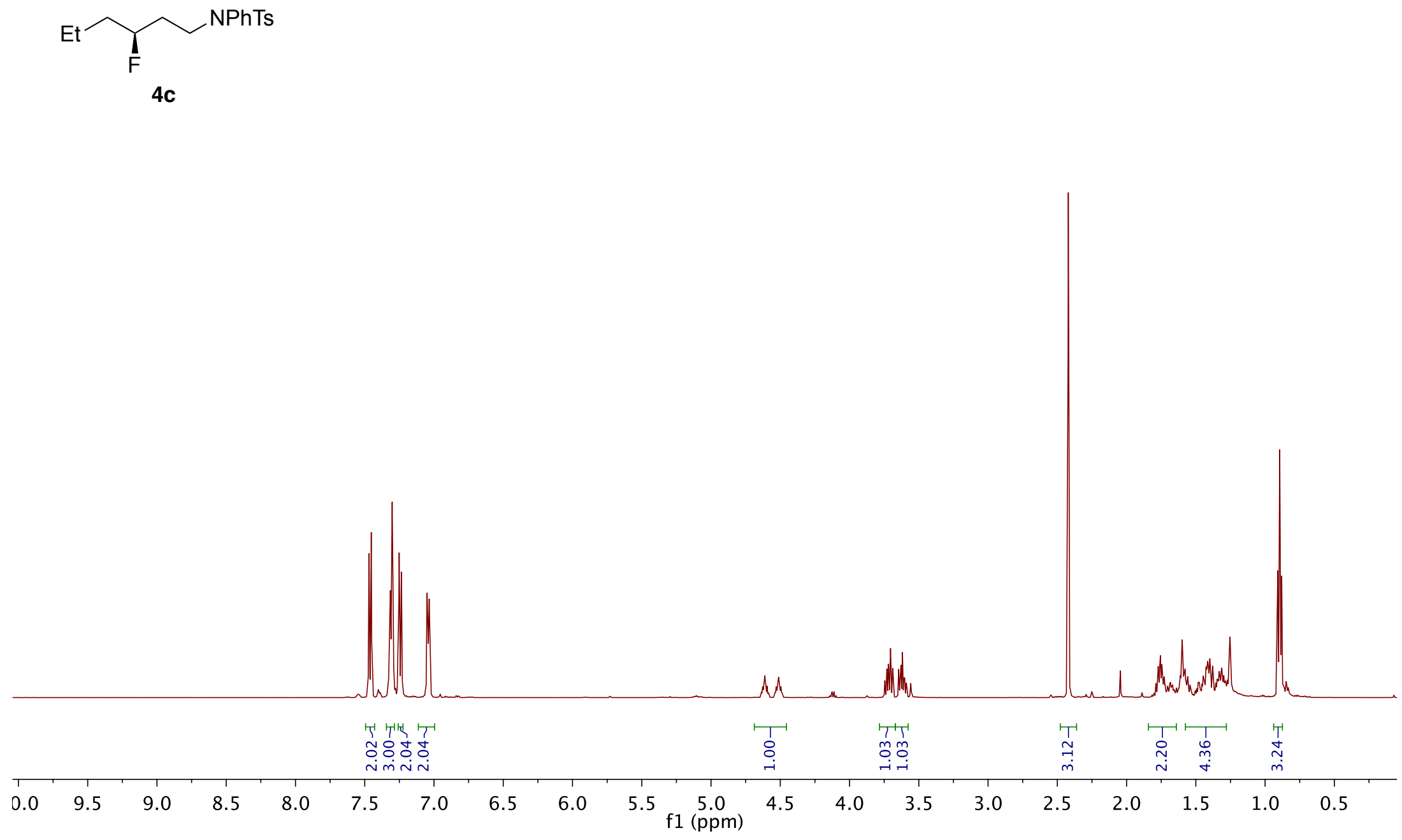
YX-4-73-13C-P.1.fid

$12 / 21 / 10$ CC AV-600 ZBO

$A Q \_M O D=D Q D$

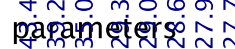

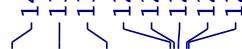

ธิ ตุ?

กัธ

11

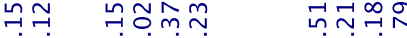

fं
$\mathrm{NPhTs}$

4c

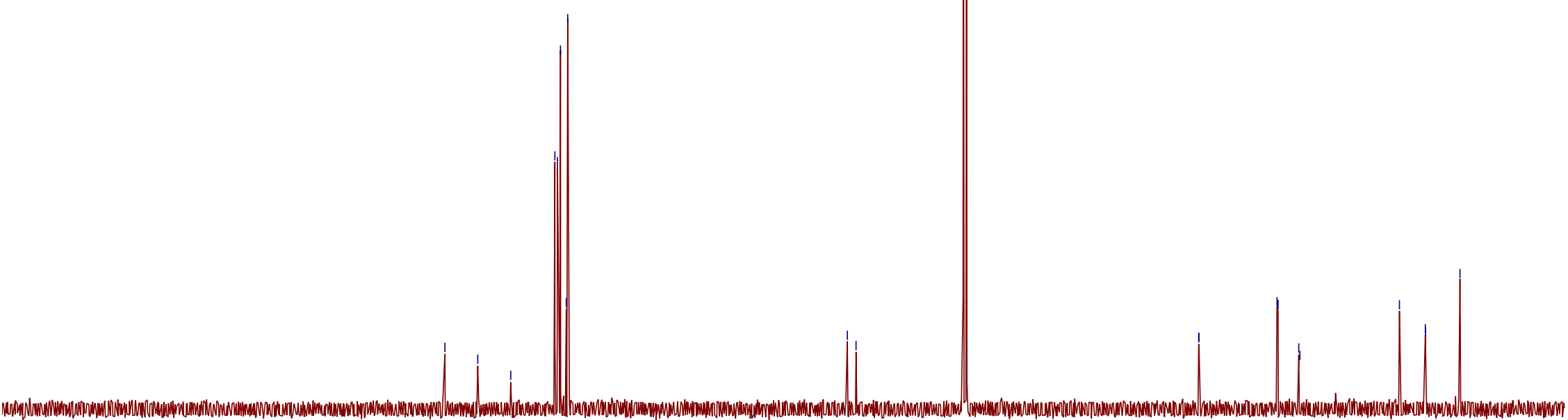


AVQ-400 QNP Probe 19F starting parameters. (revised P1, 2/12/04 RN)

chemical shifts relative to $\mathrm{CFCl} 3$ at $0 \mathrm{ppm}(082103 \mathrm{HvH})$

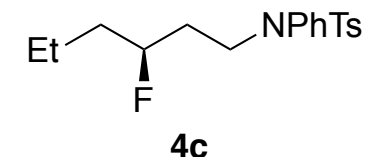

4c

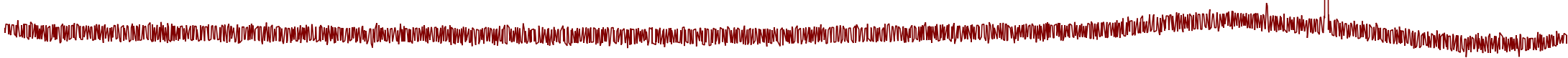

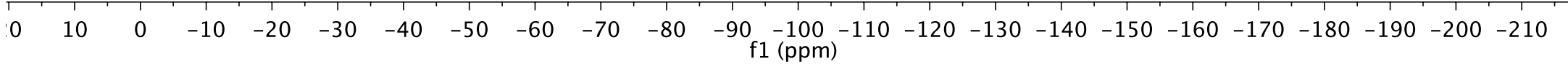


YX-3-244-1H.1.fid

CC: 12182012 AV-500 TBIP probe

1H 1D NMR
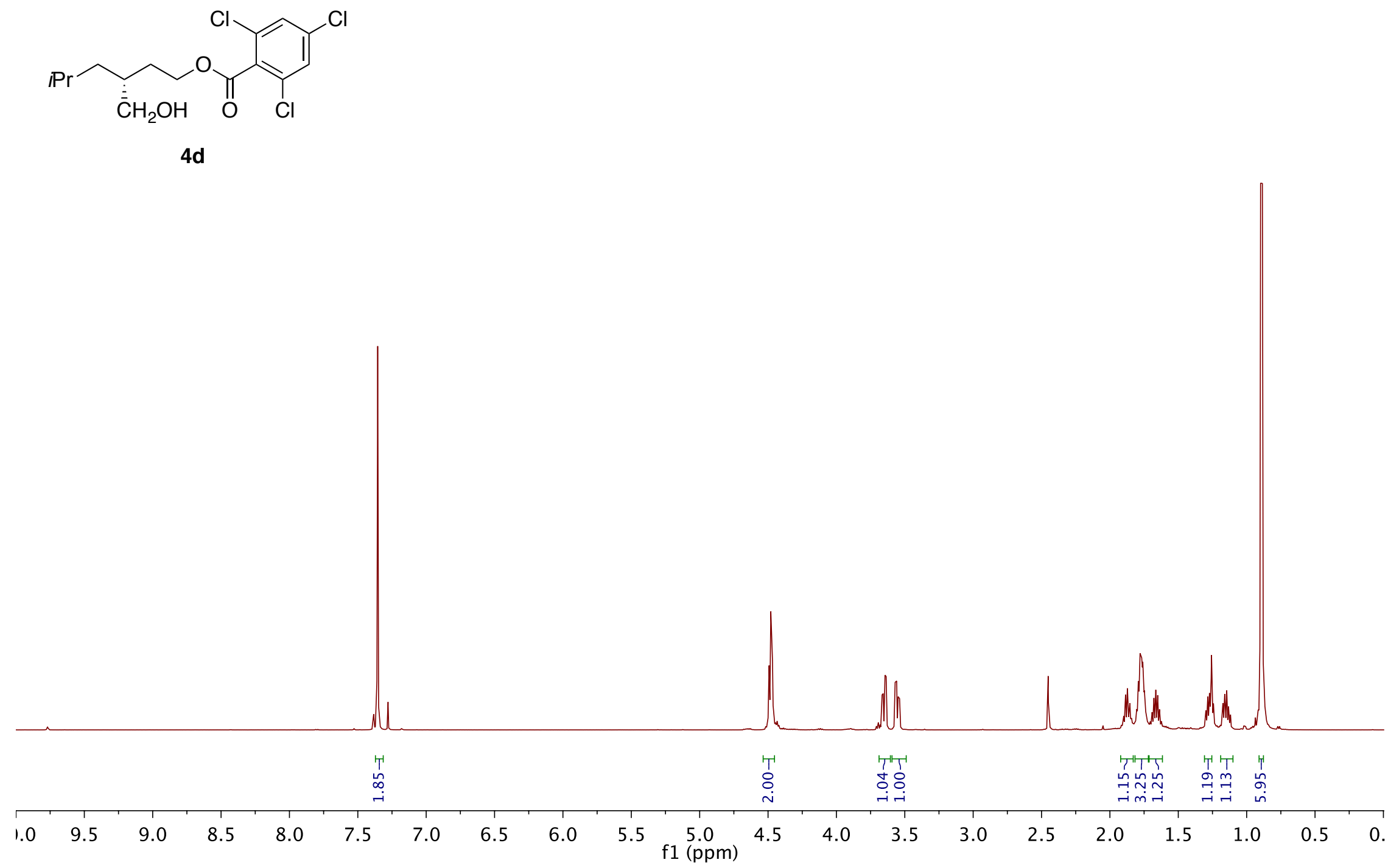


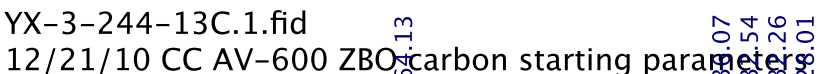

$A Q M O D=D Q D$

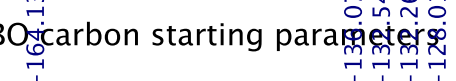

1

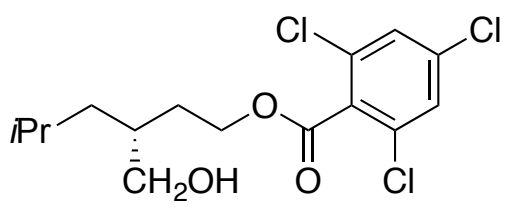

4d กุg

ஸीं

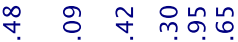

i

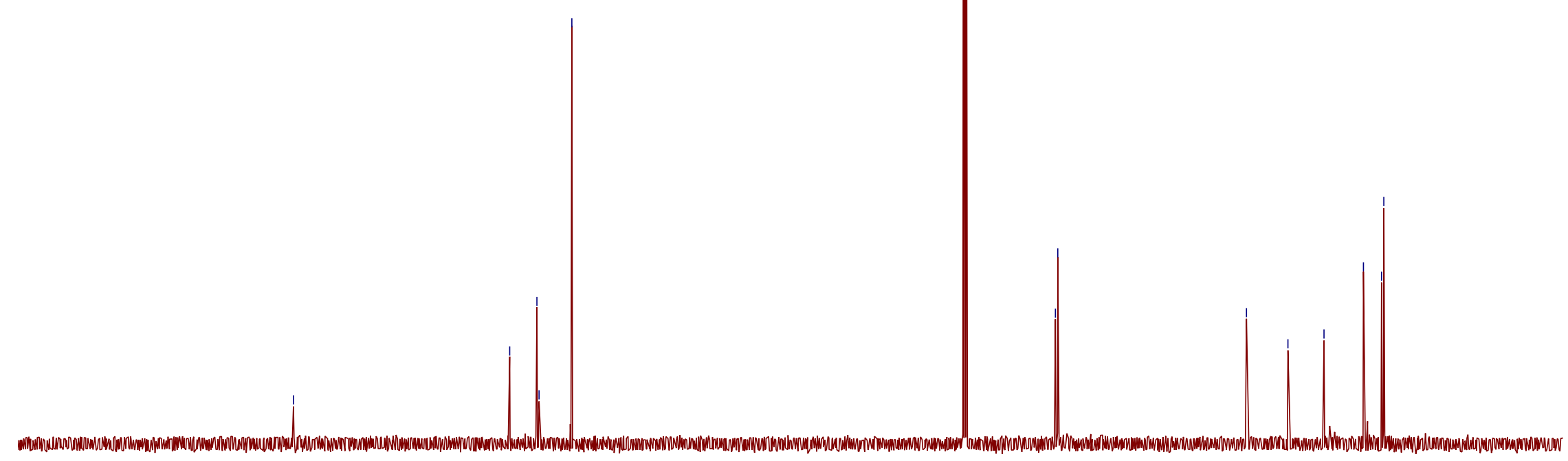


YX-3-273B-1H.1.fid

CC: 12182012 AV-500 TBIP probe

1H 1D NMR

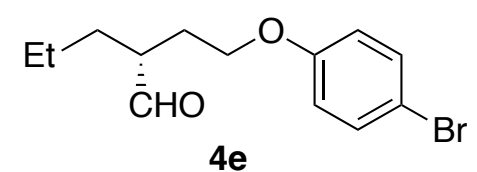

$4 e$

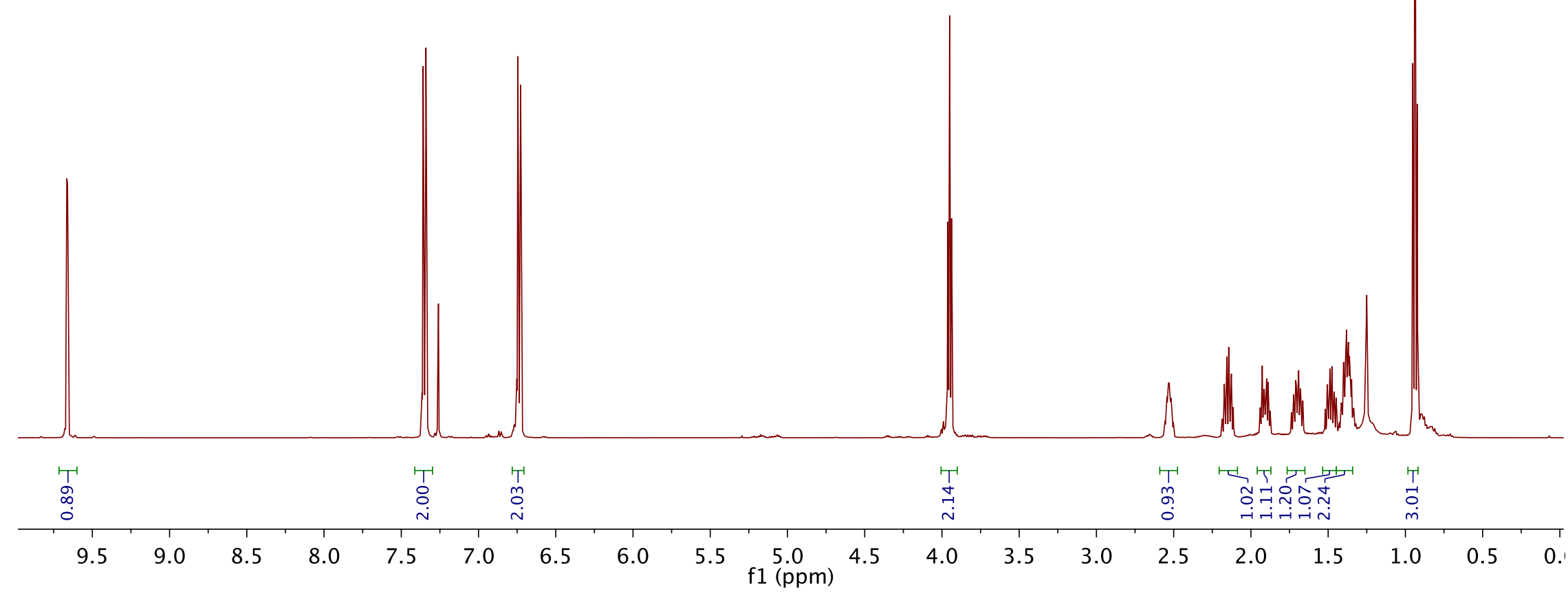




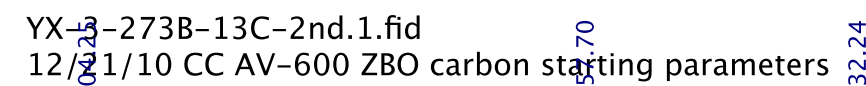
$A Q M O D=D Q D$

丝保

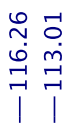

0
1
0
0

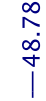

\%ำ

$\stackrel{m}{i}$

$\stackrel{+}{\stackrel{+}{+}}$<smiles>CC[C@H](C)CCOc1ccc(Br)cc1</smiles>

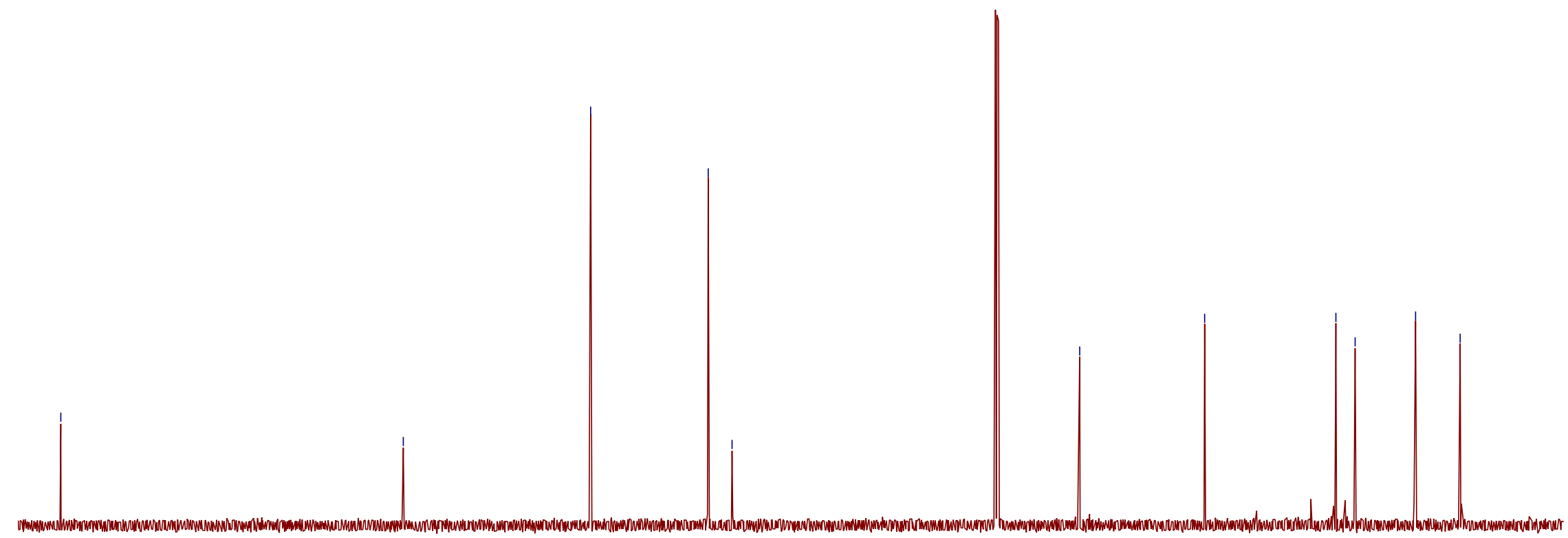

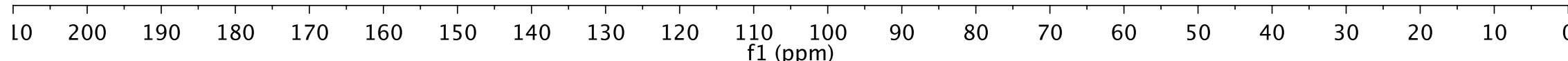


YX-3-290-1H.2.fid

AV-600 ZBO proton starting parameters $11 / 16 / 08 \mathrm{RN}$

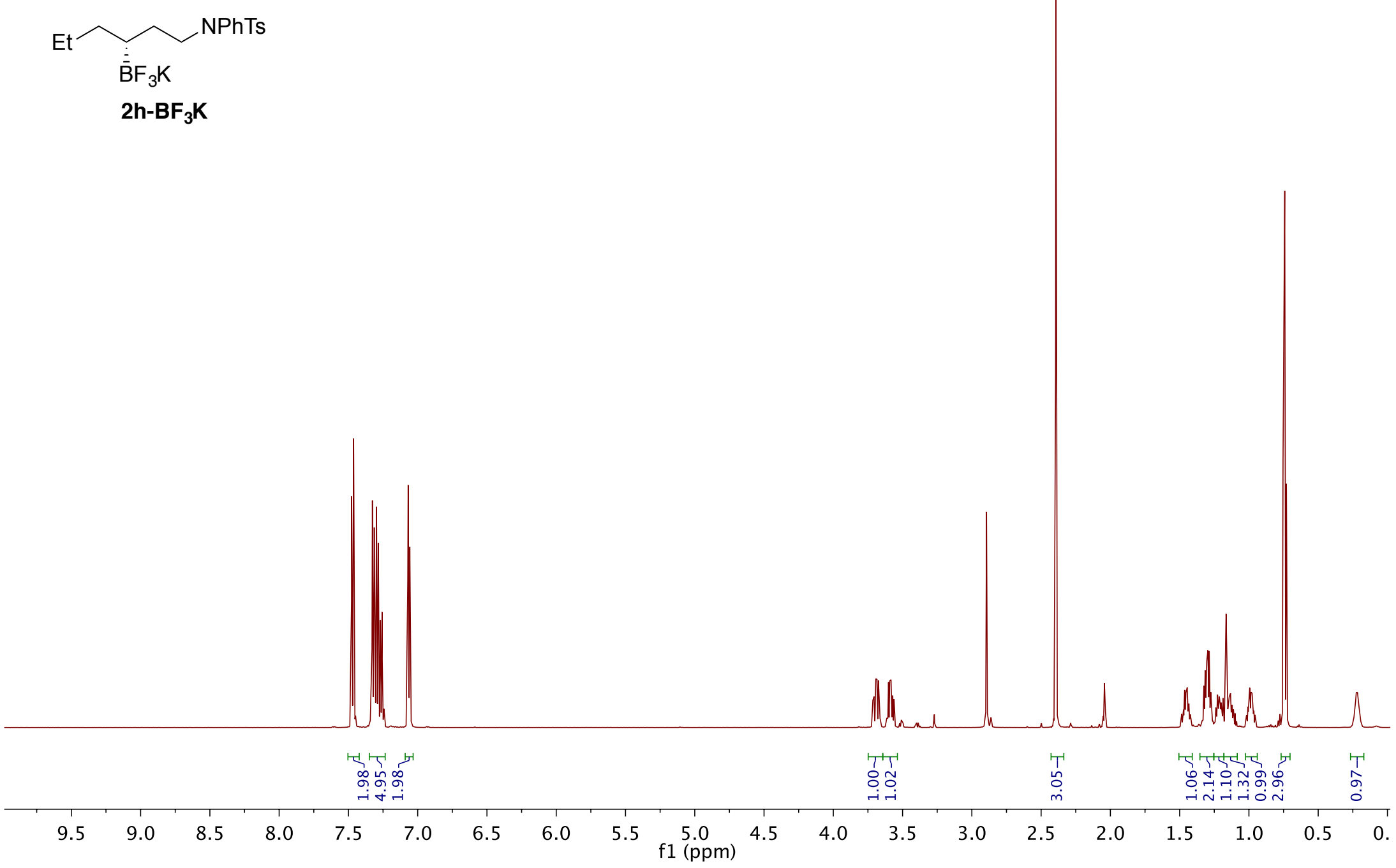


YX-3-290-13C.2.fid

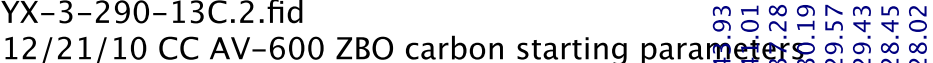
$A Q \_M O D=D Q D$

$2 \mathrm{~h}-\mathrm{BF}_{3} \mathrm{~K}$

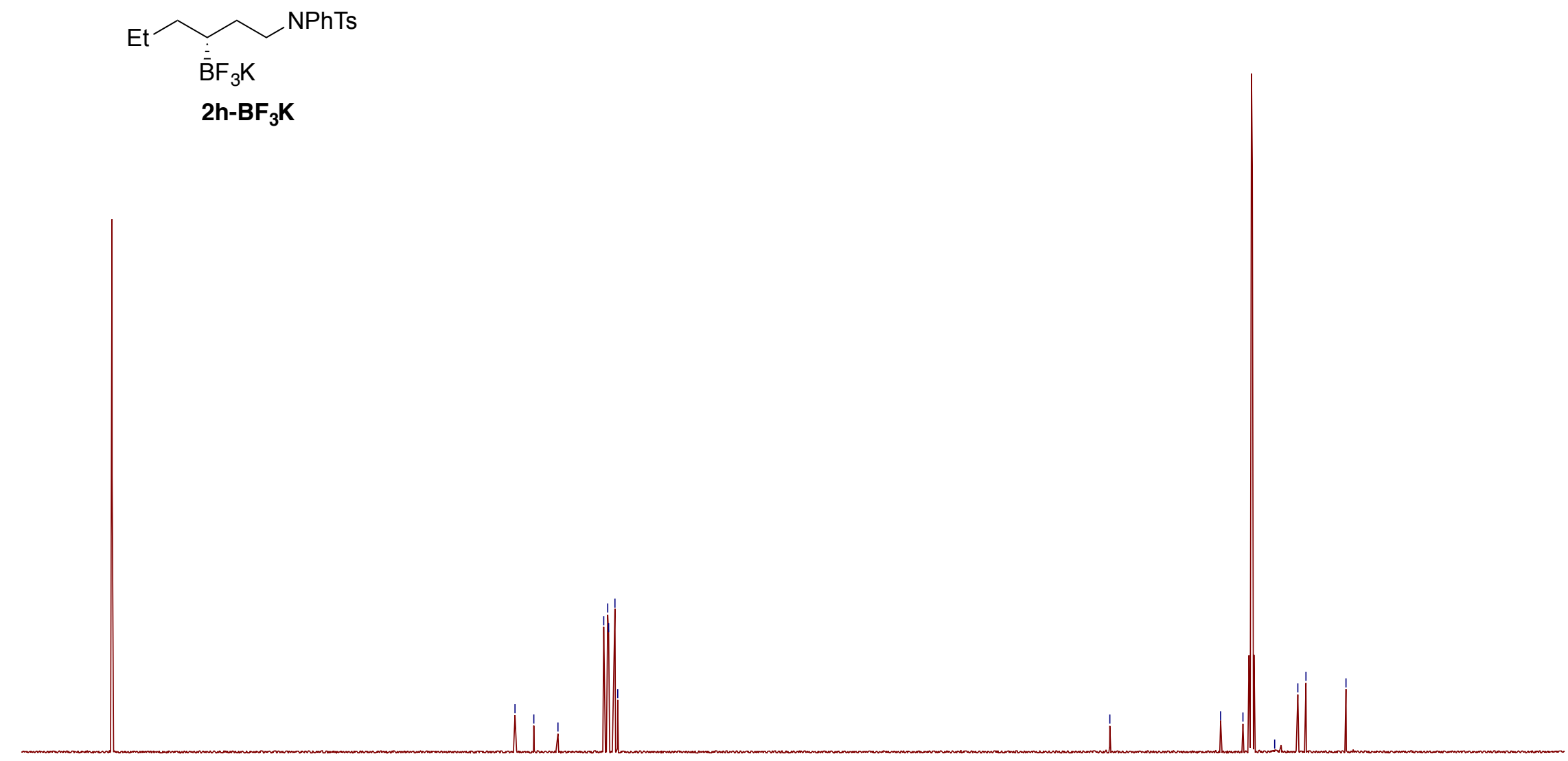

\section{क \\ mํํํ츄}


YX-3-290-11B.2.fid

AV-600 11B starting parameters

with $1 \mathrm{H}$ decoupling

Using 90 pulses (for T1 $<0.7$ sec or less).

$\mathrm{SW}=265 \mathrm{ppm}$, O1P $=40 \mathrm{BF} 3$. Et2O $=0 \mathrm{ppm}$.

For $1 \mathrm{H}$ coupled experiments use ased to change pulse prog. to $\mathrm{zg}$

Use linear prediction (ME_mod=LPbc) to remove probe background

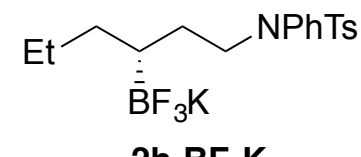

$2 \mathrm{~h}-\mathrm{BF}_{3} \mathrm{~K}$

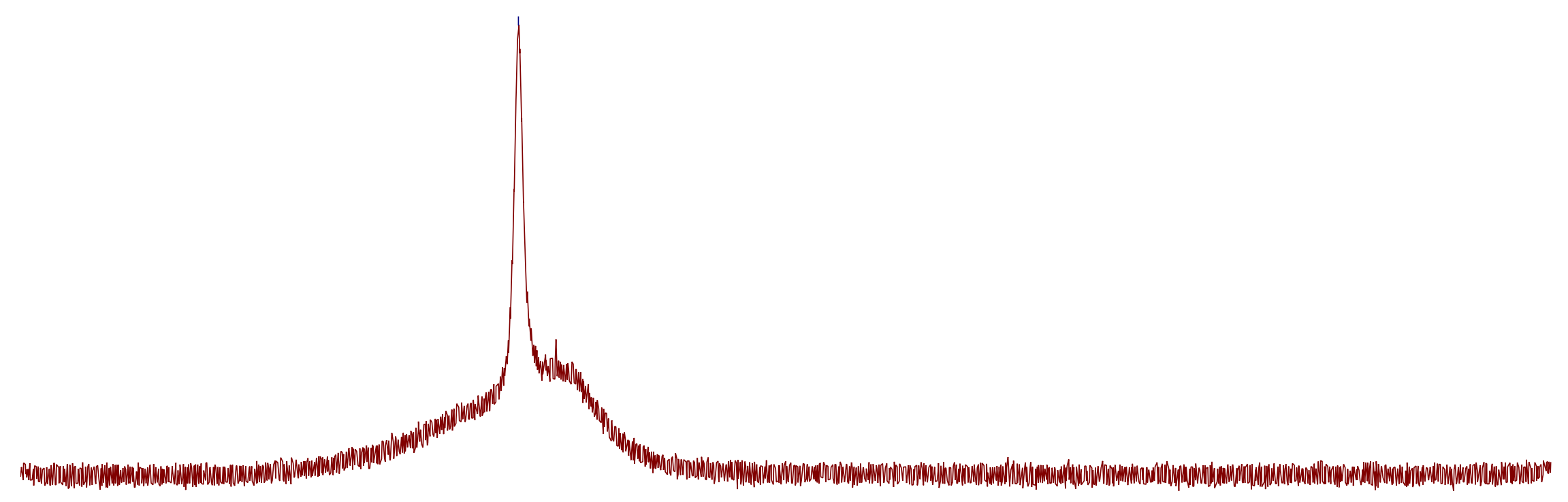

\begin{tabular}{|c|c|c|c|c|c|c|c|c|c|c|c|c|c|}
\hline 30 & 70 & 50 & 30 & 10 & -10 & -30 & m) & -70 & -90 & -110 & -130 & -150 & -170 \\
\hline
\end{tabular}


YX-3-290-19F.1.fid

AVQ-400 QNP Probe 19F starting parameters. (revised P1, 2/12/04 RN)

chemical shifts relative to $\mathrm{CFCl} 3$ at $0 \mathrm{ppm}(082103 \mathrm{HvH})$

sw 239.28 ppm; o1p 0 ppm

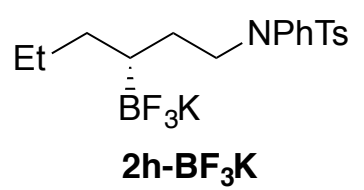

0 
YX-4-59-tube63-1H.1.fid

AV-600 ZBO proton starting parameters $11 / 16 / 08$ RN

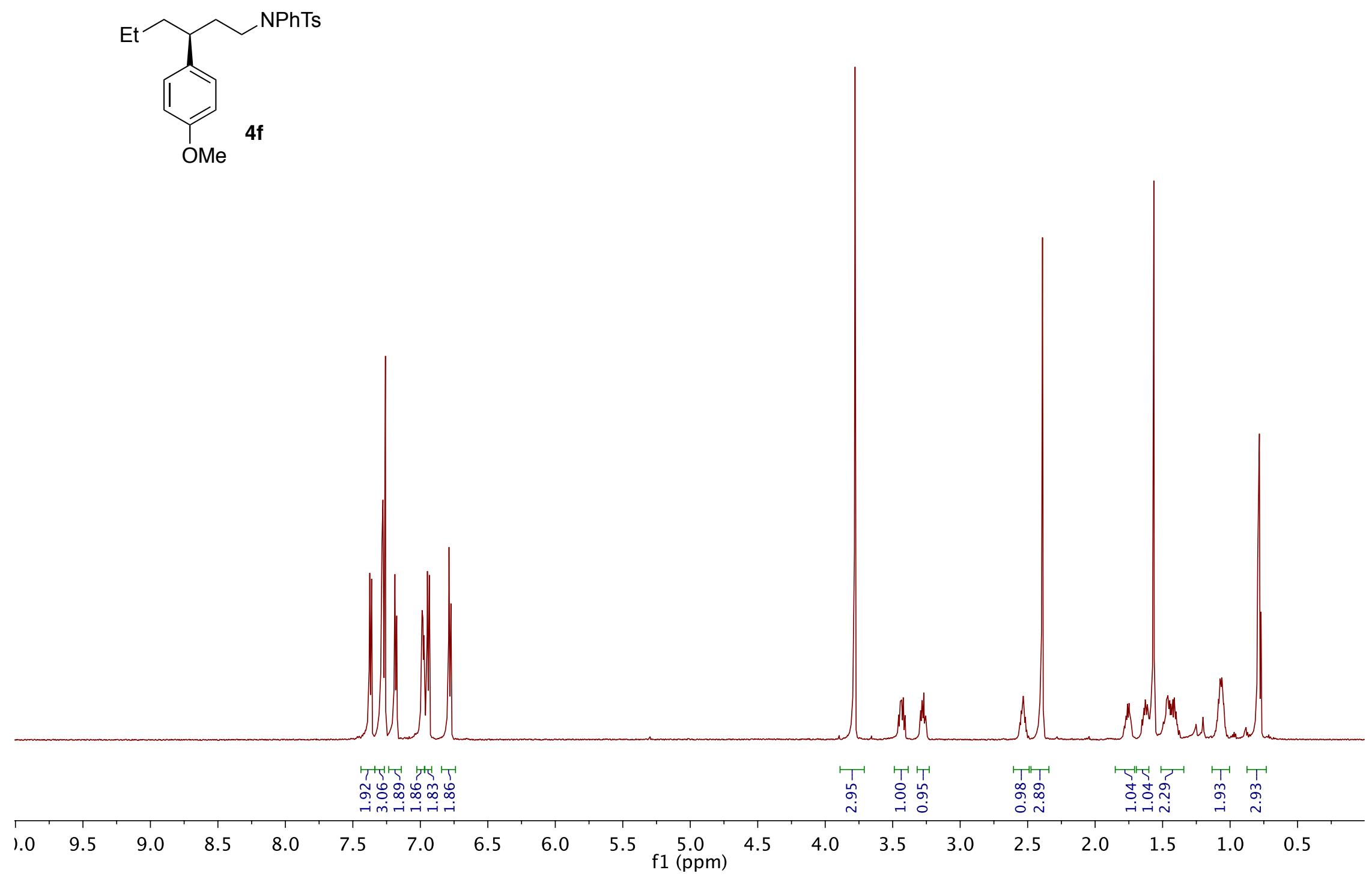



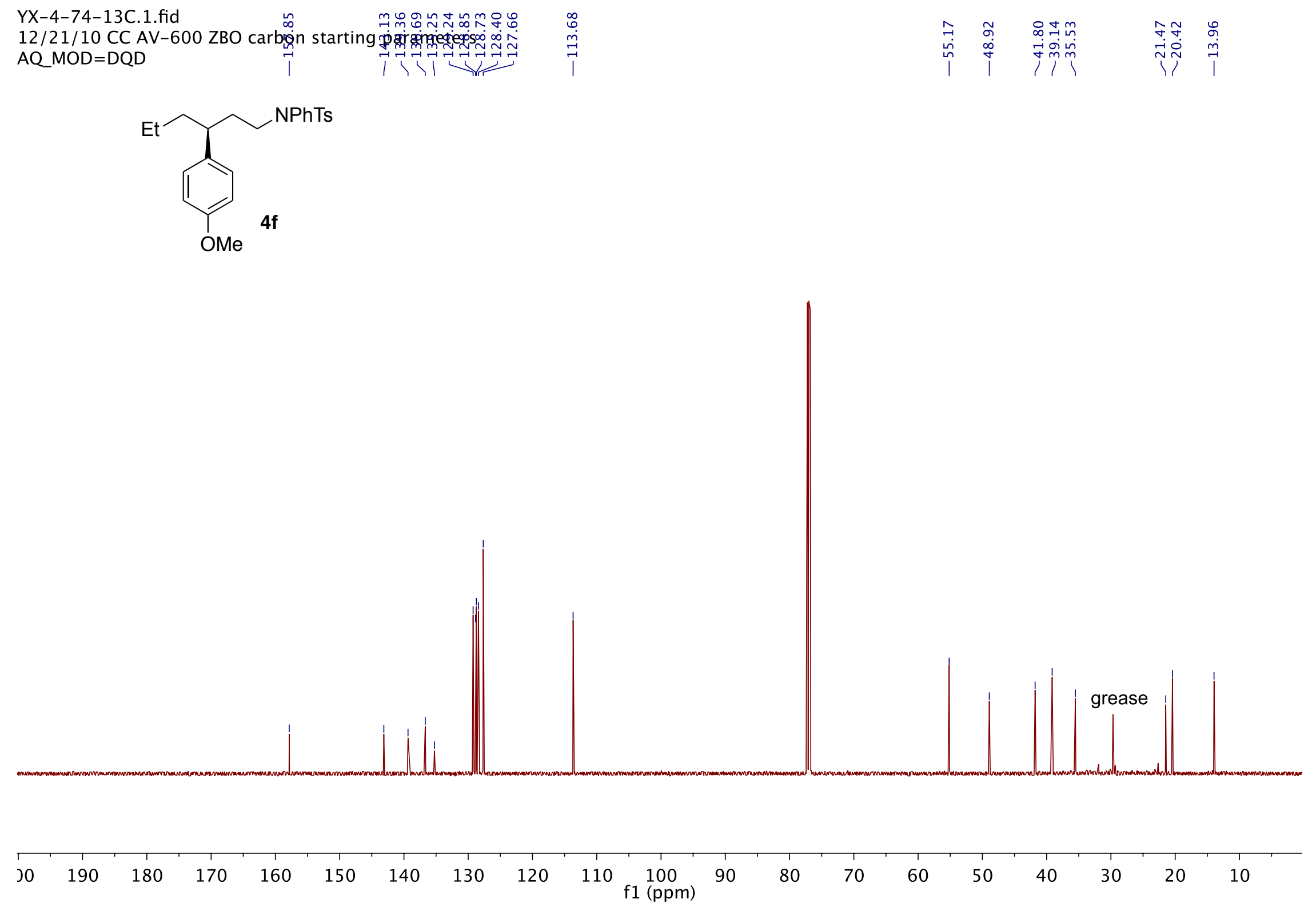
YX-4-48-1H.1.fid

CC: 12182012 AV-500 TBIP probe

1H 1D NMR

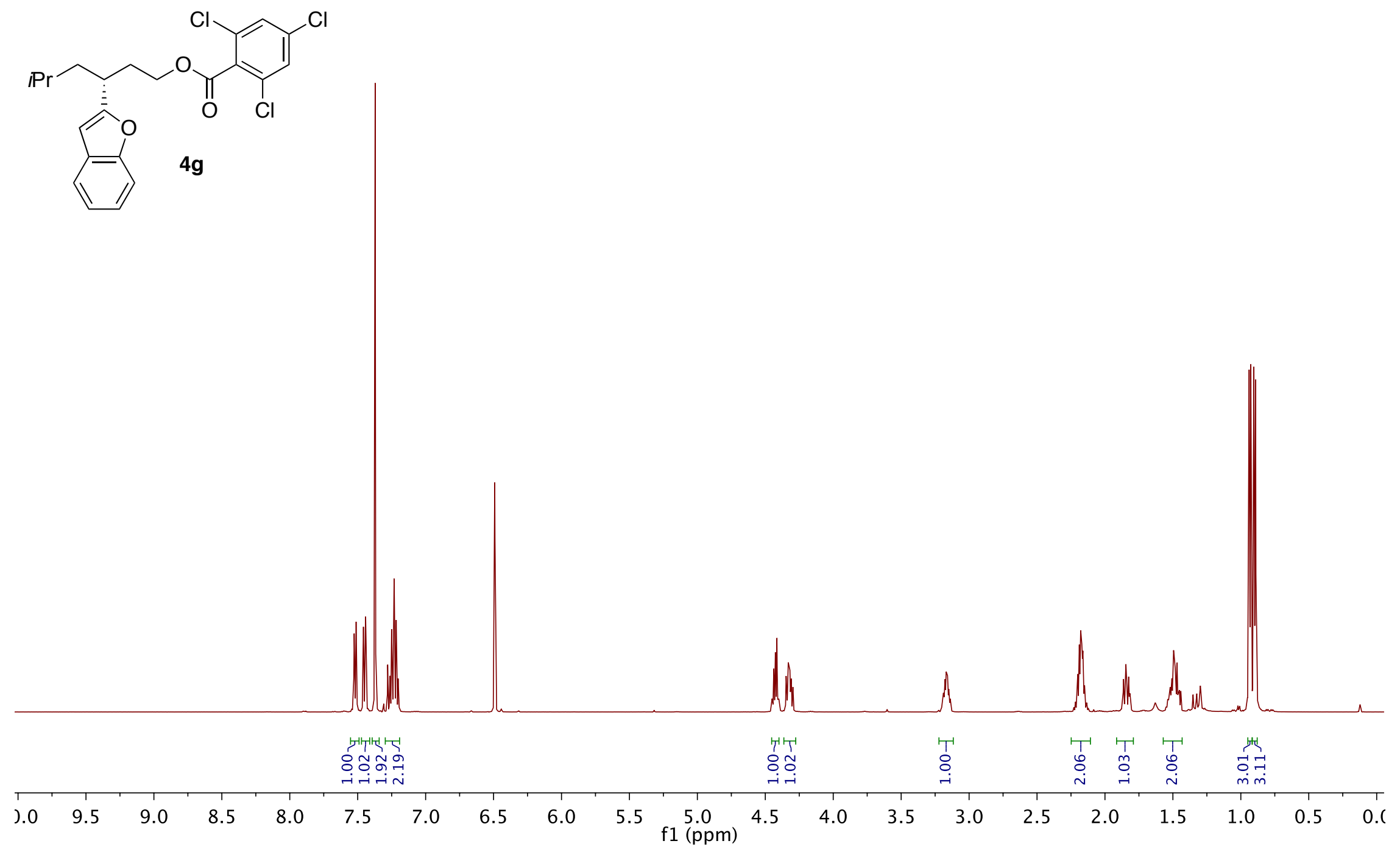




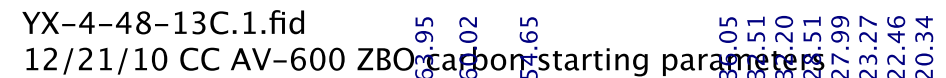
$A Q \_M O D=D Q D$ ।

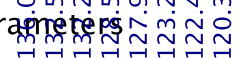

$\stackrel{0}{\infty} \quad \stackrel{\circ}{\rightarrow}$

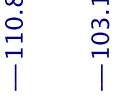<smiles>CC(C)C[C@H](CCOC(=O)c1c(Cl)cc(Cl)cc1Cl)c1cc2ccccc2o1</smiles>

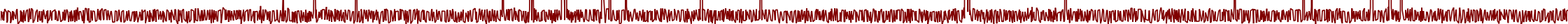

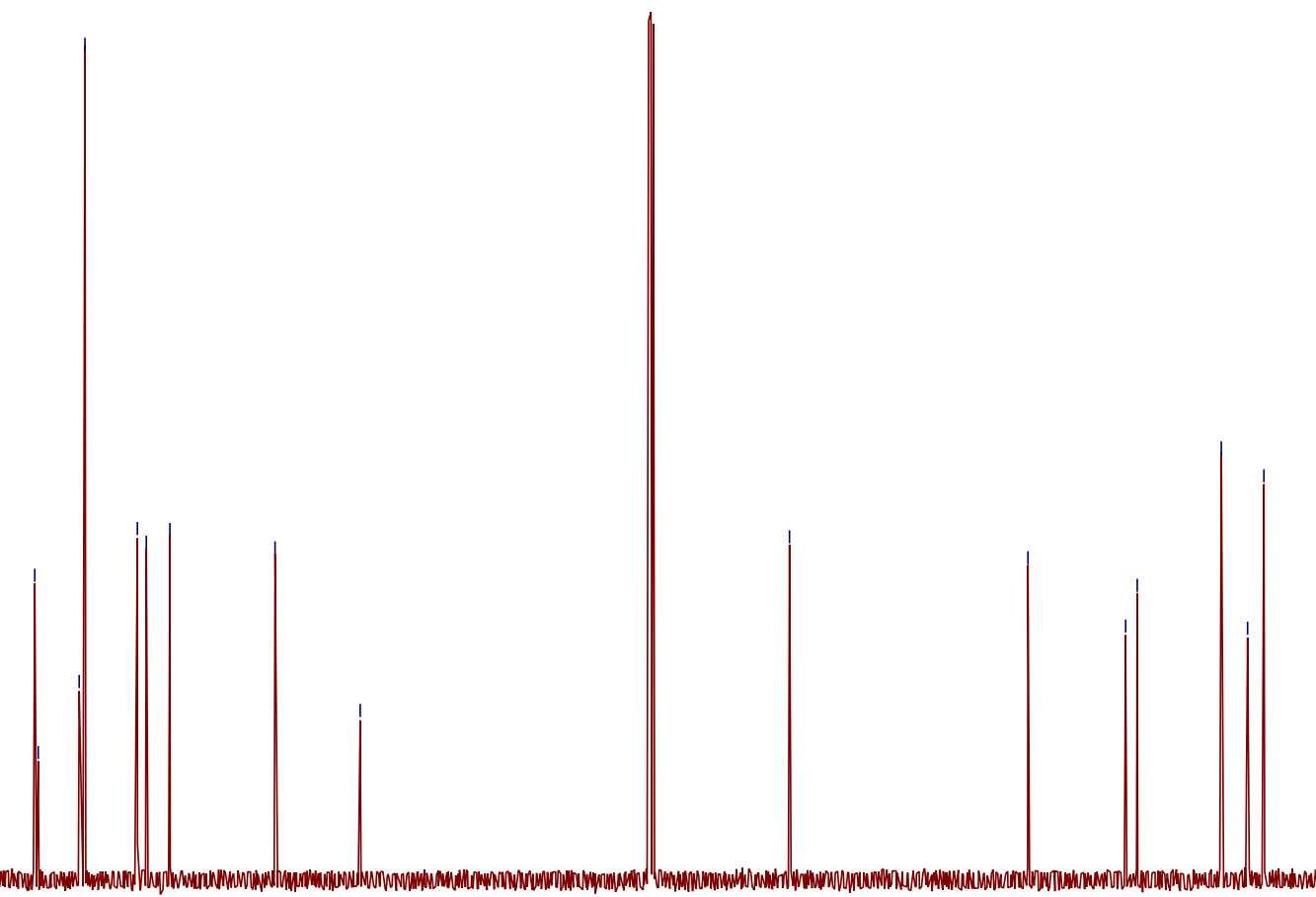

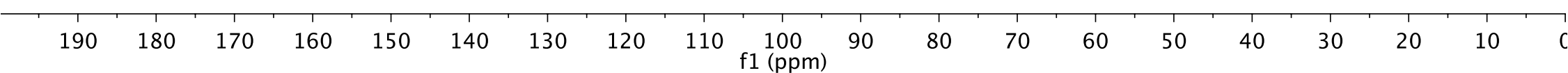


YX-3-285-1H.1.fid

CC: 12182012 AV-500 TBIP probe

1H 1D NMR
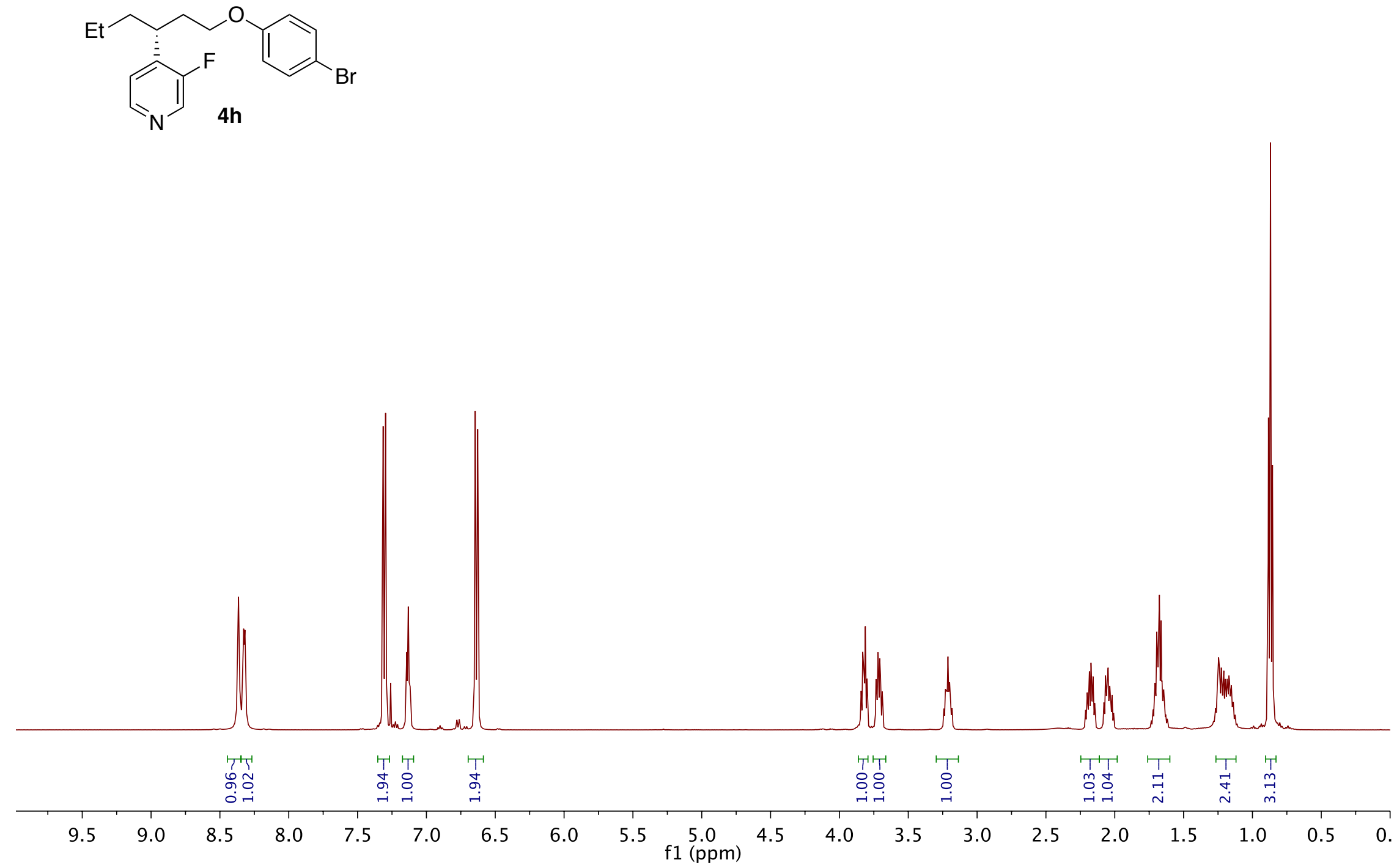


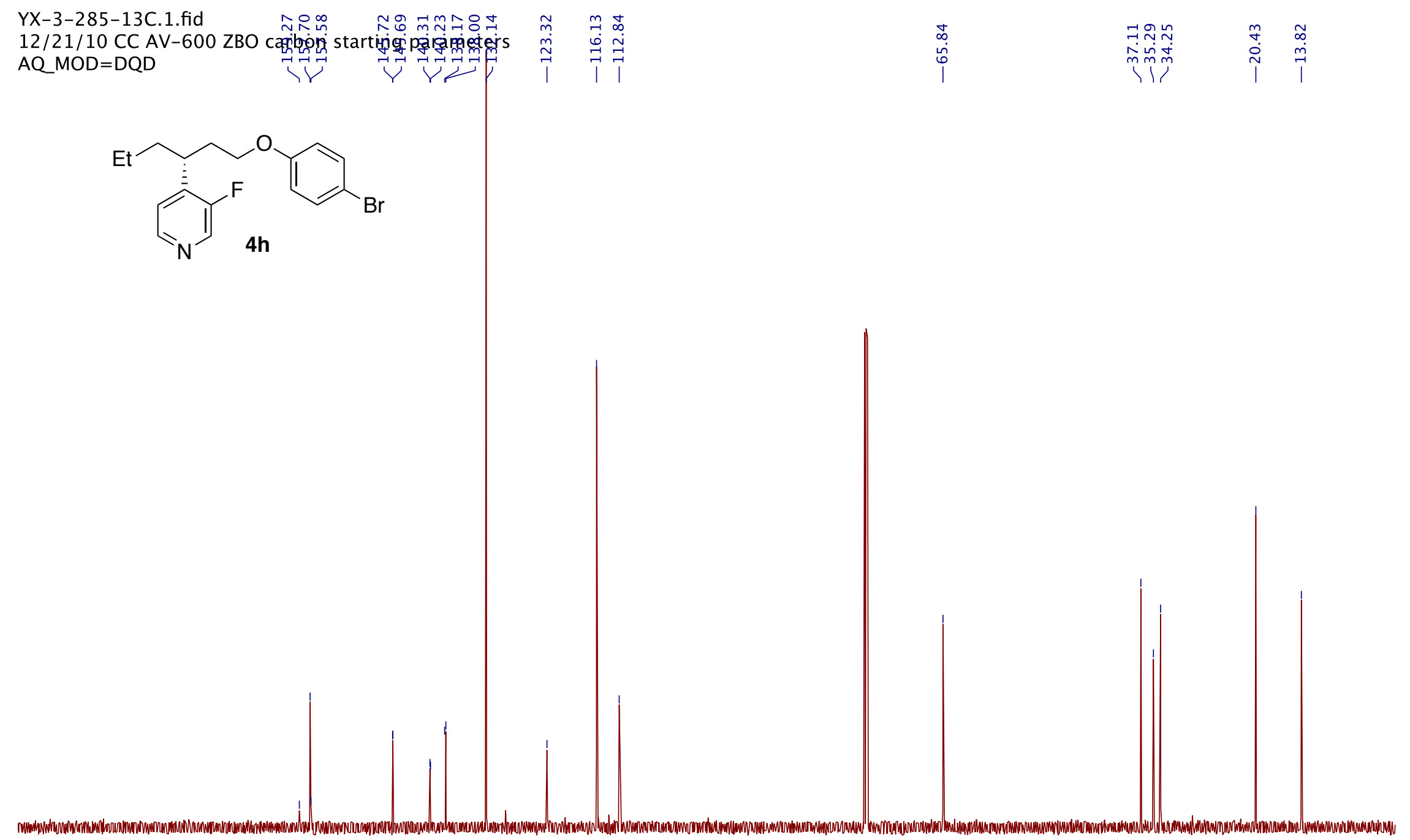

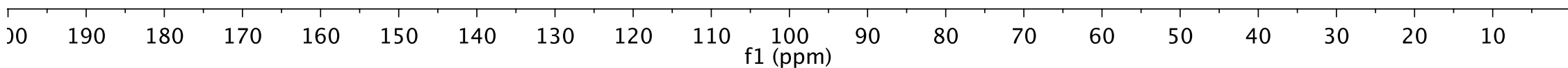


YX-3-285-19F.1.fid

AVQ-400 QNP Probe 19F starting parameters. (revised P1, 2/12/04 RN)

chemical shifts relative to $\mathrm{CFCl} 3$ at $0 \mathrm{ppm}(082103 \mathrm{HvH})$

sw 239.28 ppm; o1p 0 ppm

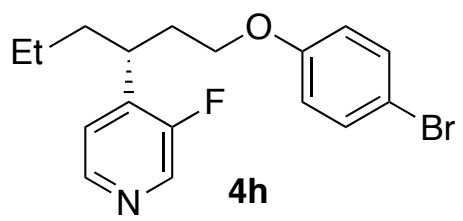

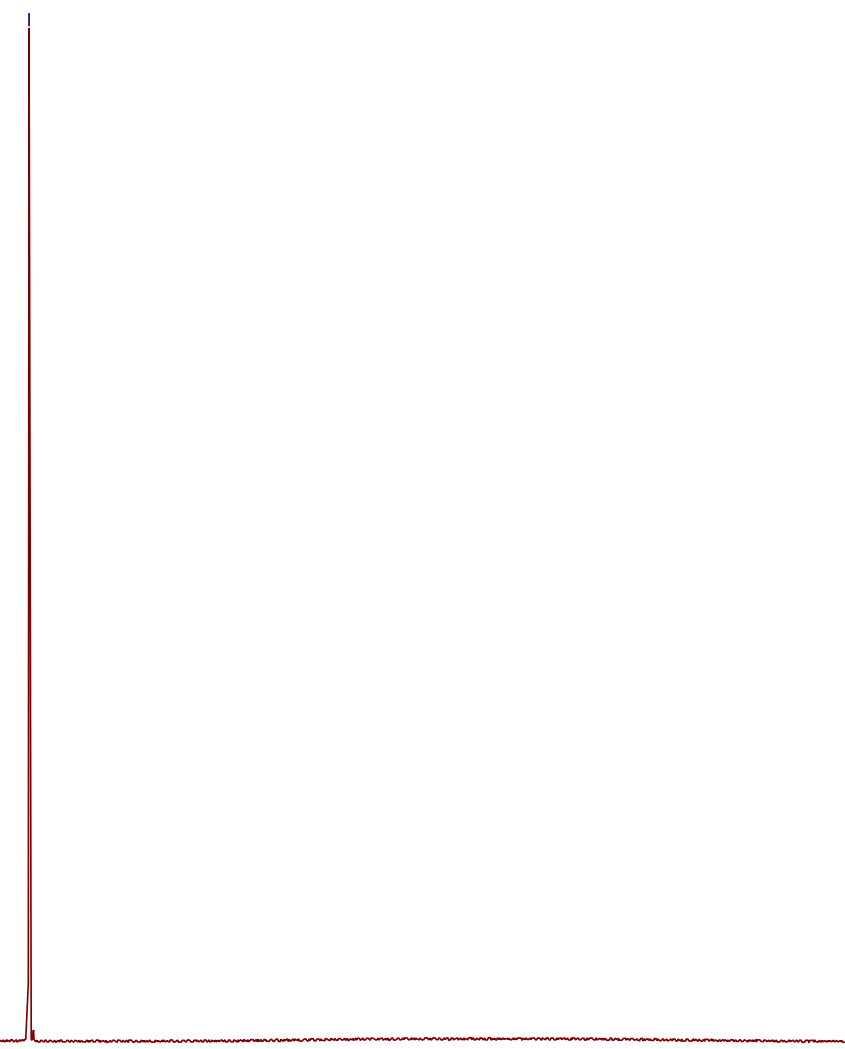

0 
YX-3-139-1H.1.fid

AV-600 ZBO proton starting parameters $11 / 16 / 08 \mathrm{RN}$
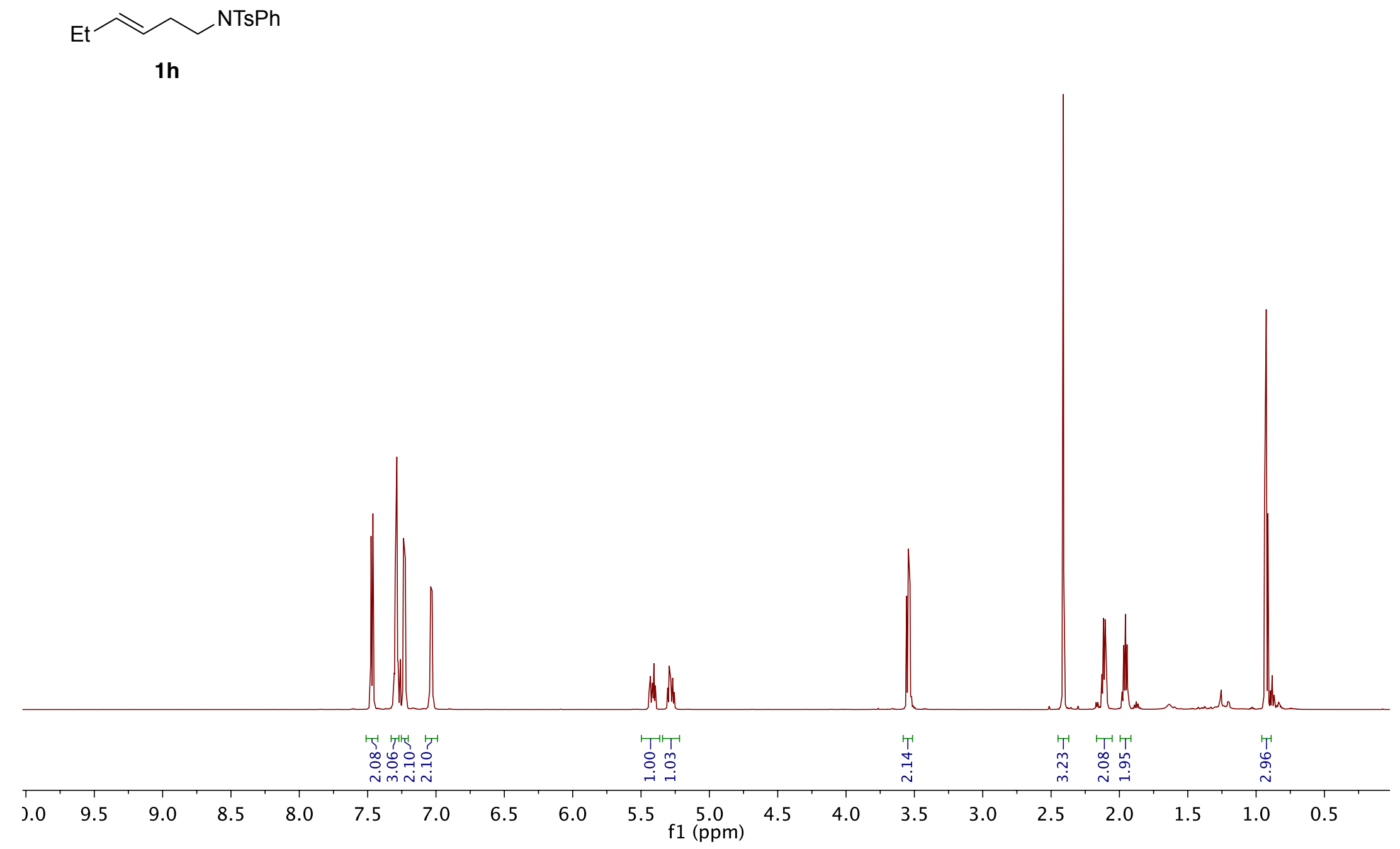
YX-3-139-13C.1.fid

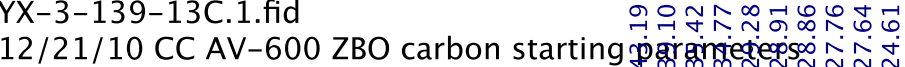

$A Q \_M O D=D Q D$

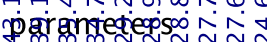

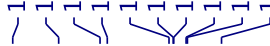

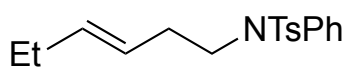

1h

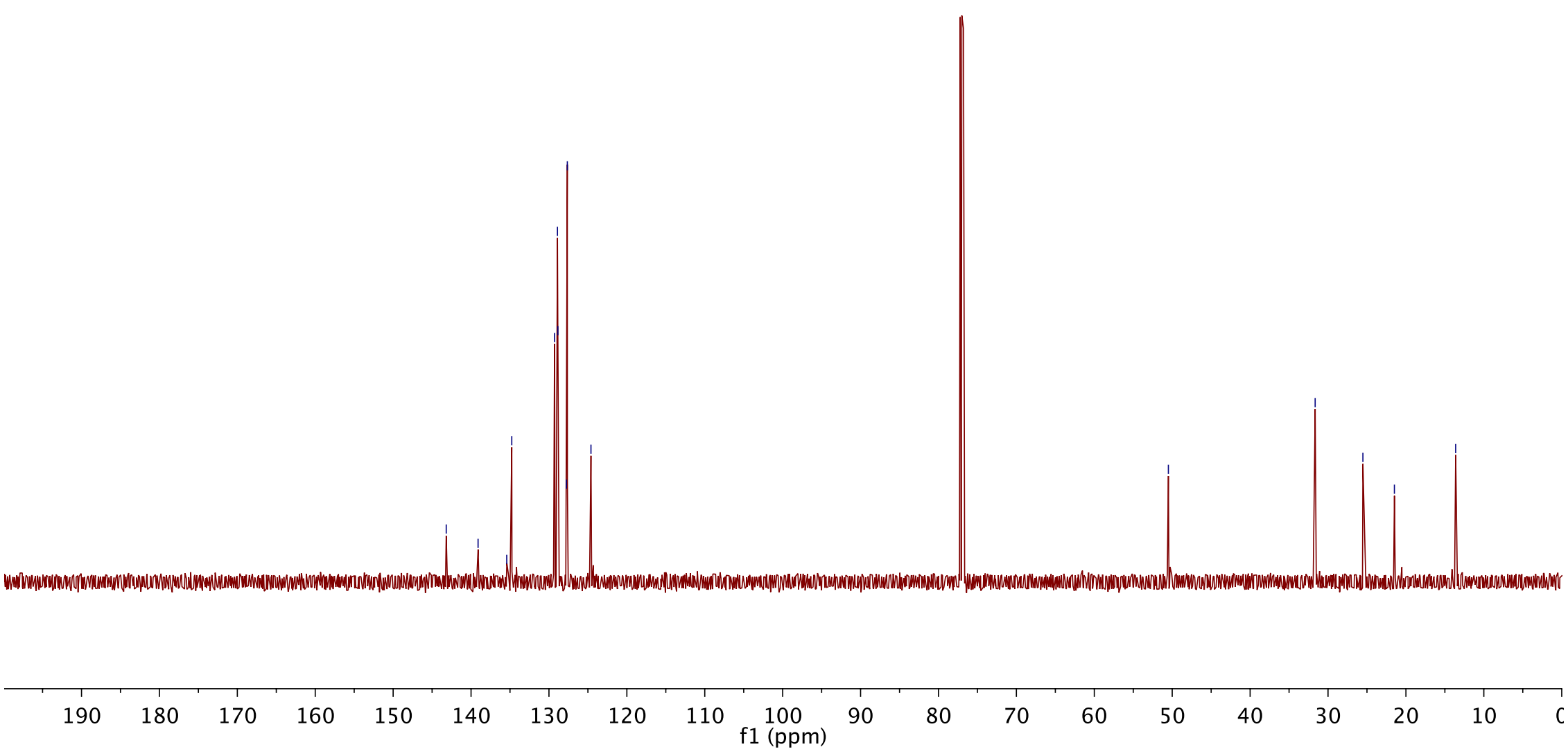


YX-3-149-1H.1.fid

AV-600 ZBO proton starting parameters $11 / 16 / 08 \mathrm{RN}$

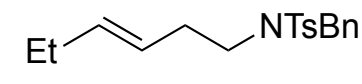

$1 \mathbf{i}$

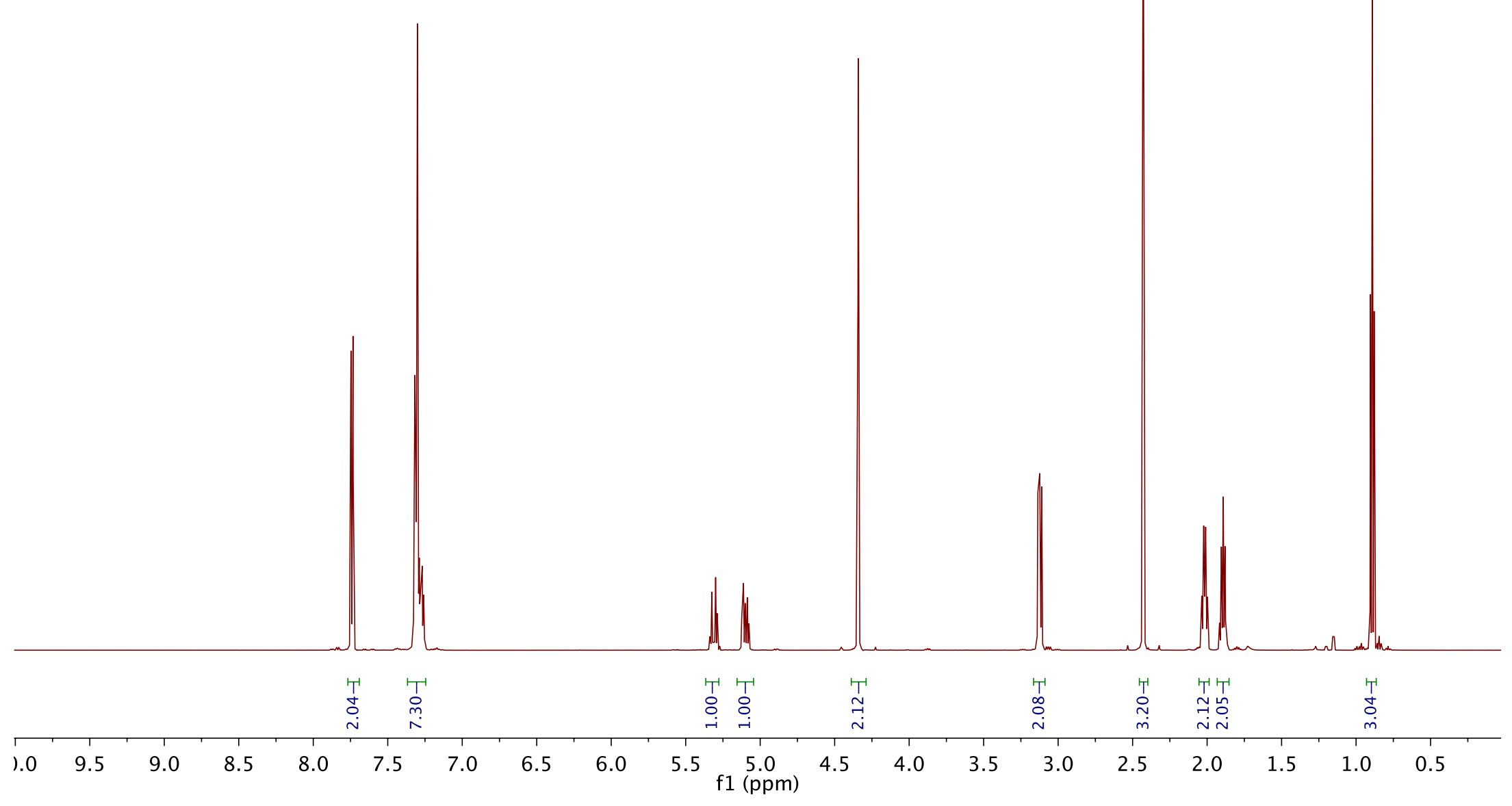


YX-3-149-13C.1.fid

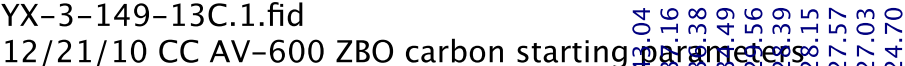

$A Q \_M O D=D Q D$

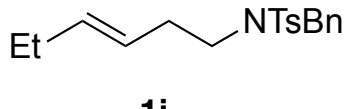

$1 i$

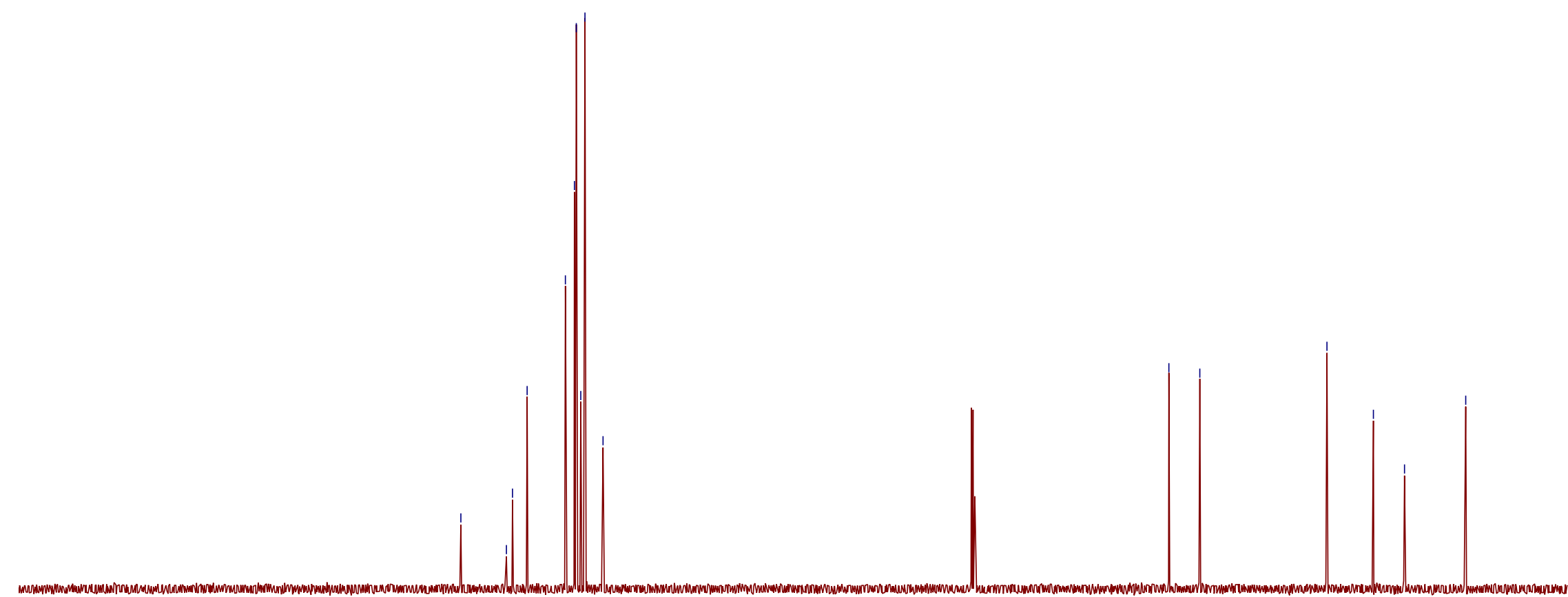

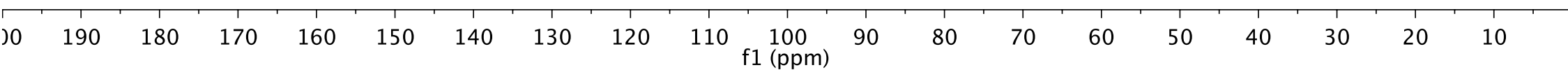


YX-3-279-1H.1.fid

AV-600 ZBO proton starting parameters $11 / 16 / 08 \mathrm{RN}$
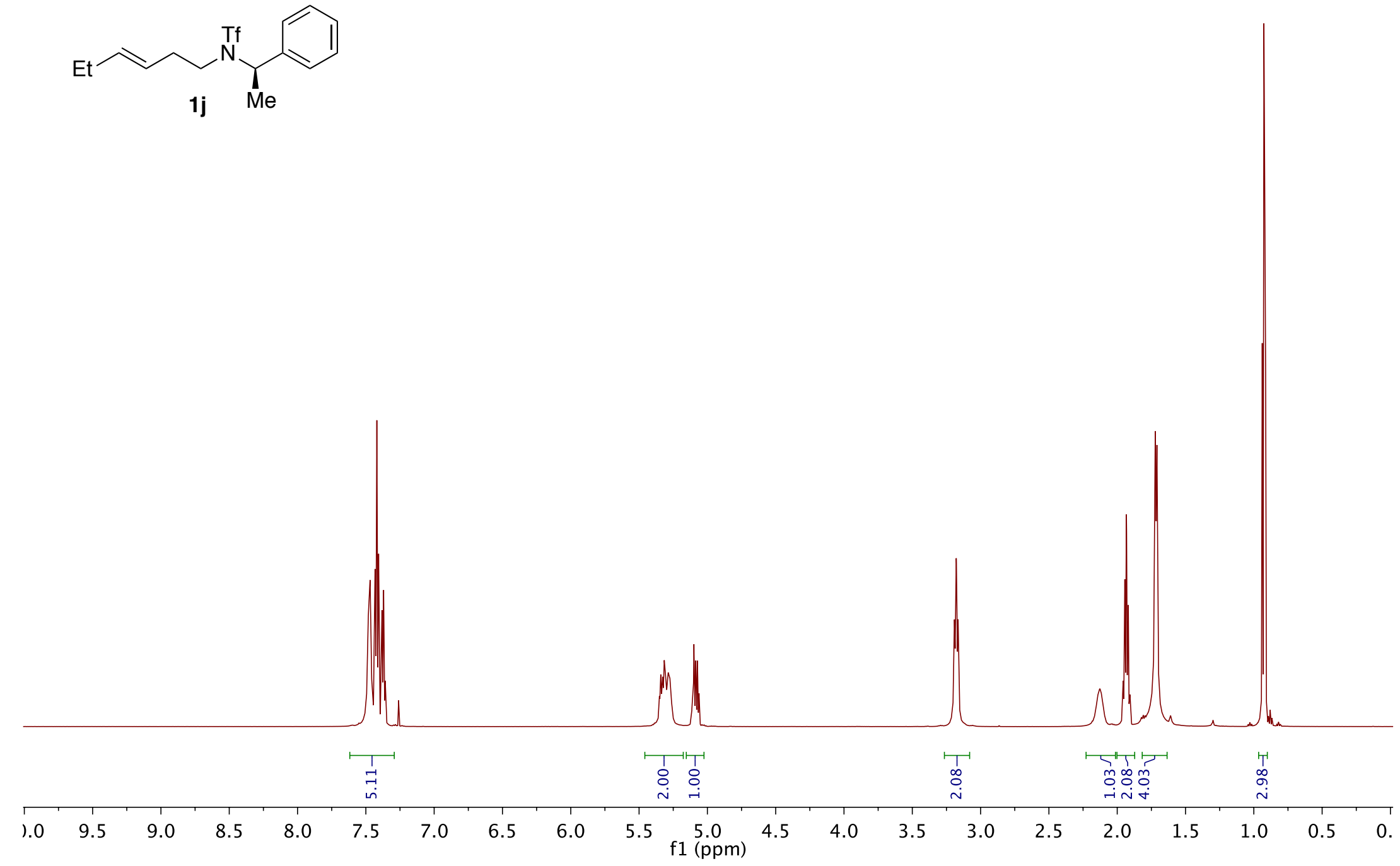
YX-3-279-13C.1.fid $A Q \_M O D=D Q D$

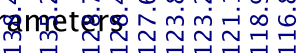

।<smiles>CC/C=C/CCN([Tl])[C@H](C)c1ccccc1</smiles>

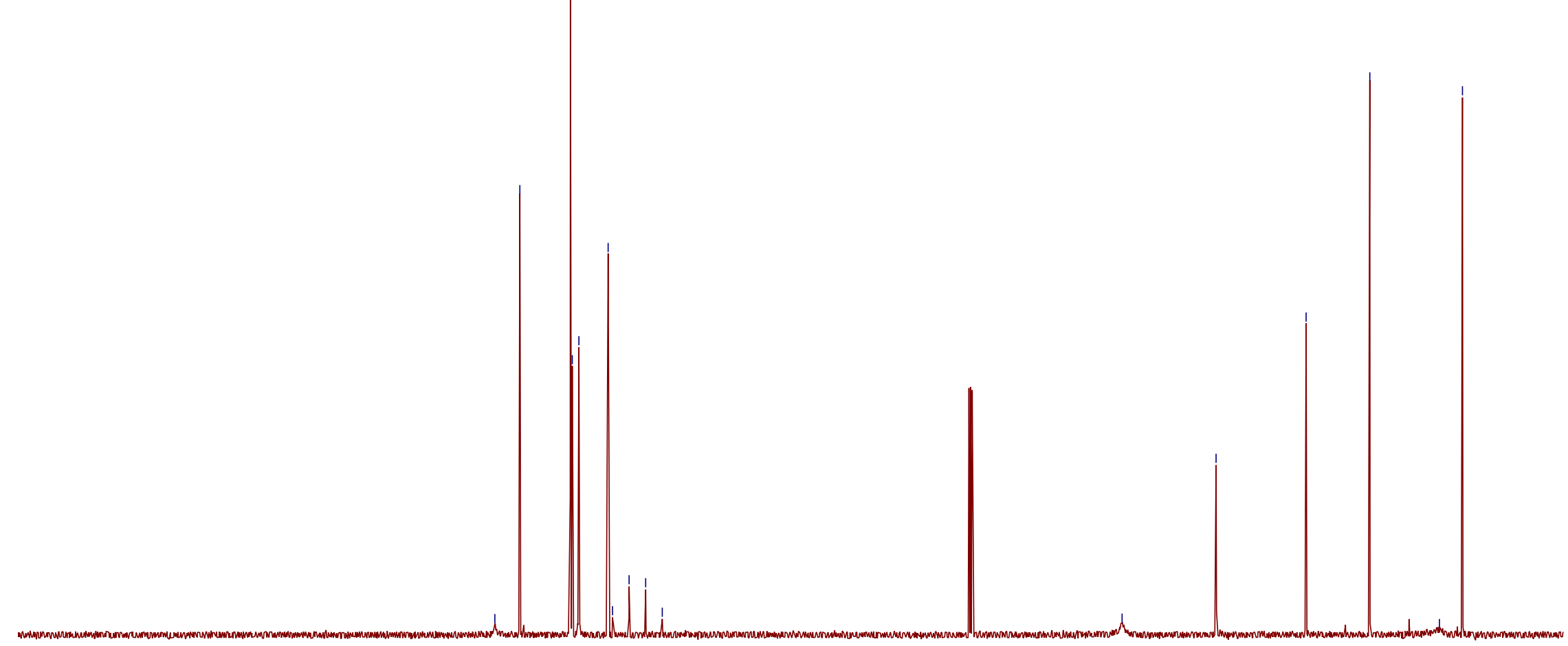

$$
30
$$

$190 \quad 180$

170

160

150

140

130

120

110

100

$90 \quad 80$

70

60

50

40

30

20

10 
YX-3-279-19F.1.fid

AVQ-400 QNP Probe 19F starting parameters. (revised P1, 2返12/04 RN)

chemical shifts relative to $\mathrm{CFCl} 3$ at $0 \mathrm{ppm}(082103 \mathrm{HvH})$

sw 239.28 ppm; o1p 0 ppm
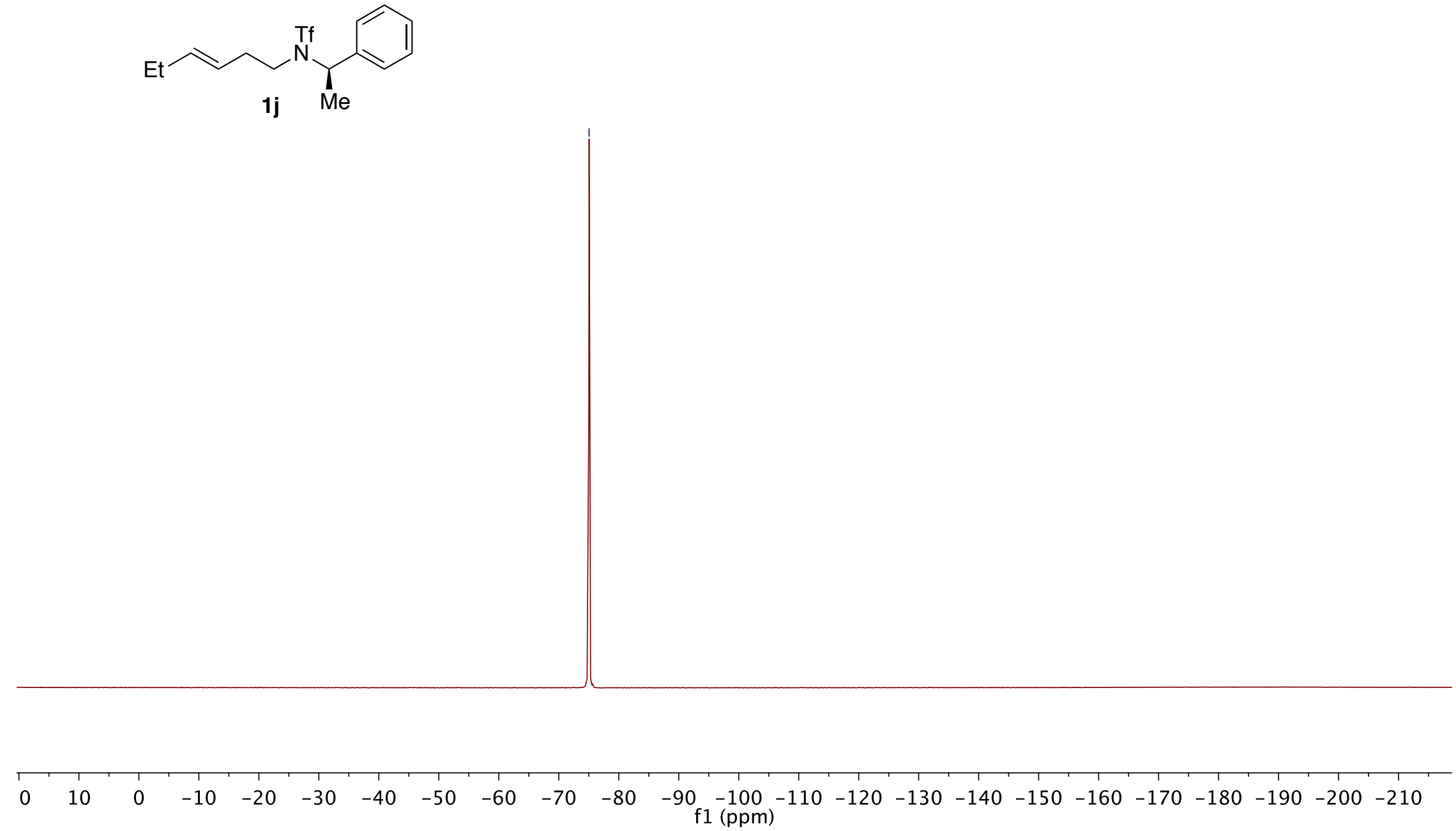
YX-2-266-1H.1.fid

CC: 12182012 AV-500 TBIP probe

1H 1D NMR

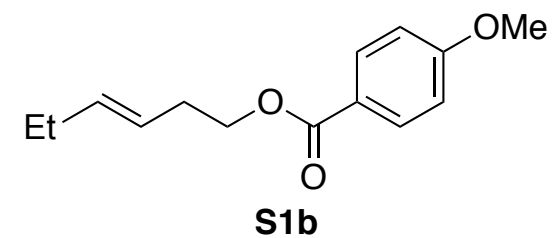

n.

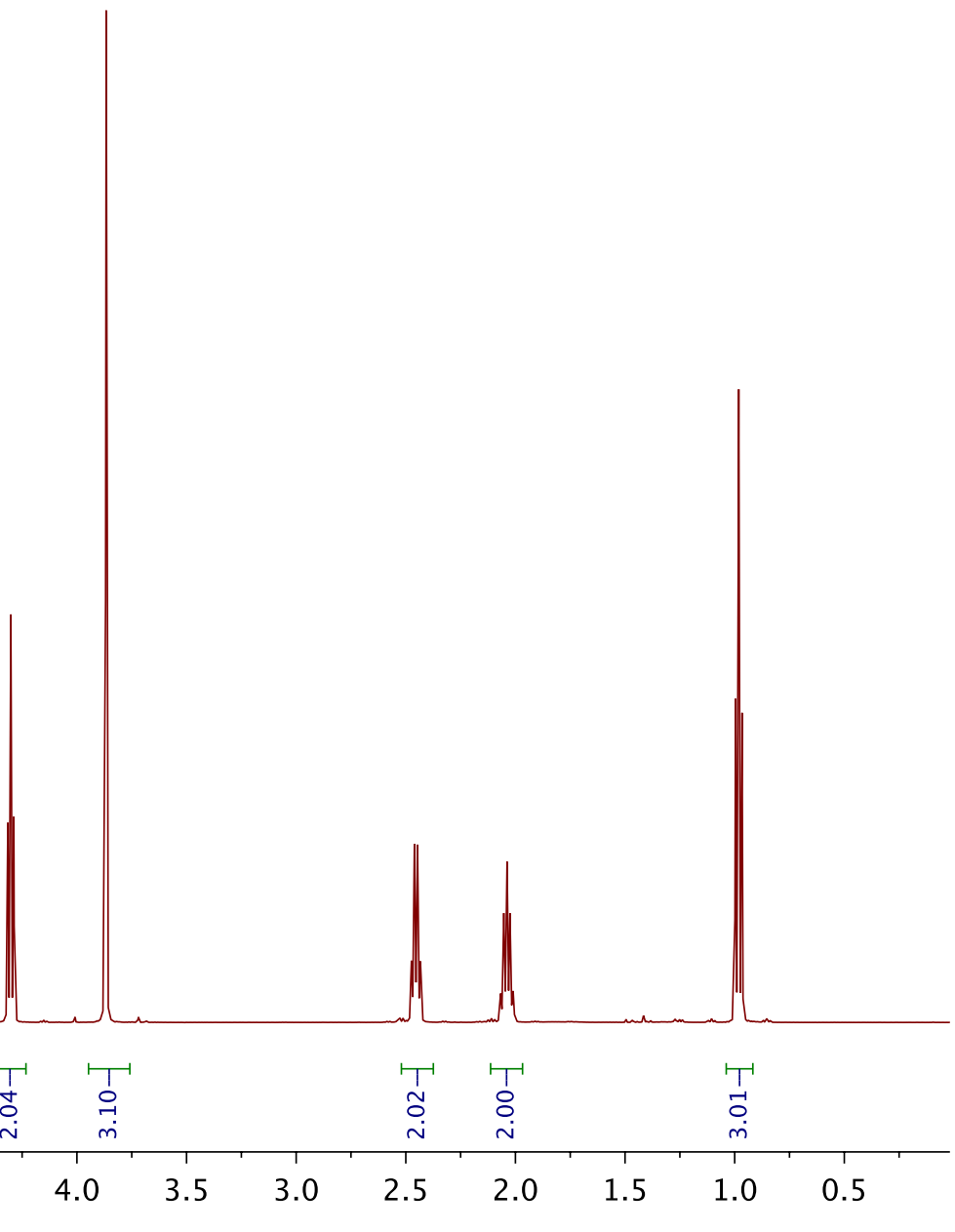


YX-2-266-13C.1.fid

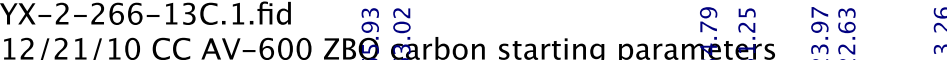

$\mathrm{AQ} M O D=\mathrm{DQD}$

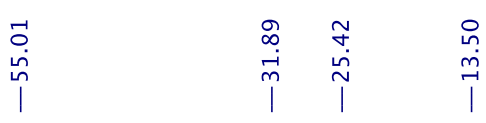

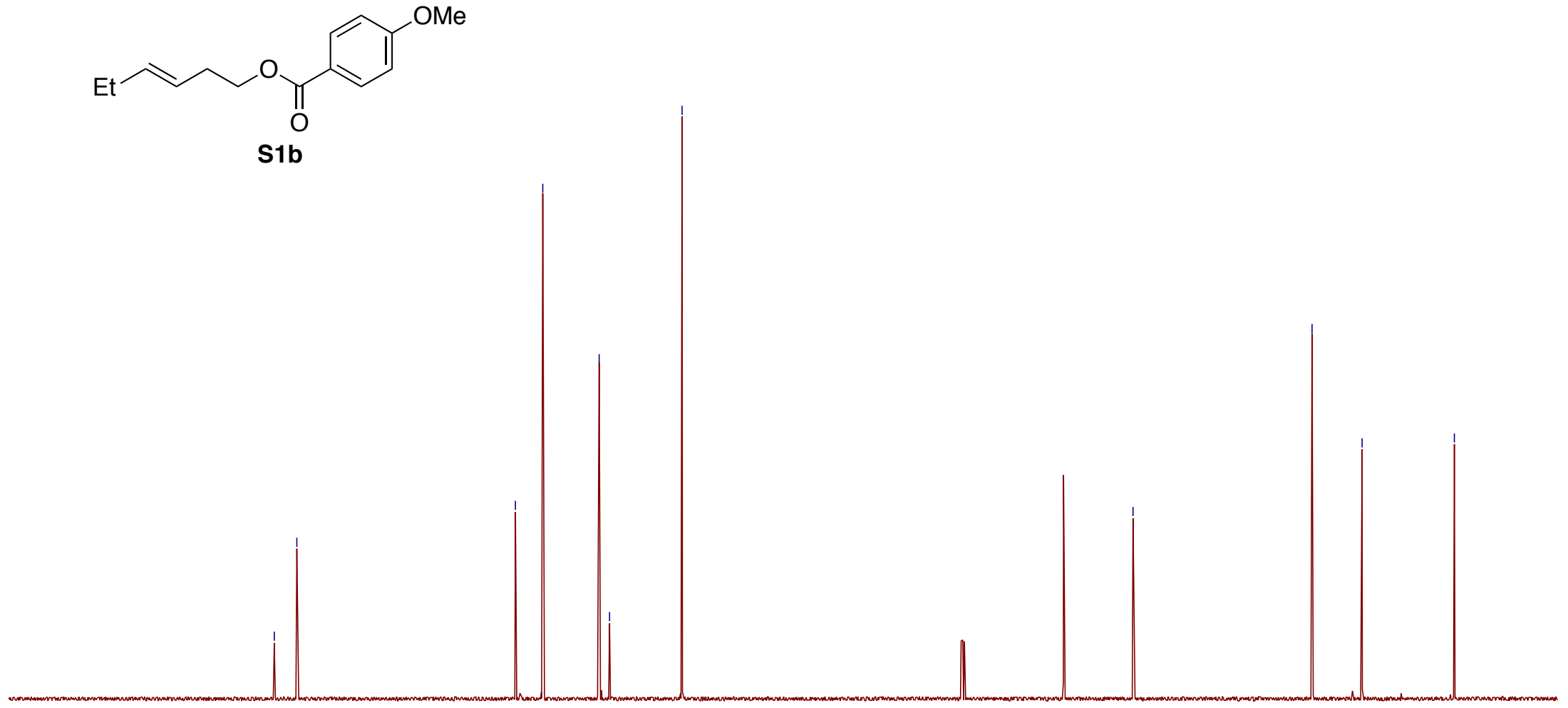

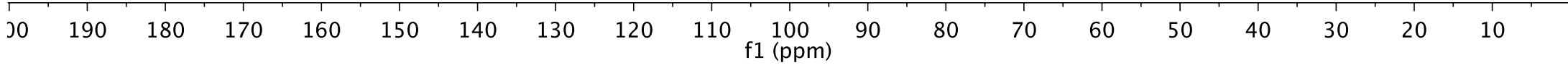


<smiles>CC/C=C/CCOC(=O)c1c(C)cc(C)cc1C</smiles>
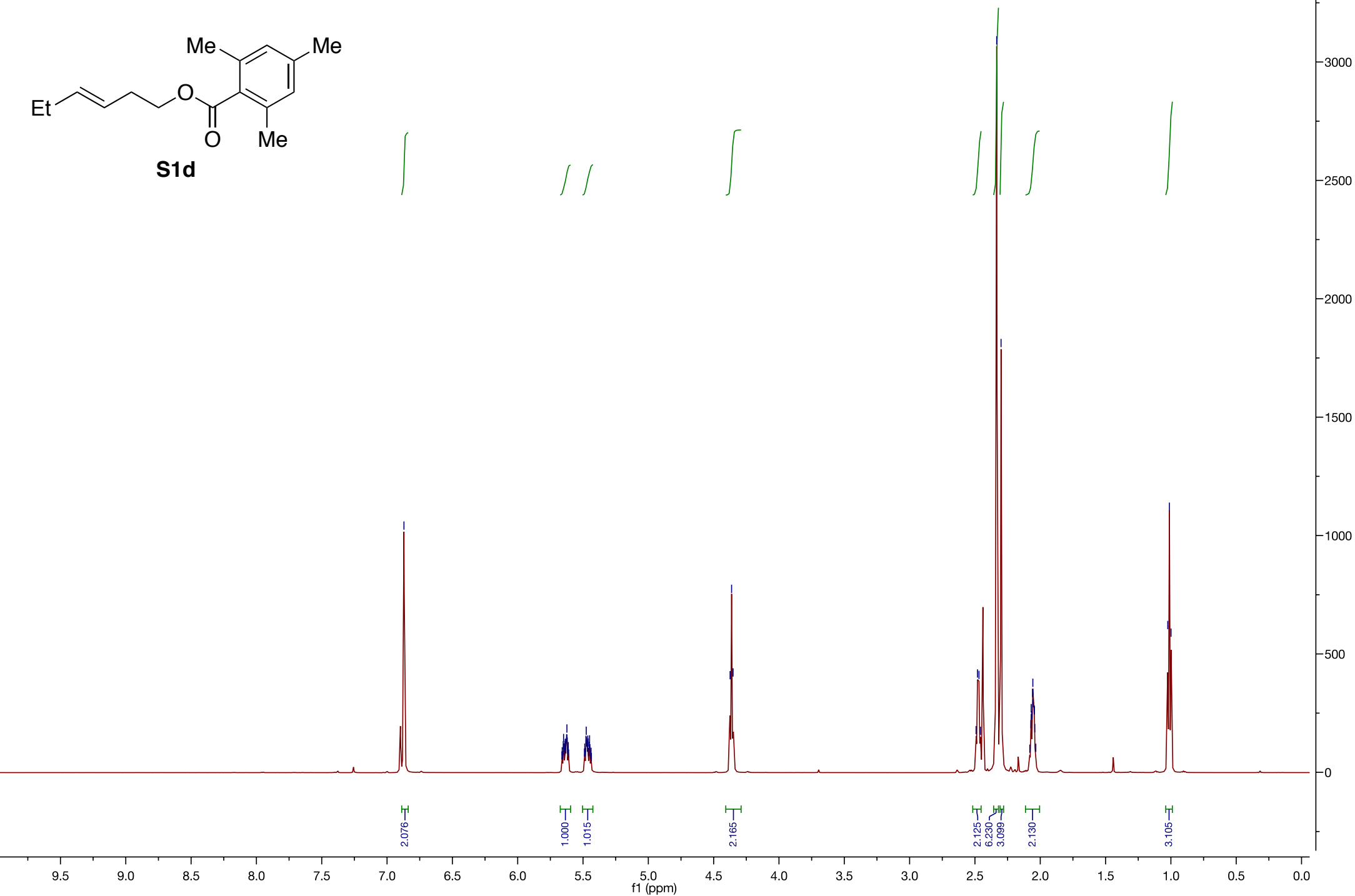
<smiles>CC/C=C/CCOC(=O)c1c(C)cc([N+](=O)[O-])cc1C</smiles>

S1d

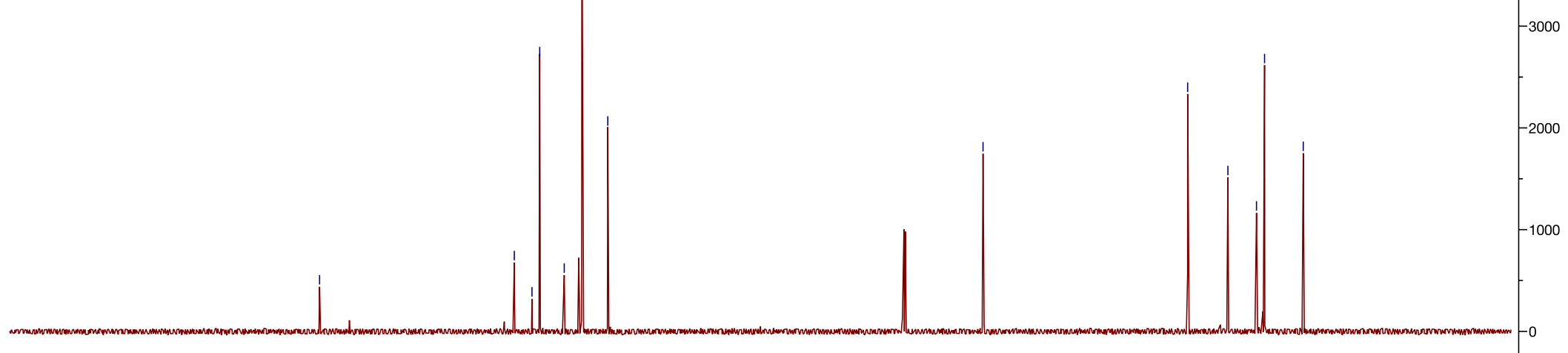

210

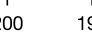

180
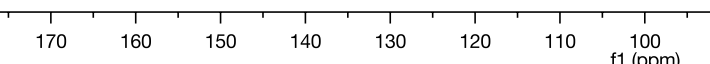

100
$\mathrm{f} 1(\mathrm{ppm})$ 
YX-2-267-1H.1.fid

CC: 12182012 AV-500 TBIP probe

1H 1D NMR

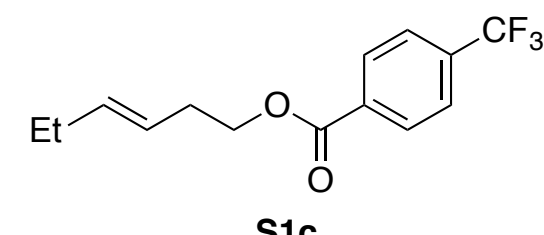

S1c

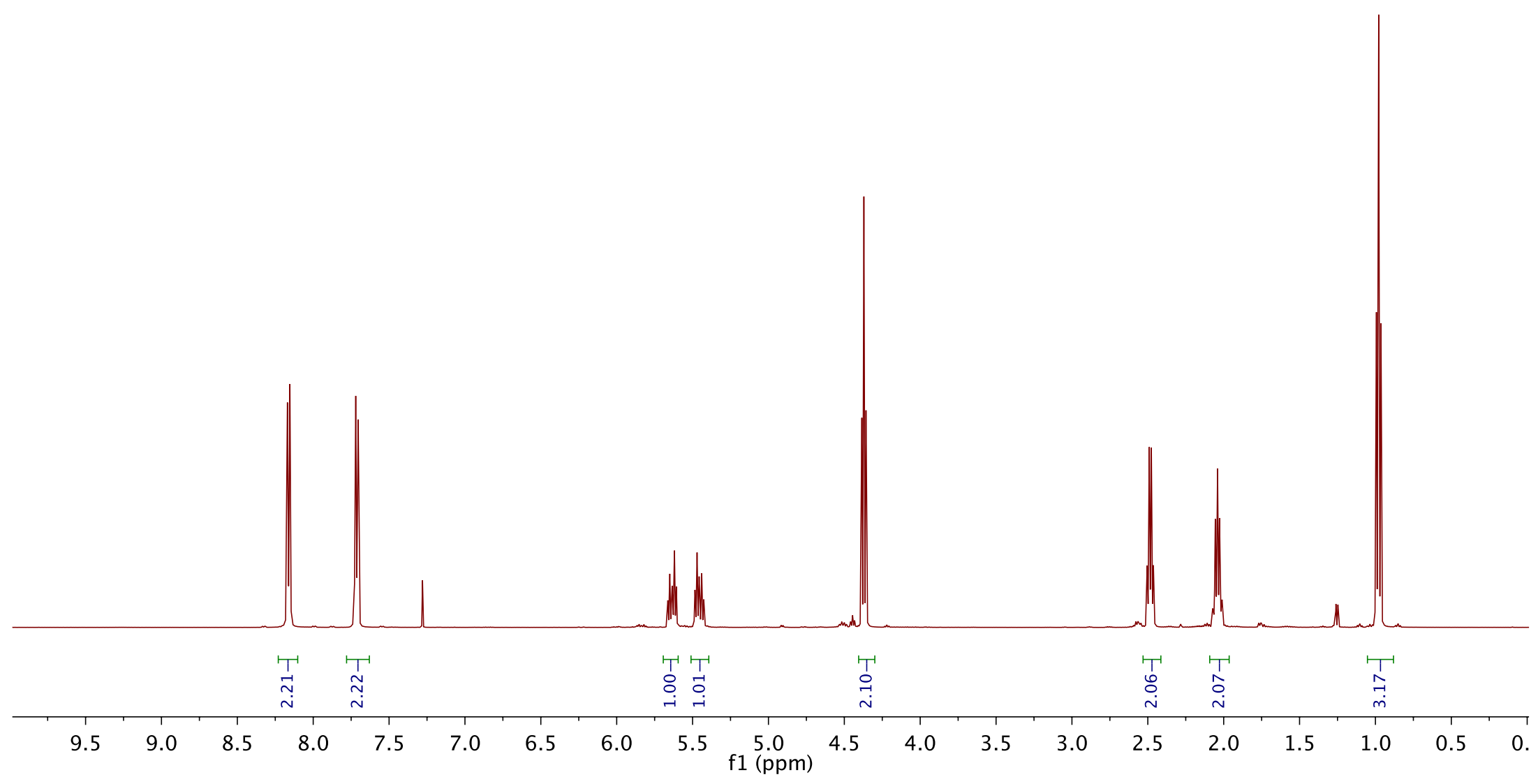




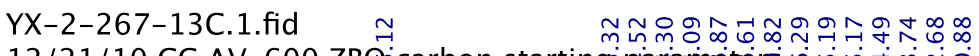

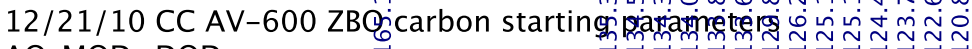

$A Q M O D=D Q D$<smiles>CC/C=C/CCOC(=O)c1ccc(C(F)(F)F)cc1</smiles>

S1c

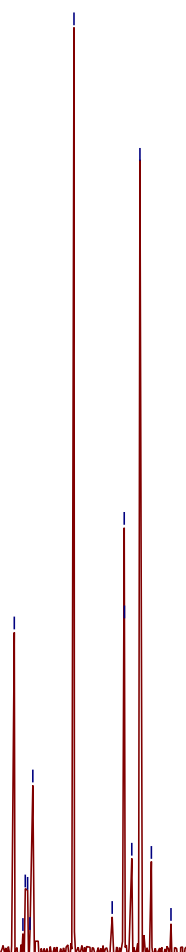

